\section{Poster Session II}

\section{December 6, 2016}

Sponsorship Statement: Publication of this supplement is sponsored by the ACNP.

Presenter disclosures are found within the abstracts. Disclosure Format: Company Name (Financial Contributor), Type of Financial Disclosure, Funding Received for Self or Spouse. Asterisks in the author lists indicate presenter of the abstract at the annual meeting.

\section{T1. Brain Activation in Mid-Insula Dynamically Tracks Cardiorespiratory Interoception}

Sahib Khalsa*, Mahlega Hassanpour, Qingfei Luo, Justin Feinstein, W Kyle Simmons, Jerzy Bodurka, Martin Paulus

Laureate Institute for Brain Research, Tulsa, Oklahoma, United States

Background: Interoceptive sensations, such as palpitations and dyspnea, are fundamental symptoms of panic anxiety. While leading theories emphasize key roles for the insular cortex in the central representation of interoceptive sensations, it is unclear whether this region responds dynamically to changes in cardiovascular state. To modulate cardiorespiratory afferent processing in healthy individuals we recently used bolus infusions of isoproterenol, a rapidly acting peripheral beta-adrenergic agonist similar to adrenaline. We observed dose-related increases in BOLD activation of the right mid dorsal insula (Hassanpour et al, in press). In the current study, we measured arterial spin labeling (ASL) during stimulation with isoproterenol. We selected ASL for this study because it is more quantitative and localized to the parenchyma than BOLD (Kim \& Ogawa. 2012), and is commonly used in pharmacological studies (Wang et al, 2011). We hypothesized that the insular cortex would show increased ASL activation during peripheral adrenergic stimulation with isoproterenol.

Methods: 23 healthy participants (11 female, mean age: 26.3 +/-6.9 years) underwent fMRI scanning during bolus intravenous administrations of isoproterenol and saline. Infusions contained either saline or isoproterenol (1 or 2 micrograms $(\mathrm{mcg})$ ). Each dose was repeated twice (six infusion scans). Infusion administration was double blinded, delivered 60 seconds into the scan, and infusion order was randomized across participants. During each infusion participants continuously rated their experience of cardiorespiratory sensation intensity by rotating a dial. Anatomical and functional MRI data were acquired using a 3 Tesla GE MR750 MRI scanner with an 8-channel receive-only head coil. Perfusion images were acquired using a pseudo continuous ASL (pCASL) pulse sequence with a single- shot accelerated gradient echo EPI readout $(\mathrm{SENSE}=2, \mathrm{FOV} /$ slice $220 / 5 \mathrm{~mm}$, matrix $64 \times 64,24$ slices, TR/TE $=4000$ $/ 13 \mathrm{~ms}$, labeling duration/post-labeling delay $=1800 / 1500$ $\mathrm{ms})$. Pulse oximetry and respiration waveforms were concurrently recorded, and RETROICOR (Glover et al, 2000) was applied separately on tag and control pCASL images to reduce physiological noise artifacts due to respiratory and cardiac pulsations. Perfusion images were calculated using pair-wise subtraction between control and label images, and cerebral blood flow (CBF) was quantified using a general kinetic model (Buxton et al, 1998). CBF data were normalized to MNI152 atlas using a linear affine transformation. A voxelwise whole brain analysis was used to map the brain's response to each infusion. Response maps were generated using a block averaging method, by subtracting brain activation during each baseline period (0-60 seconds) from the corresponding peak response period (80-140 seconds). Group-level statistical maps were generated using a random effects analysis.

Results: As expected, isoproterenol infusions elicited dosedependent increases in cardiorespiratory sensation $(p<0.001)$, with $100 \%$ of participants endorsing increased palpitations and dyspnea at the $2 \mathrm{mcg}$ dose. Cardiorespiratory intensity ratings with the dial correlated significantly with observed heart rate changes during the $1 \mathrm{mcg}$ $(\mathrm{R} 2=0.25, p=0.013)$ and $2 \mathrm{mcg}$ dose $(\mathrm{R} 2=0.20, p=0.03)$. A voxelwise whole brain analysis of the saline and $1 \mathrm{mcg}$ infusions did not reveal any significant clusters of activation in the insula or other somatosensory brain regions. During the $2 \mathrm{mcg}$ infusion, a significant cluster of activation was observed in the mid dorsal insula on the right $(p<0.005$, uncorrected). A time series analysis of the right insula revealed an increase in CBF corresponding to the time course of both the objective heart rate changes and the subjective dial ratings.

Conclusions: These findings, which represent a replication of our previous BOLD imaging study, confirm that the insular cortex, particularly the right mid dorsal insula, is a key region that responds dynamically to changes in cardiorespiratory interoceptive state. Given the prominent role of interoceptive sensations in anxiety and eating disorders, future studies examining whether abnormal insula activity explains interoceptive processing deficits in these disorders are warranted.

Keywords: Interoception, Palpitation, Dyspnea, Anxiety and Insula.

Disclosure: Nothing to disclose.

\section{T2. Functional and Anatomical Organization of Amygdala Neural Populations Encoding Emotional Valence}

Anna Beyeler*, Margaux Silvestre, Praneeth Namburi, Gwendolyn Calhoon, Amy Sutton, Gordon Glober, Craig Wildes, Kay Tye

Massachusetts Institute of Technology, Cambridge, Massachusetts, United States

Background: Inappropriate assignment of emotional valence and dysfunctions of the circuits that govern valence processing are thought to underlie many psychiatric diseases 
including anxiety, depression, addiction and compulsive disorders. From a fundamental perspective, understanding how the brain attributes valence to contexts and elements of our environment is one of the main challenges in neuroscience. The basolateral amygdala (BLA) is known to play a critical role in the formation of associative memories of both positive and negative valence and contains populations of projection neurons, including populations targeting the nucleus accumbens (BLA-NAc), the central nucleus of the amygdala (BLA-CeA) and the ventral hippocampus (BLA-vHPC). We previously showed that the BLA-vHPC projection regulates anxiety-related behaviors and that learning experiences of opposite valence differentially regulate the synaptic inputs onto BLA-NAc and BLA-CeA projector populations. However, the topographical organization of these populations, their local connectivity and their naturally-occurring firing patterns during information processing are incompletely understood.

Methods: To characterize the topographical organization of BLA-NAc, BLA-CeA and BLA-vHPC projector populations within the BLA, we used a retrograde as well as anterograde tracing approaches. To retrogradely fill the projectors we injected three different retrograde tracers (fast blue, choleratoxin-B (CTB) coupled to a red fluorophore (AF-555) or coupled to a far red fluorophore (AF-647)) in the three downstream targets (NAc, CeA or vHPC). Which fluorophore was injected to which region was counterbalanced between animals. One week after injection we collected the brains and processed them with classical histology protocols. To anterogradely label the projectors we used a dual virus strategy. We injected a retrograde viral vector carrying the gene coding for the Cre recombinase (cre) in one downstream target and injected a Cre-dependent vector coding for an enhanced yellow fluorescent protein (eYFP) in the BLA. Five weeks after viral expression we collected the brains and made them optically transparent by using CLARITY before imaging the entire brain for quantification. To stimulate BLA projectors, we also used a dual virus strategy. In this case, we injected a retrograde viral vector carrying the gene coding for cre in one downstream target and injected a Cre-dependent vector coding for the light sensitive protein channelrhodopsin-2 (ChR2) fused to eYFP within the BLA. To identify the local connectivity of BLA-vHPC population with BLA-NAc and BLA-CeA population, we performed ex vivo whole-cell patch-clamp recordings from visually identified projectors and photo-stimulated one of the two other projector populations expressing ChR2. Finally, to reveal the coding properties of the three populations we used in vivo optogenetic-mediated phototagging in combination with large-scale electrophysiological recordings during the retrieval of positive or negative associative memories.

Results: Our anatomical tracing experiments showed that BLA-NAc projectors are denser in the medial BLA while BLA-CeA projectors are denser in the lateral part of the BLA. The BLA-vHPC are evenly represented in the medial and lateral BLA but are denser in the dorsal amygdala (LA). Although there is heterogeneity in response profiles within each of these projection target defined populations, we found that BLA subpopulations differentially encode the retrieval of positive or negative associative memories. Specifically, we found that the proportion of BLA-NAc neurons excited during presentation of a reward predictive cue was overrepresented relative to the entire population of BLA neurons. Contrastingly, the relative proportion of BLA-CeA neurons excited during the presentation of an aversive cue was enhanced compared to the entire population of BLA neurons. Finally, BLA-vHPC did not preferentially code for learned associations of positive or negative valence.

Conclusions: We observed that BLA populations defined by their downstream target (NAc, CeA and vHPC) are topographically organized in the amygdala and have local synaptic interactions. During memory retrieval of emotional associations, we found heterogeneous responses to the conditioned stimuli within the BLA projector populations, yet we provided evidence that information regarding cues of positive and negative valence is routed to divergent circuits. Although the BLA-vHPC projection has been shown to drive negative valence in innate - rather than learned - behaviors, these neurons did not preferentially code for negative valence memories, supporting a model of distinct routing for learned and innate valence-related information. Together, these findings suggest that valence encoding in the BLA is at least partially accomplished through the divergent activity of anatomically defined neuronal populations.

Keywords: Anxiety, Valence, Amygdala, Neural Populations and Photoidentification.

Disclosure: Nothing to disclose.

\section{T3. Rethinking Extinction: Utilizing Advances in Computational Learning Theory and Neuroscience to Augment Traditional Fear Extinction Protocols}

\section{Joseph Dunsmoor*}

\section{New York University, New York, New York, United States}

Background: Once acquired, it is often necessary to update a long-term memory with new information in order to regulate behaviors that are no longer relevant. Research on overcoming unwanted behavior relies extensively on the principles of extinction-in which omission of expected events diminishes learned behavior. However, as a technique to permanently reduce fear behaviors, extinction is unsatisfactory as extinguished behaviors return under a variety of circumstances. Moreover, research on extinction focuses on one component of aversive memories-the behavioral expression-and it remains unclear whether extinction has any consequence on memory for episodic details of a fearful experience. The studies presented here tested novel methods for enhancing extinction and reducing relapse in healthy adults, and, for the first time, examined whether extinction has any effect on episodic memory for details of a fearful experience.

Methods: The first study (Study 1) improved upon standard models of extinction by replacing-rather than merely omitting-aversive outcomes with novel outcomes. To examine the effects of extinction on episodic memory, the second study used a validated category-conditioning paradigm in which subjects view unique (non-repeating) images from two semantic categories. Images from one category are paired with an aversive electrical shock and the other category is safe. Fear conditioning was identical across multiple groups who then underwent different forms of extinction: immediate extinction, delayed extinction, massive 
extinction, or novelty extinction (as in Study 1), or no extinction. Subjects all returned 24-hours later for a surprise recognition memory test for items they had seen during fearconditioning and extinction.

Results: First, the 'novelty-facilitated extinction' technique successfully prevented relapse of conditioned fear in humans and in rats, providing cross-species evidence that replacing expected threats is more effective than simply removing them. Second, we found that neither immediate, delayed, nor massive extinction diminished recognition memory for items associated with an aversive outcome during fear conditioning This finding is predicted by contemporary models of extinction and animal neuroscience research suggesting that extinction is not a form of unlearning. However, noveltyfacilitated extinction did diminish the episodic memory enhancements for fear-conditioned stimuli.

Conclusions: These findings provide novel nonpharmacological behavioral strategies for enhancing fear extinction that can be straightforwardly adapted and implemented in clinical situations. That episodic information associated with a feared event is not altered by extinction training provides new clues as for why fear behaviors are prone to relapse over time, as the memory of the original fear memory is preserved despite new safety learning. We discuss ongoing work examining the crossover between novel approaches to optimize extinction and reducing the emotional enhancement for negative memories. Keywords: Fear Conditioning, Fear extinction, Memory Consolidation and Extinction.

Disclosure: Nothing to disclose.

\section{T4. Brain Mechanisms and Predictors of Response to Prolonged Exposure Therapy in PTSD}

Gregory Fonzo*, Madeleine Goodkind, Desmond Oathes, Yevgeniya Zaiko, Meredith Harvey, Kathy Peng, Elizabeth Weiss, Colleen Mills-Finnerty, Allison Thompson, Sanno Zack, Steven Lindley, Bruce Arnow, Booil Jo, James Gross, Barbara Rothbaum, Amit Etkin

\section{Stanford University, Stanford, California, United States}

Background: Trauma-focused exposure therapies such as prolonged exposure (PE) are the most efficacious interventions for post-traumatic stress disorder (PTSD), but our understanding of the neural mechanisms underlying their efficacy is lacking. This knowledge gap is due partly to a lack of well-controlled imaging studies that report findings over a range of relevant neural processes. Moreover, no PTSD treatment studies have utilized causal manipulations of brain circuits, such as combining transcranial magnetic stimulation (TMS) and functional imaging, to deepen understanding of how functional brain changes during emotion tasks reflect changes in the causal dynamics of integrated circuits. Here, we report final analyses from a two-arm, RCT-style imaging intervention study in PTSD investigating the mechanisms underlying efficacy of PE therapy. We focused specifically on tasks that capture constructs theoretically relevant to PTSD and exposure therapy-emotional reactivity and regulation. We investigated therapeutic effects on brain function using a patient waitlist (WL) control condition and full intent-to- treat linear mixed models. We furthermore brought causality-focused interventions to bear on the findings by interrogating the causal connections of cortical substrates conveying a beneficial response to treatment and those showing treatment-related changes using single-pulse TMS and fMRI.

Methods: Sixty-six individuals meeting criteria for PTSD were randomized to 9-12 sessions of PE treatment $(N=36)$ or WL $(N=30)$ for a comparable time period. Both before and after treatment or WL, individuals completed a clinical assessment and battery of three functional imaging tasks assessing the identification, processing, response, and regulation of emotional responses as well as resting state fMRI. Full intent-to-treat voxelwise linear mixed models were utilized to identify brain activation and connectivity predicting a favorable therapeutic outcome to treatment as well as that which showed differential treatment-related changes not due to the passage of time or repeated measurements. Single-pulse TMS with concurrent fMRI was utilized to probe causal connections of select brain foci showing relevant treatment effects.

Results: When reacting to fear cues, individuals displaying greater anterior insula, dorsomedial, and dorsolateral prefrontal activation and less amygdala activation at baseline displayed a more favorable treatment response. Greater functional decoupling of the right dorsolateral prefrontal cortex and the left amygdala during emotional reactivity and greater TMS-induced right dorsolateral prefrontal causal inhibition of the left amygdala at rest also predicted more favorable treatment response. During regulation of emotional conflict, individuals displaying greater ventromedial prefrontal (vmPFC)/ventral striatal activation at baseline also displayed a more favorable response to treatment and better regulation of emotional conflict. Activation during cognitive reappraisal did not predict treatment response. Regarding treatment-related brain changes, there were no effects of treatment on brain function during emotional reactivity or regulation of emotional conflict. However, treatmentrandomized individuals displayed a selective increase in left lateral frontopolar (LatFP) activation during cognitive reappraisal, which was associated with greater improvement in hyperarousal symptoms and psychological well-being and was anti-correlated with changes in dorsal and ventrolateral regions typically recruited by reappraisal. LatFP change occurred concomitant with increased task-dependent connectivity with the same vmPFC/ventral striatal region that predicted therapeutic response during regulation of emotional conflict at baseline. Moreover, greater LatFP activitation during emotional conflict regulation at baseline predicted a greater increase in LatFP activation during reappraisal following treatment. Single-pulse TMS in healthy individuals during fMRI revealed that manipulation of left LatFp exerts a causal influence on vmPFC/ventral striatal activation. Both the LatFP and vmPFC/ventral striatum displayed treatment-related increases in entropy at rest, i.e. more flexible and less predictable time courses of signal fluctuations.

Conclusions: One's capacity to benefit from prolonged exposure for PTSD is gated by the degree to which prefrontal resources can be spontaneously engaged to control amygdala-mediated threat orienting, attune to relevant emotional information, and equilibrate visceral state to 
emotional salience, but not when attempting to reduce negative emotionality. Moreover, the frontopolar cortex, implicated in gating the balance of attention between the internal and external world, is a key locus of adaptive psychotherapeutic change. We propose this change reflects greater flexibility in switching attention away from a provocative external stimulus and towards an internal signal of equilibrium when consciously reducing negative emotion. TMS and task findings implicate the right dorsolateral prefrontal cortex and left LatFP as potential sites for causal intervention development.

Keywords: Post-Traumatic Stress Disorder, BOLD Imaging, Psychotherapy, Interleaved TMS/fMRI and Anterior Prefrontal Cortex.

Disclosure: Nothing to disclose.

\section{T5. Fatty Acid Amide Hydrolase Overexpression in the Basolateral Nucleus of the Amygdala Induces Paradoxical Effects on Anxiety and Fear Memory in Rats}

Maria Morena*, Kira Leitl, Asim Rashid, Sheena Josselyn, Matthew Hill

University of Calgary, Calgary, Canada

Background: A growing amount of evidence has demonstrated that elevations in the endocannabinoid anandamide (AEA) signaling within the basolateral complex of the amygdala (BLA) attenuate stress responses such as anxiety and fear expression. Specifically, inhibition of AEA hydrolysis by the enzyme fatty acid amide hydrolase (FAAH) within the BLA has been shown to reduce anxiety, neuroendocrine responses to stress and promote fear extinction. To determine if impairments in AEA signaling within the BLA would produce the opposite effects, and induce a stress-like state characterized by heightened anxiety and sustained fear, we examined the effects of overexpression of FAAH locally within the BLA on behavioural indices of anxiety and fear memory dynamics.

Methods: Male adult Sprague Dawley rats were bilaterally infused in the BLA with a Herpes simplex virus type 1 vector, which preferentially infects principal neurons, containing FAAH and green fluorescent protein (HSV-FAAH-GFP) or a control vector containing only GFP (HSV-GFP). Rats were then tested for biochemical measurements, anxiety or fear memory behaviour. All experimental procedures were in compliance with protocols approved by the University of Calgary Animal Care Committee and guidelines from the Canadian Council on Animal Care.

Results: $72 \mathrm{~h}$ following HSV administration, a time point in which protein transfection is maximal, we found increased FAAH-mediated AEA hydrolysis together with decreased AEA levels within the BLA, confirming that the virus did successfully increase FAAH expression. At this same time point, a separate cohort of rats was tested for anxiety behaviour in an elevated plus maze, a light/dark box and an open field task. Quite surprisingly, we found that the overexpression of FAAH induced consistent anxiolytic effects in all three behavioural tasks we performed, relative to HSV-GFP rats. An additional cohort of animals was tested for fear memory extinction in an auditory fear conditioning paradigm. To prevent any effects of FAAH overexpression on the initial fear memory consolidation, animals were bilaterally cannulated in the BLA, a week later were fear conditioned and $24 \mathrm{~h}$ after conditioning, the animals were injected with HSV-FAAH-GFP or its control vector. $72 \mathrm{~h}$ following HSV administration rats were re-exposed to the tone alone for 4 consecutive days to examine fear extinction dynamics. Unexpectedly, rats infused with the HSV-FAAHGFP vector exhibited a dramatic reduction in fear expression during the extinction training and first extinction retrieval sessions when exposed to the tone, as compared to their HSV-GFP control rats. Moreover, both HSV-GFP and HSVFAAH-GFP animals showed reinstatement of fear memory, when given unsignaled foot shocks after the extinction had occurred in both groups and presented $24 \mathrm{~h}$ later the tone alone, thus demonstrating that the effects of the FAAH overexpression were selective for the fear and extinction memory retrieval, without interfering with the animal ability to correctly acquire the conditioned-unconditioned stimulus association and to express freezing behaviour.

Conclusions: These findings suggest that the exact modes of action of AEA within the amygdala in the regulation of emotional states and memory are still far from being clear, thus, opening the avenue to investigate new potential mechanisms by which these processes may occur.

Keywords: Endocannabinoids, Fear extinction and Anxiety. Disclosure: Nothing to disclose.

\section{T6. Theta-Frequency Oscillatory Stimulation of vHPC Inputs to the mPFC Increases Anxiety-Like Behavior}

Nancy Padilla-Coreano*, Sarah Canetta, Richard Warren, Christoph Kellendonk, Joshua Gordon

\section{Columbia University, New York, New York, United States}

Background: Anxiety states are distinguished by an increase in theta-frequency $(4-12 \mathrm{~Hz})$ synchrony in the amygdalahippocampal-prefrontal circuit. Recently, we demonstrated that optogenetically inhibiting the ventral hippocampal (vHPC) input to the medial prefrontal cortex (mPFC) decreases anxiety-like behavior and theta synchrony between the $\mathrm{mPFC}$ and vHPC, without affecting other frequencies (Padilla-Coreano et al, Neuron. 2016). These data suggest the hypothesis that theta-frequency input from the vHPC plays a causal role in anxiety-like behavior.

Methods: We therefore asked whether stimulating the vHPC-mPFC at a theta frequency was sufficient to increase avoidance behavior in the elevated plus maze, and whether any such effects might be frequency- and/or pattern-specific. CamKIIa-hChR2-eYFP was expressed bilaterally in the vHPC and optical fibers implanted in the mPFC. In a subset of mice, single-unit recording electrodes were also implanted in the MPFC.

Results: Stimulating the vHPC-mPFC pathway with a sinusoidal light pattern at $8 \mathrm{~Hz}$ significantly increased avoidance behavior, while stimulating with brief pulses of light at $8 \mathrm{~Hz}$ or sinusoidal light at $20 \mathrm{~Hz}$ had no effect. These experiments demonstrate that the anxiogenic effect of vHPC terminal stimulation is frequency- $(8 \mathrm{~Hz}$ but not $20 \mathrm{~Hz})$ and pattern- (sinusoids but not pulses) specific. This result was surprising, as pulses of light are the most common light 
pattern used to stimulate neurons and terminals optogenetically. To understand how pulses and sinusoidal light modulate $\mathrm{mPFC}$ neurons differentially, mPFC pyramidal neurons were recorded both in vitro and in vivo while stimulating vHPC terminals with the same sinusoidal or pulsatile patterns. In vitro, sinusoidal stimulation increased the rate of spontaneous EPSCs, while pulses evoked strong, time-locked EPSCs. In vivo, sinusoidal stimulation of vHPC terminals were capable of increasing the phase-locking of mPFC single units to the optical stimulation during anxiety behaviors.

Conclusions: These results suggest that sinusoidal stimulation at $8 \mathrm{~Hz}$ increases spontaneous activity in a rhythmic, naturalistic manner that enhances theta-frequency activity in $\mathrm{mPFC}$ neurons as well as anxiety-related behavior. Moreover, they suggest that theta-frequency components of neural activity play a privileged role in vHPC-mPFC communication and hippocampal-dependent forms of anxiety.

Keywords: Optogenetics, Hippocampus-mPFC Pathway and Anxiety Circuitry.

Disclosure: Nothing to disclose.

\section{T7. Network Mechanisms of $5 \mathrm{~Hz}$ Repetitive Transcranial Magnetic Stimulation in Patients With Comorbid Posttraumatic Stress and Major Depressive Disorders}

Noah Philip*, Jennifer Barredo, Mascha van 't Wout, Jorge Almeida, Audrey Tyrka, Lawrence Price, Linda Carpenter

\section{Brown University, Providence, Rhode Island, United States}

Background: Treatment with repetitive transcranial magnetic stimulation (TMS) can selectively modulate pathological functional connectivity of the default mode network in patients with major depressive disorder (MDD). Posttraumatic stress disorder (PTSD) is often comorbid with MDD, and research indicates that TMS can also alleviate symptoms of PTSD. This is the first study to evaluate TMS-associated changes in connectivity in a sample of patients with comorbid PTSD and MDD. We predicted that clinically efficacious TMS would be associated with reduced default network connectivity and uncoupling of salience and memory regions associated with PTSD.

Methods: Resting state functional connectivity (RSFC) was acquired using a 3T MRI on 32 participants from an openlabel trial of $5 \mathrm{~Hz}$ rTMS to the left dorsolateral prefrontal cortex (up to 40 daily sessions, 3-4000 pulses per session). $5 \mathrm{~Hz}$ was selected based on our prior report of efficacy for comorbid PTSD and MDD. Imaging analyses used a priori seeds identified from prior research in TMS and PTSD, including the subgenual anterior cingulate (sgACC), dorsolateral prefrontal cortex (DLPFC), hippocampus (anterior and posterior components) and the basolateral amygala. These seeds were used to compare pre- vs. post-treatment RSFC associated with clinical changes, adjusting for age and baseline symptom severity. To examine if changes in functional connectivity between a priori seed regions and extracted clusters were predictive of meaningful clinical outcomes, the mean change in beta values across sessions for each cluster and subject was entered into a logistic regression of symptomatic recovery for both disorders. Model fit statistics are reported as Nagelkerke R2. To address concerns of insufficient thresholding and validity in imaging studies, a voxel height threshold $(p<0.005)$ was applied to all whole brain imaging results, plus cluster-based false discovery rate thresholding (i.e., FDR-corrected cluster size of $p<.05$ ). Furthermore, a leave-one-out cross-validation procedure was implemented as a stringent test of cluster validity. Clusters where the cross-validated $t$-value fell below the critical value were considered invalid and excluded from the logistic regression.

Results: Twenty-five participants had usable data available prior to and following TMS. Compared to positive connectivity with DMN regions prior to treatment, after treatment, the sgACC demonstrated anticorrelated RSFC with a broad range of regions within the default mode network, dorsomedial prefrontal cortex and insula (all corrected $p<.001$ ). The logistic regression model indicated that changes in sgACC to default mode regions were predictive of remission $(p<.001 ; \mathrm{R} 2=.63)$. DLPFC connectivity increased to the right prefrontal cortex (corrected $p<.001$ ) that was more modestly predictive of remission $(p=.008, \mathrm{R} 2=.32)$. Compared to positive RSFC at baseline, after TMS there was anticorrelated connectivity between the anterior hippocampal seed and frontal corticolimbic regions, including the dorsal anterior cingulate cortex and putamen (corrected $p<.001$ ), which was also predictive of remission $(p=.006 ; \mathrm{R} 2=.52)$. Posterior hippocampal connectivity with the right DLFPC was increased following treatment (corrected $p<.001$ ); this modestly predicted remission $(p=.03, \mathrm{R} 2=.32)$. No statistically significant changes in connectivity were observed from the basolateral amygdal

Conclusions: These results are consistent with prior imaging studies of TMS therapy in MDD that found reduced default network connectivity and more modest effects of TMS on executive network connectivity. Furthermore, these results highlight that TMS can uncouple hippocampal connectivity from salience network regions implicated in PTSD. These results show that TMS can correct clinically relevant network pathology, with results relevant to both disorders under study.

Keywords: Repetitive Transcranial Magnetic Stimulation, Resting State Functional Connectivity and PTSD Depression. Disclosure: Neuronetics: Grant support, Self.

\section{T8. Basomedial Amygdala Mediates Top-Down Control of Anxiety and Fear}

Avishek Adhikari*, Talia Lerner, Joel Finkelstein, Sally Pak, Joshua Jennings, Thomas Davidson, Emily Ferenczi, Lisa Gunaydin, Julie Mirzabekov, Li Ye, Sung-Yon Kim, Anna Lei, Karl Deisseroth

Stanford University, Palo Alto, California, United States

Background: Anxiety-related conditions are among the most difficult neuropsychiatric diseases to treat pharmacologically, but respond to cognitive therapies. There has therefore been interest in identifying relevant top-down pathways from cognitive control regions in medial prefrontal cortex (mPFC). Identification of such pathways could contribute 
to our understanding regarding cognitive regulation of affect, and provide pathways for intervention. Prior studies suggest dorsal and ventral mPFC subregions exert opposing effects on fear, as do subregions of other structures. However, precise causal targets for top-down connections among these diverse possibilities have not been established.

Methods: We optogenetically targeted the vmPFC-amygdala projection in mice during learned fear conditioning and innate anxiety behavioral assays. We also monitored the effect of this manipulation in physiological measures of high anxiety states (respiratory rate and heart rate) through pulse oxymetry. We applied in vivo and in vitro assays of functional connectivity to identify which amygdala nuclei are recruited by the vmPFC.

Results: We show the basomedial amygdala (BMA) represents the major target of ventral mPFC (vmPFC) in amygdala. Moreover, BMA neurons differentiate safe and aversive environments, and BMA activation decreases fearrelated freezing and high anxiety states. Lastly, the vmPFCBMA projection implements top-down control of anxiety state and learned freezing, both at baseline and in stressinduced anxiety.

Conclusions: Our data show activity in the vmPFCamygdala projection is sufficient and necessary to suppress high anxiety states, as measured by multiple behavioral and physiological parameters. Multiple streams of evidence including viral tracing, patch clamping, in vivo physiology and immediate early gene expression indicate that these effects were mediated by the basomedial amygdala (BMA). Accordingly, activation of the BMA also was anxiolytic. These results establish the vmPFC-BMA as a key node that mediates cortical-subcortical top-down anxiety suppression. Considering that abnormalities in MPFC-amygdala connectivity is commonly displayed in patients with diverse anxiety disorders, our results indicate that the vmPFC-BMA is a node that may be targeted to ameliorate these disorders.

Keywords: Medial Prefrontal Cortex, Mood and Anxiety Disorders, Fear Conditioning and Post-Traumatic Stress Disorder.

Disclosure: Nothing to disclose.

\section{T9. Group Differences and Treatment Outcome Prediction With Resting State and Task-Based Connectivity in Pediatric Anxiety}

David Pagliaccio*, Caroline Swetlitz, Lauren White, Daniel Pine

National Institute of Mental Health, Bethesda, Maryland, United States

Background: Anxiety disorders have been characterized by alterations in connectivity between the amygdala and prefrontal and other regions, both at rest and during task. Particularly, recent work has shown robust group differences and test-retest of task-based connectivity during a dot-probe attentional task. Additionally, efforts have been made to use functional connectivity as a potential predictor of treatment outcomes in anxiety disorders. Yet, it is unclear what relationship may exist between resting state connectivity and connectivity modulated by task and how this related to our understandings of anxiety disorders.
Methods: The current study examines data from 71 children and adolescents (8-18 years old), 33 with anxiety and 38 healthy volunteers. Anxious patients were scanned prior to an 8-week treatment trial. Participants completed two runs of an fMRI dot-probe task followed by a 10-minute resting state scan. Anatomically defined amygdala seeds were used to examine functional connectivity during the dot-probe using generalized psychophysiological interaction and during rest using standard processing in AFNI.

Results: Preliminary analyses indicate that more negative resting state connectivity between the right amygdala and medial prefrontal cortex (Monte Carlo voxel-wise correction $p<.001, \mathrm{k}>23$ ) strongly predicts greater reduction in anxious symptoms following treatment over and above pretreatment anxiety and demographic control factors $(=0.65, \mathrm{t}=3.55, p=.002)$.

Conclusions: These results suggest key relationships between amygdala connectivity and successful treatment outcomes. On-going analyses will examine the relationship between resting state and task-based connectivity, group differences in connectivity, and further prediction of treatment outcomes.

Keywords: Anxiety, Children and Adolescents, Connectivity, Functional MRI (fMRI) and Amygdala.

Disclosure: Nothing to disclose.

\section{T10. Glucocorticoid, Immune and Genomic Mediators of Amygdalaactivation Following Extreme Stress/Trauma}

Nikolaos Daskalakis*, Hagit Cohen, Janine Flory, Iouri Makotkine, Joseph Buxbaum, Charles Marmar, Owen Wolkowitz, Marti Jett, Rasha Hammamieh, Bin Zhang, Rachel Yehuda

Icahn School of Medicine at Mount Sinai, New York, New York, United States

Background: Delineating the molecular basis of individual differences in the stress response is critical to understanding the pathophysiology of post-traumatic-stress-disorder (PTSD), with the ultimate goal of identifying biomarkers.

Methods: We analyzed genome-wide expression of peripheral blood mononuclear cells (PBMCs) and functional neuroendocrinology and immunology plasma/serum data from a cross-sectional biomarker study with and without PTSD ( $n=83$ /group). In addition, gene expression in blood and brain (amygdala, hippocampus and prefrontal cortex) and neuronal morphology were analyzed from a PTSD rat model, in which vulnerable and resilient phenotypes are identified according to the long-term behavioral response to predator scent stress (PSS). Analyses of the human and animal data were performed separately, and then compared and integrated with the use of gene co-expression network analyses.

Results: Differentially expressed (DE) genes were identified in association with PTSD. These DE genes were consistent with peripheral downregulation of glucocorticoid receptor (GR) signaling and upregulation of pro-inflammatory cytokine signaling. The gene expression differences in these pathways were validated in cultured PBMCs incubated in varying doses of dexamethasone (DEX). These findings were also replicated by the DE-signatures identified in rat blood 
and brain, in association with exposure-related individual differences. Gene co-expression networks were then identified in the human and rat samples. Among the most promising networks, there was a large ( $>100$ genes) PTSD co-expression module showing a high level of dysregulation (high modular differential connectivity in the two groups) and high level of conservation in the blood and brain of PTSD-like rats. Functionally, this module is enriched with the genes related to innate immune response, and its eigengene expression associates deferentially with the PBMC lysozyme inhibition by DEX and plasma cortisol decline in the dexamethasone suppression test in the two groups. Finally, GR-agonist administration in rats shortly after PSS prevented PTSD-like phenotypes by reversing amygdala vulnerability-associated pro-inflammatory cytokine signaling and neuronal morphology patterns.

Conclusions: We identified genes, pathways and gene coexpression networks in the blood of combat veterans with PTSD. GR-dependent immune pathways and networks are associated with trauma-related individual differences in blood and amygdala, and can be the basis of treatment for PTSD.

Keywords: PTSD, Gene Network Analysis, Glucocorticoids, Immune Mechanisms and Amygdala.

Disclosure: Nothing to disclose.

\section{T11. Exposure to Inner-City Violence Associated With Inhibition-Related Activation in the Developing Brain}

Sanne van Rooij ${ }^{*}$, Jennifer Stevens, Ye Ji Kim, Timothy Ely, L. Alexander Vance, Bekh Bradley, Tanja

Jovanovic

Emory University School of Medicine, Atlanta, Georgia, United States

Background: Exposure to trauma and stress in early life is a well-known risk factor for the development of psychiatric disorders across the lifespan. As most studies are retrospective, however, the developmental effects of trauma exposure during childhood are not well understood. This study investigated the effects of trauma exposure on the developing brain in children growing up in neighborhoods with high levels of inner-city violence.

Methods: Mothers with a child between ages 8-14 were recruited through the Grady Trauma Project, an ongoing study on the effects of trauma exposure in an at-risk population. A subset of children $(N=31)$ from this sample completed an fMRI scan while they performed an emotional Go/NoGo task. During this task, fearful and neutral faces are presented in pseudo-randomized order. The participants were asked to press a button for neutral faces and not press for fearful faces. This contrast was used to measure emotional response inhibition (NoGo Fear $>$ Go Neutral). Trauma exposure was assessed with self-report measures of violence exposure (VEX-R). The relationship between inhibition-related brain activation and violence exposure (frequency) was investigated. First, exploratory whole brain analyses were performed, and secondary small volume correction was performed for our regions of interest: the amygdala, the hippocampus and the rostral ACC.
Results: After quality control, data from 16 children were available for preliminary whole brain analyses $(p<0.01)$. Exposure to violence correlated positively with inhibitionrelated activation in limbic structures (amygdala, hippocampus), motor control regions (basal ganglia, right inferior frontal gyrus), and the salience network (anterior cingulate cortex, insula). Small volume correction showed significant positive correlations between violence exposure and the right amygdala (FWE-corrected $p<0.05$ ), the left and right hippocampus (FWE-corrected $p<0.05$ ), and the rostral ACC (FWE-corrected $p<0.05)$. Further analyses investigating the effects of gender and age are of high interest when the sample size of this ongoing study increases.

Conclusions: Although preliminary, our results provide some evidence for a relationship between trauma exposure and alterations in the neurocircuitry underlying fear and response inhibition in children. Higher levels of exposure to violence were associated with more activation in the amygdala, hippocampus and rostral ACC during the combined process of viewing fearful faces and suppressing a behavioral response. Given that this neurocircuitry has been implicated in many psychiatric disorders, increased understanding of the effects of trauma exposure on the developing brain is essential for early detection of individuals at risk for developing psychiatric disorders.

Keywords: Childhood Trauma, Emotional Response Inhibition, Violence, Functional MRI (fMRI).

Disclosure: Nothing to disclose.

\section{T12. Fractional Anisotropy in Cingulum-Hippocampus} Tracts Predicts Suicidal Ideation in Young Adolescents

Tiffany $\mathrm{Ho}^{*}$, Sarah Ordaz, Josiah Leong, Daniel Lowet, Meghan Goyer, Manpreet Singh, Ian Gotlib

Stanford University, Stanford, California, United States

Background: Suicide is the second leading cause of death in adolescents worldwide (CDC, 2014). Therefore, it is imperative that we identify individuals at risk and intervene as early as possible. Suicidal ideation is an important precursor to attempt; increased severity of suicidal ideation is associated with a greater likelihood of subsequent suicide attempt (Sampasa-Kanyinga et al, 2015). The peak onset of suicidal ideation occurs during adolescence (Nock et al, 2013), which is also a period of significant neurodevelopment (Dahl. 2004). While investigators have identified suicide-related endophenotypes-notably aberrant frontal, limbic, and cingulate gray and white matter-the neurobiological markers associated with early suicidal ideation are still unknown (Cox Lippard et al, 2014). Furthermore, no studies to date have examined whether neural structure can prospectively predict the development of suicidal ideation in adolescents. Given that adolescence is a sensitive period for both neurodevelopmental maturation and increased suicidal risk, successful identification of neural risk factors for suicidal ideation has important implications for the development of neural models of suicide and of novel early interventions. In the present study, we collected diffusionweighted imaging data from young adolescents at a baseline time point, and performed tractography to identify the uncinate fasciculus (UF) and the cingulum (CG). The UF 
and CG are two major white matter tracts that connect limbic structures with frontal and with cingulate regions, respectively. We computed fractional anisotropy (FA) across the entirety of these tracts as an index of tract coherence, and tested if tract coherence at baseline was associated with severity of suicidal ideation 18 months later.

Methods: Forty-six youth (14 males; mean \pm SD age: 11.42 \pm 1.03 years) recruited from the San Francisco Bay Area completed MRI scanning at Stanford University using a 3T Discovery MR750 (GE Medical Systems, Milwaukee, WI) equipped with a 32-channel head coil (Nova Medical). For all participants, 3D T1-weighted images were acquired with a SPGR sequence $(\mathrm{TR} / \mathrm{TE} / \mathrm{TI}=6.24 / 2.34 / 450 \mathrm{~ms}$; flip angle $=$ $12^{\circ}$; 186 sagittal slices; $0.9 \mathrm{~mm}$ isotropic voxels) and diffusion-weighted images were acquired with an EPI sequence $\left(\mathrm{TR} / \mathrm{TE}=8500 / 93.5 \mathrm{~ms}\right.$; flip angle $=90^{\circ} ; \mathrm{b}=2000$ $\mathrm{mm} 2 / \mathrm{s}, 64$ axial slices, 60 gradient directions, 6 b0 images; 2 $\mathrm{mm}$ isotropic voxels). Diffusion-weighted data were preprocessed using standard methods and a continuous tensor field was estimated with trilinear interpolation of the tensor elements. We used Automatic Fiber Quantification (AFQ; Yeatman et al, 2012) software to identify and segment each participant's brain into 20 white matter fiber groups, including bilateral UF and CG. Candidate fibers for UF and CG were defined using an automated 2 region-ofinterest approach, and fibers were estimated using a deterministic streamlines tracking algorithm (Basser et al, 2000; Mori et al, 1999). Tracts were then refined by comparing each fiber within the tract to a probabilistic white matter atlas (Wakana et al, 2007). Finally, each tract was represented as a 3D Gaussian, and outlier fibers (defined as those that exceeded a maximum Gaussian distance of 5 or that deviated more than 4 SDs from the mean of the fiber group center) were excluded (Yeatman et al, 2012). Tract coherence was then calculated along the trajectory of each tract by interpolating the FA value at 100 evenly spaced nodes along each fiber, and then averaged across the entire tract. Importantly, we conducted automated tractography from the AFQ software given that these methods have been validated in the age range of our participants (Yeatman et al, 2012; Lebe et al, 2012). Suicidal ideation and depression symptom severity were assessed with self-report measures (SIQ-15; Reynolds \& Mazza. 1999 and CDI; Kovacs. 1992, respectively) at an 18-month follow-up session. Linear regression models were used to predict severity of suicidal ideation at follow-up from FA of left and right UF and CG, controlling for baseline age, gender, and depression symptom severity at follow-up. Given evidence of higher rates of suicidal ideation in female adolescents (Nock et al, 2013), we also modeled the interaction effect of gender and FA from each tract as a predictor of SIQ-15 scores.

Results: Tract coherence of the left cingulum bundles connecting the cingulate with the hippocampus showed a significant interaction with gender in predicting severity of suicidal ideation $(\mathrm{F} 1,42=3.66 ; p<0.05)$. Specifically, females with higher FA in the cingulum-hippocampal tracts at baseline endorsed higher levels of suicidal ideation. Tract coherence of the right cingulum and of bilateral UF (with and without the interaction of gender) did not significantly predict severity of suicidal ideation (all p's $>0.05$ ).

Conclusions: This study is the first to demonstrate that brain structure prospectively predicts the severity of suicidal ideation in young adolescents. Fractional anisotropy is thought to reflect axonal myelination, which is known to undergo major changes throughout early development. The cingulum is one of the last white matter tracts to develop and is implicated in emotion regulation and broad cognitive functioning (Lebel et al, 2012). Thus, greater cingulum tract coherence among females during early adolescence may represent aberrant neurodevelopment and serve as a risk factor for the onset of suicidal behaviors.

Keywords: Adolescence, DTI, Suicide Assessment, Cingulum, Hippocampus.

Disclosure: Nothing to disclose.

\section{T13. Subcortical Shape Deformation Associations Related to Substance Use in Youth With Perinatally- Acquired HIV}

Christine Paula Lewis-de los Angeles*, Kathryn I Alpert, Paige L Williams, Kathleen Malee, Yanling Huo, John Csernansky, Ram Yogev, Russell B Van Dyke, Eilzabeth R Sowell, Lei Wang, Pediatric HIV/ AIDS Cohort Study (PHACS)

Northwestern University, Chicago, Illinois, United States

Background: As youth with perinatally-acquired HIV (PHIV) enter adolescence, they may engage in substance use. Prior work has demonstrated that worse HIV disease severity is associated with substance use (Williams 2010). It is important to understand the effects of PHIV and substance use on brain structure.

Methods: 40 PHIV youth were recruited from one site (Ann \& Robert Lurie Children's Hospital of Chicago) of the NIH Pediatric HIV/AIDS Cohort Study (PHACS). Structural T1weighted magnetic resonance imaging was collected. FreeSurfer-Initiated Large Deformation Diffeomorphic Metric Mapping was used to quantify shape deformation of subcortical brain structures. Historic HIV disease severity measures (peak HIV RNA load, nadir CD4 percentage) were obtained from medical charts. Audio Computer-Assisted Self-Interview (ACASI) was used to obtain self-reported substance use. Substance use was coded as $\mathrm{Y} / \mathrm{N}$ for prior use for alcohol, marijuana, or tobacco. Multiple linear regression was used to obtain clusters of significant $(p<0.05)$ relationships between deformation and substance use on subcortical surfaces. Multiple comparisons were corrected with random field theory. Models were adjusted for age at scan and sex, with further adjustment for HIV disease severity measures. Results: Mean age was 16.7 years. Median log peak HIV RNA load was 5.7 copies/mL (IQR: 5.2,5.9) and nadir CD4 percentage was $16.5 \%$ (IQR: 8.0, 23.8). Forty-five percent reported substance use (25\% tobacco, 35\% alcohol, 35\% marijuana); substance use was unknown for two subjects who were excluded from further neuroimaging analyses. In models including age at scan and sex, significant relationships between surface shape deformation and substance use were found in the hippocampus, nucleus accumbens, caudate, and putamen. In models including log peak HIV RNA viral load and log of age at peak viral load, significant relationships between surface shape deformation and substance use were found only in the hippocampus. In models including nadir CD4 percentage and age at nadir CD4 
percentage, significant relationships between surface shape deformation and substance use were found in the hippocampus and caudate.

Conclusions: Substance use was significantly related to deformation of the brain's subcortical structures. Future work should be aimed at understanding the relationships among different types of substance use, adherence to combinational anti-retroviral therapy, HIV disease severity, and structural brain changes.

Keywords: HIV, Human Neuroimaging, Substance Abuse, Adolescence.

Disclosure: Nothing to disclose.

\section{T14. Novel fMRI Approaches Reveal Developmental Changes in Frontoamygdala Circuitry With Implications for the Emergence of Psychiatric Disorders During Development}

Dylan Gee ${ }^{\star}$, Leyla Calgar, Colleen Mills-Finnerty, Bonnie Goff, Laurel Gabard-Durnam, Dominic Fareri, Christina Caldera, Daniel Lumian, Jessica Flannery, Catherine Hanson, Stephen Hanson, Nim Tottenham

Yale University, New Haven, Connecticut, United States

Background: Interactions between the prefrontal cortex (PFC) and amygdala are fundamental to emotion regulation. Substantial changes in frontoamygdala circuitry take place during development, and this circuitry has been implicated in myriad psychiatric disorders including anxiety, depression, and schizophrenia. Given dynamic neurodevelopmental changes and the emergence of many psychiatric disorders during childhood and adolescence, delineating developmental changes in prefrontal cortex and amygdala function is critical to understanding the onset and treatment of mental illness in youth. However, extant research has been limited by reliance on correlational fMRI data.

Methods: Here we delineate developmental changes in frontoamygdala connectivity through two novel approaches: experimental manipulation of this circuitry, and Bayes network analyses. Experimental manipulation of prefrontal function has been shown to have sustained and lasting effects on amygdala reactivity during adulthood. The current study took advantage of PFC-mediated effects to investigate the nature of the amygdala-PFC relationship during development. Forty healthy children, adolescents, and young adults (4-19 years) completed an fMRI task that manipulates the effects of cognitive load on subsequent brain activation to emotional stimuli, facilitating the examination of temporal effects of prefrontal engagement on subsequent amygdala reactivity.

Results: Behavioral performance confirmed the effectiveness of the cognitive load manipulation. Specifically, participants responded more slowly and with lower accuracy to incongruent versus congruent words. High versus low cognitive load increased dorsal ACC recruitment across development $(p<.01$, corrected), but ACC engagement had differential effects on activation to emotional faces for children (reduced medial PFC, increased amygdala) versus adolescents (increased medial PFC, reduced amygdala). Network analyses demonstrated a shift in effective connectivity, such that the amygdala more strongly influenced the mPFC during childhood, but the mPFC more strongly influenced the amygdala during adolescence.

Conclusions: The cognitive load manipulation and network analyses reveal qualitative differences in the nature of frontoamygdala interactions during childhood and adolescence, with evidence for a shift from bottom-up to top-down frontoamygdala connectivity. These findings indicate that experimental activation of prefrontal cortex may serve to regulate amygdala reactivity during adolescence, but not during childhood. Our findings may serve as a foundation for understanding aberrations in the maturation of frontoamygdala connections across neurodevelopmental disorders in which this circuitry has been implicated. Furthermore, this study has important implications for differential risk for anxiety and mood disorders at unique developmental periods and may inform efforts to optimize types and timing of interventions to target the biological state of the developing brain.

Keywords: Children and Adolescents, Brain Development, Emotion Regulation, Amygdala, Prefrontal Cortex.

Disclosure: Nothing to disclose.

\section{T15. Altered Prefrontal Activation During Response Inhibition in Women Remitted From Bulimia Nervosa: The Influence of Metabolic State}

Laura Berner*, Amanda Bischoff-Grethe, Christina E Wierenga, Alan N Simmons, Ursula F Bailer, Alice V Ely, Walter Kaye

University of California, San Diego, La Jolla, California, United States

Background: Bulimia nervosa (BN) is characterized by recurrent episodes of binge eating, most saliently defined by a sense of "loss of control" over eating, and compensatory behaviors. Between binge-purge episodes, extreme dietary restriction or fasting is common. Hunger normally increases the motivational aspects of rewards, and some evidence suggests that strict dieting increases the likelihood of binge eating in BN. Despite brief periods of successfully restricted intake, compromised inhibitory control in a fasted state may increase binge eating risk and contribute to BN. Conversely, individuals report feeling less hungry before binges than non-binge eating episodes, and binge eating is defined by "difficulty stopping eating once one has started." Therefore, particularly compromised inhibitory control in a fed state could instead drive BN symptoms. Extant behavioral and neuroimaging evidence suggests inhibitory control impairments and associated frontostriatal dysfunction in BN, which may explain "loss of control" eating and other impulsive behaviors characteristic of the disorder; however, the extreme overcontrol this population displays between binge-purge episodes remains unexplained, and no study to date has manipulated metabolic state before neurocognitive testing or scanning. To address this gap in the literature, the current study examined whether being in a fasted or fed state abnormally impacts the function of self-regulatory control circuits in BN.

Methods: Women remitted from $\mathrm{BN}(\mathrm{RBN} ; n=23)$ and healthy control women (CW; $n=22)$ of equivalent age, body mass index, and full-scale IQ completed a parametric Stop 
Signal Task during fMRI on two counterbalanced visits: one after a 16-hour fast, and one after a standardized meal $(30 \%$ of daily caloric needs). This task requires the participant to inhibit a button-press response after it is initiated. On "hard" stop trials, the delay between the go and stop signals is longer, and stop signals occur shortly (0-200 ms) before a participant's pre-scan mean reaction time (MRT). On "easy" trials, stop signals occur farther in advance (300-500 ms) of MRT. To examine the influence of metabolic state on neural activation associated with successful inhibition, BOLD fMRI data for correct inhibitory trials were analyzed using Group (RBN, CW) x Visit (fasted, fed) x Difficulty (easy, hard) linear mixed effects analyses (LMEs) computed in R. To examine neural activation associated with inhibition failures, neural response during errors of commission were analyzed using a Group (RBN, CW) x Visit (fasted, fed) LME. Only hard trials were included in this analysis, as errors during easy trials were infrequent. Primary analyses were conducted in bilateral ROIs associated with self-regulatory control: lateral prefrontal cortices, cingulate, insula, and striatum. Each ROI was treated as a search region. Exploratory voxelwise analyses were also conducted. Correction for multiple comparisons was determined with Monte Carlo simulations using AFNI's 3dClustSim (ROI-wise and voxelwise corrected ps $<0.05$ for all comparisons). Post-hoc pairwise comparisons were FDR corrected for multiple comparisons.

Results: Groups performed similarly on the task, regardless of metabolic state. During correct inhibitory responses, a significant Group x Visit x Difficulty interaction was found in left dorsolateral prefrontal cortex (DLPFC). Post-hoc analyses revealed that when fed, RBN women relative to CW showed greater inhibitory activation during hard trials $(p=0.016)$. RBN also showed greater inhibitory activation in this region during hard trials when they were fed relative to when they were fasted $(p=0.047)$. Exploratory voxelwise analyses revealed a similar three-way interaction in right DLPFC. Analysis of inhibitory failures indicated that, regardless of metabolic state, the RBN group compared with $\mathrm{CW}$ showed increased activation during errors in left DLPFC $(p=0.008)$ and right anterior cingulate (ACC; $p=0.003)$.

Conclusions: Given equivalent task performance, these findings suggest that $\mathrm{BN}$ women require fewer lateral prefrontal resources to successfully inhibit responses when fasted, but greater effort to maintain performance when fed. This pattern may partially explain BN symptoms: the ability to inhibit eating and restrict intake to an extreme degree between binge episodes, but difficulty stopping once eating begins. These results provide initial support for therapeutic interventions for $\mathrm{BN}$ that are specific to metabolic state. Increased ACC activation during inhibitory control errors has been found to predict development of $\mathrm{BN}$ in adolescence and has been documented in women ill with BN. Our error-related results in women remitted from BN suggest that increased ACC activation during inhibitory failures may represent a trait biomarker for the disorder.

Keywords: bulimia nervosa, Inhibitory Control, Metabolic State, Functional MRI (fMRI).

Disclosure: Nothing to disclose.
T16. Psychophysiological Source Activity and Coherence During an Aggression Paradigm

\section{Jennifer Fanning*, Royce Lee, Mitchell Berman, Emil} Coccaro

\section{University of Chicago, Chicago, Illinois, United States}

Background: Previous research implicates neural circuits involved in emotion regulation and cognitive control in aggressive behavior. In a previous fMRI study in our lab, healthy subjects and subjects with pathological aggression (Intermittent Explosive Disorder; IED) viewed a series of emotional faces while undergoing fMRI. Compared to healthy subjects, subjects with IED showed enhanced BOLD activity in the left amygdala, diminished activity in the orbitofrontal cortex (OFC), and a lack of coupling between the OFC and amygdala when viewing angry faces (Coccaro et al, 2007). These findings implicate hyperactive amygdala and deficient prefrontal control in aggressive behavior. The purpose of this study was to test whether these patterns would characterize escalating (aggressive) behavior (versus de-escalating behavior) in response to provocation during a laboratory-controlled aggression paradigm. Other research using laboratory-based measures has found engagement of the anterior cingulate cortex (rostral and dorsal), insula, and mediofrontal gyrus (Kramer et al, 2007), as well greater theta EEG power over frontal scalp regions (Kramer et al, 2009), related to response selection under provocation.

Methods: Right-handed men and women $(\mathrm{HC}=26)$ participants with no past or current psychiatric disorders completed the Taylor Reaction Time Task modified for electroencephalogram (EEG). An aggressive interaction was simulated in the laboratory such that participants believed they were competing in a reaction time task against another participant. During the course of the task the participant and opponent exchanged electric shocks of varying intensity to the fingertips. Participants' behavior (shock intensity selection) was coded as escalating, matching or de-escalating in response to the opponent's shock selection. During the task, EEG was recorded using a BioSemi 128 channel EEG acquisition system. A model was fit to activity in the time frame around the button press response. The model included sources in temporal lobes, anterior and posterior cingulate (ACC and PCC), and dorsolateral prefrontal cortex (DLPFC), among others. Time frequency power analysis was conducted on source waveforms and coherence was computed between sources in the OFC, temporal regions, and ACC.

Results: Participants showed greater theta power in dACC/ supplementary motor area, dorsolateral prefrontal cortex (DLPFC), angular gyrus, and posterior cingulate $\sim 400 \mathrm{~ms}$ prior to the response on de-escalating trials relative to escalating trials. Subjects also showed greater alpha power in a source near the angular gyrus during the time frame of the response when de-escalating. In general, healthy subjects showed greater coherence between $\mathrm{OFC}$ and other sources (including bilateral temporal lobes, DLPFC, and dACC) when escalating against the opponent.

Conclusions: Previous research points to the involvement of emotion regulation and cognitive control in aggressive behavior. Prior research on oscillatory EEG activity during an aggression paradigm shows that subjects who are less 
aggressive on the task on average show greater theta power over frontal electrode sites compared to those who are more aggressive. Research has also shown that healthy subjects show inverse coupling of the OFC and amygdala when viewing angry faces while aggressive participants show a lack of coupling. The current data examines neural oscillations on a trial-by-trial basis and finds that healthy subjects exhibit greater theta power in several key regions prior to their deescalating responses. More unexpectedly, healthy subjects show greater coherence between sources when escalating aggressively against the opponent. This finding may reflect the non-normative nature of the response for this population. The current results are discussed light of previous studies using alternative paradigms and neuroimaging methods.

Keywords: Quantitative Electroencephalography (qEEG), Aggression, Cognitive Control, Emotion Regulation, Cognitive Neuroscience.

Disclosure: Nothing to disclose.

T17. Methylation of the Dopamine Transporter Gene as a Biomarker for Dopamine Transporter Binding in Unmedicated ADHD Patients

Corinde Wiers*, Falk Lohoff, Christine Muench, Elena Shumay, James Swanson, Gene-Jack Wang, Nora Volkow

National Institute on Alcohol Abuse and Alcoholism, Bethesda, Maryland, United States

Background: Attention deficit hyperactivity disorder (ADHD) is characterized by symptoms of inattention, hyperactivity and impulsivity. Dopamine transporters (DAT) have been shown to be decreased in adult ADHD patients compared to control groups (Volkow et al, 2007; 2009), and upregulated by chronic treatment with methylphenidate (Wang et al, 2013). Methylation of the DAT gene (SLC6A3) extracted from brain was negatively associated with DAT protein expression in the striatum of rats (Kim et al, 2014), and from DNA extracted from blood with DAT availability in the basal ganglia and impulsivity in monkeys (Rajala et al, 2014). However, it remains unknown whether methylation of SLC6A3 obtained from DNA extracted from blood is associated with DAT availability in the brain and with symptomatology in ADHD patients.

Methods: Here, we tested the association of DAT promoter methylation variation in peripheral blood with DAT availability in the brain in $n=13$ unmedicated ADHD patients (mean age $=35.2,6.0 \mathrm{SD}$ ) and in $n=34$ healthy volunteers (mean age $=34.1,8.2 \mathrm{SD}$ ). All participants underwent a Positron Emission Tomography (PET) scan with [11C]Cocaine to measure DAT availability, provided blood DNA methylation analyses of SLC6A3, and were assessed with the CAARS for ADHD symptomatology. Groups were matched for age, education, BMI and socioeconomic status (all $p>.33 \mathrm{NS}$ ). There were, however, more females in the ADHD group $(n=7)$ than the healthy group $(n=1)(\chi 2=17.7, p<.001)$.

Results: While CAARS inattention, impulsivity and hyperactivity scores were higher in ADHD subjects compared to normal volunteers (NV) (all $p \leq 0.001$ ), there were no between-group differences in DAT promoter methylation or striatal DAT availability, and neither factors were associated with age or gender. First, degree of methylation in the promoter region of SLC6A3 correlated with DAT binding potential in striatal regions in ADHD subjects only (Caudate: $\mathrm{r}=-0.80, p=.001$; Putamen: $\mathrm{r}=-0.66, p=.014$, VS: $\mathrm{r}=-0.52$, $p=.069)$. DAT availability in the VS further correlated with CAARS inattention scores in ADHD subjects only $(r=-.59$, $p=.035)$. Second, several differentially methylated sites in exon7 of SLC6A3 (average of CPG sites 553-555) correlated negatively with DAT availability in both groups in caudate (NV: $\mathrm{r}=-.478, p=.001$, ADHD: $\mathrm{r}=-0.61, p=.03$ ), putamen (NV: $\mathrm{r}=-.36, p=.049$, ADHD: $\mathrm{r}=-.53, p=.065)$ and VS in normal volunteers $(\mathrm{r}=-.47, p=.052)$. For normal volunteers only, DAT exon 7 methylation correlated positively with CAARS hyperactivity scores $(r=0.53, p=.006)$.

Conclusions: These findings suggest that peripheral DAT methylation may be predictive for striatal DAT availability in $\mathrm{ADHD}$ subjects and normal volunteers, and may be associated with inattention in ADHD cases and hyperactivity in non-ADHD individuals. Methylation of SLC6A3 extracted from DNA obtained from blood may therefore be a relevant biomarker for DAT expression in brain in ADHD.

Keywords: ADHD, Dopamine Transporter, DNA Methylation, Positron Emission Tomography Imaging.

Disclosure: Nothing to disclose.

\section{T18. Functional Connectivity in the Default Mode Network: Establishing Reproducibility and Individual Norms}

Tali Ball*, Andrea N Goldstein-Piekarski, Justine Gatt, Alex Fornito, Leanne M Williams

\section{Stanford University, Stanford, California, United States}

Background: The Default Mode Network (DMN) is defined by medial prefrontal, posterior cingulate, and temporal lobe regions that co-activate when the brain is at rest, and show reduced activation during tasks. The DMN is an intrinsic resting state network implicated in self-reflective functions, and is therefore important for understanding dimensions of health and psychopathology. Although group-level analyses have found that DMN connectivity differs in healthy adults compared to those with psychiatric conditions (see Broyd et al, 2009; Whitfield-Gabrieli \& Ford. 2012, for review), each study uses a new and typically small sample of healthy controls. We do not yet have a reproducible summary metric of healthy normative DMN function. Such a metric is essential for meaningful communication about normal vs. abnormal function at the individual patient level. Using three sizeable samples of healthy adults, our aim was to develop a summary metric of DMN connectivity and to assess its reproducibility and internal consistency. We considered topological properties (clustering, path length) as well as average DMN connectivity, aiming to identify a parsimonious metric that still captures sufficient network information, suited to future translation.

Methods: 310 healthy adults from three samples completed functional magnetic resonance scanning. Resting DMN functional connectivity was calculated in each participant 
as the absolute value of a Fisher-transformed Pearson correlation between each pair of DMN nodes (defined by prior parcellation; Gordon et al, 2015). We computed topological properties of DMN-wide weighted global clustering and weighted characteristic path length, thresholding each individual's network such that $70 \%$ of connections were retained. In addition, average connectivity across all pairs of nodes was computed. We assessed the internal consistency and reproducibility of all metrics across samples. Regional estimates of connectivity within medial prefrontal cortex and between posterior cingulate and medial prefrontal cortex were also compared against estimates for the full DMN. Finally, normative data were computed for the best-performing metrics and applied to a case example with major depressive disorder to illustrate their translational use.

Results: DMN-wide global clustering, characteristic path length, and average connectivity were all normally distributed and replicated well across samples. Internal consistency was highest and most consistent for average connectivity (Cronbach's $\alpha=0.69-0.72$ across samples). Regional connectivity averages had less consistent estimates (medial prefrontal cortex Cronbach's $\alpha=0.62-0.80$, posterior cingulate to medial prefrontal Cronbach's $\alpha=0.54-0.74$ ). Combining across all 310 healthy adult participants, mean DMN connectivity strength, in units of Fisher's Z, was 0.39 (standard deviation $[\mathrm{SD}]=0.07$ ). Mean medial prefrontal connectivity was $0.63(\mathrm{SD}=0.13)$, and mean posterior cingulate cortex to medial prefrontal connectivity was 0.47 $(\mathrm{SD}=0.14)$. A 39-year-old single male with major depressive disorder provides an illustrative clinical case example. Overall DMN connectivity was within normal limits (0.2 SD below the mean), however, regional averages showed that while medial prefrontal cortex connectivity was within normal limits ( $0.7 \mathrm{SD}$ above the mean), posterior cingulate to medial prefrontal connections showed hypoconnectivity (1.9 SD below the mean). Connectivity did not change substantially in this patient following successful treatment with Escitalopram 10mg over eight weeks (all posttreatment values within $0.2 \mathrm{SD}$ of pre-treatment), despite a 13-point reduction in Hamilton Depression Rating Scale scores.

Conclusions: Across three samples of healthy adults totaling over 300 people, we found that a simple average of functional connectivity performed as well as, or better than, more complex topological properties (i.e., global clustering and characteristic path length). The norms presented here allow for a new patient's DMN connectivity to be expressed in a single summary metric in units of standard deviations outside the mean, as in the case example. Thus, these norms have clear clinical relevance, paving the way for functional imaging measures to be used similarly to cognitive and self-report testing in understanding an individual patient's dysfunction relative to normative peers.

Keywords: Resting State Functional Connectivity, Default Mode Network, Reproducibility, Design, Replication.

Disclosure: One Mind Institute: Psyberguide Division, Consultant / Independent Evaluator, Self.
T19. Nocturnal Wakefulness as a Clinical Correlate of Next-Day Suicidal Thoughts and Antisuicidal Response to Ketamine in Depressed Patients

Elizabeth Ballard*, Jennifer Vande Voort, David Luckenbaugh, Rebecca Bernert, Erica Richards, Mark Niciu, Rodrigo Machado-Vieira, Lawrence Park, Wallace Duncan, Carlos Zarate

National Institute of Mental Health, Bethesda, Maryland, United States

Background: The suicide rate has increased since 1999, and there are few clinical options for the 400,000 Americans who present to the emergency department each year with suicidal thoughts. Sleep may represent an important modifiable risk factor for suicide; as the hours between 12- 5 AM are a particularly high risk time for suicidal behavior. Ketamine, a glutamate modulator, has been associated with rapid reductions in suicidal thoughts within minutes to hours in depressed patients. Ketamine's antidepressant response has also been linked to changes in sleep. Identifying biomarkers of ideation response can aid in understanding ketamine's mechanism of action and may provide insights into the neural underpinnings of suicidal thoughts.

Methods: Data from 65 participants with suicidal ideation and treatment-resistant MDD or BP in ketamine clinical trials will be presented. These patients underwent clinical ratings and polysomnography as part of their participation. Sleep-related biomarkers were evaluated in two areas: 1.) Baseline predictors of suicidal thoughts, and; 2.) Clinical correlates of ideation response to ketamine. Ideation response was measured using the suicide item from Hamilton Depression Rating Scale. The timeframe of interest was one day after ketamine infusion.

Results: First, nocturnal wakefulness, as defined by polysomnography, over the course of the night was associated with next morning suicidal ideation. Specifically, wakefulness during the 4-5 AM hour was associated with suicidal thoughts the next morning, when controlling for age, diagnosis, gender and depression severity, $\mathrm{F}(4,136)=3.65$, $p=.007$. Second, nocturnal wakefulness was associated with suicide ideation response, with improved sleep quality demonstrated in individuals with a reduction in suicidal thoughts after ketamine infusion, $\mathrm{F}(1,22)=5.04, p=.04$.

Conclusions: Nocturnal wakefulness was associated with both baseline suicidal thoughts and antisuicidal response to ketamine. While it is likely that suicidal ideation is response to ketamine is multifactorial, wakefulness may represent an important biomarker of suicide risk. Results underscore the importance of a translational approach to suicide research. Future directions to further evaluate the relationship between nocturnal wakefulness, suicide ideation response and ketamine will be discussed.

Keywords: Suicide, Ketamine, Sleep.

Disclosure: Nothing to disclose. 
T20. The Role of Dentate Gyrus Activin Signaling in the Antidepressant Treatment Response

Christine Yohn, Marjorie Levinstein, Rene Hen, Benjamin Samuels*

Rutgers University, Piscataway, New Jersey, United States

Background: Approximately 32-35 million adults in the US population (16\%) experience an episode of major depression in their lifetime, and commonly used treatments, such as selective serotonin reuptake inhibitors (SSRIs), are not ideal since only a subset of patients $(\sim 33 \%)$ achieves remission with initial treatment. The reasons why some individuals remit to antidepressant treatments while others do not are unknown. Our overall research program addresses this question by assessing antidepressant treatment resistance in mice. Proper assessment of the antidepressant response in mice first requires manipulations that will yield behaviors associated with negative valence constructs that can then be reversed by antidepressant treatment. Chronic treatment of mice with corticosterone (CORT) effectively induces multiple changes in behavior associated with enhanced responses to potential harm and sustained threats. Subsequent chronic treatment with antidepressants such as fluoxetine (FLX) reverses these behavioral changes in some, but not all, of the mice, permitting stratification into responders and non-responders to FLX. We found that there are differences in expression of Activin signalingrelated genes between responders and non-responders to FLX in the dentate gyrus, a region that we recently reported is critical for the beneficial effects of FLX on behavior and the hypothalamic-pituitary-adrenal (HPA) axis (Samuels et al, 2015).

Methods: Our studies were aimed at confirming our gene expression data in multiple stress paradigms and then determining whether manipulation of dentate gyrus Activin signaling can alter the behavioral response of mice to FLX.

Results: Mice were grouped into responders and nonresponders to CORT+FLX by assessing behavior in both the novelty suppressed feeding and forced swim tests. RNA was then prepared from dentate gyrus tissue and used for qPCR. We found decreases in expression of the Activin signaling components Acvr1, Acvr1c, Acvr2b, and Smad4 in nonresponders relative to responders. In addition, we found increased expression of the inhibitory Smad7 in nonresponders relative to responders. We next confirmed these results in mice exposed to Social Defeat+FLX. Finally, we found that activation of Activin signaling, achieved via chronic infusions of Activin peptide into dentate gyrus, converted behavioral non-responders to CORT+FLX into responders.

Conclusions: Our data suggest that molecular manipulation of the dentate gyrus may provide an effective augmentation strategy for non-responders to SSRI treatment. These data indicate that the dentate gyrus is a critical component of the neural circuitry underlying the antidepressant response and that local Activin signaling manipulations are a potential avenue for novel treatments.

Keywords: Antidepressant Response, Behavioral Pharmacology, Mice, Dentate Gyrus.

Disclosure: Nothing to disclose.

\section{T21. Withdrawn}

\section{T22. Shifting Priorities: Brain Network Adaptations to Chronic Stress}

Yuliya Nikolova*, Keith Misquitta, Brad Rocco, Jacob Ellegood, Jason Lerch, Ahmad Hariri, Mounira Banasr, Etienne Sibille

\section{Centre for Addiction and Mental Health, Toronto, Canada}

Background: Human in vivo magnetic resonance imaging (MRI) studies have associated depression with changes in the morphology, function, and connectivity of a distributed corticolimbic circuitry including the amygdala, hippocampus, and prefrontal cortex. Studies in stress-based rodent models have begun to provide complementary insight into the disorder's putative molecular mechanisms. To help bridge these parallel lines of work, we used MRI to assess regional brain volume and global structural covariance patterns in the unpredictable chronic mild stress (UCMS) mouse model of depression and a human sample of Duke Neurogenetics Study (DNS) participants reporting high and low levels of childhood trauma.

Methods: Male Balb/c mice were exposed to six weeks of UCMS and behaviorally characterized. High-resolution 7T structural MRI images were collected ex vivo from fixed brains ( $n=12$ UCMS-exposed, $n=9$ controls), followed by an immunohistochemistry analysis of the postsynaptic density protein 95 (PSD95), vesicular glutamate transporter 1 (Vglut1) and the microtubule-associated protein 2 (MAP2) in amygdala sections to index synaptic number and strength. Volumetric comparisons between groups were carried out on MRI data from 26 regions of interest (ROIs) pre-selected based on broad cross-species relevance to behavioral and emotion regulation. Graph theory analysis using whole-brain (i.e., 155 ROIs) structural covariance metrics was applied to assess group differences in overall brain network organization. We evaluated mean connection strength, shortest path length (indicative of global efficiency), and local clustering on the whole-network level. In addition, on the node level, we assessed degree (i.e., number of connections) and connection strength for the regions showing strongest stress-related volumetric changes in the univariate analysis. Permutation testing ( $n=10,000$ iterations) across a range of density thresholds (10-30\%) was applied to assess the significance of the observed between-group network and node attribute differences. The same structural covariance analysis was conducted in DNS participants scoring in the top 25\% ( $n=299,177$ women) and bottom $25 \%(n=237,143$ women) of the observed range of total Childhood Trauma Questionnaire (CTQ; $>37$ and $<27$, respectively) scores.

Results: UCMS-exposed mice showed relative volume increases in the amygdala, as well as the frontal association cortex, prelimibic, medial and dorsomedial orbital cortices $(p<0.05$, FDR corrected) in the absence of differences in total brain volume. Furthermore, stressed animals showed a trend toward having a lower average network connection strength and significantly reduced local network clustering, 
concurrent with higher degree and connection strength of the amygdala, particularly at densities greater than $20 \%$. These changes occurred in the absence of any differences in shortest path length. No consistent differences in degree and connection strength emerged for any of the other ROIs showing volumetric changes as a function of stress. Furthermore, stress-exposed mice had higher levels of PSD95 $(p=0.025)$, but not Vglu1 or MAP2 $(p>0.1)$ in the amygdala. A convergent structural covariance pattern was observed in the human in vivo sample where individuals reporting high childhood trauma had reduced network clustering and increased amygdala degree and connection strength, particularly in the $10-12 \%$ density range.

Conclusions: The increase in amygdala in UCMS is consistent with human work showing larger amygdala volumes in first-episode depression or following early life stress. Furthermore, the increased number and strength of the amygdala's structural covariance connections may indicate a greater ability of the amygdala to "drive" behavior through enhanced connectivity with regions of the brain not normally part of its subconnectome. This is further supported by the observed increase in PSD95, which suggests greater number and strength of synapses in the amygdala of UCMS-exposed animals. The concurrent decrease in mean network connection strength and local clustering suggests a global reorganization pattern favoring the selective strengthening of nodes involved in the avoidance of threat at the expense of other nodes of potential behavioral relevance, reflecting a network adaptation to shifting behavioral priorities. The convergent pattern we observe in humans lends cross-species translational utility of the proposed model and offers promise for further synergy between basic preclinical work and in vivo human neuroimaging in depression treatment and prevention.

Keywords: Stress Models, Amygdala, MR Imaging, Depression.

Disclosure: Nothing to disclose.

\section{T23. Early Life Stress and Glutamate Neurotransmission in Major Depressive Disorder}

Lynnette Averill*, Chadi Abdallah, Mark Niciu, Lisa Fenton, Madonna Fasula, Lihong Jiang, Douglas Rothman, Graeme Mason, Gerard Sanacora

National Center for PTSD, West Haven, Connecticut, United States

Background: Early life stress (ELS) and glutamate neurotransmission have been implicated in the pathophysiology of major depressive disorder (MDD). In non-human primates, ELS was positively correlated with cortical Glx (i.e. glutamate + glutamine). However, the relationship between ELS and cortical glutamate in adult patients with MDD is not fully known.

Methods: Using $1 \mathrm{H}$ Magnetic Resonance Spectroscopy (MRS), we measured occipital cortical glutamate and glutamine levels in 36 medication-free patients with MDD. In a subsample $(n=11)$, we measured dynamic glutamate/ glutamine cycling (Vcycle) using advanced 13C MRS methods. ELS history was assessed using Early-life Trauma Inventory (ETI).
Results: We found a significant positive correlation between ETI scores and occipital glutamine ( $r s=0.39, p=0.017$ ), but not glutamate. Post-hoc analyses showed that the association with glutamine was driven by ETI emotional abuse (ETI-EA) subscale ( $r s=0.39, p=0.02$ ). Vcycle was not significantly correlated with ETI ( $r s=0.55, p=0.087)$, but positively correlated with ETI-EA ( $\mathrm{rs}=0.67, p=0.03$ ).

Conclusions: Patients with childhood emotional abuse appear to have increased occipital glutamate neurotransmission as reflected by increased glutamate/glutamine cycling and glutamine level. Future studies would be needed to confirm this pilot evidence and to examine whether ELS effects on glutamate neurotransmission underlie the relationship between ELS and psychopathology.

Keywords: Early Life Stress, Major Depressive Disorder (MDD), Glutamate, MR Spectroscopy.

Disclosure: Nothing to disclose.

\section{T24. Potential Antidepressant Mechanism of Quetiapine in the Chronic Mild Stress Rodent Model of Depression}

Jared Moreines*, Zoe Owrutsky, Anthony Grace

University of Pittsburgh School of Medicine, Pittsburgh, Pennsylvania, United States

Background: Quetiapine and other second-generation dopamine (DA) D2 receptor antagonists (D2RA), e.g. lurasidone, are increasingly popular monotherapy and adjunct antidepressant pharmacologic strategies in treatmentresistant patients. However, the therapeutic mechanism of these agents in depression is unknown. Specifically, given that the therapeutic effect of D2RA in schizophrenia is thought to be a reduction in DA transmission, how such a mechanism could counteract depressive symptoms remains poorly understood given evidence that, in contrast to in schizophrenia, the DA system is underactive in depression. Thus we sought to elucidate the effects of quetiapine on the mesolimbic DA system to identify possible mechanisms for its antidepressant effect. We examined the effect of quetiapine administered acutely and repeatedly in both the short (10-day) and long (21-day) term. These effects were studied both in normal rats and rats exposed to the chronic mild stress (CMS) rodent model of depression, which we have previously shown to inhibit ventral tegmental area (VTA) DA neuron activity.

Methods: Single-unit extracellular recordings of identified VTA DA neurons were performed in anesthetized SpragueDawley rats to quantify DA neuron population activity, i.e. the number of DA neurons that are spontaneously active in the baseline state. This is significant because only spontaneously active DA neurons can burst fire to environmentally salient stimuli (e.g. unexpected reward). Furthermore, DA neuron population activity, quantified as the average number of spontaneously active DA neurons encountered during each passage of an electrode through the VTA, is consistently reduced in rats exposed to CMS. We quantified the effects of quetiapine on VTA DA neuron population activity in normal rats and rats exposed to the CMS model of depression, allowing us to compare the impact of quetiapine on a naïve DA system to that in a hypoactive state more relevant to individuals with depression. We used a quetiapine dose of 
$10 \mathrm{mg} / \mathrm{kg}$, which has previously been shown to induce antidepressant effects in rodent models.

Results: Following acute administration of quetiapine to normal, stress-naïve rats, we observed a significant $(p<0.05)$ increase in the number of spontaneously active VTA DA neurons. This effect persisted in normal rats exposed to 10 days of repeated quetiapine administration. However, following longer term repeated administration, i.e. $>21$-days, VTA DA neuron population activity returned to a level comparable to normal vehicle-treated rats. In contrast, in rats that were exposed to CMS beginning 5 weeks prior to the initiation of quetiapine and continuing throughout the 21-day course of repeated administration, VTA DA neuron population activity was significantly increased compared to vehicle-treated CMS-exposed rats and was indistinguishable from that of unstressed vehicle-treated control rats.

Conclusions: These data lend insight into the effects of quetiapine on the dopamine system. In normal rats, quetiapine acutely increases VTA DA neuron population activity; however, this effect does not persist following chronic administration. In contrast, chronic administration of quetiapine to CMS-exposed rats restored normal DA system function, suggesting that in the presence of a hypodopaminergic state, there is a persistent increase in DA population activity with chronic administration. These findings raise important questions about how chronic treatment with novel D2RA therapy differentially impacts the DA system of healthy individuals compared to patients in a depressive state. Moreover, in contrast to the higher dose second generation drug treatment for schizophrenia, which reduces DA neuron population activity due to depolarization block, at doses used in treating depression repeated administration does not induce depolarization block, but instead eventually normalizes DA neuron population activity. We propose that with repeated administration of quetiapine, the resultant D2 supersensitivity offsets the DA blockade produced by the antagonist, allowing the increased population activity to reverse the CMS-induced diminished reward-related responsivity of the DA system. In summary, our data indicate that quetiapine's antidepressant effect may be mediated through the DA system, via weak antagonism of DA D2 receptors resulting in a compensatory up-regulation of DA neuron activity. This suggests that enhancing DA system activity via selective D2 autoreceptor antagonism may be a potential avenue for future antidepressant therapeutic development.

Keywords: Dopamine, Antipsychotic Agents, Animal Models, Chronic Stress.

Disclosure: Nothing to disclose.

\section{T25. Cognitive Behavioral Therapy to Sustain the Antidepressant Effects of Ketamine}

Samuel Wilkinson*, Robert Ostroff, Madonna Fasula, DaShaun Wright, Matthew Griepp, Gerard Sanacora

Yale University School of Medicine, New Haven, Connecticut, United States

Background: Ketamine has been shown to be a rapid and robust antidepressant, though effects of a single treatment are short-lived. Repeated infusions may prolong the effect somewhat, but worrisome cognitive effects of repeated exposure in rodents and substance abusers give pause to indefinite treatment. Thus, there is a great need to identify alternative augmentation/maintenance strategies capable of sustaining the ketamine's rapid antidepressant effects. Ketamine has also been noted to enhance learning and memory within a 24-hr window following administration in humans and rodents, suggesting the drug may create a window of enhanced plasticity. Cognitive behavioral therapy (CBT) is an effective treatment to augment classic antidepressant medications and is highly effective in relapse prevention. In a sense, the goals of CBT are to re-learn nonpathologic thought patterns and core beliefs, leading us to speculate that patients may be particularly able to engage in CBT in the period following ketamine exposure. This pilot study aims to explore the efficacy and feasibility of combining cognitive behavioral therapy (CBT) and intravenous ketamine infusions for treatment-resistant depression. Our hypothesis is that the state of neural hyperplasticity induced by ketamine infusions provides an opportune period for the therapeutic aims of CBT, namely extinction and modification of negative core beliefs.

Methods: Eighteen subjects with a Major Depressive Episode will be recruited to undergo a brief course of 4 intravenous infusions of ketamine, given twice weekly for two weeks. Concurrent with ketamine, they will receive CBT twice weekly for two weeks, then weekly for the following 8 weeks (12 total sessions in 10 weeks). Efficacy will be assessed using measures of sustained response ( $>50 \%$ decrease in Montgomery-Asberg Depressive Rating Scale [MADRS]) for 8 weeks following the last infusion. Among responders, relapse is defined as $<50 \%$ improvement on the MADRS scale compared to baseline for two concurrent visits. As a measure of target engagement (i.e., that ketamine renders subjects better able to engage in CBT), cognition will be assessed (within-subject comparison) pre- and 24-hours post-ketamine.

Results: To date, 16 subjects have enrolled and initiated or completed the protocol with a mean age of 42.2 years (SD $13.3)$. Most $(12 / 16,75 \%)$ are women and $5(38 \%)$ had a history of ECT. The mean length in months of the current episode is 46.7 months (SD 75.6) and the mean number of antidepressant trials in the current episode was 2.6 (SD 2.0). Half of the subjects (8/16) achieved response within the ketamine treatment period. Among completed responders, $75 \%(6 / 8)$ retained response through 8 weeks following the last infusion. On longer-term follow-up of responders, the median time to relapse was 12 weeks.

Conclusions: Preliminary data demonstrate the feasibility of combining ketamine and CBT, with suggestions that CBT may sustain antidepressant effects following 4 ketamine infusions. Additional large and well-powered randomized controlled studies are warranted to definitively demonstrate efficacy.

Keywords: Ketamine, Cognitive Behavior Therapy, Relapse, Antidepressant, Major Depressive Disorder.

Disclosure: Nothing to disclose. 
T26. Sex-Specific Transcriptional Signatures in Human Depression

Benoit Labonté*, Olivia Engmann, Immanuel Purushothaman, Caroline Menard, Junshi Wang, Chunfeng Tan, Joseph Scarpa, Gregory Moe, Eddie Loe, Michael Cahill, Zachary Lorsch, Hamilton Peter, Erin Calipari, Georgia Hodes, Orna Issler, Hope Kronman, Madeline Pfau, Aleksander Obradovic, Yan Dong, Rachael Neve, Scott Russo,

Andrew Kazarskis, Carol Tamminga, Naguib

Mechawar, Gustavo Turecki, Bin Zhang, Li Shen, Eric Nestler

Icahn School of Medicine at Mount Sinai, New York, New York, United States

Background: Major depressive disorder (MDD) is a severe debilitating mental illness, affecting yearly 350 million people worldwide, inducing major economic and medical burdens on societies. While it affects both males and females, MDD is characterized by a strong sexual dimorphism. Compared to males, females are 2-3 times more susceptible to develop MDD, exhibit higher symptom severity, tend to associate with greater functional impairments, atypical depressive symptoms and higher rates of co-morbid anxiety. Furthermore, males and females with MDD have been reported to respond differently to antidepressant treatment. However, while the incidence, symptoms and treatment of MDD all point toward major sex differences in MDD, the molecular mechanisms underlying this sexual dimorphism remain largely unknown.

Methods: Transcriptional profiles from male and female post-mortem brains with MDD and controls were scanned in 6 brain regions (BA25, BA11, BA8/9, anterior insula, HPC and NAc) using RNAseq. Transcriptional profiles from the NAc and PFC in both male and female mice after chronic variable stress (CVS) were also assessed using RNAseq. Sex and brain region transcriptional profiles were analyzed through differential analysis and weighted gene coexpression network analysis (WGCNA). Human and mouse transcriptional profiles were overlapped to identify points of interspecies convergence in the genes and pathways commonly affected by chronic variable stress (CVS). We capitalized on human/mouse converging pathways to define the molecular and physiological mechanisms underlying the expression of stress susceptibility in males and females. Viral-mediated gene transfer was used to assess the behavioral relevance of our findings. We identified the cellular substrate in which these effects take place in humans and mice. We used electrophysiological measures to define the physiological impact sex-specific genes have on cellular activity and then used RNAseq to relate these changes to the structure of gene networks.

Results: Our results show a major rearrangement of transcriptional patterns, with male and female transcriptional profiles sharing less than $5 \%$ of overlap in genes differentially expressed across brain regions. This small overlap was reproduced in male and female mice after CVS. The comparison between our human MDD and stress mice transcriptional profiles revealed sex-specific genes and functional pathways commonly affected in the PFC and NAc. The strong sexual dimorphism characterizing MDD in males and females was confirmed through our gene network analysis. Our findings suggest that MDD in males and females arise in part from the activity of similar gene modules, which share cellular and biological specificity, but which are organized and expressed differently across brain regions in the two sexes. We identified male and female hub genes and confirmed their sex-specific impact as stresssusceptibility mediators. Using viral-mediated gene transfer in the PFC, we showed that the downregulation of the female-specific hub gene DUSP6, a cytoplasmic dualspecificity phosphatase, increases stress susceptibility in females but not in males. We confirmed that this effect associates with increased neuronal excitability defined by higher frequency of spontaneous excitatory post-synaptic currents (sEPSC) in pyramidal neurons. We further showed that these effects take place in a subpopulation of CamKII expressing pyramidal neurons in the PFC of both females with MDD and stress female mice. Consistent with the role of DUSP6, these effects were associated with higher phosphorylation levels of ERK in the PFC of females with MDD and stress female mice. Using RNAseq, we showed that DUSP6 downregulation induces its behavioral and molecular effects by interfering with ERK translocation to the nucleus thus changing the global organization of transcriptional profiles in the PFC of females. Furthermore, we provide evidence for interactions between DUSP6-ERK and other signaling members including small GTPases and ATP-dependent signaling pathways in mediating stress susceptibility in females.

Conclusions: In this study, we combined network-based and differential gene expression approaches to provide an unbiased and comprehensive characterization of the transcriptional sexual dimorphism in MDD. We further characterized the effects of stress in males and females by identifying specific functional pathways preserved in a mouse model of variable stress. Besides defining the transcriptional profiles of males and females with MDD, we also provided solid evidence uncovering the molecular and cellular mechanisms underlying sex-specific stress susceptibility. Together, this study represents a great resource for the investigation of sex-differences in mood disorders and the identification of molecular pathways which may be significantly involved in mediating the effect of stress in males and females and which may be considered as potential targets for the treatment of MDD.

Keywords: Major Depressive Disorder (MDD), Sex Difference, Transcriptome, ERK, Coexpression Network.

Disclosure: Nothing to disclose.

\section{T27. Artificially Modulating Memories to Alleviate Psychiatric Disease-Like States}

Steve Ramirez ${ }^{*}$

Harvard University, Cambridge, Massachusetts, United States

Background: Chronic stress affects numerous brain areas involved in memory, emotion, and motivation, such as the 
hippocampus, amygdala, and prefrontal cortex; it abnormally alters a variety of cellular events, including dendritic morphology and gene expression patterns; and, it can precipitate several maladaptive states, such as depression- and anxiety-like behaviors. Traditionally, reversing these conditions has relied on drug-based interventions, which by their nature produce brain-wide nonspecific effects and rely on drugs that are iterations of, and without improved efficacy over, their 1960s counterparts. These sobering results highlight the need for novel interventions in the biomedical community. In both mice and humans, the hippocampus has been implicated in storing and retrieving positive memories and in modulating stress-related states. Promisingly, our recent work has demonstrated that artificially stimulating cells in the hippocampus that previously were active during positive memory formation are sufficient to increase reward-like behavior and motivation. Here, we seek to leverage these findings by testing whether or not directly activating endogenous neuronal processes supporting memory is an effective means to intervene with and prevent stress-induced psychiatric disease-like states.

Methods: We utilized a recently developed virus system in which the promoter of the immediate early gene c-Fos drives the expression of the light-sensitive ion channel channelrhodopsin-2 in a manner that is under the control of the antibiotic Doxycycline. In the absence of Doxycycline (Dox), learning-induced neuronal activity selectively labels active c-Fos-expressing neurons with channelrhodopsin-2, thus conferring activity-dependent and inducible labeling of, in addition to optical control over, hippocampus cells and their corresponding axon terminals. Animals were taken off Dox and exposed to a social reward or to contextual fear conditioning to label hippocampus cells active during each process. These cells were next either acutely or chronically activated and the results behavioral phenotypes were assayed in a variety of depression, anxiety, and memory-related tasks.

Results: We find that acute activation of hippocampus cells that were previously active during the formation of negative or positive memories is sufficient to mimic or to alleviate the effects of chronic stress in a battery of depression and anxiety-related assays. Moreover, chronic optical activation of negative memories via the dorsal hippocampus was sufficient to induce memory extinction in a contextspecific manner. Unexpectedly, we observed that chronic stimulation of negative memories via the ventral hippocampus produced a context-specific enhancement of the associated memory.

Conclusions: Overall, our conclusions are twofold: we demonstrate the feasibility of harnessing the brain's endogenous plasticity mechanisms by means of positive and negative memory activation to resolve its therapeutic potential as well as its role in generating maladaptive behavior, respectively; and, we demonstrate that chronic stimulation protocols offer effective means to lastingly suppress the return of fear.

Keywords: Hippocampus, Memory and Learning, Neuropsychiatric Disorders, Depression, Anxiety.

Disclosure: Nothing to disclose.
T28. Treatment Mechanism of Magnetic Seizure Therapy for Major Depression: Insights From TMS-EEG Measures

Yinming Sun*, Faranak Farzan, Benoit Mulsant, Tarek Rajji, Paul Fitzgerald, Mera Barr, Jonathan Downar, Willy Wong, Daniel Blumberger, Zafiris Daskalakis

University of Toronto, Toronto, Canada

Background: Magnetic seizure therapy (MST) is a promising intervention for treatment resistant depression (TRD). While MST has been shown to be effective, its mechanism of action is still not clear. Increased neural plasticity, which can arise from activation of large brain networks through a seizure, has been proposed as a therapeutic mechanism following ECT-induced seizures. To evaluate if neuroplasticity is enhanced through MST, we measured changes in cortical evoked activity (CEA) and cortical inhibition (CI) using combined transcranial magnetic stimulation and electroencephalography (TMS-EEG).

Methods: Twenty TRD patients (11 females, mean age 44.8 [13.0] years) with pre and post TMS-EEG measures were part of this study. Clinical symptoms were assessed with the 24-items Hamilton Depression Rating Scale (HDRS-24) and Scale for Suicidal Ideation (SSI). All subjects received single and paired pulse stimulation delivered to the dorsolateral prefrontal cortex (DLPFC) and motor cortex as a control site. Both clinical and TMS-EEG measures were collected at baseline and after a course of MST treatment. CEA was assessed by the area under the curve of the single pulse TMS evoked potential (TEP). CI was assessed by the suppression of the paired pulse TEP relative to the single pulse TEP (i.e. long interval cortical inhibition [LICI]). Subjects were divided into responders $(n=8$, HDRS-24 change $\geq 50 \%)$ and non-responders $(n=12)$. Subjects who had a nonzero SSI score at baseline $(n=15)$ were also divided into suicidal ideation remitters $(n=7$, post SSI $=0)$ and non-remitters $(n=8$, post SSI $>0)$. All comparison results were corrected using cluster based statistics.

Results: CEA was increased in the theta frequency range (4-7 $\mathrm{Hz}$ ) after MST treatment for the frontal central electrodes (cluster $p<0.005$; $\max$ at FC3 and C3, t-score $=4.0$ and 4.1) and was higher in responders than non-responders over the same region (cluster $p<0.05$; $\max$ at $\mathrm{C} 1$, t-score $=3.3$ ). The change in LICI was found to correlate with the decrease in SSI over the frontal and central electrodes (cluster $p<0.05$; $\max$ at $\mathrm{CZ}$, spearman rho $=0.77)$. Moreover, based on the LICI change from the electrode showing the maximum correlation with SSI change (i.e. CZ), suicidal ideation remitters and non-remitters can be correctly identified with $85.7 \%$ sensitivity and $100 \%$ specificity (area under curve, $0.98 ; p=0.002$ ).

Conclusions: These results suggest that neural plasticity is enhanced through MST. Our findings also suggest that enhanced neural plasticity may account for differences in treatment response. Changes in neural plasticity predicted remission of suicidal ideations and may point to the therapeutic mechanism that underlies the treatment.

Keywords: Magnetic Seizure Therapy, Treatment Resistant Depression, Transcranial Magnetic Stimulation, Quantitative Electroencephalography (qEEG).

Disclosure: Nothing to disclose. 
T29. Role of Blood-Brain Barrier Permeability and Tight Junction Protein Claudin-5 in Vulnerability to Social Stress and Major Depressive Disorder

Caroline Menard*, Madeline Pfau, Veronika Kana, Victoria Wang, Georgia Hodes, Sylvain Bouchard, Hossein Aleyasin, Meghan Flanigan, Aki Takahashi, Sam Golden, Mitra Heshmati, Matthew Campbell, Cheuk Ying Tang, Miriam Merad, Scott Russo

Icahn School of Medicine at Mount Sinai, New York, New York, United States

Background: Multiple clinical studies suggest that heightened peripheral inflammation contributes to major depression disorder (MDD) pathogenesis. Co-morbidity of inflammatory diseases is highly prevalent in MDD patients, and MDD patients have a higher risk of developing those diseases. It has been hypothesized that circulating inflammatory molecules are released following chronic stress, penetrate the blood brain barrier (BBB), and affect neural circuits, mediating stress vulnerability and depression.

Methods: In this study we investigated the effect of chronic social defeat stress (CSDS), a mouse model of depression, on $\mathrm{BBB}$ permeability and regulation of tight junction protein Cldn5. Briefly, experimental mice are exposed to a larger dominant aggressor for $10 \mathrm{~min}$ each day for 10 consecutive days. Following CSDS most of the mice will develop depressive-like behaviors such as social avoidance and anhedonia. Tight junction proteins are produced by endothelial cells of the blood vessels and establish the paracellular barrier that controls the flow of molecules circulating between these cells. Consequently, Cldn 5 proteins actively contribute to the maintenance of $\mathrm{BBB}$ impermeability to circulating inflammatory markers. We evaluated the effect of CSDS on tight junction expression in the nucleus accumbens (NAc). NAc is a key brain region in reward, aversion and motivation processes and has been linked to the circuitry underlying depression symptoms.

Results: We found that after 10 days of CSDS, Cldn 5 mRNA and protein expression is reduced in the NAc of stresssusceptible mice when compared to resilient mice and unstressed controls. In fact, lower permissive acetylation and enhanced repressive methylation was measured along the Cldn5 gene promoter in stress-susceptible mice in comparison to resilient animals. We found a similar decrease of Cldn5 mRNA in the NAc of depressed patients. Interestingly, in mice, chronic treatment with the antidepressant imipramine promotes resilience and rescues Cldn 5 expression in the NAc. Moreover, chronic down-regulation of Cldn5 expression with an adeno-associated virus including a short hairpin RNA specific to CLDN5 was sufficient to induce social avoidance and depression-like behaviors as assessed with sucrose preference and forced swim tests. Magnetic resonance imaging scans revealed higher penetration of a gadolinium-based contrasting agent in stress-related brain regions of defeated animals suggesting reduced $\mathrm{BBB}$ integrity.

Conclusions: Our study highlights a functional role for the tight junction protein Cldn5 in social defeat-induced changes in $\mathrm{BBB}$ permeability and the development of depression-like behaviors related to MDD. By understanding how chronic stress affects the $\mathrm{BBB}$ we may be able to augment current antidepressant treatment or design new therapeutic strategies targeting BBB permeability.

Keywords: Major Depression, Blood-Brain Barrier, Immune Mechanisms.

Disclosure: Nothing to disclose.

T30. Genome-Wide Markers of Illness Vulnerability in Bipolar Disorder: A Preliminary Study in Youth at High Risk

Gabriel Fries*, Joao Quevedo, Cristian P Zeni, Giovana Zunta-Soares, Danielle E Spiker, Charles L Bowden, Consuelo Walss-Bass, Jair C Soares

University of Texas Health Science Center at Houston, Houston, Texas, United States

Background: First-degree relatives of patients with bipolar disorder (BD) have a higher risk of developing $\mathrm{BD}$ and other mental illnesses than the general population. However, the biological underpinnings of this increased risk are still unknown, particularly because most of the studies so far have been conducted in chronically ill adults and not in unaffected youth at high-risk. DNA methylation, which is the most commonly studied epigenetic marker and is responsive to both genotype and environmental exposure, may underlie this risk and act as a trigger for $\mathrm{BD}$. In addition, it can lead to changes in the expression of key genes, potentially contributing to disease susceptibility. In this sense, we hypothesized that DNA methylation and gene expression alterations in peripheral pathways would discriminate between unaffected subjects at high-risk for BD and healthy controls and could be promising biomarkers to monitor early presentation in youth at risk for developing BD.

Methods: In this preliminary cross-sectional study we analyzed genome-wide expression and methylation levels in peripheral blood mononuclear cells from children and adolescents from three matched groups: BD patients $(n=6)$, unaffected offspring of bipolar parents (high-risk, $n=6$ ), and healthy controls (low-risk, $n=6$ ). Subjects were recruited as part of the Houston Area Pediatric Bipolar Registry, and informed consent was obtained from all participants and parents/guardians. Peripheral blood mononuclear cells were isolated from blood and subjected to the isolation of RNA and DNA using commercial kits. These were interrogated by the Human HT-12 v4 Expression BeadChip array (Illumina) and the Infinium HumanMethylation450 BeadChip array (Illumina), respectively. Gene expression data were adjusted for background and quantile normalized in GenomeStudio software v2011.1, followed by differential expression analysis in the software JMP Genomics 7. Student's $t$ test was used to analyze values, with a Benjamini Hochberg false discovery rate correlation of $1 \%$, alpha $=0.05$, and a cutoff of $-\log 10(p$-value $)>1.5$. A few genes shown to be differentially expressed between groups were selected for validation by quantitative real-time PCR. Genome-wide methylation data were processed using the GenomeStudio v2011.1 software, where quality control checks, normalization (controls), and background adjustment were performed. Illumina custom was employed as the error model and false discovery rate was computed. Probes 
with a detection $p$-value $>0.01$, located on the $\mathrm{X}$ or $\mathrm{Y}$ chromosomes, and containing single nucleotide polymorphisms were removed from the analysis. Ingenuity Pathway Analysis (Qiagen) was used for the assessment of enrichment in canonical pathways and networks in the list of genes identified in the previous analyses. Cell-based assays were also performed in lymphoblastoid cell lines from 10 healthy controls and 10 adult $\mathrm{BD}$ patients matched for age, gender, ethnicity, and race. Cells were treated for $96 \mathrm{~h}$ with either 1 or 5uM 5-aza-2'-deoxycytidine (5AzadC), a DNA methyltransferase inhibitor, after which the levels of 5-methylcytosine and genes from the glucocorticoid receptor pathway were assessed.

Results: By integrating gene expression and DNA methylation data and comparing the lists of differentially expressed genes and differentially methylated probes between groups, we were able to identify 43 risk genes that discriminate patients and high-risk youth from controls. Pathway analysis with the list of risk genes showed an enrichment of the glucocorticoid receptor pathway $(p=0.00194)$ with the genes MED1, HSPA1L, GTF2A1, and TAF15, which might underlie the previously reported role of stress response in the risk for $\mathrm{BD}$ in vulnerable populations. Cell-based assays suggest that these genes can be modulated by DNA methylation in cells from adult BD patients and controls, which suggests that manipulating their expression could counteract the familial risk presented by those subjects. Specifically, treatment with $5 \mathrm{AzadC}$ significantly reduced the expression levels of MED1 and TAF15 and increased the expression of HSPA1L in both patients and controls $(p<$ 0.05 for all comparisons). Finally, in order to identify the potential impact of the risk genes in clinical measures, we correlated the expression of MED1, GTF2A1, HSPA1L, and TAF15 with variables related to family environment (cohesion and conflict). Family cohesion scores were positively correlated with the expression levels of HSPA1L $(p=0.049)$, GTF2A1 $(p=0.042)$, and MED1 $(p=0.011)$, but not significantly with TAF15 $(p=0.066)$. In contrast, family conflict scores were negatively correlated with the expression of the four genes ( $p<0.05$ for all correlations). Of note, the correlations for each gene clearly discriminated controls from $\mathrm{BD}$ patients and high-risk offspring.

Conclusions: Although preliminary, our results suggest the use of peripheral measures in the identification of biomarkers of risk in vulnerable populations. While our data report baseline findings in this at-risk cohort, an in-depth longitudinal investigation of this sample - which is being yearly followed up in our Department as subjects transition through the peak period of risk for developing $\mathrm{BD}$ - will be able to determine if these markers are useful in future risk prediction for the development of BD.

Keywords: Bipolar Disorder, DNA Methylation, Gene Expression, Glucocorticoid Receptor, Risk.

Disclosure: Nothing to disclose.
T31. Symptomatology and Predictors of Antidepressant Efficacy in Extended Responders to a Single Ketamine Infusion

Mark Niciu*, Steven Pennybaker, David Luckenbaugh, Carlos Zarate

National Institute of Mental Health, Bethesda, Maryland, United States

Background: The antidepressant response to a single subanesthetic dose infusion of the glutamatergic modulator ketamine is transient in most depressed patients; however, a minority continue to experience an extended response. This study examined depressive symptoms and potential clinical predictors of extended response to ketamine in subjects with treatment-resistant major depression in order to identify such symptoms and predictors.

Methods: All subjects were diagnosed with either Diagnostic and Statistical Manual of Mental Disorder (DSM)-IV-Text Revision (TR) major depressive disorder (MDD) or bipolar depression. All subjects were treatment-resistant and in a current major depressive episode of at least moderate severity. MDD subjects were unmedicated and those with bipolar depression were receiving therapeutic-dose lithium or valproate. All subjects received a single $0.5 \mathrm{mg} / \mathrm{kg}$ ketamine infusion. Data were collected pre-infusion (baseline) and at days one, 14 , and 28 post-infusion.

Results: Twelve of 93 (12.9\%) participants continued to meet response criteria [as defined by a $50 \%$ reduction in Montgomery-Asberg Depression Rating Scale (MADRS) score] at two weeks. All depressive symptoms were improved at two weeks in ketamine responders except for sleep duration/depth. Family history of alcohol use disorder in a first-degree relative (FHP) and dissociation during the infusion were both associated with antidepressant response at two weeks. Improved apparent sadness, reported sadness, inability to feel, and difficulty concentrating at day 1 correlated most strongly with antidepressant efficacy at two weeks.

Conclusions: The correlation between improvements in specific depressive symptoms at day one with overall twoweek antidepressant efficacy may provide a clinically useful way to gauge the likelihood of extended antidepressant response to a single ketamine administration in individual subjects. Unchanged sleep symptoms at two weeks suggests that sleep may be one of the more refractory symptoms in glutamatergic antidepressant trials (as previously observed with monoamine-based antidepressants). FHP and increased intra-infusion dissociation, also previously reported to correlate with ketamine's antidepressant efficacy at earlier time points, may be critical predictors to improve our understanding of ketamine's mechanism of action. In sum, our findings may assist future discoveries of glutamate-based antidepressant mechanisms as well as assist clinicians in predicting which subjects are more likely to experience extended antidepressant response to ketamine. These predictors should also be considered in the design and analysis of multiple ketamine infusion studies in major depression.

Keywords: Major Depressive Disorder, Bipolar Depression, Ketamine, Antidepressant, Predictors of Response.

Disclosure: Nothing to disclose. 
T32. Parsing Heterogeneity in the Brain Connectivity of Depressed and Healthy Adults During Positive Mood

Rebecca Price*, Stephanie Lane, Kathleen Gates, Thomas Kraynak, Michelle Horner, Michael Thase, Greg Siegle

University of Pittsburgh, Pittsburgh, Pennsylvania, United States

Background: There is well-known heterogeneity in affective mechanisms in depression that may extend to positive affect. We used data-driven parsing of neural connectivity to reveal subgroups present across depressed and healthy individuals during positive processing, informing targets for mechanistic intervention.

Methods: 92 individuals (68 depressed patients, 24 neverdepressed controls) completed a sustained positive mood induction during fMRI. Directed functional connectivity paths within a depression-relevant network were characterized using Group Iterative Multiple Model Estimation, a method shown to accurately recover the direction and presence of connectivity paths in individual participants. During model-selection, individuals were clustered using community detection on neural connectivity estimates. Subgroups were externally tested across multiple levels of analysis.

Results: Two subgroups emerged: Subgroup A, characterized by weaker connectivity overall, and Subgroup B, exhibiting hyperconnectivity (relative to Subgroup A), particularly among ventral affective regions. Subgroup predicted diagnostic status (Subgroup B contained $81 \%$ of patients; $50 \%$ of controls; $\chi 2=8.6, p=.003)$ and default mode network connectivity during a separate resting state task. Among patients, Subgroup B members had higher self-reported symptoms, lower sustained positive mood during the induction, and higher negative bias on a reaction time task (p's <.05). Symptom-based depression subgroups did not predict these external variables.

Conclusions: Neural connectivity-based categorization travels with diagnostic category and is clinically predictive, but not clinically deterministic. Both patients and controls showed heterogeneous, and overlapping, profiles. The larger, and more severely affected patient subgroup was characterized by ventrally-driven hyperconnectivity during positive processing. Data-driven parsing suggests heterogeneous substrates of depression, and possible resilience in a large proportion of controls in spite of biological overlap.

Keywords: Depression, Positive Mood, Neural Network Connectivity, fMRI, Community Detection.

Disclosure: Nothing to disclose.

\section{T33. Anxious Depression as a Potential Biomarker for Ketamine's Antisuicidal Effects}

Dawn Ionescu*, Lee Baer, Samuel Petrie, Abigail Archibald, Maurizio Fava, Cristina Cusin

Massachusetts General Hospital, Boston, Massachusetts, United States

Background: Patients with anxious depression generally respond more poorly to traditional monoaminergic antidepressants compared to those with nonanxious depression. Ketamine, a glutamatergic modulator that acts rapidly to reduce symptoms of depression in patients with treatment-resistant unipolar and bipolar depression, represents a mechanistic departure from the status quo of antidepressant medications. Previously, medication-free patients with anxious depression exhibited a superior antidepressant response to a single infusion of ketamine compared to those with nonanxious depression, with longer time-to-relapse, and a similar side effect profile. However, the extent to which anxious depression represents an antisuicidal response advantage in medicated patients with depression and suicidal thinking remains unknown. We predicted that, consistent with the existent literature on ketamine, patients with anxious depression would have a superior antisuicidal response.

Methods: Between January 2013 and November 2015, 26 medicated outpatients with severe major depressive disorder (MDD) with current, chronic ( $\geq 3$ months) suicidal ideation were enrolled and randomized in a double-blind fashion to six infusions over three weeks of ketamine $(0.5 \mathrm{mg} / \mathrm{kg}$ over 45 minutes) or saline placebo. A post-hoc analysis compared antisuicidal treatment response (ketamine vs. placebo) between patients with anxious $(n=12)$ vs. nonanxious $(n=14)$ depression using the Concise Health Risk Tracking (CHRT) scale; antidepressant treatment response was measured with the Hamilton Depression Rating Scale (HDRS). Anxious depression was defined as MDD plus a Hamilton Depression Rating Scale Anxiety/Somatization Factor Score $\geq 7$ at baseline.

Results: A fixed effects model analysis of the CHRT revealed a significant three-way group by time by treatment interaction $(p<0.01)$. Post-hoc testing revealed that patients with anxious depression in the ketamine group had significantly greater score decreases from baseline through the final infusion on the CHRT compared to those with anxious depression on placebo or those with nonanxious depression on ketamine or placebo. Interestingly, for the HDRS, a mixed model analysis also revealed a significant three-way group by time by treatment interaction $(p<0.001)$. Post-hoc tests indicated that patients with anxious depression in the ketamine group had significantly greater decreases in depression symptoms from baseline compared to those with anxious depression in the placebo group and nonanxious depression in either treatment group over the course of the 6 infusions.

Conclusions: Baseline anxious depression predicted a superior antisuicidal response (as measured by the CHRT) and antidepressant response (as measured by the HDRS) to repeated-doses of ketamine compared to those with anxious depression on placebo and those with nonanxious depression, regardless of treatment group assignment. These data provide further support for the use of anxious depression as a potential clinical biomarker for ketamine's superior antidepressant and antisuicidal effects.

Keywords: Ketamine, suicide, Treatment Resistant Depression, Anxious Depression.

Disclosure: Nothing to disclose. 
T34. Oxytocin Receptors Regulate Social Cognition and Arc Response to Social Stress Level

Hailian Xiao, Jeff Sanders*

Emory Univeristy, Atlanta, Georgia, United States

Background: The oxytocin receptor (Oxtr) plays influential roles in social cognition and in resilience to social stress. We studied the Oxtr regulation of social cognition and of activity regulated cytoskeleton-associated protein (Arc) within a social stress paradigm.

Methods: Male C57Bl/6J mice (WT) and Oxtr knockout mice (Oxtr-KO) were divided into 'socially housed' or 'socially stressed' experimental groups. Socially stressed mice were isolated for 6 weeks. During this period, they were tested for social investigation and discrimination at week 5, and repeatedly exposed to an intruder during week 6 . The brains of these socially stressed mice were removed after their last intruder encounter and assayed for Arc mRNA with in situ hybridization. All experimental protocols in these studies were approved by the Institutional Animal Care and Use Committee and were conducted in accordance with the National Institutes of Health Guide for the Care and Use of Laboratory Animals.

Results: Socially stressed WT mice had impaired social investigation and discrimination and decreased Arc mRNA within many brain regions. Oxtr deletion created many of these social stress-induced alterations within socially housed animals. Socially housed Oxtr-KO had impaired social discrimination and decreased Arc mRNA within several brain areas. Social stress decreased the social investigation of Oxtr-KO to a greater extent than WT, but it did not alter their baseline social discrimination impairments. Moreover, in contrast to decreased Arc mRNA found in socially stressed WT, socially stressed Oxtr-KO either showed no change in Arc mRNA or displayed increases from its baseline level.

Conclusions: These data show that the Oxtr is critical for regulating baseline levels of social cognition and Arc mRNA. Furthermore, these data indicate that the Oxtr plays a crucial role in organizing both behavioral and brain plasticity in response to social stress.

Keywords: Oxytocin, Stress, Plasticity, Arc.

Disclosure: Nothing to disclose.

T35. Genome Wide Changes in Long Noncoding RNA, Transcript Splicing, and Transcriptional Patterning in Autism Brain

Neelroop Parikshak*, Vivek Swarup, T Grant Belgard, Manuel Irimia, Gokul Ramaswami, Christopher Hartl, Luis de la Torre-Ubieta, Virpi Leppa, Jennifer Lowe, Benjamin Blencowe, Steve Horvath, Daniel Geschwind

University of California, Los Angeles, Los Angeles, California, United States

Background: Autism spectrum disorder (ASD) is a neurodevelopmental disorder characterized by deficits in social communication and mental flexibility. Genetic risk factors contribute substantially to ASD risk, and recent studies support the potential contribution of more than a thousand genetic loci. However, given the shared cognitive and behavioral features across the autism spectrum, one major hypothesis is that diverse risk factors may converge on common molecular, cellular, and circuit level pathways to result in the shared phenotype. Analysis of the transcriptome has supported this model by identifying common molecular pathways in the cerebral cortex from postmortem human brain tissue in individuals with ASD. However, there has been no systematic evaluation of long noncoding RNA (lncRNA) or splicing in ASD brain and most studies of the protein coding transcriptome lack independent replication and do not assess gene expression patterns across brain regions.

Methods: We performed rRNA-depleted RNA-seq to evaluate whole transcriptomes, including $\operatorname{lncRNA}$ and splicing alterations, from a large set of ASD and control (CTL) human brain samples including neocortex (frontal and temporal, FC and TC) and cerebellum across 97 individuals (48 ASD, 49 CTL, 235 unique samples across regions). We evaluated differential gene expression, differential alternative splicing, and transcriptional patterning across cortical regions.

Results: Investigation of transcriptome-wide differential gene expression in ASD revealed robust alterations in the noncoding transcriptome, including several dozen primate-specific lncRNA. We also identified an alteration in splicing of neuronal genes in the cerebral cortex in ASD and implicated three splicing regulators that contribute to the observed changes. Evaluation of transcriptomic alterations in an independent subset of individuals with a genetically defined syndrome causing ASD, Duplication 15q Syndrome (dup15q), revealed that transcriptome-wide gene expression and splicing alterations from idiopathic ASD were highly reproducible in this genetically defined population. We also characterized cortical patterning between FC and TC in CTL, and identified the transcriptional regulator SOX5 as a driver of this pattern. We demonstrate that a loss of patterning in SOX5 is associated with a loss of patterning in its targets between FC and TC in ASD.

Conclusions: We provide the first comprehensive picture of largely unexplored aspects of transcription in ASD. We show reproducible global transcriptomic alterations in protein coding genes, noncoding RNAs, and alternative splicing. We identify similar changes in dup15q, which suggests that the observed transcriptional alterations in ASD can be the consequence of a primary genetic alteration. We also show that the loss of patterning in SOX5 contributes to a loss of cortical patterning in ASD. Taken together, these analyses implicate novel transcriptional alterations and identify their upstream molecular regulators in ASD.

Keywords: Autism Spectrum Disorder, Transcriptomics, RNA-seq, Alternative Splicing, Long Noncoding RNA.

Disclosure: Nothing to disclose. 
T36. Disruption of Medial Geniculate Nucleus Connectivity to Auditory Cortex in Patients With Schizophrenia

Jared Van Snellenberg*, Guillermo Horga, Roberto Gil, Juan Sanchez-Pena, Seth Baker, Rachel Rosengard, Anissa Abi-Dargham

Columbia University Medical Center, New York, New York, United States

Background: Recent work in a 22q11 deletion syndrome (22q11DS) mouse model of schizophrenia identified a specific disruption of synaptic transmission from the medial geniculate nucleus (MGN) of the thalamus to auditory cortex, which was sensitive to treatment with antipsychotic medication (Chun et al, 2014. Science). However, no direct evidence for such a disruption exists in clinical samples of patients with schizophrenia.

Consequently, we employed high-resolution multiband functional Magnetic Resonance Imaging (fMRI) during a resting state and during a novel task designed to identify MGN and lateral geniculate nucleus / pulvinar (LGN/P) voxels in fMRI images, in order to directly assess the connectivity of MGN and LGN/P to auditory and visual cortices (AC and VC) in unmedicated patients with schizophrenia.

Methods: Eighteen unmedicated patients with schizophrenia and 18 healthy controls participated in the study. The below methods failed to identify anatomically plausible MGN or LGN/P regions-of-interest (ROIs) in 4 patients and 2 controls, leaving 14 patients (10 male, mean age 36.4 years) and 16 controls (11 male, 28.8 years). All participants were scanned on a 3 Tesla GE MR 750. We acquired functional EPI volumes with $2 \mathrm{~mm}$ isotropic voxels and $850 \mathrm{~ms}$ TR with a multiband acceleration factor of 6 , no in-plane acceleration, $192 \mathrm{~mm}$ FOV, 66 slices, $60^{\circ} \mathrm{FA}$, and $25 \mathrm{~ms}$ TE. The localizer task used a sparse temporal sampling sequence, with acquisition clusters of 3 volumes $(2550 \mathrm{~ms})$ followed by a $9450 \mathrm{~ms}$ inter-cluster interval, during which the scanner was not collecting data and either auditory or visual stimuli were presented in pseudo-random order. Each participant completed 4 runs of 16 acquisition clusters each. Auditory stimuli consisted of $900 \mathrm{~ms}$ segments of music followed by $100 \mathrm{~ms}$ of silence, amplitude normalized and presented in random order. The visual stimulus was a circular checkerboard alternating between black and white at $7.5 \mathrm{~Hz}$, with maximum contrast. Participants also completed 4 resting state runs (with fixation) of 7.5 minutes each.Data were preprocessed through the Human Connectome Project pipeline. MNI template aligned images were smoothed with a $4 \mathrm{~mm}$ FWHM Gaussian filter. Template masks were obtained from the SPM WFU PickAtlas for Brodmann's Areas (BAs) 41 and 42 in each hemisphere (AC mask), BAs 17 and 18 (VC mask), and medial and lateral geniculate bodies (ST mask). The VC and AC masks were dilated by 1 voxel in 3 dimensions while the ST mask was dilated by 2 voxels, but with voxels showing greater than $97.5 \%$ probability of white matter in SPM tissue probability maps removed, along with voxels within $2 \mathrm{~mm}$ of the PickAtlas hippocampus. An Auditory - Visual condition contrast was calculated for each subject, and voxels showing contrast values in the top $10 \%$ within each mask were identified. A single contiguous cluster of at least 10 voxels in each hemisphere was retained as an ROI for auditory or visual cortex for each subject. ROIs for auditory thalamus were then identified as the largest contiguous cluster of voxels within the ST mask showing a correlation with the average signal in the AC mask that was in the top $7.5 \%$ of ST mask voxels, but that was not also in the top $7.5 \%$ of voxels in terms of their correlation with the VC mask. The visual thalamic ROI was identified in the converse manner. These correlations were determined after regressing out run- and volume-specific regressors, to account for run effects as well as T1 relaxation effects. The MGN and LGN/P ROIs were then used as seeds to evaluate whole-brain connectivity during resting state, by averaging the time series of all voxels within each ROI to create a single time series. Connectivity data was 'scrubbed' for both high-motion volumes and volumes during which participants closed their eyes, and bandpass filtered between 0.008 and $0.09 \mathrm{~Hz}$. Pearson correlations between each thalamic ROI and every voxel in the brain were calculated for each subject, Fisher r-To-Z transformed, and averaged together for right- and lefthemispheres. Voxels exhibiting significant connectivity were assessed within each group, as well between-group differences in connectivity, using robust regression and correction for multiple comparisons (alphasim corrected at $P<0.05$ ). Results: Both groups exhibited significant connectivity between MGN and primary and secondary auditory cortex, as well as between LGN/P and primary and secondary visual cortex. Moreover, patients with schizophrenia showed significantly reduced connectivity between MGN and primary auditory cortex in the right hemisphere, as compared to healthy control participants, confirming our hypothesis. A similar finding was not observed between the nearby LGN/P and auditory cortex, suggesting this result is specific to MGN. In addition, patients demonstrated a reduction in connectivity between LGN/P and primary and secondary visual cortex, although a similar reduction was also observed between MGN and visual cortex.

Conclusions: We demonstrate a reduction in MGN-auditory cortex connectivity in unmedicated patients with schizophrenia, which was directly hypothesized on the basis of a 22q11DS mouse model of schizophrenia. These findings directly support the notion that the mechanism of impaired synaptic transmission identified in this model is also impaired in human patients with schizophrenia.

Keywords: Schizophrenia, Thalamus, Connectivity, Auditory Cortex, Medial Geniculate.

Disclosure: Nothing to disclose.

\section{T37. Interneuron Circuit-Specific Interaction With Mental Disorder Risk Factor During Adult Hippocampal Neurogenesis}

Juan Song*, Eunchai Kang, Hechen Bao, Guo-li Ming, Hongjun Song

University of North Carolina at Chapel Hill, Chapel Hill, North Carolina, United States

Background: Schizophrenia etiology is thought to involve the interaction between genetic and environment factors during brain development. Despite significant progress in 
understanding both genetic susceptibility and neuronal circuit dysfunction in schizophrenia, fundamental gaps exist in our knowledge about how circuitry mechanisms may interact with genetic susceptibility to affect neuronal development. Disrupted-in-schizophrenia 1 (DISC1), a risk gene for major mental disorders, regulates various processes of neuronal development and DISC1-deficiency in adultborn dentate granule neurons alone leads to cognitive and affective behavior deficits.

Methods: Using a combination of optogenetic, electrophysiological, retroviral and morphological approaches, we examine the role of distinct local interneuron circuits in regulating discrete processes of normal and aberrant development of newborn neurons labeled by retrovirus coexpressing GFP and shRNA-control or shRNA-DISC1 in the adult hippocampus.

Results: Using a combination of optogenetic, electrophysiological, retroviral and morphological approaches, we examine the role of distinct local interneuron circuits in regulating discrete processes of normal and aberrant development of newborn neurons labeled by retrovirus co-expressing GFP and shRNA-control or shRNA-DISC1 in the adult hippocampus.

Conclusions: Distinct neuronal circuit interacts with the genetic susceptibility to manifest different aspects of aberrant neuronal development.

Keywords: Adult Hippocampal Neurogenesis, Neural Circuitry, Mental Disorder, Genetic Risk Factor.

Disclosure: Nothing to disclose.

\section{T38. Cell Type-Specific Transcriptional and Ultrastructural Analyses of Oxidative Phosphorylation in the Prefrontal Cortex of Schizophrenia Subjects}

Jill Glausier*, Dominique Arion, John Enwright, Zhiguang Huo, George Tseng, David Lewis

University of Pittsburgh, Pittsburgh, Pennsylvania, United States

Background: Working memory, a core cognitive function impaired in schizophrenia, depends upon gamma oscillatory neuronal activity in the prefrontal cortex (PFC). Accordingly, individuals diagnosed with schizophrenia show lower power of gamma oscillations in the PFC during tasks that involve working memory. Gamma oscillations emerge from the coordinated activity of layer 3 excitatory pyramidal cells and layer 3 inhibitory parvalbumin (PV) cells. This reciprocal microcircuit involves dense excitatory innervation from local axon collaterals, and robust innervation of those pyramidal cells by PV cells. As such, gamma oscillations have a particularly high energetic demand that is met by adenosine triphosphate (ATP) production via oxidative phosphorylation (OXPHOS) within pyramidal and PV cell mitochondria. In the PFC of schizophrenia subjects, layer 3 pyramidal cells have prominent reductions in OXPHOS-related gene pathways. Importantly, OXPHOS can be regulated by two distinct processes: ATP demand to support neuronal firing, or upstream deficits in OXPHOS enzyme expression. Layerand cell type-specific analyses of select OXPHOS enzyme subunits and mitochondrial morphology can help to distinguish between these two possibilities. Expression of cytochrome c oxidase (COX), the terminal and rate-limiting OXPHOS enzyme, is controlled by the level of neuronal excitation. Transcription of all COX subunits is directly coupled to excitation by multiple transcription factors, including Nuclear Respiratory Factor 1 (NRF1). Thus, persistent reductions in neuronal excitation lower NRF1 expression, decreasing expression of all COX subunits. During low neuronal firing and ATP demand, mitochondria have an Orthodox conformation that reduces cristae area as a consequence of reduced OXPHOS enzyme production. During high neuronal firing and ATP demand, mitochondria increase cristae area to accommodate increased enzyme expression and have a Condensed conformation. On the other hand, defective OXPHOS enzyme expression lowers the expression of few COX subunits, has no effect on NRF1 expression, and results in a distinct Impaired conformation of mitochondria. To determine which upstream factor is likely operative in the illness, we quantify COX-related transcripts in layer 3 pyramidal and PV cells, and perform ultrastructural analyses of mitochondrial abundance and conformation within pyramidal and PV axon boutons in layer 3 of the PFC in schizophrenia and unaffected comparison subjects.

Methods: For mRNA analyses, frozen tissue sections containing area 9 from 36 pairs of schizophrenia and comparison subjects were stained with thionin for Nissl substance to identify pyramidal cells, or labeled using immunoperoxidase for aggrecan to identify PV cells. Layer 3 pyramidal and PV somata were dissected using a Leica laser microdissection (LMD) system. Transcriptome profiling was performed by microarray using Affymetrix GeneChips specific to the human genome. Expression levels of COX subunits and NRF1 in each neuronal population across diagnostic groups was assessed at $p<0.05$ in covariate- and multiple comparisons-corrected analyses. Electron microscopic analyses were performed in area 46 of matched pairs of schizophrenia and comparison subjects. Single-label immunoperoxidase using mouse anti-PV (Swant) antibody was performed, and samples from layer 3 were analyzed for each subject. Established ultrastructural criteria were used to identify pyramidal cell axon boutons forming synapses, and PV axon boutons were identified by presence of immunoreactivity. Mitochondria in each population of boutons were counted, and classified as Orthodox, Condensed, or Impaired. Chi-square analysis was used to examine whether the percentages of each type differ across diagnostic groups. Results: In layer 3 pyramidal cells, probes for all 10 COX subunits encoded by nuclear DNA showed $11-30 \%$ reductions in schizophrenia relative to unaffected subjects. All COX subunits except COX6C $(-20 \%, p=0.1)$ and COX8A $(-20 \%, p=0.2)$ were significantly lower (all $p \leq 0.03)$ in schizophrenia subjects. In contrast, expression of probes for NRF1 were not altered in layer 3 pyramidal cells (all $p>0.2$ ) in schizophrenia. Analyses of gene expression in LMDcollected PV cells are ongoing. Initial electron microscopic analyses indicate that significantly more PV boutons contained mitochondria than did pyramidal cell boutons $(\chi 2=20.1, p<0.00001)$, consistent with the fast-spiking characteristics of $\mathrm{PV}$ cells. In unaffected comparison subjects, nearly all mitochondria in pyramidal or PV cell boutons were Orthodox or Condensed, consistent with the absence of OXPHOS impairments in these subjects. 
Additional studies of mitochondrial abundance and conformation within PV and pyramidal cell boutons in both diagnostic groups are ongoing.

Conclusions: Accumulating evidence indicates that $\mathrm{OX}$ PHOS is lower in the PFC of subjects with schizophrenia. Determining whether this finding reflects the consequence of lower ATP production due to reduced neuronal activity, or an impaired capacity to produce ATP due to deficient OXPHOS enzyme expression is a critical first step in the development of mechanistically novel treatments. Our continued targeted analyses of select OXPHOS-related genes and ultrastructural analyses of mitochondria within specific neuronal populations may differentiate between these alternatives.

Keywords: Mitochondria, Pyramidal Neuron, Parvalbumin Interneurons, Electron Microscopy, Schizophrenia.

Disclosure: Nothing to disclose.

\section{T39. Neuronal Circuitry Dependent DNA Methylation Variation Within the Human Hippocampus}

William Ruzicka*, Francine Benes

Harvard Medical School, Belmont, Massachusetts, United States

Background: Chromatin modification contributes to the generation and maintenance of diverse neuronal phenotypes within the brain, and regulation of neuronal physiology in health and disease. Recent studies describe highly distinct DNA methylomes among phenotypic subclasses of neurons within the human brain, but variation in DNA methylation among neuronal populations defined by their function and location within neural circuits remains an unexplored concept. Studies able to resolve epigenetic profiles at the level of microcircuits, beyond homogenized whole brain structures, are needed to illuminate chromatin dynamics in the regulation of specific neuronal populations and circuits mediating normal and abnormal behaviors.

Methods: The Illumina HumanMethylation450 BeadChip was used to assess genome-wide DNA methylation differences between layer-specific populations of GABAergic interneurons laser-microdissected from two discrete microcircuits along the trisynaptic pathway in postmortem human hippocampus from eight control, eight schizophrenia, and eight bipolar disorder subjects. Data was normalized by stratified quantile normalization using the minfi Bioconductor package in $\mathrm{R}$ software version 3.1.0. Group differences were assessed both as single differentially methylated positions with Bonferroni corrected $p$ value $<0.05$, and as differentially methylated regions using the bumphunter algorithm with FWER $<0.05$.

Results: We identified 11 highly significant differentially methylated regions associated with a group of genes with high construct-validity, including multiple zinc finger of the cerebellum gene family members and WNT signaling factors. While the genomic location of differently methylated regions was highly similar between diagnostic categories, there were significantly more sites of methylation difference between circuit locations in bipolar disorder cases than in schizophrenia or control.
Conclusions: These findings identify distinct DNA methylomes among phenotypically equivalent populations of GABAergic interneurons performing discrete functions in separate hippocampal subfields. These data compliment recent studies that describe highly diverse epigenotypes among separate neuronal subclasses, extending this concept to distinct epigenotypes within equivalent neuronal phenotypes functioning in distinct tissue environments at separate microcircuits within the human brain.

Keywords: DNA Methylation, Hippocampus, Psychosis, Epigenetics, Postmortem Human Brain.

Disclosure: Nothing to disclose.

\section{T40. 7T Proton Magnetic Resonance Spectroscopy of the Anterior Cingulate Cortex in Minimally-Treated First-Episode Schizophrenia}

\author{
Meredith Reid*, Nouha Salibi, Thomas Denney, \\ Adrienne Lahti
}

Auburn University, Auburn University, Alabama, United States

Background: Recent magnetic resonance spectroscopy (MRS) studies suggest abnormalities of the glutamatergic system in schizophrenia are dependent on illness stage and medication status. Glutamate levels are elevated in the prodromal and early stages of schizophrenia but unchanged or reduced below normal in chronic, medicated patients. However, few of these studies have measured metabolites at $7 \mathrm{~T}$, which offers higher signal-to-noise ratio and better spectral resolution than $3 \mathrm{~T}$ and facilitates separation of glutamate and glutamine into distinct signals. In this study, we examined glutamate and other metabolites in the dorsal anterior cingulate cortex (ACC), a region known to be functionally altered, of minimally-treated first-episode schizophrenia patients.

Methods: 21 individuals with schizophrenia and 18 age- and sex-matched healthy controls were recruited from the University of Alabama at Birmingham psychiatric clinics and the general community. Imaging was performed at the Auburn University MRI Research Center on a MAGNETOM 7T MRI scanner (Siemens Healthcare, Erlangen, Germany) equipped with a 32-channel head coil (Nova Medical). 3D MPRAGE structural images $(0.7 \mathrm{~mm}$ isotropic resolution) were obtained for voxel placement. Spectra were acquired from the dorsal ACC (27x20x10 mm) using an ultra-short TE STEAM sequence (TR/TE/TM $=10,000 / 5 / 45 \mathrm{~ms}, 32$ averages), FASTESTMAP shimming, and VAPOR water suppression. MRS analysis was performed in LCModel using a simulated basis set. Spectra were eddy current corrected and quantified using the unsuppressed water signal (4 averages). Cramer-Rao lower bounds (CRLB) were used as a measure of fit, and only metabolites with CRLB $<20 \%$ were included in further analysis. Univariate ANOVA covarying for age, sex, and smoking was used to compare CSF-corrected metabolite levels between the groups. Pearson correlation coefficients were used to assess relationships among metabolites, symptom severity, and neuropsychological status.

Results: Glutamate and N-acetylaspartate (NAA) were significantly lower in schizophrenia versus controls. No 
differences were observed in levels of glutamine, GABA, creatine, or choline. Glutamate was positively correlated with GABA and NAA in both groups. GABA was negatively correlated with the total score on the Repeatable Battery for the Assessment of Neuropsychological Status (RBANS) in schizophrenia but not controls.

Conclusions: Our finding of lower glutamate in the dorsal ACC suggests glutamatergic alterations are not only state specific but also regionally specific. NAA is generally considered to be a marker of neuronal health or integrity. Our finding of reduced NAA is consistent with neuroimaging and postmortem evidence of reduced cortical thickness, neuronal density, and dendritic density in the prefrontal cortex of people with schizophrenia. The negative correlation between GABA and RBANS is consistent with a recent report showing a negative relationship between GABA and IQ in schizophrenia. These findings suggest that abnormal neuronal physiology and integrity underlie the functional alterations observed in schizophrenia.

Keywords: First Episode Schizophrenia, 1H MRS, Glutamate, Anterior Cingulate Cortex.

Disclosure: Nothing to disclose.

T41. Molecular Subtypes of Schizophrenia Accounting for Ancestry and Sex Show Different Symptom Profiles: Application of Pathway-Based Polygenic Scoring Methods to the Wellcome Trust Schizophrenia Case-Control Sample

\section{Anna Docherty ${ }^{\star}$, Silviu-Alin Bacanu}

Virginia Commonwealth University, Richmond, Virginia, United States

Background: Identifying genetic subtypes of schizophrenia (SZ) could reduce genetic heterogeneity prior to quantitative phenotypic profiling. Thus far, genetic subtyping analyses of SZ have been largely unsuccessful, mainly due to using all markers in the genome and not controlling for critical covariates such as population stratification and sex. The authors developed, tested, and present here methods to identify putative genetic subtypes of SZ.

Methods: We used the implicated human biological pathways from the Psychiatric Genomics Consortium (PGC2) SZ GWAS to calculate pathway-based risk scores in the Wellcome Trust case-control sample. Two algorithms, both accounting for population stratification and sex, were considered feasible: a preferred method used model-based clustering in $\mathrm{R}$, and an alternative that used model-based recursive partitioning, which constructs its trees using only cuts which are statistically significant after adjusting for multiple testing. We then examined the resulting subtypes with respect to SZ symptom dimensions and age at onset. Results: While hierarchical clustering consistently resulted in only one genetic subtype, recursive partitioning resulted in 4 genetic subtypes with sufficient sample sizes. Notably, emergent subtypes were based on robust effects of sex and on pathways specific to mRNA and to DNA repair. When testing these methods on the PGC subsample of SZ cases and controls, we observed that individuals in one of the genetic subtypes had elevated positive symptoms, and in another, earlier age at onset. Because of its size, the large PGC2 sample will provide optimal data for final application of the genetic subtyping methods; thus, forthcoming analyses by this awardee will apply these methods to the largest phenotyped SZ sample to date.

Conclusions: Previous applications focused on classification algorithms of whole, high dimensional genotypic data, and neglected to account for sex and ancestry. The application of significance-based recursive partitioning methods to PGC2 biological pathway-based polygenic risk scores, to model genetic subtypes in GWAS, could significantly aid in reducing the genetic heterogeneity of SZ risk. These analyses highlight the power of a careful, stepwise genetic subtyping framework, that is both computationally feasible and accounts for covariates, to provide new potential avenues for prediction and prevention of psychosis.

Keywords: Molecular Mechanisms, Subtypes, Schizophrenia, Genetic, Polygenic.

Disclosure: Nothing to disclose.

\section{T42. Should Single Strains Suffice?}

Laura Sittig*, Peter Carbonetto, Kyle Engel, Kathleen Krauss, Camila Barrios-Camacho, Abraham Palmer

\section{University of California, San Diego, La Jolla, California,} United States

Background: Genome wide association studies (GWAS) have now identified numerous loci that influence risk for psychiatric and neurological diseases. Genetically engineered mice are increasingly being employed to explore the role of genes implicated by GWAS for a variety of traits. Most such studies utilize inbred strains and are based on the assumption that observed genotype-phenotype relationships will generalize to humans. This assumption implies that the results would at least generalize to other inbred mouse strains. Given current concerns about experimental reproducibility in biomedical science, we sought to directly test this assumption.

Methods: We examined the phenotypic effects of null alleles of Cacna1c and Tcf712, which have been robustly implicated in numerous disease traits by GWAS. We produced F1 crosses between C57BL/6J +/- males and wild-type females from 30 commonly used inbred laboratory strains. Mice in the Cacnalc cohort $(N=723)$ were tested for anxiety, methamphetamine sensitivity, depression-like behavior, and acoustic startle response. Mice in the Tcf7l2 cohort $(N=630)$ were tested for several behavioral traits: anxiety, fear conditioning, and sensorimotor gating, as well as several metabolic traits: body weight, baseline blood glucose levels and fasted blood glucose levels. Thus, we obtained data for 15 phenotypes, 12 of which were behavioral.

Results: We found extremely strong interactions between genetic background and both mutant alleles. In several cases the same mutation increased a trait in one background but decreased the same trait in another.

Conclusions: These results do not negate the invaluable contributions of mouse genetics to biomedical science, but they do show that genotype-phenotype relationships cannot be reliably inferred by studying a single genetic background, and thus constitute a major challenge to the status quo. 
Keywords: Inbred Mouse Strains, Mouse Behavior, Genetic Mouse Models.

Disclosure: Nothing to disclose.

\section{T43. Mapping Cortical Functional Networks in Individuals}

Danhong Wang, Randy Buckner, Dost Ongur, Justin Baker, Hesheng Liu*

Harvard Medical School, Charlestown, Massachusetts, United States

Background: A major obstacle for exploring subtle aspects of network organization and translating insights into clinical applications is the lack of tools for mapping networks in individual subjects. Until recently, most studies of human brain organization have focused on averaging data over many individuals to estimate network properties. However, marked inter-individual variability has been demonstrated in the organization of functional systems of the brain, particularly in higher order association areas. A functional mapping technique with high sensitivity to individual variations is needed to facilitate discovery of meaningful biomarkers for cognitive ability or disease states and provide greater statistical power for investigating behavioral or genetic associations.

Methods: We developed a novel brain parcellation approach to accurately map functional organization at the individual level using resting-state fMRI. A population-based functional atlas and a map of inter-individual variability were employed to guide the iterative search for functional networks in individual subjects. The individual-level functional parcellation was then used as a basis for cross-subject alignment according to functional characteristics, instead of macroscopic anatomical landmarks, to improve group-level analyses. Finally, individual-level cortical networks of schizophrenia patients and healthy control subjects were compared.

Results: Functional networks mapped by this approach were highly reproducible within subjects and effectively captured the variability across subjects, including individual differences in brain lateralization. The algorithm performed well across different subject populations and data types including task fMRI data. The resulting parcellation networks were significantly more reliable than networks localized by traditional task-evoked response.

Aligning subjects based on the individual-level functional parcellation could improve group analysis. Functional connectivity strength between the regions of interest (ROIs) of the same network was significantly increased if the ROIs were individually-specified compared to population-based. On average, within-network connectivity strength increased by $25.32 \%$ if the ROIs were specified in individuals whreas between-network connectivity values decreased by $10.94 \%$. The cortical networks of schizophrenia patients and matched healthy control subjects were then localized using the individual-level cortical network parcellation technology. We found that functional connectivity strength and the size of some networks, including the sensorimotor and frontoparietal networks, were significantly altered in schizophrenia.
Conclusions: An individual-level functional parcellation can not only be used as the 'localizer' for specific functions, but can also provide a basis for cross-subject alignment to improve group-level functional analyses. The technology allows the estimate of size, location and connectivity strength of the functional nodes in each individual and may help to identify specific changes in functional networks associated with psychiatric disorders.

Keywords: Functional Connectivity, Connectivity-Based Parcellation, Schizophrenia.

Disclosure: Nothing to disclose.

\section{T44. Functional Connectivity Underlying Dynamic Scene Assessment During Naturalistic Social Viewing in Schizophrenia}

Gaurav Patel ${ }^{\star}$, Sophie Arkin, Nicole Strauss, Heloise De Baun, Casimir Klim, Rebecca Berman, David Leopold, Daniel Javitt

Columbia University, New York, New York, United States

Background: Deficits in social cognition are important determinants of functional outcome in schizophrenia patients (SzP), and are predicted by impairments in both auditory and visual sensory function. Here we looked at auditory and visual contributions to social cognitive impairment using the The Awareness of Social Inference Test (TASIT). As opposed to other social cognition measures, the TASIT uses video-based stimuli that can be assessed using dynamic scene assessment approaches. In the TASIT, two categories of information are presented, 1) sarcasm/sincerity, which is conveyed by tone of voice ("prosody') and facial expression, and 2) lies/truthfulness, which is conveyed by evaluation of information content of current statements vs. prior events. Dynamic scene assessment was evaluated using continuous eye tracking during TASIT processing. We predicted that SzP would have significant deficits in detection of sarcasm/sincerity vs. truthfulness/lie as compared to healthy controls (HC), and that this would be related, in part, to impairment in dynamic scene assessment abilities. Underlying these operations are face-processing areas, dorsal/ventral attention networks, and theory of mind networks. SzP have been shown to be impaired in potentially all of these networks, especially in dynamic situations, all of which have key nodes in or near the temporoparietal junction/posterior superior temporal sulcus (TPJ-pSTS). We studied the integrity of these networks using restingstate functional connectivity to determine what degree each contributes to overall social cognition deficits.

Methods: $31 \mathrm{SzP}$ and $25 \mathrm{HC}$ performed TASIT with eyetracking, along with perceptual/cognitive measures of face emotion recognition (ER-40), processing speed (PSI), and understanding of auditory sarcasm (attitudinal prosody). For each subject, the eye-position on each TASIT video frame was automatically scored for the distance from the mean $\mathrm{HC}$ eye-position, which was then used to calculate the percentage of time spent fixating (HC eye-position was compared to all other HC). The perceptual/cognitive measures and eyetracking measure were used to predict the TASIT performance using a linear mixed-effects model (LME), with the auditory sarcasm measure controlling for the contribution of 
the auditory modality. The subjects also underwent both resting-state and task functional magnetic resonance imaging (fMRI). Task fMRI data were used to localize the visual, faceprocessing, attention network, and theory of mind areas, which were then used to calculate interareal functional connectivity at rest. Connectivity was then used to calculate network topology measures.

Results: As predicted, SzP performed significantly worse in answering questions about TASIT videos involving sarcasm vs. lies $(p<.05)$. With PSI controlling for general cognitive deficits in Sz, TASIT sarcasm performance in the two groups was predicted by a combination of attitudinal prosody and eye-tracking performance $(p<.01)$; adding ER-40 to the model did not improve prediction. Eye-tracking patterns appear to differ most during video frames with multiple faces with moving facial expressions mixed with other biological motion, such as hands grasping objects. Functional connectivity between TPJ-pSTS nodes of the face-processing and ventral attention networks to the other networks were reduced in SzP, resulting in reduced node degree/betweenness centrality of these areas.

Conclusions: The difference in eye-tracking patterns may reflect impaired detection of and orientation to socially relevant features of the environment, such as facial expressions of emotion, leading to poor understanding of complex social situations. Decreased connectivity of TPJ-pSTS nodes of face processing and ventral attention networks may underlie these deficits. Overall, this study demonstrates the utility of using eye-tracking during naturalistic stimuli to study social cognition deficits in Sz.

Keywords: Resting State Functional Connectivity, Social Cognition, Face Emotion Processing, Dorsal Attention Network, Ventral Attention.

Disclosure: Nothing to disclose.

\section{T45. The Role of Muscarinic M1-KCNQ Signaling in Working Memory Circuits of the Prefrontal Cortex}

Taber Lightbourne*, Veronica Galvin, Yang Yang, Constantinos Paspalas, Min Wang, Amy FT Arnsten

Yale University School of Medicine, New Haven, Connecticut, United States

Background: Schizophrenia (SZ) is associated with profound cognitive deficits, including impairments in working memory that drive occupational, social and economic disabilities (Barch and Ceaser. 2012; Keefe and Harvey. 2012). Patients with SZ have significant deficits in the working memory functions of the dlPFC that correlate with symptoms of thought disorder (Perlstein et al, 2001).

Persistent activity across a delay period is considered the neuronal basis of working memory, which is generated by recurrent activity among a group of layer III PFC pyramidal cells, that excite each other via NMDA receptor synapses on dendritic spines. NMDAR requires a depolarized membrane to open; in some areas of the brain (e.g. visual cortex) this is done by AMPAR, but acetylcholine performs this function in layer III PFC, through actions at nicotinic a7 receptors (Yang et al, 2013), and possibly, through muscarinic M1 receptor (M1R) closing of M-type KCNQ potassium channels in the synaptic membrane. Immunoelectron microscopy data has demonstrated the presence of both M1R and KCNQ in glutamate-like synapses in layer III of primate dlPFC. This study aimed to test the hypothesis that stimulation of the M1R would enhance working memory though closure of hyperpolarizing KCNQ channels. To examine the role of the M1-KCNQ signaling pathway in working memory, we tested the effect of agents that modulated M1R and the KCNQ channel on neuronal activity in dlPFC.

Methods: Single unit neuronal recordings were performed in non-human primates performing a working memory task. Drugs manipulating M1R and KCNQ were iontophoresed onto the recording site and the effect on firing was captured by a carbon fiber electrode.

Results: We found that both M1R activation and KCNQ blockade enhanced working memory related activity in the monkey dlPFC while M1R blockade and KCNQ channel opening reduced neuronal activity in the dlPFC. Finally, the effect of the M1R was found to be influenced by the open state of the KCNQ channel. Reduced firing from M1R blockade in the dlPFC was reversed by closure of KCNQ channel.

Conclusions: The current study shows that both M1R and KCNQ channels regulate working memory circuitry at the neuronal level. The effects of M1R on neural activity in the working memory circuitry, is mediated by the open state of KCNQ channel. These findings suggest that M1R activation is a viable strategy to enhance working memory circuits and maybe be a potential target for agents developed to improve cognition. This strategy would have special relevance to cognitive dysfunction in schizophrenia, as patients have been found to have working memory deficits and downregulation of the M1R in working memory circuitry of the dlPFC (Dean et al, 2002).

Keywords: Working Memory, dlPFC, Schizophrenia, Synaptic Aberrations, Muscarinic Acetylcholine Receptor, Electrophysiology.

Disclosure: Nothing to disclose.

T46. Control of Drug-Related Plasticity by the Activity-Regulated Cytoskeleton-Associated Protein (Arc)

Laura Smith*, Rachel Penrod, Morgane Thomsen, Michelle Doyle, Yuhong Guo, Jaswinder Kumar, Jakub Jedynak, Makoto Taniguchi, Rajeev Desai, Christopher Cowan

Harvard Medical School, Belmont, Massachusetts, United States

Background: Long-lasting synaptic alterations in brain reward regions accompany repeated exposure to drugs of abuse, suggesting that addiction is promoted and sustained through the coercion of normal synaptic plasticity mechanisms including those involved in learning and memory. Understanding the molecules that mediate such responses to drug experience is critical to developing successful treatment and prevention strategies. The immediate early gene, activity-regulated cytoskeleton-associated protein (Arc), is critical for certain types of plasticity associated with longterm memory. Arc mRNA and protein expression are also transiently upregulated by acute cocaine exposure in reward- 
related brain regions of rodents, an effect that becomes more persistent after multiple exposures. As a key regulator of structural and functional synaptic features that are altered by cocaine treatment and withdrawal, including AMPA receptor surface expression, and as an early responder to cocaine exposure, Arc is well positioned to mediate aspects of drug addiction. In the past, we have observed several differences in anxiety-, depression-, and addiction-related behaviors between mice lacking Arc and their wild-type (WT) littermates. In particular, prior cocaine experience appears to facilitate reward function in the absence of Arc (increased cocaine preference following previous experience); however, this addiction-relevant alteration in behavior is not explained sufficiently by changes in surface glutamatergic AMPA receptor expression in the nucleus accumbens (NAc), a major reward-related brain region.

Methods: Here, using the intravenous drug selfadministration (IVSA) assay, we show Arc KO and WT mice responding over a range of cocaine concentrations following acquisition, and give particular emphasis to shifts in the pattern of cocaine taking observed between groups and sessions. In addition, we tested the mice on more demanding IVSA schedules of reinforcement. Given behavioral responses to cocaine in Arc KO mice across several tasks, and the lack of behaviorally relevant surface AMPA receptor changes in NAc, we suspected that Arc KO mice may have enhanced glutamatergic and/or dopaminergic transmission. To test this possibility, a separate group of mice were pretreated with cocaine and, at a behaviorally relevant time point following withdrawal, underwent microdialysis to measure neurotransmitter release in the NAc both basally, as well as in response to increasing doses of cocaine.

Results: Here we show that Arc KO mice acquire cocaine IVSA normally. Much like our prior finding in place preference, IVSA-trained Arc KO mice later respond differently to lower doses of cocaine than WT mice; however, in seeming opposition to our previous finding of reward facilitation, in this task mice lacking Arc had significant reductions in cocaine responding. Examination of patterns of responding over each trial suggest that Arc KO mice perceive lower doses of cocaine like higher doses, indicating that prior cocaine experience enhances cocaine sensitivity in the absence of Arc. Preliminary microdialysis data in mice treated with cocaine and allowed to withdraw suggest that dopamine release is decreased in NAc after cocaine challenge in Arc KO mice, while baseline glutamate levels are enhanced compared to control mice.

Conclusions: Overall, our results are consistent with an enhanced predisposition for cocaine sensitivity in mice lacking Arc. While this tendency is evident in locomotor behavior upon the first exposure, in reward-related tasks its development may require prior cocaine experience. Coupled with our previous findings of increased basal surface AMPA receptor expression, our results suggest overall enhanced NAc glutamatergic transmission in KO mice, which may contribute to the observed behavioral responses to cocaine. A better understanding of the development of cocaine sensitivity, particularly as it relates to reward, could inform therapeutic approaches to addiction. As such, future studies in this area, such as those addressing the role of Arc in cueinduced craving and relapse, are warranted.
Keywords: Cocaine, Addiction, Arc, Intravenous Drug SelfAdministration, Microdialysis.

Disclosure: Nothing to disclose.

\section{T47. Circuit Dynamics of in Vivo Dynorphin Release in the Nucleus Accumbens Shell}

Ream Al-Hasani*, Jenny Wong, Jordan McCall, Omar Mabrouk, Kirsten Porter-Stransky, Gavin Schmitz, Brandon Aragona, Robert Kennedy, Michael Bruchas

Washington University, St. Louis, Missouri, United States

Background: The nucleus accumbens (NAc) and the dynorphinergic system are widely implicated in motivated behaviors. Prior studies have shown that activation of the dynorphin-kappa opioid receptor (KOR) system leads to aversive, dysphoria-like behavior. KOR agonists induce place aversions, depression-like behavior, and dysphoria in both human and animal models. However, the mechanisms and role of endogenous dynorphin in the regulation of KORmediated negative affective behaviors remain unresolved. We recently used an optogenetic approach to demonstrate that stimulation of dynorphinergic cells in the ventral nucleus accumbens shell (vNAcSh) elicits robust aversive behavior and photostimulation of dorsal NAcSh dynorphin (dNAcSh) cells induces a place preference and is positively reinforcing. Both of which appear to be dependent on kappa opioid receptor (KOR) activation. To follow these recently published findings, we are investigating how KOR is able to mediate these opposing behaviors in two distinct regions of the NAcSh.

Methods: We virally targeted channelrhodopsin-2 to NAcSh dynorphinergic neurons and photostimulated neuronal populations in both the dorsal and ventral NAc shell. We used an opto-dialysis approach which combines optogenetics with microdialysis for use in awake, freely moving mice. This system allows quantification of neuropeptide release while directly modulating cell-type specific neuronal firing in the NAcSh. Samples were analysed using liquid chromatography-mass spectrometry (LC-MS) detection. We injected modified rabies virus and cholera toxin retrograde tracers in both the dorsal and ventral region NAc to trace the neuronal projections that may act to mediate the aversion and preference.

Results: We have identified that the amount of dynorphin and met-enkephalin released during optogenetic stimulation is equal in the dNAc and vNAc. Interestingly, release of leuenkephalin and dopamine is only detectable following photostimulation in the $\mathrm{dNAc}$ release. To further understand the circuitry driving the opposing unique behaviors and distinct neuropeptide release profiles, we are mapping the projections to and from discrete regions with the dynreporter mouse (dyn-CretdTomato) and using tracing approaches (Rabies, canine adenovirus and cholera-toxin B). Conclusions: Thus far we have identified projections from the lateral septum, dorsal and ventral tegmental area (VTA) in addition to a unique GABAergic projection from the VTA to the vNACSh that drives a preference behavior. Together these experiments will help us understand how these distinct populations of dynorphin neurons in the NAcSh are 
engaged, altered, and recruited in stress and reward-related behaviors.

Keywords: Kappa Opioid Receptor, Dynorphin, Nucleus Accumbens.

Disclosure: Nothing to disclose.

T48. Intermittent Access to Cocaine Confers a Stronger Addiction-Like Phenotype Than Long Access: Effects Dependent on Orexin-1 Receptor Signaling

Morgan James*, Colin Stopper, Nikki Koll, Benjamin Zimmer, Gary Aston-Jones

Rutgers University, Piscataway, New Jersey, United States

Background: Intermittent access (InTA) to cocaine is a novel self-administration paradigm that restricts cocaine intake to brief ( $5 \mathrm{~min}$ ) access periods every $30 \mathrm{~min}$ (Zimmer et al, 2012). InTA results in a 'spiking' pattern of cocaine intake that is thought to mimic the pattern of drug intake in human addicts more accurately than other self-administration paradigms (Beveridge et al, 2012). Behavioral economic (BE) analyses revealed that InTA to cocaine increases motivation for drug compared to animals given 6-h long access (LgA) to cocaine (Zimmer et al, 2012). It is unknown if InTA to cocaine also produces greater changes in other indices of addiction such as resistance to punishment and reinstatement. Further, the neural mechanisms that underlie InTA-induced changes in motivation are not understood. We previously found that the orexin-1 receptor (Ox1R) antagonist SB-334867 (SB) decreases motivation for cocaine measured with $\mathrm{BE}$ in high-demand animals (Bentzley \& Aston-Jones. 2015), indicating that the orexin system may drive pathological drug-seeking behavior. Here, we compared animals given InTA versus LgA to cocaine on a range of addiction indices, including economic demand, compulsive (punished) responding for cocaine, extinction responding, as well as cued and cocaine-primed reinstatement. Secondly, we assessed whether differences in addiction behaviors between InTA and LgA are orexin-dependent.

Methods: Male Sprague-Dawley rats were implanted with jugular catheters for i.v. cocaine self-administration. After initial FR1 self-administration training, rats were trained until stable on a BE procedure to obtain baseline demand values (Bentzley et al, 2013). Rats were then trained for $14 \mathrm{~d}$ on InTA (5 min access every $30 \mathrm{~min}$ for $6 \mathrm{~h}$ ), LgA (uninterrupted access for 6h) or short access (ShA; uninterrupted access for 1h) FR1 self-administration. Following InTA, LgA or ShA self-administration, animals were again re-stabilized on the $\mathrm{BE}$ procedure before being tested for compulsive (punished) responding for cocaine whereby infusions of cocaine were paired with footshock of increasing intensity. Rats then underwent extinction training, and were tested for cued and or cocaine-primed reinstatement. All behavioral tests were carried out in a within-subjects fashion, with each animal receiving counterbalanced systemic injections of the Ox1R antagonist SB $(0,10,30 \mathrm{mg} / \mathrm{kg})$.

Results: InTA decreased demand elasticity (alpha parameter; increased motivation) and increased the maximum price animals paid for a mg of cocaine (Pmax) compared to ShA. A similar trend was observed following $\operatorname{LgA}$ but to a lesser extent than for InTA. In all InTA animals, SB dose- dependently altered cocaine demand towards baseline values. In contrast, SB only normalized demand in animals that exhibited high demand for cocaine following LgA. InTA animals accepted a greater amount of footshock than either LgA or ShA animals, and this was attenuated by pretreatment with SB30. InTA animals showed higher levels of responding on the first day of extinction, and tended to take longer to reach extinction criteria, compared to $\operatorname{LgA}$ and $\mathrm{ShA}$ animals. InTA animals also showed higher levels of both cued- and cocaine-primed reinstatement, compared to LgA and ShA animals, and both types of reinstatement were decreased by SB in InTA animals.

Conclusions: InTA to cocaine induces a greater magnitude of addiction-like behavior across several endophenotypes compared to ShA or LgA. This suggests that the pattern of intake, rather than the total amount of cocaine consumed, may be important in producing changes in motivation for cocaine. These findings are interesting given recent evidence that the pattern of human intake may more closely resemble intermittent administration (Beveridge et al, 2012). We also show that InTA-induced changes are mediated at least in part by Ox1R signaling, pointing to a potential role for pharmacotherapies that target the orexin system in the treatment of addiction.

Keywords: Cocaine Addiction, Orexin, Behavioral Pharmacology, Orexin Receptor Antagonist, Self-Administration.

Disclosure: Supported by PHS grant 1-R01 DA006214 and NHMRC fellowship 1072706.

\section{T49. Effects of Opioids on Responses to Acute Psychosocial Stress in Healthy Young Adults}

\section{Anya Bershad*, Harriet de Wit}

University of Chicago, Chicago, Illinois, United States

Background: The opioid system plays an important role in the regulation of responses to social stress. Deactivation of the $\mu$-opioid system is associated with social rejection and negative social experiences, and in animal models, activation of the system, via opioid agonists, is associated with dampened responses to isolation distress. Despite evidence implicating the opioid system in mediating responses to psychosocial stress in laboratory animals, relatively few studies have investigated these effects in humans. Here we examined the effects of buprenorphine, a $\mu$-opioid partial agonist and $\kappa$-opioid antagonist, and hydromorphone, a pure $\mu$-opioid agonist, on subjective and physiological responses to a stressful public speaking task in healthy volunteers. We hypothesized that both drugs would reduce subjective and physiological stress responses.

Methods: In Study 1, healthy adult volunteers were randomly assigned to receive placebo $(N=18), 0.2 \mathrm{mg}$ buprenorphine $(N=15)$, or $0.4 \mathrm{mg}$ buprenorphine $(N=15)$, under doubleblind conditions. They attended two sessions, during which they performed either a stressful speaking task or a nonstressful control task, in counterbalanced order. During the sessions the participants completed self-report questionnaires, a measure of task appraisal, and provided measures of salivary cortisol, heart rate, and blood pressure at regular intervals. In Study 2, participants received placebo $(N=13)$, 
$2 \mathrm{mg}(N=12)$, or $4 \mathrm{mg}(N=12)$ hydromorphone using a similar design as Study 1.

Results: In both studies, the stress task produced its expected increase in heart rate, blood pressure, salivary cortisol, and subjective ratings of anxiety and negative mood in the placebo groups. The higher doses of each drug produced comparable increases ratings of "feel drug." In Study 1, buprenorphine (both doses) reduced salivary cortisol responses to stress. Although buprenorphine did not affect subjective measures of anxiety, it dose-dependently reduced how threatening participants found the tasks, and increased performance satisfaction. In Study 2, hydromorphone dosedependently dampened cortisol responses to stress, but, unlike buprenorphine, it did not affect ratings of threat appraisal.

Conclusions: These results support the idea that $\mu$-opioid agonists dampen physiological responses to acute stress in humans, in line with results from preclinical studies. The combined actions of buprenorphine at $\mu$ - and $\kappa$ - receptors may more effectively reduce psychological responses compared to a pure $\mu$-agonist alone. The potential anti-stress effects of buprenorphine may contribute to its effectiveness during the treatment of opioid abuse.

Keywords: Buprenorphine, Acute Stress, Cortisol, Opioid System, Anxiety.

Disclosure: This research was supported by NIDA DA02812.

\section{T50. Ventral Pallidum Roles in Cue-Elicited Reward Seeking and Reinforcement}

Jocelyn Richard*, Benjamin Saunders, Patricia Janak

Johns Hopkins University, Baltimore, Maryland, United States

Background: The mechanisms by which neutral cues elicit reward seeking are a critical area of inquiry in the neurobiology of motivation. Recently we showed that activity of ventral pallidum (VP) neurons in response to discriminative cue encodes both the likelihood and latency of subsequent instrumental reward-seeking actions. Additionally, inactivation of VP neurons during the cue reduces the likelihood and rapidity of reward-seeking behavior, suggesting that VP neurons encode and functionally contribute to the incentive motivational properties of cues. More recently, we have begun to probe whether VP neuron activity plays a similar role for conditioned responses to Pavlovian cues predicting delivery of sucrose or alcohol, and what aspects of incentive motivation may be driven by activation of VP neurons.

Methods: To assess the degree to which VP neurons encode the incentive value of Pavlovian cues for sucrose or alcohol, male and female Long Evans rats were trained to associate one auditory cue (the CS+) with delivery of $20 \%$ alcohol or $10 \%$ liquid sucrose reward and an alternative auditory cue (CS-) with no delivery of reward. Once rats met training criteria (port entries during $>70 \%$ of CS+ presentations and $<30 \%$ of CS- presentations), they were implanted with drivable electrode arrays, and activity of VP neurons was recorded during the task. Next, we assessed the degree to which activation of VP neurons was reinforcing, by measuring optogenetic intracranial self-stimulation (ICSS) of the VP, in female Long Evans rats expressing virus for channelrhodopsin-2 (ChR2) or YFP control in VP. During 60 min ICSS sessions, responses at the "active" nosepoke resulted in optical stimulation of VP (20 pulses, $5 \mathrm{~ms}$ duration, $20 \mathrm{~Hz}, 473 \mathrm{~nm}$ ), along with LED illumination of the nosepoke port.

Results: We found that $\sim 25 \%$ of VP neurons (42/180) are excited by the $\mathrm{CS}+$, and that these excitations are greater to the CS+ than the CS-, and greater on trials when rats make a port entry during the cue, suggesting that VP excitations functionally contribute to cue-elicited port entries. We also found that photo-illumination of VP supported robust ICSS in rats expressing ChR2 in VP (active nokepokes on day 3: mean $1208+/$ - 413 SEM, $n=6$ ), but not in YFP control rats $(8.8+/-2.9, n=4)$.

Conclusions: In addition to encoding and contributing to the ability of cues to invigorate instrumental reward-seeking actions, our results suggest that VP neuronal activity during cue presentations also contributes to Pavlovian responses to reward cues. Furthermore, our observation of robust optogenetic self-stimulation of VP suggests that activation of VP neurons is itself reinforcing. Ongoing experiments are aimed at determining whether activation of VP neurons is sufficient to support conditioned approach and conditioned reinforcement.

Keywords: Ventral Pallidum, Incentive Motivation, Electrophysiology, Reinforcement.

Disclosure: Nothing to disclose.

\section{T51. Quantify Morphine-Induced Mu-Opioid Receptor Desensitization Using Simultaneous PET/MRI}

Hsiao-Ying Wey*, Jacob Hooker, Michael Placzek, Bruce Rosen, Joseph Mandeville

Harvard Medical School, Charlestown, Massachusetts, United States

Background: $\mu$-Opioid receptor (MOR) agonists are the most effective analgesics for pain. However, the development of opioid tolerance results in less effective pain relief and prompts an increase in the dose needed over time. MOR agonists-induced receptor desensitization is a pivotal mechanism leading to opioid tolerance (1). Recent in vitro studies showed that desensitized MORs have an increased affinity for agonist binding (2). Therefore, it is anticipated that MOR agonists-induced desensitization and drugradiotracer competition result in an increase and a decrease in PET binding potential signal, respectively. With conventional PET techniques alone, it is impossible to differentiate the contribution of two competing signaling mechanisms, i.e. drug-radiotracer competition vs. receptor desensitization. In the current study, we propose and demonstrate a simultaneous PET/MRI method that enables in vivo quantification of morphine-induced MOR desensitization with the addition of a concurrent fMRI measurement.

Methods: PET/MRI images were acquired from two macaques (male, $\sim 12 \mathrm{~kg}$ ) using a $3 \mathrm{~T}$ Siemens BrainPET and an 8-channel coil. Animals were anesthetized with isoflurane and ventilated. PET/MR images were acquired from each animal using a $\mu$-opioid selective radiotracer, [11C]carfentanil ( 8 mCi; specific activity: $\sim 3 \mathrm{mCi} / \mathrm{nmol}$ ), 
given as a bolus-infusion. PET data were stored in list mode and binned into 1-min frames. CBV-fMRI data were obtained following an iron oxide (Feraheme, $10 \mathrm{ug} / \mathrm{kg}$, i.v.) injection (3). Graded doses of an MOR agonist, morphine (baseline, $0.2,0.5$, and $1.0 \mathrm{mg} / \mathrm{kg}$ ) were given intravenously at 35 min post radiotracer administration. PET data was analyzed for binding potentials referenced to a nondisplaceable compartment (BPND) using the simplified reference tissue model (4). A gamma-variant function was used to model the PET and fMRI temporal response to drug challenge. Changes in fMRI signal were converted to $\mathrm{CBV}$ changes (3).

Results: Baseline time-activity curves (TACs) show that PET signal reached a steady-state with bolus/infusion of [11C] carfentanil (Fig 1a). TACs of the reference tissue are comparable between baseline and morphine scans. Apparent increases in TACs in high-binding regions following morphine injection (Fig 1a) was observed, suggesting a potential increase in receptor affinity. The largest BPND increases were observed in the thalamus, basal ganglia, amygdala, and the brainstem (Fig 1b). Percent increases in BPND (defined as) is about $26 \%$ at $0.2 \mathrm{mg} / \mathrm{kg}, 41 \%$ at $0.5 \mathrm{mg} /$ $\mathrm{kg}$, and $49 \%$ at $1.0 \mathrm{mg} / \mathrm{kg}$. We observed negative morphineinduced CBV changes as expected $(-3.1 \%$ at $0.2 \mathrm{mg} / \mathrm{kg},-1.5 \%$ at $0.5 \mathrm{mg} / \mathrm{kg}$, and $-1.1 \%$ at $1.0 \mathrm{mg} / \mathrm{kg}$ ) because MOR is inhibitory. Dose-dependent changes in BPND and CBV were found between $0.2 \mathrm{mg} / \mathrm{kg}$ and $0.5 \mathrm{mg} / \mathrm{kg}$ of morphine, but a dose of $0.5 \mathrm{mg} / \mathrm{kg}$ and $1.0 \mathrm{mg} / \mathrm{kg}$ caused similar BPND and CBV responses.

Conclusions: In this study, we demonstrated an increase in PET BPND, which potentially reflects morphine-induced MOR desensitization. An increased in PET BPND following MOR agonist challenges cannot be attributed to drugradiotracer competition. In addition, morphine is known to be deficient to internalize MORs. Because [11C]carfentanil is an agonist radiotracer, it is possible to detect a change in affinity. With the help of simultaneously collected fMRI data, it is possible to quantify the amount of morphine-induced MOR desensitization in vivo. Additional experiments are ongoing to complete the dose-response study that will allow us to fit a PET/MRI model to quantitatively differentiate competition vs. desensitization.

Keywords: Simultaneous PET-MR, Mu-Opioid Receptors, Desensitization, Morphine.

Disclosure: Nothing to disclose.

\section{T52. Granulocyte-Colony Stimulating Factor (G-CSF) Modulates Neuronal and Behavioral Plasticity in Response to Cocaine}

Drew Kiraly*, Erin Calipari, Benoit Labonte, Deena Walker, Orna Issler, Scott Russo, Eric Nestler

Icahn School of Medicine at Mount Sinai, New York, New York, United States

Background: Pathologic use of illicit drugs represents a major public health concern and creates significant economic and social costs. Addiction to cocaine and other psychostimulants remains a major cause of this morbidity. The pathophysiological mechanisms that lead to persistent and dysregulated drug use remain incompletely understood, and there are currently no FDA-approved pharmacotherapies for treatment of psychostimulant use disorders. There is growing evidence that dysregulation of the immune system plays a role in the pathophysiology of multiple psychiatric disorders including major depressive disorder and schizophrenia. While cocaine is known to have immunomodulatory effects, the link between these immune interactions and pathological use behaviors has only recently been investigated. To further clarify this link, we performed broad characterization of serum cytokines after self or experimenter-administered cocaine in mice, and used this information to interrogate how changes in cytokines expression may affect cocaine-mediated neuronal and behavioral plasticity.

Methods: Serum profiling was performed on male C57/BL6 mice treated with experimenter-administered $(20 \mathrm{mg} / \mathrm{kg} /$ day $)$ or self-administered $(0.5 \mathrm{mg} / \mathrm{kg} /$ infusion $\mathrm{x} 2 \mathrm{hr} /$ day $)$ cocaine for ten days. Quantitative analysis of 32 cytokines from each sample was performed using Luminex multiplex immunoassay technology. Cocaine-induced c-Fos expression was analyzed in mice pre-treated with G-CSF $(50 \mathrm{mcg} / \mathrm{kg})$ or saline, who then received a single injection of saline or cocaine (20mg/kg i.p.). Animals were killed 90 minutes later and multiple brain regions dissected out and processed for qPCR analysis. For locomotor sensitization and conditioned place preference (CPP) testing, all animals were treated with a subcutaneous injection of saline or G-CSF $(50 \mathrm{mcg} / \mathrm{kg})$ on the morning of each day. Locomotor testing was performed with two days of saline injections followed by five days of cocaine $(7.5 \mathrm{mg} / \mathrm{kg}$ i.p.). Conditioned place preference tests were performed using an unbiased design. Extinction and reinstatement experiments were performed on adult male Sprague-Dawley rats trained to self-administer cocaine $(0.8 \mathrm{mg} / \mathrm{kg} /$ infusion) for seven days of stable responding. Animals were then split into two separate groups for extinction training, and were injected with saline or G-CSF $(50 \mathrm{mcg} / \mathrm{kg})$ on each day of extinction. Once both groups had extinguished responding, they were returned to home cages for 7 days before being brought back for a single cue-induced reinstatement session.

Results: Multiplex analysis of serum from cocaine-treated animals showed regulation of multiple cytokines, but only levels of IL-1 $\beta$ and G-CSF were significantly upregulated in animals treated with experimenter and self-administered cocaine, and only G-CSF showed significant positive correlation with the behavioral change in both paradigms. To assess effects of G-CSF on cocaine-induced neuronal activation we analyzed expression of c-Fos in animals pretreated with systemic G-CSF injections. Animals treated with G-CSF alone had similar levels of c-Fos expression to saline controls in all brain regions examined, and treatment with cocaine produced the expected induction of c-Fos. However, treatment with G-CSF and cocaine in combination produced a more robust increase in c-Fos in both the nucleus accumbens and prefrontal cortex, but not in other brain regions examined. To examine if elevated levels of G-CSF had direct effect on behavioral plasticity response to cocaine we performed several analyses. In locomotor analyses G-CSF treatment had no effect on baseline locomotor activity, but resulted in a markedly enhanced locomotor sensitization response. Similarly, pre-treatment with G-CSF caused a marked shift in the dose response curve on the CPP assay. 
G-CSF-treated animals showed enhanced preference for lower dose (3.75 \& $7.5 \mathrm{mg} / \mathrm{kg})$ cocaine while exhibiting normal preference for high dose $(15 \mathrm{mg} / \mathrm{kg})$ cocaine. Finally, in rats previously trained to stably self-administer cocaine, those animals treated with injections of G-CSF prior to extinction training demonstrated an accelerated extinction curve, and showed decreased reactivity to a cue-induced reinstatement paradigm.

Conclusions: Our results demonstrate that the pleiotropic cytokine G-CSF is elevated after cocaine treatments, and is a potent regulator of cocaine-induced behavioral and neuronal plasticity. Given that G-CSF enhances extinction processes and reduces reinstatement of cocaine seeking, treatment with G-CSF or one of its downstream signaling pathways may represent an important possible therapeutic treatment for cocaine use disorder.

Keywords: Cytokine, Cocaine, Neuroimmune Interaction. Disclosure: Nothing to disclose.

\section{T53. Week-to-Week Fluctuations in Risky Decision Making Track Heroin Use in Treatment-Seeking Opioid} Users

Anna Konova*, Silvia Lopez-Guzman, Adelya Urmanche, John Messinger, Soteri Polydorou, Stephen Ross, Kenway Louie, John Rotrosen, Paul Glimcher

New York University, New York, New York, United States

Background: The degree to which opioid replacement therapy (e.g., methadone), the gold standard in opioid addiction management, is effective at reducing illicit opioid use depends on how well titrated it is for the current needs of an individual. However, good proximal predictors of when an individual is at risk for relapse-and therefore in need of additional behavioral and/or pharmacological interventionare currently lacking. Here, we use standard computationally driven neuroeconomic measurements to decompose the risk taking behavior of opioid users undergoing opioid replacement therapy, as a way to identify behavioral markers that might predict illicit opioid use.

Methods: We had individuals starting opioid replacement therapy (i.e., who were within 4 weeks of treatment initiation) perform simple and easy-to-automate monetary decision making tasks weekly (and then every other week) over several months of treatment. We established when our subjects returned to illicit opioid (or any drug) use by both self-report and randomly administered (at least 1/week) urine toxicology tests. A matched sample of drug-free community controls also completed the decision making tasks. These subjects both served as a baseline control group as well as allowed us to assess the test-retest reliability of our measurements. A subset of subjects from both groups also completed the tasks while we acquired functional magnetic resonance imaging (MRI) data at two time points: once at the beginning of the treatment and again 8-12 weeks later. The measurements we used are based on a standard neuroeconomic model that decomposes the behavior of each subject into two parameters: "risk attitude" and "ambiguity attitude", indexing how sensitive that subject is to known and unknown risks, respectively. We computed these parameters for each subject at each study session, and using generalized linear mixed models, we examined how fluctuations in these parameters related retrospectively and prospectively to illicit opioid use events.

Results: We find a high degree of test-retest reliability across the study sessions for both parameters. Attesting to the distinct aspects of risky decision making captured by these parameters, we find that only sudden increases in an opioiddependent subject's willingness to take risks in our task correlated with, and in some cases preceded, illicit opioid use. But importantly, we find both parameters are not stationary in the opioid-dependent subjects: both parameters fluctuate as individuals approach and recover from opioid use events in a way not seen in controls. Both risk and ambiguity tolerance increases surrounding opioid use, albeit at different rates.

Conclusions: These data suggest that risk attitudes, which can be quickly and easily measured by our behavioral tasks, might be suitable behavioral markers-and perhaps even predictors-of relapse in opioid addiction. Our ongoing work seeks to examine the neurobiological basis of this relationship between risky decision making and drug use. Based on previous findings in health with these tasks, we anticipate a common neural mechanism of the risk and ambiguity parameters to include activation in regions that form the brain's valuation network (striatum, ventromedial prefrontal cortex), and distinct mechanisms to include activation in the insular cortex (for risk attitudes) and amygdala (for ambiguity attitudes).

Keywords: Opioids, Treatment, Reward-Based DecisionMaking, Risk and Resilience, Relapse and Treatment Outcome.

Disclosure: Nothing to disclose.

\section{T54. Exaggerated Reactivity to Uncertain Threat is a} Familial Vulnerability Factor for Alcohol Use Disorder

Stephanie Gorka*, Stewart Shankman, K Luan Phan

University of Illinois at Chicago, Chicago, Illinois, United States

Background: Exaggerated anticipatory anxiety, or reactivity, in response to uncertain threat (U-threat) is associated with risk for fear-based anxiety disorders. There is some preliminary evidence to suggest that this same affective response tendency exists in individuals with remitted alcohol use disorder (AUD) and thus reflects a shared vulnerability factor for AUD and fear-based psychopathology. To date, no study has attempted to disentangle whether reactivity to U-threat is a risk factor or acquired factor for AUD and importantly, what neural processes underlie this aberrant response tendency.

Methods: The current study had three aims to address three fundamental questions: 1) Do current problematic drinkers display exaggerated reactivity to U-threat?; 2) Do individual differences in reactivity to U-threat aggregate within families and relatedly, is exaggerated reactivity to U-threat associated with risk for AUD?; 3) What are the neural correlates of exaggerated reactivity to U-threat? Participants for all three aims were drawn from two large community samples of adults. All individuals completed the same well-validated threat-of-shock behavioral task designed to probe responses 
to U-threat and predictable threat (P-threat) (total $n=295)$. During the task, startle eyeblink response was collected as an index of aversive responding. To assess familial aggregation, one biological sibling of a subset of participants also completed the behavioral threat task ( $n=157$ sibling dyads). To assess neural responding, a subset of participants $(n=32)$ also completed an analogous threat-of-shock task during functional magnetic resonance imaging (fMRI).

Results: For Aim 1, current, problematic drinkers displayed exaggerated reactivity to U-threat, but not P-threat, relative to non-problematic drinkers $(b=16.87, t=4.44, p<0.001)$. For Aim 2, startle potentiation to U-threat, but not P-threat, was positively associated with risk for AUD, defined by first-degree family density of AUD $(\beta=0.21, t=2.61, p<0.01)$; moreover, within biological siblings, startle potentiation to U-threat (intraclass correlation $[\mathrm{ICC}]=0.35$ ) was significantly correlated. For Aim 3, problematic drinkers displayed hyperactive dorsal anterior cingulate (dACC; MNI peak [4, 34, 34], $\mathrm{Z}=3.28, p<0.05$, corrected) response to U-threat, but not P-threat, compared with non-problematic drinkers; additionally, startle response to U-threat was positively correlated with dACC response to U-threat $(r=0.37, p<0.01)$.

Conclusions: These findings demonstrate that similar to individuals with remitted AUD, current problematic drinkers display an exaggerated reactivity to U-threat. In addition, this response tendency aggregates within families and is associated with risk for AUD. In other words, exaggerated reactivity to U-threat is familial and evident in healthy individuals at-risk for problematic drinking. Lastly, the findings show that exaggerated reactivity to U-threat is driven by hyperactive dACC responding and the dACC may therefore be a neural correlate of threat reactivity as transmitted in families. Taken together, the evidence highlights the role of exaggerated reactivity to U-threat as a vulnerability factor for AUD. This behavioral-brain response tendency may be a novel target for AUD prevention and/or intervention.

Keywords: Alcohol Use Disorder, Threat of Shock, fMRI, Anxiety, Threat.

Disclosure: Nothing to disclose.

\section{T55. Translating Mesocortical Dopamine Into an Aversive Signal via Specific Downstream Projections}

Cody Siciliano*, Caitlin Vander Weele, Gillian Matthews, Edward Nieh, Eyal Kimchi, Isabella Espinel, Ehsan Izadmehr, Evelien Schut, Romy Wichmann, Kay Tye

Massachusetts Institute of Technology, Vassar Street, Massachusetts, United States

Background: A great deal of research has highlighted the role of the medial prefrontal cortex (mPFC) in encoding motivational value and decision making. Similarly, the dopamine system has been implicated in these processes; however, the large majority of these studies have focused on the encoding of positive rewarding stimuli in mesolimbic and nigrostriatal dopamine projections. More recently, emerging studies have suggested that dopaminergic innervation of the mPFC from the ventral tegmental area may play a role in aversively motivated behaviors. Here, we sought to dissect the role of mesocortical dopamine projections in aversively motivated behaviors.

Methods: To examine the role of dopaminergic innervation of mPFC we used tyrosine hydroxylase (TH)-Cre rats as well as wildtype C57BL/6J mice. Voltammetry experiments were performed in TH-Cre rats under urethane anesthesia, and response to tail-pinch was recorded. To examine the role of sub-populations in the mPFC, a cre-dependent genetically encoded calcium indicator GCaMP was virally delivered to the mPFC of wildtype mice and a retrogradely traveling virus carrying cre-recombinase was infused into downstream projection targets. Calcium dynamics were visualized and recorded using chronically implanted gradient-index lenses and Inscopix mini-microscopes. For optogenetic experiments, channelrhodopsin-2 was expressed in a projection-specific manner using the same viral strategy, and $473 \mathrm{~nm}$ light was delivered through chronically implanted optic fibers.

Results: Using fast-scan cyclic voltammetry, we found that aversive stimuli produced rapid increases in dopamine release in the mPFC. To investigate how this signal may be influencing activity to encode aversive stimuli, we used single-cell calcium imaging to record from projectiondefined sub-populations within the mPFC during exposure to either foot shock or sucrose. We found that mPFC cells projecting to the periaqueductal gray area (PAG) were robustly activated by foot shock, while having fewer responses to sucrose. Conversely, mPFC cells projecting to the nucleus accumbens (NAc) showed modest responses to foot shock and were activated during sucrose drinking bouts. To determine if these encoding patterns were causally related to behavior, we photostimulated these projector populations. We found that stimulation of PFC-PAG neurons produced robust avoidance, supporting a role for these cells in encoding of aversive stimuli. Finally, to determine the role of dopaminergic innervation of these sub-populations, we performed wholecell patch-clamp electrophysiology paired with optical stimulation of dopamine terminals in mPFC. We found that dopamine inhibited cells in the mPFC that project to the NAc, while having no effect on cells projecting to the PAG.

Conclusions: Together, these results support a model by which dopamine in the MPFC encodes allows for the encoding of innately aversive stimuli through differential action on projection defined populations. We postulate that through inhibition of NAc projectors in the $\mathrm{mPFC}$, which encode appetitive stimuli, dopamine acts as a permissive signal by allowing aversive stimuli to be encoded through PAG projecting cells without competition from populations encoding opposing valence.

Keywords: Dopamine, Medial Prefrontal Cortex, Reward and Aversion, Calcium Imaging, Optogenetics.

Disclosure: Nothing to disclose.

\section{T56. Epigenome and Transcriptome Analysis for Tobacco Smoking in a Community Population}

\section{Ke Xu*, Xinyu Zhang, Ying Hu, Rajita Sinha}

Yale University School of Medicine, New Haven, Connecticut, United States

Background: Tobacco smoking has significant health adverse impacts and increases mortality and morbidity in 
the world. The molecular mechanisms of how smoking impacts health is largely unknown. Previous epigenome and transcriptome studies have revealed differentially methylated and expressed genes between smokers and non-smokers at individual gene level. However, the relationship of these genes at the system level has not been understood. Whether differential gene expression for smoking is modulated by epigenetic modification is still unknown. Our goal is to systematically evaluate transcriptomes between smokers and non-smokers and to identify epigenetically regulated gene expression for tobacco smoking in a community sample.

Methods: We recruited 506 healthy subjects with and without current smoking from a community sample in New Haven, Connecticut area. Subjects with medical and psychiatric diagnoses except nicotine dependence were excluded. Subjects who took any medications for medical or psychiatric conditions were also excluded from the study. DNA and total RNA were extracted from whole blood for each individual. Epigenome-wide DNA methylation was profiled using Illumina HumanMehtylation 450K Beadchip. Generalized linear regression model was applied to identify differential DNA methylation between smokers and nonsmokers. For gene expression, array-based transcriptome expression was performed using Illumina HumanHT-12 v4 Expression BeadChip. To identify co-expression gene network, we applied an unsupervised network analysis approach, weighted gene co-expression network analysis (WGCA), to identify gene expression clusters and to test the relationship between co-expressed genes and smoking. We then examined correlations between smoking-associated DNA methylation and gene co-expressed modules.

Results: Epigenetically, we replicated previously reported $17 \mathrm{CpG}$ sites differentially methylated between smokers and non-smokers including 3 hypomethylated $\mathrm{CpG}$ sites on AHRR (Aryl-Hydrocarbon Receptor Repressor) (cg05575921: $\mathrm{t}=-10.9, p=1.34 \mathrm{E}-24 ; \operatorname{cg} 26703534: \mathrm{t}=-8.22$, $p=2.33 \mathrm{E}-15$; and cg21161138: $\mathrm{t}=-7.42, p=6.18 \mathrm{E}-13)$. AHRR is involved in regulation of cell growth and differentiation and is associated with lung cancer. For transcriptome expression, we identified 25 co-expression modules from top 5,000 variable genes in all 506 samples. Interestingly, only one co-expression module differentiated smokers and nonsmokers $(p=0.013)$. This module contained 88 genes that enriched on the adaptive immune response, regulation of defense, and regulation of stress responses by a hypergeometric test (FDR $q<0.05$ ). This smoking-associated coexpression module contained 2 hub genes, which were highly connected with other genes in the same module. Two hub genes were RNF13 (ring finger protein 13) and GCA (EFhand calcium-binding protein). RNF13 is a critical mediator that facilitates stress-induced apoptosis by activating the IRE1 $\alpha$-TRAF2-JNK signaling pathway. To examine the relationship between DNA methylation and gene expression in the smoking-associated co-expressed gene module, we tested the correlation of methylated $\mathrm{CpG}$ sites with gene expression. In this module, we found that methylation of 8 genes were inversely correlated with gene expression (RNF13, PICALM, RPS6KA5, EIF4E3, IPS2, ST2SLA4, EXOC6 and DIP2B) (ps adjusted $<0.05$ ). Particularly, methylation of the hub gene, RNF13, was negatively correlated with expression, indicating that methylation mediates gene co-expression of this smoking-associated module. We further identified 4 differentially expressed individual genes (ps adjusted $<$ 0.05) (SGK1, SLC31A2, LOC728319, AOAP2) between smokers and non-smokers in this module.

Conclusions: Our results suggest that tobacco smoking alters DNA methylation on multiple genes that are involved cell growth and differentiation. Smoking-altered transcripome acts as a functional group that is partially modulated by DNA methylation. Such co-expressed genes are enriched on biological pathways related to immune adaptation and stress responses. These findings provide novel molecular insights of smoking in healthy individuals.

Keywords: DNA Methylation, Transcriptome, Tobacco Smoking, Gene Network Analysis.

Disclosure: Nothing to disclose.

\section{T57. Neural Structure in Substance and Behavioral Addictions: Diagnostic and Transdiagnostic Findings}

Sarah Yip*, Patrick Worhunsky, Jiansong Xu, Kristen Morie, R Todd Constable, Robert Malison, Marc Potenza

Yale University School of Medicine, New Haven, Connecticut, United States

Background: Alterations in neural structure have been reported in both cocaine-use disorder and gambling disorder, separately, suggesting similarities across addiction diagnoses. Individual variation in neural structure has also been associated with impulsivity, a dimensional construct implicated in addictions.

Methods: Categorical (diagnosis-based) and dimensional (transdiagnostic) approaches were combined to identify neural structural alterations linked to addiction subtypes and trait impulsivity, respectively, across individuals with gambling disorder $(n=35)$, individuals with cocaine-use disorder $(n=37)$ and healthy comparison individuals $(n=37)$. High-resolution T1-weighted data were analyzed using modulated voxel-based morphometry (VBM). Statistical analyses were conducted using whole-brain generallinear models, corrected for family-wise error ( $\mathrm{pFWE}<.05$ ). A conjunction analysis was conducted to identify areas of anatomical overlap between categorical versus dimensional findings.

Results: Whole-brain categorical analyses indicated a main effect of diagnostic group on prefrontal (dorsal anterior cingulate, ventromedial prefrontal cortex) gray matter volumes (GMVs), involving decreased GMVs among cocaine-use disorder participants only. Follow-up correlational analyses indicated a negative linear association between GMVs in these regions and years of cocaine-use. Whole-brain dimensional analyses indicated a negative association between trait impulsivity and cortical (insula) and subcortical (amygdala, hippocampus) GMVs across all participants. Conjunction analysis indicated little anatomical overlap between regions identified as differentiating diagnostic groups and regions co-varying with impulsivity.

Conclusions: These data provide, in direct comparison, first evidence of neural structural differences between gambling disorder and an illicit substance-use disorder. They further indicate largely dissociable effects of diagnostic groupings and trait impulsivity on neural structure among individuals 
with behavioral and drug addictions. Study findings highlight the importance of considering both categorical and dimensional (e.g., Research Domain Criteria; RDoC) analysis approaches within the context of addictions research.

Keywords: Cocaine Addiction, Gambling Disorder, VoxelBased Morphometry (VBM), Impulsivity, Research Domain Criteria (RDoC).

Disclosure: Nothing to disclose.

\section{T58. Signaling Kinetics of Stimulated Dopamine Release in the Nucleus Accumbens Core and Shell Are Differentially Altered Following Abstinence From Cocaine Self-Administration in Behaving Rats}

\section{Michael Saddoris* \\ University of Colorado Boulder, Boulder, Colorado, United States}

Background: Repeated experience with drugs of abuse can induce persistent changes in neural signaling properties even after weeks of abstinence from the drug-taking episodes. We and others have shown that these alterations are associated with impairments in motivated and goal-directed behavior, including deficits in Pavlovian-to-instrumental transfer, conditioned approach, and second-order conditioning. At the neural level, these changes are correlated with alterations in the appropriate phasic dopamine (DA) signals in the nucleus accumbens (NAc), and these impairments showed distinct changes between the NAc core and shell. However, in these tasks, impaired signaling was investigated relative to task stimuli during the performance of a motivated task. Thus, impairments may be due to either an inability to appropriately signal the relevance of the stimuli (indicating a general motivational or learning deficit), or instead to fundamental alterations in the DA release and reuptake kinetics that occur as neuroadaptations following drug experience.

Methods: Rats were first trained to self-administer cocaine intravenously $(\sim 1 \mathrm{mg} / \mathrm{kg})$ in daily $2 \mathrm{hr}$ sessions for $14 \mathrm{~d}$. Controls self-administered water to a foodcup and yoked i.v. vehicle infusions. Following at least $30 \mathrm{~d}$ of enforced abstinence from self-administration and at least $1 \mathrm{wk}$ prior to recordings, rats were implanted with guide cannulas over the NAc core ( $n=7$ Cocaine, $n=17$ Controls) or shell $(n=6$ Cocaine, $n=12$ Controls) and a bipolar electrical stimulating probe into the ventral tegmental area (VTA). On the day of recording, an acutely-placed carbon fiber electrode was lowered into the region of interest in awake and behaving rats. Electrical stimulation of the VTA was performed at a variety of frequencies $(12-60 \mathrm{~Hz})$ and pulse number (1-24) with a $4 \mathrm{~ms}$ pulsewidth; rapid DA release and reuptake kinetics were measured via fast scan cyclic voltammetry.

Results: In Controls, we confirmed previous findings demonstrating a slower release rate to peak and slower reuptake rate following peak (e.g., Vmax) in the shell compared to the core. However, cocaine experienced rats showed significantly altered signaling kinetics. Shell DA kinetics were less sensitive to changes in applied stimulation rates, while core DA kinetics slowed and came to resemble normal NAc shell kinetics. Indeed, the distributions of peak
[DA] in the both Cocaine subjects were nearly indistinguishable from Control shell recordings.

Conclusions: While both NAc core and shell in cocaineexperienced rats display altered phasic release relative to drug-naive controls, the general effect of abstinence from drug self-administration was to induce a shell-like state of DA release/reuptake kinetics throughout the NAc. Thus, cocaine experience appears to alter DA signaling of both task relevance and terminal function. For example, while taskrelated DA release during behavior in the shell is largely eliminated (Saddoris et al, 2016), stimulated DA is relatively intact. In contrast, DA signaling in the core during behavior is robust but displays abnormal signaling of salient stimuli, while also showing more shell-like signaling kinetics following stimulation. These findings demonstrate persistent and region-specific changes in DA synaptic properties following cocaine self-administration related to abnormal task signaling and terminal function, both of which likely contribute to behavioral deficits in motivated learning well after the cessation of drug-taking episodes.

Keywords: Dopamine, Voltammetry, Nucleus Accumbens Shell, NAc core.

Disclosure: Nothing to disclose.

\section{T59. Changes in Social Rank and Dopamine Levels in Cerebrospinal Fluid: Findings From a Novel Pig Model}

Hui Cheng*, Abiy Mohammed, Anthony Pease, Joshua Gehrke, George Bohart, Michael Nader, James Anthony

\section{Michigan State University, East Lansing, Michigan, United States}

Background: Social rank, defeat, and 'coming out on top or on bottom' are interwoven with human mental life and behavior, with analogues in other species. Evidence from socially housed non-human primates suggests selective changes in dopaminergic systems (e.g., downward regulation of D2 receptors after defeat and upward regulation after win) and dampened cocaine self-administration among winners. Here, a rank-andre-rank crossover design with just-weaned piglets (Sus scrofus) is described. Preliminary results are shown for hypothesized change in endogenous CSF DA explained by social rank change, versus an expectation of no change in 'control' monoamines from CSF or peripheral blood.

Methods: We randomly assigned 16 unrelated males to 4 identical social housing conditions for 2 weeks, with videorecorded interactions, followed by standardized ratings of social rank to identify each group's alpha, B, C, D status. Then, 4 at observed alpha rank were housed together, as were the $4 \mathrm{Bs}$, the $4 \mathrm{Cs}$, and the $4 \mathrm{Ds}$, for 2 weeks, and videorecorded re-ranking. HPLC assays for CSF and circulating blood monoamines were measured at pre- ('baseline'), mid-, and post- time points. Hypothesis-testing was via regression (2-tailed alpha $=0.05)$. Animal housing, handling and all experimental procedures followed 2011 National Research Council Guidelines for the Care and Use of Mammals in Neuroscience and Behavioral Research, with Animal Care and Use Committee approval.

Results: Neither baseline weight nor monoamine levels predict 1st social ranking outcome (regression coefficient 
$\beta=-0.002$ to 0.195 ; all $p>0.09, n=15)$. As hypothesized, for each unit increase in social mobility at re-ranking, there was increase in CSF dopamine (DA) levels, while decreases in social mobility predicted decreases in CSF DA concentrations $(\beta=17.4 \mathrm{pg} / \mathrm{ml} ; 95 \% \mathrm{CI}=1.2,33.7 ; p=0.04)$. In contrast, with baseline level held constant, there were no increases in 'control' monoamine values. For example, rank predicted neither CSF norepinephrine (NE) levels ( $\beta=10.2$ $\mathrm{pg} / \mathrm{ml} ; 95 \% \mathrm{CI}=-113.4,133.7 ; p=0.85)$ nor peripheral blood DA and NE levels $(p>0.05)$.

Conclusions: The rank-and-re-rank crossover design with just-weaned male piglets is a shift in species that helps confirm and extend prior non-human primate research on how change in social rank might influence DA processing. Systematic replication with piglets is needed (including females). Analogous experiments for less complex species with DA and social rank would be helpful (e.g., anoles, zebrafish). In time, post re-ranking ethanol, cocaine, cannabinoid, and other drug self-administration components will push these preliminary studies forward, as will before and after monoamine receptor imaging. Eventually, these same study parameters in human social re-ranking experiments can be estimated in extensions of ongoing psychosocial stress research with paired-subject competitive games during neuroimaging, designed to include post-game ethanol, nicotine, and cannabinoid self-administration patterns. Keywords: Social Stress, Dopamine, Swine Model. Disclosure: Nothing to disclose.

\section{T60. Neural Activity in Childhood Predicts Adolescent Substance use Initiation}

Lora Cope*, Jillian Hardee, Michael Angstadt, Joseph Heffernan, Robert Zucker, Chandra Sripada, Mary Heitzeg

University of Michigan, Ann Arbor, Michigan, United States

Background: Substance use at an early age conveys substantial risk for later substance-related problems. One study found that those who began using drugs before age 14 had a lifetime dependence rate that was twice as high as those who started after 21. A better understanding of the early risk factors could result in more timely and effective intervention. The aim of this study was to investigate the predictive utility of neural functioning as a risk factor for early substance use initiation.

Methods: Subjects were 51 children (15 female) from an ongoing longitudinal functional magnetic resonance imaging study, scanned at a mean age of 10.5 years (SD 1.1). Eighteen subjects later initiated substance use (mean age 13.6, SD 1.3; users); 33 subjects did not (non-users). We used monetary incentive delay and go/no-go tasks to examine the hemodynamic response to reward anticipation and failed inhibitory control, respectively. Independent components analysis and logistic regression were used to test the hypothesis that brain response patterns would have predictive utility over and above two known risk factors for substance use problemsexternalizing behavior and family history of substance use disorder (SUD) - in the differentiation of users and nonusers.
Results: Nucleus accumbens activation during reward anticipation significantly predicted group membership ( $p=$ .024), whereas failed inhibitory control components did not. Using the likelihood ratio test, the model that also included neural data was significantly better than the model that had only externalizing and family history variables $(p=.003)$.

Conclusions: Heightened reward responsivity in the nucleus accumbens may predispose individuals to early substance use, beyond the risk conveyed by other known factors. In contrast, failed inhibitory control appears to be less influential at this age. Future studies should investigate the possibility that, later in development, both reward responsivity and inhibitory control components are predictive of SUD in young adults.

Keywords: Functional Magnetic Resonance Imaging, Nucleus Accumbens, Reward, Amygdala, Adolescence.

Disclosure: Nothing to disclose.

\section{T61. Input-Specific Mechanisms of Drug-Evoked Plasticity in the Mesolimbic Dopamine System}

Johannes de Jong, Seyedeh Atiyeh Afjei, James Peck, Ignas Cerniauskas, Vivian Han, Stephan Lammel*

University of California, Berkeley, Berkeley, California, United States

Background: Drug addiction is a major public issue worldwide because it strongly affects a person's health, behavior and places a costly burden upon society. Drugs of abuse can hijack synaptic plasticity mechanisms in brain circuits that are central to reward processing. One of the most well established synaptic modifications caused by in vivo administration of drugs of abuse is an increase in the strength of excitatory synapses onto ventral tegmental area (VTA) dopamine (DA) neurons. We have previously shown that a single injection of cocaine induces a strong and long-lasting (>21 days) potentiation of excitatory synapses onto Ihlacking VTA DA neurons projecting to the medial shell of the nucleus accumbens (DA $\rightarrow$ mShell) (Lammel et al, 2011; Neuron). However, the anatomical origin of the inputs to this population of neurons which undergo synaptic potentiation following cocaine administration is largely unknown. Two prominent inputs to the VTA, which have been associated with motivated behaviors, arise from the lateral hypothalamus (LH) and dorsal raphe (DR). Here, we tested the hypothesis that in vivo cocaine exposure induces synaptic adaptations in the LH $\rightarrow$ VTA and/or DR $\rightarrow$ VTA pathways. Methods: We used a multidisciplinary approach combining synaptic electrophysiology, in vivo and ex vivo optogenetic manipulations, viral-mediated tracing and immunohistochemistry in adult (8-12 weeks old) mice.

Results: We first examined the functional role of the $\mathrm{DR} \rightarrow \mathrm{VTA}$ and $\mathrm{LH} \rightarrow$ VTA pathways using a real-time place preference assay. As expected, and consistent with recent studies, in vivo optogenetic stimulation of the DR $\rightarrow$ VTA pathway induced real-time place preference, confirming its role in mediating reward-related behaviors. However, when stimulating the $\mathrm{LH} \rightarrow \mathrm{VTA}$ pathway, we observed robust real-time place avoidance behavior, suggesting that activation of this pathway is involved in aversion-related behaviors. Next, we studied the studied the functional 
synaptic connectivity of LH and DR inputs to mesolimbic DA neurons. By combining ex vivo optogenetics and synaptic electrophysiology, we found that light stimulation of LH and DR terminals in the VTA induced large excitatory postsynaptic currents (EPSCs) in DA $\rightarrow$ mShell neurons. We assessed the synaptic strength of each pathway by measuring the relative contributions of AMPA receptors (AMPARs) and NMDA receptors (NMDARs). We found that a single in vivo injection of cocaine $(15 \mathrm{mg} / \mathrm{kg}$, IP) increased the synaptic strength of DR inputs in a small proportion of $\mathrm{DA} \rightarrow \mathrm{mShell}$ neurons when assessed 24 hours later. However, a robust increase in the AMPAR/NMDAR ratio of DR inputs to DA $\rightarrow$ mShell neurons was observed following 5 consecutive days of cocaine treatment. In addition, we found that the rectification index (RI) of the AMPAR current was significantly increased, suggesting an increase in GluR2lacking AMPARs, which show a characteristic inwardly rectifying current at positive potentials. Surprisingly, however, when stimulating LH inputs to DA $\rightarrow$ mShell neurons, a single injection of cocaine induced a significant decrease in the AMPAR/NMDAR ratio and no change in the RI.

Conclusions: Our results suggest that in vivo cocaine administration reinforces synaptic transmission in brain pathways that mediate reward-related behaviors (DR $\rightarrow$ VTA) and weakens synaptic transmission in pathways that mediate aversion-related behaviors (LH $\rightarrow$ VTA). Thus, by reorganizing synaptic transmission in distinct excitatory inputs to the VTA, drug exposure can fundamentally alter how mesolimbic DA neurons are activated. The druginduced shift in balance between pathways that mediate reward and aversion might be the first critical step in the cascade of neural circuit remodeling that ultimately could lead to addiction. Combined, we provide a novel framework for the neural circuit basis of addiction, which may inspire novel treatment strategies that could involve reprogramming of specific VTA afferent pathways for treating substance abuse disorder.

Keywords: Cocaine Addiction, Dopamine, Synaptic Plasticity, Ventral Tegmental Area (VTA).

Disclosure: Nothing to disclose.

\section{T62. Lithium Monotherapy Effects on the Brain's Functional and Structural Connectome in Bipolar Disorder}

\section{Amit Anand*, Jeffrey Spielberg}

Cleveland Clinic Lerner College of Medicine, Cleveland, Ohio, United States

Background: Lithium is one of the most specific and effective treatments for bipolar disorder (BD) yet its mechanism of action remains unclear. Neuroimaging studies have reported a number of abnormalities in bipolar disorder but the effect of lithium on these neuroimaging measures has been much less studied. Importantly, until now, suitable tools have not been utilized to integrate lithium's multiple effects on the brain in terms of network-property metrics. In this study, we addressed these important gaps in knowledge by measuring brain functional and structural connectomics before and after acute and longer term treatment with lithium.
Methods: Thirteen medication free bipolar type II subjects underwent resting state functional magnetic resonance imaging (fMRI) scans and diffusion weighted structural imaging scans at baseline and after 8 weeks of lithium treatment. Thirteen matched healthy controls were also imaged at the same time but did not receive any treatment. Functional and structural scans were analyzed using graph theory analytics. Graph theory metrics move beyond simple description of brain connectivity and provide insight into network function (e.g., BD is related to less efficient propagation of information).

Results: A significant effect of lithium on these metrics and correlation of the changes in these metrics to clinical improvement was seen. Specifically, lithium treatment increased Assortativity $(p=0.02)$ and decreased orbitofrontal cortex (OFC) Brokerage Centrality $(p=0.07)$ within brain networks and these changes correlated $(P=0.003$ and $p=0.03$ respectively) with decrease in depression scores. Lithium also led to changes in the structural connectome: increased global Assortativity $(p=0.04)$ which showed a trend level correlation with time $(p=0.08)$. Furthermore, increases in white matter connectivity in a network centered around the insula correlated with symptom improvement $(p=0.03)$.

Conclusions: The findings from this study indicate that lithium monotherapy effects in the treatment of bipolar depression can be measures using graph theory connectomic markers and that these markers may provide novel biomarkers to monitor and predict lithium effects.

Keywords: Bipolar Disorder, Resting State Functional Connectivity, Lithium Treatment, Lithium, Functional MRI (fMRI).

Disclosure: This project was funded by a NIMH grant to AA (R01MH075025).

\section{T63. Depression and Anxiety Scores Predict In Vivo Plaque and Tangle Burden in Aging and Dementia}

Florence Roussotte*, Prabha Siddarth, Linda Ercoli, Natacha Donoghue, David Merrill, Helen Lavretsky, Jorge Barrio, Gary Small

David Geffen School of Medicine, Los Angeles, California, United States

Background: Positron emission tomography (PET) scans with 2-(1-\{6-[(2-[F-18]fluoroethyl)(methyl)amino]-2naphthyl\}ethylidene)malononitrile (FDDNP) provide in vivo measurement of brain amyloid plaque and tau tangle binding levels. We previously observed an association between depression and anxiety symptoms and FDDNP-PET binding in a small sample of non-demented middle-aged and older adults. APOE-4 genetic risk increases FDDNP-PET binding, and APOE- 4 carriers are more susceptible to neuropsychiatric symptoms, particularly late-life depression. Thus, the small sample size and lack of control for APOE genotype limited conclusions from our earlier study. In the present study, we address this knowledge gap in a larger cohort that includes subjects with and without dementia, and determine if depression and anxiety symptoms predict in vivo plaque and tangle burden independently of APOE genotype and diagnosis. 
Methods: A total of 102 middle-aged and elderly subjects (aged 64.39 +/- 11.64 years, 41 men/61 women) who received FDDNP-PET scans, clinical assessments, and a neuropsychological battery that included the Geriatric Depression Scale (GDS) and the State-Trait Anxiety Inventory, were drawn from a larger study of cognitive decline. The sample comprised 34 normal aging controls, 52 patients with mild cognitive impairment (MCI), and 16 with dementia. The primary outcome measure was global plaque and tangle binding from an FDDNP-PET scan, which was calculated by averaging binding levels from five brain regions of interest (parietal, medial temporal, lateral temporal, posterior cingulate, and frontal). General Linear Models determined if the GDS and Trait Anxiety scores were associated with FDDNP binding, controlling for age, sex, diagnosis, and APOE-4 status. We then conducted post-hoc tests to examine whether these mood and anxiety measures were associated with regional FDDNP binding. We used the same set of covariates across all models.

Results: In non-demented participants $(N=86)$, greater depressive symptoms were significantly associated with higher global plaque and tangle load $(p=0.004, \mathrm{~F}=8.78)$ after controlling for age, sex, diagnosis (normal aging vs. $\mathrm{MCI}$ ), and APOE genotype. Higher levels of trait anxiety were also associated with global plaque and tangle load $(p=0.009, \mathrm{~F}=7.18)$. In the entire sample $(N=102)$, higher depression and anxiety scores were likewise associated with higher global FDDNP binding $(p=0.002, F=10.01$ and $p=0.003, \mathrm{~F}=9.01$, respectively) after controlling for age, sex, diagnosis (normal aging vs. MCI vs. dementia), and APOE-4 status. Post-hoc analyses in non-demented subjects revealed that depression and anxiety symptoms were associated with FDDNP binding in the frontal lobes $(p=0.002, \mathrm{~F}=10.74$ and $p=0.01, \mathrm{~F}=6.20$, respectively) and in the posterior cingulate $(p=0.01, \mathrm{~F}=6.83$ and $p=0.01, \mathrm{~F}=6.99)$, but not in the parietal cortex $(p=0.18, \mathrm{~F}=1.80$ and $p=0.23, \mathrm{~F}=1.46)$ or in the medial $(p=0.17, \mathrm{~F}=1.94$ and $p=0.14, \mathrm{~F}=2.2)$ and lateral temporal lobes $(p=0.15, \mathrm{~F}=2.08$ and $p=0.26$, $\mathrm{F}=1.29$ ). We observed similar results when including patients with dementia in the analyses. Specifically, depression and anxiety scores were associated with plaque and tangle burden in the frontal lobes $(p=0.001, \mathrm{~F}=11.79$ and $p=0.005, F=8.34$, respectively) and in the posterior cingulate $(p=0.01, \mathrm{~F}=6.78$ and $p=0.01, \mathrm{~F}=6.40)$, but not in the parietal cortex $(p=0.08, F=3.18$ and $p=0.11$, $\mathrm{F}=2.58)$ or in the medial $(p=0.09, \mathrm{~F}=2.94$ for both measures) and lateral temporal lobes $(p=0.29, \mathrm{~F}=1.14$ and $p=0.29, \mathrm{~F}=1.11$ ).

Conclusions: This is the first study to show that depression and anxiety symptoms are significantly related to in vivo plaque and tangle burden in middle-aged and older adults, independently of APOE genotype and dementia status. While these results will need to be replicated in separate cohorts with larger proportions of demented participants, our findings are consistent with previous reports suggesting a link between neuropsychiatric symptoms and accelerated brain aging. As FDDNP-PET binding also correlates with cognitive function in dementia and normal aging, our next steps will consist of determining whether cognitive decline mediates the link between depression and anxiety scores and in vivo plaque and tangle accumulation, and whether these associations vary across brains regions and cognitive domains. Addressing these questions will provide an investigational framework for identifying critical moderating factors and may provide new leads for early intervention or prevention strategies.

Keywords: Aging and Dementia, Late-Life Depression, Anxiety, Amyloid, Tau.

Disclosure: Nothing to disclose.

\section{T64. Selective Estrogen Enhancement of Cholinergic- Related Cognitive Performance in Women With or Without Subjective Cognitive Decline After Menopause}

Kimberly Albert, Julie Dumas, Savannah Boyd, Brenna McDonald, Andrew Saykin, Joon Hyuk Park, Magdalena Naylor, Paul Newhouse*

Vanderbilt University Medical Center, Nashville, Tennessee, United States

Background: Changes in cognitive performance noticed by an individual, so-called subjective cognitive decline (SCD), has begun to emerge as identifiable risk factor or marker for increased risk of late life objective cognitive decline and dementia. Women appear at higher risk for late life cognitive impairment and thus hormonal changes that occur at menopause have emerged as a target of investigation. Cognitive symptoms reported by postmenopausal women may be linked to the loss of estradiol (E2) support to basal forebrain cholinergic systems. The cholinergic system has been implicated in many aspects of the cognitive effects shown after E2 administration including improving attention, working memory, and improved performance on effort-demanding tasks such as verbal memory. The cholinergic system is also an important site of action for estradiol in the brain and E2 appears to modulate cholinergic neurotransmission. We have shown previously that 3 months of estradiol administration blunts the detrimental effects of cholinergic antagonists on cognitive function. Recently we examined the brain activity associated with working memory tasks in postmenopausal women with significant SCD (Dumas et al, 2013) and showed that women with SCD showed increased working memory-related activity compared to non-SCD women. To further clarify potential sources of these brain activity differences in SCD, we examined the effects of 3 months of E2 administration on the response to cholinergic blockade compared to nonSCD women.

Methods: We enrolled 36 non-smoking young (50-60 year old) postmenopausal women (mean age 56.03 years, $\mathrm{SD}=$ 2.84), 18 of whom who reported a significant decline in cognitive abilities after menopause, as confirmed by at least $20 \%$ endorsement rate on a comprehensive battery of cognitive symptoms as well as 18 women without such postmenopausal symptoms. Subjects underwent baseline structural and functional brain imaging and were then placed on oral $17-\beta$ estradiol (1mg/day) or placebo for three months and scanned again. Subjects then completed four pharmacological challenge days, receiving one of the following medications on each day: $2.5 \mu \mathrm{g} / \mathrm{kg}$ of the antimuscarinic scopolamine (IV), $20 \mathrm{mg}$ of the antinicotinic mecamylamine (oral), a combination of scopolamine and mecamylamine, or placebo. Scopolamine placebo was an 
injection of saline. Mecamylamine placebo consisted of identical capsules filled with lactose. A double placebo system was used such that on each day. These challenge day conditions were randomized among subjects and double blinded. Working memory performance was assessed during cholinergic challenge days through the use of the N-Back Task (NBT).

Results: d' was calculated for each condition of the NBT ( 0 , 1, 2, and 3 back). Repeated measures mixed-effects ANOVA was used to examine the effects SCD status, E2 treatment, task load, and challenge medication $(2 \times 2 \times 4 \times 4)$. As expected, there was a main effect of $\mathrm{N}$-Back task condition ( $\mathrm{F}$ $(3,288)=179.20, p<0.001)$ with decreasing mean d' with increasing working memory load. There was also a main effect of challenge medication $(\mathrm{F}(3,288)=40.26, p<0.001)$, with worse mean performance during scopolamine $(\mathrm{t}(35)=$ 3.48, $p<0.05)$ and combined scopolamine and mecamylamine $(\mathrm{t}(35)=5.77, p<0.01)$ compared to placebo. Separate (for each challenge medication) post hoc univariate mixedeffects ANOVAs for the effects of SCD status, E2 treatment, and task load on performance ( $2 \times 2 \times 14)$ were then performed. During the mecamylamine challenge there was a significant main effect of SCD status $(F(3,96)=92.74$, $p<0.001)$ and a significant interaction effect between SCD status and $\mathrm{E} 2$ treatment $(\mathrm{F}(3,96)=5.04, p<0.005)$. There were no significant differences between participant groups during placebo challenge. During mecamylamine challenge women with SCD who received E2 treatment had worse performance than women who did not receive E2 treatment $(\mathrm{t}(16)=-3.68, p<0.05:$ E2 d' mean $=2.13$, $\mathrm{SD}=0.14$; no $\mathrm{E} 2 \mathrm{~d}$ ' mean $=2.72, \mathrm{SD}=0.14)$. Women without SCD who received E2 treatment had better 3-back performance during mecamylamine challenge than women who did not receive estradiol $(\mathrm{t}(16)=2.45, p<0.05$ : $\mathrm{E} 2 \mathrm{~d}$ ' mean $=2.41 \mathrm{SD}=0.49$; no $\mathrm{E} 2 \mathrm{~d}$ ' mean $=1.82, \mathrm{SD}$ $=0.53$ ).

Conclusions: A significant beneficial effect was seen on working memory performance after chronic E2 treatment following nicotinic blockade, but only in women who had not experienced postmenopausal cognitive changes. No such beneficial effect was seen in women with postmenopausal SCD. We have hypothesized that women who notice significant changes in cognitive performance or cognitive effort after menopause may represent a higher risk group for later life cognitive dysfunction. These results suggest that the basis of such subjective dysfunction may be alterations in cholinergic system activity, specifically nicotinic receptor changes. Estradiol has salutary effects on cholinergic system functioning in normal postmenopausal women but may not in women with SCD. These results suggest that beneficial effects of the E2 administration on cholinergic-related cognitive functioning may be restricted to women without menopausal-related cognitive changes, consistent with the healthy cell bias of estrogen effect hypothesis (Brinton 2008), and point towards further needed investigation of women with postmenopausal SCD to further understand the underlying neurobiology and potential treatment or prevention of late-life cognitive impairment.

Keywords: Estrogen, Acetylcholine, Aging, Nicotinic.

Disclosure: Nothing to disclose.
T65. Association of Anxious-Depression Symptomatology With Neuro-Cognition Among MiddleAged and Older Hispanic/Latino Adults; Results From the Hispanic Community Health Study/Study of Latinos (HCHS/SOL)

Alvaro Camacho*, Wassim Tarraf, Daniel Jimenez, Linda Gallo, Robert Kaplan, Melissa Lamar, Martha Daviglus, Patricia Gonzalez, Sylvia Wassertheil-Smoller, Hector Gonzalez

University of California, San Diego, Imperial, California, United States

Background: Anxious-depression is a constellation of anxiety and depressive symptoms that do not meet criteria for a major depressive or an anxiety disorder1-4. We previously found a significant inverse association between depressive symptoms and verbal learning, word fluency and processing speed5. Therefore, we hypothesized an inverse association of moderate to severe anxious-depression with cognitive domains among Hispanics/Latinos.

Methods: This cross-sectional study included 9,357 Hispanic/Latino adults 45-74 years recruited from four HCHS/SOL centers (Bronx,NY; Chicago,IL; Miami,FL; San Diego,CA). An anxious-depression construct was derived from a latent class analysis of symptoms from the 10-item-Center for Epidemiological Studies for Depression (CESD-10) and the Spielberger State-Trait Anxiety Inventory (STAI) measures, defined as low, moderate and high as previously reported1. Cognitive measures included standardized scores on the Brief Spanish English Verbal Learning Test (B-SEVLT trials and recall), phonemic Word Fluency (WF), Digit Symbol Substitution (DSS) test, and a global cognitive score (GCS; average $\mathrm{z}$-score across the four cognitive tests). The associations between anxiousdepression and cognitive function were tested using survey linear regression models.

Results: Mean age was 56.4 years; among men 71\% reported low, 24\% moderate and 5\% high anxious-depression symptomatology. Among women 54.7\% reported low, $33.2 \%$ moderate and $12.2 \%$ high anxious-depression symptomatology, respectively. After controlling for age, sex, weight, sociodemographic, cardiovascular risk factors and antidepressant use, we found significant and consistent inverse associations between moderate and high anxiousdepression (ref:low) and B-SEVLT (3-trials: $\beta=-0.18$, $p<0.05 ; \beta=-0.40, p<0.05$ ) (recall: $\beta=-0.17, p<0.05 ; \beta=$ $0.45 ; p<0.05)$, DSS $(\beta=-0.19, p<0.05 ; \beta=-0.26, p<0.05)$ and GCS $(\beta=-0.16, p<0.05 ; \beta=-0.31, p<0.05)$. Moderate, but not high, anxious-depression was also inversely associated with WF $(\beta=-0.10, p<0.05)$.

Conclusions: Elevated anxious-depression symptomatology was associated with poorer neurocognitive function among middle-aged and older Hispanic/Latinos. Longitudinal studies are needed to establish if the association of anxiety and depression symptomatology with neurocognitive function changes overtime in order to guide clinical interventions.

Keywords: Anxiety State, Late-Life Depression, Neurocognition, Hispanic/Latinos.

Disclosure: Nothing to disclose. 
T66. Genome-Wide Mapping of Conditioned Fear in the Diversity Outbred Mouse Population

Clarissa Parker*, Daniel Gatti, Troy Wilcox, Elissa Chesler, Andrew Holmes

Middlebury College, Middlebury, Vermont, United States

Background: Mice offer a powerful tool for elucidating the genetic basis of behavioral and physiological traits relevant to anxiety disorders; yet conventional experimental crosses have only been able to identify large chromosomal regions rather than specific genes. Recent advances have led to genetically diverse, highly recombinant mouse populations. These characteristics allow for the opportunity to observe a wider range of phenotypic variation, offer greater mapping precision, and thus increase the potential for efficient gene identification.

Methods: We have taken advantage of the newly developed Diversity Outbred (DO) mouse population to identify and map narrow quantitative trait loci (QTL) associated with conditioned fear. We phenotyped 587 JAX Diversity Outbred mice (DO) for the acquisition, extinction, and renewal of conditioned fear using a three-day paradigm. We genotyped a subset of these mice at $\sim 150 \mathrm{k}$ markers across the genome and performed high precision QTL mapping using the R program DOQTL.

Results: A one-way repeated measures ANOVA found a significant increase in freezing following each tone-shock pairing during acquisition, (F 1.8, $892.8=799.5, p<0.0001$; $\eta \mathrm{p} 2=0.612$ ), demonstrating the ability to learn to associate the tone and foot-shock. Freezing behavior in response to the tone significantly decreased across trial-blocks during extinction training (F 6.2, 3619.4 = 145.6, $p<0.0001$; $\eta \mathrm{p} 2$ $=0.199$ ) suggesting mice were able to successfully extinguish the fearful association over time. On the renewal test, mice displayed less freezing relative to the first trial-block of extinction training $(\mathrm{t}(586)=13.7, p<0.0001)$. QTL analyses identified numerous suggestive and significant QTLs associated with conditioned fear on chromosomes $2,3,7$, and 12 . Importantly, we observed tremendous variation in all three traits which enables genetic mapping of naturally occurring genetic variation that is associated with trait variation.

Conclusions: With increased sample size to improve mapping power and resolution, and the inclusion of RNASeq and other molecular profiling we will be able to apply a systems genetic strategy to construct the network of correlations that exist between DNA sequence, gene expression values and anxiety-related phenotypes. This information can in turn be used to identify alleles that contribute to anxiety disorders in humans, elucidate causative biological mechanisms, or assist in the development of putative treatment strategies.

Keywords: GWAS, Fear Conditioning, Outbred Mice.

Disclosure: Nothing to disclose.
T67. IGFBP2 Produces Rapid-Acting and Long-Lasting Effects in Rat Models of Post-Traumatic Stress Disorder via a Novel Mechanism Associated With Structural Plasticity

Jeffrey Burgdorf*, Elizabeth Colechio, Nayereh Ghoreishi-Haack, Amanda Gross, Christopher Rex, Xiao-lei Zhang, Patric Stanton, Roger Kroes, Joseph Moskal

Northwestern University, Evanston, Illinois, United States

Background: Post traumatic stress disorder is an anxiety disorder characterized by deficits in the extinction of aversive memories. Insulin-like growth factor I (IGF1) is the only growth factor that has shown anxiolytic and antidepressant properties in human clinical trials. In animal studies, insulinlike growth factor binding protein 2 (IGFBP2) shows both IGF1-dependent and IGF1-independent pharmacological effects, and IGFBP2 expression is upregulated by roughand-tumble play which induces resilience to stress.

Methods: IGFBP2 was evaluated in Porsolt, contextual fear conditioning and chronic unpredictable stress models of PTSD. The dependence of IGFBP2 effects on IGF1- and AMPA-receptor activation was tested using selective receptor antagonists. Dendritic spine morphology was measured in the dentate gyrus and the medial prefrontal cortex $24 \mathrm{hrs}$ after in vivo dosing.

Results: IGFBP2 was 100 times more potent than IGF1 in the Porsolt test. Unlike IGF1, effects of IGFBP2 were not blocked by the IGF1-receptor antagonist JB1, or by the AMPAreceptor antagonist NBQX in the Porsolt test. IGFBP2 $(1 \mathrm{Eg} / \mathrm{kg})$ and IGF1 (100 Eg/kg IV) each facilitated contextual fear extinction and consolidation. Using a chronic unpredictable stress paradigm, IGFBP2 reversed stress-induced effects in the Porsoltnovelty induced hypophagia, sucrose preference, and ultrasonic vocalization assays. IGFBP2 increased mature dendritic spine densities in the medial prefrontal cortex and hippocampus 24 hrs post-dosing.

Conclusions: These data suggest that IGFBP2 has therapeutic-like effects in multiple rat models of PTSD via a novel IGF1 receptor-independent mechanism. These data also suggest that the long-lasting effects of IGFBP2 may be due to facilitation of structural plasticity at the dendritic spine level. IGFBP2 and mimetics may have therapeutic potential for the treatment of PTSD.

Keywords: PTSD, Insulin-Like Growth Factor 1, Dendritic Spines, Resilience.

Disclosure: Aptniyx: Consultant fee and stock, Self.

\section{T68. Sleep and HPA Axis Responses to Metyrapone in} Posttraumatic Stress Disorder

Sabra Inslicht*, Madhu Rao, Anne Richards, Aoife O'Donovan, Carolyn Gibson, Thomas Metzler, Thomas Neylan

University of California, San Francisco, San Francisco, California, United States

Background: Disturbed sleep is a core feature of posttraumatic stress disorder (PTSD). Patients often report non-restorative sleep, frequent awakenings, and nightmares. 
Objectively measured sleep disturbance, including decreased delta sleep, have also been observed. The decrease in delta sleep in PTSD may be related to stress-related alterations in corticotropin releasing factor (CRF) and the hypothalamicpituitary-adrenal axis. The aim of this study was to examine the neuroendocrine factors mediating sleep disturbances in patients with PTSD. Metyrapone, which blocks the enzymatic conversion of 11-deoxycortisol to cortisol and increases the release of hypothalamic CRF, was used as an experimental probe of these neuroendocrine factors. We predicted that metyrapone would result in an increase in ACTH and a decrease in delta sleep in PTSD subjects. Further, given the possible relationship between CRF and delta sleep, we predicted that the ACTH response to metyrapone would be associated with a decreased delta sleep response, and that this relationship differs in PTSD subjects vs. controls. A secondary aim was to examine sex differences in these relationships.

Methods: Sixty-six medically healthy medication-free men and women with chronic PTSD (16 Women, 17 Men; mean age $=29.6(5.3))$ and age- and sex-matched controls (14 Women, 19 Men; mean age $=30.3(8.4)$ ) participated in a study to examine the effects of PTSD status on sleep and ACTH responses to a metyrapone challenge. Participants were admitted into a General Clinical Research Center (GCRC) for 3 nights. PTSD status was determined using DSM-IV criteria for PTSD with the Clinician Administered PTSD Scale (CAPS) interview. Women participated during the follicular phase of their menstrual cycle, and female groups were blocked on the presence of menopause symptoms. On the second morning, subjects were given an oral dose of metyrapone $(750 \mathrm{mg})$ every 4 hours starting at 12 hours before habitual sleep onset, for a total of 3 doses, and one dose of $2.5 \mathrm{~g}$ at habitual sleep onset along with $30 \mathrm{ccs}$ of an antacid. Two hours before habitual sleep onset, a catheter was inserted in an antecubital vein for repeated sampling of blood on nights 2 \& 3 (5.5 ccs q 15 minutes providing 32 samples for ACTH (16- pre and 16- post metyrapone)). Samples were analyzed using radioimmunoassay, and delta sleep was quantified using visual electroencephalographic analysis. Regression analyses were conducted on baseline-adjusted change in delta sleep (log transformed) predicted from baseline-adjusted change in ACTH (log transformed), sex, PTSD status, and interactions of ACTH, PTSD status, and sex.

Results: Regression analyses revealed a significant relationship between baseline-adjusted change in ACTH and delta sleep that was negative in PTSD, but not in controls, B = $16.3(\mathrm{SE}=4.3), p<.001$ and $\mathrm{B}=4.4(\mathrm{SE}=4.9), p=.375$, respectively. The difference between slopes in PTSD was significant, $F(1,56)=10.11, p=.002$. The relationship between ACTH and delta sleep was negative in males but not in females: $\mathrm{B}=-13.0(\mathrm{SE}=5.2), p=.016$ and $\mathrm{B}=2.5$ ( $\mathrm{SE}$ $=3.4), p=.473$, respectively. The difference in slopes in males vs. females was significant: $\mathrm{F}(1,56)=6.17, p=.016$. There was no ACTH by sex by PTSD interaction, $\mathrm{F}(1,56)=$ $0.45, p=.515$.

Conclusions: Our findings suggest that PTSD status moderates the association between ACTH and delta sleep response to metyrapone. We previously found that the ACTH response to metyrapone was higher in PTSD subjects compared to controls in plasma sampled immediately and at intervals over 8 hours. Previous studies have shown that elevations in both hypothalamic (neurohormonal) and extrahypothalamic (neurotransmitter) CRF release are associated with decreased delta sleep activity. There is evidence of elevated CSF corticotrophin-releasing hormone in PTSD, and a link between single nucleotide polymorphisms of the CRF type 1 receptor gene with PTSD. Decreased delta sleep in PTSD could be related to changes in pituitary ACTH or adrenal cortisol release. The increase in PVN CRF may also affect other brain areas involved in sleep or arousal, such as through projections to the locus ceruleus. Sex-specific effects may result from modulation by reproductive hormones. An implication of these findings is that CRF antagonists may increase delta sleep in stressed subjects. Further studies are needed to address whether hormones that are affected by metyrapone (i.e., progesterone) may modulate these observed sleep changes.

Keywords: PTSD, Sleep, Metyrapone, Sex Differences. Disclosure: Nothing to disclose.

\section{T69. Corticotropin Releasing Factor Regulation of Forebrain Cholinergic Nuclei Impairs Attention and Learning in Rats}

Debra Bangasser*, Kimberly Wiersielis, Brittany Wicks, Madeleine Salvatore, Sarah Cohen, Attilio Ceretti, Joy Bergmann, Nina Duncan, Hanna Lefebo

Temple University, Philadelphia, Pennsylvania, United States

Background: Stress can impair a variety of cognitive processes, including attention, learning, and memory. Previous studies in rodents have demonstrated that often these disruptions in cognition can be mimicked by a central infusion of the stress-neuropeptide, corticotropin releasing factor (CRF). However, where CRF is working within the brain to regulate attention and memory is largely underexplored. Candidate regions for direct CRF regulation include the nucleus basalis of Meynet (nbM) and medial septum (MS), because these forebrain cholinergic nuclei are critical for attention and mnemonic processes, respectively, and both contain CRF receptors. Here we begin to assess whether administering CRF directly into these regions impairs cognition in rats.

Methods: The first study examined the effect of CRF in the $\mathrm{nbM}$ on attention. To this end, adult male and female rats were trained on a touchscreen, operant, sustained attention task in which they had to discriminate visual signals from non-signaled events. After attaining criterion $(70 \%$ correct responses on signal and non-signal trials), they were surgically implanted with cannulas into the nbM. Following recovery and reacquisition of baseline, one of two doses of CRF (30ng, 100ng) or vehicle (artificial cerebrospinal fluid, $\mathrm{aCSF}$ ) were administered directly into the $\mathrm{nbM} 10$-min prior to the task onset. The doses were administered in a counterbalanced fashion using a within-subjects design (successive infusions were separated by at least a week). The second study used an object location task to assess the effect of CRF in the MS on spatial memory, which is modulated by the MS cholinergic projection to the hippocampus. Additionally, hippocampal-independent 
memory was tested using the novel object recognition task. Specifically, male and female rats with cannulas implanted in the MS, received one of two doses of CRF (30ng, 100ng) or aCSF $10 \mathrm{~min}$ prior to the administration of the object location task (where one of two familiar objects was moved to a new location), or the novel object recognition task (where one of two familiar objects was replaced with an unfamiliar object).

Results: For the attention task, there was a sex $\times$ drug interaction for the vigilance index, a measure of overall attentional performance $[\mathrm{F}(2,16)=5.22, p=.037]$, with the high dose of CRF impairing attention in male $(p=.002)$ but not female $(p=.179)$ rats. For the memory tasks, there was an effect of CRF in the MS on the object location task $[\mathrm{F}(2,29)=$ 5.22, $p=.012]$, such that the low and high doses of CRF impaired spatial memory in both sexes (ps $<.05$ compared to aCSF). In contrast, there was no effect of CRF in the MS on object recognition memory $[\mathrm{F}(2,28)=2.64, p=.09]$.

Conclusions: The first study revealed that CRF in the nbM impairs sustained attention in male but not female rats, suggesting that attention in males would be more susceptible to disruption by stressors that increase CRF in the basal forebrain. The second study revealed that CRF in the MS impaired spatial memory in both males and females, but not recognition memory. Given the dense cholinergic projections of the MS to the hippocampus, it was not surprising that CRF in the MS impaired hippocampal-dependent memory, while leaving hippocampal-independent memory unaffected. Together, the studies reveal that direct infusions of CRF into forebrain cholinergic nuclei can impair attention and memory, but that these effects, at times, are sex-specific. $\mathrm{CRF}$ regulation of the cholinergic system may be an important, yet unexplored, mechanism by which stress can regulate cognition. Finally, these findings suggest that drugs that block the effects of CRF could represent a viable therapeutic option to treat cognitive impairments during times of stress.

Keywords: Attention, Memory and Learning, Sex Difference, Acetylcholine, Corticotropin-Releasing Factor (CRF).

Disclosure: Nothing to disclose.

\section{T70. Forebrain Glutamatergic, but Not GABAergic, Neurons Mediate Anxiogenic Effects of the Glucocorticoid Receptor}

Jakob Hartmann*, Nina Dedic, Max L Pöhlmann, Alexander Häusl, Henk Karst, Clara Engelhardt, Sören Westerholz, Klaus V Wagner, Christiana Labermaier, Lianne Hoeijmakers, Maurice Kertokarijo, Alon Chen, Marian Joëls, Jan M Deussing, Mathias V Schmidt

Harvard Medical School, Belmont, Massachusetts, United States

Background: Anxiety disorders constitute a major disease and social burden worldwide, however many questions concerning the underlying molecular mechanisms still remain open. Besides the involvement of the major excitatory (glutamate) and inhibitory (GABA) neurotransmitter circuits in anxiety disorders, the stress system has been directly implicated in the pathophysiology of these complex mental illnesses. The glucocorticoid receptor (GR) is the major receptor for the stress hormone cortisol (corticosterone in rodents) and is widely expressed in excitatory and inhibitory neurons, as well as in glial cells. However, currently it is unknown which of these cell populations mediate GR-actions that eventually regulate fear and anxiety-related behaviors.

Methods: In order to address this question, we generated mice lacking the receptor specifically in forebrain glutamatergic (GRGlu-CKO mice) or GABAergic (GRGABA-CKO mice) neurons by breeding GRflox/flox mice to Nex-Cremice or Dlx5/6-Cre-mice, respectively. We assessed neuroendocrine and physiological parameters in both mouse lines, as well as fear and anxiety-related behavior using, classical fear conditioning, open field, dark-light and elevated plus maze tests. Miniature excitatory postsynaptic currents were recorded in BLA neurons of GRGlu-CKO mice and littermate controls. In addition, we investigated the specific contribution of the GR in glutamatergic BLA neurons to fear and anxiety-related phenotypes via AAV-mediated knockout of the receptor in GRflox/flox mice.

Results: GR deletion specifically in glutamatergic, but not in GABAergic neurons induced hypothalamic-pituitary-adrenal axis hyperactivity, and reduced fear and anxiety-related behavior. This was paralleled by reduced GR-dependent electrophysiological responses in the basolateral amygdala. Importantly, viral-mediated GR deletion additionally showed that fear expression, but not anxiety, is regulated by GRs in glutamatergic neurons of the BLA.

Conclusions: Our findings suggest that pathological anxiety likely results from altered GR signaling in glutamatergic circuits of several forebrain regions, while modulation of fear-related behavior can largely be ascribed to GR signaling in glutamatergic neurons of the BLA. Collectively, our results reveal a major contribution of GRs in the brain's key excitatory, but not inhibitory neurotransmitter system in the regulation of fear and anxiety behaviors, which is crucial to our understanding of the molecular mechanisms underlying anxiety disorders.

Keywords: Glucocorticoid Receptor, Anxiety, Glutamate, GABA, Amygdala.

Disclosure: Nothing to disclose.

\section{T71. Skin Conductance Response as a Marker of Conditionability: Do Poor Conditioners' Brains Respond Differently During Fear Conditioning?}

Marie-France Marin*, Florentine Barbey,

Blake L Rosenbaum, Scott P Orr, Mohammed R Milad

Harvard Medical School, Montreal, Canada

Background: Fear conditioning and extinction paradigms have been used to study the biological mechanisms underlying threat and safety learning. Skin conductance response (SCR) is a measure that has been widely used as an index of conditioned fear. SC reactivity is highly variable across individuals and various demographic and personality variables have been shown to influence its conditioning. Data from subjects who exhibit very small SCRs are commonly excluded from analysis. Small SCR may be blamed on technical issues such as faulty electrodes or connections. However, this very low SC reactivity may result from non- 
technical factors and convey important information about the biological mechanism underlying individual differences in SCR conditioning. The present study examined the neural correlates associated with SCR conditionability.

Methods: Data from 109 healthy adults aged between 18 and 60 years were pooled. All subjects participated in a validated 2-day fear conditioning and extinction protocol. During conditioning (on Day 1), subjects were exposed to three colored lamps that served as conditioned stimuli (CSs). Of these three cues, two were partially reinforced (CS+), i.e. paired with an electrical shock, and one was never reinforced (CS-). The procedure took part in a functional magnetic resonance imaging (fMRI) environment, during which blood-oxygenated-level-dependent (BOLD) signal and SCR were measured. Subjects were ranked as a function of the magnitude of their SCR to the threat cues. Three groups were created based on that ranking: High Conditioners (largest SCRs to the threat cues), Medium Conditioners (medium SCR to the threat cue), and Low Conditioners (smallest SCR to the threat cue). The neuroimaging analysis of interest during fear conditioning was to contrast the CS+ trials with the CS- trials. Based on the fear conditioning literature, the following regions of interest were examined: amygdala, hippocampus, insular cortex, dorsal anterior cingulate cortex (dACC) and ventromedial prefrontal cortex (vmPFC).

Results: The three groups did not differ statistically in terms of sex distribution, age, years of education, and ethnicity. Moreover, all three groups selected an equivalent level of shock. Importantly, magnitudes of the SCRs that were elicited by the shock (unconditioned response) did not differ between the groups. Examination of the CS+ vs. CS- contrast during fear conditioning yielded significant Group main effects for dACC and amygdala. The extracted beta-weights revealed that the Low Conditioners group showed significantly less activation in both of these regions, compared to the Medium and High Conditioners groups.

Conclusions: Our findings suggest that low SCR conditionability has a distinct neural signature, with hypoactivation of brain regions known to promote fear learning and expression. The hypoactivations are not attributable to differences on demographic and personality factors known to influence SCR conditioning or to the magnitude of the unconditioned response, as the groups were comparable across these variables. Our findings suggest that low SCR conditionability likely reflects an individual difference that has a discernable neurobiological basis. This highlights the need to be cautious when excluding SCR non-conditioners and to consider the potential implications of such exclusion when interpreting the findings from studies of conditioned fear.

Keywords: Skin Conductance Responses, fMRI, Fear Conditioning, Amygdala, dACC.

Disclosure: Nothing to disclose.

\section{T72. Endocannabinoid Mechanisms Promoting Resilience to Acute Traumatic Stress}

Rebecca Bluett, Baldi Rita, Sachin Patel*

Vanderbilt University, Nashville, Tennessee, United States

Background: Stress is a ubiquitous risk factor for the exacerbation and development of affective disorders including major depression and posttraumatic stress disorder, however, stress-resilience is protective against the development of psychopathology. Understanding the neurobiological mechanisms conferring resilience to the adverse consequences of stress could have broad implications for the treatment and prevention of mood and anxiety disorders. Anxiety and tension reduction are highly cited motives for chronic cannabis use, and cannabis use is highly co-morbid with stress-related psychiatric disorders including posttraumatic stress disorder. However, neither the biological basis for this co-morbidity, nor the role of endogenous cannabinoid signaling in the regulation of intra-individual responses to stress are well understood.

Methods: We developed and validated a modified noveltyinduced hypophagia assay, combined with tradition cue-fear conditioning, to detect individual differences in the susceptibility to develop generalized anxiety responses to traumatic stress exposure. We utilized behavioral pharmacology, novel genetic approaches, and ex vivo optogenetic electrophysiology to test the role of the endogenous cannabinoid, 2 -arachidonoylglycerol (2-AG), in promoting resiliency to traumatic stress exposure.

Results: Acute pharmacological 2-AG augmentation (and acute THC treatment) promoted the expression of a stressresilient phenotype, and enhanced resilience in previously susceptible mice. In contrast, $\mathrm{CB} 1$ receptor blockade prevented the resiliency promoting effects of 2-AG augmentation and increased stress-induced generalized anxiety in previously resilient mice. Moreover, amygdala-specific 2-AG depletion, using conditional genetic approaches, impaired successful adaptation to repeated stress. Mechanistically, stress-resilience was associated with increased phasic, but not tonic, 2-AG-mediated retrograde synaptic suppression at hippocampal-amygdala glutamatergic synapses.

Conclusions: Our data indicate amygdala 2-AG signaling mechanisms promote resilience to adverse effects of acute traumatic stress and facilitate adaptation to repeated stress exposure. Based on our work, we propose 2-AG deficiency states represent a stress-susceptibility endophenotype predisposing to the development of stress-related neuropsychiatric disorders, and that such a state could partially explain the high rates of cannabis use in patients with anxiety disorders. Our data suggest pharmacological augmentation of 2-AG signaling could represent a novel treatment approach for stress-related psychiatric disorders and co-morbid cannabis use disorders in self-medicating patients.

Keywords: Endocannabinoids, Acute Stress, PTSD.

Disclosure: Nothing to disclose.

\section{T73. Placebo Effects in a PTSD Trial of a CRH Antagonist: Not Limited to Clinical Measures}

Gabrielle Hodgins*, Jared Blommel, Boadie Dunlop, Dan Iosifescu, Sanjay Mathew, Thomas Neylan, Charles Nemeroff, Philip Harvey

University of Miami Miller School of Medicine, Miami, Florida, United States

Background: In order to receive FDA approval, generally a treatment must be more efficacious than a placebo. For example, over the past few years, trials for multiple drugs, 
including selective serotonin reuptake inhibitors (SSRIs) failed due to increased placebo response. Not only has the placebo effect always been a significant presence in trials, but seems to be increasing within patient populations. These increased placebo responses appear to be present for clinician rated and self-reported measures. The present study is based on data collected from participants in a trial for a novel drug treatment for post-traumatic stress disorder (PTSD). The body of literature suggests that PTSD, amongst other anxiety related illness, might be associated with chronically increased activity of corticotropin releasing hormone $(\mathrm{CRH})$. This trial examined the effects of a $\mathrm{CRH}$ antagonist and examined self-reported, clinically-rated, and performance based assessments.

Methods: Women with chronic PTSD were randomized to six weeks of double-blind treatment with either GSK561679 or placebo in a 1:1 manner. Subjects completed clinical and functional assessments prior to beginning the trial and these were repeated after five weeks on the medication to evaluate clinical change. The clinician-based measures used in the trial included the Clinician-Administered PTSD Scale (CAPS) and the Montgomery-Asberg Depression Rating Scale (MADRS). Self-reported PTSD symptoms were examined with the PTSD Symptom Scale, Self-Report (PSS-SR), self-reported depression severity was assessed with using the Quick Inventory of Depressive Symptoms, Self-Report (QIDS-SR), and self-reported disability was examined with the Sheehan Disability Scale (SDS). An infrequency scale was also administered as a component of the PSS-SR. Finally, to assess neuropsychological impairment and performancebased functional capacity, the MATRICS Consensus Cognitive Battery (MCCB) and UCSD Performance-Based Skills Assessment (UPSA) were administered.

Results: Drug GSK561679 failed to produce any significant improvement in the participants of the trial, in that active treatment and placebo did not separate on any measures. There was a substantial placebo effect seen in self-report and clinical measures. The effect sizes for placebo effects were greater than 1.5 SD across the self-report and clinical measures. For neuropsychological test performance, the effect size for change was $0.5 \mathrm{SD}$, while for the UPSA there was a minimal change of 0.1 SD. Analyses found that the clinical change scores were more highly intercorrelated with each other than with changes in the MCCB. No single variable predicted placebo-related changes, with baseline scores on each clinical variable consistently being the largest predictor of placebo related change. Despite the smaller effect size for changes in cognitive performance, this is a larger retest associated change than previous reports with the MCCB, including healthy individuals and patients with severe mental illness. In fact, $25 \%$ of the participants improved by more than $0.5 \mathrm{SD}$ on the MCCB, a level of change that would be viewed as clinically significant if actually induced by treatment. In contrast, less than $10 \%$ of participants had scores at retest that were worse than baseline and no patient worsened by $0.5 \mathrm{SD}$ or more.

Conclusions: The placebo effect encountered in this PTSD drug trial was substantial, with large changes in clinical symptoms and some participants manifesting an increase in cognitive performance equivalent to over 10 IQ points. Thus, using performance-based assessments does not obviate placebo effects, although these changes were small in comparison to the clinical symptom changes. The PTSD population has fewer pharmacological treatment options than other mental illnesses, and it is likely that patients are desperate for further treatment modalities to be available and to be able to participate in research. The women participating in the trial manifested particularly substantial retest effects. One possibility for this finding is that they had minimal previous experience with performance-based psychological assessments and taking the test in the first week of the trial lead to a substantial increase in familiarity and comfort with the second assessment. This may be unique amongst drug trials aimed at cognition, where in many other conditions participants have been exposed to the tests before, thus diminishing the novelty and exposure effects. Schizophrenia patients have many trials to participate in; PTSD patients have few to none. Although it must be considered that the patients were not exerting adequate effort on the initial round of tests, the baseline performance-based scores correlated with lifetime functional status, suggesting that the participants were exerting their best effort on the initial UPSA and MCCB. These findings have implications for future studies using completely novel performance based assessment paradigms, such as would be expected in the rDOCS initiative. If lack of previous experience with assessment is associated with increased retest effects, such completely novel paradigms might be particularly vulnerable to these outcomes.

Keywords: Placebo Response, PTSD, Placebo-Controlled Trial, Randomized Clinical Trial.

Disclosure: Nothing to disclose.

\section{T74. Inflammation in the Neurocircuitry of Obsessive Compulsive Disorder}

Sophia Attwells*, Elaine Setiawan, Alan A Wilson, Pablo M Rusjan, Romina Mizrahi, Laura Miler, Sarita Sharma, Margaret Anne Richter, Sylvain Houle, Jeffrey Meyer

\section{Centre for Addiction and Mental Health, Toronto,} Canada

Background: An autoimmune model within the basal ganglia was proposed for a small percentage of obsessive compulsive disorder (OCD) cases after exposure to Group A Beta-Hemolytic Streptococcus. It is possible that elevated neuroinflammation, which may be induced by a more diverse range of mechanisms, could be broadly important in OCD. Microglial activation, a key component of neuroinflammation, may be measured with positron emission tomography (PET) imaging applying the new advance in radioligands binding to the translocator protein (TSPO). TSPO distribution volume (TSPO VT), an index of TSPO level, is increased when microglia are activated. The primary aim of the present study is to determine if TPSO VT is elevated within the dorsal caudate, orbitofrontal cortex, thalamus, anterior cingulate cortex, and ventral striatum in OCD.

Methods: Eighteen OCD subjects and 18 healthy controls were recruited. All were drug and medication free, were nonsmoking and had no additional psychiatric or medical illnesses. Cases and controls were matched for age. [18F] 
FEPPA PET was applied to measure TSPO VT in the dorsal caudate, orbitofrontal cortex, anterior cingulate cortex, thalamus, and ventral striatum. Participants were also genotyped for the rs6971 polymorphism which influences binding of [18F] FEPPA (and all second generation PET radiotracers) to TSPO.

Results: TSPO VT was elevated in OCD subjects in the dorsal caudate, orbitofrontal cortex, thalamus, anterior cingulate cortex, and ventral striatum by $33.1 \%, 25.5 \%$, $31.4 \%, 21.8 \%$, and $32.7 \%$ respectively (multivariate analysis of variance: effect of OCD versus health: F5,28 $=5.6, P=$ .001 ; effect of genotype: F5,28 = 11.6, $P<.001$ ).

Conclusions: The best explanation for the elevation in TSPO $\mathrm{VT}$ is that it represents microglial activation. This is the first study to find strong evidence of neuroinflammation in OCD. Whether microglial activation is helpful or harmful in OCD will be an important direction for future study as it is plausible that microglial activation represents a new immunomodulatory target in OCD.

Keywords: Obsessive Compulsive Disorder, TSPO VT, Positron Emission Tomography Imaging, [18F]-FEPPA.

Disclosure: Nothing to disclose.

\section{T75. Temporal Dynamics and Dose Dependency of Intranasal Oxytocin Effects on the Humanamygdala Response to Emotional Faces}

Rene Hurlemann*, Franny Spengler, Johannes Schultz, Wolfgang Maier, Dirk Scheele

University of Bonn, Bonn, Germany

Background: There is broad interest in developing the neuropeptide oxytocin (OXT) into a treatment for anxiety and social deficits. Most studies in humans have assessed behavioral and neural effects of intranasal OXT (IN-OXT) with a standard single dose of 24 IU and a latency of $45 \mathrm{~min}$. The objective of the present study was to determine whether IN-OXT effects on blood oxygenation level-dependent functional magnetic resonance imaging (BOLD fMRI) activation in the amygdala vary as a function of dose and latency.

Methods: In this double-blind, placebo-controlled, randomized study, 104 healthy men underwent imaging after receiving 12, 24 or 48 IU of IN-OXT or placebo. Data were acquired at 15-40, 45-70 and 75-100 min after IN-OXT administration. During fMRI, subjects completed an emotion face assessment task.

Results: Our results show that the IN-OXT-induced suppression of fear-evoked responses in the amygdala was strongest in a time window between 45 and $70 \mathrm{~min}$ after administration of a 24 IU dose.

Conclusions: Our findings confirm the widely established protocol of IN-OXT administration (24 IU and $45 \mathrm{~min}$ latency) in humans and may help to support the development of IN-OXT into an anxiolytic medication.

Keywords: Oxytocin, Amygdala, fMRI.

Disclosure: Nothing to disclose.
T76. Acute Exercise Improves Working Memory While Decreasing Anxiety in Humans

Tiffany Lago*, Abigail Hsiung, Brooks Leitner, Courtney Duckworth, Kong Chen, Christian Grillon, Monique Ernst

National Institute of Mental Health, Bethesda, Maryland, United States

Background: Exercise reduces anxiety and sharpens cognitive function (for review DeBoer et al, 2012; Chang et al, 2015; Ensari et al, 2015), two effects that may be functionally related. To our knowledge, no studies have yet examined how exercise modulates cognition and anxiety in the same subjects. A better understanding of the mechanisms underlying these modulatory effects could help generate insights into the nature of anxiety-cognition interactions, but also into potential novel avenues for treatment. Interactions of anxiety with cognitive function have been amply demonstrated. While anxiety can impair cognition, cognitive processes can also decrease anxiety (Vytal et al, 2012). Working memory (WM) tasks are especially efficient at reducing anxiety, although the mechanisms are unclear. This study examines the modulatory effects of exercise on the interactions between anxiety and WM performance, through orthogonal manipulations of all three factors (exercise, anxiety and cognition) in a within-subject design. Exercise was executed at two levels of difficulty, moderate and light (30 min biking at $60-70 \%$ vs. $10-20 \%$ of maximum exertion). Task comprised 3 levels of difficulty. Anxiety was experimentally manipulated through anticipation of unpredictable electrical shocks. Compared to light exercise, moderate exercise was expected to improve WM performance, while decreasing self-reported anxiety and physiological measures (anxiety-potentiated startle) of anxiety.

Methods: At this point of this ongoing study, 15 healthy volunteers completed 3 outpatient visits 5-8 days apart. The first visit consisted of a VO2 max test to assess fitness and determine target heart rate of exercise. The next two visits consisted of the completion of a moderate or light exercise for $30 \mathrm{~min}$ (including warm-up and cool-down) followed 30 min later by a WM task performance tasks (n-back task) under shock threat and safe conditions. Exercise visits were counterbalanced. WM performance, subjective anxiety, and startle variables were analyzed using 3-way ANOVAs, with Condition (threat, safety), Load (0-back, 1-back, 3-back), and Exercise (light, moderate) as within-subject factors.

Results: Regardless of exercise level, participants exhibited worse cognitive performance at high vs. low load. Moderate vs. light exercise speeded up reaction time $(\mathrm{F}(1,13)=5.02$, $p<.05)$. This performance improvement was most beneficial for the high-load WM level (3-back) $(F(1,13)=7.07, p<.05)$. Finally, subjective anxiety was significantly lower in the moderate vs. light exercise session $(\mathrm{F}(1,12)=5.34, p<.05)$, especially during threat, accounting for the significant interaction of condition $\mathrm{x}$ exercise $(\mathrm{F}(1,12)=4.97, p<.05)$. Anxiety-potentiated startle results did not reach statistical significance.

Conclusions: The present findings support the beneficial effects of moderate (vs. light) exercise on anxiety self-reports to threat. The 3-back task was the cognitive load that was the most sensitive to the effects of moderate exercise, suggesting 
a stronger blockade of the effect of threat on the most difficult level of cognitive performance. If this pattern strengthens with the full sample $(n=40)$, the next steps will be to (1) move to a patient population, and (2) apply this protocol to a neuroimaging study.

Keywords: Anxiety, Working Memory, Exercise.

Disclosure: Nothing to disclose.

\section{T77. Resting State Functional Connectivity of the BNST vs. Amygdala Cea at Ultra-High Field Imaging}

\section{Monique Ernst*, Adam Gorka, Salvatore Torrisi,} Christian Grillon

National Institute of Mental Health, Bethesda, Maryland, United States

Background: The neural substrates of defensive responses to threat consist of widely distributed networks that comprise two main circuitries, one that subserves fear or the response to imminent proximal threat, and one that subserves anxiety, or the response to uncertain distal threat (Davis et al, 2009). These circuitries are partly overlapping and partly distinct. Although much progress has been made in understanding the mechanisms underlying defensive behaviors in animals, how these circuitries in humans differ from one another, and, particularly how they are differentially modulated, remains unclear. A critical obstacle to addressing this question has been the difficulty of imaging small structures, e.g., those identified as key nodes in the coding of fear (amygdala sub-nuclei) and anxiety (bed nucleus of the stria terminalis [BNST]). The advent of ultra-high field 7T-fMRI can help circumvent this limitation. Accordingly, we recently used 7T fMRI to examine the BNST intrinsic functional connectivity (iFC) (Torrisi et al, 2015) and found that the BNST was strongly coupled with the CeA, which motivated the present study. Here, we use 7T-fMRI to compare wholebrain iFC of the BNST with that of the central nucleus of the amygdala (CeA).

Methods: 27 healthy adults completed a 10 min resting state fMRI study on a 7T Siemens Magnetom MRI with a 32channel head coil. Participants passively viewed a white fixation cross on a black screen $(\mathrm{TR}=2.5 \mathrm{~s}, 1.3 \mathrm{~mm}$ isotropic voxels). High resolution $(0.7 \mathrm{~mm}$ isotropic) structural T1weighted MPRAGE images were collected for the handtracing of the BNST masks. The CeA mask was derived from Nacewicz et al, 2006. Preprocessing and analyses were conducted on AFNI (ANATICOR, Jo et al. 2010). FreeSurfer was used for MPRAGE image segmentation. Non-linear normalization into MNI space was employed, and the resulting parameters were applied to the functional data. Importantly, timecourses from ROIs were extracted before smoothing. Nuisance variance was removed from both functional images and ROI timecourses. A $2.6 \mathrm{~mm}$ FWHM Gaussian kernel was applied for smoothing functional images, and the residual timecourses were entered into a multiple regression model at the single subject level (3dDeconvolve). Group-level paired $\mathrm{T}$ tests were used to analyze the brain regions where the intrinsic connectivity of the BNST significantly differed from that of the CeA. Corrected threshold (3dClustSim) was set at $p<0.0001, \mathrm{k}=15$.
Results: Whole brain iFC maps of the BNST and CeA were consistent with prior reports demonstrating mostly positive coupling across cortical and subcortical regions. The novel findings consisted of the iFC differences between the BNST and CeA maps. Two broad significant patterns emerged from this analysis: (1) The CeA, vs. the BNST, was more strongly coupled with the dorsal anterior cingulate cortex, anterior insula, thalamus, hippocampus, and areas of sensory cortex. (2) The BNST, vs. the CeA, was more strongly coupled with the caudate nucleus.

Conclusions: The CeA exhibited relatively stronger coupling with neural regions that are involved in information gating (thalamus), conflict monitoring, salience coding and interoceptive awareness (dorsal anterior cingulate cortex/anterior insula), and sensory perception (auditory and visual cortex). This CeA iFC pattern fits well with the prominent impact of fear on motor responses. Relative to the CeA, the BNST showed stronger coupling with the caudate nucleus, an important structure for action-outcome learning. Abnormalities in striatal function have been noted in patients with anxiety disorders as well as individuals at higher risk for developing clinical anxiety. The stronger coupling of the $\mathrm{BNST}$ with the caudate nucleus, relative to the $\mathrm{CeA}$, might suggest a specific contribution of the BNST to the striatal hypersensitivity demonstrated in clinical anxiety. Given the role of dopamine in obsessive compulsive behavior and in drug addiction, the iFC patterns identified herein might also inform the compulsive symptoms observed in certain anxiety disorders and the behaviors that maintain drug addiction. Replication and extension of these findings to functional mapping of task-related processes using ultra-high field 7T fMRI methodology are warranted to further elucidate the differential role of these two key structures (CeA and BNST) in defensive behaviors.

Keywords: Resting State Functional Connectivity, Bed Nucleus of the Stria Terminalis, Amygdala, Anxiety Circuitry.

Disclosure: Nothing to disclose.

\section{T78. Effect of a Novel NMDA Receptor Modulator, Rapastinel (Formerly GLYX-13) in OCD: Proof-of- Concept}

Carolyn Rodriguez ${ }^{\star}$, Jordana Zwerling, Eyal

Kalanthroff, Hanyang Shen, Maria Filippou, Booil Jo, Helen Simpson, Ronald Burch, Joseph Moskal

Stanford University School of Medicine, Stanford, California, United States

Background: A single intravenous dose of ketamine, a $\mathrm{N}$-methlyl D-aspartate receptor (NMDAR) full antagonist, produces robust and rapid anti-obsessional effects in obsessive-compulsive disorder (OCD), but ketamine's side effects, including dissociation and nausea, may limit clinical use. Rapastinal (formerly GLYX-13), a putative NMDAR functional glycine-site partial agonist, has shown rapid antidepressant activity without ketamine-like side effects, and may be a new therapeutic strategy for OCD. We conducted the first test of the tolerability and potential efficacy of rapastinel administration in OCD. Specifically, we explored the drug's acute effects on obsessive-compulsive symptoms, 
depression and anxiety at 90 and 230 minutes post-infusion and at one-week post-infusion.

Methods: With IRB approval, seven unmedicated OCD outpatients (aged 18-55) were recruited (March, 2014 March, 2015) and provided written informed consent. Patients met criteria for OCD (both DSM-IV and DSM-5) with at least moderate symptoms (Yale-Brown ObsessiveCompulsive Scale [Y-BOCS] score $\geq 16$ ). Exclusion criteria included severe depression (Hamilton Depression Rating Scale [HDRS] >25), current cognitive behavioral therapy, and other comorbid psychiatric or medical conditions that made participation unsafe.

Patients $(n=7)$ received a single 3-5 minute IV push of rapastinel (dose $=10 \mathrm{mg} / \mathrm{kg}$ ).

At baseline, 90, and 230 minutes post-infusion, patients selfrated the severity of their obsessions and compulsions ( $\mathrm{Y}$ BOCS Challenge Scale [YBOCCS], a 10 item self-report form that assesses OCD symptoms [i.e., time spent, degree of control, severity] over the previous 60 minutes [range 0-40]), anxiety (Beck Anxiety Inventory [BAI]), and depression (Beck Depression Inventory [BDI]). Side effects of dissociation, mania, and psychosis were assessed at baseline, 90, and 230 minutes post-infusion. At baseline and one-week postinfusion, an independent evaluator, blind to study design, evaluated patients using the Y-BOCS, which appraises obsessive and compulsive symptoms over the prior week, and patients self-rated anxiety (BAI) and depression (BDI). Treatment response was defined a priori as $\geq 35 \%$ Y-BOCS reduction. Outcomes were analyzed using a non-parametric Wilcoxon signed-rank matched-pairs test $(\alpha=.05$, twotailed) without adjustment for multiple comparisons given the exploratory nature of this study.

Results: All seven patients who received rapastinel completed the infusion, which was well tolerated and without adverse events. Assessments of dissociation, mania, and psychosis were not significantly changed from baseline. Compared to baseline, YBOCCS, BAI, and BDI scores were significantly lower at 90 and 230 minutes post-infusion (all $p$ values $<.05)$. From baseline to one-week post-infusion, OCD severity, as measured by the Y-BOCS, was not significantly decreased $(p=.20)$, BDI was not significantly decreased $(p=.20)$, although BAI was significantly decreased $(p=.02)$. No patient met the treatment response criterion ( $\geq 35 \%$ Y-BOCS reduction) at one-week postinfusion.

Conclusions: The findings suggest that rapastinel is well tolerated in unmedicated OCD patients, as it is in patients with depression. Specifically, it did not increase psychotomimetic effects following dosing in this open-label sample of OCD patients, unlike ketamine in prior studies. In this small sample, rapastinel had acute effects on obsessions and compulsions, anxiety and depression. However, rapastinel did not have significant effects on OCD symptoms one-week post-infusion. To have clinical utility, glutamate modulators should refine molecular targets for rapid and sustained action while minimizing side effects. Mechanistic preclinical data suggests drugs that act on AMPA receptor modulation pathways like rapastinel and ketamine's metabolite hydroxynorketamine may be promising therapeutic strategies.

Keywords: OCD, Glutamate, Human Clinical Trial, GLYX-13, Depression.

Disclosure: Nothing to disclose.
T79. Obstructive Sleep Apnea Syndrome Impacts Dentate Gyrus and Neurocognitive Development in Youth

Jiook Cha*, Johanna Zeahernandez, Sanghun Sin, Katharina Graw-Panzer, Eileen Moran, Mark Wagshul, Jonathan Posner, Molly Zimmerman, Raanan Arens

Columbia University, New York, New York, United States

Background: Obstructive sleep apnea syndrome (OSAS) is a respiratory disorder that manifest in all age groups from early infancy to adulthood. OSAS causes detrimental longterm effects such as hypertension, metabolic derangements of glucose intolerance, and neurological impairments. Impact of OSAS may be particularly damaging to a child's brain because disrupted neurodevelopment is hardly reversible after a critical period. The dentate gyrus in the hippocampus may be the most important affected region with respect to neurocognitive development, because it is a central region not only for learning and memory, but also for neurogenesis. However, to date, few studies have tested the impact of OSAS on the dentate gyrus or the hippocampus in youth. In this study, we tested the hypothesis that the influence of OSAS on the dentate gyrus microstructure mediates the effects of childhood OSAS on cognitive development.

Methods: 12 youths with OSAS and 12 age- and sex-matched control youths without OSAS were recruited. Polysomnography was conducted to diagnose OSAS using standard criteria. Once a diagnosis was made, subjects underwent magnetic resonance imaging (MRI) sessions. To assess microstructure and macrostructure of the dentate gyrus, we measured diffusion anisotropy and volumes, respectively, using diffusion tensor imaging and T1-weighted structural MRI. To then segment the dentate gyrus, based on structural MRI, we conducted a recently developed automated subdivision segmentation method using the Freesurfer MRI analysis suite. After preprocessing, diffusion MRI was used to calculate diffusion anisotropy measures using a diffusion tensor model in FSL (Functional MRI of the Brain Software Library). If the neurogenesis in the dentate gyrus was affected by OSAS, it would affect volumes of other brain regions where new born neurons would migrate. To test this, we assess grey matter volumes across the whole brain and correlate them with dentate gyrus mean diffusivity using structural MRI and voxel-based morphometry (VBM) in FSL. Neuropsychological evaluation was performed on the day of the MRI scan. Tests included verbal learning and memory (using the International Shopping List Task), and visual learning and memory (using the Groton Maze Test). Statistical analyses were conducted using general linear model (GLM) and non-parametric bootstrapping (biascorrected and accelerated) based mediation analysis in "mediation" $\mathrm{R}$ package, and GLM combined with permutation tests in randomize in FSL.

Results: We found a significant impact of OSAS on mean diffusivity of the left dentate gyrus with a large effect size ( $t$ $=3.36, P=0.003$, partial $\eta 2=0.386$, adjusting for effects of age, sex and BMI). That is, childhood OSAS was linked to a decrease in dentate gyrus mean diffusivity. An exploratory analysis showed no significant effects in other subdivisions of the hippocampus (P's $>0.12$ ). This suggests a specific effect 
OSAS on mean diffusivity of the dentate gyrus. Next, we tested functional correlates of decreased mean diffusivity of the dentate gyrus in childhood OSAS. Decreased mean diffusivity of the dentate gyrus was positively correlated both with Apnea (pauses in breathing)-Hypopnea (shallow breathing) Index (Spearman's $r=-0.495, P=0.016$, twosided) and with a decrease in non-verbal learning and memory (Spearman's $r=0.548, P=0.007$, two-sided). Youths with OSAS showed neuropsychological measures of learning and memory comparable to that of control youths (P's >0.1).

A path model revealed a likely causal pathway: a decrease in mean diffusivity of the dentate gyrus mediated the association between OSAS and a decrease in non-verbal learning and memory (average causal mediation effect, ACME = -6.732, $P=0.02$, Bias-corrected \& accelerated CI).

Lastly, we tested an association between the dentate gyrus mean diffusivity and development of other brain regions. Whereas no significant impact of OSAS on the brain volumes were found at alpha of 0.05 (whole brain correction), a significant correlation between a decrease in dentate gyrus mean diffusivity and a decrease in volumes of the hippocampus, striatum, and thalamus was found $(P<0.01$, whole brain correction). This reflects the link between microstructure of the dentate gyrus and volumes of other brain regions.

Conclusions: This pediatric OSAS study, in combination with neuroimaging and neuropsychological testing, show that a disruption of the microstructure of the dentate gyrus mediates the effects of childhood OSAS on development of learning and memory. OSAS effects were only significant in the dentate gyrus mean diffusivity, but not in other brain regions or structural measures, or on learning and memory. The specific impact on dentate gyrus mean diffusivity and its associations with volumes of neighboring regions and cognition, suggest disrupted neurogenesis in the dentate gyrus as a potential mechanism of the impact of OSAS on neurocognitive development.

Keywords: Sleep Disturbance, Dentate Gyrus, Neurogenesis, Learning and Memory, Diffusion Weighted Imaging.

Disclosure: Nothing to disclose.

T80. Establishing the Reproducibility of Autism-Related Differences in the Brain Connectome: A Large-Scale Evaluation Using the Second Edition of the Autism Brain Imaging Data Exchange (ABIDE-II)

Adriana Di Martino*, Dorothea Floris, David

O'Connor, Bosi Chen, Michael Milham

NYU Child Study Center, New York, New York, United States

Background: Multiple sources of evidence, including functional neuroimaging, have supported models of autism spectrum disorder (ASD) as a condition characterized by abnormal connections among brain regions. Yet, both the complexity of the brain connectome and the striking heterogeneity of ASD have hampered efforts to specify the nature of putative dysconnections. For example, while resting state functional resonance imaging (R-fMRI) studies have substantiated the dysconnection model of ASD, notable variation exists in findings of hyper vs. hypo connections53, 61 and on the extent of the circuitry involved. To address these challenges, the Autism Brain Imaging Data Exchange (ABIDE) in 2012 openly shared 1112 R-fMRI scans of individuals with ASD and typical controls (TC). Initial analyses of this dataset - now termed ABIDE I demonstrated the feasibility of analyzing such an aggregate, and weighed in on the controversy regarding the contribution of hypo- vs. hyperconnections (Di Martino et al, 2014). Results from whole-brain intrinsic functional connectivity (iFC) analyses of 360 ASD vs. 403 TC revealed that both hypo and hyperconnectivity existed in ASD, but they varied as a function of the circuits involved. Hypoconnectivity mostly encompassed cortico-cortical circuits, while hyperconnectivity was primarily involved on sensory motor circuits anchored on subcortical regions (e.g., thalamus). Here, we aim to explore the extent to which these findings can be replicated in ABIDE II - a recently released multi-site dataset of over 1000 new independent datasets (ASD $N=$ 487; TC $N=557$ ).

Methods: Consistent with the initial ABIDE I study, we selected R-fMRI and corresponding structural imaging data from males with 1) full-scale IQ (FIQ) within 2 s.d. of the overall new sample mean $(111 \pm 15), 2)$ anatomical images providing near full brain coverage and successful registration, and 3) functional data motion within 2 s.d. of the sample mean (i.e., mean framewise displacement [FD] $0.08 \pm 0.46 \mathrm{~mm})$. Finally, we included only data from sites with FIQ available for at least $75 \%$ /diagnostic group and with at least 10 participants per diagnostic group after the above exclusions. This yielded a total of 637 individual datasets across 13 sites $(N=298$ ASD and $N=339$ TC; groups matched for $M$ age; $M$ age across data $=16 \pm 10$ yrs). Standard preprocessing including removal of 24 motion derivatives and nuisance regression using Compcor was conducted via the Configurable Pipeline for the Analysis of Connectomes. We examined whole-brain iFC of the parcellation units defined by the Harvard-Oxford Atlas. To do so, we extracted the mean time series for each of the 110 units from the preprocessed time series data in MNI space and calculated whole-brain iFC matrices using Pearson's correlations. Group comparisons accounted for age, FIQ, site, mean FD and global mean connectivity. Multiple comparisons were controlled for by applying the false discovery rate $(\mathrm{q}<0.05)$.

Results: Similar to prior findings obtained in ABIDE I, analyses in ABIDE II suggested that both hypo- and hyperconnectivity coexisted in ASD, and varied as a function of the circuit involved. The number of connections exhibiting ASD-related hyperconnectivity in the ABIDE sample was similar to that previously found in ABIDE I. A similar localization to subcortical connections was also revealed. In particular, iFC between each, the thalamus and globus pallidus, and primary parietal sensorimotor regions was significantly increased in ASD relative to TC. While findings of ASD-related hypoconnectivity for the ABIDE II sample were similar to those previously reported for ABIDE I with respect to their localization to cortico-cortical iFC, they were notably less prominent.

Conclusions: Leveraging ABIDE II we were able to identify an appropriately sized replication subsample to examine the reproducibility of the ASD-related iFC patterns previously 
observed using ABIDE I. Findings of subcortical hyperconnectivity with sensorimotor circuitry, were robustly replicated. They highlight the relevance of sensorimotor processes in ASD, which are commonly underexplored in the literature. In contrast, findings regarding hypoconnectivity, a prominent theme in the literature, were notably less robust, though similar in spatial distribution. Future efforts would benefit from parsing potential sources of heterogeneity in this novel resource, which may have limited the extent reproducibility of prior findings.

Keywords: Autism, Resting State Functional Connectivity, Data Sharing.

Disclosure: Nothing to disclose.

\section{T81. Trauma Exposure Predicts Greater Episodic Memory Function in the Hippocampus and Amygdala in Children}

Jennifer Stevens*, Sanne van Rooij, Ye Ji Kim, Timothy Ely, L Alexander Vance, Minhnguyen Cao, Bekh Bradley, Tanja Jovanovic

Emory University School of Medicine, Atlanta, Georgia, United States

Background: Childhood trauma is a primary risk factor for multiple mental and physical health problems, including intrusive memories associated with traumatic stress. Nonhuman animal findings indicate the hippocampus is highly sensitive to the damaging effects of stress, but little previous research has addressed effects of childhood traumatic stress on human hippocampal function. Here we investigated the effects of trauma exposure on memory encoding-related fMRI activation, in a sample of school-age children at risk for experiencing high levels of inner-city violence.

Methods: Mother-child pairs were recruited from the Grady Trauma Project, an epidemiological sample of participants recruited through the primary care waiting rooms of a large publicly funded urban hospital. We have previously found this population (both children and adults) to be exposed to high levels of community violence. Children ages 8-14 $(N=37)$ participated in an fMRI study; this age window targets a time of peak childhood trauma exposure, as well as substantial maturational change in medial temporal lobe connections with the prefrontal cortex. After quality control, data from 31 children were included in the analyses. In the fMRI task, children viewed static scene stimuli with emotional content of negative, positive, or neutral valence. After a 30-minute delay, children completed a cued recall task to indicate their memory for each scene from the scanning session. FMRI analyses focused on memory encoding-related activation. Trauma exposure was measured using the Traumatic Events Screening Inventory for Children (TESI-C).

Results: Children showed greater memory for negative and positive scenes, relative to neutral $(\mathrm{ps}<.05)$. Age was positively associated with overall recall performance $(\mathrm{R} 2=.07, p<.05)$ and the enhancing effect of emotion on recall (negative-neutral scene recall; $\mathrm{R} 2=.08, p<.05$ ). Trauma exposure was positively associated with recall performance, particularly for negative scenes (all scenes: $\mathrm{R} 2=.14$, negative scenes: $\mathrm{R} 2=.25$, ps $<.05)$. Furthermore, trauma exposure was positively associated with encoding- related activation in the left hippocampus $(\mathrm{R} 2=.25, p<.05)$ and amygdala $(\mathrm{R} 2=.11, p<.05)$ for negative stimuli. Girls showed better recall performance than boys across all 3 emotional valence categories (overall Mgirls $=.36$, Mboys $=$ $.23, p<.05)$. Girls and boys did not differ in amygdala or hippocampal activation, but girls showed less entorhinal cortex activation than boys.

Conclusions: Findings point to a surprising increase in hippocampal activity with greater childhood trauma load, which may be particularly relevant to negative emotional stimuli. We posit that this may represent a neurodevelopmental adaptation for children in a high-trauma environment, facilitating memory for negative stimuli in the environment. However, such an adaptation may increase later risk for impairing intrusive memory symptoms. Investigation of how early trauma exposure influences the developing brain will be critical to our understanding of why trauma and other forms of chronic stress have such strong impacts on mental health when experienced early in life, and will help us to identify developmental time windows that will be key targets for intervention.

Keywords: Childhood Trauma, Hippocampus, Violence, Amygdala, Episodic Memory.

Disclosure: Nothing to disclose.

T82. Common Genetic Variation in Oxytocin Receptors is Differentially Associated With Social Abilities Across Neurodevelopmental Disorders

Danielle Baribeau*, Annie Dupuis, Tara A Paton, Stephen W Scherer, Russell J Schachar, Paul D Arnold, Peter Szatmari, Rob Nicolson, Stelios Georgiades, Jennifer Crosbie, Jessica Brian, Alana Iaboni, Evdokia Anagnostou

Univeristy of Toronto, Toronto, Canada

Background: Children with autism spectrum disorder (ASD) or attention deficit hyperactivity disorder (ADHD) share many overlapping symptoms, including difficulties with social information processing. A large body of human and animal research has demonstrated associations between social abilities/behaviors and the oxytocin and/or vasopressin neuropeptide systems. Common genetic variation in the oxytocin or vasopressin receptors (OXTR and AVPR1a) may contribute to differences in social abilities in typically developing humans. The degree to which genetic differences in these receptors modify the severity of social impairments in children with neurodevelopmental disorders, such as ASD or ADHD, is less well studied. Whether the magnitude or direction of the effects of genetic differences in neuropeptide receptors on social abilities varies depending on the diagnosis of the individual is also unknown.

Methods: In this study, social abilities were assessed in a cohort of children with either autism spectrum disorder (ASD, $n=339$ ) or attention deficit hyperactivity disorder (ADHD, $n=275$ ) using the Reading the Mind in the Eyes Test (RMET) and the Social Communication Questionnaire (SCQ). Genotype was determined for four OXTR single nucleotide polymorphisms (SNPs) (i.e. rs53576, rs237887, rs13316193 and rs2254298), and AVPR1a RS3 microsatellite length, from blood/saliva samples. Social abilities were compared by 
genotype within and across diagnostic groups using regression models while accounting for ethnic differences.

Results: Several significant associations between neuropeptide receptor genotype and social abilities were detected; findings survived correction for multiple comparisons for OXTR rs237887, rs2254298 and rs53576. For these SNPs, interactions between genotype and diagnosis were highly significant. For OXTR rs53576 and rs2254298, the opposite direction of an association between genotype and social abilities was observed in the ASD group as compared to $\mathrm{ADHD}$ group; specifically, the risk-conferring variant in ASD was advantageous to social abilities in ADHD.

Conclusions: Results suggest that neuropeptide receptor gene polymorphisms may modulate the severity of social deficits in ASD and ADHD. However, specific common variants may not be inherently risk-conferring with respect to their impact on social abilities. Whether a genotype is advantageous or not may vary depending on the diagnostic context. Our findings therefore contribute to the often-conflicting literature regarding these SNPs and behavioral-genetic studies more generally, implicating potential diagnostic differences as one explanation for heterogeneity in findings. Keywords: Oxytocin, Social Behavior, Autism, Attention Deficit Hyperactivity Disorder.

Disclosure: Nothing to disclose.

\section{T83. Critical Role of Brain-Derived Neurotrophic Factor-Trkb Signaling in the Function of Cortistatin- Positive Inhibitory Interneurons}

Julia Hill*, Dennisse Jimenez, Nicholas Hardy, Ming Ren, Huei-Ying Chen, Kristen Maynard, Feng Yang, Brady Maher, Robert Schloesser, Keri Martinowich

Lieber Institute for Brain Development, Baltimore, Maryland, United States

Background: Activity-dependent brain-derived neurotrophic factor (BDNF) signaling via tropomyosin kinase receptor $\mathrm{B}$ (TrkB) plays a critical role in synaptic plasticity and the maturation of cortical inhibition. We previously showed decreased expression of several interneuron markers, including the neuropeptide cortistatin (CST), in mice with selective loss of activity-dependent BDNF signaling. CST is selectively expressed in hippocampal and cortical inhibitory interneurons. CST influences brain excitability-it has anticonvulsant effects and it promotes slow-wave sleep via antagonizing cholinergic signaling. Genes that are misregulated in the brain of individuals with autism spectrum disorder (ASD) as well as clinically identified candidate risk genes are over-represented in the CST interneuron transcriptome, strongly suggesting their impairment in ASD. We hypothesized that CST interneurons are important in controlling hyperexcitability and that BDNF-TrkB signaling plays a critical role in this function. Decreased BDNFTrkB signaling across the lifespan and in a number of neuropsychiatric disorders is linked to changes in inhibitory signaling. Hence, it is important to elucidate the mechanisms by which BDNF-TrkB signaling controls the function of specific subpopulations of inhibitory interneurons.

Methods: We used several genetic mouse models to interrogate the functional role of CST interneurons and the cell autonomous role of TrkB signaling in these interneurons.
To investigate the role of decreased activity-dependent BDNF expression upon hyperexcitability, we used a transgenic knock-in mouse model in which transcription of BDNF derived from promoter IV is inhibited by insertion of an enhanced-green fluorescent protein (eGFP)-stop cassette following exon IV (BDNF-KIV). To investigate the function of CST-positive interneurons, we selectively ablated this population by crossing CST cre-expressing mice (CSTcre) to mice carrying a loxP-flanked STOP cassette associated with an attenuated diphtheria toxin cassette. In these mice, diphtheria toxin expression is activated by cre expression in CST-positive cells, leading to their ablation. Finally, to elucidate the specific effects of BDNF-TrkB loss in CST cells, CST cre-expressing mice were crossed to mice harboring a floxed TrkB allele (CSTcre/TrkBflox/flox). We further crossed CSTcre/TrkBflox/flox mice to mice with a floxed TdTomato reporter to visualize and quantify CST-positive cells. We quantified the presence of spontaneous seizures using automated home cage recordings and video EEG and assessed seizure threshold in response to chemoconvulsants. We used standard behavioral analysis to assess social interaction and routine locomotor measures. We utilized immunohistochemical techniques and confocal microscopy to quantify location and number of CST-expressing cells in animals lacking expression of TrkB in these cells.

Results: BDNF-KIV mice exhibit decreased latency to seizure onset ( $n=8$ /group, $p=0.0002$ ) and increased seizure severity following injection with a chemoconvulsant agent $(p<0.0001)$. Mice with complete ablation of CST-positive cells showed onset of tremors by $\mathrm{P} 11$, and death following status epilepticus by P23 ( $n=22 /$ genotype, $p<0.0001)$. Finally, CSTcre/TrkBflox/flox mice, also died as a result of spontaneous seizures, with the majority living only 5-6 weeks ( $n=16 /$ genotype, $p=0.0173$ ). Additionally, mice with a heterozygous deletion of TrkB (CSTcre/TrkB+/flox) exhibited abnormal social interaction ( $n=5$ /group, $p=0.01)$. We found that there was no decrease in the number of CSTpositive cells in CSTcre/TrkBflox/flox mice.

Conclusions: These findings demonstrate that CSTexpressing cells are critical in maintaining excitatoryinhibitory balance, and that disruption of this population results in seizure-induced death. Proper functioning of CSTexpressing cells appears to be largely dependent upon BDNFTrkB signaling, as deletion of the TrkB receptor also resulted in epilepsy. We are working to elucidate the specific biological mechanism by which BDNF-TrkB signaling results in this epileptic phenotype.

Keywords: BDNF, TrkB, Epilepsy, GABAergic Interneurons, Cortistatin.

Disclosure: Nothing to disclose.

\section{T84. Are Children Treated With Second-Generation Antipsychotics at Higher Risk of Weight Gain Than Adults?}

Celso Arango*, Covadonga M Diaz-Caneja, Laura Pina-Camacho, David Fraguas

Hospital Gregorio Marañon, Madrid, Spain

Background: Second-generation antipsychotics (SGAs) have been consistently associated with weight gain and metabolic 
disturbances in young people and adults [1-2]. Children and adolescents treated with SGAs seem to be at higher risk of some these adverse effects, but no prospective studies have specifically compared weight gain between antipsychoticnaïve pediatric and adult patients in the same representative sample.

Methods: Six-month naturalistic longitudinal study assessing antipsychotic-naïve or quasi-naïve (lifetime exposure to antipsychotics $\leq 10$ days) pediatric and adult patients prescribed SGAs. Anthropometric and metabolic parameters were measured at baseline, 6-week, 3- and 6-month followup. In children, age- and sex-adjusted body mass index (BMI) z-scores were calculated using Spanish normative data. In adults, sex-adjusted BMI z-scores were calculated based on an age- and sex-matched healthy control sample recruited within the same study. Repeated measures mixedmodel analyses were used to compare 6-month trajectories of weight gain between children and adults in each of the main antipsychotic groups (risperidone, olanzapine and quetiapine), controlling for potential confounders (sex, socioeconomic status, diagnosis of psychotic disorder, drug use, concomitant psychotropic treatment and cumulative antipsychotic dose).

Results: One hundred and fifty-seven adult patients (age $44.22 \pm 18.01$ years, $51.6 \%$ male) and 226 pediatric patients (age $15.2 \pm 2.7$ years, 64.2\% male) comprised the study sample. In the adult sample, 46 patients received risperidone, 44 olanzapine, 58 quetiapine and nine other antipsychotics. Among children and adolescents, 140 received risperidone, 37 olanzapine, 44 quetiapine and five other antipsychotics. $56.1 \%$ of adults and $53.4 \%$ of youth were diagnosed with a psychotic disorder. BMI z-scores increased significantly both in adult (mean increase: 0.43 , $\mathrm{F}=17.64, p<0.001$ ) and pediatric (mean increase: 0.74 , $\mathrm{F}=27.58, p<0.001)$ patients and in all antipsychotic groups (risperidone: $\mathrm{F}=11.2, \quad p<0.001$; olanzapine: $\mathrm{F}=49.6$, $p<0.001$; quetiapine: $\mathrm{F}=10.6, p<0.001)$ during the first six months of treatment with SGAs. In children, treatment with olanzapine was significantly associated with greater weight gain at 3- and 6-month follow-up than treatment with risperidone (mean difference $(\mathrm{MD})=0.41, p<0.001$ and $\mathrm{MD}=0.62, p<0.001$, respectively) or quetiapine $(\mathrm{MD}=0.50$, $p<0.001 ; \mathrm{MD}=0.83, p<0.001)$. In adults, olanzapine was associated with significantly greater weight than risperidone and quetiapine in all visits. In all antipsychotic groups, pediatric patients showed significantly greater weight gain than adults during follow-up (risperidone: $\mathrm{F}=14.4$, $p<0.001$; olanzapine: $\mathrm{F}=18.7, \quad p<0.001$; quetiapine: $\mathrm{F}=25.5, \quad p<0.001)$. Trajectories of weight gain were significantly different in pediatric and adult patients treated with olanzapine $(\mathrm{F}=11.2, \quad p<0.001)$; even if there was significant weight gain between all visits in both age groups, weight gain was significantly in children between the 6-week and 3-month visits and between the 3- and 6-month visits.

Conclusions: Antipsychotic-naïve children treated with SGAs seem to be at higher risk of significant weight gain than adults during the first months of treatment. Careful evaluation of the risk-to-benefit balance should guide the choice of an SGA for children and adolescents. Close monitoring of weight and metabolic disturbances in young people and adults treated with SGAs is warranted.

Keywords: Antipsychotic Induced Weight Gain, Children and Adolescents, Drug Naive, Longitudinal.

Disclosure: Janssen, Otsuka, Astrazeneca, Lilly: Consultant and Honoraria, Self.

\section{T85. Lymphocytic Extracellular Signal Related Kinase Activation in Autism Spectrum Disorder}

Craig Erickson*, Logan Wink, Rebecca Shaffer, Ernest Pedapati, Tori Schaefer, Kelli Dominick, Hilary Meyer, Michael Hong, Amie Duncan, Carrie Thomas, Kaela O'Brien, Charles Tessier, John Sweeney

Cincinnati Children's Hospital Medical Center, Cincinnati, Ohio, United States

Background: The extracellular signal-related kinase (ERK) signaling cascade plays critical roles in brain development, learning, and memory. In neurons, the ERK cascade is activated by synaptic activity and ERK in turn phosphorylates numerous proteins involved in a diverse number of cellular processes including translational and transcriptional regulation, long-term potentiation and depression, and synaptogenesis. Evidence supporting potential ERK dysregulation in autism spectrum disorder (ASD) includes ASDassociated copy number variation at 1611.2 including the ERK1 locus and known excessive ERK activation in the RASopathies including Noonan syndrome, Costello syndrome and cardio-facio-cutaneous syndrome, all disorders associated with increased autistic traits. ERK activation (phosphorylation) has been evaluated in peripheral blood in humans with fragile X syndrome (FXS), a known single gene cause of ASD. In persons with FXS, peripheral lymphocytic ERK activation kinetics have been shown to be delayed compared to match typically developing control samples. To date, lymphocytic ERK activation kinetics has not been assessed in persons with idiopathic ASD.

Methods: We enrolled persons aged 3 to 25 years with a diagnosis of ASD confirmed by clinical interview using DSM-V criteria and testing with the Autism Diagnostic Observation Schedule (ADOS). Control group subjects included age- and gender-matched neurotypical participants (typically developing control group (TDCG)) and for subjects with ASD and an IQ of less than 90, an age- and IQ-matched subject without an ASD diagnosis was additionally enrolled (developmental delay control group (DDCG)). All subjects additionally underwent IQ testing and completion of the Social Communication Questionnaire (SCQ), a screening measure for features associated with ASD. Blood samples were drawn at Cincinnati Children's Hospital and shipped chilled overnight to the flow cytometer lab at Indiana University School of Medicine-South Bend. At the lab, lymphocytes from whole blood were purified by density gradient centrifugation and activated with $20 \mathrm{nM}$ phorbol myristate acetate (PMA) to activate protein kinase C and stimulate ERK phosphorylation. Aliquots of cells were removed from activation at 1 minute intervals over a 15 minute time course, fixed with $2 \%$ paraformaldehyde, and 
stained with anti-ERK1/2(pT202/Y204) antibody conjugated to Alexa Fluor 488. Phospho-ERK levels were then assayed by flow cytometry. Background unstained median fluorescence intensity was first subtracted from the antibody stained median fluorescence intensity at each time point. These values were then divided by the background subtracted median fluorescence intensity of a non-activated sample in order to obtain the phospho-ERK fold change of each time point after PMA activation. Each fold change time course was then fit to a sigmoidal curve to calculate the time to half maximum activation ( $t 1 / 2$ activation) value for each subject. All lab analysis was completed blinded to participant group assignment. We completed a 1-way ANOVA test analysis of t1/2 activation (reported in minutes) values among the three groups followed by use of the post-hoc Least Significant Difference (LSD) test to possible pair-wise comparisons of $t 1 / 2$ activation means.

Results: Subjects we enrolled as follows: ASD, $n=73$, mean age $12.0 \pm 5.9$ years; TDCG, $n=65$, mean age $13.8 \pm 14.1$ years; DDCG, $n=36$, mean age $10.2 \pm 5.8$ years. Mean $t 1 / 2$ activation were $5.81 \pm 1.49$ minutes (ASD), $6.35 \pm$ 1.04 minutes (TDCG), and $6.78 \pm 1.59$ minutes (DDCG). The ANOVA result was significant with a $F$ ratio of 6.57 and a $p$ value of 0.002 . Post-hoc LSD testing noted no t1/2 activation difference between the developmental delay control group and the neurotypical control group $(p=0.14)$. Significant $t 1 / 2$ activation differences between the ASD group and the developmental disability $(p=0.001)$ and neurotypical $(p=0.02)$ groups were noted. As expected the TDCG mean IQ $(103.8 \pm 9.2)$ was significantly higher than the mean IQ of the ASD (76.3 \pm 28.3$)$ or DDCG groups $(71.4 \pm 14.0)$ and the ASD and DDCG mean IQ values did not differ.

Conclusions: The reduced time to half maximum lymphocytic ERK activation noted in ASD points to a potentially hyper-responsive cellular state in ASD. The use of two matched controlled groups designed to essentially control more pointedly for the impact of ASD and not general developmental delay highlights the potential specificity of this finding. This work is also potentially consistent with our prior work analyzing baseline or static ERK activation in peripheral lymphocytes in persons with ASD that noted enhanced phosphorylated or activated ERK without efforts to stimulate cells. Future work will be required to validate the signaling abnormality noted in this study including efforts to correlate the dysregulation with phenotypic features and obtain test-retest data to evaluate the consistency of the result. The impact of potential pharmacotherapy on this finding also must be considered in the future.

Keywords: Autism Spectrum Disorder, ERK, Lymphocytes, Biomarker.

Disclosure: Takeda, Fulcrum Therapeutics, Neurotrope: Consultant, Self; Confluence Pharmaceuticals: Consultant/ Equity, Self; Cincinnati Children's Hospital Research Foundation, Indiana University School of Medicine: Patents, Self.
T86. Mutations in Mitochondrial Enzyme Glutamate Pyruvate Transaminase 2 (GPT2) Cause a Novel Neurogenetic Disorder

Eric Morrow*, Qing Ouyang, Tojo Nakayama, Ozan Baytas, Shawn Davidson, Chendong Yang, Michael Schmidt, Sophia Lizarraga, Sasmita Mishra, Malak El-Quessny, Saima Niaz, Mirrat Gul Butt, Syed Imran Murtaza, Afzal Javed, Haroon Rashid Chaudhry, R Sean Hill, Jennifer Partlow, Muna Al-Saffar, Anna Rajab, Ralph DeBerardinis, David Housman, Ganesh Mochida

\section{Brown University, Providence, Rhode Island, United} States

Background: We have identified mutations in the mitochondrial enzyme glutamate pyruvate transaminase 2 (GPT2) in a novel neurogenetic syndrome involving postnatal microcephaly. Postnatal microcephaly likely reflects failures in processes driving postnatal brain growth such as neuronal arborization, synaptogenesis, and gliogenesis. In needing to support these critical processes, the metabolic demands are substantial. To this end, mitochondria play an essential role in neurons and glia of the developing brain given the high energy demands of these cells. The mitochondrial tricarboxylic acid (TCA) cycle is a hub of metabolism, with central importance in both energy production and biosynthesis. TCA cycle intermediates may be extracted for biosynthetic metabolism through a process termed cataplerosis, and TCA intermediates are replenished via anaplerosis. The enzyme glutamate pyruvate transaminase 2 (GPT2), also known as alanine transaminase 2 (ALT2), is one of two related transaminases that catalyze the reversible addition of an amino group from glutamate to pyruvate yielding alanine and $\alpha$-ketoglutarate. GPT2 and highly related GPT are among several transaminases that regulate key metabolic processes, including amino acid metabolism and the TCA cycle. In addition to being the main excitatory neurotransmitter in CNS, glutamate is also a substrate in the synthesis of glutathione, an important neuroprotective mechanism in brain.

Methods: We have studied two large pedigrees with a new neurodevelopmental condition using linkage analysis and high-throughput sequencing. We have identified autosomal recessive GPT2 mutations. Biochemically, we characterized the enzyme activity of control and disease-relevant mutant forms of GPT2. Using techniques of molecular biology and cell biology, we then determined the localization of control and mutant forms of GPT2 in cultured cells and their partitioning into specific cellular fractions, namely, a cytosolic fraction vs. a mitochondrial fraction. We also generated a Gpt2-null mouse strain in order to model the genetic mutations in brain. We also applied state-of-the-art methods in metabolomics to cells and brains derived from our Gpt2-null mouse.

Results: We report autosomal recessive mutations in GPT2 in large kindreds initially ascertained for intellectual and developmental disabilities (IDD). In addition to IDD, all affected individuals show postnatal microcephaly and approximately $80 \%$ of those followed over time show progressive motor symptoms, a spastic paraplegia. Homozygous nonsense p.Arg404* and missense p.Pro272Leu 
mutations are shown biochemically to be loss-of-function. At the cellular level, GPT2 protein localizes to mitochondria and partitions to a mitochondrial fraction. Akin to the human phenotype, Gpt2-null mice exhibit reduced brain growth, as well as defects in synaptogenesis. Through metabolomics and direct isotope tracing experiments, we find a number of metabolic abnormalities associated with loss of GPT2. These include defects in amino acid metabolism, such as low alanine levels and elevated essential amino acids. Also, we find defects in anaplerosis, the metabolic process involved in replenishing TCA cycle intermediates. Finally, Gpt2-mutant mouse brains demonstrate misregulated metabolites in pathways implicated in neuroprotective mechanisms previously associated with neurodegenerative disorders.

Conclusions: The study of newly identified genetic mutations relating to brain development such as those we are studying in GPT2 is a powerful approach for investigating critical metabolic pathways, including those involving mitochondrial and glutamate metabolism in brain. Overall, our data reveal an important role for the GPT2 enzyme in mitochondrial metabolism with relevance to developmental as well as potentially to neurodegenerative mechanisms.

Keywords: Neurodevelopmental Disorders, Neurodegenerative Disease, Glutamate, Mitochondria, Metabolism.

Disclosure: Nothing to disclose.

\section{T87. Intranasal Vasopressin Treatment Improves Social Abilities in Children With Autism}

Karen Parker*, Ozge Oztan, Robin Libove, Raena Sumiyoshi, Jacqueline Summers, Kyle Hinman, Lawrence Fung, Kara Motonaga, Dean Carson, Jennifer Phillips, Joseph Garner, Antonio Hardan

\section{Stanford University, Stanford, California, United States}

Background: There are currently no medications that target autism spectrum disorder (ASD)'s core social deficits. However, neurobiological systems that are critical for social functioning are arguably one of the most promising for ASD therapeutic target discovery. Arginine vasopressin (AVP) is one such candidate; it plays a critical role in promoting social behavior and experimental dysregulation of the AVP signaling pathway produces social deficits in animal models. Here we present preliminary data from a double-blind randomized placebo-controlled trial which tested the effects of 4-week intranasal AVP administration to children with ASD.

Methods: Participants were medically healthy outpatients ( $N=18$ males, $N=4$ females), aged 6 to 12 years. Participants underwent comprehensive diagnostic and behavioral testing, and blood samples for safety monitoring and biomarker quantification were obtained. Participants were then randomized to receive either AVP treatment (a maximum of 12 IU BID or 16 IU BID based on age) or placebo treatment. The primary outcome measure was change in social ability as assessed by parent ratings on the Social Responsiveness Scale 2 (SRS-2) between baseline and after treatment. Throughout the trial, drug safety was assessed. Upon completion of treatment, SRS-2 ratings and participants' blood samples were again obtained. Blood AVP levels and oxytocin receptor (OXTR) and AVP receptor vla
(AVPRvla) gene expression levels were quantified via enzyme immunoassay and qPCR, respectively.

Results: Using a general linear model, including treatment (drug vs. placebo) and the biomarker measures, we found that treatment efficacy depended on pre-treatment AVP levels $(\mathrm{F} 1,9=7.3544 ; P=0.0239)$. Further analysis revealed that pre-treatment AVP levels predicted treatment response in participants receiving drug $(P=0.0099)$, but not in those receiving placebo, suggesting that pre-treatment AVP levels may be useful in discerning children most likely to respond to AVP treatment. The treatment $\mathrm{x}$ AVP levels interaction therefore informed our comparison of drug- and placebotreated participants. AVP-treated participants improved by an average of $12.8 \pm 3.5$ points on the SRS-2 Total Score $(P=0.0102)$, but placebo-treated participants' SRS-2 Total Scores did not significantly differ from a 0-point improvement. We also observed a pre-treatment "biomarker signature" that predicted treatment efficacy. Thus, in addition to the effect of pre-treatment AVP levels, participants with lower OXTR gene expression, and higher AVPRvla gene expression, showed greater improvement in SRS-2 Total Scores, particularly when relative expression was contrasted in each participant $(\mathrm{F} 1,9=8.060 ; P=0.0194)$. Finally, there were no significant differences in adverse events between treatment groups, nor any significant changes from baseline in electrocardiogram, vital sign, or clinical laboratory measurements during AVP treatment.

Conclusions: This is the first study to show that intranasal AVP treatment is well tolerated and improves social abilities in individuals with ASD. Findings from this study also suggest that pre-treatment neuropeptide measures may help identify patients most likely to benefit from AVP treatment. This research has high potential to lead to development of the first effective and personalized medication to treat ASD's currently intractable social deficits.

Keywords: Autism Spectrum Disorder, Vasopressin, Randomized Clinical Trial, Social Functioning, Biomarkers.

Disclosure: Nothing to disclose.

\section{T88. Variation in the Infant Gut Microbiome is Associated With Cognitive Development and Brain Morphometry}

Alexander Carlson, Kai Xia, Andrea Azcarate-Peril, Barbara Goldman, Martin Styner, Amanda Thompson, Xiujuan Geng, John Gilmore, Rebecca Knickmeyer ${ }^{*}$

University of North Carolina at Chapel Hill, Chapel Hill, North Carolina, United States

Background: Studies conducted in rodents provide compelling evidence that the gut microbiome influences brain development and function. In particular, experimental manipulations which alter the intestinal microbiota impact exploratory, social, and communicative behaviors, as well as cognitive performance. In humans, altered gut microbiota have been reported in individuals with autism and depression, but no studies have addressed when these relationships emerge or directly examined which brain regions may be involved. Early development is likely of critical importance as the first year of life is the foundational period for microbial colonization of the gut and the most rapid and dynamic 
phase of postnatal brain development. The objective of the current study was to determine how microbial composition at 1 year of age impacts cognitive development and to identify neural circuits mediating this relationship using high resolution magnetic resonance imaging (MRI), diffusion tensor imaging (DTI) and resting state fMRI (rfcMRI).

Methods: Fecal samples were collected from 89 typically developing one-year old infants. 16s rRNA amplicon sequencing was used for identification and relative quantification of bacterial taxa. Measures of alpha diversity were generated and distance metrics and cluster scoring methods were used to identify genus level enterotypes. Cognition was assessed at 1 and 2 years of age using the Mullen Scales of Early Learning. Brain scans were acquired on a Siemens head-only 3T TIM-Trio scanner (Siemens Medical System, Erlangen, Germany) during unsedated natural sleep.

Results: There was moderate support for clustering subjects into three enterotypes by the relative abundance of different bacterial genera. One cluster was characterized by high levels of Faecelibacterium, one by high levels of Bacteroides, and one by high levels of an unclassified genus of Ruminococcaceae. Mullen scores at age 2 differed significantly between clusters with the cluster characterized by Bacteroides abundance showing higher cognitive ability on the overall Composite score as well as the Expressive Language Scale. Alpha diversity measures were also significantly associated with overall Composite score at age 2 and the Expressive Language Scale. Lower diversity was associated with better performance. Exploratory analyses of regional gray matter volumes in 46 1-year-old children suggested that the gut microbiome influences neural circuits for visual processing, emotion regulation, and reward. We are current testing whether these relationships are also evident when assessing anatomical and functional connectivity.

Conclusions: Microbial colonization of the gut at 1 year of age predicts later cognitive development particularly in the area of communicative behavior, a key domain disrupted in many psychiatric disorders. Ongoing studies by our group are aimed at determining whether patterns of microbial colonization at 2 weeks and 1 year of age are related to other behaviors relevant to psychiatric risk including fear reactivity. We are also actively investigating the role of immune signaling molecules and tryptophan metabolism in mediating microbial effects on neurodevelopment in human infants. Ultimately this line of research is expected to identify early interventions and therapeutics to promote a healthy microbiome, thereby improving cognitive outcomes and reducing risk for psychiatric disorders.

Keywords: Gut Microbiome, Infancy, Human Neuroimaging, Cognition, Language.

Disclosure: Pfizer: Grant, Self.

\section{T89. Spectroscopic Glutamate Correlates of Trauma and Anhedonia in Adolescents}

Paul Croarkin*, Charles Lewis, Jennifer Vande Voort, Jasmin Kohli, Frank Kozel, Mark Frye, John Port

Mayo Clinic, Rochester, Minnesota, United States

Background: Prior work suggests that adolescents with mood disorders have dysregulated glutamatergic neurotransmission. The clinical correlates and implications of this are still poorly understood. A trauma history and ensuing anhedonia may be important dimensional characteristics to consider. This study examined cortical proton magnetic resonance spectroscopy scans, a self-reported inventory of trauma, and a dimensional measure of anhedonia in depressed and healthy control adolescent participants. We hypothesized that adolescents with elevated trauma scale scores would have increased levels of anhedonia. Further, depressed adolescents with elevated trauma and anhedonia scale scores would have decreased cortical glutamate metabolite levels as compared to participants with low trauma and anhedonia scale scores.

Methods: Healthy control $(n=15)$ and depressed adolescents $(n=33)$ underwent a semi-structured diagnostic interview and completed the Childhood Trauma Questionnaire (CTQ). A threshold of 30 and above on the combined subscales of the CTQ was used to define a trauma history for categorical analyses. Depressive symptom severity was assessed with the Children's Depression Rating Scale-Revised (CDRS-R), and the Quick Inventory of Depressive SymptomatologyAdolescent (QIDS-A17). A composite anhedonia scale included items 2 and 3 on the CDRS-R and item 14 on the parent and self-report QIDS-A17. Proton magnetic resonance spectroscopy scans of the anterior cingulate cortex (ACC) and left dorsolateral prefrontal cortex (L-DLPFC) were collected at $3 \mathrm{~T}$ with $8 \mathrm{~cm} 3$ voxels. A TE-optimized PRESS $(\mathrm{TE}=80 \mathrm{~ms}, \mathrm{TR}=2000 \mathrm{~ms}$, No. of excitations $=8$, No. of acquisitions $=128$ ) and a 2-dimensional J-resolved averaged PRESS (TE35-195 ms in 16 steps, TR $=2000 \mathrm{~ms}$, No. of excitations $=8$ ) sequences were collected in the ACC and L-DLPFC. Glutamate (Glu), glutamine (Gln), and Glx (corrected to creatine) were examined in each voxel of interest. Mann-Whitney $U$ tests were run to examine differences in cortical glutamate metabolite differences among groups. Spearman's rank-order correlations were run to assess the putative relationship between CTQ measures and cortical metabolites.

Results: The composite anhedonia scale and the CTQ trauma index had a significant positive correlation in this sample of adolescents (Spearman's rho $=.492, p<.001$ ). Thirty-two participants had a threshold trauma score on the CTQ. Participants with a threshold trauma score had significant increased anhedonia ratings compared to participants without trauma (Mann-Whitney U Test, $p<.001$ ). Adolescents with a threshold CTQ score had significantly elevated Glx/Cr (Mann-Whitney U Test, $p=.042$ ) and Glu/ Cr (Mann-Whitney U Test, $p=.015$ ) in the L-DLPFC (assessed with optimized PRESS) as compared to adolescents with subthreshold CTQ scores. There were nonsignificant, negative correlations between the overall CTQ trauma index and L-DLPFC Glu/Cr assessed with optimized PRESS (Spearman's rho $=-.306, p=.062$ ) and L-DLPC Glx/Cr (Spearman's rho-.284, $p=0.84$ ). However, the CTQ physical abuse scale had significant correlations with L-DLPFC Glu/ Cr (Spearman's rho $=-.479, p=.002$ ) and L-DLPC Glx/Cr (Spearman's rho-.444, $p=0.005$ ) both assessed with optimized PRESS. The composite anhedonia scale had an insignificant correlation with ACC $\mathrm{Glx} / \mathrm{Cr}$ assessed with the optimized PRESS sequence (Spearman's rho $=.314$, $p=.055)$. However, the parental report of anhedonia on the QIDS-A17 had a positive correlation with ACC Glx/Cr 
assessed with the optimized PRESS sequence (Spearman's rho $=.46, p=0.004$ ).

Conclusions: The present findings suggest that historical physical abuse may be an important clinical correlate of cortical glutamate dysregulation in adolescent depression. Childhood trauma may have important clinical and neurobiologic relationships with subsequent anhedonia which warrant further study. Dimensional measures of trauma and anhedonia may have utility in spectroscopic biomarker work with adolescents. Further study of glutamate neurochemistry in depressed adolescents with a history of trauma could guide intervention development and drug discovery for adolescent mood disorders and post-traumatic stress disorder.

Keywords: Adolescent Depression, Childhood Trauma, Anhedonia, Glutamate, MR Spectroscopy.

Disclosure: Nothing to disclose.

\section{T90. High Maternal Serum Choline at 16 Weeks Gestation Protects Against Infection-Associated Impairments in Infant Auditory P50 Sensory Gating}

Randal Ross*, M Camille Hoffman, Sharon Hunter, Robert Freedman

University of Colorado School of Medicine, Aurora, Colorado, United States

Background: Prenatal maternal infection is associated with increased risk in the offspring for a variety of neuropsychiatric illnesses including schizophrenia, autism, and ADHD. Impaired cerebral inhibition is associated with schizophrenia and other mental illnesses and has been proposed as a mechanistic contributor. Prenatal infection predicting postnatal cerebral inhibition deficits would be consistent with this model, yet has never been assessed. In both animal and human models, prenatal stimulation of the alpha7 nicotinic cholinergic receptor with dietary choline supplementation leads to improved development of sensory gating, improved memory, and decreased anxiety; however, whether that relationship extends to non-supplemented women and their children has not been assessed. Using P50 sensory gating as a measure of infant cerebral inhibition, this report examines whether maternal prenatal infection and/or maternal serum choline levels predict infant cerebral inhibition, and whether there is an interaction between these two factors.

Methods: 155 initially healthy pregnant women were surveyed at 16 and 28 weeks gestation as to whether they had experienced moderate-to-severe symptoms consistent with any infection in the previous six weeks. Serum was drawn for choline levels at the same gestational time points. P50 sensory gating was assessed during active sleep in the resultant infants at a mean adjusted age of (SD) 28 (10) days. Results: At 16, but not 28 weeks gestation, women who report moderate-to-severe symptoms of any infection $(n=61)$ birth infants with more impaired P50 sensory gating $(\mathrm{t}=2.285, p=.024$, standardized $\sigma=.187$ and $\mathrm{t}=0.374$, $p=.709$, standardized $\sigma=.030$ respectively). At 16 , but not 28 weeks gestation, lower maternal serum choline levels are associated with more impaired infant P50 sensory gating $(\mathrm{t}=2.068, p=.040$, standardized $\sigma=.167$ and $\mathrm{t}=1.169$, $p=.244$, standardized $\sigma=.094$ respectively). At 16 weeks gestation, there is an interaction between maternal reported infection and serum choline $(\mathrm{t}=2.921, p=.004$, standardized $\sigma=.343)$ : in mothers who report infection, maternal serum choline is inversely correlated with infant sensory gating ratio (Pearson $r=-.405, p=.001$ ); no similar correlation is identified for women who did not report such an infection (Pearson $r=-.003, p=.975$ ).

Conclusions: Prenatal exposure to maternal infection in the late first trimester/early second trimester period has previously been associated with increased risk for schizophrenia and other psychiatric disorders. These results support a similar relationship between infection and development of cerebral inhibition raising the possibility that cerebral inhibition is a mediator between prenatal infection and later onset of psychiatric illness. Higher maternal serum choline, which impacts cerebral inhibition development via stimulation of the alpha7 nicotinic cholinergic receptor, compensates for maternal infection. Prenatal choline supplementation remains a potential primary prevention strategy.

Keywords: Pregnancy, Infection, Infant, P50, Choline. Disclosure: Nothing to disclose.

\section{T91. Age-Dependent Effects on Social Interaction of Nmda GluN2A Receptor Subtype-Selective Antagonism}

Torrian Green, Jessica Burket, Stephen Deutsch*

Eastern Virginia Medical School, Norfolk, Virginia, United States

Background: The heterotetrameric NMDA receptor (NMDAR) is composed of two obligatory GluN1 and either two modulatory GluN2A or GluN2B receptor subunits. GluN2A and GluN2B-containing receptors differ in their developmental expression, distribution between synaptic and extrasynaptic locations, and channel kinetic properties, among other differences. Clarifying the relative contributions of GluN2A- and GluN2B-containing NMDARs to the regulation of sociability will have important translational implications for the development of selectively targeted NMDAR agonist interventions for the treatment of impaired sociability. The current study explored a possible regulatory role of the GluN2A subtype-selective NMDAR in normal mouse sociability. Specifically, PEAQX, an NMDAR antagonist, was used to explore effects of the GluN2A-containing NMDAR on the salience of a stimulus mouse, social interaction, stereotypic behaviors emerging spontaneously during social interaction, and spatial working memory in 4and 8-week old male Swiss Webster mice.

Methods: 4- and 8-week old male, outbred Swiss Webster test mice were individually weighed prior to drug administration $(N<20$ mice in each condition). Stimulus mice were 4 -week old male ICR mice. PEAQX dissolved in $0.85 \%$ PBS was injected intraperitoneally in a volume of $0.01 \mathrm{~mL} / \mathrm{g}$ of body weight $20 \mathrm{~min}$ prior to behavioral testing. Dose selection of PEAQX was determined by preliminary rotarod data; $32.0 \mathrm{mg} / \mathrm{kg}$ of PEAQX was the highest administered dose devoid of motor incoordination and ataxic effects, and did not affect locomotor activity in the sociability apparatus. Sociability was tested using a 3-compartment apparatus. Spatial working memory was assessed using the Y-maze 
spontaneous alternation test. Paired t-tests were used to determine effects of PEAQX on the salience of the enclosed stimulus mouse for Swiss Webster mice (Session II). A twoway ANOVA was used to examine effects of age (i.e., 4-week vs 8-week), treatment (i.e., PEAQX vs vehicle), and their interaction on social and stereotypic behaviors observed during session III, and percentage of spontaneous alternations. A Mann-Whitney $U$ test was used to evaluate differences in discrete episodes of mounting between PEAQX-treated and vehicle-treated 8-week old mice during session III. A one-way ANOVA was used to explore the effects of PEAQX on the rotarod performance of 4-week old mice. In all instances, when ANOVA was significant, posthoc comparisons were made where appropriate.

Results: Vehicle-treated 4- and 8-week old Swiss Webster test mice preferred the social compartment containing the enclosed stimulus mouse to the compartment containing the empty inverted cup $(p<0.01$ and $p<0.001)$, and spent more time exploring/sniffing the enclosed stimulus mouse than the empty inverted cup $(p<0.001$ and $p<0.0001)$. 8-Week old test mice treated with PEAQX preferred the social compartment containing the enclosed stimulus mouse to the compartment containing the empty inverted cup $(p<0.01)$, and both 4 - and 8-week old test mice treated with PEAQX spent more time exploring/sniffing the enclosed stimulus mouse than the empty inverted cup $(p<0.001$ and $p<0.0001)$. Thus, the stimulus mouse retained salience for Swiss Webster mice treated with PEAQX. Interestingly, in contrast to Session II measures, when test and stimulus mice were allowed to interact freely during Session III, 8-week old Swiss Webster mice treated with PEAQX showed diminished social interaction compared to age-matched, vehicle-treated mice on several measures, including: discrete episodes of social approach $(p<0.0001)$, discrete episodes of anogenital sniffing $(p<0.01)$, discrete episodes of mounting $(p<0.0001)$, discrete episodes of social pursuit $(p<0.0001)$ and total time spent engaged in pursuit behavior $(p<0.0001)$. Although PEAQX worsened the social interaction of 8-week old Swiss Webster mice, it diminished the intensity of rearing emerging spontaneously during social interaction in both 4 - and 8-week old mice $(p<0.0001)$ and total time spent engaged in grooming in the 4 -week old mice $(p<0.01)$. Thus, directionally-opposite adverse (i.e., disruption of social interaction) and beneficial (i.e., attenuation of intensity of stereotypic behaviors) effects of PEAQX occur, which may be influenced by age. Comparisons of agematched mice showed that PEAQX did not significantly affect spatial working memory assessed in the Y-maze.

Conclusions: The data implicate an age-dependent contribution of GluN2A-containing NMDARs to the regulation of normal social interaction in mice. At a dose of PEAQX devoid of any effect on locomotor activity and mouse rotarod performance, the social interaction of 8 -week old mice was disrupted without any effect on the social salience of a stimulus mouse. Moreover, PEAQX attenuated stereotypic behavior emerging during social interaction in 4- and 8-week old mice. However, PEAQX had no effect on spontaneous alternations, a measure of spatial working memory, suggesting that neural circuits mediating sociability and spatial working memory may be discrete and dissociable from each other. The data suggest that the regulation of stereotypic behaviors and sociability may occur independently of each other. Because expression of GluN2A-containing NMDARs occurs at a later developmental stage, they may be more involved in mediating the pathogenesis of ASDs in patients with histories of "regression" after a period of normal development than GluN2B receptors.

Keywords: NMDA Receptor, GluN2A Recepter Subunit, Sociability, Stereotypic Behavior, Cognition.

Disclosure: Nothing to disclose.

\section{T92. Copy Number Elevation of 22q11.2 Genes Arrests the Developmental Maturation of Working Memory Capacity and Adult Neurogenesis}

Shuken Boku*, Seiji Abe, Takeshi Izumi, Tomohisa Takahashi, Takeshi Hiramoto, Yasuhiko Naka, Hiroko Nomaru, Akira Nishi, Gina Kang, Akitoyo Hishimoto, Gilberto Duran-Torres, Kenji Tanigaki, Jinghang Zhang, Kenny Ye, Shigeki Kato, Kazuto Kobayashi, Pekka Mannisto, Noboru Hiroi

\section{Kobe University Graduate School of Medicine, Kobe, Japan}

Background: Working memory capacity, a critical component of executive function, developmentally expands from childhood to adulthood. This developmental process halts its upward trajectory during adolescence and increasingly lags behind toward adulthood in individuals with autism spectrum disorder (ASD), suggesting that this process is functionally relevant to the developmental trajectory of neuropsychiatric disorders. However, the cellular and neuronal substrates contributing to this process are not understood. As duplication/triplication of human chromosome $22 \mathrm{q} 11.2$ is one of the copy number variants (CNVs) consistently and robustly associated with developmental neuropsychiatric disorders, we used this genetic variant as an entry point to delve into the cellular substrates for this developmental atypicality.

Methods: Using a region- and cell-specific gene expression approach, we over-expressed catechol-O-methyl-transferase (COMT) or Tbx1, two 22q11.2 CNV-encoded genes, in adult neural stem/progenitor cells in the hippocampus of C57BL/ $6 \mathrm{~J}$ mice. Mice were tested for spontaneous alternation in a T-maze, a measure of spatial working memory, at either 1 month or 2 months of age. Following behavioral analysis, mice were sacrificed and the location of gene-transduced cells within the granule cell layer of the hippocampal dentate gyrus was examined. In a separate experiment, cells were taken from the hippocampus of C57BL/6J mice and cultured and passaged to isolate adult neural stem/progenitor cells. Cells in culture were then tranfected with plasmid carrying either COMT or Tbx1, and the rate of proliferation and apoptosis was examined.

Results: Mice increased working memory capacity from 1 to 2 months of age, corresponding to adolescence to young adulthood. Over-expression of COMT in adult neural stem/ progenitor cells reduced the maximum working memory capacity at 2 months, but not at 1 month of age. Similarly, over-expression of Tbx1 in the same cell population at 2 months reduced working memory capacity. Moreover, over-expression of COMT or Tbxl reduced the rate of 
migration of progenies of adult neural stem/progenitor cells in the hippocampus granule cell layer. When COMT or Tbx1 was over-expressed in adult neural stem/progenitor cells in vitro, their proliferation was reduced without elevating the rate of apoptosis.

Conclusions: Our data provide evidence for the novel notion that elevated levels of these 22q11.2 genes deter the typical developmental maturation of working memory capacity via an altered rate of proliferation-and migration of progenies of-adult neural stem/progenitor cells in the hippocampus.

Keywords: 22q11.2 CNV, Working Memory, Adult Hippocampal Neurogenesis, COMT, Tbx1.

Disclosure: Nothing to disclose.

\section{T93. The Ginkgo Special Extract EGb761 ${ }^{\circledR}$ Improves Mitochondrial Dysfunction and Impaired Neuroplasticity: A Possible Explanation for Clinical Efficacy Over the Whole Continuum of Age-Associated Cognitive Disorders}

Walter Mueller*, Kristina Friedland

University Frankfurt, Frankfurt, Germany

Background: After the failure of all attempts to treat Alzheimer's disease (AD) by reducing $B$-amyloid deposits $(A B)$ in the brain, alternative concepts to explain cause and progression of the disease become more and more relevant. As probably the most important example, the "mitochondrial cascade hypothesis", put forward more than 10 years ago by Swerdlow and coworkers, assumes mitochondrial dysfunction as the major underlying pathomechanism of $\mathrm{AD}$. Driven by genetic and environmental factors, mitochondrial dysfunction cumulates in susceptible patients over years, slowly increasing by age-associated elevation of free radical (ROS) production, leading at some point to further mitochondrial deficits due to elevated $A B$ levels, which finally result in $A B$ aggregation and plaque formation. The major aspect of this concept relates to mitochondrial dysfunction as the major pathomechanism directly driving neurodegeneration and psychopathology independent of $A B$ deposits. It finally leads to impaired cognition from aging over mild cognitive impairment (MCI) to dementia. To confirm this concept pharmacologically, we show that the ginkgo special extract EGb761 reduces mitochondrial dysfunction and improves impaired neuroplasticity in animal and cell models of aging and AD.

Methods: Since many different ginkgo extracts have been investigated over the years with sometimes, we only report data using the specific standardized ginkgo extract EGb761@. This extract has been used all of the preclinical work presented and for nearly all clinical studies published over the last 10 years.

Results: $\mathrm{EGb}{ }^{\oplus} 1^{\oplus}$ has been demonstrated to improve mitochondrial function and to protect mitochondria against oxidative stress by its radical scavenging properties as well as by direct effect at the respiratory chain of mitochondria. These properties have not only been show in a large number of in vitro experiments using many different cell lines but also in many animal models. Its effect on mitochondrial function are mainly seen when mitochondrial function is impaired. Similarly, in many animal models improvement of cognition as well as of synaptic plasticity was observed, again much more pronounced when these mechanisms were impaired by aging, reduced vascular function, or by overexpression of human $A ß$.

Conclusions: In patients, EGb761@ improved cognition and activities of daily living in cases of mild to moderate dementia ( $\mathrm{AD}$ and vascular dementia) as indicated in recent meta-analyses. Moreover, it shows improvement of impaired cognition in Minimal Cognitive Impairment (MCI) as well in elderly patients with very mild cognitive symptoms.

The data presented show that EGb761 ${ }^{\circ}$ improves mitochondrial dysfunction in animal models of aging and vascular as well as Alzheimer's dementia. This parallels improved cognition and activties of daily living in man over the whole continuum of age-associated cognitive disorders from very mild memory problems in aging up to mild to moderate dementia. These data confirm the hypothesis of mitochondrial dysfunction as an important mechanism for the initiation and the progression of a cascade of events finally leading to dementia.

Keywords: Mitochondrial Dysfunction, Ginkgo Extract, Neuroplasticity, Cognitive Impairment.

Disclosure: Dr. W Schwabe: Research Grant, Speakers Honorarium, Self.

\section{T94. Validation of a Novel Computerized Self-} Administered Memory-Screening Test With Automated Reporting (SAMSTAR) in Patients With Mild Cognitive Impairment and Normal Control Participants: A Randomized, Crossover, Controlled Study

Randall Morrison*, Huiling Pei, Gerald Novak, Daniel Kaufer, Kathleen Welsh-Bohmer, Stephen Ruhmel, Vaibhav A Narayan

Janssen Research \& Development, LLC, Titusville, New Jersey, United States

Background: Mild cognitive impairment (MCI) is a transitional state between normal cognition and dementia or Alzheimer's disease. Decline in verbal episodic memory is a hallmark feature observed in patients with MCI. Reliable detection of episodic memory impairment is therefore crucial in assessing disease progression and success of potential treatments. The self-administered memory-screening test with automated reporting (SAMSTAR) is a newly developed word list recall test (WLR) and is an adaptation of the Rey Auditory Verbal Learning Test (RAVLT) designed for use with portable electronic devices (i.e., iPad). The present study evaluates the validity and feasibility of SAMSTAR vs the standard examiner-administered RAVLT in quantifying deficits in verbal episodic memory in patients with MCI and cognitively normal control (NC) individuals. Methods: In this randomized, two-site, crossover study, patients with MCI (Montreal Cognitive Assessment [MoCA] score, 24 to 27) and NC individuals (MoCA score $\geq 28$ ) aged 55 to 84 years, who were English-speaking with normal or corrected visual and hearing acuity were enrolled. Enrollment was based on stratification and even distribution by site, age and sex for NC and by site for patients with MCI (approximate ratio 3:1, NC: MCI). Randomization was balanced and all participants underwent either conventional 
$\overline{S 346}$

examiner-administered WLR (RAVLT) or computeradministered WLR (SAMSTAR) during period 1. After a 7 to 14-day memory washout period, each participant was crossed-over to the alternate test (period 2). Primary efficacy endpoint was WLR scores (number of words successfully recalled from15-word lists) based on 8 trials (I to $\mathrm{V}$ acquisition trials [List $\mathrm{A}$ ], distractor trial [List $\mathrm{B}$ ], postdistraction recall trial and 20-minute delayed recall trial [List A]) administered as either RAVLT or SAMSTAR to evaluate learning efficiency, retroactive interference effects and immediate/ delayed verbal memory. Primary analysis to establish equivalence between SAMSTAR (based on independent rater scorings of audio recordings) and RAVLT was performed using repeated measure mixed model, with dependent variable (WLR scores from the 8 trials), fixed effects (period, evaluation sequence, evaluation group [computer or examiner], trial, interaction of evaluation group and trial), and participants as random effect. Equivalence was established if the $90 \%$ confidence intervals (CI) of least square (LS) mean difference were well within the pre-specified range of $(-1.35,1.35)$. Three sensitivity analyses were performed (1) using primary analysis model with only scores from Trials I to $\mathrm{V}$ as dependent variable; (2) adding demographic factors (NC/MCI status, age and sex) to the primary analysis model; (3) using WLR scores from automated scoring (speech recognition engine: Nuance Speech Anywhere) as dependent variable. Secondary endpoints included further analysis of WLR scores to assess total learning (total score from Trials I to V), learning over trials (LOT) index and serial position analysis of recalled words (primacy, middle region and recency). Adverse events (AEs) were recorded for safety evaluation.

Results: Of the 161 participants screened, 153 (SAMSTAR: $n=75$; RAVLT: $n=78)$ were randomized and $148(97 \%)$ participants completed the entire crossover study. Randomized participants had mean (SD) age of 69.9 (7.86) years and were mostly women (68.6\%). Measures of verbal learning and memory based on WLR scores from the 8 trials were comparable between the 2 tests. Pearson's correlation coefficients for the 8 trials ranged from 0.12 to 0.70 . The overall LS mean difference between SAMSTAR and RAVLT was $-0.84(90 \% \mathrm{CI},-1.15 ;-0.54)$, which was within the pre-specified equivalence limit and suggested significant equivalence (equivalence margin adjusted $P$ value $=0.003)$ between the 2 tests. Model factor estimates indicated trial $(P<0.001$; suggestive of learning effect from trial-to-trial), period $(P<0.001)$ and evaluation sequence $(P=0.038)$ as significant factors. Higher mean scores for each trial were noted in period 2 vs period 1 , suggestive of learning effect from period 1 to period 2, despite the washout period. Sensitivity analyses from repeated measure mixed model supported equivalence between the 2 tests, and analysis using automated scoring from Nuance speech recognition system showed similar results but did not support equivalence claim for SAMSTAR. The 2 tests were also comparable on LOT index and serial position effects with primacy and recency being superior to middle-region in both tests. AEs were reported in 7 (5\%) participants and none led to study discontinuation. One serious AE and no deaths were reported.

Conclusions: Findings from the current study demonstrated equivalence between the iPad computer-administered
SAMSTAR and the examiner-administered RAVLT in terms of the participant's verbal recall performance. Collectively, SAMSTAR provided a comprehensive profile of cognitive abilities related to verbal episodic memory, including learning, recall and susceptibility to proactive interference. Thus, computerized measurements of cognitive attributes using tools such as SAMSTAR may enhance the prospects of yielding standardized, sensitive and reproducible endpoints, assisting large-scale screening during clinical research and optimizing treatment choices in mainstream clinical practice. Keywords: Automated Memory-Screening Test, Mild Cognitive Impairment, Verbal Episodic Memory, Word List Recall Test.

Disclosure: Janssen Research \& Developement, LLC: Full Time Employee, Self.

\section{T95. Upregulation of Glutamate Release in Cortex of Aged PDAPP and tg4510 Mice: Implications for Therapeutics in Alzheimer's Disease}

\section{Xia Li, Scott Geason, James Monn, Jeffrey Witkin*}

Eli Lilly and Company, Carmel, Indiana, United States

Background: Murine models of Alzehimer's disease such as PDAPP and $\operatorname{Tg} 4510$ mice recapitulate some aspects of human disease and disease progression. Hence they have been used to help elucidate the biological underpinnings of this neurodegenerative disorder and to ascertain methods for disease correction. The precise mechanism of neurodegeneration is still not definitively defined. It is, however, well established that glutamate can be neurotoxic. We postulated that excessive glutamate release might be associated with aged and diseased transgenic mice.

Methods: We utilized female PDAPP and Tg4510 mice at young and older ages to create cortical synaptosomes that were from disease-progressing and disease established mice. Cortical synaptasomes were prepared by using the method described in Nature Protocols (3). Briefly mouse cortex were homogenized in $0.32 \mathrm{M}$ sucrose containing protease inhibitors. The homogenate was first centrifuged at $1000 \mathrm{~g}$ for $10 \mathrm{~min}$; the resulting supernatant was layered onto discontinuous sucrose-Percoll gradients and centrifuged at 20,000g for $5 \mathrm{~min}$. Purified synaptosome was collected and resuspended in $\mathrm{HB}$ buffer with composition (in $\mathrm{mM}$ ) (140 $\mathrm{NaCl}, 5 \mathrm{KCl}, 5 \mathrm{NaHCO} 3,1.2 \mathrm{NaH} 2 \mathrm{PO} 4,1 \mathrm{MgCl}$, 10 Hepes, 10 glucose, $\mathrm{pH}$ 7.4). Protein content was measured. Synaptosomal solution were diluted to obtained a final concentration of $0.2 \mathrm{ug} / \mathrm{ml}$ for glutamate evaluation. 50ul samples of synaptosomes were incubated at $370 \mathrm{C}$ for $60 \mathrm{~min}$ with $25 \mathrm{ul}$ of either testing compounds or control per well in a 96 well plate. Synaptosomes were stimulated using $25 \mathrm{ul}$ of $35 \mathrm{mM}$ of $\mathrm{KCl}$ in the presence of $0.2 \mathrm{mM} \mathrm{Ca} 2$ and incubated for a further 8 mins at 370C. Samples were then filtered with $0.22 \mathrm{uM}$ filters and $50 \mathrm{ul}$ of the filtrate was used for detecting of glutamate release by using Amplex Red Glutamic Acid/ Glutamate Oxidase Assay Kit (ThermoFisher) according to the manufactures instruction.

Results: Basal release of glutamate efflux from synaptosomes was increased in both strains and these increases were remarkably exacerbated by age. An mGlu2/3 receptor agonist, LY354740.H20, was able to dampen K+-evoked 
glutamate release. Likewise, LY354740.H20 dosedependently dampened the excessive age-dependent hyperactivity observed in $\operatorname{tg} 4510$ mice, an effect comparable to the antipsychotic drug chlorpromazine.

Conclusions: Enhanced basal glutamate release from cortical synaptasomes is a common age-related phenotype in two commonly used murine models of Alzheimer's disease. The enhanced glutamate release predicted the ability of a presynaptic glutamate braking system to dampen age- and diseaseassociated behavioral abnormalities. Consequently, glutamate dampening methods might function to help symptomatic control of Alzheimer's disease and slow disease progression.

Keywords: Alzheimer's Disease, mGlu 2/3, Behavioral Pharmacology, Neurodegenerative Disease.

Disclosure: Eli Lilly and Company, Employee, Self.

\section{T96. Perinatal Acetaminophen Risk: Characterizing Potential Developmental Risk}

Shona Ray-Griffith*, Prit Gill, Sudeepa Bhattacharyya, Richard Frye, Laura James, D Jeffrey Newport, Zachary Stowe

University of Arkansas for Medical Sciences, Little Rock, Arkansas, United States

Background: The role(s) of perinatal events and fetal exposures on neurodevelopmental trajectory have garnered increased scrutiny. Remarkably, the most common pharmacologic exposure in pregnancy, acetaminophen (APAP), has no established treatment guideline and is often presumed innocuous. Recent studies have found associations with maternal APAP use and fetal respiratory disorders, small testes, and behavioral aberrations. This project sought to 1) elucidate the placental passage of APAP and cysteine protein adducts, 2) explicate if fetal APAP exposure alters sex steroids, and 3) investigate the risk of early neurobehavioral aberrations with maternal APAP use.

Methods: The project uses a clinical biobank of prospectively collected biological samples (maternal serum and urine, cord blood) from a well characterized cohort of women. Women were enrolled prior to 16 weeks' gestation and followed through delivery and the early postpartum period at 4-8 week intervals. At each visit, exposures (prescription, over-the-counter, and environmental) were documented and biological samples were obtained. The Brazelton Neonatal Behavioral Assessment Scale (NBAS) was completed at 7 days of age (corrected for gestational age at delivery), and the women completed the Child Behavior Checklist (CBCL) at 18-36 months postpartum. Across pregnancy, exposure to APAP will be objectively confirmed and quantified in order to separate APAP exposed and non-exposed subjects. Assay results will be utilized as both categorical and continuous (ng/ml) variables. Repeated measures of APAP results will be used to estimate cumulative exposure as area under the curve (AUC) for each subject. APAP exposed and non-exposed groups will be compared regarding demographic characteristics, lifetime psychiatric diagnoses, depression symptoms, and other exposures. To isolate the potential effects of APAP exposure on neurobehavioral outcomes, subjects with major obstetrical complications, thyroid disorders, substance use disorders, and tobacco use will be excluded. APAP exposed and non-exposed subjects will be matched 1:1 for maternal age, maternal education, infant gender, gestational age at delivery, and method of delivery to limit confounds in examining the potential impact of APAP exposure. We will incorporate the extent of exposure, timing (trimester), and peak exposure ( $\mathrm{ng} / \mathrm{ml}$ ) into analyses of the available NBAS and CBCL assessments. Lastly, the relationship between fetal APAP exposure and sex steroids will be elucidated by assaying paired maternal/cord blood collected at delivery from male children for cord blood testosterone and APAP cysteine protein adducts.

Results: Full assay completion is pending at time of submission. Preliminary data derived from a single measure in pregnancy $(n=608)$ indicate: 1$)$ No differences in demographic characteristics or lifetime psychiatric diagnoses between the APAP exposed $(n=143)$ and non-exposed groups $(n=466)$; 2) APAP exposed group demonstrated elevated depressive scores $(\mathrm{BDI}=15.96 \pm 0.90)$ compared to non-exposed $(\mathrm{BDI}=13.51 \pm 9.62)(p=0.015)$; and 3$)$ The APAP exposed group had greater number of exposures to additional classes of medications $(p=0.011)$, including opiates $(p<0.001)$ and sedative-hypnotics $(p<0.001)$. In a pilot sample of paired maternal/cord samples, the cysteine protein adduct concentration in $\mathrm{nM} / \mathrm{ml}$ demonstrated a highly variable placental passage with a higher concentration in the cord blood compared to maternal plasma (ratio [cord] $/[$ maternal plasma] $=2.51 \pm 1.9)$ in the majority of samples (16/18). Upon completion of all assays, remaining analyses will be completed.

Conclusions: The results of the ongoing study have yet to identify a particular demographic profile predictive of APAP utilization. A pattern of additional exposures associated with APAP further confounds the ability to isolate potential independent effects of in utero pharmacological exposures. The extent of placental passage confirms that maternal APAP use is associated with fetal exposure warranting the formulation of clinical guidelines with a focus on administration of APAP during the perinatal period.

Keywords: Pregnancy, Neurodevelopment, Acetaminophen. Disclosure:The project described was supported by 1) the Susan A Hickman Research Award from Postpartum Support International; 2) a NARSAD Young Investigator Grant from the Brain \& Behavior Research Foundation; and 3) the Translational Research Institute, grants UL1TR000039 and KL2TR000063 through the NIH National Center for Research Resources and the National Center for Advancing Translational Sciences. The content is solely the responsibility of the authors and does not necessarily represent the official views of the NIH.

\section{T97. ASD-Associated De Novo Mutations in POGZ Impair the DNA-Binding Activity of POGZ}

Takanobu Nakazawa*, Kensuke Matsumura, Kazuki Nagayasu, Atsushi Kasai, Atsuko Hayata-Takano, Norihito Shintani, Kazuhiro Takuma, Hidenaga Yamamori, Yuka Yasuda, Ryota Hashimoto, Hitoshi Hashimoto

Osaka University, Osaka, Japan

Background: Autism spectrum disorders (ASDs) are neurodevelopmental disorders characterized by impairments in 
social interactions, reduced verbal communication abilities, stereotyped repetitive behaviors and restricted interests. Although genetic studies have identified numerous candidate genetic variants, the genetic etiology of ASD remains poorly understood. Recent studies have demonstrated that de novo mutations contribute to the risk of ASD and often produce large effects. Currently, de novo mutations in multiple unrelated patients have been identified in several genes. Among these high-confidence ASD risk genes, POGZ is one of the most recurrently mutated genes in ASD patients; we and other groups have recently identified at least 18 independent de novo possible loss-of-function mutations in POGZ. Therefore, de novo mutations in POGZ can be possible cause of ASD pathogenesis; however, the biological effects of these mutations remain largely unknown. Here, we present the functional characterization of de novo POGZ mutations identified in sporadic ASD cases.

Methods: POGZ encodes a heterochromatin protein 1 alphabinding protein that contains a zinc-finger cluster, an HP1-binding motif, a centromere protein-B-like DNAbinding (CENPB-DB) domain and a transposase-derived DDE domain. We recently identified a de novo Q1042R amino acid substitution within the CENPB-DB domain of POGZ in a sporadic ASD case. The CENPB-DB domain is likely to be involved in CENP-B box sequence-specific DNA-binding, therefore, this substitution may affect the DNA-binding activity of POGZ. To examine the possibility, we performed DNA-binding experiments using wild-type and Q1042R-mutated and ASD-associated R1008Xmutated POGZ.

Results: We found that wild-type POGZ co-precipitated well with the DNA fragment carrying the CENP-B box sequence, indicating that wild-type POGZ binds the CENP-B box sequence. The Q1042R mutation resulted in a reduction of approximately $60 \%$ in DNA-binding, suggesting that Q1042 is involved in the DNA-binding activity of POGZ. We also examined the DNA-binding activity of ASD-associated R1008X de novo mutated POGZ that lacks the entire CENPB-DB domain. We found that R1008Xmutated POGZ did not co-precipitate with the DNA fragment, indicating that the CENPB-DB domain is important for the DNA-binding activity of POGZ in vitro. Furthermore, we also found that POGZ is highly expressed in the neuron and that POGZ is involved in the neural development.

Conclusions: It has been suggested that POGZ regulates chromatin structure and gene expression. Our results indicate that $\mathrm{ASD}$-associated de novo mutations disrupt the DNA-binding activity of POGZ, an effect likely to result in the perturbation of chromatin function and the neuronal transcription network. Chromatin regulation plays an important role in the ASD-related central nervous system function, therefore, the disruption of chromatin-related mechanisms can cause ASD pathogenesis. Further analysis of the function of de novo mutations in POGZ will provide important clues to understand the molecular link between chromatin remodeling and ASD.

Keywords: Autism Spectrum Disorders, De Novo Mutation, POGZ, Neural Development.

Disclosure: Nothing to disclose.

\section{T98. Identifying Markers to Individualize DBS Targeting in a Rodent Model of Binge Eating}

Wilder Doucette*, Lucas Dwiel, Jared Boyce, Amanda Simon, Jibran Khokhar, Alan Green

Dartmouth Hitchcock Medical Center, Lebanon, New Hampshire, United States

Background: Binge eating is a difficult to treat and problematic behavior that complicates the management of obesity and other psychiatric disorders. The therapeutic potential of neuromodulation targeted to the brain reward circuit in binge eating has been demonstrated in pre-clinical models and in a clinical study using repetitive transcranial magnetic stimulation (rTMS) of the prefrontal cortex. Unfortunately, these studies have demonstrated significant inter-individual variability in treatment outcomes, thus indicating the need to develop individualized neuromodulation-based treatment approaches. This study leverages our prior work which demonstrated that a single site for neuromodulation was not effective at reducing binge sizes across all animals in a rodent model of binge eating. Our prior work demonstrated that the optimal DBS target (nucleus accumbens [NAc] core or shell) varied between animals, with rats responding to DBS in only one of the two targets. The goal of this study is to better understand the source of our previously observed heterogeneity in DBS outcomes. Here we assess the relative contributions of two potential sources of DBS outcome variation: 1) individual differences in brain reward circuit function; and 2) variation in electrode targeting within the NAc sub-regions.

Methods: All experiments were carried out in accordance with the National Institute of Health Guide for the Care and Use of Laboratory Animals and approved by the Institutional Animal Care and Use Committee of Dartmouth College. Adult male Sprague-Dawley rats were implanted bilaterally in both the NAc core and shell $(N=13)$ using custom-built electrode arrays. Following recovery from surgery, rats acquired binge eating through a limited access paradigm until a stable binge baseline was established (previously defined as $<26 \%$ variance from baseline average [2 Std]). Rats were then stimulated bilaterally (biphasic, monopolar, $130 \mathrm{~Hz}, 90 \mu \mathrm{s}, 200 \mu \mathrm{A}$ ) in either core or shell for three intervention binge sessions followed by 2 weeks of sham sessions to re-established baseline. This was then followed by 3 intervention binge sessions in the alternate target. If DBS produced $>26 \%$ ( $>2 \mathrm{Std}$ ) reduction in binge size from baseline on all three intervention days, the rat was characterized as a "responder" to stimulation at that site (core or shell). Animals were then tested on a small battery of reward related tasks (locomotor response to novelty; conditioned place preference; and food-deprived bingeing) and relationships to DBS outcomes were evaluated using linear regression analysis. In addition, local field potentials were recorded from bilateral NAc core and shell during 2 separate recording sessions with rest intervals selected for analysis (video scoring). Total power per frequency band (theta $=4-7 \mathrm{~Hz}$, alpha $=8-13 \mathrm{~Hz}$, beta $=15-30 \mathrm{~Hz}$, low gamma $=45-65 \mathrm{~Hz}$, and high gamma $=70-90 \mathrm{~Hz}$ ) was calculated from each electrode and average coherence per band was calculated between all possible electrode combinations (50 LFP features per animal).Given that there were 
many more predictor variables (LFP features) than observations (DBS outcomes), we used a penalized regression method (elastic net) with a 5-fold cross-validation repeated 1000 times to identify possible predictors and provide an overall misclassification error for each model (core or shell response). Lastly, animals were sacrificed and underwent histological analysis to determine electrode tip coordinates for each animal that were then correlated with DBS outcomes.

Results: Overall, $15 \%$ of rats had significant binge reductions with DBS in both the NAc core and shell, while $69 \%$ had significant reductions with DBS to one of the 2 sites only (shell [38\%] and core [31\%]). Of the behavioral measures, food-deprived bingeing correlated with core DBS outcomes $(\mathrm{R}=0.62, p=0.015)$, and performance in the conditioned place preference task correlated with shell stimulation outcomes $(\mathrm{R}=0.056, p=0.042)$. When DBS outcomes were classified binomially ("responder" and "non-responder") only conditioned place preference showed a trend toward differentiating shell responders from non-responders (unpaired t-test, $p=0.082$ ). Interestingly, the local field potential derived features were able to produce a model that could predict shell DBS response with a $12 \%$ misclassification error and core response with a $4 \%$ misclassification error (core and shell analyzed with separate models). Lastly, while electrode targeting variation along the anterior posterior axis did not correlate with core or shell DBS outcomes (response or non-response), among of the responder sub-group, more anterior electrode placements correlated with larger binge reductions.

Conclusions: Our findings highlight the potential of neuromodulation (DBS) targeted to the reward circuit (NAc) to regulate binge eating. These findings suggest that direct and indirect measures of individual brain reward circuit function could serve as potential biomarkers for individualized target selection. They also suggest that the majority of inter-individual variation in DBS outcomes is likely due to differences in brain function and not variations in precise electrode targeting within each NAc sub-region.

Keywords: Deep Brain Stimulation, Binge Eating, Local Field Potentials, Machine Learning.

Disclosure: Nothing to disclose.

\section{T99. Dysfunction of the Orbitofrontal Cortex in Diet-Induced Obesity}

\section{Lindsay Naef*, Corey Baimel, Stephanie Borgland \\ University of Calgary, Calgary, Canada}

Background: The orbitofrontal cortex (OFC) is involved in the cognitive control of reward processing. It keeps information online and updates behaviour based on changing reward contingencies. Human studies have demonstrated that obesity is associated with lower behavioural adaptation to reward devaluation. The goal of the present experiments was to examine the function of the OFC and to assess reward devaluation in an animal model of dietinduced obesity.

Methods: Mice were maintained on a high-fat $(60 \%$ fat $)$ or low-fat (10\% fat) diet for 12 weeks. They were then trained to lever press for liquid sucrose. Once stable lever pressing was achieved, they were trained on random ratio (RR) schedules of reinforcement and the reward devaluation test was performed. We used in-vitro patch clamp electrophysiology to assess inhibitory synaptic transmission and excitability of lateral OFC neurons. To determine if decreased inhibitory input onto pyramidal neurons leads to impairment in reward devaluation in normal weight animals, we expressed an inhibitory Designer Receptor Exclusively Activated by Designer Drugs (DREADD) in VGAT ires cre mice and performed reward devaluation as described above.

Results: Obese mice display deficits in reward devaluation. This behavioural impairment is associated with reduced GABAergic transmission onto pyramidal neurons in the lateral OFC and increased excitability of pyramidal neurons. Furthermore, inhibition of GABAergic transmission in the lateral OFC by clozapine $\mathrm{N}$-oxide (in DREADD-expressing animals) impairs reward devaluation.

Conclusions: Together, these results demonstrate that obesity induces neuroadaptations in the lateral OFC and alters the cognitive control of reward processing.

Keywords: Obesity, Lateral Orbitofrontal Cortex, Reward Devaluation.

Disclosure: Nothing to disclose.

\section{T100. Cytoplasmic FMR1-Interacting Protein 2 is a} Major Genetic Factor Underlying Binge Eating

Stacey Kirkpatrick, Lisa Goldberg, Neema Yazdani, Richard Babbs, Jiayi Wu, Eric Reed, David Jenkins, Amanda Bolgioni, Kelsey Landaverde, Kimberly Luttik, Karen Mitchell, Vivek Kumar, William Johnson, Megan Mulligan, Pietro Cottone, Camron Bryant*

Boston University School of Medicine, Boston, Massachusetts, United States

Background: Eating disorders are lethal and heritable; however, the underlying genetic factors are unknown. Binge eating is a highly heritable trait associated with eating disorders, obesity, and food addiction and is comorbid with mood and substance use disorders. Furthermore, compulsive binge eating shares several features with addictive behaviors, including an escalation in consumption, physiological and emotional-affective dependence, continued use despite known harm, cue-induced craving, and relapse. Understanding the genetic basis of binge eating will inform therapeutic development and treatment and could be relevant to improving several comorbid neuropsychiatric and physiological conditions.

Methods: We developed a binge eating paradigm in outbred CFW mice in a conditioned place reference (CPP) paradigm of intermittent, limited access $(30 \mathrm{~min})$ to palatable food (PF; 5-TUL diet) over four weeks. Next, we assessed binge eating in closely related $\mathrm{C} 57 \mathrm{BL} / 6 \mathrm{~J}$ and $\mathrm{C} 57 \mathrm{BL} / 6 \mathrm{NJ}$ mouse substrains and in an F2 cross $(N=156)$ between these strains to identify quantitative trait loci (QTL) associated with binge eating. We then used gene-targeted knockout mice in a $2 \times 2$ design (Genotype and Treatment as factors) to validate the causal genetic factor. In this case, we extended our protocol to include the light/dark conflict test to assess compulsive eating and concomitant behaviors. Finally, we used transcriptome analysis of the striatum via mRNA sequencing 
(RNA-seq) from a subset of knockout and wildtype mice to identify the premorbid transcriptome as a function of Genotype and the binge-induced transcriptome as a function of Treatment (PF versus Chow pellets) to inform molecular mechanisms that mediate binge eating susceptibility and establishment, respectively.

Results: Outbred CFW mice showed a significant escalation in PF intake and PF-CPP that correlated highly with increasing $\mathrm{PF}$ consumption. C57BL/6NJ mice, but not $\mathrm{C} 57 \mathrm{BL} / 6 \mathrm{~J}$ mice showed a rapid and robust escalation in $\mathrm{PF}$ consumption and conditioned PF reward. We mapped a single genome-wide significant QTL on chromosome 11 $(\mathrm{LOD}=7.4)$ in both female and male F2 mice to a missense mutation in cytoplasmic FMR1-interacting protein 2 (Сyfip2). Female F2 mice were much more prone to escalating their PF intake and showed the strongest QTL signal as well as increased freezing behavior in the elevated plus maze test of anxiety-like behavior. We subsequently validated Cyfip2 as a causal genetic factor underlying binge eating and compulsive behavior in heterozygous knockout mice on a C57BL/6N background that showed reduced binge eating toward a C57BL/6J-like level. Striatal transcriptome analysis of premorbid genetic risk identified several KEGG biological enrichment terms relevant to the addictions, including "morphine addiction", "retrograde endocannabinoid signaling", "glutamatergic synapse", and "dopaminergic synapse". Furthermore, enrichment analysis of binge eating identified a large set of downregulated genes enriched for decreased myelination, oligodendrocyte differentiation, and expression. Conclusions: We identified Cyfip2 as a genome-wide significant genetic factor underlying binge eating and compulsive behavior and provide a behavioral paradigm for additional genome-wide association studies in mouse populations with increased genetic complexity. Cyfip2 was recently identified in the same genetic cross to underlie differences in cocaine neurobehavioral sensitivity and has been implicated in neurodevelopmental/neuropsychiatric disorders, including schizophrenia and autism. The closely related human gene CYFIP1 is deleted in a subset of patients with a more severe symptomatology in Prader-Willi Syndrome, a neurodevelopmental disorder characterized by extreme hyperphagia. Thus, our results implicate for the first time both CYFIP2 and CYFIP1 in disordered eating in humans. Interestingly, the results of the binge-induced transcriptome suggest that decreased myelination could be a crucial neurobiological adaptation that supports binge eating. In support, multiple human imaging studies have found evidence for perturbations in white matter integrity in patients with eating disorders. Experiments are currently underway to examine binge-induced changes in myelinassociated proteins and whether manipulating these changes can be used to affect the development and treatment of binge eating.

Keywords: GWAS, Binge Eating, Compulsive eating, RNAseq, Oligodendrocytes.

Disclosure: Nothing to disclose.
T101. Relationships Between the Clinical Global Impressions Scale, Binge Eating Days, and the Yale-Brown Obsessive Compulsive Scale Modified for Binge Eating in Adults With Moderate to Severe Binge Eating Disorder in Two Phase 3 Studies

\section{Leslie Citrome*, Judith Kando, Caleb Bliss, Barry Herman}

New York Medical College, Suffern, New York, United States

Background: In two randomized, placebo-controlled, phase 3 studies, lisdexamfetamine dimesylate (LDX) reduced binge eating days/week in adults with moderate to severe binge eating disorder (BED) and was associated with improvement on the Clinical Global Impressions-Improvement (CGI-I) scale. Here, we describe relationships between global clinical disease severity and improvement, as measured by the Clinical Global Impressions-Severity (CGI-S) and CGI-I scales, and the number of binge eating days/week and YaleBrown Obsessive Compulsive Scale Modified for Binge Eating (Y-BOCS-BE) total score in individuals who participated in the aforementioned studies. Similar analyses examining the relationship between clinical scale changes and changes in global clinical disease severity and improvement have been conducted in other disease states, but no such analyses have examined this relationship in BED. The objective of these post hoc analyses was to understand the clinical significance of the baseline relationship and the relationship for the change from baseline between a clinical observation (ie, the number of binge eating days/week) and a clinical rating scale (ie, the CGI-S or CGI-I) in individuals with BED.

Methods: Two 12-week, double-blind, placebo-controlled studies randomized (1:1) adults meeting DSM-IV-TR BED criteria (study $1, N=383$; study $2, N=390$ ) to placebo or dose-optimized LDX (50 or $70 \mathrm{mg}$ ). Eligible participants had protocol-defined moderate to severe BED (defined as a binge eating frequency of $\geq 3$ binge eating days/week for 2 consecutive weeks before baseline and a CGI-S score at screening and baseline of $\geq 4$ ). Assessments included the number of binge eating days/week based on daily binge eating diaries, scores on the CGI-S (1 [normal, not at all ill] to 7 [among the most extremely ill]) and CGI-I (1 [very much improved] to 7 [very much worse]), and Y-BOCS-BE total score (range: 0-40, with higher scores indicating worse symptoms). For these post hoc analyses, data at baseline or at study endpoint (weeks 11-12 for number of binge eating days/week; week 12/early termination for CGI-I; week 12 for Y-BOCS-BE total score) were pooled across studies and treatment arms from participants in the full analysis set (those taking $\geq 1$ dose of study drug and having $>1$ postbaseline primary efficacy assessment) of each study with nonmissing values for both variables in each relationship. Statistical assessments included Spearman correlations and equipercentile linking analyses to obtain scores having the same percentile rank for each variable. Reported $p$ values are not adjusted for multiplicity and are presented for descriptive purposes only.

Results: At baseline, nominally significant correlations were reported for the number of binge eating days/week and CGI$\mathrm{S}$ score $(n=724$, Spearman $\mathrm{r}=0.374, p<0.001)$ and for 
Y-BOCS-BE total score and CGI-S score $(n=721$, Spearman $r=0.319, p<0.001)$. Equipercentile linking analyses at baseline indicated that CGI-S scores of 4 (moderately ill), 5 (markedly ill), and 6 or 7 (severely ill/extremely ill), respectively, corresponded to numbers of binge eating days/week ranging from 1.995 to $4.501,4.502$ to 6.997 , and 6.997 to 7.005 and to Y-BOCS-BE total scores ranging from 7.5 to $21.6,21.6$ to 28.0 , and 28.1 to 39.5 . Nominally significant correlations were reported for changes from baseline at study endpoint in the number of binge eating days/week and CGI-I score $(n=606$, Spearman $\mathrm{r}=0.647$, $p<0.001)$ and for the change from baseline at study endpoint in Y-BOCS-BE total score and CGI-I $(n=617$, Spearman $\mathrm{r}=0.741, p<0.001)$. Equipercentile linking analyses indicated that CGI-I scores of 1 (very much improved), 2 (much improved), 3 (minimally improved), and 4 to 7 (not improved) at study endpoint, respectively, corresponded to changes in the number of binge eating days/week from baseline at study endpoint ranging from -7.005 to -3.502 , 3.502 to $-2.500,-2.499$ to -1.497 , and -1.496 to 3.005 and to changes from baseline in Y-BOCS-BE total score at study endpoint ranging from -36.5 to $-13.9,-13.8$ to $-7.8,-7.7$ to 2.9, and -2.8 to 10.5 .

Conclusions: These post hoc analyses from two phase 3 studies indicate that indices of global disease severity and improvement positively correlate with binge eating behavior and with the obsessive and compulsive features of BED as measured with the Y-BOCS-BE. A decrease in binge eating days/week of approximately 3.5-7.0 days/week from baseline corresponded with a CGI-I score of 1 ("very much improved"). When interpreting these findings, it is important to note that the use of the CGI-S as a proxy for clinical severity could limit the precision of the findings because functional impairment is not taken into account. Furthermore, interpretations should be considered in light of potential ceiling effects, because relatively few participants were categorized as "severely ill" or "among the most severely ill" at baseline, and potential floor effects, because study entry required a binge eating frequency of $\geq 3$ binge eating days/week and a CGI-S score of $\geq 4$.

Keywords: Binge Eating Disorder, Lisdexamfetamine, Rating Scales, Clinical Trial Rating Methods.

Disclosure: Acadia, Alkermes, Allergan, Janssen, Lundbeck, Merck, Otsuka, Pfizer, Shire, Sunovion, Takeda, Vanda: Consultant \& Speaker, Personal Fees, Self; Eli Lilly, Forum, Neurocrine, Noven, Reviva, Teva: Consultant, Self.

T102. Discovery of the First Evidence for a Direct Association Between the Inattentive Symptoms of Attention Deficit Hyperactivity Disorder (ADHD) and Binge Eating: Mediation by Mood and Eating in Response to Internal Hunger and Satiety Signals

Colin Dourish*, Panagiota Kaisari, Suzanne Higgs

P1vital, Wallingford, United Kingdom

Background: Attention Deficit Hyperactivity Disorder (ADHD) has been associated with disordered eating, in particular Binge Eating. However, whether core symptoms of ADHD are specifically associated with subtypes of disordered eating remains unclear. In addition, there has been little or no investigation of mediating factors that may have important implications for treatment. Mood disorders are often associated with both $\mathrm{ADHD}$ and disordered eating. In addition, inattentiveness to internal signs of hunger and satiety may provide another pathway that links ADHD and disordered eating. However, to the best of our knowledge, no study to date has investigated these potential mediation pathways. This study investigated the potential relationships between ADHD symptomatology and binge/disinhibited eating versus restrictive eating and assessed for first time, whether mood and/or reliance on internal hunger and satiety signals mediate any relationship between ADHD symptomatology and disordered eating. The potential influence of gender on the direction and/or strength of any relationship between ADHD symptomatology and disordered eating was also investigated.

Methods: Adult men and women, aged 18-60 years, were invited to take part in an online study through postings on the web and advertisements at the University of Birmingham, UK. Recruitment took place between November 2015 and March 2016. The final sample consisted of 237 individuals (27.4\% male) with a mean age of 26.79 years $(\mathrm{SD}=8.96)$. The majority of the respondents were resident in either Europe (57.4\%) or North America (35.0\%). Symptoms of ADHD were assessed using the Conner's Adult ADHD Rating Scale Self Report-Screening Version. The Binge Eating Scale (BES), the Bulimic Investigatory Test, Edinburgh (BITE), the Loss of Control over Eating Scale (Brief version) the Dutch Eating Behaviour Questionnaire (DEBQ) and the Eating Attitudes Test (EAT-26) were used to assess disordered eating. To evaluate the mediating role of mood in any relationship between ADHD symptomatology and disordered eating a composite measure was created based on the combined score of the Hospital Anxiety and Depression scale (HADS) and the Perceived Stress Scale (PSS) which were used to assess current levels of anxiety and depression and current levels of stress, respectively. The Reliance on Hunger/Satiety cues sub-scale of the Intuitive Eating Scale was used to assess eating in response to internal hunger and satiety signals.

Results: Principal component analysis reduced the disordered eating measures to two components: "binge/disinhibited eating" and "restrictive eating". Together these components explained $73 \%$ of the variance observed. Composite scores were created for each of the two components based on the sum score of the measures, which had their primary loadings on each component. Mediation analyses, controlling for important confounds (age, gender, Body Mass Index, socio-economic status, comorbidities, alcohol use and ADHD medication) revealed that both inattentive and hyperactive/impulsive symptoms of ADHD related to both of the two components of disordered eating. However, a significant direct relationship was identified only for the association between the inattentive symptoms of $\mathrm{ADHD}$ and binge/disinhibited eating $\mathrm{b}=0.36, \mathrm{BCa} \mathrm{CI}[0.02$, 0.70]. Both mood and eating in response to internal hunger and satiety signals were found to be significant mediators of the relationship between the inattentive symptoms of ADHD and disordered eating $(p<0.05)$. Hyperactivity/impulsivity symptoms were indirectly associated with binge/disinhibited and restrictive eating, relationships that were mediated by mood, $b=0.18$, BCa CI $[0.07,0.35]$ and $b=0.13$, BCa CI 
$[0.06,0.23]$ respectively, but not by responses to internal hunger and satiety cues. Gender had no influence on any of the relationships identified between ADHD symptomatology and disordered eating.

Conclusions: This is the first study that was specifically designed to assess the role of the core symptoms of ADHD in the development of disordered eating and the potential mediating factors of any relationships identified. The results show that both the inattentive and hyperactive/impulsive symptoms of ADHD are associated with disordered eating and that these effects are independent of gender. However, most importantly, only the inattentive symptoms of ADHD were found to be directly related to binge eating whereas both the inattentive symptoms and the hyperactive symptoms of the disorder were indirectly related to binge eating and restrictive eating. Mood was identified as a significant mediator of the relationship between ADHD symptomatology and disordered eating suggesting that treatment interventions specially designed to target mood disturbances in ADHD could prevent the development of disordered eating. Our findings show for first time that binge eating is directly associated with inattentive symptoms of ADHD and that this may relate to disturbances in mood and eating in response to internal signals of hunger and satiety. Further investigation of the role of the inattentive symptoms of ADHD in disordered eating may be helpful in developing novel treatments for both ADHD and binge eating.

Keywords: ADHD, Binge Eating, Inattentive Symptoms, Mood, Hunger and Satiety.

Disclosure: P1vital Limited: Employee, Director and Shareholder, Self.

\section{T103. Cortical Morphology in Hair Pulling Disorder: Multi-Site Mega-Analysis}

Samuel Chamberlain*, Dan Stein, Christine Lochner, Nancy Keuthen, Jon Grant

University of Cambridge, Cambridge, United Kingdom

Background: Hair pulling disorder (trichotillomania) is a common but neglected neuropsychiatric disorder, characterized by the repetitive pulling out of one's own hair, leading to significant functional impairment. Trichotillomania may constitute a particularly useful model for understanding brain-behavior relationships in psychiatry, given the relatively specific nature of the symptoms, and the availability of animal models with ostensibly good face validity. Previous studies have explored morphometric brain abnormalities in trichotillomania, but have typically comprised relatively small sample sizes, which would have limited statistical power.

Methods: We contacted authors of all available structural MRI studies of adult trichotillomania patients, based on a PubMed review of the existing literature (conducted August 2016). Authors were invited to contribute de-identified MRI scans from patients and controls for a neuroimaging megaanalysis. Available scans were pooled and pre-processed using standard techniques (skull stripping, identification of grey matter, warping to standard template). Cortical thickness across the whole brain was compared between patients and controls using permutation analysis, as well as volumes of sub-cortical structures, as implemented in Freesurfer software. Relationships between cortical thickness and symptom severity were explored using correlational analyses.

Results: Scans were available from total 61 patients and 59 matched healthy controls. Trichotillomania was associated with morphometric abnormalities across distributed frontostriatal circuitry.

Conclusions: In the largest pooled analysis of trichotillomania MRI data to date, patients exhibited morphometric cortical abnormalities in regions involved in error monitoring and habit generation. Data are discussed in relation to prevailing neurobiological models of Obsessive Compulsive and Related Disorders, and animal models such as the hoxb8 knockout mouse. Future work will evaluate white matter tract abnormalities in patients, and seek to establish a rolling platform for collection of new data from international collaborations in this area.

Keywords: Impulsivity, Structural MRI, Meta-Analysis. Disclosure: Nothing to disclose.

\section{T104. A Double-Blind, Placebo-Controlled Study of Inositol in Trichotillomania}

\section{Jon Grant*}

\section{University of Chicago, Chicago, Illinois, United States}

Background: Trichotillomania is characterized by repetitive pulling that causes noticeable hair loss. Data on the pharmacological treatment of trichotillomania are limited with no clear first-line agent. The goal of the current study was to determine the efficacy and tolerability of inositol in adults with trichotillomania.

Methods: 38 individuals ( 35 women; mean age $=28.9 \pm$ 11.4) with trichotillomania entered a 10 -week, double-blind, placebo-controlled trial to evaluate the safety and efficacy of inositol (dosing ranging from 6 grams/day to $18 \mathrm{grams} /$ day). Subjects were assessed with the Massachusetts General Hospital Hair Pulling Scale, the NIMH Trichotillomania Severity Scale, Clinical Global Impression scale, and measures of depression, anxiety, and psychosocial functioning. Outcomes were examined using a linear mixedeffects model.

Results: Subjects assigned to inositol failed to demonstrate significantly greater reductions on primary or secondary outcomes measures compared to placebo. At study endpoint, $42.1 \%$ of subjects were "much or very much improved" on inositol compared to $35.3 \%$ on placebo.

Conclusions: This study, the first to examine the efficacy of inositol in the treatment of trichotillomania, found no differences in symptom reductions between inositol and placebo. Future studies will have to examine whether inositol may provide promise in controlling pulling behavior in a subgroup of individuals with trichotillomania.

Keywords: Pharmacotherapy, Myoinositol, Trichotillomania. Disclosure: Nothing to disclose. 
T105. Neuronal Correlates of Motivational Sensitivity to Natural and Drug Rewards

Bernadette O'Donovan, Srimal Samaranayke, Rhiannon Robke, Parastoo Hashemi, Pavel Ortinski*

University of South Carolina, Columbia, South Carolina, United States

Background: Individual differences in motivation for natural rewards may predict future response to psychostimulants. Regulation of motivated behavior is known to be dependent on dopamine signaling in the nucleus accumbens (NAc) shell. However, the neuronal mechanisms underlying motivation remain largely unexplored. This study evaluates the mechanisms underlying individual differences in motivation for natural reward and the effect of motivational state on neuronal response to cocaine.

Methods: Rats were identified as high (HighS) and low (LowS) motivated responders based on their performance on a progressive ratio sucrose self-administration task. Following the final behavioral session, dopamine levels in the NAc shell were measured using fast scan cyclic voltammetry (FSCV) and fast scan adsorption controlled voltammetry (FSCAV), or slices containing NAc were prepared and whole-cell patch clamp recordings were performed from NAc shell medium spiny neurons (MSNs). Separate groups of HighS and LowS rats were exposed to "binge" cocaine treatment (5 days of 3 daily injections at 1 hour intervals, $15 \mathrm{mg} / \mathrm{kg}$ i.p) or underwent 14 days of cocaine self-administration training on a fixed ratio schedule of reinforcement (2 hour daily sessions).

Results: HighS rats had elevated levels of phasic and tonic dopamine and slower dopamine clearance in the NAc shell when compared to LowS rats. HighS rats also had more spontaneous dopamine transients. In whole-cell patch clamp recordings from NAc shell MSNs in the slice, MSNs in LowS rats were significantly more excitable than MSNs in HighS rats. This excitability was suppressed following cocaine exposure in both "binge" and cocaine selfadministration paradigms.

Conclusions: We propose that individual differences in motivation for a sucrose reward are linked to alterations in dopamine signaling in the NAc shell and are also associated with susceptibility to cocaine-induced intrinsic plasticity. We are exploring whether motivation for natural reward predicts a pattern of cocaine self-administration and altered dopamine responses to cocaine in the NAc shell.

Keywords: Cocaine, Motivation, Patch Clamp Recording, Voltammetry, Reward System.

Disclosure: Nothing to disclose.

T106. Multimodal Assessment of the Positive Valence Network in Major Depressive Disorder

Sara Weisenbach*, Patrick Pruitt, Marta Pecina, Maggie Sikora, Tiffany Love, Erich Avery, Jon-Kar Zubieta, Robert Welsh

University of Utah/VA Salt Lake City, Salt Lake City, Utah, United States

Background: The Positive Valence System (PVS) is one of the NIMH Research Domain Criteria (RDoC) constructs. It is relevant to aspects of hedonic functioning, including constructing stimulus reward-associations, anticipating reward, motivation to attain a reward, effort (sustained energy) to attain a reward, consummatory pleasure, and integrating feedback regarding reward presence and value (Kring \& Barch. 2014; Rizvi et al, 2016). Neural structures relevant to PVS functioning include the anterior cingulate (ACC), orbital prefrontal cortex, nucleus accumbens (NAcc), ventral pallidum, and midbrain dopamine neurons (Haber \& Knutson. 2010). Disruption to PVS functioning, including devaluation of rewards, difficulty initiating goal-directed activity, and reduced flexibility in learning stimulus-reward contingencies is a well-documented finding in the depression literature (see Sakina et al, 2016 for review). Depression decreases the ability to work hard for desirable rewards (Sherdell et al, 2012) and results in a decreased likelihood of integrating reinforcement history over time when making choices (Pizzagalli et al, 2009). During the resting state, connectivity of the VS with other key regions in the PVS is reduced in the context of depression, and this reduction is positively correlated with depression severity (Satterthwaite et al, 2015) as well as with apathetic symptoms in older adults with Major Depressive Disorder (MDD) (Alexopoulos et al, 2013). While findings are somewhat mixed, fMRI studies of individuals with MDD generally demonstrate hyporesponsivity of striatal brain regions (Pizzagalli et al, 2009; Smoski et al, 2009; Stoy et al, 2011) and hyperresponsivity of prefrontal regions (Knutson et al, 2008) during reward anticipation and outcome. While disruption to the PVS is well documented in MDD, little is known about how multimodal measures of the PVS relate to one another in MDD. The aim of the current study was to measure the PVS multimodally, using self-report, behavioral (i.e., performance-based), and neuroimaging (i.e., resting state and task-based fMRI).

Methods: Forty-one adults with MDD ( $\mathrm{M}$ age $=34.1$ ) completed a battery of self-report questionnaires and neuropsychological tests, then underwent fMRI, during which measurements were acquired at rest and while performing two tasks. For the current study, we measured PVS functioning with the Apathy Evaluation Scale (AES; self-report), the Iowa Gambling Task (IGT; behavioral), activation of the NAcc during anticipation of reward (Monetary Incentive Delay Task), and connectivity of the NAcc with the ACC during the resting state. We then assessed relationships of these measures with one another using Pearson product-moment correlations to ascertain how well they are associated in a depressed sample. Masks for the NAcc $(9,10,-7,8 \mathrm{~mm}$ spheres) and ACC (using Brodmann Areas) were created using the Wake Forest Pickatlas. Activation/connectivity values were extracted using MarsBaR.

Results: Participants reported symptoms of apathy that were, on average, higher than the suggested clinical cut-off of 39-41 (AES $M=46.31, S D=9.2$; Marin et al, 1991). At the same time, performance on the IGT, which measures rewardbased learning, revealed that participants chose a greater number of advantageous $(\mathrm{M}=54.68, \mathrm{SD}=12.14)$ than disadvantageous $(\mathrm{M}=45.32, \mathrm{SD}=12.14)$ decks. Significant activation at the group level during anticipation of monetary rewards was shown in bilateral NAcc (ps $<.001$, uncorrected). During the resting state, the left NAcc demonstrated 
significant connectivity with the left ACC, while the right NAcc showed significant connectivity with the right ACC (ps $<$.001, uncorrected). Relationships among self-report (AES Total Score), performance-based (Advantages-Disadvantages on IGT), resting state fMRI (extracted connectivity values between NAcc and ACC), and task-based fMRI (activation in NAcc) were not significantly correlated with one another. In fact, the correlations were weak, ranging from -.03 to .30 ( $p=.07$, right NAcc connectivity with right ACC and IGT performance).

Conclusions: Despite high self-report of symptoms related to amotivation (i.e., "wanting), depressed individuals demonstrated engagement of the NAcc during anticipation of reward, and showed intact connectivity within the reward system during rest. They also demonstrated the ability to learn in the face of reward on the IGT. The four measures of the PVS used did not relate to each other. This may suggest that each of these measures is tapping a different aspect of PVS functioning that does not predict other aspects. Consistent with RDoC, these findings suggest that multimodal measurement of the PVS is essential to fully comprehend the different facets of this construct in a given individual.

Keywords: Depression, fMRI, Behavior.

Disclosure: Nothing to disclose.

\section{T107. Transcranial Photobiomodulation for the Treatment of Major Depressive Disorder: The ELATED-2 Clinical Trial}

Paolo Cassano*, Samuel Petrie, David Mischoulon, Dawn Ionescu, Cristina Cusin, Husam Katnani, Albert Yeung, Luis De Taboada, Abigail Archibald, Eric Bui, Lee Baer, Trina Chang, Justin Chen, Paola Pedrelli, Lauren Fisher, Amy Farabaugh, Michael Hamblin, Jonathan Alpert, Maurizio Fava, Dan Iosifescu

Massachusetts General Hospital, Boston, Massachusetts, United States

Background: Transcranial Photobiomodulation (t-PBM) with red and near-infrared light can increase brain metabolism and neuroplasticity; it also modulates endogenous opioids, while decreasing inflammation and oxidative stress. $\mathrm{t}-\mathrm{PBM}$ has been shown to penetrate deeply into the cerebral cortex, to modulate cortical excitability and improve cerebral perfusion and oxygenation. t-PBM can also significantly improve cognition in healthy subjects, and in subjects with traumatic brain injury. The safety of t-PBM has been studied in a sample of 1410 stroke patients, where there were no significant differences in rates of adverse events between t-PBM and sham exposure. Preliminary, uncontrolled evidence suggested both an antidepressant and anxiolytic effect of t-PBM in subjects suffering from major depressive disorder (MDD). For the first time we rigorously tested the antidepressant effect of t-PBM.

Methods: We conducted a double-blind, sham-controlled study on the safety and efficacy of t-PBM delivered to the forehead (dorso-lateral prefrontal cortex, dlPFC) twice a week in subjects with MDD. Standard clinical trials exclusionary criteria applied; subjects were allowed into the study if they had failed at most one antidepressant medication and psychotherapy during the current MDD episode. The treatment course was 8 weeks of t-PBM (Omnilux New U - LED, 830nm; 33.2mW/cm2; up to 60J/ $\mathrm{cm} 2 ; 3.4 \mathrm{~kJ}$ per session vs. sham for a total of 16 sessions). The HAM-D17 total score was the primary outcome measure. All reported analyses are unpaired t-test and chisquared test.

Results: Eighteen subjects (9 vs. 9) had received at least 4 $\mathrm{t}-\mathrm{PBM}$ sessions and a post-treatment assessment and were included in the analyses. There were no statistical differences between groups in age $(44.0 \pm 13.1$ (SD) vs. $49.3 \pm 14.2$ in the NIR- and sham-mode groups, respectively), gender (56\% vs. $44 \%$ females), or baseline severity of depression (HAM-D17 was $20.4 \pm 3.3$ versus $19.1 \pm 3.5)$. The NIR-mode group had a slightly earlier age of onset of MDD $(15.3 \pm 7.1$ years versus $30.5 \pm 20.9 ; \mathrm{df}=16, \mathrm{t}=-2.06, p=.055)$, and a higher number of MDD episodes $(4.4 \pm 1.7$ versus $2.7 \pm 1.8 ; \mathrm{df}=16, \mathrm{t}=2.00$, $p=.06)$. Most subjects were not on an antidepressant treatment - else than t-PBM-, in both the NIR- (56\%) and sham-mode (67\%) groups. At endpoint, the mean change in HAM-D17 total score in subjects receiving t-PBM in NIR-mode $(n=9)$ was significantly greater than in subjects receiving sham-mode $(n=9):-11.7 \pm 7.47(\mathrm{SD})$ vs. $-5.3 \pm 7.03$ (LOCF, $\mathrm{df}=16, \mathrm{t}=1.85, p=.04$ ). In the completers, at endpoint, the mean change in HAM-D17 total score in subjects receiving t-PBM in NIR-mode $(n=6)$ was also significantly greater than in subjects receiving shammode $(n=7):-15.7 \pm 4.41(\mathrm{SD})$ vs. $-6.1 \pm 7.86(\mathrm{LOCF}, \mathrm{df}=11$, $\mathrm{t}=2.62, p=.01)$. The effect size for the antidepressant effect of t-PBM, based on change in HAM-D17 total score at endpoint, was 0.87 (Cohen's d). At endpoint, response and remission per the HAM-D17 occurred in 5 out of $9(55 \%)$ subjects in the NIR-mode; in the sham-mode response and remission occurred in 3 and 2 subjects out of 9 , respectively (33\% and $22 \%)$. The timing of response in the NIR-mode was after 2 weeks of t-PBM $(n=3)$ and after 3 and 4 weeks ( $n=1$ for each time point); in the sham-mode it was after 3 , 4 and 5 weeks of t-PBM ( $n=1$ for each time point). At endpoint, $67 \%$ vs. $22 \%$ of the subjects were at least "much improved" according to the CGI, respectively in the NIRand sham-mode. The t-PBM was well tolerated. All adverse events were mild to moderate; none was serious and all but one had resolved at study end. Only one subject in the NIRmode group required dose adjustment due to her irritability, which became first apparent at day 35 (after 8 t-PBM sessions). Only 2 subjects receiving the sham-mode developed an adverse event at least "possibly related" according to the investigators: insomnia at day 28 and headaches at day 14. Instead, in the NIR-mode 5 subjects developed one or more adverse events at least "possibly related" to the intervention: 3 subjects experienced insomnia (at day 0, 2, 14); 3 subjects experienced illusions such as "seeing vivid colors" or "tasting from an ashtray" (at day 0, 0, 7); 2 subjects experienced irritability (at day 14 and 35) and 1 subject experienced headaches at day 2 and abdominal bloating at day 10.

Conclusions: These findings suggest that t-PBM with nearinfrared light could be a novel intervention for patients with MDD. t-PBM is mechanistically different from other existing device-based treatments for MDD, typically employing electro-magnetic modulation. Numerous LED devices are 
FDA-cleared for sale over-the-counter; they are safe for personal use and would represent an inexpensive option for the treatment of MDD. If $\mathrm{t}-\mathrm{PBM}$ were to be confirmed as an effective and safe treatment for MDD, it could be swiftly adopted.

Keywords: Depression, Light Therapy, Neuromodulation. Disclosure: Janssen: Consultancy, Self, Janssen: Patent Filing (not the technology presented in the abstract), Self.

\section{T108. JNJ-54175446, a CNS Penetrant P2X7 Antagonist in Clinical Development for Mood Disorders}

Anindya Bhattacharya*, Brad Savall, Brian Lord, Pascal Bonaventure, Leah Aluisio, Tatiana

Koudriakova, Kia Sepassi, Freddy Schoetens, Marc Ceusters, Nicholas Carruthers, Timothy Lovenberg, Michael Letavic

Janssen, San Diego, California, United States

Background: The ATP-gated ion channel P2X7 has emerged as a potential central nervous system (CNS) drug target based on the hypotheses that pro-inflammatory cytokines such as IL-1 $\beta$ that are released by microglia, may contribute to the etiology of various disorders of the CNS including mood disorders. Emerging science has strengthened the role of P2X7-IL-1 $\beta$ signaling in animal models of depression (Iwata et al 2016. Biol Psychiatry 80(1): 12-22). To that end, we disclose a CNS penetrant, high-affinity and selective P2X7 antagonist JNJ-54175446, that has progressed into clinical studies in healthy volunteers.

Methods: Pharmacology of JNJ-54175446 was evaluated in a series of in vitro, ex vivo, and in vivo assays. Radioligand binding was carried out to evaluate affinity (pKi) using [3H]A804598 and [3H]-JNJ54232334, as described elsewhere (Lord et al 2015. Eur J Pharmacology 765: 551-59). Binding to human and rat brain tissue was assessed by autoradiography. IL-1b release was measured by standard ELISA. Standard ADMET studies were carried out according to industry standards. Anhedonia was measured by sucrose intake in rats subjected to chronic mild stress paradigm.

Results: JNJ-54175446 is a high-affinity, CNS penetrant $\mathrm{P} 2 \mathrm{X} 7$ antagonist. The affinity $(\mathrm{pKi})$ of the compound at the recombinant human and rat $\mathrm{P} 2 \mathrm{X} 7$ was $8.0 \pm 0.1$ and $8.3 \pm$ 0.05 , respectively. The affinity at the native rat brain tissue (cortex) was 8.0 \pm 0.1. As such, in human and rat brain slices, the compound demonstrated concentration dependent binding to brain P2X7 channels. Since LPS primed monocytes release IL-1 in a P2X7 dependent manner, JNJ-54175446 was tested and found to attenuate ex-vivo IL-1 release in human blood with a pIC50 of $8.1 \pm 0.1$. The compound exhibited good drug-like properties and dosed orally, JNJ-54175446 produced excellent bioavailability, good pharmacokinetic properties in rodents, dogs and non-human primates, with a brain:plasma ratio of unity. Brain target engagement was assessed by autoradiography and IL-1 release: for ex-vivo autoradiography, JNJ-54175446 demonstrated an ED50 of $0.46 \mathrm{mg} / \mathrm{kg}$ with plasma concentration of $100 \mathrm{ng} / \mathrm{ml}$; for brain IL-1 modulation, the compound demonstrated dose dependent suppression of IL-1release. JNJ-54175446 eventually progressed into a phase I clinical study after demonstrating adequate safety margins in preclinical toxicology studies. In order to better understand the profile of P2X7 occupancy in the brain needed for efficacy, we dosed rats subjected to chronic stress with oncedaily oral dosing of P2X7 antagonists with different duration of occupancy profiles; compounds that had sustained occupancy of brain P2X7, produced efficacy in the chronic mild stress model of anhedonia. Considering the overall preclinical profile, JNJ-54175446 is a suitable candidate to be evaluated in clinical proof-of-concept studies in mood disorders.

Conclusions: JNJ-54175446 is a CNS penetrant P2X7 antagonist with good drug-like properties, demonstrated dose-dependent target engagement with adequate safety margins to progress into clinical development. The molecule has completed a successful phase I clinical study and will progress into proof-of-concept studies in the near term.

Keywords: Mood Disorder, P2X7, IL-1b, Chronic Mild Stress.

Disclosure: Nothing to disclose.

\section{T109. Molecular Insights of Dysregulated Microrna Network in Locus Coeruleus of Suicide Subjects}

Yogesh Dwivedi*, Bhaskar Roy, Qingzhong Wang, Miklos Palkovits, Gabor Faludi

University of Alabama at Birmingham, Birmingham, Alabama, United States

Background: Norepinephrine (NE), one of three catecholamine neurotransmitters in the brain, is produced primarily by neurons in the locus coeruleus (LC). Retrograde and ultrastructural examinations reveal that the core of the LC and its surrounding regions receive afferent projections from several brain areas which provide multiple neurochemical inputs to the LC with changes in LC neuronal firing making it a highly coordinated event. On the other hand, NE containing fibers from the LC innervate nearly the entire brain. Although NE, NE receptors, and mediated signaling system have been studied in relation to suicide as well as psychiatric disorders that significantly increase the risk of suicide but less is documented for corresponding changes in molecular network within LC. Molecular and cellular events like epigenetic modifications influencing gene expression are the major focus of research in stress-related disorders including major depression and suicide. MicroRNA (miR$\mathrm{NA}$ ) is one of the candidate epigenetic modifiers from small non coding RNA family which has the innate ability to induce disease phenotype by regulating expression of a large number of genes in a cohesive and coordinated fashion. In this study, we examined miRNA networks in LC of depressed-suicide subjects and matched healthy controls.

Methods: The study was approved by the IRB of University of Alabama at Birmingham. Brain tissues were collected from the Lenhossek Human Brain Program, Semmelweis University, Budapest, Hungary. miRNAs in LC of 9 suicide subjects and 11 healthy controls were by analyzing by Low density qPCR based expression array (TLDA). For global analysis, the statistics significance was calculated using the paired t.test. SAM analysis, which estimates statistical significance by subjecting the data to multiple random permutations, was used to analyze significant differences in 
individual miRNAs. Finally, igraph package in $\mathrm{R}$ was used analyze pairwise coexpression relationships where nodes represent miRNAs and edges connect pairs of miRNAs. Two sets of target mRNAs were analyzed: (1) a set of mRNA target gene was prepared using TarBase, Ingenuity Expert Finding and miRecords with experimental validation; (2) another set of mRNA target gene was prepared based on number of conserved targeting site and total context score prediction value with a high-to-moderate degree of $3^{\prime}$ untranslated region-binding specificity with the miRNA seed sequence using TargetScan. The short listed target genes were further analyzed with IPA core analysis module for functional enrichment of target genes deciphering their role in canonical pathway, molecular network along with disease pathway using Fisher Exact Test and $P$-value threshold set at $\leqslant 0.05$. The initial data output from canonical pathway were further filtered by setting the criteria stringently to represent only a few selected pathways related to stress, and psychiatric disorder related pathophysiology.

Results: Global analysis indicated a mixed trend between suicide and control group, however, the trend was towards upregulation especially for plate A $(p=0.000393)$. When tested individually, a total of thirteen miRNAs achieved significance at $p=0.05$ or better. Perturbation analysis, which takes testing of multiple miRNAs into account, identified eight of these miRNAs as significant at a very stringent false discovery rate of $1.52 \%$ (miR-17-5p, miR-20b5p, miR-330-3p, miR-330-3p, miR-541-3p, miR-582-5p, miR-890, miR-1179, miR-1197). Out of a total of 367 pairs of miRNAs that were included in the co-expression analysis displayed a network for the core set of 25 miRNAs that were each pairwise specifically correlated with suicide group, but not with healthy control group. On the other hand, a set of 30 microRNAs formed a very extensive inter-connected network in the control group which was not present in the suicide group. Interaction between altered miRNAs and target genes showed dense interconnected molecular network, in which multiple genes were predicated to be targeted by the same miRNAs. Functional clustering of predicated target genes yielded stress induced disorders such as anxiety, hyperactive behavior, post traumatic disorders that collectively showed the complex nature of suicidal behavior.

Conclusions: Altogether, our study reveals the involvement of LC based dysregulated miRNA network in disrupting cellular pathways associated with suicidal behavior. In addition, correlation analysis revealed the existence of factors that drive co-expression of a group of miRNAs across individuals specifically in the suicide group.

Keywords: Suicide, Depression, Postmortem Human Brain, Locus Coeruelus, MicroRNA.

Disclosure: Nothing to disclose.

\section{T110. Circuit-Specific Role of Ventral Hippocampal} $\Delta$ FosB in Resilience to Social Defeat Stress

Andrew Eagle, Claire Manning, Paula Gajewski, Rachael Neve, Alfred Robison*

Michigan State University, East Lansing, Michigan, United States
Background: The dorsal hippocampus (dHipp) is essential for spatial learning and memory, while ventral hippocampus (vHipp) appears to regulate emotional and motivated behaviors. vHipp efferents modulate reward circuitry and emotional behavior through projections to nucleus accumbens (NAc) and amygdala (Amy), and the vHipp-NAc circuit is critical for resilience to chronic social defeat stress (CSDS; Bagot et al, 2015). However, it is unknown how CSDS alters vHipp gene expression and function, and whether such alterations may underlie resilience. Although the transcription factor $\Delta$ FosB is induced in the hippocampus by CSDS, and its expression in other brain regions regulates mood, the role of $\Delta$ FosB in vHipp function remains unknown. Therefore, we explored the induction and function of $\Delta$ FosB in vHipp efferents to both NAc and Amy. Using a novel dual-virus CRISPR system, we show that silencing of the FosB gene specifically in vHipp-NAc cells increases susceptibility to CSDS, and provide evidence that this occurs through decreases in neuronal excitability.

Methods: Male, C57Bl6/J mice from Jackson Labs were used in this study and all experiments were performed in accordance with Michigan State University IACUCapproved protocols. Chronic Social Defeat Stress (CSDS) and subchronic SDS were performed essentially as previously described (Vialou et al, 2010). Briefly, mice were exposed to aggressors for $5 \mathrm{~min}$ sessions once per day for 10 days while being co-housed but physically separated from the aggressor for the remaining time each day (CSDS), or were exposed to aggressors for three $5 \mathrm{~min}$ sessions in a single day (subchronic SDS). Social interaction tests were performed one day after the final defeat session. The open field apparatus consisted of a custom-made, square white polyvinylchloride foam box $(38 \mathrm{~cm} \times 38 \mathrm{~cm} \times 35 \mathrm{~cm})$ and elevated plus maze was performed using a custom built apparatus based on plans from ANY-maze (www.anymaze. com). Movement was recorded with a digital CCD camera and automated video tracking software package (CleverSys, Inc.). Viral vectors were injected by stereotaxic surgery into vHipp ( $3^{\circ}$ angle; $-3.2 \mathrm{~mm}$ anteroposterior (AP), $\pm 3.4 \mathrm{~mm}$ mediolateral (ML) and $-4.8 \mathrm{~mm}$ dorsoventral (DV)), NAc $\left(10^{\circ} ;+1.6 \mathrm{AP} ; \pm 1.5 \mathrm{ML} ;-4.4 \mathrm{DV}\right)$, or Amy $\left(0^{\circ} ;-1.3 \mathrm{AP} ; \pm 3.4\right.$ ML; -4.5 DV). For immunohistochemistry, animals were transcardially perfused with (and brains were postfixed in) $10 \%$ formalin and $35 \mu \mathrm{m}$ slices were made on a fixed-blade microtome. Slices of vHipp were stained with a polyclonal FosB antibody (5G4, Cell Signaling), and positive cells were counted by a double-blind experimenter. Western blots were performed on tissue punches from vHipp using the same antibody and quantified using ImageJ (www.NIH.gov). For electrophysiology, coronal slices containing vHipp $(250 \mu \mathrm{m}$ thick) were cut in ice-cold sucrose artificial cerebrospinal fluid (ACSF), and recordings were performed at $30-32{ }^{\circ} \mathrm{C}$ in ACSF. vHipp was identified under visual guidance using infrared differential interference contrast video microscopy with a $40 \times$ water-immersion objective (Olympus BX51-WI). Whole-cell voltage-clamp recordings were performed with a computer-controlled amplifier (MultiClamp 700B), digitized (Digidata 1440), and acquired with Axoscope 10.1 (Molecular Devices) at a sampling rate of $10 \mathrm{kHz}$.

Results: We find that multiple FosB gene products, including $\triangle \mathrm{FosB}$, are induced in dentate gyrus (DG), CA1, and CA3 of vHipp by CSDS, and that this induction also occurs in vHipp 
cells projecting to either NAc or Amy. We show that general inhibition of $\Delta$ FosB function throughout the vHipp (but not dHipp) promotes susceptibility to subchronic (microdefeat) stress. Importantly, we find that specific silencing of FosB gene expression in vHipp cells projecting to NAc promotes susceptibility to CSDS, while inhibition in vHipp-Amy cells does not. Conversely, silencing FosB in vHipp-Amy cells promotes anxiety behaviors in the absence of stress, while vHipp-NAc silencing does not. Finally, we show that overexpression of $\Delta$ FosB in both DG and CA1 cells reduces excitability, both in the dorsal and ventral Hipp.

Conclusions: $\triangle F o s B$ is a unique candidate factor for mediating long-term responses to stress and antidepressant treatment because, unlike other immediate early genes, it possesses remarkable stability (weeks in a living brain). Here, we demonstrate that $\Delta$ FosB is induced in the mouse vHipp by stress, and that its expression in vHipp-NAc projecting cells is required for resilience to CSDS. Because we find that $\Delta$ FosB reduces the excitability of Hipp neurons, and because reduced function of vHipp-NAc projections promotes resilience (Bagot et al, 2015), we suggest that vHipp-NAc $\Delta$ FosB may drive the expression of genes critical for cell excitability and subsequent resilience to stress and/or antidepressant function. Thus, vHipp $\Delta$ FosB and its downstream targets may represent novel therapeutic inroads for the treatment or prevention of depression.

Keywords: Ventral Hippocampus, $\Delta$ FosB, Social Defeat Stress, CRISPR, Neurocircuits.

Disclosure: Nothing to disclose.

T111. Cognitive Behavioral Therapy Restores Prefrontal Cortex Activity in Major Depression and Post-Traumatic Stress Disorder: Evidence from Longitudinal Task-Based fMRI

Zhen Yang, Desmond Oathes, Steven Bruce, Theodore Satterthwaite, Phillip Cook, Emma Satchell, Russell Shinohara, Haochang Shou, Yvette Sheline*

University of Pennsylvania, Philadelphia, Pennsylvania, United States

Background: Patients with anxiety disorders endorse elevated depressive symptoms and likewise depressed patients endorse heightened levels of anxiety. Traditionally, major depressive disorder (MDD) and post-traumatic stress disorder (PTSD) have been considered distinct disorders each with its own psychopathology. In the present study, the authors examined whether depression and anxiety share a common dimensional neural substrate, and whether such imaging biomarkers responded similarly to cognitive behavioral therapy. The key question was what are the brain indices of dimensional symptoms in MDD and PTSD and are they corrected by CBT treatment?

Methods: Functional MRI data were collected on the same Siemens 3T Trio scanner during a cognitive/affective task in 19 healthy control subjects as well as in 27 patients with major depressive disorder and 50 posttraumatic stress disorder patients. Patients received 12 weeks of manualized cognitive behavioral therapy by a skilled neuropsychologist (SEB) and both patients and controls were scanned again at 12 weeks using the same task and protocol (17 control, 15
MDD and 16 PTSD at Time 2). For each participant, fMRI task timeseries were process using standard methods in FSL. Primary analyses assessed dimensional brain associations with depression and anxiety symptoms using a voxel-wise Linear Mixed Effects model. We followed up with three ROIbased LME analyses to test for 1) whether the baseline brainsymptom associations differ between MDD and PTSD; 2) whether the brain disruptions in patients identified at baseline were normalized by $\mathrm{CBT}$; and 3) whether CBT effects on brain activation differed between MDD and PTSD. Results: The two primary patient groups showed highly similar patterns of baseline brain relationships with symptoms and brain activation predictors of response to treatment. Task related fMRI BOLD activation was correlated with anxious arousal (MASQ-AA) and depression (MADRS) symptoms in patients in an overlapping region of the lateral prefrontal cortex. An additional region in the medial thalamus was related to depressive symptoms only. These regions all normalized (showed increased activation) following CBT among patients. Adjacent bilateral prefrontal cortex activation at baseline predicted reduced symptoms and response times on the task following treatment. CBT effects on brain activation did not differ between MDD and PTSD with or without comorbid depression.

Conclusions: The results suggest common dimensional deficits associated with anxiety and depression in MDD and PTSD. Further, the results delineate a common set of lateral prefrontal areas across these two disorders contributing to treatment outcome. These results provide empirical evidence to support the value of dimensional approaches to characterize trans-diagnostic symptoms and contributions to CBT treatment outcome. We had the explicit goal of understanding what biological processes are targeted and treated with CBT across affective/anxiety disorders. We provide evidence supporting the need for additional RDoC analyses across disorders and treatment modalities to better inform diagnostic nosology and to improve treatment decisions.

Keywords: Cognitive Control, Depression, PTSD, CBT, Research Domain Criteria (RDoC).

Disclosure: Nothing to disclose.

\section{T112. Ketamine-Mediated Antidepressant-Like Activity} in Mice is Enhanced by Melatonin

Gloria Benitez-King ${ }^{\star}{ }^{\star}$ Rosa Estrada-Reyes, Daniel Quero-Chávez, Ana Dorantes-Barrón, Marcela Valdés-Tovar, Citlali Trueta-Segovia, Margarita Dubocovich

Instituto Nacional de Psiquiatría Ramón de la Fuente Muñíz, Ciudad de México, Mexico

Background: Major depressive disorder (MDD) is a recurrent and disabling psychiatric illness characterized by anhedonia, cognitive impairment, low mood and diminished ability to think and concentrate (Pittengier C. and Duman RS. 2008). Patients with MDD also have low levels of circulating nocturnal plasma melatonin (MEL) and disturbances in circadian rhythms (Bumb JM et al, 2016; Robillard R et al, 2013). MEL mediates neuroprotective actions in rodent experimental models of oxidative stress (Reiter R et al, 2007; 
Gupta YK et al, 2003) and its chronic administration produces antidepressant-like effects in mice (Solberg LC et al, 1999; Ramírez-Rodríguez $\mathrm{G}$ et al, 2014). Current available antidepressants require long term administration (6-8 week) to reach full efficacy, yet a high proportion of patients are resistant to treatment (Trivedi et al, 2006). Ketamine (KET) at non-anesthetic doses induces antidepressant effects within hours after administration (Murrough et al, 2013). However, adverse dissociative and psychotomimetic effects seriously limit its use in the treatment of MDD and resistant MDD (Cooper et al, 2016; Krystal et al, 1994). This study tested the hypothesis that a sub-effective KET dose in combination with MEL would induce antidepressant-like activity in two predictive mice models of depression, the forced swimming test (FST) and the tail suspension test (TST).

Methods: Male Swiss Webster mice (25 - $35 \mathrm{~g})$ obtained from the local vivarium were maintained in an inverted 12:12h light:dark cycle with lights on at 08:00 pm and lights off at 08:00 am. Mice were treated with various combinations of MEL (ip) and/or KET (ip) before the FST or TST performed at ZT18.5 [Zeitgeber Time (ZT); ZT $0=$ Lights $\mathrm{ON}$ ) using the following protocols: a) Single doses of MEL (4 $\mathrm{mg} / \mathrm{kg}$ or $16 \mathrm{mg} / \mathrm{kg})$ or $\operatorname{KET}(1.5,3,10,20$ and $30 \mathrm{mg} / \mathrm{kg})$ administered at ZT18; b) Administration of three doses of vehicle or MEL (ip) $24.5 \mathrm{~h}, 17.5 \mathrm{~h}$ and $0.5 \mathrm{~h}$ before the start of the FST (ZT 18.5) (Martínez-Vazquez et al 2012. J Ethnopharmacol 139: 164-170) with mice receiving (ip) at ZT 18 either b1) vehicle, or b2) a submaximal dose of KET (1.5 mg/ $\mathrm{kg}$ ). Mice were handled in strict accordance with national and international bioethical policies and the general principles of laboratory animal care. All protocols were approved by the Institution's ethics committee. Results were analyzed by One Way ANOVA followed by Bonferroni's test.

Results: Administration of MEL (4 or $16 \mathrm{mg} / \mathrm{kg}$ ) at ZT18 did not affect mice immobility time in the FST $(\mathrm{F}(2,24)=0.0017$, $\mathrm{df}=2, p=0.983$ ), while a single administration of $3,10,20$, and $30 \mathrm{mg} / \mathrm{kg}$ KET significantly decreased immobility time in the FST in a dose-dependent manner. Administration of three doses of MEL (16 mg/kg) 24.5h, 17.5h and $0.5 \mathrm{~h}$ before the FST decreased the duration of immobility by $50 \%$ (F $(2,31)=11.57, \mathrm{df}=2, \mathrm{p} \leq 0.001)$. In the TST both $16 \mathrm{mg} / \mathrm{kg}$ MEL and either dose of KET (3 or $10 \mathrm{mg} / \mathrm{kg}$ ) were able to significantly decrease the immobility time similarly to imipramine (12.5 and $25 \mathrm{mg} / \mathrm{kg}$ ), the antidepressant drug used as reference. A single administration of MEL (4 or 16 $\mathrm{mg} / \mathrm{kg}$ ) in combination with the sub-effective dose of KET $(1.5 \mathrm{mg} / \mathrm{kg})$ at $0.5 \mathrm{~h}$ before the FST induced an antidepressant-like effect in mice compared with the group treated with vehicle $(p<0.001)$. Administration of either dose of MEL under the three- dose administration scheme (4 or $16 \mathrm{mg} / \mathrm{kg}$ ) in combination with $\mathrm{KET}(1.5 \mathrm{mg} / \mathrm{kg})$ produced a significant decrease in the immobility time on the FST $(\mathrm{F}(2,31)=39.00, p<0.001)$. MEL at $4 \mathrm{mg} / \mathrm{kg}$ produced an optimal effect. Neither MEL (4 and $16 \mathrm{mg} / \mathrm{kg}$ ), KET (1.5 and $3 \mathrm{mg} / \mathrm{kg}$ ) or the combination treatments produced changes on ambulatory activity in mice subjected to the open field test $(\mathrm{F}(2,23)=0.545, p=0.589)$.

Conclusions: MEL administered in a single dose is not effective to elicit the antidepressant effect in the FST. However, administration of MEL following a three- dose schedule showed an antidepressant-like effect in both the FST and TST. KET induced antidepressant-like effects in both FST and TST in a dose-response manner. Administration of a sub-effective dose of KET in combination with MEL in either a single or multiple administration scheme significantly augmented the antidepressant-like effect. The results suggest that the use of KET/MEL combination in patients with resistant depression could induce a rapid antidepressant-like effect without behavioral stereotypies, freezing, and/or the ambulatory excitatory behavior caused by KET.

Keywords: Melatonin, Ketamine, Major depression, Forced Swim Test.

Disclosure: Supported by SEP/CONACYT/ Mexico Grants No. 178075 and 252935.

${ }^{\star}$ Equal contribution to the work as first author

T113. A Comparison of Cortical Inhibition in a Sample of Treatment Resistant Depression Patients Pre and Post rTMS Treatment

Daphne Voineskos*, Andrea Levinson, Nigel Rogasch, Yinming Sun, Faranak Farzan, Tarek Rajji, Daniel Blumberger, Zafiris J. Daskalakis

Centre for Addiction and Mental Health, Toronto, Canada

Background: Dysfunctional cortical inhibition (CI) has been postulated as a mechanism through which MDD symptoms are mediated. To index neurophysiological measures of CI (long-interval cortical inhibition (LICI) and the N100 response), TMS is combined with electroencephalography (TMS-EEG) and applied to the cortex. CI deficits have been established in MDD as a whole, however, require replication and more investigation in treatment resistant depression (TRD) specifically how brain stimulation such as rTMS may affect these indices. We hypothesize that indices of CI will TRD patients will exhibit abnormal baseline indices of CI compared to healthy subjects.

Methods: Subjects with TRD were recruited to a clinical trial comparing different forms of rTMS. Prior to the trial, cortical inhibition measures were assessed in the dorsolateral prefrontal cortex through the N100 and LICI TMS-EEG paradigms.

Results: The N100 measure was performed on 35 subjects with TRD. The LICI measure was performed on 21 subjects with TRD. TRD subjects were recruited from a clinical rTMS trial. Statistical analyses are currently being performed to examine cortical inhibition measures before and after a therapeutic course of rTMS.

Conclusions: The N100 measure was performed on 35 subjects with TRD. The LICI measure was performed on 21 subjects with TRD. TRD subjects were recruited from a clinical rTMS trial. Statistical analyses are underway to examine differences in cortical inhibition measures between healthy subjects and those with TRD at baseline, as well as difference in cortical inhibition between rTMS responders and nonresponders.

Keywords: rTMS, Treatment Resistant Depression, Cortical Inhibition.

Disclosure: Nothing to disclose. 
T114. Pharmacogenetic Modulation of the Behavioral Effects of Buprenorphine in a Mouse Model of the OPRM1 (A118G) Polymorphism

Caroline Browne $e^{\star}$ Rebecca Erickson, Julie Blendy, Irwin Lucki

Uniformed Services University of the Health Sciences, Bethesda, Maryland, United States

Background: Pharmacogenetic studies have identified the non-synonymous single nucleotide polymorphism (A118G) in the human mu opioid receptor (MOR) gene (OPRM1) as a critical genetic variant capable of altering the efficacy of opioid therapeutics including morphine and fentanyl. To date few studies have explored the potential impact of the OPRM1 gene on the therapeutic efficacy of buprenorphine (BPN), a potent MOR partial agonist and kappa opioid receptor (KOR) antagonist. As BPN is approved by the FDA for the treatment of opioid addiction and chronic pain, the lack of information pertaining to the OPRM1 A118G SNP in relation to BPN's effects is surprising. Therefore, the goal of this study was to determine whether the common single nucleotide polymorphism in the OPRM1 gene, A118G, alters the efficacy of BPN in behavioral paradigms mediated by MORs.

Methods: All studies were approved by the Institutional Animal Care and Use Committee of the University of Pennsylvania. Mice were generated on a C57BL/6 genetic background, and bred using a heterozygous breeding paradigm. Briefly site-directed mutagenesis introduced the polymorphism into exon 1 of the Oprm 1 gene, where the adenine (A) nucleotide at position 112 was changed to a guanine $(G)$. This substitution is equivalent to that in the human OPRM1 gene at position 118. Female mice with AA, AG and GG genotypes were used for all studies. BPN produced significant antinociception in the hot plate test, reduced the latency to approach and start consuming a palatable food in the novelty induced hypophagia (NIH) test and induced robust psychomotor stimulant hyperactivity immediately following administration. These behavioral effects of BPN are mediated by MORs. Therefore, the impact of the Oprm1 A112G of BPN's effects was evaluated in these tasks and also in the forced swimming test (FST), where BPN produces significant reductions in immobility mediated by KORs.

Results: The maximal analgesic effect of BPN in the hot plate test was obtained in AA mice and significantly blunted in AG and GG mice (Genotype* Treatment Interaction; $F(8$, $156)=2.608, p=0.010$, AG v's AA $p<0.01$ at $3 \mathrm{mg} / \mathrm{kg}$ and GG v's $A A p<0.05$ at 1 and $3 \mathrm{mg} / \mathrm{kg}$ ). Similarly, the BPNinduced reduction of latency to consume food in the NIH test in AA mice was blocked entirely in both heterozygous AG and homozygous GG littermates (Genotype ${ }^{\star}$ Treatment Interaction; $F(2,40)=3.394, p=0.0435$, AA BPN v's AA Vehicle $p<0.05)$. In addition, GG mice exhibited marked reductions in psychomotor stimulant locomotor activity compared to the AA group, diminishing both horizontal (F $(2,72)=3.29, p=0.043$, GG v's AA $p<0.01)$ and vertical activity $(\mathrm{F}(2,72)=4.165, p=0.019 \mathrm{GG}$ v's AA $p<0.05)$. In contrast, reduced immobility in FST, an effect of BPN mediated by kappa opioid receptors, was not affected by genotype (main effect of treatment; $F(2,56)=5.913$, $p=0.018$ ).

Conclusions: These studies demonstrate the ability of the Oprm1 A112G SNP to attenuate the analgesic, anxiolytic and hyperlocomotor effects of BPN. Overall, these data suggest that the OPRM1 A118G SNP will significantly impact the therapeutic efficacy of BPN, especially if employed for chronic pain and opioid dependence. As such, clinical studies are required to fully explore the impact of OPRM1 A118G SNP in different patient populations. These studies will be timely, given the recent effort to repurpose BPN for use as a therapeutic for major depressive disorder. Considering the increasing number of individuals prescribed BPN for its many indications and the large number of individuals diagnosed with depression and comorbid pain/ addiction, such pharmacogenetic studies will provide invaluable assistance in improving clinical outcomes.

Keywords: Pharmacogenetics, OPRM1 A118G, Buprenorphine.

Disclosure: Nothing to disclose.

\section{T115. Real-World Effectiveness of Pharmacological Treatments in Severe Unipolar Depression in a Nationwide Cohort of 123,712 Patients}

Jari Tiihonen*, Markku Lähteenvuo, Fabian Hoti, Pia Vattulainen, Antti Tanskanen

Karolinska Institutet, Stockholm, Sweden

Background: Very little is known about the comparative effectiveness of long-term pharmacological treatments of severe unipolar depression.

Methods: We studied the risk of re-hospitalization during 1996-2012 among all patients who had been hospitalized because of unipolar depression in Finland $(N=123,712$; mean follow-up time 7.7 years), by using nationwide databases for hospitalization and purchased medications. The primary analysis was within-individual analysis, in which each individual was used as his/her own control to eliminate selection bias. The effect of concomitant psychotropic medications and the temporal order of exposure and non-exposure periods were adjusted.

Results: Lithium use was associated with a markedly lower risk of re-hospitalization due to mental disorders (HR 0.47, 95\% CI 0.40-0.55), while antidepressant treatments (1.10, $1.06-1.13)$ and antipsychotic treatments $(1.16,1.12-1.20)$ were not associated with any beneficial effect in this regard. Risk of re-hospitalization was lower during sole lithium therapy $(0.31,0.21-0.47)$ than its concomitant use with other antidepressants $(0.50,0.43-0.59)$. Analysis among incident cases $(N=30,004)$ showed slightly lower re-hospitalization risks during antidepressant $(0.87,0.82-0.93)$ and antipsychotic $(1.03,0.94-1.13)$ use, and markedly lower risks for lithium $(0.31,0.18-0.54)$ than in the total cohort. The only specific agent approaching the effectiveness of lithium was clozapine (HR 0.65, 0.46-0.90 in the total cohort; 0.33, 0.190.58 in the incident cohort). A sensivity analysis for all-cause hospitalizations showed the same rank order as the primary analysis.

Conclusions: Lithium, and especially without concomitant antidepressant, is the most effective long-term treatment for 
severe unipolar depression. Within-individual analysis eliminates selection bias, but since treatments are started when clinical state deteriorates, the HRs may be somewhat overestimated for all treatments. In any case, it is obvious that the effectiveness of antidepressants and ordinary antipsychotics in the maintenance treatment of severe unipolar depression is substantially lower when compared to lithium or clozapine.

Keywords: Depression, Pharmacology, Antidepressant, Lithium, Relapse and Hospitalization.

Disclosure: The Finnish Medicines Agency Fimea, F. Hoffman-La Roche, Organon: Consultant, Self; AstraZeneca: Consultant, Giving expert testimony, Lecture fees, Advisory Board Member, Self; Bristol-Myers Squibb, Lundbeck: Consultant, Giving expert testimony, Lecture fees, Self; Eli Lilly, Janssen-Cilag: Consultant, Giving expert testimony, Lecture fees, Advisory Board Member, Research Collaboration, Self; GlaxoSmithKline: Giving expert testimony, Lecture fees, Self; Otsuka: Giving expert testimony, Lecture fees, Advisory Board Member, Self; Pfizer: Giving expert testimony, Lecture fees, Self; Novartis: Lecture fees, Self; Stanley Foundation, Sigrid Jusélius Foundation: Grant, Self.

T116. Efficacy and Safety of Aripiprazole Once-Monthly in the Maintenance Treatment of Bipolar I Disorder: A Double-Blind, Placebo-Controlled, Randomized Withdrawal Study

Joseph Calabrese*, Raymond Sanchez, Na Jin, Joan Amatniek, Kevin Cox, Brian Johnson, Pamela P Perry, Peter Hertel, Pedro Such, Phyllis M Salzman, Robert D McQuade, Margaretta Nyilas, William H Carson

Case Western Reserve University School of Medicine, Cleveland, Ohio, United States

Background: Bipolar I Disorder (BP-I) is a lifelong illness that requires long-term treatment to prevent future mood episodes. Atypical antipsychotics are widely used in the treatment of BP-I and guidelines support adjunctive as well as monotherapy uses of aripiprazole as a maintenance therapy for bipolar disorder, but primarily in the manic phase of the illness. Oral aripiprazole has demonstrated efficacy in delaying time to recurrence of mood episodes; and it improves clinical outcomes and has a favorable tolerability profile. Aripiprazole once-monthly $400 \mathrm{mg}$ (AOM 400) is an atypical long-acting injectable antipsychotic approved by the FDA in 2013 for the treatment of schizophrenia and is hypothesized to act through partial agonism at dopamine D2 and serotonin 5-HT1A receptors as well as antagonism at serotonin 5-HT2A receptors. With monthly injections, AOM 400 may mitigate the risks associated with treatment nonadherence in BP-I. The objective of this study was to evaluate the efficacy, safety, and tolerability of AOM 400 in the maintenance treatment of BP-I.

Methods: This double-blind, placebo-controlled, randomized withdrawal study (NCT01567527) was designed to assess the time to recurrence of any mood episode in patients with BP-I stabilized on AOM 400 treatment (with optional dose reduction to $300 \mathrm{mg}$ for tolerability). The study included inpatients and outpatients, 18-65 years of age, with a diagnosis of BP-I (DSM-IV-TR criteria). Patients were required to be experiencing a manic episode (per DSM-IVTR criteria) with a Young-Mania Rating Scale (YMRS) total score $\geq 20$ at study entry as well as at least 1 previous manic or mixed episode of severe enough to require hospitalization or treatment with a mood stabilizer or antipsychotic agent. Patients currently experiencing a mixed or depressive episode were excluded. The study comprised a screening phase followed by conversion to oral aripiprazole monotherapy (if needed), an oral aripiprazole stabilization phase of 2-8 weeks, an AOM 400 stabilization phase of 12-28 weeks, and a 52-week double-blind, placebo-controlled, randomized withdrawal phase. The primary efficacy endpoint was the time from randomization to recurrence of any mood episode defined by any of the following criteria: hospitalization for any mood episode, YMRS total score $\geq 15$, Montgomery Asberg Depression Rating Scale total score $\geq 15$, Clinical Global Impression - Bipolar Version-Severity (CGI-BP-S) score $>4$, serious treatment-emergent adverse event (TEAE) of disease worsening, discontinuation due to lack of efficacy/disease worsening, clinical worsening with the need for pharmacological treatment of symptoms, or active suicidality. Time to recurrence was analyzed using a log-rank test while hazard ratios (HR, AOM 400 vs placebo) with 95\% confidence intervals (CI) were estimated using Cox proportional hazards modeling. The proportion of patients with recurrence was analyzed using Fisher's exact test. Adverse events were summarized by frequency and seriousness.

Results: A total of 1175 patients were screened for inclusion with 731 patients enrolling into the study. Of these, 632 patients entered the oral stabilization phase, and 425 patients entered the AOM 400 stabilization phase. Following stabilization, 266 patients were randomized to AOM 400 or placebo, and 102 patients (AOM 400: 48.1\%; placebo: 28.6\%) completed the 52-week randomized phase. In the primary analysis, AOM 400 significantly delayed the time to recurrence of any mood episode during 52 weeks' treatment compared with placebo (HR: 0.451; 95\% CI: [0.299-0.678]; $p<0.0001)$. Additionally, the proportion of patients with recurrence of any mood episode in the randomized phase was significantly lower $(p<0.0001)$ in the AOM 400 group $(35 / 132 ; 26.5 \%)$ than in the placebo group $(68 / 133 ; 51.1 \%)$. The time to recurrence defined by hospitalization was significantly delayed with AOM 400 vs placebo (HR: 0.137 ; 95\%CI: [0.040-0.465]; $p=0.0002)$. Significantly better adjusted mean changes from baseline to week 52 of the randomized phase were observed for AOM 400 vs placebo in CGI-BP-S mania scores with a mixed model repeatedmeasures (least squares mean difference: -0.43 ; 95\%CI [-0.69; -0.17]; $p=0.0011)$. AOM 400 was generally well-tolerated in patients with BP-I, and serious TEAEs in the randomized phase were reported by $10 / 132(7.6 \%)$ patients in the AOM 400 and in $25 / 133(18.8 \%)$ patients in the placebo group. Specific TEAEs reported at rates $\geq 5 \%$ in randomized phase and more frequently with AOM 400 than placebo treatment where weight increased (AOM 400: 23.5\%; placebo: $18.0 \%$ ), akathisia (AOM 400: 21.2\%; placebo: 12.8\%), insomnia (AOM 400: 7.6\%; placebo: 7.5\%), and anxiety (AOM 400: 6.8\%; placebo: $4.5 \%)$.

Conclusions: AOM 400 was efficacious in the treatment of BP-I, preventing recurrence of mood episodes and 
maintaining stability in recently manic patients, and treatment was well-tolerated. The efficacy and tolerability profile for monthly injection of AOM 400 in BP-I is consistent with that of aripiprazole as daily oral administration. These results suggest that AOM 400 is a viable once-monthly option for maintenance treatment in BP-I, preventing reoccurrence of mood episodes.

Keywords: Bipolar I Disorder, Aripiprazole Once-Monthly, Maintenance Treatment, Long-Acting Injectable Antipsychotic.

Disclosure: Otsuka Pharmaceutical Development \& Commercialization, Inc.: Employee, Consultant/Advisory Board Member/Speaker, Self; H. Lundbeck A/S: Employee, Grant Support, Consultant/Advisory Board Member/Speaker, Self; Abbott Laboratories, Pfizer, Inc, AstraZeneca, Bristol-Myers Squibb Company, Teva Pharmaceutical Industries Ltd, Dainippon Sumitomo Pharma Co., Ltd, GlaxoSmithKline, Janssen Pharmaceuticals, Inc., Sunovion Pharmaceuticals Inc., Takeda Pharmaceutical Company Limited: Grant Support, Consultant/Advisory Board Member/Speaker, Self; Eli Lilly and Company, Intra-Cellular Therapies, Inc.: Grant Support, Self; Allergan, Pfizer, Inc., Repligen Corporation, Servier, Solvay Pharmaceuticals, Inc.: Consultant/Advisory Board Member/Speaker, Self; Department of Defense, Health Resources Services Administration, National Institute of Mental Health: Federal Funding, Self.

\section{T117. Electroconvulsive Therapy Modulates Neural Response to Emotional Faces in Depressed Patients: A Randomized Controlled fMRI Study}

Kamilla Miskowiak*, Lars Kessing, Caroline Ott, Julian Macoveanu, Catherine Harmer, Rasmus Revsbech, Hartwig Siebner, Martin Jorgensen

Copenhagen University Hospital, Rigshospitalet, Copenhagen, Denmark

Background: Negative bias in the neurocognitive response to emotional information is a core feature of major depressive disorder (MDD) that is associated with depression severity and risk of relapse. Emerging evidence suggests that reversal of negative bias is a common mechanism for pharmacological and psychological treatments for depression. This study aimed to investigate with functional magnetic resonance imaging (fMRI) whether electroconvulsive therapy (ECT) modulates neural and cognitive processing of emotional information in MDD.

Methods: Twenty-nine MDD patients were randomized to receive one active or a sham ECT session at the beginning of their ECT course in a double-blind, between-groups design. The following day, patients underwent whole-brain fMRI at $3 \mathrm{~T}$ during which time they viewed emotional faces and were given facial expression recognition and faces dot-probe tasks after the scan. Mood symptoms were assessed at baseline, on day 1 and after six ECT sessions.

Results: Data was lost for two patients (sham) and analyses therefore included 27 patients (ECT: $N=15$, sham: $N=12$ ). Electroconvulsive therapy reduced parahippocampal activity, increased superior frontal activity to fearful vs. happy faces and enhanced fear-specific functional connectivity between the amygdala and dorsolateral prefrontal cortex (dlPFC), orbitofrontal and occipito-temporal regions. Across the entire cohort, stronger fear-specific amygdala-occipital coupling was associated with lower fear vigilance. Greater fear-specific amygdala-dlPFC coupling correlated with better subsequent clinical response. These effects occurred in the absence of differences between groups in cognitive performance, mood symptoms or medication.

Conclusions: This exploratory study shows for that first time that ECT modulates neural response to emotional information. The findings suggest that early positive shift in neural response to emotional information may be a common mechanism of distinct treatments for MDD.

Keywords: Functional MRI (fMRI), Major Depression, Electroconvulsive Therapy, Facial Emotional Processing.

Disclosure: Lundbeck: Consultancy fees, Self.

\section{T118. Repetitive Transcranial Magnetic Stimulation in the Olfactory Bulbectomy Mouse Model of Depression}

\section{Alesha Heath*, Avalon Young, Sofia Rinaldi, Kalina Makowiecki, Jennifer Rodger \\ University of Western Australia, Crawley, Australia}

Background: Depression is one of the leading causes of disability worldwide. With $30 \%$ of sufferers not responding to conventional antidepressant treatments there is a growing need for the development of novel therapies to alleviate symptoms in these patients. One of the most promising of these is repetitive transcranial magnetic stimulation (rTMS), however, the mechanisms of action and most effective treatment parameters remain unknown. This study aimed to replicate clinically used methods of rTMS in an animal model of depression and assess behavioural and neurobiological changes.

Methods: C57B1 $\backslash 6 \mathrm{~J}$ mice underwent either surgery to aspirate the olfactory bulbs ( $n=38$, olfactory bulbectomy) or sham surgery ( $n=11$, craniotomy but no aspiration). Mice with olfactory bulbectomy received either rTMS $(n=11)$, sham rTMS $(n=9)$, fluoxetine $(n=9)$ or a vehicle treatment $(n=9)$ administered for four weeks starting two weeks after surgery. rTMS was delivered at $10 \mathrm{~Hz}$ for 3 minutes (1800 pulses) daily. Fluoxetine $(18 \mathrm{mg} / \mathrm{kg})$ was given orally in cookie dough daily. A subset of animals in each group received an injection of EdU two weeks after the start of treatment. The forced swim behavioural test was carried out at three time points: before surgery (baseline), two weeks after surgery to confirm the bulbectomy model, and at the end of the treatment period to assess efficacy. After the final behavioural test, mice were euthanased $(150 \mathrm{mg} / \mathrm{kg}$ pentobarbitone, i.p.) and, serotonin levels in the prefrontal cortex was assessed using an enzyme immunoassay and cell proliferation in the dentate gyrus was assessed using EdU histochemistry.

Results: With respect to baseline values; after the olfactory bulbectomy mice showed significantly higher activity levels in the forced swim test and this was rescued only by rTMS, fluoxetine showed no significant effect in this test. In the prefrontal cortex serotonin levels were significantly increased only in mice treated with fluoxetine, whereas rTMS appeared to increase the survival of newly differentiated cells in the dentate gyrus. 
Conclusions: These results indicate a replication of the therapeutic effect of rTMS treatment in a specific animal model of depression, providing a foundation for exploring the mechanisms of rTMS antidepressant effects in human patients. Our findings showing improved behavioural outcomes in the forced swim test without a significant increase in serotonin levels suggest that rTMS may have a mechanism of action that is distinct from serotonin-related antidepressant treatments, consistent with its beneficial effects in drug resistant patients.

Keywords: Neurostimulation, Depression, Animal Models. Disclosure: Nothing to disclose.

\section{T119. Genome-Wide Association Study of Major Depressive Disorder in European Americans and African Americans}

Li Wen, Yinghao Yao, Thomas Payne, Jennie Ma, Ming $\mathrm{Li}^{\star}$

Seton Hall University, South Orange, New Jersey, United States

Background: Major depressive disorder (MDD) is a common complex mental illness, which poses one of leading causes of preventable deaths and disabilities throughout the world. To date, genome-wide association study (GWAS) for MDD and its-related phenotypes, have not yielded consistent results.

Methods: We conducted a GWAS for the Center for Epidemiological Studies Depression (CES-D) score, a widely used questionnaire for measuring $\mathrm{MDD}$, in European American (EA) and African American (AA) populations, with age, gender, ancestry-informative principal component scores and smoking status as covariates. Considering there existed no evidence for the presence of heterogeneity between the AA and EA samples, we analyzed two samples together with a total sample size of 4,817 .

Results: Our results revealed a genome-wide significant association between a nonsynonymous SNP rs61753730 in FZD6 (frizzled class receptor 6; functions as a negative regulator of the canonical Wnt/beta-catenin signaling cascade) gene that gives rise to a glutamine to glutamate substitution and the CES-D score $(P=8.46 \times 10-9)$. Such a finding has also received independent support from a molecular study using animal model where knockdown of FZD6 gene expression lead to a depressive effect on rat. Due to there were a few SNPs included in the Illumina Infinium HumanExome BeadChip for FZD6 gene, we performed imputation analysis for the gene with IMPUTE2 using 1000 Genomes as references and then conducted gene-based association study using SNP-set Kernel Association Test (SKAT) in the pooled AA and EA samples, which revealed a significant association of the FZD6 gene with the CES-D score $(P<0.01)$. Additional gene-bygene interaction analysis using a generalized multifactor dimensionality reduction (GMDR) algorithm showed a significant interaction between FZD6 and CREB3L1 $(P=$ $0.0016)$ or FZD6 and CREB3L4 $(P=0.002)$. Finally, we showed that tobacco smoking plays a significant role in the association of FZD6 gene with depression where the significant association of the gene with CES-D score was only detected in smoker samples, but not in nonsmoker samples.

Conclusions: Taken together, this study showed that FZD6 gene represents a plausible candidate gene for MDD and further study with a large sample size is warranted.

Keywords: Major Depressive Disorder (MDD), GWAS, CESD Score.

Disclosure: Nothing to disclose.

T120. Selective Dietary Supplementation in Early Postpartum is Associated With High Resilience Against Depressed Mood

Yekta Dowlati*, Arun Ravindran, Zindel Segal, Donna Stewart, Meir Steiner, Jeffrey Meyer

Centre for Addiction and Mental Health, Toronto, Canada

Background: Postpartum depression, the most common complication of childbearing has a $13 \%$ prevalence, but there are no widespread prevention strategies and no nutraceutical interventions have been developed. Postpartum blues is often a prodromal state for postpartum depression, since severe postpartum blues strongly elevates risk for postpartum depression. To counter the effects of the greater than $40 \%$ elevation of monoamine oxidase-A levels that occur during postpartum blues, a dietary supplement kit consisting of monoamine precursor amino acids, tryptophan and tyrosine, and dietary antioxidants was created and key ingredients were shown not to affect their total concentration in breast milk. In a series of studies, we show that oral tryptophan and oral tyrosine supplements do not increase their total concentrations in breast milk; despite the significant increase in maternal plasma. In contrast, some dosing regimen of oral cysteine supplements increase total cysteine concentrations in breast milk yet have a lesser effect on maternal plasma levels. The aim of this open-label study was to assess whether a dietary supplement consisting of tryptophan, tyrosine and blueberry extract + blueberry juice reduces the vulnerability to depressed mood at day- 5 postpartum, the typical peak of postpartum blues.

Methods: Forty-one healthy day- 5 postpartum women successfully completed all study procedures. One group $(n=21)$ received the dietary supplement composed of 2 grams tryptophan, 10 grams tyrosine and blueberry juice with blueberry extract. The control group $(n=20)$ did not receive any dietary supplement. Postpartum blues severity was quantitated by the elevation in depressed mood on the visual analogue scale and the profile of mood state depression scores following the sad mood induction procedure.

Results: Following the sad mood induction procedure, univariate analysis of variance (ANOVA) demonstrated a robust induction of depressed mood on the visual analog scale in the controls but no effect in the supplement group (F $(1,39)=88.33, \quad p<0.001 ; \quad 43.85 \pm 18.98 \mathrm{~mm}$ versus $0.05 \pm 9.57 \mathrm{~mm}$ shift, effect size 2.9). Moreover, the effect of the dietary supplement was assessed as a predictor of change in profile of mood state depression scores and this showed a highly significant effect of group (ANOVA, F $(1,39)=19.81, p<0.001)$. 
Conclusions: This is the first study to investigate the effect of a combination of monoamine precursors, tryptophan and tyrosine, and blueberry extract/juice on the intensity of postpartum blues. This dietary supplement designed to counter functions of elevated monoamine oxidase-A activity virtually eliminated the vulnerability to depressed mood during the peak of postpartum blues. This suggests that this nutraceutical intervention is a highly promising approach to target this prodromal state of postpartum depression.

Keywords: Postpartum Blues, Prodrome, Prevention, Monoamine Oxidase-A, Monoamines.

Disclosure: Nothing to disclose.

\section{T121. Metabolic Outcomes in Response to Chronic Stress and Antidepressant Treatment}

Ma-Li Wong*, Suhyun Lee, Ethan Dutcher, Martin Lewis, Claudio Mastronardi, Julio Licinio

\section{South Australian Health, Adelaide, Australia}

Background: Antidepressant prescribing has risen 400\% since the late 1980s; in parallel, obesity rates have doubled in adults and tripled in childhood during this period. Antidepressants treatment can lead to significant weight gain but the interplay between MDD, obesity and antidepressant treatment is complex. It is conceivable that nearly $25 \%$ of the cases of obesity may be attributable to the association with MDD and antidepressant use. Our lab has developed an animal model to understand the mechanisms of weight recovery after chronic stress, as we hypothesized that the effects of antidepressants on body weight would be unmasked by environmental factors, such as an obesogenic high-fat diet.

Methods: Male Sprague-Dawley rats were subjected to shortterm exposure to recurrent restraint stress and antidepressants (fluoxetine or imipramine) for 2 weeks, followed by long-term high-fat diet intake for 295 days. Animals were then sacrificed and various organs were collected and weighed. Measurements: Body weight, food intake ratio, behavioural testing, and bone weight.

Results: Obesity-prone rats, defined as those within the upper $50 \%$ of body weight gain, treated with fluoxetine (FX) had increased body weight, in comparison to the control group treated with saline and non-restraint control group. The antidepressant-treated groups had significantly lower food intake ratio in comparison to the non-restraint control group. Obesity-prone FX rats had significantly longer body length in comparison to all other groups. Rats in the FX group were significantly less anxious and had heavier bones. New metabolic (leptin, adipose tissue gene expression) and IGF-1 data will be presented.

Conclusions: We present the conceptually novel and medically relevant, data-based conclusion that the interaction of stress, antidepressant treatment, and high-fat diet may have long-lasting body, bone allometric, and metabolic effects. In the context of CDC data showing that $11 \%$ of the US population over 12 years of age is on antidepressants, the clinical and public health implications of these findings are substantial.

Keywords: Antidepressants, Chronic Stress, Body Weight, Adipose Tissue.

Disclosure: Nothing to disclose.
T122. Deep Brain Stimulation in the Neuroanatomical Framework of Medial Forebrain Bundle Generates Antidepressant-Like Phenotype in Rats

Albert Fenoy*, Manoj Dandekar, Vijayasree

Giridharan, Dustin Luse, Carson Hoffmann, Travis Peery, Christian Ruiz, Patrick Cotton, Jair Soares, Joao de Quevedo

University of Texas Health Science Center at Houston, Houston, Texas, United States

Background: Deep brain stimulation (DBS) is currently being investigated as a one of the therapeutic modalities for depressive disorders and treatment-resistant depression (TRD). In recent years, the superolateral branch of the medial forebrain bundle (MFB) has attracted much attention in the treatment of depression. One uncontrolled study targeting the superolateral branch of the MFB reported a rapid antidepressant effect and $85 \%$ response rate. MFB is a key structure of the mesolimbic-dopamine system for appetitive motivation and euphoric feelings, connecting the ventral tegmental area (VTA) to the nucleus accumbens (NAc) and prefrontal cortex (PFC). However, the mechanism underlying such rapid antidepressant effect due to MFB modulation remains speculative. The objective of this study was to examine if DBS of the MFB is an effective treatment modality for depression and to perform biochemical analysis of reward mechanisms associated with dopaminergic system modulation.

Methods: A concentric bipolar electrode (Plastics One, VA) was stereotactically implanted into the left MFB of male Wistar rats. Animals were divided into three groups, shamoperated, DBS-Off (no stimulation), or DBS-On (stimulation for 1 week, $8 \mathrm{hrs}$ /day). Exploratory activity and depressionlike behavior were evaluated using the open-field and Porsolt's forced swim test (FST), respectively, in the rat. Bilateral rat brain samples of the medial prefrontal cortex (PFC), hippocampus, amygdala and NAc were processed with Western blot analysis of markers of dopaminergic activity that included the tyrosine hydroxylase, dopamine transporter (DAT) and dopamine receptors D1 through D5. Protein expressions were then correlated to immobility time during the FST.

Results: Animals undergoing stimulation of the MFB showed significant increases $(p<0.05)$ in swimming time without altering the locomotor activity, relative to the DBSOff and sham stimulation groups. This indicated an antidepressant-like phenotype in the animals. Interestingly, we have seen trends towards an increase in protein levels of dopamine D1, D2, D4 and D5 receptors in the medial PFC following MFB DBS. However, we did not observe an appreciable difference in tyrosine hydroxylase expression in the medial PFC, hippocampus, amygdala and NAc.

Conclusions: MFB-DBS showed behavioral improvement in depression-like phenotype and biochemical alterations of the PFC that demonstrate changes within the circuitry of the brain different from the target area of stimulation. Such observed neurochemical alterations may underlie the therapeutic efficacy of this treatment. In the future, further testing of MFB-DBS in TRD models for antidepressant-like effect will help us advance this intervention for ultimate clinical use. 
Keywords: Deep Brain Stimulation, Treatment Resistant Depression, Medial Forebrain Bundle, Rat Model.

Disclosure: Nothing to disclose.

\section{T123. Neurocognitive Effects of Combined Electroconvulsive Therapy and Venlafaxine in Elderly Adults With Major Depressive Disorder}

Sarah Lisanby, Shawn McClintock*, Rebecca Knapp, Martina Mueller, Robert Greenberg,

Matthew Rudorfer, Mustafa Husain, Richard Weiner, Robert Young, W. Vaughn McCall, Peter Rosenquist, Georgios Petrides, Shirlene Sampson, Joan Prudic, C. Munro Cullum, George Alexopoulos, Samuel Bailine, Elisabeth Bernhardt, Kristen Tobias, Zhi-De Deng, Lauren Liebman, Mimi Briggs,

Emma Geduldig, Styliani Kaliora, Vassilios

Latoussakis, Abeba Teklehaimanot, Charles Kellner

University of Texas Southwestern Medical Center, Dallas, Texas, United States

Background: Major depressive disorder is a chronic neuropsychiatric disease that results in significant health consequences. The rate of MDD in elderly adults has been steadily increasing and unfortunately results in cognitive difficulties, debilitation, morbidity, and mortality. Electroconvulsive therapy (ECT) is a safe and effective antidepressant treatment for MDD, particularly in elderly adults. When combined with pharmacotherapy, ECT may produce greater antidepressant benefits. Also, while ECT is associated with neurocognitive adverse effects, ECT parameters such as right unilateral (RUL) electrode configuration ultrabrief pulse width (UB) help to minimize the impact on cognitive functions. However, there is limited information regarding the neurocognitive effects of combined pharmacotherapy and ECT, particularly with newer parameters, in geriatric depression. Thus, the purpose of this study was to determine the neurocognitive effects of an acute course of right RULUB ECT combined with venlafaxine in elderly adults with MDD.

Methods: The Prolonging Remission in Depressed Elderly (PRIDE) study was a two-phase, multicenter, randomized study of an individualized continuation ECT schedule combined with pharmacotherapy to enhance long-term outcomes in elderly adults with depression. The study design involved two phases. In Phase 1, patients received acute ECT $3 \mathrm{x}$ weekly combined with VLF. In the randomized phase (Phase 2), those who remitted in Phase 1 were randomized to receive pharmacotherapy (VLF and lithium) alone or the combined modalities (pharmacotherapy and continuation ECT). Elderly adults (age $>60$ ) with major depressive disorder, based on the SCID-I or MINI, were recruited and enrolled in the study. All participants provided written informed consent for this IRB approved investigation before completing study procedures. Patients discontinued psychotropic medications within 1-week of starting Phase 1. ECT was provided 3x per week and procedures were standardized as follows: RUL electrode placement using a Somatics Thymatron System IV (Somatics, LLC, Lake Bluff, IL) with an ultrabrief pulse width of $0.25 \mathrm{~ms}$ and current of $0.89 \mathrm{Amps}$ or a MECTA SPECTRUM (MECTA Corporation, Portland,
OR) device with an ultrabrief pulse width of $0.3 \mathrm{~ms}$ and current of 0.8Amps. Dose titration to determine seizure threshold was conducted at the first ECT session. Subsequent treatments were administered at $6 \mathrm{x}$ the seizure threshold. Multiple cognitive domains including attention and processing speed, verbal fluency, verbal learning and memory, autobiographical memory consistency, and executive function were assessed with a neurocognitive battery. Specific neurocognitive instruments included the Autobiographical Memory Interview-Short Form (AMI-SF), California Verbal Learning and Memory Test-II (CVLT-II), Delis-Kaplan Executive Function System (DKEFS) Verbal Fluency Test, Dementia Rating Scale-2nd Edition Initiation Perseveration Index (DRS-2 IP), Stroop Color and Word Test, and Trail Making Test Parts A and B (TMT A and B). The neurocognitive battery was administered at baseline and then within 72 hours following the last ECT session. Descriptive statistics were used to describe the sociodemographic and clinical characteristics of the study population. Means and standard deviations are presented for continuous variables, and frequency distributions are presented for discrete variables. Paired $t$ tests were computed to determine there was a significant change in neurocognitive measure performance from baseline to end and corresponding 95\% confidence intervals were calculated for the difference in means between baseline and end. Statistical significance was defined as a two-sided $p$-value of less than 0.05.

Results: Changes in performance were statistically significantly lower from baseline to end on neuropsychological measures of psychomotor $(p<0.0001)$ and verbal $(p=0.0002)$ processing speed, autobiographical memory consistency $(p<0.0001)$, short-term verbal recall $(p=0.001)$ and recognition $(p=0.0006)$ of learned words, phonemic fluency $(p<0.0001)$, inhibition $(p=0.05)$, and complex visual scanning and cognitive flexibility $(p=0.0004)$. However, in terms of qualitative changes from baseline to end, performance across most neurocognitive measures remained relatively stable and ranged from intact/average to mildly impaired.

Conclusions: This is the first study to characterize the neurocognitive effects of combined RUL-UB ECT and venlafaxine in elderly adults with MDD. While changes in performance on some neuropsychological measures were statistically significant, the majority of neuropsychological indices remained stable. Indeed, only autobiographical memory consistency, phonemic fluency, and complex visual scanning and cognitive flexibility qualitatively decreased from low average to mildly impaired. These findings are consistent with prior research and provide new evidence for the relative neurocognitive advantage of RUL-UB ECT parameters. Future research is warranted to confirm the neurocognitive effects of combined ECT and psychotropic medications, and to determine the association with underlying neural mechanism, and clinical and functional outcomes.

Keywords: Electroconvulsive Therapy, Neurocognition, Major Depression, Late-Life Depression.

Disclosure: Nothing to disclose. 
T124. Change in Leukocyte Telomere Length Over Two Years in a Sample of Individuals With Major Depressive Disorder and Controls

Mary Zeng, Eric Bui, Benjamin Kovachy, Mireya Nadal, David Mischoulon, Maurizio Fava, Immaculata de Vivo, Kwok-Kin Wong, Naomi Simon*

Massachusetts General Hospital, Boston, Massachusetts, United States

Background: Reduced leukocyte telomere length (LTL) has been found to be associated with multiple common diseases of aging, including heart disease, diabetes, and cancer (e.g., Price et al, 2013). More recently, a link has also been suggested between shortened LTL and psychiatric conditions, especially major depressive disorder (MDD) (e.g., Lin et al, 2016; Ridout et al, 2016). These findings suggest that MDD may be a disease of accelerated aging. Prior studies on the association between MDD and LTL were limited by a cross-sectional design, use of banked blood from investigations conducted for other purposes, and use of self-report measures or clinician-rated measures assessed by nondoctoral-level clinicians. The present prospective longitudinal study aims to examine the association between MDD diagnosis at baseline and change in LTL over two years in a well-characterized sample of individuals with or without MDD at baseline.

Methods: Participants were $n=67$ individuals with MDD (M $(\mathrm{SD})$ age $=42.7(13.2) ; 52 \%$ women $)$ and $n=50$ psychiatrically healthy controls $(\mathrm{M}(\mathrm{SD})$ age $=44.1(14.0) ; 58 \%$ women) who participated in a prior study (Simon et al, 2016), and were reassessed at two-year follow-up. Diagnoses were assessed by doctoral-level raters using the Structured Clinical Interview for DSM-IV (SCID; First et al, 2002). At both time points, participants were also assessed for: demographic and clinical characteristics, including smoking status, body-mass index (BMI), and exercise level; MDD symptom severity using the Montgomery Asberg Depression Rating Scale (MADRS; Montgomery and Asberg, 1979); anxiety symptoms using the Hamilton Anxiety Rating Scale (HAM-A; Hamilton. 1959); childhood trauma exposure using the short form of the Early Trauma Inventory SelfReport (ETISR-SF; Bremner et al, 2007); lifetime trauma exposure using the Traumatic Events Questionnaire (TEQ; Vrana \& Lauterbach. 1994); and perceived stress using the Perceived Stress Scale (PSS; Cohen et al, 1983). Participants were asked to provide blood samples at baseline and two years, and LTL was assayed with qPCR. T-tests were used to examine whether change in LTL (calculated with $\mathrm{z}$-scores to allow comparison between LTL measurements at different time points). Pearson's correlations were used to examine whether change in LTL over two years in participants with baseline MDD was significantly associated with baseline, follow-up, and change covariates.

Results: At baseline, there were no significant differences between depressed and non-depressed participants in age, sex, race, ethnicity, or highest educational level. Change in LTL between baseline and 2-year follow-up was significantly different between individuals with MDD and the control group $(\mathrm{M}(\mathrm{SD})=-0.34(1.2)$ vs. $\mathrm{M}(\mathrm{SD})=0.25(1.4), \mathrm{t}(115)=$ $2.45, p<0.05)$. While this change in LTL significantly differed from zero change in the MDD group $(\mathrm{t}(49)=$
$-2.07, p=0.04)$, this was not the case for the this was not true for control group. Further, Pearson's correlation analyses in the MDD group failed to show any significant association between LTL change, and baseline, follow-up, change covariates including age, sex, marital status, MDD and anxiety symptom severity, lifetime depression duration, lifetime substance use disorder, months of antidepressant use, cigarette smoking pack-years, body mass index, exercise level, childhood and lifetime trauma exposure, and perceived stress.

Conclusions: Despite limitations including a relatively small sample size, and the use of z-scores to compare LTL assayed at two different time points, our results provide further support for MDD as a disease of accelerated aging, in a wellcharacterized sample using validated, clinician-rated measures, and suggest an independent mechanism by which MDD might shorten telomeres length.

Keywords: Leukocyte Telomere Length, Depression, Chronic Stress.

Disclosure: Nothing to disclose.

\section{T125. Vortioxetine Versus Placebo in Major Depressive Disorder Comorbid With Social Anxiety Disorder}

Michael Liebowitz*, Jason Careri, Kyra Blatt, Ann Draine, Rita Hanover

Columbia University, New York, New York, United States

Background: Both major depressive disorder (MDD) and social anxiety disorder (SAD) are common psychiatric conditions that can at times be highly disabling. Moreover, they often occur together; SAD is the most common anxiety disorder found in patients with MDD, and individuals with SAD are at increased risk for MDD. Given the prevalence and severity of MDD comorbid with SAD, it is striking that no drug approved for MDD or SAD has been subject to a placebo controlled trial in patients with both disorders. Vortioxetine is a recently marketed antidepressant with activity at the serotonin transporter as well as at several serotonin receptor subtypes. It has documented efficacy in a number of MDD trials, positive findings in one of two GAD trials, and was found effective in reducing anxiety as well as depressive symptoms in depressed patients. We therefore hypothesized that it would be effective in patients with both MDD and comorbid SAD.

Methods: Forty outpatients between the ages of 18 and 70 who met criteria for both MDD and SAD per DSM-5 were randomized on a 1:1 basis to vortioxetine $10-20 \mathrm{mg} /$ day or matching placebo in a 12 week double blind trial. The primary outcome measure was the investigator rated composite CGI-I, with a rating of responder indicated by an endpoint rating of much improved (2) or very much improved (1). These ratings were operationalized as follows: much improved- at least moderate benefit in both MDD and SAD features, or at least marked benefit in of of the two domains if there was no or only minimal benefit in the other; very much improved- marked benefit in both MDD and SAD. Secondary endpoints included the the Montgomery Asberg Depression Rating Scale (MADRS), the Liebowitz Social Anxiety Scale (LSAS), the Hamilton Anxiety Scale 
(HAM-A), the self-rated Sheehan Disability Scale (SDS) and a subject rated global improvement scale (PGIC).

Results: The final patients have entered the trial, and when they complete, vortioxetine and placebo will be compared on the following variables: responder rates (1 or 2 ) on the composite CGI-I, change from baseline to endpoint on MADRS, LSAS, HAM-A, SDS, and PGIC, and time course to response $(50 \%$ or greater reduction in MADRS) and to remission (MADRS 7 or less) for MDD and for remission of SAD (LSAS or 30 or less). Adverse event and medical safety data will also be reported.

Conclusions: The findings should be significant in several ways. In planning this trial, we found that there was no established methodology for conducting treatment trials in MDD comorbid with an anxiety disorder such as SAD. A previous open trial had rated change in each disorder separately, but did not provide any composite measure of overall improvement in individual patients. The present study allowed us to pilot a method for assessing overall improvement in individual patients with MDD comorbid with SAD, and could provide a model for future investigations in this area. If successful, this model should also be transferrable to MDD comorbid with other anxiety disorders, as well to other comorbid conditions in general, a neglected but important field for treatment studies. In addition to guiding future investigations, the study is sufficiently powered to provide informative effect size data that could influence current clinical decision making about the treatment of MDD comorbid with SAD.

Keywords: Psychiatric Comorbidity, Major Depressive Disorder, Social Anxiety, Clinical Trial Methodology.

Disclosure: Takeda Pharmaceuticals: Financial support for the trial via funding of investigator initiated proposal, Self, Holds copyright to the LSAS, Self.

\section{T126. Histone Serotonylation: A Novel Mechanism of Epigenetic Plasticity}

Lorna Farrelly, Robert Thompson, Shuai Zhao, Ashley Lepack, Yang Lu, Olivier Berton, Haitao Li, Tom Muir, Ian Maze*

Icahn School of Medicine at Mount Sinai, New York, New York, United States

Background: Alterations in gene expression promote physiological adaptations implicated in a wide variety of human diseases. More recently, histone mechanisms have been shown to regulate transcriptional programs contributing to neurodevelopmental, neurodegenerative and psychiatric disorders; however, our understanding of how these mechanisms mediate persistent patterns of transcriptional dysfunction remains limited. Monoaminergic signaling in brain plays a critical role in neuronal plasticity, with alterations in monoamine production being implicated in the development and treatment of numerous brain disorders. Although vesicular packaging of monoamines is essential for neurotransmission, recent data have demonstrated the additional presence of 'reserve' pools of extravesicular monoamines in the nucleus of monoaminergic neurons; it remains unclear, however, whether nuclear monoamines play roles independent of neurotransmission. Serotonin, as well as other monoamines, has previously been shown to form covalent bonds with certain cytoplasmic proteins via transamidation by the tissue Transglutaminase 2 enzyme, a modification that alters the signaling properties of modified proteins. We therefore hypothesized that nuclear proteins may similarly be modified to control distinct aspects of their function.

Methods: We employ a large variety of biochemical, biophysical and molecular approaches to fully delineate the functions of protein serotonylation in brain. These approaches include, but are not limited to, enzymatic analyses of covalent serotonin transfer to substrate proteins, mass spectrometry, biophysical interrogations of serotonyl interacting proteins, genome-wide ChIP-/RNA-seq sequencing analyses, molecular manipulations of serotonylation in vitro and in brain, and behavioral analyses.

Results: We have identified histone proteins, specifically histone $\mathrm{H} 3$, as robust substrates for monoaminylation in brain. Our data indicate that $\mathrm{H} 3$ serotonylation acts to facilitate the establishment of permissive histone posttranslational modifications, such as $\mathrm{H} 3 \mathrm{~K} 4 \mathrm{me} 3$, potentiate binding of specific chromatin interacting proteins, and plays a critical role in regulating active transcription via alterations in the deposition/removal of nearby histone/DNA modifications. Furthermore, histone serotonylation appears to be critical during both early neurodevelopment in the establishment of cell-type identity in brain, as well as in the adult central nervous system in the regulation of environmentally-induced neural plasticity (e.g., behavioral responses to stress).

Conclusions: Human neurological diseases associated with monoaminergic dysfunction affect millions of individuals worldwide; however, treatments for these neurodevelopmental, neurodegenerative and/or psychiatric disorders remain inadequate. Employing this unique combination of biochemical, molecular and behavioral approaches, we are examining novel, nuclear-specific roles for monoamines in brain in the direct regulation of neuronal gene expression. Our data suggest that alterations in the expression of these epigenetic marks likely contribute to deficits in neuronal plasticity and the subsequent precipitation of disease states. Therefore, our work aims to fully characterize these so-called histone monoaminylation states in brain, as well as their aberrant regulation in neurological disease, in order to identify novel targets for the development of more effective future therapeutics.

Keywords: Epigenetics, Serotonin, Neuroplasticity.

Disclosure: Nothing to disclose.

\section{T127. Chronic Unpredictable Stress Exposure Augments Habenular 2-Arachidonoylglycerol Signaling}

Anthony Berger, Janelle Lugo, Martin Sticht, Maria Morena, Matthew Hill, Ryan McLaughlin*

Washington State University, Pullman, Washington, United States

Background: The societal and economic burden of major depressive disorder (MDD) is currently at an all-time high, and more efficacious treatment strategies are urgently needed. In recent years, there has been a resurgence of interest in the phylogenetically ancient, epithalamic 
habenular nuclei as a novel putative target for the treatment of MDD. The habenula receives multiple converging inputs from cortical and subcortical structures involved in emotional processing and action selection, the summation of which determines the activity of principal output neurons that negatively regulate midbrain monoaminergic transmission. The lateral subdivision of the habenula $(\mathrm{LHb})$ has been shown to be hyperactive in preclinical models of learned helplessness and clinically depressed individuals (see Proulx et al, 2014, Nat Neurosci for review), while LHb deep brain stimulation produces robust antidepressant-like effects in both rodents (Meng et al, 2011, Brain Res) and treatmentresistant MDD patients (Sartorius et al, 2010, Biol Psychiatry). Moreover, synaptic potentiation within the LHb is positively correlated with the degree of despair-like behavior in preclinical studies via enhanced presynaptic release probability (Li et al, 2011, Nature). Despite these intriguing findings, the mechanisms that confer hyperexcitability of the $\mathrm{LHb}$ remain unknown. We have recently shown that the endocannabinoid (ECB) system in the LHb controls the selection of behavioral coping strategies to aversive environments in preclinical paradigms (Berger et al, under review). However, the impact of chronic stress, which is inextricably linked to MDD pathology, on ECB signaling in the LHb has yet to be empirically evaluated.

Methods: The objectives of the current study were to assess whether exposure to chronic unpredictable stress (CUS) elicits alterations in 1) content of the primary ECBs anandamide (AEA) and 2-arachidonoylglycerol (2-AG), 2) the hydrolytic efficacy of the 2-AG catabolizing enzyme monoacylglycerol lipase (MGL), and 3) mRNA expression for enzymes responsible for the synthesis (NAPE-PLD, DGL $\alpha$ ) and degradation (FAAH, MGL) of AEA and 2-AG in the habenula of adult male and female Sprague Dawley rats. Rats were subjected to six weeks of CUS (2-3 randomized stressors/day) as described previously (McLaughlin et al, 2013, Behav Brain Res). On the morning following the final stressor, rats were rapidly decapitated and the habenula (comprised of both the medial and lateral subdivisions) was dissected, flash-frozen, and stored at $-80^{\circ} \mathrm{C}$ until analysis. ECB content was measured using liquid chromatography-tandem mass spectrometry (LC-MS/MS) as described previously (Qi et al, 2015, Rapid Commun Mass Spectrom). The maximal velocity of 2-AG hydrolysis (Vmax) and binding affinity of MGL for 2-AG (Km) were determined using membrane fractions prepared from homogenized habenula tissue as previously described (Hill et al, 2009, Neuropsychopharmacology). Expression of mRNA for ECB-related enzymes was determined via realtime quantitative polymerase chain reaction (RTqPCR) using a QiaCube robot (Qiagen) and TaqMan primers targeted at ECB proteins, and stress and non-stress groups were compared using the $\Delta \Delta \mathrm{Ct}$ method (Ct values normalized to housekeeping gene Rn45s). All experimental protocols were approved by the Institutional Animal Care and Use Committee and conducted in accordance with the National Institutes of Health Guide for the Care and Use of Laboratory Animals.

Results: Preliminary results indicate that 2-AG content was significantly elevated in the habenula of female (but not male) rats exposed to CUS compared to non-stressed control rats. Although CUS exposure did not significantly affect habenular
AEA content in male or female rats, AEA content was significantly elevated in female rats compared to male rats under basal, non-stress conditions. Moreover, concentrations of AEA and 2-AG were also strongly correlated with adrenal gland weights and body weight gain. With respect to MGL activity, male rats subjected to CUS had significantly elevated Vmax and $\mathrm{Km}$ compared to non-stressed controls, which implies augmented MGL activity but reduced binding affinity of MGL for 2-AG. Lastly, mRNA concentration for MGL was significantly decreased in the habenula of CUS-exposed male rats compared to non-stressed rats. There was also a trend for CUS exposure to decrease both DGL $\alpha$ and NAPE-PLD expression in male rats, although neither reached statistical significance. Studies exploring the effect of CUS exposure on habenular MGL activity and ECB-related mRNA expression in female rats are currently underway.

Conclusions: Our preliminary data suggest that chronic stress exposure preferentially augments 2-AG signaling in the habenula, likely via modulation of MGL-mediated catabolism. These data support the hypothesis that recruitment of ECB signaling in the habenula has detrimental consequences that may contribute to the adoption of a despair-like phenotype. Future work is required to fully delineate the functional impact of augmented habenular 2AG signaling, its influence on the excitability of this nucleus, and its physiological, emotional, and behavioral implications. Keywords: Endocannabinoids, Chronic Stress, Habenula, Rat. Disclosure: Nothing to disclose.

\section{T128. HIV and Symptoms of Depression are Independently Associated With Impaired Glucocorticoid Signaling}

Gretchen Neigh*, Mandakh Bekhbat, C Christina Mehta, Igho Ofotokun, Jennifer Felger, Gina Wingood, Kathryn Anastos, Tracey Wilson, Seble Kassaye, Joel Milam, Bradley Aouizerat, Kathleen Weber, Elizabeth Golub, Michelle Floris Moore, Ralph Diclemente, Margaret Fischl, Mirjam-Colette Kempf, Pauline Maki

Virginia Commonwealth University, Richmond, Virginia, United States

Background: Chronic inflammation caused by HIV infection may lead to deficient glucocorticoid (GC) signaling, thus predisposing people living with $\mathrm{HIV}$ (PLWH) to psychiatric disorders linked to GC resistance, such as depression. The female bias in depression renders women living with HIV particularly vulnerable to developing depression. We hypothesized that comorbid symptoms of depression in PLWH would synergistically associate with deficits in GC signaling without stimulation and when stimulated with a synthetic GC, dexamethasone (Dex).

Methods: This cross-sectional study used PBMCs obtained from participants in the Women's Interagency HIV Study in one of four groups: 1) HIV-neg, non-depressed $(n=32) ; 2$ ) HIV-neg, depressed $(n=32) ; 3)$ HIV-pos, non-depressed $(n=34)$; and 4) HIV-pos, depressed $(n=35)$. Participants with depressive symptoms had a CES-D score $\geq 16$ at the time of sample collection. Following stimulation of PBMCs with 10-8 M Dex for 12 hrs, expression of the target genes 
Fkbp5 and Nr3c1 (GR) was assessed via qPCR. A generalized estimating equation model was used to examine the association between gene expression and HIV status and depressive symptoms, controlling for current pot use.

Results: When unstimulated, cells from patients with depressive symptoms showed increased FKBP5 $(p=0.036)$ and GR $(p=0.005)$, these associations were also present in PLWH $(p=0.041 ; p=0.008)$. Decreased unstimulated GR $(p=0.004)$, but not FKBP5 $(p=0.164)$, was observed in cells from PLWH and symptoms of depression compared to HIV-neg nondepressed participants. In Dex-stimulated cells, depression was associated with decreased FKBP5 $(p=0.006)$ and GR expression $(p=0.001)$ in HIV-neg individuals. In subjects with elevated depressive symptoms, HIV was associated with elevated FKBP5 and GR expression ( $p=0.020 ; p=0.049)$.

Conclusions: These data suggest that both HIV and depressive symptoms are associated with GR and FKBP5 expression but do not appear to exacerbate the impact of either condition when they co-occur.

Keywords: HIV, Depression, Glucocorticoid Receptor, Inflammation, Cytokines.

Disclosure: Nothing to disclose.

T129. Placebo-Induced Changes in Peripheral Levels of $\beta$-Endorphins are Associated With the Neural Representation of Placebo Responses

Marta Pecina*, Joseph Heffernan, Erich Avery, Kristen Villalobos

University of Pittsburgh Medical Center, Pittsburgh, Pennsylvania, United States

Background: The placebo effect is a striking example of how expectations shape outcomes in medicine. A neural and molecular signature of the placebo effect has been previously described in conditions such as pain (Pecina and Zubieta, 2015) and major depression (Pecina et al, 2015), where placebo effects were observed in response to the central release of endogenous opioids. Still, the association between placebo-induced changes in peripheral levels of endogenous opioids and the neural representation of placebo responses in the brain has not been demonstrated. Here, we aimed to investigate whether peripheral measures of endogenous opioids were associated with: 1) the subjective mood improvement in response to an intravenous (i.v.) placebo with expectations of fast-acting antidepressant effects and 2) placebo-induced changes in blood-oxygen-level dependent (BOLD) during an fMRI scanning session. We hypothesized that peripheral increases in $\beta$-endorphins will be associated with: 1) greater placebo responsiveness during the fMRI task and 2) increased BOLD responses in placebo-related networks, mainly the rostral anterior cingulate cortex (rACC), the orbitofrontal cortex (OFC), dorsolateral prefrontal cortex (dlPFC), the anterior and posterior insular cortex (aIns, pIns), the nucleus accumbens (NAcc), the amygdala (Amy), and the periaqueductal grey (PAG).

Methods: Twenty patients (15 females; mean age $=27, \mathrm{~s}$. d. =8.3) with a DSM-5 diagnosis of major depression underwent the "simulated real-time neurofeedback (NF) fMRI task" during the i.v. infusion of a fast-acting antidepressant treatment (i.v. saline: "the placebo"). Briefly, the task included six 12 trial runs, where each trial began with a 10 second timer cue reflecting the anticipation period of the expected drug infusion (infusion/no infusion) followed by 12 seconds of NF signal of different valance (positive/ negative) along with a filling bar graph, representing the drug infusion/no-infusion. After both, the anticipation of drug infusion and the simulated NF signal/drug infusion period, subjects rated their expected and actual mood improvement, respectively. A continuous measure of placebo responsiveness was created based on the patient's subjective mood improvement in response to the positive simulated NF during the administration of the i.v. placebo. $\beta$-endorphin plasma levels were measured before and after the simulated real-time NF task. Neuroimaging data and blood samples were analyzed according to standard procedures. Regions a priori hypothesized were deemed significant at a $p<0.001$ uncorrected (others at $p<0.05$ FWE-corr).

Results: We found no significant relationships between BOLD responses during the placebo real-time NF fMRI task and the overall changes in peripheral $\beta$-endorphin plasma levels. As hypothesized, greater increases of $\beta$-endorphin plasma levels were associated with greater placebo responsiveness $(r=0.55, p=0.02)$. During the anticipation of the drug infusion compared to the no-infusion period, greater placebo-induced increases in $\beta$-endorphin plasma levels were associated with increased BOLD responses in the bilateral DLPFC (left: -34, 26, 50; right: 44, 24, 44). During the i.v. placebo administration + the presentation of the simulated positive NF, greater placebo-induced increases in $\beta$-endorphins plasma levels were associated with decreased BOLD responses in the bilateral aINS (left: $-48,12,-10$; right: 58,12 , 4) and the bilateral DLPFC (left: $-40,40,30$; right: $42,38,32$ ). Conclusions: These results suggest that placebo-induced changes in $\beta$-endorphins plasma levels contribute to understanding the overall neural signature of placebo responses in patients with depression. Increases in $\beta$-endorphins plasma levels correlated positively with BOLD responses in the DLPFC during the anticipation of drug infusion and negatively with BOLD responses in the DLPFC and aINS during the administration of the i.v. placebo + presentation of the simulated positive NF. This evidence suggests a complex role of the opioid system in the modulation of the expectations and outcomes during the administration of placebos. Still, peripheral measures of $\beta$-endorphins may be a potential predictor of brain levels of endogenous opioid release in response to the administration of placebos.

Keywords: Placebo Response, Endogenous Opioids, Major Depressive Disorder.

Disclosure: Nothing to disclose.

T130. Altered Levels of Apoptotic and Anti-Apoptotic Factors in Peripheral Mononuclear Cells of Bipolar Disorder Patients

Joao de Quevedo*, Giselli Scaini, Gabriel Fries, Samira Valvassori, Giovana Zunta-Soares, Jair Soares

University of Texas Health Science Center at Houston, Houston, Texas, United States

Background: Bipolar disorder (BD) is a progressive psychiatric disorder characterized by recurrent changes of mood 
and is associated with cognitive decline. Currently, studies have reported that numbers and sizes of glia and neurons are reduced in brain areas, suggesting the involvement of apoptosis in the pathophysiology of BD. However, the specific processes of apoptosis in BD have not been fully elucidated. To test this hypothesis, we measured protein levels of the pro-apoptotic (Bax, Bad, Bak, Smac, caspase-3) and anti-apoptotic factors (Bcl-xL, Bcl-xL/Bak dimer, survivin, Mcl-1) in peripheral blood mononuclear cells (PBMCs) from BD patients and healthy controls.

Methods: 16 patients with $\mathrm{BD}$ type I and 16 age- and sexmatched healthy controls were recruited from outpatient clinics at the University of Texas Health Science Center at Houston. Human blood samples were collected in heparin collection tubes. Then, PBMCs were separated using LeucoPREP brand cell separation tubes, and intrinsic pathway of apoptosis was assayed using multiplex fluorescent immunoassay kits (Bio-Plex Pro RBM apoptosis assays).

Results: Our results showed that the levels of anti-apoptotic proteins, $\mathrm{Bcl}-\mathrm{xL}$, survivin and $\mathrm{Bcl}-\mathrm{xL} / \mathrm{Bak}$ dimer were significantly decreased in PBMCs from BD patients when compared with HC. With regard to active caspase-3 protein levels, the levels were significantly increased in PBMCs from $\mathrm{BD}$ patients when compared with $\mathrm{HC}$. In contrast, no differences in protein levels of pro-apoptotic factors Bad, Bax, Bad and Smac, as well as in anti-apoptotic proteins $\mathrm{Mcl}$ 1 and $\mathrm{Mcl}-1 / \mathrm{Bak}$ dimer were found between the control and $\mathrm{BD}$ group.

Conclusions: Overall, the data reported here are consistent with the working hypothesis that apoptosis may contribute to cell dysfunction, brain atrophy and progressive cognitive in $\mathrm{BD}$. Moreover, our results indicate that the anti-apoptotic factors are decreased in $\mathrm{BD}$, suggesting that mechanism to compensate for increased neuronal apoptosis fails in $\mathrm{BD}$ patients.

Keywords: Bipolar Disorder, Apoptosis, Peripheral Blood Mononuclear Cells.

Disclosure: Nothing to disclose.

T131. Glutamate Homeostasis in the Adult Rat Medial Frontal Cortex is Modified by Dietary Omega-3 Fatty Acid Intake During Peri-Adolescent Development: An in Vivo 1H MRS Study

\section{Robert McNamara*, Richard Komoroski, Diana Lindquist}

University of Cincinnati College of Medicine, Cincinnati, Ohio, United States

Background: Although $1 \mathrm{H}$ magnetic resonance spectroscopy $(1 \mathrm{H}$ MRS) studies have found that different psychiatric disorders are associated with abnormalities in regional glutamate and glutamine concentrations, the etiological mechanisms remain poorly understood. Psychiatric disorders are also associated with deficits in the long-chain omega-3 fatty acid docosahexaenoic acid (DHA, 22:6n-3) which is the most abundant omega-3 fatty acid in cortical membranes. DHA promotes the functional maturation of cortical astrocytes which regulate glutamatergic homeostasis and recent in vitro evidence suggests that DHA directly modulates astrocyte-mediated glutamate uptake. To investigate the role of cortical DHA on glutamatergic homeostasis in vivo, the present study determined the effects of dietaryinduced alterations in cortical DHA accrual during periadolescent development on glutamate homeostasis in the adult rat brain by $1 \mathrm{H}$ MRS.

Methods: From P21-P90 male rats were fed a diet with no n-3 fatty acids (Deficient, $n=20$ ), a diet fortified with preformed DHA (fish oil, FO, $n=20$ ), or a control diet fortified with alpha-linolenic acid (18:3n-3, $n=20$ )(HarlanTEKLAD, Madison, WI). On P90 1H MRS data were acquired from voxels in the mPFC and thalamus using a 7T Bruker Biospec system (Bruker BioSpin, Ettlingen, Germany). Spectra were imported into LCModel for quantitation and absolute concentration calculated. Primary measures of interest were glutamate, glutamine, glutamate +glutamine (Glx), and the glutamine/glutamate ratio, an index of glutamine synthetase activity. Postmortem PFC and red blood cell (RBC) DHA composition were determined by gas chromatography.

Results: Compared with controls, PFC and RBC DHA levels were significantly lower in rats fed the $\mathrm{n}$-3-free diet and significantly higher in rats fed the FO diet. Among all rats $(n=60)$ PFC and RBC DHA levels were positively correlated $(\mathrm{r}=+0.96, p=0.0001)$. In the mPFC, there was a significant main effect of diet for glutamate $(p=0.006)$ which was significantly higher in DHA-deficient rats compared with rats fed the FO diet $(+12 \%, p=0.003)$ and controls $(+7 \%$, $p=0.05$ ). There were no significant group differences for glutamine $(p=0.69)$ and there was a trend for Glx $(p=0.09)$. The glutamine/glutamate ratio was lower in DHA-deficient rats compared with controls $(-10 \%, p=0.05)$ and rats fed the FO diet $(-10 \%, p=0.04)$. Among all rats $(n=60)$ glutamate concentrations in the mPFC were inversely correlated with PFC ( $\mathrm{r}=-0.57, p=0.0002)$ and RBC $(\mathrm{r}=-0.36, p=0.009)$ DHA levels. In the thalamus, there were no significant group differences in glutamate $(p=0.54)$, glutamine $(p=0.78)$, Glx $(p=0.56)$, or the glutamine/glutamate ratio $(p=0.72)$. We are currently investigating whether alterations in mPFC glutamate concentrations are associated with changes in the expression of astrocyte glutamate transporters and glutamine synthetase.

Conclusions: Cortical DHA accrual during peri-adolescent development is an important determinant of prefrontal, but not thalamic, glutamatergic homeostasis in adult rats. Deficits in rat cortical DHA accrual recapitulates elevated glutamate concentrations frequently observed in patients with psychiatric disorders, and increasing cortical DHA accrual may represent a novel strategy to promote glutamate homeostasis. Studies are warranted to determine whether increasing DHA intake and biostatus can modify prefrontal glutamate activity in adolescents with or at risk for psychiatric disorders.

Keywords: Omega-3 Fatty Acid, Medial Prefrontal Cortex, 1H MRS.

Disclosure: Nothing to disclose. 
T132. A Randomized, Double-Blind, Parallel-Group, Placebo- and Active-Controlled Study to Evaluate the Efficacy and Safety of MIN-117 in Patients With Major Depressive Disorder

Michael Davidson*, Corinne Staner, Jay Saoud, Nadine Noel, Sandra Werner, Elisabeth Luthringer, Joe Reilly, Remy Luthringer

\section{Tel Aviv University, Tel Aviv, Israel}

Background: The usefulness of the currently available SSRIs and SNRIs is limited by their delayed onset of action, relatively poor tolerability and large proportion of nonresponders. Attempts to prove the efficacy of ketamine-like drugs and electrophysiological interventions are still under investigation. Hence, broadly effective, well-tolerated antidepressants with rapid onset of actions are an urgent priority. MIN-117 is a potential antidepressant drug with a differentiated mechanism of action targeting adrenergic alpha 1a, alpha $1 \mathrm{~b}, 5-\mathrm{HT} 1 \mathrm{~A}$, and 5-HT2A receptors, as well as serotonin and the dopamine transporter.

Methods: This four-arm, parallel group, randomized double blind, placebo and positive-controlled trial tested two doses of MIN-117: $0.5 \mathrm{mg}$ and $2.5 \mathrm{mg}$. The study included 84 patients (21 per arm) with moderate to severe MDD in three European countries. The antidepressant, paroxetine, was used as an active control to confirm assay sensitivity. Change on the Montgomery-Asberg Depression Rating Scale (MADRS) was used as the main outcome measurement. As established prospectively in the statistical analysis plan, this trial was designed for signal detection and effect size estimation. As such, it was not powered to demonstrate statistically significant differences between MIN-117 and placebo.

Results: A dose-dependent superiority of MIN-117 over placebo as measured by change in the MADRS was demonstrated. MIN-117 at the $0.5 \mathrm{mg}$ daily dose had an effect size (ES) as compared to the placebo group of 0.23 while the $2.5 \mathrm{mg}$ dose had an ES of 0.33 . These ES(s) are similar to those observed with currently marketed antidepressants. Improvement in MADRS with MIN-117 against placebo was observed at two weeks. Furthermore, $24 \%$ of the patients treated with $2.5 \mathrm{mg}$ of MIN-117 achieved remission as prospectively defined. Paroxetine also differentiated from placebo, confirming assay sensitivity. Both doses of MIN-117 demonstrated a favorable tolerability profile, and the incidence and types of side effects did not differ significantly between the MIN-117 group and the placebo group. No unexpected adverse events were reported. Treatment with MIN-117 was not associated with cognitive impairment, sexual dysfunction, suicidal ideation or weight gain. MIN-117 preserved sleep continuity and architecture and therefore is not expected to have detrimental effects on rapid eye movement sleep distribution and duration unlike most marketed antidepressants.

Conclusions: MIN-117 may be an effective, well tolerated antidepressant with a novel mechanism of action that addresses certain shortcomings of currently available antidepressants.

Keywords: Depression, Treatment, Alpha 1A, Alpha 1B, 5-HT1A, and 5-HT2A Receptors.

Disclosure: Minerva Neuroscience: Consultant fee and stock, Self.
T133. Verbal Memory Impairments in Bipolar Disorder; Effect of Type of Word Learning Tasks

Tomiki Sumiyoshi*, Atsuhito Toyomaki, Naoko Kawano, Tomoko Kitajima, Ichiro Kusumi, Norio Ozaki, Nakao Iwata, Kazuyuki Nakagome

National Center of Neurology and Psychiatry, Tokyo, Japan

Background: Disturbances of cognitive functions, including verbal (learning) memory, in bipolar disorder have attracting interest. The degree of the impairment varies across studies, depending on several factors, including stage of the illness, mood state, medications, and etc. The present study was conducted to determine if type of word learning tasks affects severity of cognitive decline in patients with bipolar disorder. Methods: Thirty-six patients with bipolar disorder with mild symptoms and 42 healthy volunteers participated in the study. We first compared effect sizes for memory deficits in patients among the California Verbal Learning Test (CVLT)II, Brief Assessment of Cognition in Schizophrenia (BACS) List-Learning, and Hopkins Verbal Memory Tests-Revised (HVLT-R). We next evaluated the correlations between scores of the CVLT-II vs. those of the BACS and HVLT-R. Bipolar patients were re-assessed with the same (standard) or alternate forms of the CVLT-II and HVLT-R one month later.

Results: Scores on the CVLT-II 1-5 Free Recall and Longdelay Free Recall, and the HVLT-R Immediate Recall, but not the BACS List Learning were significantly lower for patients compared to control subjects. The effect sizes for cognitive decline due to the illness were comparable when measured by the CVLT-II and HVLT-R, ranging from 0.5 to 0.6. CVLT-II scores were significantly correlated with those of the HVLT-R and BACS.

Conclusions: These results suggest the degree of verbal memory deficits in bipolar disorder depends on type of word learning tasks. The ability of the CVLT-II and HVLT-R, but not BACS List Learning to discriminate between patients and control subjects may be related to the use of memory organization strategy specific to the former word list tasks.

Keywords: Bipolar Disorder, Learning and Memory, Memory Organization, California Verbal Learning Test.

Disclosure: Nothing to disclose.

T134. Efficacy and Safety of Intranasal Esketamine for the Rapid Reduction of Symptoms of Major Depressive Disorder, Including Suicidal Ideation, in Patients Assessed to be at Imminent Risk for Suicide: A Proof-ofConcept Study

Carla Canuso*, Jaskaran B Singh, Maggie Fedgchin, Larry Alphs, Rosanne Lane, Pilar Lim, Christine Pinter, Husseini Manji, Wayne Drevets

Janssen Research \& Development, LLC, Titusville, New Jersey, United States

Background: Patients with major depressive disorder (MDD) have an increased risk of suicide that is 20 times greater than that of general population. The standard of care (SoC) for patients with MDD who are at imminent risk for 
suicide includes hospitalization and treatment with conventional antidepressants for underlying depression. However, hospitalizations are temporary and do not eliminate the risk of suicide, and conventional antidepressant pharmacotherapies show a delayed onset of action that limits their utility in treating vulnerable patients. Recent clinical studies of the $\mathrm{N}$-methyl-D-aspartate receptor antagonist ketamine and one of its active isomers, esketamine (ESK), have suggested rapid onset of antidepressant action. In addition, preliminary studies of intravenous ketamine demonstrated rapid reduction of suicidal ideation (SI) in patients with MDD. Intranasal ESK is currently in development for the rapid reduction in symptoms of $\mathrm{MDD}$, including SI, in patients who are assessed to be at imminent risk for suicide.

Methods: The PeRSEVERe trial was a randomized, doubleblind (DB), placebo-controlled, multicenter, phase 2, proofof-concept study. Eligible patients (aged 18 to 64 years) who met the inclusion criteria of having a DSM-IV diagnosis of MDD as well as active SI and intent as confirmed by the Mini International Psychiatric Interview (MINI) and of being in need of acute psychiatric hospitalization, were enrolled. The study consisted of a screening phase (24 to 48 hours), a DB treatment phase (day 1 to 25), wherein patients were randomized (1:1) to intranasal ESK $(84 \mathrm{mg})$ or intranasal placebo, administered 2 times/week, and a post-treatment follow-up phase (day 26 to 81 ). All patients received SoC treatment, including hospitalization and oral antidepressants. The primary endpoint for ESK $84 \mathrm{mg}+$ SoC (ESK 84 $\mathrm{mg}$ ) vs placebo+SoC (placebo) was change from baseline (day 1, predose) to 4 hours postdose on day 1 in MADRS total score. Secondary and exploratory endpoints included change from baseline to day $2(\sim 24$ hours postdose) in MADRS total score, change from baseline to 4 hours postdose and day 2 in MADRS suicide item and in Clinical Global Judgment of Suicide Risk (CGJ-SR) measured using the Suicide Ideation and Behavior Assessment Tool (SIBAT), as well as response rate ( $\geq 50 \%$ improvement on MADRS) and remission rate (MADRS total score $\leq 12$ ) at day 2 . Safety included assessment of treatment-emergent adverse events (TEAEs), vital signs and Clinician Administered Dissociative States Scale (CADSS) total score. Statistical analyses were performed using analysis of covariance models; as a proofof-concept study, a two-sided 0.20 significance level was prespecified to indicate evidence of potential therapeutic effect.

Results: Of the 68 patients randomized, 49 (ESK: 27; placebo: 22) completed the DB treatment phase. Mean (SD) baseline MADRS total scores were: 38.5 (6.17) and 38.8 (7.02) for the ESK and placebo groups, respectively. The primary endpoint, change from baseline in MADRS total score at day 1 (4 hours postdose) was significantly greater for ESK treatment compared with placebo (Least-square [LS] mean difference: -5.3 [SE: 2.10]; 2-sided $p=0.015)$. Secondary endpoint findings showed significantly greater change from baseline in MADRS total score at day 2 for ESK treatment vs placebo (LS mean difference: -7.2 [SE: 2.85]; 2-sided $p=0.015$ ). Patients in the ESK group showed significantly greater improvement vs placebo in the MADRS suicide item at day 1 (4 hours postdose; 2 -sided $p=0.002$ ). The ESK treatment showed evidence of potential therapeutic effect vs placebo for the MADRS suicide item at day $2(2$-sided $p=0.129)$ and CGJ-SR change from baseline at 4 hours $(2$-sided $p=0.112)$ and day 2 (2-sided $p=0.150)$. A greater proportion of patients in ESK group achieved resolution of suicide risk (CGJ-SR score of 0 or 1$)$ vs placebo at 4 hours $(21.2 \%$ [7/33] vs $9.7 \%[3 / 31])$ and day $2(40 \%[14 / 35]$ vs $6.5 \%[2 / 31])$. The response rate at day 2 was higher in ESK group (54.3\%; $19 / 35)$ vs placebo group (29\%; 9/31). Similarly, the remission rate at day 2 was higher for patients treated with ESK (34.3\%; $12 / 35)$ vs placebo $(16.1 \% ; 5 / 31)$. During the DB phase, the most common $(\geq 20 \%)$ TEAEs reported in the ESK group were nausea $(37.1 \%)$, dizziness $(34.3 \%)$, dysgeusia, headache and dissociation (31.4\% each) and vomiting (20\%); 4 patients experienced serious TEAEs during the DB phase (suicide ideation: 2, agitation: 1, depressive symptoms: 1 ). No death occurred in either group. In the ESK group, the dissociative/ perceptual changes measured by CADSS had an onset shortly after the start of intranasal dosing and generally resolved by 2 hours post dose.

Conclusions: In patients with MDD who were assessed to be at imminent risk for suicide, intranasal ESK $84 \mathrm{mg}$ demonstrated statistically significant and clinically meaningful effects in reducing depressive symptoms compared with placebo at 4 hours (day 1) and $\sim 24$ hours (day 2) after initial dose. A significant improvement in suicidality (as measured by MADRS suicide item and the CGJ-SR from SIBAT) was also observed at these time points. Moreover, intranasal ESK treatment exhibited rapid onset of response and greater likelihood of remission in this vulnerable population. Treatment with intranasal ESK $84 \mathrm{mg}$ was generally tolerated.

Keywords: Antidepressant, Esketamine, Intranasal, Major Depressive Disorder, Suicide Assessment.

Disclosure: Janssen Research \& Development: Full-time employement, Self.

\section{T135. Ketamine Treatment and Global Brain Connectivity in Major Depression}

Chadi Abdallah*, Lynnette Averill, Katherine Collins, Paul Geha, Jaclyn Schwartz, Christopher Averill, Kaitlin DeWilde, Edmund Wong, Alan Anticevic, Cheuk Ying Tang, Dan Iosifescu, Dennis Charney, James Murrough

\section{Yale University School of Medicine, West Haven, Connecticut, United States}

Background: Capitalizing on recent advances in resting state functional connectivity magnetic resonance imaging (rs$\mathrm{fcMRI}$ ) and the distinctive paradigm of rapid mood normalization following ketamine treatment, the current study investigated intrinsic brain networks in major depressive disorder (MDD) during a depressive episode and following treatment with ketamine.

Methods: Eighteen medication-free patients with MDD and 25 healthy control subjects (HC) completed baseline rsfcMRI. MDD patients received a single infusion of ketamine and underwent repeated rs-fcMRI at $24 \mathrm{~h}$ post-treatment. Global brain connectivity GBC values were computed as the average of correlations of each voxel with all other gray matter voxels in the brain. Whole-brain voxel-wise fully data-driven analyses with appropriate correction were conducted. 
Results: MDD group showed reduced GBC in the prefrontal cortex (PFC), but increased GBC in the posterior cingulate, precuneus, lingual gyrus, and cerebellum. Ketamine significantly increased GBC in the PFC and reduced GBC in the cerebellum. At baseline, 2174 voxels of altered GBC were identified, but only 310 voxels significantly differed relative to controls following treatment (corrected $\alpha<0.05$ ). Over the treatment period, clinical response was associated with an increase in GBC, such that responders to ketamine showed increased GBC in the right later PFC and left anterior insula. Post treatment, responders to ketamine also showed higher GBC values in the lateral PFC and caudate. Followup seedbased analyses illustrated a pattern of dysconnectivity between the PFC/subcortex and the rest of the brain in $\mathrm{MDD}$, which appeared to normalize following ketamine treatment.

Conclusions: The extent of the functional dysconnectivity identified in MDD and the swift and robust normalization following treatment, suggest that GBC may serve as a treatment response biomarker for the development of rapid acting antidepressants. The data also identified unique prefrontal and striatal circuitry as putative marker of successful treatment and target for antidepressants development.

Keywords: Ketamine, Major Depressive Disorder (MDD), Resting State Functional Connectivity, Rapid Antidepressant, Prefrontal Circuit.

Disclosure: Genentech: Consultant, Self; Genentech: Grant, Self.

\section{T136. Resilience is Associated With More Dentate Gyrus Granule Neurons and no Change in Glia}

Maura Boldrini*, Tanya H Butt, Lauren Bonilla, Cynthia Zizola, Soo H Kim, Gorazd B Rosoklija, Andrew J Dwork, Victoria Arango, J John Mann

\section{Columbia University, New York, New York, United States}

Background: Childhood adversity increases the risk of major depression (MDD), suicidal behavior, anxiety disorders and aggression. We previously reported lower neuron density in prefrontal cortex (PFC) in MDD who died by suicide and hypothesized this may be the result of altered trophic and apoptotic mechanisms. Exposure to early life adversity (ELA) may lead to changes in BDNF expression, elevated cortisol and structural changes in the brain. Stress affects cellular plasticity, neurogenesis, cell survival, and hippocampal volume. The number of mature GNs following exposure to childhood adversity has never been studied. Genetic variability may interact with childhood stress, resulting in the development of psychopathology in adult life in a subgroup of exposed individuals. We aimed to assess the effect of ELA exposure in subjects with MDD who died by suicide and in controls with no psychiatric diagnosis lifetime. We also aimed to dissect cellular changes found in suicide versus MDD comparing GN and glial cell numbers in MDDs who died by suicide with MDD subjects who died from natural causes.

Methods: To assess the effect of ELA exposure, we quantified numbers of DG GNs and glia in age- and sex- matched subject groups ( $n=10$ per group): group 1 . MDD who died by suicide; and group 2: non-psychiatric controls, both groups included subjects with reported exposure to ELA (before 15 y of age) and subjects without ELA exposure. To dissect cellular changes related to suicide versus MDD, we included a group $(n=10)$ of untreated subjects with MDD who did not die by suicide. Adverse events included deprivation due to separation from parents (e.g. death of parents, growing up in foster care or orphanage) or abuse (physical or sexual). All subjects underwent neuropathological examination, $\mathrm{pH}$ determination, toxicology, and psychological autopsies to determine DSM-V axis I and II diagnoses. The whole hippocampal formation was dissected from frozen coronal blocks, fixed in $4 \%$ paraformaldehyde at $4^{\circ} \mathrm{C}$, cryoprotected in $30 \%$ sucrose, sectioned at $50 \mu \mathrm{m}$ on a sliding microtome (Microm HM440E) and stored in 40-wells boxes at $-20^{\circ} \mathrm{C}$ in cryoprotectant. Immunohistochemistry was performed on sections at 2-mm interval using antiNeuN Ab (1:100,000; Chemicon, Temecula, CA), Nissl stain was used to identify glia. Cells were counted using stereology (MBF Biosciences Inc., Williston, VT).

Results: Subjects with ELA who had no psychiatric diagnosis lifetime, had more anterior DG GNs compared with controls without $\operatorname{ELA}(p=0.010)$, non-suicide $\operatorname{MDDs}(p=0.021)$, suicide-MDDs with ELA $(p=0.0037)$ and suicide-MDDs without ELA $(p<0.001)$. Between these same groups, there were no differences in GN number in mid and posterior DG. There were no differences in number of GNs in anterior, mid and posterior DG between suicide MDDs with or without ELA and between suicide groups and non-suicide MDDs. There were no group differences in anterior, mid and posterior DG glial cell number.

Conclusions: More GNs in resilient individuals exposed to ELA who never developed psychiatric diseases, may provide resilient subjects with enhanced cognitive flexibility and adaptive coping, while problem solving and cognitive flexibility are reported to be impaired in suicide. More GNs are found selectively in the anterior DG in resilient individuals. The mid and anterior hippocampus in primates, and the rodent ventral hippocampus, connect with the frontal lobe and amygdala, regulating stress responses, suggesting that neuroplastic changes occurring in human anterior DG may affect emotional processing. Subjects with no psychopathology and no ELA had fewer GNs than those exposed, suggesting that their hippocampal circuit had no need to adapt to an adverse environment through the implementation of more complex brain circuits. Studies of children exposed to adversity revealed that a successful adaptation was associated with good intellectual functioning, effective self-regulation of emotions, capacity to convert traumatic helplessness into learned helpfulness, and an active coping style in confronting stressors. These cognitive abilities possibly reflect brain circuits adaptations that are the result of DG cellular plasticity, hippocampus excitability, amygdala and PFC connectivity, and final encoding of emotion-related memories. No difference in GN number in MDDs who died by suicide with and without ELA exposure and non-suicide MDDs, suggests that their psychopathology has a similar final effect on DG cell numbers. Unaltered glial cell between groups indicate that ELA, MDD and suicide primarily affect the remodeling of DG neurons, although we did not assess number of astrocytes in these groups. The major limitation of the study is that postmortem studies cannot establish 
cause and effect relationships between experiences like childhood adversity and brain biology, but this method can detect correlations of heuristic importance.

Keywords: Risk and Resilience, Hippocampus, Childhood Adversity, Suicide, Major Depressive Disorder.

Disclosure: Nothing to disclose.

\section{T137. Human Amygdala Engagement Moderated by Early Life Stress Exposure is a Biobehavioral Target for Predicting Recovery on Antidepressants}

Andrea Goldstein*, Mayuresh S Korgaonkar, Erin Green, Trisha Suppes, Alan Schatzberg, Trevor Hastie, Charles Nemeroff, Leanne Williams

Stanford University School of Medicine, Stanford, California, United States

Background: Amygdala reactivity and exposure to adverse childhood events (ACEs) are both strongly implicated in depression mechanisms in both animal and human models. The amygdala plays an important role in emotion processing, including evaluating biologically salient emotional stimuli, generating emotional states and potentiating emotional memories. It also plays a central role in the stress response, both promoting downstream hypothalamicpituitary-adrenal (HPA) axis stimulation and receiving HPA axis feedback. Engagement of the stress system can fundamentally change amygdala structure and function, especially as a result of ACEs. Moreover, the amygdala is likely a component of the neural circuit involved antidepressant action, and the antidepressant response is modified by prior stress exposure. Despite these mechanistic foundations, amygdala-ACE interactions have not been investigated as a prognostic biobehavioral therapeutic target for depression. Addressing this issue, we tested whether the interaction of these two factors can predict who would benefit from treatment in MDD patients.

Methods: 80 patients from the International Study to Predict Optimized Treatment in Depression (iSPOT-D) were scanned using fMRI when unmedicated, randomized to one of three treatments (escitalopram, sertraline and venlafaxine-XR) and re-assessed 8 weeks post-treatment. Amygdala reactivity was assessed using an established emotional face task. Adverse events were assessed using the 19-item Early Life Stress Questionnaire. Participants were split into low ( $\leq 1$ event), mid (2-5 events) and high ( $\geq 6$ events) ACE groups. Because childhood adversity produces depressive-anxious symptoms as well as stress-related adjustment problems we defined functional remission by a combined measure of clinician-rated depression symptom severity using the HRSD17, self-reported symptom severity using the 16-item Quick Inventory of Depressive Symptomatology - Self-Rated (QIDS-SR16) and observer-rated functional capacity using the Social and Occupational Functioning Assessment Scale (SOFAS) at week 8. Remitters were defined as being in the normative range on symptoms ( $\leq 7$ on the HRSD17 and $\leq 5$ on the QIDS-SR16) and with healthy adjustment ( $\geq 10$ point improvement from baseline to achieve $\geq 61$ on the SOFAS). Hierarchical logistic regression models and ROC analyses with leave-one-out cross-validation implemented in $\mathrm{R}$ were used to test the predictive performance of models.

Results: A logistic regression model including ACE interactions with pre-treatment amygdala reactivity to masked happy and fearful faces significantly predicted posttreatment outcome $\left(\chi^{2}=47.10, \mathrm{df}=9, p<0.001\right)$. This full model predicted functional remission over and above the contribution of demographics, symptom severity, ACE and amygdala reactivity alone $(\Delta \chi 2=14.9, \mathrm{df}=2, p<0.001)$. Pretreatment depression severity and age were not significant contributors to this prediction. Using ROC analysis, the predictive accuracy for the ACE-amygdala model was $92 \%$ (sensitivity, 89\%; specificity, 88\%) and the leave-one-out cross-validated model maintained a high overall accuracy of $81 \%$ (sensitivity, 84\%; specificity, 69\%), suggesting good generalizability. Our model suggests that in depressed people with high ACE, the likelihood of remission is highest with greater amygdala reactivity to socially rewarding stimuli, whereas for those with low ACE exposure, remission is associated with lower amygdala reactivity to both rewarding and threat-related stimuli.

Conclusions: Our results advance our understanding of how exposure to adverse childhood events and the amygdala function synergistically to predict subsequent depression remission with antidepressants. Moreover, we have demonstrated that a composite brain-stress metric, based on validated predictive model might be used to support decisions about individual patients. The findings demonstrate that metrics based on combined brain and life experience data hold promise for developing a neuroscience-informed approach to mental disorder and its management.

Keywords: Adverse Childhood Events, Functional MRI (fMRI), Biomarker, Major Depressive Disorder, Antidepressant Response.

Disclosure: Nothing to disclose.

\section{T138. Social Behavior Abnormalities Associated With Depression and Suicidality}

Ricardo Caceda*, Jessica Carbajal, Jordan Moore, Favrin Smith

University of Arkansas for Medical Sciences, Little Rock, Arkansas, United States

Background: Abnormalities in social function represent a cardinal finding in major depression. Suicide, at the severe end of the depression severity spectrum, is almost universally triggered by social crises. We have previously shown genderspecific abnormalities in reciprocity behavior in depressed patients with and without suicidal ideation. Perception of social mistreatment or neglect is commonly found in depressed individuals. Hence, we aimed to characterize response to unfairness in depressed and acutely suicidal individuals.

Methods: We examined four groups of adult individuals of both genders: a) recent suicide attempters; b) suicidal ideators; c) non-suicidal depressed patients; and d) healthy controls $(n=135)$ with the Ultimatum Game- a behavioral economic task which examines the behavioral response to fairness/unfairness, driven by the interaction of an emotional 
reaction and a rational utilitarian perspective. We also measured demographic, cognitive and clinical variables.

Results: All depressed groups rejected lower (unfair) offers than healthy controls $(\mathrm{t}=13.9, p<0.01)$, with no difference between the three depressed groups. The higher acceptance of low offers with higher amounts in healthy controls was maintained in the three depressed groups. There were no gender differences in offer rejection rates or amounts between the study groups. Rejection of offers correlated with education years and hopelessness.

Conclusions: Depressed individuals showed proclivity of increased emotional response to unfairness. In contrast to reciprocity behavior in depression, response to unfairness does not seem to be influenced by gender or depression severity. A detailed characterization of social behavior can provide targets for psychotherapeutic interventions for depression and improvement of quality of life.

Keywords: Suicide, Social Behavior, Depression.

Disclosure: Nothing to disclose.

\section{T139. Hippocampal Subfield and Medial Temporal Cortical Volumes in Late-Life Depression}

\section{Warren Taylor* \\ Vanderbilt University Medical Center, Nashville, Tennessee, United States}

Background: Structural and functional alterations of the hippocampus are observed with aging and across numerous neuropsychiatric disorders. These differences are particularly important in late-life depression, where the effects of recurrent depressive episodes intersect with aging processes. Importantly, hippocampal alterations may also have prognostic value as older adults with depression have significantly higher risk for Alzheimer's disease. In this study we tested for differences in the volumes of hippocampal subfields and related medial cortex regions in older adults with and without depression.

Methods: We enrolled adults aged 60 years or older, 32 with current Major Depressive Disorder and 21 with no psychiatric history. All depressed participants were off antidepressant medications for at least two weeks. After clinical evaluation, participants completed 3T MRI that included a standard T1-weighted acquisition as well as a specialized T2-weighted acquisition obtaining coronal slices orthogonal to the long axis of the hippocampus. This T2weighted acquisition was utilized in automated medial temporal lobe volumetric processing using the ASHS (Automated Segmentation of the Hippocampal Subfields) tool. Statistical analyses tested for group differences in volumes of hippocampal subfields and medial temporal gray matter cortical regions while controlling for age, sex, and total intracranial volume. As we had no a priori hypotheses related to laterality, we examined the mean value across hemispheres for each region; for regions where we observed a statistically significant group difference, in subsequent analyses we examined subregions and hemispheres separately.

Results: There were no significant group differences in demographic variables or global cognitive performance on the Mini-Mental State Exam. After controlling for covariates, we did not observe any statistically significant group differences in volumes of the total hippocampus, the cornu ammonis (CA 1-3), the dentate gyrus or the subiculum. There was no statistically significant group difference in the thickness of the entorhinal cortex, however the depressed group exhibited smaller volumes of the perirhinal cortex (D: $1.75 \mathrm{ml}(\mathrm{SD}=0.21) ; \mathrm{ND}: 1.88 \mathrm{ml}(\mathrm{SD}=0.28) ; \mathrm{F}=4.40, p=$ 0.0405). As the perirhinal cortex consists of two regions, BA35 and BA36, we tested for group differences in these regions, observing a statistically significant group difference only for BA36 (D: 1.38ml (SD = 0.17); ND: 1.48 (SD = 0.25); $\mathrm{F}=4.55, p=0.0374)$. On further analysis, group differences were apparent in the left ( $\mathrm{D}: 1.38 \mathrm{ml}(\mathrm{SD}=0.21)$; ND: 1.56 $(\mathrm{SD}=0.31) ; \mathrm{F}=7.37, p=0.0088)$ but not right hemisphere. Conclusions: In contrast to past work associating depression with smaller hippocampal volumes, using advanced automated processing techniques we did not observe group differences in either total hippocampal volume or in subfield volumes. We did observe a group difference in the left hemisphere perirhinal cortex, a key medial temporal region involved in associative memory and recognition memory. Early atrophy in this area is reported in Mild Cognitive Impairment and very-early Alzheimer's disease and, in typical Alzheimer's disease, neurofibrillary tangle deposition begins in the perirhinal cortex before spreading to other medial temporal regions. Thus our observations are concordant with clinical reports associating late-life depression with an increased risk of dementia. Our study provides clues about the underlying neurobiology of that clinical relationship.

Keywords: Late-Life Depression, Hippocampus, MR Imaging, Aging.

Disclosure: Nothing to disclose.

\section{T140. Is Suicidal Behavior Linked to Early Aging?}

Jorge Gamboa, Pedro Delgado*, Ricardo Caceda

University of Arkansas for Medical Sciences, Little Rock, Arkansas, United States

Background: One of the barriers to implement suicide prevention measures is the lack of understanding of the biological mechanisms leading to it. Suicidal events are usually triggered by social or interpersonal crises and conflict associated with intense psychological pain. Psychological stress and major depression have been associated with increased cellular aging. We aimed to examine the relationship of cellular senescence and acute suicidality in adult depressed patients.

Methods: We examined saliva telomere length as a marker of cellular aging in four groups of adults of both genders $(n=133): 1)$ depressed patients admitted following a suicide attempt with persistent suicidal ideation; 2) depressed patients with suicidal ideation and no suicidal attempts in the last six months; 3) depressed non-suicidal patients; and 4) healthy controls. Additionally, we gathered demographic data and clinical data.

Results: There was no difference in telomere length between recent suicide attempters and suicidal ideators $(288.9 \pm 44.8$ vs. $287.3 \pm 44.9, \mathrm{t}=0.24, p=.981)$. Depressed patents showed shorter telomere length than healthy controls $(301.4 \pm 38.8$ 
vs. $202.8 \pm 46.9, \mathrm{t}=3.9, p=0.01)$. However, telomere length in recent suicide attempters and suicidal ideators did not differ from healthy controls. Telomere length was correlated with gender and use of antidepressant drugs.

Conclusions: These preliminary results suggest that acute suicidality is associated with cellular senescence in a direction opposite than in depression and acute psychological stress. Replication of this findings and analysis of mitochondrial DNA as a second marker of cellular aging are currently underway.

Keywords: Suicide, Telomere, Depression.

Disclosure: Nothing to disclose.

\section{T141. Complement Component 3 Signaling in PFC Mediates Depressive-Like Behavior}

Chirayu Pandya, Amanda Crider, Anthony Ahmed, Gustavo Turecki, Anilkumar Pillai*

\section{Medical College of Georgia, Augusta, Georgia, United} States

Background: Accumulating evidence suggest that immune system plays an important role in the pathophysiology of Major depressive disorder (MDD). Although many CNS functions, such as cognition and mood, are affected by cytokines, but less is known about the regulation and functional effects of innate immune system in the CNS associated with depression. The complement system is part of the innate arm of immunity, and complement proteins are widely expressed in neurons and glia in the brain. Among the various components, component 3 (C3) plays an important role as a converging point of the activation pathways of complement system. Given the important role of $\mathrm{C} 3$ in neuroplasticity, and the implications of altered immune signaling and synaptic plasticity in depression, we investigated whether C3 has regulatory effects on the susceptibility to depression.

Methods: We used real time PCR to analyse gene expression of complement components in postmortem prefrontal cortex samples from depressed suicide and age and gender-matched control subjects. The C3 expression in PFC and hippocampus was determined in mice following chronic unpredictable stress. The role of $\mathrm{C} 3$ in depressive-like behavior was further determined using C3 agonist, C3a antagonist, C3 knock out mice and lentiviral C3siRNA approaches.

Results: We found significant increase in $\mathrm{C} 3 \mathrm{mRNA}$ in the prefrontal cortex of depressed-suicide subjects. The examination of mRNA levels of other members of complement system revealed that mRNA levels of C1q, C4, MASP2, and MBL were increased in the PFC of depressed suicide subjects. However, we did not find any significant change in the mRNA levels of C1r, C1s, C2 and MSP between depressed suicide and control subjects. C3 expression was increased in mouse PFC following chronic stress. Selective activation of $\mathrm{C} 3 \mathrm{aR}$ using a potent C3a agonist induced depressive-like behavior in mice. Moreover, inhibition of C3a signaling using a C3aR anatgonist attenuated CUS-induced depressivelike behavior. Further studies using C3 knockout mice showed that C3 deficiency attenuated CUS-induced depressive-like behavior. In addition, C3aR inhibition in PFC using
siRNA lentiviral particles blocked CUS-induced depressive behavior in mice.

Conclusions: These postmortem and preclinical studies identify C3 signaling as a key factor in MDD pathophysiology and as a new target for therapeutic interventions.

Keywords: Complement, Depression, Behavior.

Disclosure: Nothing to disclose.

\section{T142. Periphery-To-Brain Immune Communications in Brain Function and Behavior: Potential Role for Circulating Extracellular Vesicles}

Eisuke Dohi, Rei Mitani, Takashi Imai, Indigo Rose, Eric Choi, Shin-ichi Kano*

\section{Johns Hopkins University, Baltimore, Maryland, United} States

Background: Peripheral immune alterations have been described in psychiatric disorders such as schizophrenia, depression, and autistic spectrum disorders. In addition, behavioral changes have been observed in various immunodeficient animal models. However, the mechanisms by which peripheral immune system influences brain development and function are poorly understood. In this study, we have explored the mechanisms by which adaptive immune cellderived extracellular vesicles influence brain cellular phenotypes and behaviors.

Methods: Mice deficient for Rag1 gene (Rag1 KO mice) were used as a model to study the effects of loss of $\mathrm{T}$ and $\mathrm{B}$ cells on brain cellular phenotypes and behaviors. Brain cellular phenotypes were assessed by immunofluorescent staining and qRT-PCR analysis. Behavioral phenotypes of Rag1 KO and WT mice were examined in the following paradigms: open field test (OFT), marble burying test (MBT), and elevated plus maze (EPM). Circulating extracellular vesicles (EVs) were analyzed in peripheral blood by using electron microscopy, nanoparticle tracking assay, and Western blotting.

Results: Microglia in the frontal cortex of Rag1 KO mice substantially differed in morphology from those of WT mice. c-Fos immunoreactivity, an indicator of neuronal activity, was also enhanced in the frontal cortex of these mice. In addition, expression of genes related to neuronal activities and microglial function was increased in Rag1 KO mice compared to WT mice. These cellular phenotypic changes were accompanied by behavioral alterations in OFT and in MBT. Unexpectedly, the amount of circulating immune cellderived EVs was decreased in Rag1 KO mice. Further analysis revealed that a set of microRNAs (miRNAs) in circulating EVs were lost in Rag1 KO mice. Interestingly, the expression of target gene(s) of such EV-associated miRNAs was altered in the frontal cortex of Rag1 KO mice. Further characterization of distribution and effects of EVs in the brain is in progress.

Conclusions: Our data suggest that peripheral immune cellderived EVs may reach the brain, modify its function, and influence behaviors. This study provides a novel biological insight into the mechanisms underlying peripheral-to-brain immune communications and may eventually have a broad impact on psychiatric disorders and other brain diseases. 
Keywords: Neuroimmunology, Adaptive Immunity, Extracellular Vesicles, MicroRNA.

Disclosure: Nothing to disclose.

\section{T143. Understanding the Mechanisms of Cognitive- Behavioral Therapy: Treatment-Related Changes in Brain Connectivity in Obsessive Compulsive Disorder}

Jamie Feusner*, Teena Moody, Gigi Cheng, Joseph O'Neill

Semel Institute for Neuroscience \& Human Behavior, Los Angeles, California, United States

Background: Cognitive-behavioral therapy (CBT) is an effective treatment for reducing symptoms of obsessivecompulsive disorder (OCD). Previous studies in OCD have found changes in brain activity with CBT both within and outside of classic cortico-striatal-thalamo-cortical circuits, yet the mechanisms are still poorly understood and no study has examined regional connectivity changes. Here we applied a data-driven approach to determine regional functional connectivity changes as a result of treatment with intensive CBT.

Methods: We acquired resting state functional magnetic resonance image data in 43 medicated and unmedicated adults with OCD. All were scanned before and after 4 weeks of intensive exposure and response prevention, a form of CBT; 21 of the 43 were additionally scanned before and after a 4-week minimal-contact waitlist period that preceded treatment. As an additional control, we scanned 24 matched healthy controls before and after 4 weeks of no treatment. Image preprocessing included parcellation of the brain into 160 nodes, previously derived from meta-analyses of functional studies. We assessed whole-brain resting state functional connectivity using the network-based statistics approach to identify nodes that significantly changed from pre- to post-treatment. We additionally compared connectivity in OCD to healthy controls before and after treatment, and assessed for relationships between changes in connectivity in the OCD group and changes in symptomatology on the Yale-Brown Obsessive Compulsive Scale (YBOCS).

Results: OCD participants showed significant clinical symptom improvements pre- to post-CBT $(41.0 \%$ decrease in YBOCS; $\mathrm{t} 42=12.4, p<.001$ ), but not pre- to post-waitlist $(.03 \%$ decrease; $\mathrm{t} 20=1.3, p=.211)$. Pre- to post-CBT there were significant increases in connectivity in 8 networks ( $t$ statistics ranging from 6.0 to $7.3 ; p<.01$, corrected), predominantly involving prefrontal, striatal, and cerebellar regions. Strongest increases were in networks involving connectivity between cerebellum and caudate/putamen, and between cerebellum and dorsolateral and ventrolateral prefrontal cortex. There were no significant associations between connectivity changes and YBOCS scores. Pre-CBT there were no significant differences in connectivity patterns between the OCD and healthy control group. However, after treatment the OCD group demonstrated significantly stronger connectivity than $\mathrm{HC}$ in three networks ( $\mathrm{t}$-statistics ranging from 4.0 to $5.0 ; p<.05$, corrected), partially overlapping with pre- to post-CBT increases in connectivity involving the caudate, thalamus, and occipital cortex. There were no significant changes in connectivity in the pre- to post-waitlist control condition. Likewise, there were no significant changes in connectivity in the healthy controls before and after 4 weeks of no intervention.

Conclusions: This represents the first study of the effects of CBT on regional brain connectivity in OCD. CBT resulted in increases in resting state functional connectivity in multiple networks both within and outside of classic cortico-striatalthalamo-cortical circuits, in a predominantly anteriorposterior pattern. Given the strong behavioral emphasis of the treatment, increased connectivity between cerebellar and striatal regions, and between cerebellar and prefrontal regions, may reflect the effect of CBT on strengthening patients' control over compulsive motor behaviors and thoughts, respectively. The observation that there were no significant connectivity differences between OCD and healthy controls pre-treatment, but significant differences post-treatment, could therefore reflect strengthening of processes specific to the mechanism of CBT rather than normalization of pathophysiological circuits.

Keywords: Obsessive Compulsive Disorder, Resting State Functional Connectivity, Cognitive-Behavioral Therapy, Cerebellum, Cortico-Striatal Plasticity.

Disclosure: Nothing to disclose.

\section{T144. Shared Economic Roles of Subgenual and Dorsal Anterior Cingulate Cortices in Decision Making}

Benjamin Hayden*

University of Rochester, Rochester, New York, United States

Background: Theories of dorsal anterior cingulate cortex (dACC) function generally emphasize its cognitive and regulatory functions, while theories of subgenual ACC (sgACC) emphasize its emotional, limbic, and arousalrelated roles. But how different are these areas when compared directly?

Methods: We recorded neuronal responses in both regions in a task with cognitive and limbic aspects, a token gambling task (using tokens allowed us to compare wins and losses). We used rhesus macaques and standard electrophysiological recording techniques.

Results: Both regions phasically encoded several important economic variables including offer values, remembered values, chosen values, and outcome values. We found that dACC (but not sgACC) signals value difference and choice probability, suggesting it contributes directly to choice. Neurons in sgACC showed enhanced firing for losses and dACC showed enhanced firing for outcome magnitude, regardless of valence.

Conclusions: These results highlight the common economic functions of the anterior cingulum and suggest that domains of specialization do not necessarily correspond to cognitive vs. emotional.

Keywords: Subgenual, Anterior Cingulate Cortex, Neuroeconomics.

Disclosure: Nothing to disclose. 
T145. Cross-Species Tests of the Cognitive-Enhancing Potential of Low-Dose Nicotinic Antagonism

Britta Hahn*, Marie Yuille, Carolyn Reneski, Ashleigh Wells, Olmstead Cory

University of Maryland School of Medicine, Baltimore, Maryland, United States

Background: Several preclinical studies suggested paradoxical performance-enhancing effects of nicotinic acetylcholine receptor (nAChR) antagonists at very low doses. Early reports employed paradigms of learning and memory; later studies showed beneficial effects also in tasks of attention. Our own data suggested enhanced response accuracy in the rat 5-Choice Serial Reaction Time Task (5-CSRTT) of attention with low doses of the alpha 7-selective nAChR antagonist methyllycaconitine (MLA), and preliminary data suggested a similar effect with the non-selective nAChR antagonist mecamylamine. A human study testing low doses of mecamylamine in adult non-smokers with ADHD (Potter et al 2009. Hum Psychopharmacol 24: 309-17) reported slowed reaction time in some tasks but enhanced recognition memory under specific task conditions. The present series of experiments aimed at systematically evaluating the cognitiveenhancing potential of low doses of mecamylamine in healthy human non-smokers, and of low doses of mecamylamine and MLA in rats performing the 5-CSRTT.

Methods: The human study employed a double-blind within-subject design. Over four separate days, 23 healthy adult non-smokers were tested with placebo and three trace doses of mecamylamine $(0.25,0.5,1 \mathrm{mg}$, p.o.), adjusted for body weight. Participants performed three computerized tasks: a task of spatial selective attention and stimulus detection; the Rapid Visual Information Processing Task (RVIPT) taxing sustained attention and working memory; and a change detection short-term memory task. Subjective state and vital signs were assessed repeatedly.

For the preclinical studies, 46 male Wistar rats were trained in the 5-CSRTT for approximately 4 months. The task required the animals to detect 1-s light stimuli, presented randomly in one of five apertures located along the curved rear wall of the operant conditioning chambers. Rats were food-reinforced for nose-poking into a lit hole. All studies employed a within-subject design, with different doses tested on different days. Four separate mecamylamine doseresponse curves were established ( $N=19-24$ per experiment), the first two testing $0,0.02,0.04, \& 0.08 \mathrm{mg} / \mathrm{kg}$, the last two testing $0,0.02,0.04,0.08, \& 0.16 \mathrm{mg} / \mathrm{kg}$ (s.c.). MLA was tested at $0,0.2,0.4,0.8, \& 1.6 \mathrm{mg} / \mathrm{kg}$ (i.p.), with each dose tested twice $(N=15)$.

Doses of mecamylamine in both the human and the rat experiments were approximately one order of magnitude lower than those typically reported to impair performance.

Results: Mecamylamine did not improve performance in any of the tasks. Instead, even at the trace doses tested, mecamylamine was found to increase omissions errors in the spatial attention task, and to decrease stimulus detection sensitivity and slow reaction time in the RVIPT. Other than a subtle increase in sleepiness at the largest dose, mecamylamine had no effects on subjective state, or vital signs.

Similarly, mecamylamine did not improve performance of rats on any response index of the 5-CSRTT. At the largest dose tested $(0.16 \mathrm{mg} / \mathrm{kg})$, there was a trend for mecamylamine to reduce anticipatory responding in the intertrial interval, suggesting subtle rate-suppressant effects. The preclinical setting allowed us to test whether low-dose nAChR antagonism may have beneficial effects on performance when selectively targeting the alpha 7 nAChR subtype. However, MLA also had no effects on any measure. Conclusions: No performance-enhancing effects were observed with low doses of mecamylamine in either human participants or rats, with the largest tested dose causing subtle impairment or rate suppression in both species. The lack of MLA effect in rats suggests that selective low-dose antagonism at alpha $7 \mathrm{nAChRs}$ also does not engender beneficial cognitive effects. The dose ranges chosen for all experiments included doses at which some types of performance-enhancing effects had been reported previously, in studies employing smaller sample sizes with no replication. The present results do not support the hypothesized cognitive-enhancing potential of low-dose nAChR antagonism and speak against it as a novel avenue for treating cognitive deficits.

Keywords: Nicotinic Acetylcholine Receptors, Low-Dose Antagonism, Cognitive Enhancement, Humans, Rats.

Disclosure: Nothing to disclose.

\section{T146. Sex and BMI-Related Alterations in Intrinsic Brain Activity and Connectivity in Reward and Interoceptive Regions}

Arpana Gupta*, Emeran Mayer, Jennifer Labus, Tiffany Yu, Aubrey Love, Amanat Bal, Kirsten Tillisch, Bruce Naliboff, Claudia Sanmiguel, Lisa Kilpatrick

University of California, Los Angeles, Los Angeles, California, United States

Background: Neuroimaging studies in obese individuals have identified morphological and evoked functional brain alterations in networks that process interoceptive and reward-related information. We aimed to identify: 1.BMIrelated differences in intrinsic activity of the brain. 2. Sex related differences in the association between BMI and intrinsic brain activity.

Methods: Functional resting state magnetic resonance imaging was acquired in 43 male (range $=22.71-33.60 \mathrm{~kg}$ / $\mathrm{m} 2$ ) and 43 female (range $=22.23-34.78 \mathrm{~kg} / \mathrm{m} 2$ ) healthy subjects. Fractional amplitude of low frequency fluctuations was computed and using partial least square analyses, sex differences and commonalities were investigated in BMIrelated alterations in intrinsic connectivity.

Results: 1. BMI-related differences: Greater BMI was associated with increased low frequency power in reward regions including the globus pallidus (GP) and ventral tegmental area/substantia nigra (VTA/SN). 2. Sex-related differences: Mid frequency power in these regions also increased with BMI for women but not for men. In men only, frequency-specific sex differences in the relationship between BMI and VTA/SN and GP functional connectivity was demonstrated, particularly with right medial orbital frontal gyrus and middle frontal gyrus in the low frequency range, and with bilateral insula (primary interoceptive cortex) and somatosensory regions (bilateral precentral 
gyrus and supplementary motor area) in the mid frequency range.

Conclusions: These results demonstrate sex differences as well as commonalities in BMI-related altered intrinsic activity of brain regions of the mesolimbic reward system. In addition, BMI-related alterations in functional connectivity with interoceptive and somatosensory regions were observed in men but not women. These insights may be useful to identify sex-based specific targets for future mechanistic studies and treatments aimed at abnormal ingestive behavior and obesity.

Keywords: Obesity, Sex Differences, Extended Reward Network, Resting State Intrinsic Connectivity, Somatosensory Network.

Disclosure: Nothing to disclose.

\section{T147. Increased Incidence of Parkinson's Disease in Patients With a History of Attention-Deficit/ Hyperactivity Disorder}

Annette Fleckenstein*, Karen Curtin, Brooks Keeshin, Glen Hanson

University of Utah, Salt Lake City, Utah, United States

Background: Attention-deficit/hyperactivity disorder (ADHD; ICD-9-CM 314.0-314.2, 314.8/9) is a brain disorder marked by an ongoing pattern of hyperactivity-impulsivity and/or inattention that interferes with functioning and/or development. Although amphetamine (AMPH) and methylphenidate (MPD)-based psychostimulant medications are the most commonly prescribed agents to treat ADHD, relatively little is known regarding long-term consequences of treatment by these agents in ADHD patients, particularly as related to Parkinson's Disease (PD). Of interest are our findings and those of others that abuse of AMPH/ methamphetamine (AMPH/METH; ICD-9-CM 304.4, 305.7, 969.7, 854.2) increases the likelihood of PD/parkinsonism/essential tremor (PD/PT; ICD-9-CM 332.0/1, $333.0 / 1)$. Because psychostimulants are often prescribed for primary treatment of $\mathrm{ADHD}$, the purpose of the present study was to investigate potential links among AMPH/ $\mathrm{METH}, \mathrm{ADHD}$ and PD/PT.

Methods: Statewide medical records data from 1996 through 2013 were retrieved from the Utah Population Database (i.e., the same database used in our previous study that demonstrated the association between AMPH/METH abuse and PD/PT; Curtin et al, Drug and Alcohol Dependence, 2015). A retrospective cohort design was used to determine incident $\mathrm{PD} / \mathrm{PT}$ in 34,749 ADHD diagnosed patients (inclusion criteria: no prior $\mathrm{PD} / \mathrm{PT}$ at baseline, age 20 or older at last follow-up, and no abuse history of AMPH/ METH, other illicit drugs, or alcohol) compared to 173,681 random population controls matched by gender and birth year in a target 5:1 ratio. A Cox model using a competingrisks framework was used to provide hazard ratio estimates. Results: Diagnosis of ADHD was associated with a 2-fold increased risk for development of $\mathrm{PD} / \mathrm{PT}$ when compared to the population controls (95\%CI $1.8-2.3 ; P<0.0001)$. In a subset of 4,116 ADHD patients determined to have been prescribed psychostimulants (MPD and/or mixed amphetamine salts), risk of early onset PD/PT (age $\leq 60)$ was especially pronounced; that is, more than 9-fold (95\%CI 5.415.8; $P<0001)$. Based on historic ADHD prevalence adjusted to current Center for Disease Control surveys, we estimate that as many as $38-49 \%$ of early onset $\mathrm{PD} / \mathrm{PT}$ patients in Utah have an ADHD history. Excluding subjects with a diagnosis history of psychotic conditions or tobacco-use disorders did not significantly alter our findings of an increased $\mathrm{PD} / \mathrm{PT}$ risk in $\mathrm{ADHD}$ patients, and this risk did not differ between males and females.

Conclusions: Patients diagnosed with ADHD in Utah were at a 2-fold increased incident PD/PT risk. Further, those patients known to be prescribed psychostimulants exhibited a pronounced increased risk of early-onset PD/PT. Future studies designed to determine specific subgroups of ADHD phenotype and/or treatment that are most associated with development of PD/PT, and to address mechanisms underlying this phenomenon, are warranted. (Supported by DA031883, DA039145, Huntsman Cancer Foundation, University of Utah Center for Clinical and Translational Science) Keywords: Attention Deficit Hyperactivity Disorder, Parkinson's Disease, Methylphenidate, Amphetamine, Nicotine.

Disclosure: Nothing to disclose.

T148. Mining for Neurotherapeutic Targets: Genome-Wide Human Tissue Transcriptome Profiling and Ligand Screening Identifies Seven Striatum-Specific Orphan GPCRs

\section{John Allen*, Sweta Raval, Robert Yang, Simon Xi \\ University of Texas Medical Branch, Galveston, Texas,} United States

Background: The striatum serves as the primary input of the basal ganglia performing essential brain functions including movement control, regulation of attention and motivation and is a critical component of the reward system. Disruption of striatal function is associated with many neurological and psychiatric diseases including Parkinson's and Huntington's disease, addiction and psychosis. Of the approximately 350 non-sensory human G protein-coupled receptors (GPCRs), roughly 120 remain orphan receptors whose endogenous ligands and physiological functions are largely unknown; however, brain orphan GPCRs likely include valuable targets for creating future neurotherapeutics. Here we employed a genome-wide tissue transcriptomic analysis to identify GPCRs selectively expressed in the human striatum and utilized a high throughput screening platform to search for ligands active at newly identified striatal orphan receptors.

Methods: We conducted a comprehensive genome-wide survey of human tissue-selective gene expression using an unprecedented collection of 1640 high-quality RNA-seq samples from the Genotype Tissue Expression (GTEx) pilot project, covering 31 human peripheral tissues and 13 brain subregions, including the caudate, putamen and nucleus accumbens. We developed a weighted tissue-selectivity scoring method that takes into account the similarity and difference of gene expression in all tissues and variability across individual samples. To identify receptor activity and screen for new pharmacology at the identified striatal orphan GPCRs, human clones of receptors were expressed in HEK293 cells and signaling assessed using the PRESTO- 
Tango beta arrestin assay or the GloSensor cAMP assay. As a screening proof-of-concept validation, the PRESTO-Tango assay was used to screen the LOPAC library of 1280 pharmacologically active small molecules against the striatal orphan receptors.

Results: The gene expression analysis identified a total of 99 protein-coding genes and 76 non-coding RNAs selectively expressed in the human striatum. Functional annotation of striatum selective protein-coding genes showed strong enrichment in dopamine neurotransmission and signaling (e.g. DRD1, DRD2, DRD3, PDE1B, PDE10A, PPP1R1B and RGS14), consistent with the well-described function of the striatum to coordinate dopamine-dependent brain functions. Strikingly, a large number of GPCRs were selectively expressed in the human striatum with 18 out of the 99 striatum-selective genes identified as encoding GPCRs. Out of these GPCRs, 11 have known ligands including many established therapeutic or investigational drug targets (e.g. dopamine D1, D2, D3 receptors, serotonin 5-HT3, 5-HT4 and 5-HT6 receptors). Seven of the remaining human GPCRs identified were all class A orphan receptors, namely, GPR6, GPR52, GPR55, GPR88, GPR101, GPR139 and GPR149. When expressed in HEK293 cells, the human striatal orphan receptors exhibited varying degrees of basal/constitutive activity for altering cAMP levels with GPR6, GPR101 and GPR149 increasing basal or forskolinstimulated cAMP suggesting Gs/olf coupling, while GPR88 decreased basal or forskolin-stimulated cAMP suggesting Gi/o coupling. Preliminary screening results from the PRESTOTango platform indicated all orphan receptors were appropriately plasma membrane localized and they induced varying degrees of basal/constitutive activity to recruit beta arrestin.

Conclusions: Although future pharmacological and physiological studies are required and ongoing, identification of this large number of orphan receptors selectively expressed in the human striatum suggests these receptors may modulate striatal neurotransmission and they function to regulate the basal ganglia. Our findings also suggest the seven identified human orphan GPCRs are potential drug targets amenable to screening whose pharmacological modulation may be therapeutic for treating striatum-related neurological diseases.

Keywords: GPCR, Basal Ganglia, Drug Discovery - New Approaches, Transcriptomics, Beta Arrestin.

Disclosure: Nothing to disclose.

\section{T149. Novel Synergistic Actions of Multiple Stress Hormones Mediate Memory Impairments After Acute Simultaneous Stresses}

Yuncai Chen, Jenny Molet, Julie Lauterborn, Christine Gall, Gary Lynch, Tallie Z Baram*

University of California-Irvine, Irvine, California, United States

Background: Acute stress typically enhances memory, an adaptive process crucial for survival. Surprisingly, we recently found that several acute concurrent stresses provoke enduring memory problems. During stress, hippocampal synapses are bathed in a cocktail of stress-released molecules, yet it is unknown how these molecules interact to mediate the profound effects of stress on memory.
Methods: We employed in vivo and in vitro approaches including transgenic mice, slice electrophysiology and novel imaging methods such as wide-field confocal imaging and $3 \mathrm{D}$ deconvolution tomography, to examine the mechanisms of the enduring memory problems provoked by acute concurrent stresses.

Results: The multiple acute concurrent stresses led to the lost ability of hippocampal synapses to sustain a potentiated state. These physiological deficits derived from structural alterations of synapse-bearing dendritic spines. Investigating the molecular mediators of these effects of stress, we found that both physiological and structural defects were recapitulated by the combined actions of the steroid stress hormone corticosterone (CORT) and the peptide corticotropin-releasing hormone (CRH). Mechanistically, CORT and CRH converged on the stability and plasticity of the actin skeleton of dendritic spines. Specifically, CORT and CRH exerted synergistic effects on the spine actin-regulating Rho-GTPase enzyme, RhoA. Supporting the convergent actions of CORT and CRH in mediating the effect of concurrent acute stresses on memory, blocking the brain actions of both hormones, but not each alone, rescued spatial memory in stressed mice.

Conclusions: Memory impairment by concurrent acute stresses requires interactions of multiple stress hormones at hippocampal synapses, with significant clinical impact.

Keywords: Memory, Hippocampus-mPFC pathway, Acute and Chronic Stress, Long-Term Potentiation, Rho Family GTPase.

Disclosure: Supported by NIH P50MH 096889 and NS28912.

\section{T150. Patients With Autism Spectrum Disorder Have Lower Functional Connectivity Density in the Dorsal Stream of the Attention Network}

Dardo Tomasi*, Ehsan Shokri Kojori, Corinde E Wiers, Şükrü B Demiral, Gene-Jack Wang, Nora Volkow

National Institute on Alcohol Abuse and Alcoholism, Bethesda, Maryland, United States

Background: Autism spectrum disorder (ASD) is a neurodevelopmental disorder characterized by deficits in social understanding and behavior, as well as delayed verbal and non-verbal language skills. Recently studies on ASD also documented deficits in visual motion perception and proposed that these may be related to a dorsal stream ("where" pathway) dysfunction (Koldewyn, 2010). We hypothesized that patients with ASD would demonstrate lower local functional connectivity density (IFCD) than controls in brain regions of the dorsal stream of attention and language areas, which would be associated with increased symptom severity.

Methods: Resting-state functional magnetic resonance imaging (rfMRI) datasets from 237 patients with ASD (18.1 - 8.7 years old, mean - sd.; 27 females) and 318 healthy controls (NML; 18.1 -8.3 years; 50 females) of the open access Autism Brain Imaging Data Exchange (ABIDE) repository were used in this study. Subjects were included if they had low head motion (mean frame wise displacement $(\mathrm{FD})<0.2 \mathrm{~mm}$ ) during the resting state session. The anatomical MRI scans were segmented into cortical and subcortical gray matter structures with FreeSurfer. The functional scans were 
realigned and normalized to the MNI space (3-mm isotropic voxels) using FSL. Scrubbing was used to remove timeframes excessively contaminated with motion artifacts $(\mathrm{FD}>$ $0.5 \mathrm{~mm}$ and DVARS $>0.5 \%$ ). Multilinear regression was used to minimize motion related signal fluctuations and 0.01-0.08 Hz band-pass filtering was used to minimize physiological noise. IFCD was computed using the standard correlation threshold, $\mathrm{R}>0.6$. Voxelwise ANOVA (with age, gender and mean motion covariates) and simple linear regression analyses in SPM8 were used for statistical tests. A cluster-level $\mathrm{PFWE}<0.05$ (family-wise error corrected) statistical threshold was used to evaluate the statistical significance of group differences in functional connectivity. Results: As expected the ASD group had deficits in social and communication skills (measured with the Autism Diagnostic Observation Schedule, ADOS) and lower IQ than the control group. The ASD group also had lower IFCD in bilateral parietal (superior parietal, precuneus and postcentral gyrus), occipital (calcarine cortex, lingual and lateraloccipital gyri, and cuneus), temporal (superior temporal sulcus and transverse temporal gyrus) cortices and cerebellum than the control group. An inverted U-shaped aging trajectory was found for the thalamic IFCD, which reached maximum at 31 years of age in both groups. The amplitude of the U-shaped aging trajectory was 4 times larger for controls than for ASDs. The volumes of thalamus, diencephalon and cerebellum, and the pericalcarine cortex were smaller for the ASD group than for the control group. The lFCD in language (pars orbitalis), memory (parahippocampal gyrus) and visual (lingual and lateral occipital gyri) areas decreased with increased symptoms severity (ADOS total).

Conclusions: The dorsal stream (also known as the "parietal", "where", or the "how" stream) is involved in orienting attention and in the localization of objects in space. This pathway stretches from the primary visual cortex (V1) in the occipital lobe forward into the parietal lobe. It is interconnected with the parallel ventral stream (the "what" stream) which runs downward from V1 into the temporal lobe. The lower IFCD in regions of the dorsal stream is consistent with impairment in higher-level dynamic attentional processes in ASD. In addition, our results are consistent with structural and functional alterations in subcortical regions that support a range of cognitive, emotional, and motor functions in ASD. Capitalizing on enhanced statistical power of large open access image dataset, this study highlights the potential of IFCD, a graph theory metric, as a neuroimaging marker of ASD.

Keywords: Resting State, Autism, MRI, ABIDE.

Disclosure: Nothing to disclose.

T151. Preliminary Evaluation of the Effects of Electronic Cigarettes Versus Own Cigarette on Withdrawal, Craving, and Smoking Severity in Tobacco-Dependent Volunteers

Richard De La Garza*, Jin Yoon, Luba Yammine, Manuela Holst, Ramiro Salas

Baylor College of Medicine, Houston, Texas, United States

Background: In place of quitting smoking, many individuals have turned to electronic cigarettes (e-cigs) to help control withdrawal and craving for nicotine. In the current project, we evaluated the effects of Blu e-cigs, as compared to the participant's own cigarette, on baseline craving and withdrawal, virtual reality (VR)-induced cue reactivity, and smoking severity.

Methods: Using a within-subjects, placebo-controlled study design, 7 tobacco-dependent participants maintained abstinence overnight then during 4 separate visits completed distinct phases of this inpatient protocol. Specifically, participants were randomized to one of 3 e-cig doses $(0,8$ or $16 \mathrm{mg}$ ) or their own cigarette. Study sessions were $\sim 3$ hours in duration and included assessments of withdrawal (using WSWS), craving (using QSU), VR-induced cue reactivity (VAS craving), and smoking severity.

Results: On average, participants were $50.6 \pm 6.6$ (mean \pm S. D.) years of age, Black $(71 \%)$ and male $(85 \%)$. They had FTND scores of $6.4 \pm 1.3$, smoked $15.1 \pm 4.3$ cigarettes per day, and smoked for $30.3 \pm 10.1$ years. At the beginning of each session, participants abstained overnight as required $(\mathrm{CO} \leq 4 \mathrm{ppm})$. Smoking an own cigarette, but no e-cig dose, resulted in a significant increase in breath $\mathrm{CO}(p=.002)$. At baseline, participants were evenly matched on their QSU scores and after 2 distinct bouts of smoking 10 puffs of their own cigarette these symptoms were reduced $(p=.06)$, but no e-cig dose resulted in a changes in QSU scores. After smoking each e-cig dose, participants completed an e-cig questionnaire and the data showed that the $16 \mathrm{mg}$ dose reduced withdrawal symptoms as compared to $0 \mathrm{mg}$ $(p=.05)$, but participants did not report that any dose of e-cig reduced was considered "rewarding" or that they "matched the feeling" of smoking their own cigarette (all p's $>.10$ ). VR data did not reveal differences among e-cig vs. own cigarette conditions for craving a cigarette in either the party or paraphernalia conditions (all p's $>.10$ ). Finally, with regard to smoking severity, the time to first puff (in minutes) and number of cigarettes chosen in a cigarette purchasing task were not significantly different across conditions (all p's $>.10$ ).

Conclusions: The proposed experiments provide novel data on e-cig use and perceptions, and data showing whether e-cigs alter craving and withdrawal responses among cigarette smokers. As expected, smoking an own cigarette reduced withdrawal and craving, but these same effects were not observed after any e-cig dose. Despite this, participants did indicate that the high e-cig dose was "rewarding", but did not answer affirmatively to any other questions that would indicate that these products would be likely to be used and adopted by these participants. The outcomes described here are likely the result of the specific e-cig device (Blu) utilized, which has been shown by others to deliver an inferior amount of nicotine $(\sim 4 \mathrm{ng} / \mathrm{ml} ; 25 \%$ of advertised dose). Ongoing research involves use of a new e-cig device and liquid shown to deliver nicotine doses more closely approximating that of a combustible cigarette.

Funding: NCI - 5P30CA125123-09S1.

Keywords: Electronic Cigarette (e-cigarette), Tobacco Smoking, Withdrawal, Craving.

Disclosure: Nothing to disclose. 
T152. Swedish Massage Therapy Clinically and Statistically Reduced Fatigue in Breast Cancer Survivors

Mark Rapaport*, Boadie Dunlop, Becky Kinkead, Jeffrey Rakofsky, Pamela Schettler, Sherry Edwards, Mylin Torres, Erica Larsen, Leticia Allen,

Dedric Carroll, James Nettles

\section{Emory Brain Health Center, Atlanta, Georgia, United} States

Background: There are approximately 15.5 million cancer survivors in the United States. A consequence of this success is that many surviving patients suffer from long-term treatment related sequelae that diminish the quality of their lives (QOL). One of the most prevalent and debilitating of these sequelae is cancer-related fatigue (CRF). Based on our prior work, we hypothesized that Swedish Massage Therapy (SMT) would decrease symptoms of fatigue more than either the Light Touch (LT) active control or a wait list control (WLC) group.

Methods: Fifty-seven subjects completed this randomized 3arm trial that compared weekly manualized SMT; weekly manualized LT, and a 6-week wait list control group. All of the subjects were women who were between 6 months and 4 years post treatment for stage 0-III breast cancer, had completed surgery, along with chemotherapy, radiation, or the combination of the two therapies, and were experiencing significant cancer-related fatigue (Brief Fatigue Inventory, BFI, $\geq 25$ ). All subjects were assessed with the Multidimensional Fatigue Inventory (MFI), PROMIS Fatigue Short Form 7a, and the Quality of Life, Enjoyment and Satisfaction Scale Short-form (Q-LES-Q). Assessments occurred pre session 1 and after sessions 3 and 6 . WLC subjects were also assessed pre WLC period.

Results: At baseline (pre session 1) mean MFI scores were 59.35 for SMT, 54.5 for LT and 62.95 for WLC. After 6 weeks there was a significant treatment-by-time interaction with mean (sd) changes in MFI scores of -16.50 (1.46) for SMT, -8.06 (1.49) for LT and + 5.88 (1.62) for WLC; (F, 24.31; df 2, $144 ; p<0.0001)$. The standard Effect Size (ES) for the contrasts were: -3.39 SMT vs WLC; $-2.09 \mathrm{LT}$ vs WLC and -1.28 SMT vs LT. There was also a significant treatment-bytime interaction with the PROMIS Fatigue score (F, 7.55; df $2,144 ; p<0.0008)$ with a similar pattern for the ES contrasts between the 3 arms: ES -2.06 SMT vs WLC; -1.20 LT vs WLC; and -0.86 SMT vs LT. There was a similar pattern of improvement in the Q-LES-Q scores with a treatment-bytime interaction of (F, 6.54; df 2, 137; $p<0.0019)$ : ES 1.64 SMT vs WLC; 0.89 LT vs WLC; .74 SMT vs LT.

Conclusions: SMT and LT resulted in significant decreases on 2 different measures of fatigue. SMT had clinically and statistically greater benefits for subjects suffering from CRF than LT or WLC conditions. Both SMT and LT led to improve in QOL with SMT group having a more robust enhancement in QOL.

Keywords: Cancer, Massage, QOL, Fatigue, CAM.

Disclosure: Nothing to disclose.
T153. Male-Specific Elevations in ERK Phosphorylation in a Mouse Model of 16p11.2 Hemideletion

Nicola Grissom*, Sarah McKee, Hannah Schoch, Nicole Bowman, Robbert Havekes,

Thomas Nickl-Jockschat, Teresa Reyes, Ted Abel

University of Minnesota, Minneapolis, Minnesota, United States

Background: Neurodevelopmental disorders, including autism spectrum disorders (ASD), are highly male biased, but the underpinnings of this are unknown. Striatal dysfunction has been strongly implicated in the pathophysiology of neurodevelopmental disorders, raising the question of whether there are sex differences in how the striatum is impacted by genetic risk factors linked to neurodevelopmental disorders. Here, we report male-specific deficits in striatal function important to reward learning in a mouse model of 16p11.2 hemideletion, a genetic mutation that is strongly associated with risk of neurodevelopmental disorders, particularly autism and ADHD.

Methods: We examined male and female 16p11.2 deletion animals in a series of operant tasks designed to assess reward-directed learning, motivation, and executive function. Striatal tissue samples from male and female wildtype and 16p11.2 deletion mice were collected for protein and gene expression analysis.

Results: We find that male, but not female, 16p11.2 deletion animals show impairments in reward-directed learning and maintaining motivation to work for rewards. Male, but not female, deletion animals overexpress mRNA for dopamine receptor 2 and adenosine receptor $2 \mathrm{a}$ in the striatum, markers of medium spiny neurons signaling via the indirect pathway, associated with behavioral inhibition. Despite equivalent effects in males and females on the mRNA levels of genes located within the 16p11.2 region in the striatum, including the kinase ERK1, hemideletion males show increased activation in the striatum for ERK1 at baseline and in response to sucrose, a signaling change associated with decreased striatal plasticity. In contrast, we find that hemideletion females show increased protein for ERK1 as well as the related kinase ERK2, and no change in phosphorylation either at baseline or in response to sucrose. Conclusions: These data indicate male-specific vulnerability in the mechanisms regulating intracellular signaling in the brain as a result of a genetic lesion associated with autism. Keywords: Autism, Sex Differences, Striatum.

Disclosure: Nothing to disclose.

\section{T154. Withdrawn}

T155. Evaluation of Efficacy and Safety of LY2951742 in Randomized, Double-Blind, Placebo-Controlled, SingleDose and Dose-Ranging Studies in Patients With Migraine

Smriti Iyengar*, David W Dodick, Peter J Goadsby, Qi Zhang, Margaret B Ferguson, Tina M Oakes, James M Martinez, Michael R Due, Aaron L Schacht, Vladimir Skljarevski, Kirk W Johnson

Eli Lilly and Company, Indianapolis, Indiana, United States

Background: Migraine remains poorly treated with few effective preventive medications available. LY2951742, a 
monoclonal antibody that binds to calcitonin gene-related peptide, was studied in the prevention of migraine headache in two different Phase 2 trials (ART-01 and CGAB). The results of the two studies are described.

Methods: (1) In Study ART-01, subjects with 4-14 migraine headache days (MHD) per month were enrolled in a doubleblind, randomized (1:1), 12-week placebo-controlled trial of biweekly subcutaneous injections (SC) of LY2951742 (150 $\mathrm{mg}$ ) versus placebo. The primary endpoint was the mean change from baseline in number of MHD per 28-day period assessed at month 3. Secondary efficacy endpoints were mean change from baseline in the number of migraine or probable migraine (MHD + pMHD) and migraine attacks per 28-day period, and the proportion of responders. Responders were defined as patients who had a greater than $50 \%$ reduction in the number of migraine headache days in a 28-day period. (2) In Study CGAB, patients with 4-14 MHD per month were randomized $(2: 1: 1: 1: 1)$ to placebo or 1 of 4 LY2951742 dose groups $(5,50,120$, and $300 \mathrm{mg})$ given SC once monthly for 3 months. The primary objective was to assess whether at least one dose of LY2951742 was superior to placebo in the prevention of migraine headache. The primary endpoint was the mean change from baseline in the number of MHD per 28-day period assessed at month 3 . Secondary efficacy endpoints were mean change from baseline in number of MHD + pMHD and migraine attacks per 28-day period assessed at month 3 , and the proportion of responders. Responders were defined as a patient with at least $50 \%$ or greater reduction in MHD. In both studies, statistical analyses were performed using Mixed-Model Repeated Measures based mean change from baseline for all endpoints described. Treatment emergent adverse events were evaluated for those with an incidence of $\geq 5 \%$ of LY2951742 treated patients and greater than that for placebo. Analyses were conducted on an intent-to-treat population (ClinicalTrials.gov, ART-01 = NCT01625988 and CGAB = NCT02163993).

Results: (1) For Study ART-01, patients were randomized to LY2951742 $(N=107)$ or placebo $(N=110)$. LY2951742 showed greater reduction in the mean change from baseline in the number of MHD at month 3 (-4.2 vs. -3.0 days for LY2951742 vs. placebo, respectively, $p=0.003)$. LY2951742 was also superior to placebo at month 3, for MHD + pMHD, ( -4.8 vs. -3.5 days, $p=0.010)$, migraine attacks, $(-3.1$ vs. -2.3 attacks, $p=0.005)$, and $50 \%$ response rate, $(75 \%$ vs. $49 \%$ responders, $p=0.0002)$, respectively. Treatment emergent adverse events seen more frequently with LY2951742 than placebo included injection site pain, upper respiratory tract infections, and abdominal pain. (2) For Study CGAB, patients were randomized to LY2951742 $(N=273)$ or placebo $(N=137)$. Compared with placebo, LY2951742 120 $\mathrm{mg}$ showed greater reduction in mean change from baseline in the number of MHD at month 3 (-4.9 vs. -3.6 days for LY2951742 vs. placebo, $p=0.004)$. LY2951742 $120 \mathrm{mg}$ was also superior to placebo at month 3 for MHD + pMHD, (-5.9 vs. -4.0 days, $p<0.001)$, migraine attacks, $(-3.5$ vs. -2.7 attacks, $p<0.003)$, and $50 \%$ response rate, (77\% vs. $61 \%$ responders $p=0.036)$, respectively. Treatment emergent adverse events that occurred more frequently with LY2951742 than with placebo included injection site pain, upper respiratory tract infections, nasopharyngitis, dysmenorrhoea, and nausea.
Conclusions: (1) In study ART01, LY2951742,150 mg (biweekly), at month 3, significantly decreased the number of $\mathrm{MHD}, \mathrm{MHD}+\mathrm{pMHD}$, and migraine attacks, and was superior for $50 \%$ responder rate compared to placebo in subjects with migraine headaches. (2) In study CGAB, LY2951742, $120 \mathrm{mg}$ (monthly), at month 3, significantly decreased the number of MHD, MHD+pMHD, and migraine attacks, and was superior for 50\% responder rate compared to placebo in subjects with migraine headaches. These results provide evidence that LY2951742 is efficacious in the prevention of migraine at the doses described. LY2951742 is safe and well tolerated.

Keywords: CGRP, Antibody, Migraine.

Disclosure: Eli Lilly and Company: Employee, Self.

\section{T156. Regular Oral Lithium Treatment is Associated With a Reduced Prevalence of Severe Neurological Disease and Myocardial Infarction: The Lithium Archive Project}

\section{Ronald Fieve, Barbara Orlowski, James Prosser*}

Foundation for Mood Disorders, New York, New York, United States

Background: Lithium has been used as a mood stabilizer medication for bipolar disorder for more than 50 years. A variety of studies have examined the medical consequences of long-term lithium treatment. In-vitro and animal studies have demonstrated that lithium has neurotrophic and cytoprotective actions in nerve cells and other tissue. In a rat model of cerebral vascular accident, post-infarct lithium treatment has been demonstrated to reduce both stroke volume and functional deficits compared to control treatments. We are interested in examining if a disease-sparing effect of lithium may be observable in human patients taking regular oral lithium therapy. This study compares the prevalence of cardiovascular and neurological disease in psychiatric outpatients receiving and not receiving lithium. Methods: This study consists of a retrospective chart review of adult psychiatric outpatients treated at the New York State Psychiatric Institute Lithium Clinic and two affiliate lithium clinics of the Columbia University Medical Center and the Foundation for Mood Disorders. These clinics specialize in the treatment of mood disorders and the majority of patients were diagnosed with either major depression or bipolar disorders. All patient diagnoses were made by board-certified psychiatrists, and patients were assigned to lithium treatment as appropriate for their individual diagnoses. All patients in the practice underwent yearly physical exams and blood chemistries performed by an independent medical practice. Data extracted from the medical records included patient demographic information, diagnosis, treatment information, and any reported medical or neurological disorders. Odds ratios were calculated to assess the risk of having a disorder for patients receiving lithium compared to patients not receiving lithium. A logistic regression model was used to examine the predictive role of lithium in the prevalence of neurological and cardiovascular disease among the study subjects.

Results: To date, 1084 patients have been entered in the database (53.5\% female, and $46.0 \%$ male), ranging in age 
from 18 to 88 years old ( mean $=42.2 \mathrm{yrs}$; sd $=14.8 \mathrm{yrs}$ ). Of these, 611 patients $(56.4 \%)$ received lithium treatment, with the average duration of lithium therapy being 2.75 years (range $0.1-30.0 \mathrm{yrs}$; sd $=4.82 \mathrm{yrs}$ ). The frequency of any neurological disease in this group was low: there were 178 unique disorders occurring in 117 patients $(10.8 \%$ of all patients). The frequency of specific conditions ranged from 39 patients with a history of Migraine Headache, to zero patients with Huntington's Chorea, Down's Syndrome, Lewy Body Disease, and Multi-Infarct Dementia. For seizures, the OR was 0.096 (95\% CI: 0.021 - 0.428). For dementia - not otherwise specified (D-NOS), the OR was 0.112 (95\% CI: 0.017 - 0.921). For amyotrophic lateral sclerosis (ALS), the OR was 0.112 (95\% CI: 0.015 - 0.920). For myocardial infarction (MI), the OR was 0.291 (95\% CI: $0.112-0.753$ ). Odds ratios for all other disorders (Alzheimer's Disease, Cerebrovascular Disease, CNS Neoplasm, Down's Syndrome, Huntington's Disease, Lewy-Body Disease, Migraine Headache, Multi-Infarct Dementia, Multiple Sclerosis, Optic Atrophy/Neuritis, Parkinson's Disease, Stroke, Syncope) were found to be equivalent to unity. For ALS, D-NOS, Seizures, and MI, lithium treatment was found to be negative predictor of disease occurrence when patient age, duration of clinic attendance, and use of any anti-psychotic medication were controlled.

Conclusions: Patients receiving long-term lithium treatment for psychiatric illness have a significantly lower prevalence of seizure disorders, ALS, Dementia (NOS), and myocardial infarction, compared to psychiatric outpatients not receiving lithium therapy. These results suggest that long-term lithium treatment may protect against some neurological disorders and myocardial infarction in human patients taking lithium. Keywords: Lithium, Mood Disorders, Seizures, Dementia, Myocardial Infarction.

Disclosure: Nothing to disclose.

T157. Striatal Dopamine Receptor Type 2-Expressing Medium Spiny Neurons Represent a Key Domain of Apathy Pathogenesis

Iku Tsutsui-Kimura*, Hiroyuki Takiue, Kenji F Tanaka

Keio University, Tokyo, Japan

Background: Apathy is clinically defined as a lack of motivation (Marin. 1990, 1991). It is a common feature of many pathologies involving neural degeneration, lesion, or dysfunction such as Alzheimer's disease (Mega et al, 1996; Lyketsos et al, 2011), Parkinson's disease (den Brok et al, 2015), Huntington's disease (Walker 2007; Thompson et al, 2012), progressive supranuclear palsy (Burrell et al, 2014), and stroke (Hama et al, 2011; Caeiro et al, 2013). Apathy lowers activities of daily living in patients and obstructs their rehabilitation. Therefore, the understanding of apathy pathophysiology and the establishment of its treatment are pressing issues. To model neurodegeneration-associated decreased motivation and understand the pathogenesis in detail in animals, we focused on two previous findings: 1) apathy is the most common behavioral disturbance resulting from striatal lesion (Bhatia and Marsden. 1994), 2) apathy is a major neuropsychiatric symptom in the early stages of
Huntington's disease, in which dopamine receptor type 2expressing striatal medium spiny neurons (D2-MSNs) are particularly degenerated (Reiner et al, 1988; Albin et al, 1992). These findings encouraged us to address whether bilateral D2-MSN-specific ablation or dysfunction induces decreased motivation in animals.

Methods: To achieve D2-MSNs specific loss-of-function, we exploited tetracycline-controllable gene expression system to induce progressive expression of diphtheria toxin (DTA) in D2-MSNs. DTA expression always started in the ventrolateral striatum (VLS) and expanded concentrically. We combined VLS-initiated, stepwise expanding diphtheria toxin expression with food-reinforced instrumental tasks in mice. To narrow the manipulation within the VLS, we also conducted acute optogenetic inhibition and chronic optogenetic ablation.

Results: We found that a loss-of-function of a specific cell type (D2-MSNs) within a restricted region (VLS) was sufficient to produce a reduction of goal-directed behaviors. We further demonstrated that optogenetic inhibition and ablation of VLS D2-MSNs caused, respectively, transient and chronic reductions of goal-directed behaviors.

Conclusions: Our data demonstrate that the circuitry containing VLS D2-MSNs control motivated behaviors and that the loss-of-function of VLS D2-MSNs causes decreased motivation in mice, thus implicating this circuit in apathy associated with neurodegenerative diseases such as Huntington's.

Keywords: Apathy, Motivation, Ventrolateral Striatum, Dopamine Receptor Type 2-Expressing Striatal Medium Spiny Neuron, Food-Reinforced Instrumental Task.

Disclosure: Nothing to disclose.

\section{T158. Worse the Second Time Around: Sensitization to Re-Encountered Emotional Stimuli in Borderline Personality Disorder Patients}

Harold Koenigsberg*, Bryan Denny, Jin Fan, Samuel Fels, Hayley Galitzer, SaraJo Mayson, Liza Rimsky

Icahn School of Medicine at Mount Sinai, Bronx, New York, United States

Background: Extreme emotional reactivity to psychosocial cues is a defining feature of borderline personality disorder (BPD), yet the psychological and neural mechanisms underlying this affective instability are poorly understood. One possible contributor would be an inversion of the adaptive emotion regulatory mechanism of habituation resulting in sensitization, rather than habituation, when emotionally-salient stimuli are reencountered. We hypothesized that when re-encountering emotional stimuli, borderline patients, in contrast to healthy volunteers and patients with avoidant personality disorder (AvPD), would show an intensified rather than reduced behavioral response and increased rather than reduced activity in the neural salience network, which has previously been shown to include amygdala, insula, dorsal anterior cingulate cortex (dACC), and hippocampus. AvPD patients were selected as a psychopathological control group since they are highly reactive to social cues. 
Methods: $26 \mathrm{BPD}$ patients, $25 \mathrm{AvPD}$ patients and 24 healthy volunteers $(\mathrm{HC})$ were shown emotionally negative International Affective Pictures System (IAPS) pictures as fMRI data was obtained on two occasions separated by 2 to 4 days. On each day, the same 30 negative and 30 neutral images were each presented five times in a pseudorandom sequence for 3 $\mathrm{s}$ each, followed by a $3 \mathrm{~s}$ negative affect rating for each trial and a $1 \mathrm{~s}$ intertrial interval. Data were analyzed using a random effects GLM with regressors for each picture presentation iteration of each stimulus valence plus a regressor for the rating period (undifferentiated by condition) as well as 6 motion parameters. BOLD signal intensities (beta-weights) were examined in anatomically-defined regions-of-interest for amygdala, insula, dorsal anterior cingulate (dACC) and hippocampus.

Results: On the first day of repeated exposures to the same negative images, all three groups show a decrease in right amygdala activity from first to fifth presentation (all $p<0.01)$. However, when re-encountering the same pictures 2-4 days later, BPD patients, unlike the other two groups, show a marked increase in right amygdala $(p<0.01)$ and right anterior insula activity $(p<0.02)$, but not dACC activity. Behaviorally, the HC $(p<0.03)$, but not BPD or AvPD, subjects show a decrease in negative ratings of pictures from first viewing on day 1 to last viewing on the day 2. The increase in right amygdala activity from day 1 to day 2 is negatively correlated with the level of hippocampal engagement during habituation on day 1 for BPD patients ( $r$ $=-0.55$ and $\mathrm{r}=-0.58, \mathrm{R}$ and $\mathrm{L}$ hippocampus respectively). Conclusions: BPD patients, a group characterized by intense reactivity to recurring negative psychosocial stimuli, show a reversal of initial habituation and a sensitized amygdala and insula response to negative emotional pictures when the pictures are re-encountered a few days later. For BPD's, the degree of amygdala sensitization upon rechallenge is inversely related to the level of hippocampal engagement during initial habituation, potentially reflecting poor initial encoding of the emotional material being associated with greater reactivity during subsequent encounters. These findings provide a neural basis for the persistent hyperreactivity to negative psychosocial cues seen in BPD patients and have implications for psychotherapeutic work with these patients.

Keywords: Borderline Personality Disorder, Habituation, Salience Network.

Disclosure: Nothing to disclose.

\section{T159. Sequencing Identifies Candidate Genes Underlying Borderline Personality Disorder}

Colin Hodgkinson*, Mercedes Perez-Rodriguez, Marc Ferrer, Qiaoping Yuan, Ming Leung, Oscar Andion, Natalia Calvo, Monica Prat, Cristina Sanchez-Mora, Marta Ribases, Miguel Casas, Antonia New, Larry Siever, David Goldman

National Institute on Alcohol Abuse and Alcoholism, Rockville, Maryland, United States

Background: Borderline personality disorder (BPD) is a serious psychiatric disorder characterized by an inability to appropriately control emotions, reckless and impulsive behavior, and unstable relationships with others. In some instances, individuals exhibit extreme reactions to perceived abandonment by people to whom they feel close, and patterns of unstable relationships which oscillate from extreme closeness to intense dislike. Inappropriate anger directed at self or at others and reckless impulsive behaviors such as unsafe sex, gambling and substance abuse are also observed in BPD patients and self-harm or suicide is a significant risk. Often BPD symptoms are accompanied by depression, anxiety disorders, substance abuse, eating disorders or antisocial personality disorder. These comorbid disorders potentially result in under diagnosis of $\mathrm{BPD}$, estimated to have a prevalence of $1.4 \%$ amongst adults. BPD usually manifests in late adolescence to early adulthood with approximately equal numbers of males and females affected. Twin studies have shown BPD to have a high heritability $(\sim 60 \%)$ and variation at genes involved in serotonin signaling, (TPH1, 5HTTLPR) have been associated with the disorder. However, the genetic underpinnings and the etiology of BPD remain largely unknown.

The International Borderline Disorder Consortium has performed exome sequencing on a cohort of BPD patients and matched controls to identify genes or pathways in which functional coding variants are over represented in BPD patients, and followed up candidate variants in two larger cohorts.

Methods: Recruitment and ascertainment of BPD cases and control subjects was carried out by the BPD Research Group at the Psychiatry Department of the Hospital Vall d'Hebron, Barcelona. Exome sequencing was performed on 102 BPD cases along with 100 controls. Target enrichment was performed using the Ampliseq Exome kit which targets $33 \mathrm{Mb}$ of coding exons through highly multiplexed amplification. Sequencing was performed on the Ion Proton platform (Life Technologies). The reads were mapped to UCSC hg19 with tmap-f3. The variants were called with TVC plugin in Torrent Suite. The genotypes at the variant positions were called by a pipeline consisting of mpileup function within samtools (version 1.2) along with calls function and vcfutils. $\mathrm{pl}$ in bcftools (version 1.2). AB genotypes were generated by parsing the counts in the VCF files. The variant functional annotation was performed with an in-house tool (SNP.to. ucsc-functions.pl) and Ensembl's VEP. Follow up genotyping of candidate variants was performed by 5 '-exonuclease assay in an expanded cohort from Barcelona and in a Finnish cohort.

Results: Subjects were sequenced to an average depth of $89.3 x$ ( $89.4 x$ cases, $89.2 x$ controls) and on average 54,690 variants $(29,837-74,325)$ were detected in each sample. Analysis of the variant data in VEP identified a total of 254,166 SNV, 158,255 substitutions (where the length of change in the variant is the same as the reference length), 10,381 insertions, 4,719 deletions and 2,064 sequence alterations (variants that alter sequence and length). Approximately $10 \%$ of all coding variants identified are predicted to have functional consequences. A variant that introduces a premature termination codon into the gap junction protein alpha 10 (GJA10) was identified to be present in the BPD discovery samples at a frequency of $3.8 \%$, compared to $0.5 \%$ in controls. The frequency of this variant is below $1 \%$ for all populations in EXaC. When analyzed in an expanded Catalan cohort the association did not reach 
significance $(p=0.157)$. However, in a large Finnish cohort $(n=712)$ where BPD was grouped with ASPD (antisocial personality disorder), a related and possibly overlapping disorder the stop codon was associated with disease $(p=0.014)$. When the Finnish and Catalan samples are analyzed together the stop codon showed association to $\operatorname{BPD} / \operatorname{ASPD}(p=0.004)$.

Conclusions: The gap junction protein GJA10 represents a potential candidate for BPD/ASPD. The frequency at which the stop codon is observed in our sample is higher than expected given the frequencies seen in world-wide populations and the initial association is replicated in an unrelated cohort. This association to BPD/ASPD needs to be confirmed in much larger samples.

Keywords: Borderline Personality Disorder, Whole Exome Sequencing, Psychiatric Genetics.

Disclosure: Nothing to disclose.

\section{T160. Shared Architecture of Dimensional Psychotic} Symptoms Across Psychosis Spectrum Disorders

M. Mercedes Perez-Rodriguez ${ }^{\star}$, Luz Ospina, Amanda Fisher, Armando Cuesta-Diaz, George Nitzburg, Manuela Russo, Megan Shanahan, Margaret McNamara, Erin Hazlett, Harold Koenigsberg, Antonia New, Larry Siever, Katherine Burdick

\section{Icahn School of Medicine at Mount Sinai, New York,} New York, United States

Background: There is evidence that the individual diagnoses within the psychosis spectrum (e.g., schizophrenia, SZ, bipolar disorder, BD, and schizotypal personality disorder, SPD, among others) share significant overlap in symptoms, biomarkers, genetic factors, outcomes and treatment response. Moreover, within each individual disorder, there is significant heterogeneity in many aspects of these illnesses, including symptom profiles, neurocognitive performance and everyday functioning. This has lead to recent efforts, in line with the Research Domain Criteria (RDoC) framework, to develop novel classifications of patients based on dimensional measures that span the full range from normal to abnormal and cut across traditional diagnostic boundaries. While a substantial amount of research has focused on the structure of psychotic symptoms in schizophrenia and their relationship to neurocognition and functioning, whether there are homogeneous subgroups of $\mathrm{BD}$ patients with distinct profiles of psychotic symptoms has not been widely studied. Moreover, the potential impact of these subgroups on neurocognitive and everyday functioning is also unknown. In this study we aimed to cluster BD patients in homogeneous subgroups based on their profiles of psychotic symptoms. Then, we described the clinical, neurocognitive and functional characteristics of each homogeneous subgroup. Finally, to test whether these homogeneous subgroups extend across the psychosis spectrum, we aimed to replicate our results in an independent sample of patients with schizotypal personality disorder (SPD).

Methods: Rather than focusing on overt psychotic symptoms, in this study we chose to classify patients based on the schizotypal personality questionnaire (SPQ). The SPQ is a multidimensional measure of psychosis proneness that captures the full range from normal to abnormal, including subthreshold psychotic symptoms that may be missed by standard psychotic symptom scales in individuals without history of overt psychosis. We administered the SPQ to a sample of 138 patients with BD I or II and 282 patients with SPD diagnosed with DSM criteria using SCID and SIDP-IV. Neurocognitive functioning was assessed using the MATRICS Consensus Cognitive Battery (MCCB). Disability was measured using the World Health Organization Disability Assessment Schedule, 2nd Edition (WHODAS-II). We empirically tested for homogeneous subgroups in each sample (BD and SPD patients) based on multidimensional measures of psychosis (the 9 subscales of the SPQ) using hierarchical cluster analyses to determine the optimal number of clusters and assign cases to subgroups based on similar profiles. To determine the impact of psychosis symptom profile on outcome, we used ANOVAs to compare the clusters on measures of neurocognitive and everyday functioning.

Results: Our results suggest that the propensity to different dimensional profiles of psychotic symptoms is heterogeneous. In the sample of $\mathrm{BD}$ patients, we identified 3 subgroups with distinct profiles of psychotic symptoms (3 clusters): 1) A subgroup with high propensity to positive symptoms, with significantly higher scores on the ideas of reference, suspiciousness, unusual perceptions, odd beliefs, odd behavior and odd speech SPQ subscales than the other 2 clusters. This subgroup also had a significantly higher rate of lifetime history of subclinical and overt persecutory delusions assessed with SCID (Pearson Chi-Square=18.41; $\mathrm{df}=4 ; \quad p=0.001) ; 2)$ A subgroup with high negative symptoms and social dysfunction, characterized by high scores on the excess social anxiety and lack of friends subscales, and 3) A subgroup with a mild psychosis symptom profile, with the lowest scores across all SPQ subscales. The $\mathrm{BD}$ subgroup with high propensity to positive symptoms scored significantly lower on standardized scores in all cognitive areas (MATRICS composite, $\mathrm{F}=3.72 ; \mathrm{df}=2$; $p=0.027)$ and had lower premorbid IQ $(\mathrm{F}=5.6 ; \mathrm{df}=124$; $p=0.005)$ and significantly higher functional disability (WHODAS overall disability, $\mathrm{F}=9.19 ; \mathrm{df}=2 ; p<0.001$ ) as compared with the other subgroups.

When applying the same approach in the SPD sample, we found a subgroup structure that was highly consistent with that described above.

Conclusions: We found three homogeneous subgroups with distinct profiles of psychotic symptoms in a sample of patients with $\mathrm{BD}$, and replicated this finding in an independent sample of patients with SPD. The subgroup of BD patients characterized by high propensity to positive psychotic symptoms had significantly lower neurocognitive performance and everyday functioning. Our finding of a common structure of psychotic symptom profiles across different patient populations within the psychosis spectrum supports the dimensional nature of the propensity to positive symptoms in a continuum across diagnostic boundaries. Our results further highlight the potential implications that this new classification system may have on outcome.

Keywords: Psychosis Continuum, Bipolar Disorder, Schizotypy, Cognition, Functioning.

Disclosure: Nothing to disclose. 
T161. Early Clinical Evaluation of AUT00206, a Novel Selective Kv3 Channel Modulator for the Treatment of Schizophrenia

John Hutchison*, Anil Sajjala, Omair Sahgal, Bill Deakin, Oliver Howes, Colin Dourish, Giuseppe Alvaro, Charles Large

Autifony Therapeutics, London, United Kingdom

Background: It is universally acknowledged that there is a pressing need for novel treatments for schizophrenia, particularly in respect of negative and cognitive symptoms which are not well served by current therapeutic options (Keef et al Arch. Gen. Psychiatry 2007; 64:633-647). Here we present the first clinical data for AUT00206, a novel selective modulator of the Kv3 channel which has exhibited promising activity in preclinical models of schizophrenia (Large et al, Neill et al, this meeting). We also describe the translational medicine approach we have adopted to bridge from preclinical studies to clinical trials in patients.

Methods: The first in human study was a randomised, double-blind, placebo-controlled study of single and multiple ascending oral doses of AUT00206 in healthy male volunteers, which also incorporated an evaluation of food effect. The primary objective was to explore the safety, tolerability (adverse events: AEs) and pharmacokinetics (PK) of AUT00206, with pharmacodynamic (PD) biomarkers as exploratory outcome measures. In Part A, 3 cohorts of 8 subjects participated in up to 5 dosing sessions, in which they received single oral doses of AUT00206 or placebo (6:2 ratio) in a dose escalating design. Progression from one dose level to the next was made after a medical review of the safety, tolerability and PK of the previous dose. The highest dose achieved by one cohort was repeated in the first dosing session of the next. The effect of food on the PK of AUT00206 was assessed in the first cohort and all subsequent treatments were administered in the fed state. In Part B, 4 cohorts of 8 subjects (6:2 ratio) received multiple oral doses of AUT00206 once or twice daily for 14 days in the fed state. Pharmaco-EEG (PhEEG) was performed in all subjects predose and at several timepoints post dosing. The 3 higher dose groups in Part B also had cognitive function assessments (using the CANTAB battery) and assessments of Event-Related Potentials (ERPs). The ERPs included mismatch negativity (MMN), P300 oddball paradigm (P300) and gamma response to an auditory tone pre- and post- dosing, as exploratory biomarkers.

Results: AUT00206 was considered to be safe and well tolerated at all dose levels tested in Parts A and B of the study. The data remain blinded at the time of writing, however somnolence was more common following high single doses, compared to lower doses in Part A and headache was more prevalent at the higher dose levels in Part B. There were no deaths, serious AEs or severe AEs in either part of the study. Treatment-related AEs were more common in the Nervous System, compared to other system organ classes. There were no dropouts relating to treatmentemergent AEs. The PK analyses revealed a significant food effect, with Cmax and AUC0-inf increased 4 fold and 2 fold respectively in the presence of food. Following single doses in the fed state, exposure increased in a slightly less than dose proportional manner with low inter-individual variability.
Half-life ranged from 10-15 h, compatible with once daily dosing. The accumulation ratio (Racc) following multiple doses was approximately 1.5 , with steady state achieved within 2-3 days in Part B. Data from the PhEEG, cognitive battery and ERPs assessments are currently being analysed. Conclusions: The first in human data both support and inform the continued development of AUT00206, as plasma exposure in the single and multiple dose parts of the study achieved levels in the range predicted to be pharmacologically active, based on preclinical studies. Two dose levels from Part A have been chosen to explore the effects of AUT00206 on blood oxygen-level dependent (BOLD) fMRI and short term memory (N-Back) in healthy volunteers given a low i.v. dose ketamine challenge, in a randomised double-blind, placebo-controlled 4-way single dose crossover study. This study will explore whether signals identified in a previous rodent ketamine challenge model translate to humans and is due to commence shortly in Manchester, UK. The top dose level from Part B will be employed in an experimental study in patients diagnosed with schizophrenia within the last 5 years, who are taking no more than 2 recognised antipsychotics. This will be a randomised doubleblind, placebo-controlled study (part inpatient, part outpatient) with daily dosing for 28 days. The study will be conducted jointly at King's College and Hammersmith Medicines Research, both in London, UK. In addition to clinical rating scales, cognitive function (CANTAB), ERPs (MMN, P300, ASSR) and imaging (18 F-DOPA PET, BOLD fMRI) will be performed at various timepoints to investigate PD effects in the target patient population. This study will commence later in the year.

Keywords: Kv3 Family, Schizophrenia, Clinical Trials.

Disclosure: Autifony: Employee and Shareholder, Self; Autifony: Employee, Self; Hammersmith Medicines Research: Employee, Self, University of Manchester: Consultancy and Grant, Self; Kings College London: Consultancy and Grant, Self; P1Vital: Employee and Shareholder, Self; Autifony: Employee and Shareholder, Self; Autifony: Employee and Shareholder, Self.

\section{T162. Neural Complexity as a Potential Translational Biomarker for Psychosis}

Albert Yang*, Brandon Hager, Roscoe Brady, Brett Clementz, Godfrey Pearlson, John Sweeney, Carol Tamminga, Matcheri Keshavan

Harvard Medical School, Boston, Massachusetts, United States

Background: The adaptability of the human brain to the constantly changing environment is reduced in patients with psychotic disorders, leading to impaired cognitive functions. Brain signal complexity, which may reflect adaptability, can be readily quantified via resting-state functional magnetic resonance imaging (fMRI) signals. We hypothesized that resting-state brain signal complexity is altered in psychotic disorders, and is correlated with cognitive impairment.

Methods: We assessed 156 healthy controls (HC) and 330 probands, including 125 patients with psychotic bipolar disorder (BP), 107 patients with schizophrenia (SZ), 98 patients with schizoaffective disorder (SAD) and 230 of their 
unaffected first-degree relatives (76 BPR, 79 SADR, and 75 SZR) from four sites of the Bipolar-Schizophrenia Network on Intermediate Phenotypes (B-SNIP) consortium. Using multi-scale entropy analysis, we determined whether patients and/or relatives had pathologic differences in complexity of resting-state fMRI signals toward regularity (reduced entropy in all time scales), or toward uncorrelated randomness (increased entropy in fine time scales that decays as the time scale increases) and how these complexity differences might be associated with cognitive impairment.

Results: Compared to HC subjects, proband groups showed either decreased complexity toward regularity or toward randomness. SZ probands showed decreased complexity toward regular signal in hypothalamus, and BP probands in left inferior occipital, right precentral and left superior parietal regions, whereas no brain region with decreased complexity toward regularity was found in SAD probands. All proband groups showed significantly increased brain signal randomness in dorsal and ventral prefrontal cortex (PFC), and unaffected relatives showed no complexity differences in PFC regions. SZ had the largest area of involvement in both dorsal and ventral PFC. BP and SAD probands shared increased brain signal randomness in ventral medial $\mathrm{PFC}, \mathrm{BP}$ and $\mathrm{SZ}$ probands shared increased brain signal randomness in ventral lateral PFC, whereas SAD and SZ probands shared increased brain signal randomness in dorsal medial PFC. Only SZ showed increased brain signal randomness in dorsal lateral PFC. The increased brain signal randomness in dorsal or ventral PFC was weakly associated with reduced cognitive performance in psychotic probands. Conclusions: These observations support the loss of brain complexity hypothesis in psychotic probands. Furthermore, we found significant differences as well as overlaps of pathologic brain signal complexity between psychotic probands by DSM diagnoses, thus suggesting a biological approach to categorizing psychosis based on functional neuroimaging data.

Keywords: Schizophrenia, Schizoaffective Disorder, Bipolar Disorder, fMRI Resting State, Neural Complexity.

Disclosure: Nothing to disclose.

\section{T163. The In Vivo Electrophysiological Effects of Cariprazine on the Rat Midbrain Dopaminergic System}

Ken Kramer*, Sarah Delcourte, Renaud Rovera, Béla Kiss, Nika Adham, Bence Farkas, Nasser Haddjeri

\section{Allergan, Inc., Jersey City, New Jersey, United States}

Background: Schizophrenia symptoms are thought to result from the dysregulation of dopaminergic signaling. Atypical antipsychotics with partial agonist activity at dopamine receptors have demonstrated efficacy in relieving symptoms associated with this aberrant dopaminergic neurotransmission. Cariprazine, a potent dopamine D3/D2 receptor partial agonist with preferential binding to the D3 receptor, is FDA approved in the US for the treatment of schizophrenia and acute manic or mixed episodes associated with bipolar I disorder. This study used in vivo electrophysiological methods in naïve rats to investigate the acute effects of cariprazine on dopamine cell activity in the ventral tegmental area (VTA) and substantia nigra pars compacta (SNc) regions of the rat midbrain.
Methods: Extracellular recordings of individual dopamine neurons were performed on anesthetized rats via electrodes placed stereotaxically in the VTA or SNc. Rats (VTA studies, $n=5-10$ per treatment group; SNc studies, $n=5-9$ per treatment group) received intravenous injections of cariprazine (VTA, up to $70 \mu \mathrm{g} / \mathrm{kg}$; SNc, up to $80 \mu \mathrm{g} / \mathrm{kg}$ ) and/or the selective D3 receptor antagonist SB-277011A (500 $\mu \mathrm{g} / \mathrm{kg})$, selective D2 receptor antagonist L741,626 (500 $\mu \mathrm{g} / \mathrm{kg})$, D3 preferring D3/D2 receptor full agonist PD128,907 (10 $\mu \mathrm{g} / \mathrm{kg}$; VTA), and the non-selective dopamine receptor full agonist, apomorphine $(40 \mu \mathrm{g} / \mathrm{kg} ; \mathrm{SNc})$. Drug-induced changes in spontaneous neuronal firing were analyzed.

Results: In the VTA and SNc, cariprazine partially and dosedependently inhibited dopamine neuronal firing by up to $38 \%$ $(P<.01)$ and $46 \% \quad(P<.001)$, respectively. Interestingly, cariprazine $(20 \mu \mathrm{g} / \mathrm{kg})$ administration partially but significantly reversed PD128,907-induced full suppression of neuron firing activity in the VTA $(P<.05)$. In this same region, pretreatment with cariprazine $(20 \mu \mathrm{g} / \mathrm{kg}), \mathrm{SB}-277011 \mathrm{~A}$, or L741,626 partially but significantly inhibited PD128,907induced full suppression of firing activity $(P<.05)$. Similarly, cariprazine $(15-20 \mu \mathrm{g} / \mathrm{kg})$ significantly promoted the reversal of the apomorphine-induced full suppression of neuronal firing in the SNc $(P<.05)$. In both regions, pretreatment with L741,626, but not SB-277011A, counteracted the dosedependent suppressant effect of cariprazine $(5-20 \mu \mathrm{g} / \mathrm{kg})$ on dopamine neuron firing (VTA, $P<.01$; SNc, $P<.0001$ ). Low dose $(10 \mu \mathrm{g} / \mathrm{kg})$ cariprazine treatment produced long-lasting suppression of neuronal firing in the VTA, while a complete return to baseline activity was seen in the SNC.

Conclusions: In the current study, cariprazine behaved as a potent D3/D2 receptor partial agonist in the VTA and the $\mathrm{SNc}$ by both partially suppressing dopaminergic firing and by reversing dopamine receptor full agonist-induced firing inhibition. These effects of cariprazine were prevented by the selective D2 receptor antagonist L741,626, but not the selective D3 receptor antagonist SB-277011A, suggesting that dopamine D2 receptor activation was the major component contributing to the suppressive effect of cariprazine on the dopaminergic cell bodies. Due to the lack of highly selective dopamine D2 and D3 receptor agonist compound tools, future studies using D3 and/or D2 knockout mice may be necessary to determine whether D3 receptors contribute to the cariprazine-mediated suppression of dopamine neuronal firing activity in the midbrain.

Keywords: Cariprazine, Dopamine, Schizophrenia, Electrophysiology.

Disclosure: Allergan, Inc.: Employee, Self.

\section{T164. Exploring Somatic Variation in Schizophrenia}

John Fullard, Alexander Charney, Mads Hauberg, Vahram Haroutunian, Panos Roussos*

Icahn School of Medicine at Mount Sinai, New York, New York, United States

Background: Multiple lines of evidence suggest a strong genetic component to schizophrenia (SCZ), and identification the SCZassociated genetic variants may provide critical knowledge for understanding and treating the disease. Although multiple recent genetic studies have identified numerous common and 
rare SCZ risk variants, overall these genetic variants account overall for only a small portion of the disease's heritability. One potential explanation for this result is that SCZ risk variants are not inherited through the germline, but instead arise during development through spontaneous mutations within brain of affected individuals, a phenomenon known as somatic mosaicism (SM). The aim of this study is to explore the hypothesis that SM in brain tissue contributes to the pathologic mechanisms associated with the development of SCZ.

Methods: Specimens from the prefrontal cortex (PFC) and temporal muscle of $5 \mathrm{SCZ}$ cases and 4 controls were obtained from the Mount Sinai NIH Brain and Tissue Repository. Deep whole-exome sequencing (250X coverage) was performed using DNA extracted from neuronal and nonneuronal nuclei, which were isolated from cortical specimens by fluorescent-activated nuclear sorting (FANS). We followed standard protocols for mapping reads and calling somatic single nucleotide variants (SNVs). Validation of somatic variants was performed with digital PCR (dPCR) and parallel sequencing of multiple clones (clone-seq).

Results: We identified 35 somatic SNVs in SCZ cases (7 SNVs per sample) and 35 somatic SNVs in controls (8.75 SNVs per sample), including 10 and 13 exonic variants in controls and SCZ cases, respectively. Somatic variants were validated by dPCR and clone-seq experiments. The majority of exonic variants in SCZ was non-synonymous (85\% or 11 out of 13 ); in controls only $40 \%$ of exonic variants were nonsynonymous (Fisher exact two-tailed $p=0.039$ ). Moreover, based on the Combined Annotation Dependent Depletion approach, which integrates multiple annotations into one metric by contrasting variants that survived natural selection with simulated mutations, the SCZ SNVs have higher deleteriousness on protein sequence compared to the control SNVs ( $\mathrm{t}$ test: $\mathrm{t}=-3.3, p=0.0017$ ). Pathway analysis of genes affected by SCZ variants showed enrichment for pathways related to neurodevelopment and cellular response to cAMP $(p<0.001$, fold change $>60)$. No pathways were significant for variants detected in controls.

Conclusions: In this study, we identified a number of cellspecific somatic variants by performing deep whole exome sequencing of DNA from FANS-separated neuronal and non-neuronal nuclei from the PFC. While there was no difference in the SCZ vs. control burden, we found more deleterious somatic variants within genes with an important role in neurodevelopment in SCZ compared to controls. Our study supports the hypothesis that somatic mosaicism might play a significant role in the etiopathogenesis of SCZ.

Keywords: Somatic Mutation, Postmortem Brain Tissue, Whole Exome Sequencing.

Disclosure: Nothing to disclose.

\section{T165. Cariprazine Modulates Hippocampal Gamma Oscillations In Vitro: Potential Involvement in Schizophrenia Symptoms}

Nika Adham*, Clement Lemercier, Christoph Kulisch, Béla Kiss, Balázs Lendvai, Zoltan Gerevich

Allergan, Inc., Jersey City, New Jersey, United States

Background: Cortical gamma oscillations, which establish precise temporal relationships between distinct neural networks, are disturbed in patients with schizophrenia. Positive and negative schizophrenia symptoms are thought to correlate with increased and decreased gamma oscillation power, respectively. Dopamine D3 receptor activation has been shown to decrease gamma oscillation power and periodicity in vitro by reducing the firing rate of pyramidal cells. Cariprazine, a potent $\mathrm{D} 3 / \mathrm{D} 2$ receptor partial agonist with preferential binding to the D3 receptor, is FDA approved in the US for the treatment of schizophrenia and acute manic or mixed episodes associated with bipolar I disorder. This study investigated the effects of cariprazine in comparison with aripiprazole, another dopamine receptor partial agonist antipsychotic, on in vitro gamma oscillations in the CA3b hippocampal area. The dopaminergic mechanism of action of cariprazine on these effects was also explored using relatively selective D2 and/or D3 receptor compound tools.

Methods: Gamma oscillations were induced in $400 \mu \mathrm{m}$ rat hippocampal slices ( $n=6-16$ per treatment condition) using bath perfusion of acetylcholine (ACh, 10 or $5 \mu \mathrm{M}$ ) and physostigmine (Physo, 2 or $1 \mu \mathrm{M}$ ). Extracellular field potentials were recorded from the stratum pyramidale of area CA3b in the hippocampal slices. To assess the effects of dopamine D2 and D3 receptor agonists and antagonists on gamma oscillations, slices were treated at various times with the selective D3 receptor antagonist SB $277011(30 \mu \mathrm{M}), \mathrm{D} 2$ preferring D2/D3 receptor partial agonist aripiprazole $(30 \mu \mathrm{M})$, selective D2 receptor antagonist SV-156 (30 $\mu \mathrm{M})$, and/or D3 receptor full agonist pramipexole (10 and $30 \mu \mathrm{M})$. To investigate whether cariprazine affects gamma oscillations, slices were treated with cariprazine $(10 \mu \mathrm{M})$ alone or in combination with both concentrations of pramipexole. Power, frequency, and half bandwidth values were normalized to a $10-\mathrm{min}$ period after $90 \mathrm{~min}$ of $\mathrm{ACh} / \mathrm{Physo}$ treatment (prior to drug application). The effects of the last $10 \mathrm{~min}$ of compound application were compared to this baseline period. To assess the effects of cariprazine on oscillation dynamics in vitro, ACh/Physo was washed out and then reapplied to induce desynchronization and resynchronization, respectively.

Results: Pramipexole $(30 \mu \mathrm{M})$, but not SB-277011, SV-156, or aripiprazole, inhibited the stable gamma oscillation parameters induced by higher $\mathrm{ACh} /$ Physo concentrations $(10 \mu \mathrm{M} / 2 \mu \mathrm{M})$. Similar to aripiprazole and the two dopamine receptor antagonists, cariprazine did not significantly modulate stable gamma oscillations. Cariprazine demonstrated partial agonist activity on the stable gamma oscillations in the presence of low or high concentrations of pramipexole. Treatment with a high concentration $(30 \mu \mathrm{M})$ of pramipexole significantly decreased stable gamma oscillation power and increased bandwidth and frequency $(P<.05$ for each), whereas at a lower concentration $(10 \mu \mathrm{M})$ it had no significant effect on any parameter. Pretreatment with cariprazine potentiated the effect of the low pramipexole concentration, significantly decreasing power and increasing bandwidth relative to control $(P<.05)$. In contrast, cariprazine antagonized the inhibitory effect of the high concentration of pramipexole on all three parameters. The effect of cariprazine was also explored using lower ACh/Physo concentrations $(5 \mu \mathrm{M} / 1 \mu \mathrm{M})$ that produce unstable gamma oscillations, which may be more similar to the disturbed oscillations observed in patients with 
schizophrenia. Cariprazine significantly decreased the bandwidth of these unstable oscillations $(P<.05)$; power and frequency were unchanged. In the dynamic oscillation model, cariprazine treatment significantly accelerated bandwidth normalization during resynchronization compared to control.

Conclusions: Cariprazine increased the periodicity of in vitro hippocampal gamma oscillations and acted as a D3 receptor partial agonist by augmenting the effects of low receptor activation and antagonizing those of high receptor activation. These results suggest that cariprazine may have a balancing effect on gamma oscillations in the hippocampus. Furthermore, the ability of cariprazine to modulate the speed of network oscillation resynchronization and normalization of unstable gamma oscillations may contribute to the improvements in schizophrenia symptoms seen in cariprazinetreated patients.

Keywords: CaripraZINe, Dopamine, Schizophrenia, Gamma Oscillation.

Disclosure: Allergan, Inc.: Employee, Self.

T166. Two Subgroups of First Episode Antipsychotic Naïve Schizophrenia Patients Identified From a Gaussian Mixture Model Based on Cognition and Electrophysiology Data That Indicate Different Response to Treatment

Nikolaj Bak*, Bjørn H Ebdrup, Bob Oranje, Birgitte Fagerlund, Maria Jensen, Signe Dûring, Mette Nielsen, Birte Glenthoj, Lars Hansen

\section{Copenhagen University Hospital, Glostrup, Denmark}

Background: Deficits in information processing are among the most robust findings in schizophrenia patients. Previous efforts to translate group-level deficits into individualized, clinically relevant information on treatment response have been non-successful possibly explained by the existence of biologically different disease subgroups. We applied machine learning algorithms on measures of early and late information processing as measured with electrophysiology and cognition to identify potential subgroups of schizophrenia. Methods: Sixty-six antipsychotic-naïve first-episode schizophrenia patients and sixty-five healthy controls underwent an electrophysiological test battery of early information processing, and a neurocognitive test battery to measure late information processing. Patients were assessed on the positive and negative syndrome scale (PANSS) before and after six weeks of monotherapy with the relatively selective D2 antagonist, amisulpride $(280.3 \mathrm{mg} / \mathrm{d} \pm 159)$. A statistically distinct principal component subspace was found based on 19 electrophysiological variables and 26 cognitive variables, using the Akaike Information Criterion (AIC). This reduced principal component subspace was used as input for a Gaussian Mixture Model (GMM) to identify subgroups of patients. The optimal number of groups were found by calculating the mean negative log likelihood in a leave-oneout cross-validation approach. To estimate the clinical relevance of the subgroup structure identified we used support vector machines (SVMs) to explore the relation between PANSS subscores and the identified subgroups.
Results: The optimal number of principal components was found to be four so the subspace based on the first four principal components was used for the GMM. We identified two statistically distinct subgroups of antipsychotic-naïve first-episode patients with the GMM analyses. We found no significant baseline psychopathological differences between these subgroups, but with SVM analyses, group could be predicted by the effect of treatment with an accuracy of $74.3 \%$, indicating that the two groups have a differential effect of treatment. This difference was primarily driven by a difference in the effect on negative symptoms.

Conclusions: Information processing data may be used to classify subgroups of schizophrenia patients. We identified two distinct subgroups, which were psychopathologically inseparable before treatment, yet their response to dopaminergic blockade was predicted with noticeable accuracy. This proof of principle study supports the presence of biologically based, clinically relevant subgroups of schizophrenia and implies that stratification of patients is required to recognize specific treatment needs in individual subgroups. The current results encourage further endeavors to apply datadriven, multivariate and multimodal models to facilitate progress from symptom-based psychiatry towards individualized treatment regimens.

Keywords: Antipsychotic-Naïve First-Episode Schizophrenia, Subgroups, Gaussian Mixture Model, Cognition, Electrophysiology.

Disclosure: Nothing to disclose.

\section{T167. Effect of MIN-101 on Cognition of in} Schizophrenia Patient With Predominant Negative Symptoms: A 12-Week Randomized, Double Blind, Placebo-Controlled Trial

Richard Keefe* ${ }^{*}$ Anzalee Khan, Corinne Staner, Joe Reilly, Jay Saoud, Michael Davidson, Remy Luthringer

Duke University Medical Center, Durham, North Carolina, United States

Background: To explore the effect of MIN-101, a compound with high affinities for sigma 2 and 5-HT2A receptors, compared to placebo on cognitive functioning by treating negative symptoms, in patients with stable symptoms of schizophrenia.

Methods: This multi-national Phase 2b trial enrolled 244 patients diagnosed with schizophrenia who were symptomatically stable for $\geq 3$ months prior to entering the trial and had baseline scores $\geq 20$ on the 3 -factors negative subscale of the PANSS. Patients were randomized to daily monotherapy with MIN $10132 \mathrm{mg}$, MIN-101 $64 \mathrm{mg}$, or placebo in a 1:1:1 ratio. The primary endpoint was the PANSS negative symptom score based on the 5-factors (pentagonal) model. Cognitive function was evaluated using the Brief Assessment of Cognition in Schizophrenia (BACS) just prior to drug administration as well as 4 weeks, and 12 weeks after treatment initiation. BACS subscale raw scores were converted to age and gender corrected $\mathrm{z}$ and $\mathrm{t}$ scores and composite $\mathrm{z}$ and $\mathrm{t}$ scores. The Mixed-Effect Model Repeated Measure (MMRM) with Last Observation Carried Forward (LOCF) was used to compare the BACS subscales and composite scores; violation of normality assumption 
(Shapiro-Wilk W $p$-value $\leq 0.01$ ), and examination of $\mathrm{Q}-\mathrm{Q}$ plots were assessed across treatment groups.

Results: The three treatment groups were balanced on all demographic and illness-related baseline characteristics. Completion rates for randomized patients in this 12 -week study were as follows: MIN-101 $62 \mathrm{mg}=69 \%$, MIN-101 32 $\mathrm{mg}=69 \%$ and placebo $=65 \%$. Both doses of MIN-101 were superior to placebo on the PANSS negative symptom for MIN 101 (p c 0.022 for the $32 \mathrm{mg}$; $\mathrm{p} \leq 0.003$ for the $64 \mathrm{mg}$ ) in a dose-dependent way. At baseline, the age- and genderadjusted BACS composite score (T) were 30.06, 29.00, and 30.11 for the placebo, MIN-101 $32 \mathrm{mg}$, and MIN-101 $64 \mathrm{mg}$, respectively. Following LOCF, the MIN-101 $32 \mathrm{mg}$ was superior to placebo after 12 weeks of treatment on the following subtests: verbal fluency ( $p \leq 0.05$ ), and the composite $\mathrm{z}$ score ( $\mathrm{p} \leq 0.05$; Effect size: 0.439 ). The $64 \mathrm{mg}$ dose did not have a statistically significant effect compared to placebo.

Conclusions: The lower dose of $32 \mathrm{mg} /$ day demonstrated statistically significant effect on subtests of the BACS and the BACS composite $\mathrm{z}$-score. The observed improvement in cognitive performance needs to be evaluated further It is not immediately clear why only the lower dose showed an effect on cognition and further analysis of patient characteristics at baseline may help explain.in terms of patient characteristics and relation to symptom change.

Keywords: Cognition, Schizophrenia, Negative Symptoms. Disclosure: Nothing to disclose.

\section{T168. Association Between Inflammatory Markers and Negative Symptoms in Individuals With Persistent Symptoms of Schizophrenia Treated With Clozapine}

David Goldsmith*, Sarah Kopelovich, Derek Novacek, Jonathon Widener, Evanthia Wommack, Jennifer Felger, Andrew Miller, Robert Cotes

\section{Emory University School of Medicine, Atlanta, Georgia,} United States

Background: Negative symptoms of schizophrenia are characterized by absent or diminished behavior and include decreased motivation, social withdrawal, as well as poverty of speech, decreased emotional reactivity and psychomotor retardation. Importantly, negative symptoms have consistently been identified as those features of the disorder that are most predictive of functional impairment and poor outcome. Moreover, whereas antipsychotic medications are effective in treating positive symptoms (e.g., delusions and hallucinations), they are less effective in treating negative symptoms. One pathophysiologic pathway that may contribute to negative symptoms in schizophrenia is inflammation. There is a growing literature that inflammatory cytokines may be linked to negative symptoms in individuals with schizophrenia, and no one has explored this specific connection in patients on clozapine.

Methods: Ten participants $(9 \mathrm{M}, 1 \mathrm{~F})$ with diagnoses of schizophrenia or schizoaffective disorder, all treated with clozapine, were included in this analysis. All subjects were recruited from the PSTAR (Persistent Symptoms: Treatment, Assessment and Recovery) Clinic, at Grady Memorial Hospital in Atlanta, Georgia. Inclusion criteria included evidence of persistent symptoms as measured by cutoff scores on the Positive and Negative Symptom Scale (PANSS), treatment with clozapine for at least three months, a documented clozapine level $>350 \mathrm{ng} / \mathrm{ml}$, and no change in clozapine dose greater then $100 \mathrm{mg}$ at least one month prior to study enrollment. The following inflammatory markers were measured: high sensitivity c-reactive protein (hsCRP), interleukin (IL) 1beta, IL-6, IL-10, and tumor necrosis factor (TNF). Blood sampling for plasma inflammatory markers were completed at standardized times to control for circadian variations. Clinical rating scales to assess negative symptoms included the PANSS as well as the Scale for the Assessment of Negative Symptoms (SANS). To assess functional ability, the World Health Organization Disability Assessment Schedule (WHODAS 2.0) was also administered. Pearson correlation coefficients were calculated for the relationship among inflammatory markers and negative symptoms.

Results: Mean clozapine dose in this analysis was $557.5 \mathrm{mg}$ (SD 128.05). Mean clozapine level was $759.8 \mathrm{mcg} / \mathrm{L}$ (SD 601.68). One subject had an hsCRP and IL-6 $>3$ SD from the mean concentration and that subject's hsCRP and IL-6 values were excluded from analysis. Mean hsCRP concentration $(n=9)$ was $3.72 \mathrm{mg} / \mathrm{L}$ (SD 2.74). Mean IL-1beta concentration $(n=10)$ was $0.67 \mathrm{pg} / \mathrm{ml}$ (SD 0.21). Mean IL-6 concentration $(n=9)$ was $1.16 \mathrm{pg} / \mathrm{ml}$ (SD 0.18). Mean IL-10 concentration $(n=10)$ was $0.44 \mathrm{pg} / \mathrm{ml}$ (SD 0.44$)$. Mean TNF alpha concentration $(n=10)$ was $5.71 \mathrm{pg} / \mathrm{ml}$ (SD 2.48). IL-1beta was significantly correlated with the passive/apathetic social withdrawal $(\mathrm{r}=0.657, p=0.039)$ and the disturbance of volition $(r=0.686, p=0.029)$ items of the PANSS, as well as the avolition - impersistence at work or school $(r=0.644, p=0.045)$, global rating of avolition-apathy $(\mathrm{r}=0.751, p=0.012)$, and the attention social inattentiveness $(r=0.665, p=0.036)$ items of the SANS. IL-10 was negatively correlated with the emotional withdrawal $(\mathrm{r}=-0.638, p=0.047)$ and the passive/apathetic social withdrawal $(r=-0.655, p=0.04)$ items of the PANSS as well as the negative symptom total score of the PANSS ( $\mathrm{r}=-0.792, p=0.006)$. IL-10 was also negatively correlated with the total score of the WHODAS $2.0(\mathrm{r}=-0.792$, $p=0.006)$.

Conclusions: IL-1beta, a pro-inflammatory cytokine, was significantly correlated with items assessing negative symptom domains of avolition and social deficits. Moreover, IL-10, an anti-inflammatory cytokine, was negatively correlated with similar negative symptom items, including the negative symptom total score on the PANSS as well as the total score on a scale of real-world functional and role impairment. Though the sample size is small, these data suggest that inflammation may be relevant for these negative symptom domains. Indeed, administration of inflammatory stimuli has been reliably linked to deficits in reward processing and motivation via effects of inflammatory cytokines on regions of the basal ganglia, including the ventral striatum. Individuals with depression and increased inflammation also exhibit decreased functional connectivity in reward circuits in association with anhedonia. Future work should further explore the relationship between negative symptoms and inflammation in individuals with schizophrenia. 
Keywords: Schizophrenia, Negative Symptoms, Inflammation, Cytokines, Clozapine.

Disclosure: Nothing to disclose.

\section{T169. D2/D3 Dopamine Receptor Binding With [F-18] Fallypride Correlates of Executive Function in Medication-Naïve Patients With Schizophrenia}

Monte Buchsbaum*, Nora Vyas, Douglas Lehrer, Bradley Christian, Brian Merrill, Nicholas Doninger, Jogesh Mukherjee

University of California, San Diego, San Diego, California, United States

Background: Converging evidence indicates that the prefrontal cortex is critically involved in executive control and that executive dysfunction is implicated in schizophrenia. Reduced dopamine D2/D3 binding potential has been reported in schizophrenia, and the correlations with neuropsychological test scores have been positive and negative for different tasks. The aim of this study was to examine D2/D3 dopamine correlates with frontal cortical related cognitive task performance in schizophrenia.

Methods: Resting-state 18F-fallypride positron emission tomography was performed on 20 medication-naïve and 5 previously medicated (for brief earlier periods) patients with schizophrenia and 19 age- and sex-matched normal controls. Striatal and extra-striatal dopamine D2/D3 receptor levels were quantified as binding potential using fallypride imaging. Magnetic resonance images in standard Talairach position and segmented into gray and white matter were coregistered to the fallypride images, and the AFNI stereotaxic atlas was applied. Two neuropsychological tasks known to activate frontal and temporal lobe function were chosen, the Wisconsin Card Sorting Test (WCST) and the California Verbal Learning Test (CVLT).

Results: Images of the voxel-by-voxel correlation coefficient between Fallypride binding and WCST and CVLT performance showed predominantly negative correlations in patients in contrast to positive correlations in healthy volunteers. This was especially marked for the temporal lobe, Brodmann areas 46 and 47, striatum, and thalamus. Examination of the distribution of correlation coefficients between neuropsychological scores and binding potential in regions of interest showed healthy volunteer correlations significantly more positive correlations with a more positive skewed distribution. Patients with schizophrenia had a negative skew to the distribution. Permutation analysis is used to confirm correlation differences, addressing the issue of regional intercorrelations.

Conclusions: The results of this study demonstrate that lower Fallypride binding potential in patients with schizophrenia may be associated with better performance and consistent with previous studies that failed to find cognitive improvement with typical dopamine-blocking medications.

Our findings in this study reinforce the concept of distributed influence of dopamine on performance involving both striatal and extrastriatal regions. These influences are trait-like in nature extending across cognitive tasks in healthy subjects. Patients with schizophrenia show relationships between performance and binding potential that are anomalous and likely heterogeneous, leaving a disturbed distribution of regional binding potential versus performance correlations. Our findings are also consistent with the widely interconnected network patterns observed in fMRI connectivity studies rather than with a restricted network of several small, localized, and specialized neural loci.

Keywords: Dopamine (D2, D3) Receptors, Positron Emission Tomography Imaging, Neurocognition.

Disclosure: Nothing to disclose.

\section{T170. Late Adolescent Shift From Local to Distributed Monosynaptic Inputs Onto Fronto-Posterior Cortical Projection Neurons}

Elisa Nabel, Michael Demars, Sarah Lopez, Giulia Taccheri, Hiroyuki Koike, Hirofumi Morishita*

Icahn School of Medicine at Mount Sinai, New York, New York, United States

Background: Visual attention, the cognitive ability that enhances the perception of selected stimuli, develops postnatally and is disrupted in neurodevelopmental disorders such as autism. A direct long-range projection from the anterior cingulate cortex (ACC) to primary visual cortex is a key circuit mediating "top-down" modulation of visual processing, which is a hallmark component of attention. However, little is known about this projection's input sources, and if inputs undergo developmental modifications. Methods: An intersectional viral genetic technique was employed to map the monosynaptic inputs onto top-down cells in adolescence and adulthood. Whole brain maps of top-down inputs were generated, and regions of interest were further characterized by cell type.

Results: Cortical areas contributed the majority of monosynaptic inputs onto top-down cells. Local inputs from the ACC and secondary motor area comprise the large source; other major sources of cortical contributions include the retrosplenal, prelimbic, and infralimbic cortex. Non-cortical regions provide distributed inputs from the basal forebrain, dorsal thalamus, and hypothalamus. Inputs from the basal forebrain, the major neuromodulatory source for this projection, comprise of approximately $80 \%$ cholinergic, $14 \%$ putative glutamatergic, and $6 \%$ inhibitory neurons. Comparison of the adult and adolescent whole brain maps revealed a larger number of inputs from local cortical regions during adolescence lost in adulthood. No age-related differences were observed in other cortical and noncortical areas.

Conclusions: This result suggests that local pruning during late adolescence causes a relative shift in input weight from local to distal brain regions between adolescence and adulthood. This cortical maturation may be required to establish effective top-down control of attention.

Keywords: Top-Down Control, Cortical Circuit Development, Anterior Cingulate Cortex, Visual Cortex, Synaptic Pruning.

Disclosure: Nothing to disclose. 
T171. Brexpiprazole Reduces Impulsivity in Patients With Schizophrenia: A Task-Based fMRI Study

Theo GM van Erp*, Ross A Baker, Kevin Cox, Taka Okame, Yoshitsugu Kojima, Anna Eramo, Steven G Potkin

University of California, Irvine, Irvine, California, United States

Background: Patients with schizophrenia show high levels of self-reported impulsivity and demonstrate self-control performance deficits on tasks that test response inhibition such as the stop-signal task. Impulsivity is a risk factor for suicide and drug abuse, both of which are increased in schizophrenia. It is hypothesized that inhibition of serotonin may reduce impulsivity in humans, but related pharmacologic treatments have not been well studied in controlled trials of impulsive behavior. Brexpiprazole is a serotonin-dopamine activity modulator that is a partial agonist at 5-HT1A and dopamine D2 receptors, and an antagonist at 5-HT2A and noradrenaline alpha1B/2C receptors, all at similar potency. The receptor profile of brexpiprazole suggests the potential to reduce impulsivity, thus the primary objective of our study was to evaluate the effect of brexpiprazole, via functional magnetic resonance imaging (fMRI), on inhibitionassociated right ventrolateral prefrontal cortex (VLPFC) activation. Right VLPC activity is associated with inhibition of impulsive behavior. The secondary objective was to evaluate the effect of brexpiprazole on safety and tolerability in patients with schizophrenia who have impulsivity.

Methods: The study (NCT02194933) was an exploratory, multicenter, open-label, blinded-to-dose fMRI trial in adults with schizophrenia, currently stable $(\mathrm{CGI}-\mathrm{S}<=4)$ with some impulsivity as defined by Barrett Impulsivity Scale (BIS) score $>=50$ at screening. After screening/washout, eligible patients were randomized to 6-week (42-day) treatment with either $2 \mathrm{mg}$ or $4 \mathrm{mg}$ brexpiprazole. Patients received an $\mathrm{fMRI}$ scan at baseline (BL, Day 0), Week 3 (Day 21), and Week 6 (Day 42) of the treatment phase, and completed a number of tasks related to impulsivity (Go/Nogo Task, Stop Signal Reaction Time [SSRT] Task) while undergoing fMRI. Patients also completed a self-report questionnaire (BIS-11) on the same days as the fMRI scans. Right VLPFC activation at Week 6 was compared with the corresponding baseline values on the No-go - Go contrast of the Go/No-go task and on the Stop - Go contrast of the Stop Signal Reaction Time Task (SSRT). Mixed model regression analysis with repeated measures (MMRM) was used to examine baseline to Week 3 and Week 6 mean blood oxygenlevel dependent (BOLD) activation in the right VLPFC. The model predicts BOLD activation with fixed class effect terms for the treatment and visit week, the treatment by visit week interaction, age and sex included as covariates. Statistical contrasts of differential change in activation between the treatments from baseline to followup were conducted with significance level of 0.05 (2-sided).

Results: A total of 38 patients were randomized, 19 to $2 \mathrm{mg} /$ day and 19 to $4 \mathrm{mg} /$ day. Mean BIS-11 scores at baseline were 76.5 [+/- standard deviation (sd) of 15.0] for the $2 \mathrm{mg}$ group and $75.4+/-16.1$ for the $4 \mathrm{mg}$ group. Twenty-seven patients at BL and Week 3 (14 at $2 \mathrm{mg} ; 13$ at $4 \mathrm{mg}$ ) and 23 patients at $\mathrm{BL}$ and Week 6 (13 at $2 \mathrm{mg} ; 10$ at $4 \mathrm{mg}$ ) had evaluable fMRI scans. No changes in BOLD activation in the right VLPFC (fMRI) nor on the false alarm rate (behavior) on the Go/NoGo task were observed. In contrast, brexpiprazole produced significant decreases in inhibition-related rVLPFC BOLD activation $[\mathrm{t}=3.07, \mathrm{df}=23, p=0.005]$ and stop signal reaction time (SSRT; behavior) $[\mathrm{t}=3.20, \mathrm{df}=17, p=0.005]$ between baseline and Week 6. Moreover, inhibition-related rVLPFC BOLD activation on the SSRT task showed a pattern consistent with a more rapid decrease in the $4 \mathrm{mg}$ compared with the $2 \mathrm{mg}$ brexpiprazole dose. I.e., rVLPFC activation for the $4 \mathrm{mg}$ dose shows a trend-level significant decrease from baseline to Week $3[\mathrm{t}=1.81, \mathrm{df}=46, p=0.08$, two-tailed] with a continued and significant decrease from baseline by Week $6[\mathrm{t}=2.24, \mathrm{df}=47, p=0.03]$, while the rVLPFC activation for the $2 \mathrm{mg}$ dose appears to be similar to baseline at Week 3 with a trend-level decrease by Week $6[t=1.60$, $\mathrm{df}=47, p=0.12$, two-tailed]. No unexpected safety or tolerability concerns were identified; tolerability was in line with what was observed in the Phase 3 studies of brexpiprazole for the treatment of schizophrenia.

Conclusions: Baseline impulsivity in patients entering the study was confirmed by relatively high baseline BIS-11 scores. The relative ease of the Go/No-Go task may explain the lack of change in rVLPFC activity or behavior. Significant decreases in both right VLPFC activity and behavior in the more complex and adaptive SSRT support the hypothesis that brexpiprazole not only reduces impulsive behavior, but also reduces inhibition-related rVLPFC activition. We acknowledge that the brexpiprazole-induced reduction in rVLPFC activitation observed here needs to be replicated, and further elucidation of impulsive neurocircuitry is needed. Our novel combination of behavioral tasks and fMRI measures of brain activity was sensitive to pharmacologic effects, suggesting further studies using similar techiques may provide additional insights about drug effects during psychiatric drug development.

Keywords: Brexpiprazole, Schizophrenia, Impulsivity.

Disclosure: Otsuka Pharmaceutical, Inc.: Grant, Self.

\section{T172. Cortico-Striatal Circuitry Regulates Sensorimotor Gating via DISC1/Huntingtin-Mediated Axonal Transport}

Minae Niwa*, Hanna Jaaro-Peled, Kazunori Nakajima, Atsushi Kamiya, Kafui Dzirasa, Toshifumi Tomoda, Akira Sawa

Johns Hopkins University School of Medicine, Baltimore, Maryland, United States

Background: Adult brain function and behavior are influenced by neuronal network formation. Deficits in prepulse inhibition (PPI) have been linked to abnormalities of sensorimotor gating and are associated with many brain disorders, such as schizophrenia, mood disorders, and Huntington's disease. Nonetheless, the biological mechanisms underlying behavioral performance in PPI have not been well examined.

Methods: A genetically-engineered mouse model with the Disrupted-in-Schizophrenia 1 locus impairment (Disc1 LI), which has a 40-kilo base locus covering exons $1,1 \mathrm{~b}, 2$, and 3 deleted, and carries the 25-bp deletion in exon 6, was used in 
the present study. The validity of this model was initially studied with the disturbance of neural migration and progenitor proliferation, as roles for DISC1 in these biological processes are well established. As a behavioral test, PPI test was performed. Brain volume was examined by ex vivo MRI. Levels of brain-derived neurotrophic factor (BDNF) in the cortex and the striatum were measured by enzyme-linked immunosorbent assay. Trafficking velocity was examined by the transport assay. Adeno-associated virus of BDNF was injected into the striatum for the rescue experiment in PPI. Lithium, which is a representative mood stabilizer and is reportedly neurotrophic and neuroprotective by increasing levels of BDNF, was administered to mice in drinking water. To examine the roles of Huntingtin (Htt) and DISC1 in BDNF transport machinery, co-immunoprecipitation and Western blots were performed.

Results: We observed the PPI deficits in Disc1 LI model, compared with wild-type mice. Smaller striatum volume and reduced striatal BDNF, likely due to its attenuated transport along the cortico-striatal tract, were observed in the Disc1 LI model in comparison to wild-type mice. The viral vectormediated BDNF supply to the striatum tended to rescue the PPI deficits in Disc1 LI model. Chronic lithium treatment normalized the PPI deficits in Disc1 LI model, at least in part, via the augmentation of striatal BDNF. The direct interaction of $\mathrm{Htt}$ and DISC1 protein likely accounted for at least part of the integration mechanism, as DISC1 is included in the motor machinery responsible for BDNF transport.

Conclusions: In the present study, we show that corticostriatal circuitry regulates sensorimotor gating via DISC1/ Huntingtin-mediated axonal transport. The present study provides new insight on a novel, critical biological mechanism that underlies PPI and information processing.

Keywords: Cortico-Striatal Circuit, Sensorimotor Gating, DISC1, Huntingtin, Axonal Transport.

Disclosure: Nothing to disclose.

T173. A Phase 1 Functional Neuroimaging Study of SEP-363856 in Healthy Volunteers With High or Low Schizotypy

Jadwiga Nazimek, Francesca Perini, Liliana Capitao, Shane McKie McKie, Michael Browning,

Catherine Harmer, Gerard Dawson, Una Campbell, Hiroyuki Nishikawa, Seth Hopkins, Antony Loebel, Kenneth Koblan, Bill Deakin*

University of Manchester, Manchester, United Kingdom

Background: SEP-363856 is a novel compound identified using phenotypic behavioral screening to be effective in animal models of schizophrenia (positive and negative symptoms) and depression, but without any of the D2 or 5-HT2A receptor activity of currently marketed antipsychotics. The molecular target(s) responsible for the profile of effects may include agonism at 5 HT1A and TAAR1 (trace amine associated receptor 1) receptors. A study was designed to evaluate the potential for antipsychotic- or antidepressantlike effects on reward- or emotional-processing, respectively, in order to inform subsequent decisions in the clinical development of SEP-363856.
Methods: In this Phase 1, double-blind, placebo-controlled study, 96 healthy volunteers with high (HS) or low schizotypy (LS) scores on the Schizotypal Personality Questionnaire were randomized to receive a single dose of SEP-363856 (50 mg), amisulpride (400 mg) or placebo. The functional magnetic resonance imaging (fMRI) study measured blood oxygen level dependent (BOLD) signals in brain regions of interest (ROIs) during performance of a Monetary Incentive Delay (MID) and N-back task. Secondary imaging endpoints included resting state connectivity (RSC). The antidepressant-like effects of SEP-363856 on emotional processing were also assessed outside the scanner using the P1vital Oxford Emotional Testing Battery (ETB).

Results: MID. The MID task induced neural activity as measured by BOLD signal responses in pre-specified ROIs in ventral striatum (VS), medial orbitofrontal cortex (mOFC) and insula. In both LS and HS, reward anticipation activated VS and deactivated mOFC, reflecting known dopamine incentive processes. An effect of schizotypy was observed in the insula in anticipation of loss, where HS appeared to be associated with insula activations relative to deactivations in LS. Compared with placebo, SEP-363856 decreased striatal activation and induced mOFC activation. In addition, SEP-363856 prevented the insula activation in HS. During the anticipation phase, the general pattern of effects (magnitude and direction) of SEP-363856 on neural activity in striatum and insula were similar to amisulpride. During the outcome phase, in the left insula and independently of schizotypy, SEP-363856 enhanced activation to both win and loss outcomes compared to amisulpride which reduced activation.

RSC. HS participants had significantly reduced default mode $(\mathrm{DMN})$, salience (SN) and right executive control network (ECN) connectivity compared to LS. Both drugs reduced the effect of schizotypy in all networks, amisulpride significantly in DMN and SEP-363856 in the anterior SN - notably in anterior insula.

N-back. Neither drug nor HS modified activations in the dorsolateral prefrontal cortex ROI.

ETB. Compared to placebo, SEP-363856 generally reduced performance of emotional processing across the task battery that were independent of the emotional valence, and was not differentially effective in HS versus LS. The pattern of effects with SEP-363856 was qualitatively similar to those observed with amisulpride, and different from the established profile of antidepressants such as SSRI's and SNRI's.

Conclusions: The overall pattern of activity in striatum, mOFC and insula during MID task performance suggests that the novel mechanism of action of SEP-363856 modulates dopaminergic incentive circuitry in a way relevant to improvement in positive and negative symptoms of schizophrenia. This inference is encouraged by the ability of SEP-363856 to reverse the effects of HS on insula BOLD signals in both the MID task and anterior SN resting state connectivity. The signature of SEP-363856 on emotional processing was similar to the D2-based antipsychotic amisulpride and distinct from monoamine-based antidepressants. Taken together, the results indicate that SEP-363856 presents a novel mechanistic profile with potential therapeutic benefit in psychotic disorders.

Keywords: Trace Amine-Associated Receptor 1, Functional MRI (fMRI), Reward Neural Circuitry, Individual Items of Positive and Negative Syndrome Scale, Salience Network. 
Disclosure: Sunovion: Research Funding, Self; P1vital: Research Funding, Self; P1vital: Shareholder, Self.

\section{T174. The Nucleus Reuniens of the Midline Thalamus Gates Prefrontal-Hippocampal Modulation of Ventral Tegmental Area Dopamine Neuron Activity}

\section{Eric Zimmerman*, Anthony Grace}

University of Pittsburgh, Pittsburgh, Pennsylvania, United States

Background: Excess dopamine (DA) signaling, ie "hyperdopaminergic states", are thought to underlie psychosis in a variety of psychiatric conditions, including schizophrenia, schizotypal personality disorder, bipolar disorder, and temporal lobe epilepsy. Previous work from our group has described a tripartite circuit comprised of the ventral subiculum (vSub), nucleus accumbens, and ventral pallidum, which governs the proportion of ventral tegmental area (VTA) DA neurons that are spontaneously active, ie "population activity". Population activity is a key parameter of VTA DA neuron activity: DA cells can only exhibit burst firing if they are spontaneously active. Therefore, population activity serves as a means of adjusting the amplitude of rapid, phasic DA system responses. For example, our group and others have observed aberrantly high population activity in the methylaxozymethanol acetate (MAM) model of schizophrenia. More recently, our group reported that inhibition of the infralimbic subdivision of the medial prefrontal cortex (ilPFC) increases DA neuron population activity, and that this effect depends on vSub. However, there is no direct projection from ilPFC to the ventral hippocampus. We propose that communication between the two structures is mediated by the nucleus reuniens of the midline thalamus (reuniens). Several anatomical studies have characterized dense, reciprocal connections between reuniens and ilPFC/ vSub, suggesting that reuniens could be involved in controlling VTA DA neuron population activity, and that ilPFC might exert its influence on VTA via reuniens. We hypothesize that reuniens governs VTA DA neuron population activity via the ilPFC-reuniens-vSub circuit, and altered signaling in this circuitry contributes to hyperdopaminergic states.

Methods: All experimental protocols were approved by the Institutional Animal Care and Use Committee at the University of Pittsburgh and were conducted in accordance with the National Institutes of Health Guide for the Care and Use of Laboratory Animals. Electrophysiological studies: recordings of VTA DA neurons were performed in anesthetized rats following intracranial infusion of pharmacological agents into reuniens, ilPFC, and/or vSub just prior to recordings. DA neurons were identified using wellestablished electrophysiological criteria. Three parameters of DA neuron activity were measured: (1) population activity (number of spontaneously firing DA neurons per electrode track, i.e. cells/track), (2) firing rate, and (3) the percentage of action potentials occurring in bursts. Population activity was determined by counting the number of spontaneously firing DA neurons encountered while making 6-9 vertical passes (tracks) through the VTA. Behavioral studies: locomotor activity was measured in an open field immediately following amphetamine administration in animals receiving intracranial infusion of NMDA or vehicle into reuniens.

Results: We show that pharmacological stimulation of reuniens enhances VTA DA neuron population activity and amphetamine-induced hyperlocomotion, a behavioral indicator of an over-responsive DA system. This effect of reuniens stimulation on population activity is prevented if vSub is also inhibited. In addition, pharmacological inhibition of ilPFC enhances VTA DA neuron population activity, but this effect does not occur if reuniens is also inhibited. Pharmacological inhibition of ilPFC also modulates firing activity in reuniens and the reticular nucleus of the thalamus (TRN), highlighting a potential pathway mediating the effect of ilPFC manipulation on VTA DA neuron activity.

Conclusions: These findings suggest that increased DA neuron population activity following inhibition of ilPFC may occur via disinhibition of reuniens, enhancing DA system gain. Indeed, dysfunction in prefrontal cortex, as well as hyperactivity in thalamus and ventral hippocampus, are thought to underlie the psychotic symptoms of schizophrenia and other disorders, and show activation with psychotomimetic drugs. Therefore, loss of prefrontal regulation via disruption of ilPFC-reuniens or ilPFC-TRN communication could lead to a dysregulated hyperdopaminergic state, and may play a role in psychotic disorders.

Keywords: Thalamus, Schizophrenia, Ventral Tegmental Area (VTA).

Disclosure: Nothing to disclose.

\section{T175. Dysfunctional Emotion Discrimination in Schizophrenia is Associated With HSV-1 Infection and Improves With Antiviral Treatment}

Vishwajit Nimgaonkar*, Triptish Bhatia, Joel Wood, Kehui Chen, Sreelatha Narayan, Satish Iyengar, Konasale Prasad, Robert Yolken, Faith Dickerson, Ruben Gur, Raquel Gur, Smita N Deshpande

University of Pittsburgh School of Medicine, Pittsburgh, Pennsylvania, United States

Background: Dysfunction in social cognition (SC) is associated with considerable disability in schizophrenia (SZ). A recent meta-analysis indicates that deficits in SC contribute to long term outcome in SZ. Like other cognitive domains, variation in SC is likely to be multi-factorial. We investigated its association with Herpes Simplex Virus, type 1 (HSV-1), which infects over $50 \%$ of US adults and causes lifelong, latent infection in human neurons. HSV-1 rarely causes encephalitis, but over ten cross-sectional studies indicate that persistent, non-encephalitic infection is associated with cognitive dysfunction, particularly among persons with SZ. The association between HSV-1 dysfunction and cognitive dysfunction is further supported by MRI studies that indicate reduced fronto-temporal gray matter volume in HSV-1 infected individuals. One randomized controlled trial (RCT) also suggested beneficial cognitive effects of Valacyclovir (VAL), an HSV-1 specific antiviral drug, over placebo when added to antipsychotic treatment for outpatients with SZ. Therefore, we investigated whether $\mathrm{HSV}-1$ related to non-encephalitic cognitive dysfunction is progressive or remediable with VAL. 
Methods: We investigated two samples from Delhi, India following written informed consent: (i) Sample 1, prospective naturalistic follow up (PNFU): Among persons with or without SZ, temporal changes in cognitive functions were analyzed in relation to HSV-1 infection at baseline $(N=226$, SZ, $N=138$, without SZ, $N=88$ ). (ii) Sample 2, RCT. Among stabilized HSV-1 infected outpatients with SZ, VAL (1.5 G twice daily for 16 weeks) or placebo (PLA) were added to ongoing antipsychotic treatment $(N=67)$, following a 2week placebo run in. VAV/PLA were allocated using a stratified randomization design. All clinical evaluations were conducted blind to VA/PLA status. In both samples, participants were evaluated using structured diagnostic interview schedules and consensus diagnoses established by board certified psychiatrists/psychologists. HSV-1 infection (seropositivity) was estimated using sensitive and specific serum IgG antibody assays. Individuals with prior encephalitis were excluded. Eight cognitive domains were evaluated using the Hindi version of the Penn Computerized Neurocognitive Battery.

DATA ANALYSIS. The accuracy scores on each cognitive domain were standardized using a separate group of Indian control participants, and the resultant Z-scores were used in all subsequent analysis. PNFU sample: A fully conditional specification (FCS) was used to perform multiple imputations. Baseline comparisons utilized generalized linear models (GLM) with HSV-1 seropositive state as the predictor and diagnostic status, sex and age as covariates. For temporal trajectory analyses of cognitive functions, GLM analyses were conducted for each cognitive domain using as the dependent variable the change in $\mathrm{z}$ score between baseline and follow-up divided by the duration of follow up for the respective cognitive domain (i.e., slope of cognitive change). HSV-1 infection (based on elevated HSV-1 antibody titers) was used as the predictor and the correlates included diagnostic status (SZ/not SZ), sex, age and the baseline measure for the relevant cognitive domain. An interaction term between diagnostic status and HSV-1 seropositive state was added in a subsequent model. All analyses were conducted using the Statistical Package for Social Sciences (SPSS v21). RCT sample: We used intent-to-treat analysis. With three time points of assessment, baseline (week 0), after completing VAV/PLA (week 16) and one month after completing VAV/PLA (week 20), we used a mixed model repeated measures analyses. The model included arms (VAV, PLA), time (visit 2, visit 7 and visit 8 ), time ${ }^{\star}$ arm interaction, gender, age and SES. For the two intervention groups (VAV, PLA), effect sizes for the changes in cognitive domains at different assessment points in relation to baseline values were calculated by Cohen's $d$, adjusted for the covariates age, gender and SES. Changes from baseline were examined using the mixed model analysis.

Results: PNFU: At study entry, HSV-1 infected participants had significantly lower accuracy scores than uninfected participants for Emotion Identification and Discrimination (EMOD), Spatial memory and Spatial ability $(p=0.025$, 0.029 and 0.046 , respectively), regardless of SZ diagnosis. Over a mean follow up of $1.93 \pm 1.07$ years, significantly steeper temporal change for EMOD was observed in the
HSV-1 seropositive group ( $p=0.03$ ). HSV-1 seropositivity was not associated with SZ diagnosis.

RCT: VAV was well tolerated, and dropout rates were similar in the VAV/PLA groups $(N=56$ completers, VAV 25; PLA 31). EMOD improved significantly in VAV-treated patients $(p=0.048$, Cohen's $\mathrm{d}=0.43)$.

Conclusions: Dysfunction in EMOD was associated with non-encephalitic HSV-1 exposure, regardless of SZ diagnosis. It worsened over time and improved with VAV treatment in SZ patients infected with HSV-1. Since emotion identification and discrimination is proposed as a core feature of social cognition, HSV-1 infection could lead to impairments in social cognition and adjunctive treatment with VAV may improve associated social cognitive deficits. A previous study similarly noted temporal worsening of executive function over time and their improvement following VAV. Thus, the improvements observed following VAV are the domains that show progressive worsening with HSV-1 exposure.

Keywords: Cognition, Schizophrenia, Emotion. Disclosure: Nothing to disclose.

\section{T176. NeuroDam: Longitudinal Assessment of Neuronal 3D Genomes in Mouse Prefrontal Cortex}

Amanda Mitchell ${ }^{*}$, Behnam Javidfar, Lucy Bicks, Rachael Neve, Krassimira Garbett, Sharon S Lander, Karoly Mirnics, Hirofumi Morishita, Marcelo Wood, Yan Jiang, Inna Gaisler-Salomon, Schahram Akbarian

Icahn School of Medicine at Mount Sinai, New York, New York, United States

Background: Neuronal epigenomes, including chromosomal loopings moving distal cis-regulatory elements into proximity of target genes, could serve as molecular proxy linking present-day-behavior to past exposures. However, longitudinal assessment of chromatin state is challenging because conventional chromosome conformation capture assays essentially provide single snapshots at a given time point, thus reflecting genome organization at the time of brain harvest and therefore are non-informative about the past.

Methods: Here, we introduce 'NeuroDam' to assess epigenome status retrospectively. Short-term expression of the bacterial DNA adenine methyltransferase Dam, tethered to the Gad1 gene promoter in mouse prefrontal cortex neurons, resulted in stable GmethylATC tags at Gad1-bound chromosomal contacts.

Results: We show by NeuroDam that mice with defective cognition 4 months after pharmacological NMDA receptor blockade already were affected by disrupted chromosomal conformations shortly after drug exposure.

Conclusions: Retrospective profiling of neuronal epigenomes is likely to illuminate epigenetic determinants of normal and diseased brain development in longitudinal context.

Keywords: Neuronal Epigenome, Behavior, Time.

Disclosure: Nothing to disclose. 


\section{T177. Characterizing the Splicing Variants of GAD1 in Human Brain}

Ran Tao*, Kasey Davis, Chao Li, Joo Heon Shin, Bin Xie, Yuan Gao, Andrew Jaffe, Joel Kleinman, Daniel Weinberger, Thomas Hyde

Lieber Institute for Brain Development, Baltimore, Maryland, United States

Background: GAD1 gene located at chromosome 2q31.1 in human, encodes glutamic acid decarboxylase 67 (GAD67), which is the rate limiting enzyme in the synthesis of gammaaminobutyric acid (GABA), the major inhibitory neurotransmitter in mammalian brain. Abnormalities of GABAergic interneurons are one of the most consistent findings in postmortem studies for schizophrenia, especially the decreased expression of GAD67 in schizophrenia. Previous studies have suggested that GAD1 promoter genetic variants are associated with schizophrenia. Schizophrenia is a highly heritable disorder with a strong developmental component. Many risk associated schizophrenia genes manifest expression abnormalities early in development. GAD1 expression changes markedly over the course of brain development. In the fetal rodent, two GAD1 alternative transcripts, named for the insertion of two cassette exons (I80 and I86) are highly expressed in the brain, leading to two enzymatically inactive proteins, a $25-\mathrm{kDa}$ protein (GAD25) and a $44-\mathrm{kDa}$ protein (GAD44). These two truncated transcripts are preferentially expressed in the fetus, with a rapid decline after birth. On the other hand, the full length transcript, GAD67, is lowly expressed in the fetal brain and dramatically increased after birth. To better understand the role of GAD1 transcripts in both normal brain development and the pathology of schizophrenia, we conducted a serious of experiments to characterize the alternative splicing of GAD1 and the expression pattern of these variants. We also explored the role of clinical risk SNPs and DNA methylation in human prefrontal cortex and hippocampus across development and in schizophrenia.

Methods: Postmortem Brains: Postmortem brains were collected at the CBDB, NIMH with informed consent of the next kin under NIMH protocol $90-\mathrm{M}-0142$ and the BTBDD at the NICHHD under contracts NO1-HD-4-3368 and NO1-HD-4-3383.

Identify GAD1 transcripts: To find potential splicing variants, RNA sequencing was performed on two commercial (Clontech) pooled poly A+ RNA samples (human fetal brain poly A+ RNA and human adult brain poly A+ RNA). The results were mapped to GRCh37/hg19 by TopHat. Based on the previously identified GAD1 gene exons and RNA sequencing results, we designed primer pairs to amplify fulllength and portions of GAD1 transcripts using Platinum Taq DNA polymerase (Invitrogen). All PCR products were cloned into pCR4-TOPO vector (Invitrogen) and sequenced. RNA sequencing: lug DNase treated total RNA of each subjects was used to generate a cDNA library for high throughput DNA sequencing according to manufacturer's protocol. Paired-end reads of cDNA sequences obtained by the HiSeq 2000 (Illumina) were aligned to the human genome reference (UCSC hg19) by TopHat (v2.0.4). To measure mRNA expression of GAD1 transcripts, we acquired the mapped reads covering GAD1 genomic region, corresponding to chr2:171,658,206-171,748,200 on genome build GRCh37/hg19. The mapped reads on the unique junction for each transcript have been used as quantification for GAD1 transcripts.

Quantitative real-time PCR and genotyping: The expression levels of GAD1 transcripts were measured in a large postmortem DLPFC sample cohort using the TaqMan qRT-PCR method. Genotyping was conducted using the TaqMan 5' exonuclease allelic discrimination assay (Applied Biosystems).

DNA methylation: Methylation of DNA extracted from DLPFC was assessed according to the manufacturer's instructions using the Infinium HumanMethylation450 BeadChip Kit (Illumina), which measures CpG methylation across $>485,000$ probes covering $99 \%$ RefSeq gene promoters, including the GAD1 gene. The methylation data were processed and normalized using the minfi Bioconductor package.

Results: We report the discovery of 10 previously unannotated transcripts of GAD1 in human brain, produced by novel exons, exon skipping and/or alternative exonic boundaries. Among those transcripts, expression levels of four novel GAD1 transcripts (I80, I86, 8A and 8B) showed a life trajectory expression pattern that is the inverse of the expression of full length GAD1 transcript, which encodes GAD67, in PFC and hippocampus. The quantification of those alternative transcripts suggested that GAD67 and GAD25 are two most abundant isoforms in human brain. Besides confirming the decreased expression of GAD67 in the patients with schizophrenia, we have provided further evidence that GAD25/GAD67 ratio was up-regulated in the patients with schizophrenia and also associated with allelic variation at the schizophrenia-risk SNP rs3749034 in the GAD1 promoter. The methylation levels of two CpG loci within the putative GAD1 promoter were significantly associated with rs3749034. The genotype at the schizophrenia-associated risk locus in GAD1 (rs3749034) is associated with regionally specific DNA methylation at the two CpG loci in the promoter region of the gene.

Conclusions: GAD1, as a candidate gene for schizophrenia, has been intensively studied in the postmortem brain in the past decade. Our study has identified multiple novel alternative transcripts in human brain derived from GAD1, including four fetal predominant transcripts, which lead to four truncated GAD1 isoforms. We confirmed our previous findings of expression abnormalities for GAD1 in schizophrenia. However, we also have observed two DNA methylation loci in the GAD1 promoter that are significantly associated with the schizophrenia-risk SNP rs3749034 in PFC and hippocampus. Our results suggest that the splicing of GAD1 is complex in human brain and epigenetic regulation elements may contribute to the expression changes of GAD1 in patients with schizophrenia.

Keywords: Schizophrenia, DNA Methylation, Gene Expression, GAD, Alternative Splicing.

Disclosure: Nothing to disclose. 
T178. Genetic Variants Associated With Neurocognition Impact Antipsychotic Response in Patients With Chronic Schizophrenia

\section{Maju Koola*, Kristin Bigos, Fengyu Zhang}

Sheppard Pratt Health System, Baltimore, Maryland, United States

Background: Patients with schizophrenia experience a cognitive deficit, and poor cognition is associated with a poor long-term outcome in patients with schizophrenia. The objective of this study was to examine how common genetic variants associated with neurocognition predict the clinical response to antipsychotic treatment.

Methods: The sample includes 418 individuals of European ancestry from the Clinical Antipsychotic Trial of Intervention Effectiveness (CATIE) study. Clinical data and genomewide single nucleotide polymorphisms (SNPs) genotype data were acquired through the publically available CATIE schizophrenia dataset. We performed a genome-wide association analysis of five cognitive domains (working memory, processing speed, verbal memory, reasoning and problem solving, vigilance/attention) measured in patients with schizophrenia at their baseline visit in the CATIE trial. The analysis was performed using a general linear model while controlling for age, sex, years of treatment. Polygene scores based on additive model and weighted by coefficients were created for each subject based on SNPs that showed association signal with at least one of the cognitive domains. Cox proportional hazard regression model was used to test the effect of polygene scores on time to discontinuation for each antipsychotic. Positive and Negative Syndrome Scale (PANSS) and cognitive measures were analyzed using a general linear mixed model that used the data at baseline and last observation (end of phase or end of study). Individual estimates of antipsychotic drug clearance were used as covariates for all analyses related to antipsychotic response. Analyses were performed using PLINK and SAS 9.3.

Results: We observed 15862 SNPs associated with at least one of the five cognitive domains at the threshold of $p<0.01$. No SNPs were associated with cognition at the GWA level $(p<5 \mathrm{e}-08)$, but strong signals $(p<10 \mathrm{e}-07)$ were observed at rs10492093, rs6589208, rs17575566, rs4740196 and rs531269), mostly located at intergenic regions. SNPs in or nearby genes MACC1, FHIT, PCBD1, UNC5B, KALRN, HEPHL1 were associated with neurocognition at $(p<5 \mathrm{e}-06)$. Polygene scores explained a large proportion of the variability in most cognitive domains ( $\mathrm{r} 2>0.5$ ), except reasoning and problem solving $(\mathrm{r} 2=0.27)$ and was strongly associated with the neurocognitive composite score $(\mathrm{r} 2=0.87)$. Time to antipsychotic discontinuation was associated with the polygene scores for all five cognitive domains and the neurocognition composite score, as well as social cognition $(p<0.05)$. The stronger significant association between polygene scores for neurocognition and time to discontinuation was for olanzapine $(P<0.005$ for most domains) compared with all medications combined. Polygene score for social cognition was more significantly associated with time to discontinuation especially in olanzapine, quetiapine, and ziprasidone $(P<0.01)$, than in risperidone and perphenazine. Finally, fifty-eight SNPs nominally associated with at least one of the domains of neurocognition affected both discontinuation and the improvement of PANSS scores.

Conclusions: In this study, we found SNPs associated with cognition significantly predict the treatment response in patients with schizophrenia, especially in individuals treated with olanzapine. Future studies are warranted to investigate the 58 SNPs associated with cognition, which impact the clinical response to antipsychotics.

Keywords: Schizophrenia, Cognitive Impairments, Genetics. Disclosure: Nothing to disclose.

T179. Fluctuations in Hallucinatory Experiences Within a day in Relation to Dopamine D2receptor Blockade With Antipsychotics in Patients With Schizophrenia

Hiroyuki Uchida*, Teruki Koizumi, Robert Bies, Bruce Pollock, Takefumi Suzuki

\section{Keio University School of Medicine, Tokyo, Japan}

Background: Dosing schedule of antipsychotics has conventionally aimed at assuring constant delivery of the drug, based on the assumption that blockade of dopamine D2 receptors needs to be continuous to counteract positive symptoms. On the other hand, recent evidence shows that extended but regular blockade of dopamine D2 receptors with antipsychotic drugs may be sufficient for maintenance treatment, suggesting a possibility that lower blockade of dopamine D2 receptors at trough does not always result in worsening of positive symptoms. However, there has been no study to examine fluctuation in positive symptoms within a day in relation to dopamine D2 blockade with antipsychotic drugs in patients with schizophrenia in the chronic phase of the illness. Whether the fluctuation is unrelated to the degree of dopamine D2 receptor blockade with antipsychotic drugs could have significant impact on the dosing schedule; for example, burdensome twice or thrice dosing could be converted to once daily dosing irrespective of the biological half-life of the compound. This study explored this intriguing clinical question.

Methods: Out- or in-patients who fulfilled the following criteria were included: (1) having diagnosis of schizophrenia according to the International Classification of Diseases, the 10th edition, (2) having received stable treatment with antipsychotic monotherapy for 2 weeks or more with risperidone or olazanpine, (3) experiencing auditory hallucinations of at least minimal severity as assessed by the physician in charge, (4) being capable to provide informed consent, and (5) age of 18 years-old or older. Subjects were asked to record the time, frequency, and duration during 8 time periods (i.e. 00:00-03:00, 03:00-06:00, 06:00-09:00, 09:00-12:00, 12:00-15:00, 15:00-18:00, 18:00-21:00, and 21:00-24:00) for 3 days. Time periods that overlapped the time for sleep were excluded from the analysis. Time and degree of peak and trough dopamine D2 receptor blockade levels with antipsychotics were calculated from 2 sparsely collected plasma drug concentrations for each subject, using population pharmacokinetic models and our D2 prediction model (Nakajima et al. Schizophr Bull 2016). Frequencies and durations of auditory hallucinations were compared between the peak and trough time periods in estimated D2 
receptor blockade, using a paired t-test. Values are shown as mean \pm SD.

Results: 27 patients have participated in this study as of 3rd August 2016; the preliminary analysis included the data from 11 participants (11 inpatients; 7 men; age, $61.5 \pm 9.2$ years; duration of illness, $36.4 \pm 61.3$ years; Positive and Negative Syndrome Scale total score, $70.2 \pm 20.8$ ). No significant difference was found in the frequency or duration of auditory hallucinations between the peak and trough time periods (frequency, $0.36 \pm 0.50$ vs. $0.45 \pm 0.52$; duration, $21.8 \pm 30.3$ vs. $27.3 \pm 31.3$ mins). Auditory hallucination was found to occur most frequently during the time period of 18:00-21:00 at $63.3 \%$ ( $p=0.03$ by chi-squared test).

Conclusions: The preliminary results suggest that the occurrence of auditory hallucinations is not related to dopamine D2 receptor blockage with antipsychotics within a day in patients with schizophrenia; rather, it may be associated more with circadian rhythm. Although a small sample size and large SDs call for a cautions interpretation, if this finding is confirmed in further investigations, the conventional dosing schedule in the treatment of schizophrenia will need to be revisited.

Keywords: Dopamine (D2, D3) Receptors, D2 Dopamine Antagonists, Schizophrenia, Dopamine, Antipsychotics, Antipsychotics.

Disclosure: Nothing to disclose.

\section{T180. Imaging Genetic Correlations in Structure Between Brain Regions}

Aaron Alexander-Bloch*, Samuel R Mathias, Ravi Duggirala, Joanne Curran, John Blangero, David Glahn

Yale University School of Medicine, New Haven, Connecticut, United States

Background: How genetics influence human brain morphology remains an outstanding question for clinical neuroscience. The majority of research to date has investigated brain regions as relatively independent phenotypes, for example, describing regional heritability and in some cases suggesting regionally-specific genetic factors. In contrast, the study of the genetic relationships between brain regions is increasingly recognized as a critical area of investigation at the intersection of neuroimaging, genetics and network science.

Methods: This study is based on data from the Genetics of Brain Structure and Function study (GOBS). The sample consists of $\sim 1500$ brain MR images of individuals of Mexican-American heritage living in San Antonio, Texas, in the form of large families (extended pedigrees) facilitating relatively powerful genetic inference. All scans were acquired on a Siemens 3T TIM Treo at the University of Texas Health Science Center at San Antonio, using multiple highresolution T1-weighted 3D turbo-flash sequences. FreeSurfer software was used to generate regional measures of cortical thickness and cortical surface area. SOLAR software was used to estimate pairwise genetic correlations between every pair of brain regions, a measurement of the genetic contribution to inter-regional covariance, to generate largescale genetic correlation networks.
Results: The average genetic correlation (rg) is 0.45 for thickness, 0.47 for surface area. The genetic correlation matrices for thickness and surface area are significantly correlated with one another $(\mathrm{r}=0.5, p<0.001)$. The highest $\mathrm{rg}$ for each region tends to be the contralateral homologue in the opposite hemisphere. Compared to the statistical control of randomly generated networks of the same size, networks of genetically correlated brain regions have significantly higher modularity (network community structure, $p<0.001$ ) as well as significant skew in degree distribution towards a relatively small number of hub regions $(p<0.001)$. High degree hubs in these networks occur in multimodal association areas including prefrontal cortex and the lateral temporal-parietal junction. There is a significant, non-linear relationship with anatomical distance such that stronger genetic correlations tend to occur with regions in anatomical proximity to a region (or to its contralateral homologue). The modular genetic communities are formed largely of spatially contiguous regions of cortex that are symmetric between left and right hemispheres. Genetic communities differ in the modular structure (which regions belong to which modules) between cortical thickness and surface area. Conclusions: This is the first systematic study of interregional genetic correlations using the GOBS sample, replicating and extending previous work on the the genetic correlation structure between regions. Networks of genetically correlated brain regions have both similarities and significant differences from other complex networks such as functional connectivity or white matter networks. Interregional relationships may be used to facilitate gene discovery in both healthy and clinical populations at the network level.

Keywords: Imaging Genetics, Network Analysis, Structural MRI.

Disclosure: Nothing to disclose.

T181. AUT00206, a Novel and Selective Kv3 Channel Modulator for the Treatment of Schizophrenia Restores Cognitive Function in an Animal Model in 2 Tasks in the Presence of Antipsychotic Drugs

Jo Neill*, Daniela Cadinu, Mike Harte, Ben Grayson, John Gigg, Colin Dourish, Giuseppe Alvaro, Charles Large

University of Manchester, Manchester, United Kingdom

Background: Although antipsychotic drugs alleviate positive symptoms of schizophrenia, cognitive deficit and negative symptoms remain an unmet clinical need (Keefe et al 2007. Arch Gen Psychiatry 64: 633-647). Accumulating evidence supports glutamatergic dysfunction in the pathophysiology of schizophrenia, leading to disinhibition of cortical circuitry, dysregulation of gamma oscillations and reductions in the calcium binding protein parvalbumin (PV), located in fast spiking GABAergic interneurons (Tse et al 2015. Biol Psychiatry 77: 929-939, for review). The voltage gated potassium channel Kv3.1 is predominantly localized to PVpositive inhibitory interneurons and has been shown to be reduced in un-medicated schizophrenia patients (Yanagi et al 2014. Mol Psychiatry 19: 573-579). We have extensively demonstrated efficacy of a novel molecule targeting these 
Kv3.1 channels, AUT00206 to restore sub-chronic PCP (scPCP) induced cognitive and social behaviour deficits, in addition to restoration of PV in cortex and hippocampus (Neill et al, Poster M153, this meeting, 2015). Although scPCP is a well validated model for chronic schizophrenia and of particular relevance to this project as it produces robust cognitive and PV deficits (Neill et al 2010. Pharmacol \& Ther. 128(3): 419-432) in order to fully mimic the clinical condition, the animal model must include chronic antipsychotic treatment prior to, and concomitant with, treatment with any novel drug. Here we test the efficacy of AUT00206 to restore cognitive function in two tests for different domains affected in schizophrenia in scPCP treated rats that also received sub-chronic treatment with the dopamine antagonist drug for schizophrenia, haloperidol or the dopamine/serotonin antagonist drug, olanzapine at doses providing $>70 \%$ dopamine D2 receptor occupancy (Kapur et al 2003. JPET 305: 625-631).

Methods: Fifty adult female Lister Hooded rats, received vehicle or scPCP $(2 \mathrm{mg} / \mathrm{kg})$ i.p. twice daily for 7 days, followed by 7-days washout. scPCP-treated rats then received 14 days treatment with haloperidol $(0.1 \mathrm{mg} / \mathrm{kg}$, i.p. once per day) or olanzapine $(1.2 \mathrm{mg} / \mathrm{kg}$, i.p. once per day) followed by acute AUT00206 at $60 \mathrm{mg} / \mathrm{kg}$, given orally once per day (for novel object recognition-NOR testing, visual recognition memory). A separate cohort of 40 female Hooded Lister rats received 21 days' treatment with haloperidol plus AUT00206 at $60 \mathrm{mg} / \mathrm{kg}$ orally once per day for 14 days from day 7 onwards (attentional set shifting task-ASST, executive function). AUT00206 was also tested alone in these tests in scPCP-treated rats. We chose a dose of $60 \mathrm{mg} / \mathrm{kg}$ given via the oral route, which was previously shown to be most effective in our scPCP model and is predicted to be a clinically relevant dose (see Hutchison et al. this meeting).

Results: Acute treatment with AUT00206 restored NOR deficits induced by scPCP alone and after sub-chronic treatment with haloperidol $(P<0.05)$ and olanzapine $(P<0.05)$. scPCP treatment produced a significant and selective deficit in the extra dimensional shift phase of the ASST $(P<0.001)$ which was significantly attenuated by 14 day treatment with AUT00206 at $60 \mathrm{mg} / \mathrm{kg}(P<0.001)$ alone as well as by the combination of AUT00206 (14 days) and haloperidol (21 days; $P<0.001$ ).

Conclusions: Studies in animals (Dean Prog 2006. Neuropsychopharmacol Biol Psych. 30: 174-189) and in chronic schizophrenia patients (Navari and Dazzan 2007. Psychol Med. 39: 1763-1777) suggest that antipsychotics can adversely affect neuronal structure and function which may further impair cognition. Our data demonstrate the efficacy of a novel Kv3.1 channel modulator in the scPCP model for schizophrenia, in the presence of antipsychotic drugs at D2 blocking doses. These results provide support for the hypothesis that modulation of Kv3.1 channels can restore cognitive function in 2 different cognitive domains affected in schizophrenia in the presence of chronic antipsychotic treatment. Work to date with this molecule has demonstrated efficacy in in vitro models for schizophrenia (Large et al, this meeting) and efficacy in restoring functional and pathological deficits in extensive studies in our scPCP model. Phase Ia clinical evaluation of the molecule has now been successfully completed (Hutchison et al, this meeting) and
Phase Ib trials are currently underway. We continue to evaluate the efficacy of AUT00206 in an animal model for prodromal schizophrenia under development in our laboratory (Edye et al 2016. J Psychopharmacol. 30(8): A40). Parallel on-going studies in our laboratory are exploring the effects of AUT00206 on restoring fronto-parietal-striatal network functional connectivity in the scPCP model using in vivo electrophysiology.

Keywords: Kv3 Family, Cognitive Impairments, Animal Models, Parvalbumin Interneurons, Schizophrenia, Dopamine, Antipsychotics.

Disclosure: Autifony: Research Grant, Self via University of Manchester.

\section{T182. Conjoint Analysis Reveals Divergent Outcome Preferences in Patients With Schizophrenia}

Robert Zipursky*, Charles Cunningham, Bailey Stewart, Heather Rimas, Emily Cole, Stephanie McDermid Vaz

\section{McMaster University, Hamilton, Canada}

Background: Current pharmacologic approaches to the treatment of schizophrenia are effective in bringing about a remission of psychotic symptoms for the majority of patients. A small minority of patients, however, will achieve broader outcome goals such as recovery or sustained fulltime work. The factors that contribute to the gap between the number that achieve remission versus recovery or work are not yet fully delineated. Little is known, in particular, about what outcome goals patients prefer, which they consider to be most important, and how these align with the outcomes typically associated with recovery. Conjoint analysis methods such as discrete choice experiments (DCE) provide an opportunity to characterize patient preferences. DCEs involve developing a survey that requires respondents to make trade-offs between different attributes each presented with a range of levels. Conjoint surveys are more likely than traditional surveys to reflect processes that drive real-world decision-making. We developed a DCE to investigate the following questions: 1) What outcomes are preferred by patients with schizophrenia? 2) Are respondents distributed into distinct segments or classes with different outcome preferences? 3) What clinical and demographic factors are associated with different outcome preferences?

Methods: Participants were recruited from two outpatient clinics at St. Joseph's Healthcare Hamilton that specialize in the treatment of patients with psychotic disorders: The Cleghorn Early Intervention Clinic treats individuals experiencing their first episode of psychosis and the Schizophrenia Outpatient Clinic treats individuals with more longstanding psychotic disorders. Twelve outcomes were identified: psychotic symptoms, medication side effects, physical health, work, income, housing, independence, family, friends, relationships, religion, and recreational activity. Each outcome was defined at three levels and incorporated into a computerized survey with 15 choice tasks using Sawtooth Software's SSI Web version 8.3.8. Utility values and importance scores were calculated for each outcome level. Latent class analysis was carried out using Latent Gold software version 5.0 to determine whether participants were 
distributed into segments with different preferences. Multinomial logistic regression was carried out to test whether segment membership was associated with age, duration of illness, gender, education, socioeconomic status, work status, psychotic symptom severity, and psychological distress.

Results: Surveys were completed by a total of 300 subjects. Latent class analysis revealed that respondents were distributed between three segments based on their outcome preferences. We have labelled these segments as "Achievement-focused", "Stability-focused" and "Health-focused" to reflect the preferences endorsed. The Achievement-focused segment $(48 \%)$ had preferences consistent with achieving a full recovery including working full-time, living independently, being in a long-term relationship and having no psychotic symptoms. The Stability-focused segment (29\%) had a strong preference for not working, for living independently and was accepting of having some ongoing psychotic symptoms. The Health-focused segment (23\%) also had a strong preference for not working but preferred to live in a supervised setting and to be free of all psychotic symptoms. Members of all segments preferred to have more frequent involvement with family and friends, to be more active in recreational activities, and to have some involvement with their religion. Membership in the Stabilityfocused segment versus the Achievement-focused segment was predicted by greater severity of psychotic symptoms (Wald $=21.401, \exp (\mathrm{B})=2.464, p<.001)$, lower education (Wald $=8.130, \exp (\mathrm{B})=2.537, p=.004)$, and lower socioeconomic status (Wald $=7.451, \exp (\mathrm{B})=2.697, p=$ .006). Membership in the Health-focused segment versus the Achievement-focused segment was predicted by not currently working (Wald $=9.922, \exp (\mathrm{B})=2.846, p=.002)$, and lower education (Wald $=6.198, \exp (\mathrm{B})=2.259, p=$ .013). Taken together, these variables predicted $28.5 \%$ of the variance in segment membership. Neither age nor duration of illness was significantly associated with membership in the Stability-focused or Health-focused segments.

Conclusions: We have demonstrated using a DCE survey that individuals with schizophrenia and other psychotic disorders show substantial variability in their outcome preferences and priorities. One half of our sample of respondents were distributed into a segment that preferred outcomes that are typically associated with full recovery while the other half were distributed between two segments that expressed preferences that did not include working. That only a small minority of patients with schizophrenia will meet criteria for recovery may in part reflect a mismatch between patient outcome preferences and current definitions of recovery. It was striking that membership in the latter segments was not predicted by age or duration of illness. Rather, education, socioeconomic status, psychotic symptom severity and current work status proved to be the significant predictors. Our findings underscore the importance of understanding patient outcome preferences when interpreting outcomes observed from schizophrenia and other psychotic disorders. Clinical trials of both pharmacological and psychosocial interventions for schizophrenia as well as long-term outcome studies should be informed by a greater understanding of patient preferences and priorities.

Keywords: Treatment Outcome, Recovery, First-Episode Psychosis, Multi-Episode Schizophrenia.

Disclosure: Nothing to disclose.
T183. In Vitro Evaluation of AUT00206, a Novel and Selective Kv3 Channel Modulator for the Treatment of Schizophrenia

Charles Large*, Tamara Modebadze, Nadia Pilati, Fiona LeBeau, Claire Gilloughley, Giuseppe Alvaro, Mark Cunningham

\section{Autifony Therapeutics, London, United Kingdom}

Background: Accumulating evidence supports a central role of fast spiking GABAergic interneurons in the pathophysiology of schizophrenia. Dysfunction of these interneurons, which is associated with reductions in the calcium binding protein, parvalbumin (PV) leads to disinhibition of cortical circuitry, dysregulation of gamma oscillations, and is thought to contribute to cognitive deficits observed in patients with schizophrenia (Lewis et al 2012. TiNS 35: 57-67). Voltage gated, Kv3.1 potassium channels are selectively expressed by $\mathrm{PV}$ interneurons in cortical circuits, where they permit rapid and accurate firing necessary to synchronise the coordinated firing of pyramidal principle neurons at gamma frequencies. Kv3.1 channels are found to be reduced in un-medicated schizophrenia patients (Yanagi et al 2014. Mol Psychiatry 19: 573-579). Modulation of this channel may therefore provide a means to restore $\mathrm{PV}$ interneuron function in schizophrenia patients, and improve cognitive and perhaps, negative symptoms, an unmet clinical need. This study describes in vitro assessment of the pharmacology of a novel, first-in-class Kv3 channel modulator, AUT00206, which has proven effective at improving cognitive and social behavioural deficits in a sub-chronic PCP (scPCP) model of schizophrenia symptoms (see also Neill et al, this meeting), and is currently in early clinical development for the treatment of schizophrenia (see also Hutchison et al. this meeting). The present study further explores the compound's ability to enhance gamma frequency cortical network synchrony in an animal model for schizophrenia pathology, and in in vitro brain slices obtained from human patients. Methods: In vitro pharmacology studies were conducted using mammalian cell lines stably expressing human Kv3.1 and Kv3.2 channels. Standard patch-clamp recording techniques were used to determine the effects of AUT00206 on Kv3.1 and Kv3.2 channel function. For native tissue studies, cohorts of adult female Lister-Hooded (LH) rats received phencyclidine $(2 \mathrm{mg} / \mathrm{kg}, \mathrm{scPCP})$ or saline i.p. for 7 days, followed by 6 weeks washout. This procedure has been shown to produce enduring cognitive and social behavioural deficits associated with reduced PV in frontal cortex and hippocampus (Neill et al 2010. Pharmacol \& Ther. 128(3): 419-432). Rats were then tested to confirm cognitive deficits using a novel object recognition task. Prefrontal cortical slices of the prelimbic and infralimbic regions were prepared from these rats and efficacy of AUT00206 to modulate kainate/carbachol-induced fast (20$80 \mathrm{~Hz}$ ) network oscillations was examined. Human frontal or temporal neocortex slices were obtained from patients undergoing brain surgery for removal of a tumour or epilepsy focus. Effects of AUT00206 on gamma oscillations induced by kainate/carbachol in the presence or absence of PCP was investigated in these slices.

Results: In recombinant cell line experiments, AUT00206 enhanced hKv3.1 channel whole-cell currents, which was 
associated with a leftward-shift of the voltage-activation curve, but no change in maximal conductance at positive membrane potentials. Similar effects were observed for hKv3.2 channels. In rat brain slice experiments, AUT00206 at 10 microM significantly enhanced the power of fast network oscillations by $26.1 \pm 8.1 \%(n=11, p<0.01)$ in prelimbic cortex and by $19.7 \pm 8.4 \%(n=19, p<0.05)$ in infralimbic cortex from scPCP treated rats with no significant effect in slices taken from vehicle treated animals. At 10microM AUT00206 enhanced gamma oscillations by $40.4 \pm 12.5 \%(n=3, \mathrm{~ns})$ in human neocortical slices acutely pre-treated with PCP, but had no effect on oscillations in the absence PCP.

Conclusions: AUT00206 positively modulated recombinant human Kv3.1 and Kv3.2-mediated currents, primarily through effects on channel activation. The compound has a weaker effect on Kv3.3 and Kv3.4 channels, and little or no effect on other ion channels tested, as well as a wide range of other receptors, enzymes and transporters. In native tissue experiments, AUT00206 enhanced gamma oscillations in cortical slices from scPCP treated, but not normal rats, and slices acutely treated with PCP from humans. These effects are consistent with the modulation of Kv3 channels on PV neurons by AUT00206, and thus suggests potential to provide an important novel approach for improving symptoms and function in schizophrenia patients.

Keywords: Kv3 Family, Gamma Oscillation, Parvalbumin Interneurons, Human Brain Slice.

Disclosure: Autifony Therapeutics: Financial, Self.

T184. Expression Changes in Prefrontal Cortex After Neurotransmission Blocking of the Nucleus Accumbens Pathways

Takatoshi Hikida*, Shuhei Yao, Ayumi Fukakusa, Makiko Morita, Haruhide Kimura, Keisuke Hirai, Tatsuya Ando, Hiroyoshi Toyoshiba, Akira Sawa

Kyoto University Graduate School of Medicine, Kyoto, Japan

Background: The nucleus accumbens (NAc) is a key substrate in the control of motivation, cognition, and psychomotor functions. Dysfunction of the NAc is associated with several mental disorders, such as schizophrenia, depression, and drug addiction. The NAc is part of a loop circuit, including the prefrontal cortex (PFC) - NAc ventral pallidum (VP) - substatia nigra pars reticulate $(\mathrm{SNr})$ - thalamus pathway. The NAc has two projection neuron types, D1- and D2-receptor expressing medium spiny neurons (D1/D2-MSN), which have distinct roles in reward and aversive learning, respectively. However, molecular mechanisms of information processing via D1/D2-MSN pathways in the PFC-NAc-VP circuit are unknown.

Methods: We evaluated changes of the transcriptome level in the PFC and VP after blocking neurotransmission of NAc D1- or D2-MSN using a pathway-specific reversible neurotransmission blocking method (D1/D2-RNB: Hikida et al 2010. Neuron). RNA-seq data were calculated using two independent methods, edgeR and voom. The PFC includes many cell types, and gene lists enriched in specific cell lines (Gene Sets GSE13379 and GSE35766) have been published
(Doyle et al, Cell 2008; Schmidt et al, Cell 2012). We applied Gene Set Enlichment Analysis (GSEA), in which gene lists were sorted in descending order by edgeR/voom statistics (ranked gene lists), and then compared these gene lists with the published Gene Sets and calculated the Enrichment Scores (ES).

Results: GSEA using both edgeR and voom statistics revealed that the ES of two types of corticothalamic neurons, Glt25d2positive layer $5 \mathrm{~b}$ and Ntsr1-positive layer 6 neurons, were downregulated by both NAc D1- and D2-RNB. In contrast, the ES of S100a10-positive corticostriatal neurons were upregulated and downregulated by NAc D1- and D2-RNB, respectively.

Conclusions: These data show that cortical neurons are asymmetrically regulated by subcortical pathways in the PFC-NAc-VP circuit.

Keywords: Neurocircuitry, RNA-seq, Fronto-Striatal Networks.

Disclosure: Takeda Pharmacoceutical Company Limited: Research Grants, Self.

\section{T185. Effects of Extended Cannabis Abstinence on Cognitive Outcomes in Cannabis Dependent Schizophrenia Patients Versus Non-Psychiatric Controls}

Rachel Rabin*, Mera Barr, Michelle Goodman, Konstantine Zakzanis, Stephen Kish, Michael Kiang, Gary Remington, Tony George

Icahn School of Medicine at Mount Sinai, New York, New York, United States

Background: Cross-sectional studies of the effects of cannabis use on cognition in patients with schizophrenia have produced mixed results. Heavy and persistent cannabis use in people with schizophrenia is a common clinical problem, and the effects of controlled abstinence from cannabis in these patients have not been carefully evaluated. We utilized a prospective 28-day cannabis abstinence paradigm to investigate the state-dependent effects of cannabis on cognitive outcomes in cannabis dependent schizophrenia patients versus non-psychiatric controls.

Methods: Nineteen patients and 20 non-psychiatric male cannabis dependent participants underwent 28 days of cannabis abstinence. Cognition was assessed on Day 0, 14 and 28 using a comprehensive neuropsychological battery. Clinical symptoms were assessed weekly. Abstinence was facilitated using weekly therapy sessions and contingency management, confirmed by twice weekly urine assays.

Results: Forty-two percent of patients and 55\% of controls achieved end-point cannabis abstinence, which was biochemically verified by full cannabis elimination (Day 28 urinary $\mathrm{THC}-\mathrm{COOH}<20 \mathrm{ng} / \mathrm{mL}$ ). Schizophrenia-abstainers demonstrated significant improvements in performance on the Hopkins Verbal Learning Test-Revised (HVLT-R) over time $[\mathrm{F}(2,14)=4.73, p<0.03]$; improvements were not observed on other cognitive tests, or in non-psychiatric controls. In a subset of these patients, relapse to cannabis smoking reversed these abstinence-related improvements observed in HVLT-R performance.

Conclusions: Verbal memory and learning performance selectively improved in patients with schizophrenia who 
successfully abstained from cannabis for one-month. This suggests that certain cannabis-induced deficits in schizophrenia are state-dependent and may be reversible. Recovery may favor cognitive processes facilitated by brain regions rich in cannabinoid type 1 (CB1) receptors, such as the hippocampus. Our findings underscore the importance of developing effective treatment interventions for cannabis use disorders in patients with schizophrenia.

Keywords: Cannabis Dependence, Schizophrenia, Cognition. Disclosure: Nothing to disclose.

T186. Treatment of Antipsychotic-Associated Obesity With a GLP-1 Receptor Agonist: An InvestigatorInitiated Prospective, Randomized, Placebo-Controlled, Double-Blinded Intervention Study (The TAO Study)

Pelle L Ishøy, Filip K Knop, Brian V Broberg, Nikolaj Bak, Ulrik B Andersen, Niklas R Jørgensen, Jens J Holst, Birte Y Glenthøj, Bjørn Ebdrup*

Center for Neuropsychiatric Schizophrenia Research, Glostrup, Denmark

Background: Schizophrenia is associated with cardiovascular co-morbidity and a reduced life-expectancy of up to 20 years. Antipsychotics are dopamine D2 receptor antagonists and the standard of medical care in schizophrenia, but the drugs are associated with severe metabolic side effects like obesity and diabetes. Glucagon-like peptide-1 receptor agonists (GLP-1RAs) are registered for treatment of both obesity and type 2 diabetes. We investigated metabolic effects of the GLP-1RA, exenatide once-weekly, in non-diabetic, antipsychotic-treated, obese patients with schizophrenia.

Methods: Antipsychotic-treated, obese, non-diabetic, schizophrenia spectrum patients were randomized to doubleblinded adjunctive treatment with once-weekly subcutaneous exenatide $(n=20)$ or placebo $(n=20)$ injections for three months.

The primary outcome was body weight loss after treatment and repeated measures analysis of variance was used as statistical analysis. Secondary endpoints comprised blood pressure, biochemistry, and measurements of body composition.

Results: Between March 2013 and June 2015, 40 patients completed the trial. At baseline, the mean body weight was $118.3 \pm 16.0 \mathrm{~kg}$ in the exenatide group and $111.7 \pm 18.0 \mathrm{~kg}$ in the placebo group, with no group differences $(P=0.23)$.

After three months of treatment, the exenatide and placebo groups experienced significant $(P=0.004)$, but, similar $(P=0.98)$ weight losses of $2.24 \pm 3.3 \mathrm{~kg}$ and $2.23 \pm 4.4 \mathrm{~kg}$, respectively.

The exenatide group had a significant decrease in central $24 \mathrm{~h}$ systolic blood pressure of $6.8 \mathrm{~mm} / \mathrm{Hg}(P=.004)$ as well as a decrease in the pulse wave velocity (a measure of arterial stiffness) of $0.3 \mathrm{~m} / \mathrm{s}(P=.007)$. Changes in biochemistry and body composition were similar in the groups ( $P$-values $<.47$ ). Exenatide once-weekly was well-tolerated.

Conclusions: Treatment with exenatide once-weekly did not promote weight loss in obese, antipsychotic-treated patients with schizophrenia compared to placebo. This suggests that the body weight-lowering effect of GLP-1RAs involves dopaminergic signaling and implies that anti-obesity regimens effective in the general population may not be readily implemented in antipsychotic-treated patients with schizophrenia.ClinicalTrials.gov identifier: NCT01794429.

Keywords: Schizophrenia, Dopamine, Antipsychotics, Metabolic Side Effects, Antipsychotic-Associated Obesity, GLP-1 Receptor Agonist.

Disclosure: Nothing to disclose.

T187. Epigenetic-Related mRNA Levels in Lymphocytes of Schizophrenic and Non-Psychotic Controls

Robert Smith*, Henry Sershen, Mary Youssef, Muhammed Sharifi, Sylvia Boules, Hua Jin, James Auta, Abel Lajtha, John Davis, Alessandro Guidotti

NYU Medical School, Hewlett, New York, United States

Background: Epigenetic dysregulation may be involved in the underlying molecular deficits in schizophrenia (SZ). Previous research by our group has show hypermethylation of GABAergic promoter gene in and increases in DNMT1 and DNMT3A in post mortem brain samples of patients with SZ. We have also shown that differences in DNMT1 and other epigenetically related enzymes are also found in the lymphocytes of living patients with chronic schizophrenia (CSZ). We now report preliminary results on epigenetic related mRNA's in lymphocytes on a larger sample of chronic schizophrenics and controls.

Methods: CSZ $(n=29)$ and non-psychotic controls (NPC) $(n=31)$ subjects had a blood sample $(50-60 \mathrm{cc})$ drawn, and lymphocyte pellet extracted by Ficoll gradient procedure. qPCR assays were used to measure epigenetically related mRNA's - DNMT1, DMNT3A, TET1, TET2, TET3, BDNF and NR3C (glucocorticoid receptor). We also assayed several immunological-related mRNA (which appeared as strong hits from RNA sequence analysis): T-Cell Surface Glycoprotein CD4, CCR1 (C-C motif chemokine receptor 1), FPRL3 (Formyl Peptide Receptor). Assays were performed using Taqman probes with B-Actin as housekeeping gene. Patients were also evaluated with psychopathology with PANSS, for cognitive function with MATRICS, and for odor identification and discrimination with Sniff and Sticks smell test.

Results: In this sample CSZ showed significantly higher DMNT3A $(P=.048)$ than NPC. Male CSZ showed significantly higher DNMT1 than NPC $(P=.014)$, but in the total sample there was a trend, but no significant difference in DNMT1. Compared to NPC, CSZ subjects showed significantly higher levels of GABAergic enzymes measured by the GAD1 probe (which assayed GAD67 and GAD25 mRNA) $(P=.030)$, higher levels of glucocorticoid receptor measured by the NRC3 probe $(P=.006)$, and significantly lower levels of FRPRL3 $(P=039)$. Higher levels of FPRL3 and CD4 were moderately correlated with PANSS positive symptoms in CSZ (FRPL3 $\mathrm{r}=+.43 P=.019$, CD4 $\mathrm{r}=+.37 P=.054$ ) and these correlations were slightly stronger in male CSZ. Higher DNMT1 and DNMT3A levels in lymphocytes of CSZ correlated negatively with scores on MATRICs battery (DNMT1 - attention/vigilance-r $=-.41, P=.031$, working memory $\mathrm{r}=-.37, P=.048$, composite score $\mathrm{r}=-.36, P=.063$ ). 
Conclusions: CSZ demonstrate differences in epigenetically related mRNA's in their lymphocytes. In CSZ higher levels of DNMT were related to poorer cognitive performance and higher levels of immunological related mRNA to greater positive symptom scores. Some potential differences to our previously published results may be related to differences in mRNA's transcript variants detected by the Taqman probes and earlier research with specifically generated sequence probes.

Keywords: Epigenetic, Schizophrenia, Immunological. Disclosure: Nothing to disclose.

\section{T188. Cell Cycle and p53 Gate the Direct Conversion of} Human Fibroblasts to Dopaminergic Neurons

Houbo Jiang, Zhimin Xu, Ping Zhong, Yong Ren, Gaoyang Liang, Haley Schilling, Zihua $\mathrm{Hu}$, Yi Zhang, Xiaomin Wang, Shengdi Chen, Zhen Yan, Jian Feng*

State University of New York at Buffalo, Buffalo, New York, United States

Background: In a multicellular organism, different types of cells are generated in a deterministic manner during development from a single totipotent cell. This unidirectional process and the stability of cell type identity suggest that a self-reinforcing mechanism is at work to maintain distinct cell type identities, all of which are expressed from the same genome. However, the direct conversion of fibroblasts to induced dopaminergic (iDA) neurons and other cell types demonstrates the plasticity of cell fate. The low efficiency of these relatively fast conversions suggests that kinetic barriers exist to safeguard cell type identity. Identification of kinetic barriers to transdifferentiation would reveal significant mechanistic insight into cellular reprogramming in general and produce highly efficient ways to generate many types of useful cells from readily available sources such as fibroblasts. Methods: We use lentivirus to express Ascl1, Nurr1, Lmx1a, miR124 and p53 shRNA in a variety of primary human fibroblasts and screen for the optimal condition for the direct conversion of human fibroblasts to iDA neurons, which are characterized by immunostaining, qRT-PCR, electrophysiology, neurochemistry and transplantation.

Results: We find that suppression of p53, in conjunction with cell cycle arrest at G1 and appropriate extracellular environment, markedly increase the efficiency in the transdifferentiation of human fibroblasts to iDA neurons by Ascl1, Nurr1, Lmxla and miR124. The conversion is dependent on the DNA hydroxylase Tet1, as G1 arrest, p53 knockdown or expression of the reprogramming factors induces Tet1 synergistically. Tet1 knockdown abolishes the transdifferentiation while its overexpression enhances it. The iDA neurons express markers for midbrain DA neurons, support dopaminergic transmission, and extend arborization and synaptic connections when grafted in rat brains.

Conclusions: Our results suggest that overcoming kinetic barriers such as p53, cell cycle and extracellular environment may enable highly efficient epigenetic reprogramming in general. In the specific case of dopaminergic neurons, this method enables the generation of patient-specific iDA neurons for research and drug development on a variety of dopamine-related neurological and neuropsychiatric disorders.
Keywords: Dopaminergic System, Dopamine, Transdifferentiation.

Disclosure: Nothing to disclose.

\section{T189. A Concierge Model of Customized Adherence Enhancement Plus Long-Acting Injectable Antipsychotic in Individuals With Schizophrenia at Risk for Treatment Non-Adherence and for Homelessness}

Martha Sajatovic ${ }^{*}$, Luis Ramirez, Edna FuentesCasiano, Jamie Cage, Curtis Tatsuoka, Ashley Bukach, Kristin Cassidy, Jennifer Levin

Case Western Reserve University, Gates Mills, Ohio, United States

Background: People with serious mental illness (SMI) often have difficulty with medication adherence, which contributes to illness relapse and poor outcomes. Among homeless individuals, rates of highly symptomatic schizophrenia are high. Long-acting injectable antipsychotic medication (LAI) can be a practical treatment option to optimize adherence for high-risk groups such as homeless individuals with SMI. These investigators have developed a Concierge Model Customized Adherence Enhancement (CAE) approach that is practical to deliver in standard clinical settings and can improve outcomes in homeless, poorly adherent SMI individuals. This study tested CAE, which can be delivered by social workers in community settings, combined with the LAI paliperidone palmitate (CAE-L).

Methods: This was a prospective 6-month, non-controlled trial of CAE-L in 30 homeless and recently-homeless individuals with schizophrenia or schizoaffective disorder. Research assessments were conducted at screening, baseline, 3-month and 6-month follow-up. Primary outcome was medication treatment adherence as measured with the tablets routine questionnaire (TRQ) and LAI injection frequency. Additional outcomes included psychiatric symptoms, global psychopathology, social functioning, extrapyramidal symptoms, reported side effects, health resource use, and housing status. Psychiatric symptoms were assessed with the Positive and Negative Syndrome Scale (PANSS), and Brief Psychiatric Rating Scale (BPRS) and global psychopathology with the Clinical Global Impressions (CGI). Social functioning was assessed via the Social and Occupational Functioning Assessment Scale (SOFAS). Standardized measures of extrapyramidal symptoms included the Simpson Angus Scale (SAS), the Barnes Akathisia Scale (BAS), and the Extrapyramidal Symptoms Scale-Abbreviated version (ESRS-A).

Results: Mean age of the sample was 43.6 years $(\mathrm{SD}=9.53)$, mainly minorities (86.7\% African-American), mainly single/ never married $(72.4 \%)$ with a mean of 11.55 years of education. Baseline rate of substance abuse within the past year was $40.0 \%$, and rate of incarceration within the past 6 months was $32.1 \%$. Four individuals $(13.3 \%)$ terminated the study prematurely. CAE-L was associated with good adherence to LAI $(90.0 \%)$ and improved adherence on pastweek TRQ $(p=.02)$. There were significant improvements in PANSS $(p<.01)$, BPRS $(p<.01)$, CGI $(p<.01)$ and SOFAS $(p<.01)$. There were no significant changes on SAS, BAS, or ESRS at 6-months. The proportion of days spent in sub-

(1) 
optimal housing (jail, streets, shelter) was reduced from 41.7 $\%$ in the 6 months prior to study enrollment compared to $18.1 \%$ at 6 -month follow-up $(p=.003)$.

Conclusions: Although results interpretation must be tempered by the methodological limitations, CAE-L appears to be associated with improved adherence, symptoms and functioning in homeless and recently homeless individuals with schizophrenia/schizoaffective disorder. While side effects limit tolerability in some individuals and not all individuals will remain engaged, paliperidone palmitate $+\mathrm{a}$ patient-centered behavioral approach can improve outcomes for some high -risk groups with SMI.

Keywords: Schizophrenia, Antipsychotic Medication, Treatment Adherence.

Disclosure: Janssen: Research funding, Self; Woodruff Foundation, Reinberger Foundation, Reuter Foundation: Research funding, Self; Clinical and Translational Science Collaborative (Dahms Clinical Research Unit) NIH grant number UL1 RR024989: Research funding, Self.

T190. Auditory Steady State Response in Patients With Schizophrenia and Bipolar Disorder: A Neural Correlate of Clinical State, Medication, and Community Function

Tian-Hang Zhou, Nora Mueller, Sonal Mallya, Kevin Spencer, Kathryn Eve Lewandowski, Lesley Norris, Deborah L Levy, Bruce M Cohen, Dost Ongur, Mei-Hua Hall*

Harvard Medical School, Belmont, Massachusetts, United States

Background: Gamma oscillations are important concomitants of cortico-cortical transmission and the integration, or binding, of information across neural networks. The present study assembled a large clinical sample and used an auditory steady-state response paradigm (ASSR) to examine (1) ASSR dysfunction in patients with schizophrenia (SZ) and bipolar disorder (BPD) as compared to controls (HC), (2) the relationship between ASSR and clinical symptom type and severity, (3) whether psychotropic medications have any effect on cortical oscillations, and (4) whether the strength of gamma oscillations is associated with community functioning in patients.

Methods: EEGs were recorded from $156 \mathrm{HC}, 130 \mathrm{SZ}$ and 141 BPD patients during $20-30-40-\mathrm{Hz}$ binaural click trains. Clinical assessments included the Positive and Negative Syndrome Scale, Young Manic Rating Scale, Montgomery Asberg Depression Scale, Multimodal Community Ability Scale, Snaith-Hamilton Pleasure Scale, and Mood and Anxiety Symptom Questionnaire. Linear regression analysis was used to compare ASSR responses between groups. Associations between ASSR, medication regimen and clinical symptoms were examined controlling for age, sex, and education.

Results: SZ patients had selective deficits in ASSR gamma frequency, whereas BPD patients had deficits in both beta and gamma ASSR. $20 \mathrm{~Hz}$ ASSR in BPD was associated with manic symptoms. ASSR anomalies were lesser in those receiving GABA (A) receptor agonist benzodiazepine medication. Both hallucinations and depressive symptoms were significant and independent predictors of $40 \mathrm{~Hz}$ ASSR.
Finally, ASSR at gamma frequency was highly associated with real-life functional status, particularly in the domain of independence in community living and ability to manage money successfully.

Conclusions: Patients with SZ and BPD appear to share some of the same neural circuit abnormalities underlying gamma oscillation. $40 \mathrm{~Hz}$ ASSR may be informative as a biomarker for predicting functional outcome and informing studies of the pathophysiology of SZ and related disorders. Keywords: Schizophrenia, Bipolar Disorder, Functional Capacity, Gamma Oscillation, Clinical Symptom Type and Severity, Psychotropic Medications.

Disclosure: Nothing to disclose.

\section{T191. Working Memory in Individuals With Childhood Onset Schizophrenia and Their Siblings}

Siyuan Liu*, Frances Loeb, Kirsten Craddock, Judith Rapoport

National Institute of Mental Health, Bethesda, Maryland, United States

Background: Working memory (WM) deficits are consistently reported in the adult onset schizophrenia (AOS) literature and severely impair patients' functional outcomes. It is unclear if WM function is also disrupted in childhood onset schizophrenia (COS), which is a rare and severe form, as well as how it is associated with clinical severity. More critically, the neural correlates of WM impairment in COS are under-studied and a clear understanding of these would help to determine how WM deficits are developed in schizophrenia. And studying the nonpsychotic siblings allows us to assess the genetic influence on WM deficits in schizophrenia. Here, using fMRI of an n-back paradigm, we examine whether COS patients and their siblings show impairment in WM function compared with healthy controls, and examine whether WM performance is correlated with clinical symptoms in COS, and examine whether WM related brain activation patterns are abnormal. Methods: 32 COS patients $(21.3+6.1$ years $), 30$ non-psychotic siblings $(19.4+4.1)$, and 39 healthy controls (20.0+4.5), matched for age and sex, were scanned at $3 \mathrm{~T}$ and performed a block-designed paradigm. It included 4 permuted runs, one for each of 1-, 2-back letter (verbal) and location (visual) WM tasks. The total duration was $17.64 \mathrm{~min}$. COS patients met DSM-IV criteria for schizophrenia with the onset of psychosis before 13. The Scale for the Assessment of Positive Symptoms (SAPS) and Scale for the Assessment of Negative Symptoms (SANS) were used to quantify symptom severity in COS. ANOVA was used to compare groups in accuracy rates and regression was used to examine clinical relevance. A standard pipeline was used to process fMRI images. A random-effects ANOVA model was used to draw statistical inferences at the group level. A family-wise error of 0.05 was used to determine corrected significance.

Results: COS patients scored significantly lower in accuracy rate than controls in all tasks (Cohen's $\mathrm{D}>0.98, p<.01$ ). To be noted, only $40 \%$ COS patients were able to accomplish 2back tasks, which is not an issue in AOS, indicating the relative severity of their illness. In addition, their accuracy rate of 1-back location task was significantly correlated with 
SAPS $(p=.029$, R-square $=0.36)$ scores. Unlike patients, siblings showed no significantly lower accuracy rates compared to controls. However, when switching from 1- to 2-back tasks, their averaged effect sizes increased from 0.11 to 0.39 and $p$ value reached 0.16 , indicating that siblings suffer a subthreshold WM functional loss. fMRI analyses revealed significant hypo brain activations in both patients and siblings compared with controls at 2-back tasks. Patients and siblings shared hypo activations in the dorsal lateral prefrontal cortex (DLPFC), a core area performing WM functions. Furthermore, unique hypo activations were found in the caudate in COS patients, and the anterior cingulate cortex (ACC) in siblings. It suggests different mechanisms underlie WM impairment in patients and siblings.

Conclusions: Consistent with adult literature, our results support that WM dysfunction is a core feature of COS. It is worthy to note that this experiment was restricted to patients in adolescence and early adulthood because a majority of younger COS patients failed to perform even 1-back tasks. Together with the fact that a low percentage of patients accomplished 2-back tasks, it suggests that COS patients may suffer a more severe loss of WM functions than AOS peers, in alignment with our previous findings in clinics, neuroanatomy, and genetics. The association between the visualspatial WM deficits and the positive psychotic symptoms like visual hallucination highlights clinical relevance of WM dysfunction. Since siblings took a smaller but clear hit, we argue that such WM impairment at least partially is caused by genetic factors. Our imaging results support that dysfunction of the DLPFC plays an unequivocal role in WM dysfunction in both patients and siblings. The detailed mechanisms are bifurcated: executive function related frontostriatal circuit is more critical in patients, and the ACC associated with monitoring in siblings. Taken together, given that COS represents an early stage of schizophrenia, our findings elucidate that WM impairment could be a premorbid phenotype and result from abnormal development of the frontal-subcortical network.

Keywords: Childhood-Onset Schizophrenia, Working Memory, fMRI.

Disclosure: Nothing to disclose.

T192. A Diffusion Magnetic Resonance Imaging Study of Corticostriatal Brain Miswiring in Chronic Schizophrenia

James Levitt*, Marek Kubicki, Robert McCarley, Martha Shenton, Yogesh Rathi

Harvard Medical School, Brockton, Massachusetts, United States

Background: It is believed that SZ may be based on disturbances in brain connectivity which could be due to abnormalities of long-range axonal connectivity or to abnormal synaptic function. Using diffusion weighted imaging (DWI) tractography to study white matter connections between brain regions allows us to test for the possibility of structural abnormalities in long-range axonal connectivity. The primary network target assessed here is the associative striatum and its corticostriatal connections, as these structures modulate executive function, which is a core deficit in SZ. The corticostriatal circuitry in normal brains is parsed into functionally segregated, anatomically parallel and functionally integrative, anatomically converging white matter pathways that project from the cortex to the striatum. The distinction between these two types of tract input may have clinical importance as it has been suggested that information processing via segregated tracts allows for the refining of skills already learned, whereas integrative information processing allows for new reward based learning. Here we have developed a novel method, using tractography, enabling us to label the surface area on the striatum into 2 types; that receiving segregated pathways and that receiving integrative pathways.

Methods: We used MR Diffusion tractography to calculate frontostriatal pathway streamline counts, an estimate of fiber counts, and FA between the cortex and striatum in 27 chronic schizophrenia (CSZ) patients and 26 matched healthy controls (HCs). For structural and diffusionweighted imaging (DWI) measurements, images were acquired on a 3T Siemens Magnetic Resonance Imaging (MRI) scanner at the Brigham and Women's Hospital. We performed whole brain two-tensor tractography Cortical regions of interest (ROIs) were automatically acquired using FreeSurfer, and the whole striatum ROI, not subdivided, was acquired using manual tracing. All ROIs were acquired using structural MRIs and then registered to the diffusion MRI images. We performed whole brain two-tensor tractography, which, in turn, allows for the extraction of specific fiber tracts that connect any specific ROIs of choice. We separately extracted fiber streamlines connecting 4 distinct frontal ROIs with the striatum [limbic cortex (L), 2 associative cortex subregions (dorsolateral prefrontal cortex (DLPFC: A1), ventrolateral prefrontal cortex (VLPFC: A2), and sensorimotor cortex (SM), comprised of supplementary motor area, premotor area and precentral gyrus]. For each surface voxel on the striatum, we thus obtained fiber streamline count measures of 4 distinct fiber tracts projecting from 4 distinct frontal ROI origins with their destination endpoints on the surface voxels of the striatum. We chose a threshold proportion of 0.7 such that if a given surface voxel exceeded the threshold from a single cortical ROI source, then this surface voxel would be labeled as a functionally segregated voxel corresponding to the dominant input source (either L, A1, A2 or SM). However, if no single fiber input type reaches the threshold proportion of 0.7 then the voxel is labeled as a functionally integrative voxel, i.e., a mixed-input voxel (MX). We then added up the number of striatal surface voxels that fit into L, A1, A2, SM and MX categories.

Results: We found that first, mixed model ANOVA showed a group difference for surface voxel number $(p=0.045)$. Post-hoc t-tests showed that CSZ subjects had significantly fewer integrative (MX) surface voxels compared with HCs in the left $(p=0.007)$ but not right hemisphere $(p=0.2)$.

Second, to test for a group difference in the relative amount of striatal surface area allocated to receiving segregated vs integrative-mixed input we calculated a relative segregated vs integrative (RSI) surface area quotient. This represents the ratio of surface area of the striatum allocated to receiving segregated-input vs. integrative-input; i.e., the proportion of the 2 areas. A mixed model ANOVA showed a group difference in RSI quotient between groups. The RSI quotient in the striatum was larger, i.e., there is more segregated vs 
S406

integrated surface area, in both hemispheres for both groups. Further, we found a significant group by hemisphere interaction for RSI $(p=0.04)$ and post-hoc t-tests showed a left, but not right, hemisphere increase in RSI quotient in SZ vs HCs $(p=0.006 ; p=0.5)$.

Conclusions: The results show that striatal surface area as defined by frontostriatal long-tract white matter connectivity is miswired in CSZ subjects.

We concluded first, CSZs had significantly fewer integrative surface voxels in the left, but not right, hemisphere, which is of interest as such voxels serve an integrative function; and, second CSZs had significantly higher RSI quotient scores in the left, but not right, hemisphere indicating an imbalance in the proportion between segregated and integrative surface areas on the striatum, i.e., a greater surface area allocated to receiving segregated compared to integrative inputs, lateralized to left hemisphere. These finding support brain miswiring of the corticostriatal network in CSZ.

Keywords: Multi-Episode Schizophrenia, Diffusion Weighted Imaging, Associative-Striatum, Cognitive Control, Neurodevelopment.

Disclosure: Nothing to disclose.

\section{T193. CRMP2 Contributes to Dendritic Arbor Pathology in Schizophrenia}

Glenn Konopaske*, Brian Tobe, Evan Snyder, Francine Benes, Joseph Coyle

University of Connecticut Health Center, Farmington, Connecticut, United States

Background: Cortical dendritic arbor pathology in schizophrenia has been reported by several groups including our own. CRMP2 (DPYSL2) has been reported as a schizophrenia risk gene and regulates the RAC1 and RHOA signaling pathways. CRMP2 also regulates microtubule polymerization and dendrite morphology. In the current study, we sought to determine whether CRMP2 is involved in dendritic arbor pathology in schizophrenia.

Methods: Using western blotting, the protein expression of CRMP2 and phospho-CRMP2 was assessed in the DLPFC (BA 46) grey matter from subjects with schizophrenia $(n=19)$ and unaffected control subjects $(n=19)$. Protein expression data were then correlated with basilar dendrite parameters of pyramidal cells in the deep layer III of the DLPFC (BA 46) which were obtained previously in many of the subjects. CRMP2 protein expression was also assessed in the frontal lobe from rats administered haloperidol or clozapine for 28 days.

Results: Relative to controls, CRMP2 was significantly increased by $10 \%(p=0.05)$. In addition, CRMP2 protein expression was inversely correlated with dendrite length $(\mathrm{r}=-0.37, p=0.04)$. There was no difference between groups for phospho-CRMP2 protein expression or for the phosphoCRMP2:CRMP2 ratio $(p>0.05)$. This is in contrast with data from the DPLFC of bipolar disorder subjects where the phospho-CRMP2:CRMP2 ratio appears to be altered or dysregulated (Tobe et al. in preparation). Relative to controls, CRMP2 protein expression was not different in the frontal lobe of rats administered haloperidol or clozapine.
Conclusions: In the current study, CRMP2 protein expression was increased and inversely correlated with dendrite length in subjects with schizophrenia. Although the effects of antipsychotic medications cannot be ruled out, the increased CRMP2 in schizophrenia subjects appears unlikely to be due to antipsychotic medication. The findings of the current study are in contrast to prior studies which suggest that CRMP2 overexpression is associated with dendrite growth. The inverse relationship between increased CRMP2 protein expression and dendrite length might reflect ineffective functioning of CRMP2 or altered interactions with other regulators of dendritic morphology in schizophrenia. Moreover, the presence or absence of an altered phospho-CRMP2: CRMP2 ratio might provide a molecular basis to distinguish schizophrenia from bipolar disorder. In sum, CRMP2 does appear to be involved in the pathophysiology of schizophrenia and further study of CRMP2, including its posttranslational modification, might lead to novel diagnostic and therapeutic interventions.

Keywords: CRMP2, Schizophrenia, Bipolar Disorder, Postmortem Brain Tissue, Protein Expression.

Disclosure: Nothing to disclose.

\section{T194. Pharmacogenetic Study of Treatment-Resistant Schizophrenia in a Mexican Population}

Humberto Nicolini, Jose Jaime Martinez Magaña, Nuria Lanzagorta, Michael Escamilla, Alma Delia Genis Mendoza, Vanessa Gonzalez-Covarrubias, Xavier Soberon

Instituto Nacional de Medicina Genómica, Mexico City, Mexico

Background: Treatment resistant schizophrenia (TRS) has been defined mainly by severity of symptoms and response to antipsychotics derived from a relative change in the representative scales, but these definitions have been inconsistent. Suzuki et al. (2012) proposed that TRS could be defined by at least two failed adequate trials with different antipsychotics that could be retrospective or preferably include prospective failure to respond to one or more antipsychotic trials. Approximately up to $30-40 \%$ of patients with schizophrenia experience only partial remission and even more do not reach a level of full functional recovery. Methods: Fourty-eight patients of Mexican descent diagnosed with schizophrenia receiving haloperidol $(N=23)$ were classified as responders or non-responders $(N=5)$ by standard medical criteria including SAPS (Scale for Assessment of Positive Symptoms) and SANS (Scale for Assessment of Negative Symptoms). All individuals signed an informed consent, and the study protocol was approved by an Ethics Committee. For genotyping, we used the DMET (Affymetrix) microarray, which includes 1936 polymorphisms in 225 genes. Exploratory analyses to identify a potential association between genetic variants and antipsychotics responses were defined significant at $P$-value $<0.05$ after FDR correction. Regression models were developed using STATA 11.0 (StataCorp LP, TX, USA, 2009); regression models accounted for sex, age, and time of untreated psychosis; independent variables modeled were involuntary movements and response to treatment using the scales SAPS 
(Scale for Assessment of Positive Symptoms) and SANS (Scale for Assessment of Negative Symptoms).

Results: After genotyping, quality control arrangements were made and the $p$ value for the false discovery rate was adjusted; age, gender, duration of untreated psychosis and ancestry genotypes were analyzed. Six polymorphisms were associated with TRS: two were intron variants on AOX1 (rs7563682 and rs11684227, p - value 0.02183 and 0.02245); two were exon variants on ABCC6 (rs8058694 and rs8058696, p - value 0.03963 for both); one variant was upstream of CYP2E1 (rs2070672, p - value of 0.01410) and one variant was on 3'-UTR of CYP2C18 (rs2860840, p value of 0.02177$)$. Members of cytochrome P-450, including CYP2E1 and CYP2C18 are involved in phase I metabolism of several antipsychotics. Additionally, the product of ABCC6 belongs to a group of proteins putatively related to resistance to drugs directed to treat brain disorders. Regression analyses, showed a significant association between 9 variants on ABCB11, SLC22A14, GSTO1, CYP2C18, CHST3 and drug efficacy, for which the presence of the variant allele was associated to a lack of pharmacological response. Furthermore, analyses of five patients with schizophrenia that did not respond to any treatment (haloperidol, risperidone, clozapine, olanzapine, trifluoperazine, piperazine, and $\mathrm{zu}-$ clopentixol, or their combination) showed that the frequency of the variant allele of SNPs on AOX1, CYP2C18, CYP2E1, and ABCC6 was significantly higher in resistant patients.

Conclusions: Pharmacological treatment in schizophrenia is not always completely effective. It may vary depending on the therapeutic class or population studied. A lack of control over drug safety and efficacy motivates research to pinpoint markers to predict them. The use of genetic polymorphisms represents an approach, to address these predictions in a Mexican population.

Keywords: Treatment-Resistant Schizophrenia, Pharmacogenetics, Mexican Population.

Disclosure: Nothing to disclose.

\section{T195. Trajectories and Changes in Individual Items of Positive and Negative Syndrome Scale Among Schizophrenia Patients Prior to Impending Relapse}

Husseini Manji*, Dai Wang, Srihari Gopal, Qingqin Li, Gayle Wittenberg, Susan Baker, Vaibhav Narayan

Janssen Research \& Development, LLC, Titusville, New Jersey, United States

Background: Relapse in schizophrenia may lead to treatment resistance, cognitive impairment, decreased quality of life, and increased economic burden. Effective early detection of relapse through symptom monitoring may offer early interventions to prevent full relapse. The Positive and Negative Syndrome Scale (PANSS) assesses the full spectrum of schizophrenia symptoms but is resource demanding, which prevents it from being administered frequently. It is unclear which PANSS symptoms change immediately prior to relapse, and when these symptoms start to change before relapse. We analyzed 3 relapse-prevention studies conducted by Janssen to identify individual PANSS items that changed the most prior to relapse and to understand exactly when these symptoms manifested.
Methods: Data were pooled from 3 randomized withdrawal studies to determine the efficacy of paliperidone oral extended-release formulation, paliperidone palmitate 1month injectable formulation, and paliperidone palmitate 3-month injectable formulation, respectively, in delaying psychosis relapse in patients with schizophrenia. PANSS was administered every 4 weeks in these studies except that in the paliperidone oral extended-release study it was administered weekly or biweekly until 8 weeks into the double-blind phase and every 4 weeks thereafter. Relapse was defined as having either a psychiatric event (hospitalization, suicidal or homicidal ideation, or violent behavior) or a significant increase in the PANSS total score or several pre-specified individual PANSS item scores. Individual PANSS items were sorted by their changes at relapse from randomization in patients who experienced a relapse during the double-blind phase of the 3 studies to identify PANSS items that had most increases at relapse. Individual PANSS items were also sorted among patients whose relapses were defined by psychiatric events as well as by their changes from the last pre-relapse visit. Linear and non-linear mixed effect models were applied to model the trajectories of individual PANSS items from a stable state to the time of relapse.

Results: A total of 267 patients experienced a relapse during the double-blind phase of the 3 studies. Among these relapsed patients, a subset of 7 PANSS items had on average more than 1-point of increase at relapse from randomization. These 7 PANSS items included P1 [delusions] (mean change (standard error): 1.53 (0.08)), P2 [conceptual disorganization] (1.12 (0.07)), P3 [hallucinations] (1.44 (0.09)), P4 [excitement] (1.29 (0.07)), and P6 [suspiciousness] (1.49 (0.08)) from the positive symptom subscale as well as G2 [anxiety] (1.32 (0.07)) and G4 [tension] (1.24 (0.07)) from the general psychopathology subscale. Similar patterns were observed among patients whose relapses were defined by psychiatric events as well as for changes at relapse from the last pre-relapse visit. The trajectories of these items suggested that these items started to increase 7-10 days before relapse and reached on average 1-point of increase about $0.3 \sim$ 1.2 days before relapse.

Conclusions: Relapse is abrupt in schizophrenia patients. Close monitoring is needed for early detection of relapse. A subset of PANSS items (P1, P2, P3, P4, P6, G2, and G4) exhibited greater increases than other items immediately before relapse. Focusing on this subset of items may offer the opportunity to intervene prior to an actual relapse event. This work provides a basis for developing technology enabled remote assessment solutions for tracking and predicting relapse in schizophrenia patients.

Keywords: Schizophrenia, Relapse Prevention, Positive and Negative Syndrome Scale, Individual Items, Remote Assessment Solution.

Disclosure: Janssen Pharmaceutical Companies of Johnson \& Johnson: Full-time employee, Self. 
T196. Impaired Brain Reward Circuitry May Underlie Alcohol Drinking in a Rat Model of Schizophrenia and Co-Occurring Alcohol Use Disorder

Jibran Khokhar*, Hanbing Lu, Xi Chen, Barjor Gimi, Elliot Stein, Alan Green

Dartmouth College, Lebanon, New Hampshire, United States

Background: Alcohol use disorder commonly occurs in patients with schizophrenia and contributes greatly to its morbidity. We have suggested that alcohol use may transiently ameliorate a brain reward circuit (BRC) dysfunction that underlies alcohol drinking in these patients. Additionally, very few options are available for the treatment of co-occurring alcohol use disorder and schizophrenia. To understand the mechanisms underlying, and to develop medications for, co-occurring alcohol use in schizophrenia, we have recently established and validated a novel rat model of alcohol drinking in schizophrenia based upon the neonatal ventral hippocampal lesion (NVHL) rat.

Methods: All animal studies were approved by Dartmouth College's Institutional Animal Care and Use Committee and were conducted in accordance with the National Institutes of Health Guide for the Care and Use of Laboratory Animals. Sprague-Dawley rat pups $(n=240)$ on post-natal day 7 (PND7, 15-20 g) were bilaterally injected with excitotoxic ibotenic acid (or aCSF in sham [unlesioned] animals) into their ventral hippocampi (AP $-3.0 \mathrm{~mm}, \mathrm{ML} \pm 3.5 \mathrm{~mm}$, VD $\pm 5.0 \mathrm{~mm}$ relative to bregma). Rats were weaned on PND21 and then given access to $10 \%$ alcohol (v/v) in a 2 two-bottle choice design from PND28-42. Upon reaching adulthood (PND90) animals were allowed to drink $20 \%$ alcohol in a free-access two-bottle preference design until a stable drinking baseline was established. In the first experiment, rats were allowed to drink alcohol in an intermittent or continuous access paradigm until a stable drinking level was established. Rats were then divided into three groups (vehicle, $8 \mathrm{mg} / \mathrm{kg}$ clozapine and $0.8 \mathrm{mg} / \mathrm{kg}$ haloperidol) and injected daily for 28 days. Another cohort of female NVHL rats were also used in a similar design to study alcohol drinking and the effects of clozapine. For the magnetic resonance (MR) studies, one cohort of animals ( $n=9$ /group) were scanned for resting state-functional connectivity prior to alcohol drinking in adulthood under isoflurane and dexmedetomidine anesthesia. A second cohort was placed under forced abstinence for 30 days and then scanned for MR spectroscopy in three brain regions (anterior cingulate cortex, hippocampus and nucleus accumbens) under isoflurane anesthesia ( $n=5$-6/group).

Results: The male NVHL rat, like patients with schizophrenia, drinks more alcohol than sham rats (2.5-fold; if exposed to alcohol during adolescence; $p<0.0001$ main effects of time and lesion status), and reduces its alcohol drinking when treated with clozapine, and not haloperidol $(p<0.005$ main effects of time and treatment). Female NVHL rats also displayed higher alcohol drinking compared to sham rats ( 2 -fold; $p<0.005$ main effects of time and lesion status), but unlike the male rats, an escalation in alcohol drinking was not observed over time; the effects of clozapine on alcohol drinking were also transient and not as pronounced in the female NVHL rats. Importantly, prior to alcohol drinking in adulthood, male NVHL rats displayed impaired MRI resting-state functional connectivity within the BRC $(p<0.05$; hypoconnectivity between the nucleus accumbens and pre-frontocortical regions [i.e., anterior cingulate, orbitofrontal, infralimbic/prelimbic); this is consistent with a hypoconnected BRC observed in patients with schizophrenia and a substance use disorder. Moreover, like patients with schizophrenia alone, and those with alcohol use disorder alone, significantly higher glutamine and GABA levels (1.4- and 1.3-fold respectively; $p<0.05$ ) were observed in the anterior cingulate cortex after 1-month of alcohol abstinence, and the higher glutamine levels correlated significantly with alcohol intake on the last day of drinking $(\mathrm{R}=0.84 ; P=0.002)$.

Conclusions: This study describes a novel animal model of alcohol use disorder in schizophrenia and establishes that this model exhibits both face and predictive validity. Further, using translational neuroimaging techniques, these findings also suggest that connectivity and neurometabolic abnormalities in the BRC may underlie alcohol drinking in this model, and potentially in patients with schizophrenia. Future studies using this model can help to identify novel treatment targets while uncovering mechanisms underlying alcohol use in patients with schizophrenia.

Keywords: Schizophrenia, Co-Morbid, Alcohol Abuse, Adolescent Alcohol, fMRI Functional Connectivity.

Disclosure: Nothing to disclose.

\section{T197. Computerized Functional Skills Training in Older People with Schizophrenia}

\section{Philip Harvey*, Ronald Berkowsky, Sara Czaja}

University of Miami Miller School of Medicine, Miami, Florida, United States

Background: Cognitive enhancement with pharmacological or remediation strategies is a topic of major interest in severe mental illness. Despite success in enhancing cognitive performance, these intervention strategies have not been shown to improve their distal target of everyday functioning without additional skills-based interventions. In many locations, however, there is no availability of psychosocial interventions, leading us to develop a technology-based skills training system and testing its efficacy in this study. Herein we present the results of a computerized training system focused on everyday skills that require the use of technology. Methods: Twenty people with schizophrenia and 20 healthy older adults participated in this study. Two different functional skills, ATM banking and telephone voice menu utilization for prescription refill, were trained using cognitive remediation principles (strategy learning, titrated difficulty, feedback). These training tasks used "walk-up" strategies not requiring the use of a human trainer and were delivered in 4 60-minute training sessions. Dependent variables of time to complete the fixed difficulty version of the task and number of errors made were examined prior to and after the training sessions.

Results: The two subject samples had an average age of 53, with $63 \%$ male participants and $67 \%$ with a high school education or less. All participants earned less than $\$ 40,000$ per year. Both groups of participants demonstrated 
substantial and statistically significant improvements in task performance with training. Healthy controls reduced their time to completion of the ATM task by $50 \%$ ( 4 minutes to 2 ) and their performance on the refill task by $66 \%$. Patients performed more poorly at baseline on both tasks, but also reduced their performance time by $60 \%$ on both tasks. After training, there was no difference in time to completion of either of the tasks across the two subject samples.

Conclusions: Computerized skills training without a human trainer significantly improved functional capacity in healthy older people and people with schizophrenia. There was no cognitive enhancement therapy provided and performance in an abbreviated neuropsychological assessment did not improve significantly over the training period. These results suggest that computer-delivered functional skills training can improve functional capacity and that this intervention may be an important adjunct to pharmacological or remediation focused cognitive enhancement, with a goal of improving everyday outcomes through a combination of improved cognitive functioning and improvement of functional capacity.

Keywords: Schizophrenia, Technology, Functional Capacity, Technology, Cognitive Enhancement.

Disclosure: Nothing to disclose.

\section{T198. Neural Responses to Feedback in Schizophrenia Patients Predict Behavioral Inhibition Scores}

\section{James Waltz*, Ziye Xu, Elliot Brown, Rebecca Ruiz,} James Gold

University of Maryland School of Medicine, Baltimore, Maryland, United States

Background: Extreme scores on the BIS/BAS, an instrument for assessing the functioning of systems for Behavioral Inhibition (BIS) and Behavioral Activation (BAS) have been associated with psychopathology, including in psychotic disorders. Compared to healthy controls, individuals with schizophrenia report higher BIS sensitivity, despite BAS sensitivity in the normal range (Horan et al, 2006; Barch et al, 2008; Strauss et al, 2011). The neural correlates of this elevated BIS sensitivity in schizophrenia have not been definitively established. Previous work from our group has revealed robust sensitivity to losses in schizophrenia especially in patients with more severe negative symptoms, despite reduced sensitivity to gains, in the context of reinforcement learning tasks. We hypothesized that elevated BIS sensitivity observed in schizophrenia would be accompanied by relatively greater sensitivity to losses, as manifested by feedback responses in the basal ganglia (BG) and prefrontal cortex (PFC).

Methods: We acquired event-related MRI data (81 2-mm axial slices; $128 \times 128$ matrix; FOV $=22 \times 22 \mathrm{~cm} ; \mathrm{TR}=2 \mathrm{~s}$; $\mathrm{TE}=30 \mathrm{~ms} ; \mathrm{FA}=90^{\circ}$ ) from 27 participants with SZ and 27 controls performing a variant of a probabilistic reinforcement learning paradigm (Pessiglione et al. 2006). In this task, participants learned three discriminations where a choice of the better stimulus was reinforced $80 \%$, and punished $20 \%$ of the time, and a choice of the worse stimulus was reinforced $20 \%$ and punished $80 \%$ of the time. In a "Gain-Miss" (GM) pair, possible outcomes were a gain of 25 cents, or a neutral outcome. In a "Loss-Avoid" (LA) pair, outcomes were either a loss of 25 cents, or the avoidance of a loss. In a "CorrectIncorrect" (CI) pair, subjects received only "Correct" and "Incorrect" as verbal feedback. Following standard preprocessing of data, functional datasets for individual subjects were submitted to general linear models using AFNI (Cox, 1996). For group analyses, we performed whole-brain analyses (multivariate models, using the AFNI 3dMVM function; Chen et al, 2014) with factors of stimulus pair (GM, LA, or CI) and RPE valence. Whole-brain analyses were corrected for multiple comparisons with cluster size thresholds, determined with Monte Carlo simulations to provide an overall false-positive rate of 0.05 . We performed regions-ofinterest (ROI) analyses, using these functional ROIs, as well as coordinates for dorsal anterior cingulate cortex (dACC) drawn from the literature. We then examined correlations between z-transformed BIS and BAS scores, as well as their difference, and ROI contrasts.

Results: Whole-brain analyses revealed main effects of outcome valence in the BG, as well as numerous areas in the frontal and temporal cortices, including dorsolateral prefrontal cortex (DLPFC), bilaterally, dorsomedial prefrontal cortex (DMPFC), left anterior insula, right frontopolar cortex, and right superior and middle temporal gyrus. We found that, in patients with schizophrenia, the [BIS - BAS] contrast correlated significantly with the [LOSS-AVOIDANCE - LOSS] contrast in two regions of interest: dorsal ACC ( $\mathrm{r}=0.424 ; p=0.034)$ and right DLPFC ( $\mathrm{r}=0.495$; $p=0.012$ ). Thus, feedback responses in two brain regions associated with the signaling of outcomes and prediction errors were predictive the balance of behavioral inhibition and behavioral activation.

Conclusions: The results of this study help to identify the neural correlates of elevated BIS sensitivity in schizophrenia, suggesting that enhanced BIS sensitivity may emerge from enhanced sensitivity to losses. Further study is required to determine how neural signals associated with behavioral inhibition and activation systems relate specifically to the negative symptoms of schizophrenia.

Keywords: Reinforcement Learning, Motivation, Behavioral Inhibition.

Disclosure: This work supported by NIH Grant\# 5R01MH094460 (PI: Waltz).

\section{T199. A Long-Term Open-Label Study to Evaluate the Safety and Tolerability of Brexpiprazole as Maintenance Treatment in Adults With Schizophrenia}

Robert Forbes*, Mary Hobart, John Ouyang, Stephanie Pfister, Mika Hakala

Otsuka Pharmaceutical Commercialization and Development, Inc., Princeton, New Jersey, United States

Background: Brexpiprazole is a serotonin-dopamine activity modulator that is a partial agonist at 5-HT1A and dopamine D2 receptors, and an antagonist at 5-HT2A and noradrenaline alpha $1 \mathrm{~B} / 2 \mathrm{C}$ receptors, all at similar potency. Brexpiprazole was approved in 2015 in the United States for treatment of schizophrenia and for use as adjunctive treatment in MDD. The current study (NCT01397786) was designed to assess the safety and 
tolerability of brexpiprazole as maintenance treatment for patients with schizophrenia.

Methods: This was a multicenter, open-label study of oral brexpiprazole ( 1 to $4 \mathrm{mg} /$ day) as monotherapy in adults with schizophrenia. The study included rollover patients from the double-blind, phase 3 efficacy studies (ie, VECTOR, BEACON, and EQUATOR) and de novo subjects. The primary outcome variable was the safety and tolerability of brexpiprazole, which was assessed by examining the frequency and severity of treatment-emergent adverse events (TEAEs), body weight, laboratory parameters including metabolics and prolactin, vital signs and ECG. Secondary outcomes included change from baseline in PANSS total score, in CGI-S score, and in PSP total score.

Results: A total of 1044 patients entered the open-label treatment phase (de novo $n=229$; rollover $n=815$ ) and $47.4 \%$ completed the open-label treatment phase. The most common reason for discontinuation was withdrawn consent (16.5\%) and AEs (14.8\%). The discontinuation rate for lack of efficacy was $4.0 \%$. TEAEs with an incidence $\geq 5 \%$ included schizophrenia (11.6\%), insomnia (8.6\%), weight increase $(7.8 \%)$, headache $(6.4 \%)$, and agitation $(5.4 \%)$. The most commonly-reported treatment-emergent EPS-related $\mathrm{AE}$ was akathisia (4.8\%), followed by parkinsonian events (3.8\%). The incidence of somnolence was $2.1 \%$. The mean increase in body weight from baseline to the last visit was 1.0 $\mathrm{kg}$ (2.1 kg in patients who completed 52 weeks of treatment). The incidence of patients that had an increase in body weight $\geq 7 \%$ was $18.6 \%$ and the incidence of patients that had a decrease in body weight $\geq 7 \%$ was $9.2 \%$. The changes in metabolic parameters were small and there were no clinically relevant findings for events related to orthostatic hypotension, QT prolongation, and prolactin. Long- term, open-label treatment was associated with sustained and/or continued improvement in efficacy measures and functional outcomes (mean change from baseline to week 52 in PANSS total score, CGI-S score and PSP total score were -12.20 points, -0.63 points and 7.7 points respectively).

Conclusions: The data shows that up to 52-week treatment with brexpiprazole 1 to $4 \mathrm{mg}$ (flexibly dosed) was generally safe and well-tolerated. No unexpected safety or tolerability signals were observed. Efficacy measures showed that longterm exposure to brexpiprazole produced sustained/continued improvement on symptoms and patient functioning.

Keywords: Brexpiprazole, Schizophrenia, Maintenance Treatment.

Disclosure: Pharmaceutical Commercialization and Development, Inc.: Employee, Self.

\section{T200. Lower Brain $\mathrm{pH}$ as a Shared Endophenotype of Psychiatric Disorders}

Hideo Hagihara, Vibeke Catts, Yuta Katayama, Tsuyoshi Takagi, Freesia L Huang, Kuo-Ping Huang, Shunsuke Ishii, Isabella A Graef, Gerald R Crabtree, Keiichi I Nakayama, Cynthia Shannon Weickert, Tsuyoshi Miyakawa*

Fujita Health University, Toyoake, Japan

Background: Schizophrenia and bipolar disorder are severe and debilitating psychiatric disorders, affecting approxi- mately $1 \%$ of the population worldwide. Accumulating evidence indicates that a substantial part of genetic influences on schizophrenia, bipolar disorder, and autism spectrum disorder, overlap (IS Consortium, Nature, 2009; Carroll and Owen, Genome Medicine, 2009), suggesting a common biological basis underlying the diseases. Lower $\mathrm{pH}$ is a well-replicated finding in the postmortem brain of patients with schizophrenia and bipolar disorder. Interpretation of the data, however, is controversial as to whether it reflects a primary feature of the diseases or is a result of confounding factors such as medication, postmortem interval, and agonal state (Prabakaran et al 2004. Mol. Psychiatry; Halim et al 2008. J. Neurosci. Methods, ).

Methods: We first reevaluated the $\mathrm{pH}$ of the postmortem brains of patients with schizophrenia and bipolar disorder by conducting a meta-analysis of existing datasets from nine studies. Then, by using animal models of psychiatric disorders with high validity, we tested the hypothesis that lower brain $\mathrm{pH}$ exists in these brains compared to controls to provide support for the proposal that lower $\mathrm{pH}$ is part of the pathophysiology or is an endophenotype of these brains disorders. To our knowledge, this is the first study that systematically evaluates the $\mathrm{pH}$ and lactate levels in mouse models of psychiatric disorder that can be studied without confounds inherent in the human studies. We used three mouse models of schizophrenia (Schnurri-2 knockout [KO], forebrain-specific calcineurin $\mathrm{KO}$, and Neurogranin $\mathrm{KO}$ mice) and one of bipolar disorder (Camk2a heterozygous $\mathrm{KO}$ [HKO] mice), as well as an autism model (Chd8 HKO mice) to measure the $\mathrm{pH}$, lactate, and the related metabolite levels in brain homogenates. All mice were drug-naïve with the same postmortem interval and agonal state at death.

Results: Two-way ANOVA revealed that the brain $\mathrm{pH}$ was significantly lower in patients with schizophrenia $(6.35 \pm$ $0.02, P<0.0001)$ and bipolar disorder $(6.42 \pm 0.02, P=$ $0.0053)$ as compared to control subjects $(6.51 \pm 0.02)(n=$ $240,147,280$, respectively). In the animal studies, all mice used to model psychiatric disease showed significantly lower $\mathrm{pH}$ in the postmortem brains as compared to the corresponding controls (Schnurri-2 KO, 7.17 \pm 0.0060 , Con, 7.20 \pm 0.0056, $P=0.0083$; Calcineurin KO, $7.08 \pm$ 0.0057, Con, $7.13 \pm 0.0080, P=0.0014$; Neurogranin KO, $7.10 \pm 0.017$, Con, $7.16 \pm 0.0080, P=0.0090$; Camk2a $\mathrm{HKO}, 7.14 \pm 0.0093$, Con, $7.21 \pm 0.0090, P=0.0014$; Chd8 $\mathrm{HKO}, 7.08 \pm 0.0066$, Con, $7.12 \pm 0.0031, P=0.00080)$. In addition, these mouse strains had a significantly higher lactate level in their brains. There was a highly significant negative correlation between $\mathrm{pH}$ and lactate level.

Conclusions: In this study, we confirmed the lower $\mathrm{pH}$ in the postmortem brains of patients with schizophrenia and bipolar disorder by conducting a meta-analysis of existing data. Lower $\mathrm{pH}$ was also found in five different mouse models of psychiatric disorders. These mouse models also exhibited increased lactate levels in their brains. There was a highly significant negative correlation between $\mathrm{pH}$ and lactate levels in the models, suggesting that increased lactate causes lower brain $\mathrm{pH}$ in these models. These results suggest that lower $\mathrm{pH}$ and increased lactate level are a pathophysiology of the diseases rather than artifacts.

Keywords: Brain $\mathrm{pH}$, Schizophrenia, Bipolar Disorder, Animal Models, Lactate. 
Disclosure: Astellas Pharma Inc.: Grant, Self, Lundbeck: Australia Pty, Ltd.: Consultant, Self.

\section{T201. Fragile X Mental Retardation Protein Signaling Partners Display Altered Subcellular Expression in Superior Frontal Cortex of Subjects With Schizophrenia}

Timothy D Folsom, S Hossein Fatemi*

University of Minnesota, Minneapolis, Minnesota, United States

Background: Recently, our laboratory has identified reduced expression of fragile X mental retardation protein (FMRP) in subjects with schizophrenia; a finding that has subsequently been verified by other laboratories. Little, however, is known about the mechanism behind this reduction. One hypothesis is that FMRP and its downstream targets display abnormal subcellular distribution in schizophrenia.

Methods: Postmortem, superior frontal cortex samples derived from subjects with schizophrenia $(N=12)$ and matched healthy controls $(N=12)$ underwent subcellular fractionation. SDS-PAGE and western blotting were used to measure protein levels. We measured protein levels of FMRP, phosphorylated FMRP (P-FMRP), protein phosphatase 2A catalytic subunit (PP2AC), p70 S6 kinase (P70S6K), and amyloid precursor proteins $120 \mathrm{kDa}$ and $88 \mathrm{kDa}$ (APP120, APP88). All protein measurements for subjects with schizophrenia and control subjects were normalized against neuronal specific enolase (NSE). Additionally, fraction-specific markers [i.e., histone $\mathrm{H} 3$ for nucleus; postsynaptic density protein $95 \mathrm{kDa}$ (PSD95) for synapse; and Jena-Muenchen 4 (JM4) for rough endoplasmic reticulum] were used to verify the validity of our technique. Group differences were analyzed statistically using student's t-test. Significant differences were defined as those with a $p$ value $<0.05$. Confound effects (i.e., age and PMI) will be examined using Pearson's correlation test.

Results: We found that each fraction displayed enhanced expression of its biochemical marker relative to the other two, indicating that our procedure produced the desired subcellular fractions. In total homogenate of subjects with schizophrenia, we identified lower protein levels of FMRP $(p<0.015)$, P-FMRP $(p<0.025)$, and PP2AC $(p<0.0038)$ when compared with controls. In the nuclear fraction of subjects with schizophrenia we identified significantly higher levels of APP $120 \mathrm{kDa}(p<0.041)$, APP $88 \mathrm{kDa}(p<0.038)$, PP2AC $(p<0.0026)$, and p70 S6K $(p<0.044)$ when compared with controls. In the rough endoplasmic reticulum fraction, we identified significantly lower levels of APP 120 $\mathrm{kDa}(p<0.043)$ and $\mathrm{p} 70 \mathrm{S6K}(p<0.042)$ in subjects with schizophrenia vs. healthy controls. There were no significant differences in the synaptic fraction.

Conclusions: Our results are the first to demonstrate altered subcellular expression of FMRP signaling molecules in the superior frontal cortex of subjects with schizophrenia. It is likely that altered expression of these molecules may contribute to cognitive and behavioral deficits associated with schizophrenia.

Keywords: Schizophrenia, FMRP, Subcellular Fractionation, Superior Frontal Cortex.
Disclosure: Grant support by the Winston and Maxine Wallin Neuroscience Discovery Fund and the Bernstein Chair for Adult Psychiatry is appreciated.

\section{T202. Prefrontal Cortex Dysfunction Increases Susceptibility to Schizophrenia-Like Changes Induced by Adolescent Stress Exposure}

Felipe Gomes*, Anthony Grace

University of Pittsburgh, Pittsburgh, Brazil

Background: Adolescence is a developmental period of complex neurobiological changes and heightened vulnerability to psychiatric disorders. In particular, evidence suggests that stress during adolescence is an important risk factor in the etiology of schizophrenia, a developmental disorder that typically manifests in late adolescence or early adulthood. Indeed, the emergence of psychosis is often associated with stressful life events, and adolescents that are at high risk for schizophrenia experience abnormally high reactivity to stress. A dysfunction of the medial prefrontal cortex (mPFC) is proposed to interfere with stress control, increasing the susceptibility to stress and, consequently, contributing to the emergence of psychiatric disorders, including schizophrenia. Thus, we evaluated the impact of single and combined stressful events during adolescence on schizophrenia-like signs in rats as adults and whether disruption of prelimbic ( $\mathrm{pl}$ ) PFC during adolescence affects stress-induced pathology that emerges in adulthood.

Methods: Adolescent male rats $(n=10-12 /$ group $)$ were submitted to different stressful events [restraint stress (RS; $1 \mathrm{~h}$ session at postnatal day (PD) 31, PD32 and PD40); footshock (FS; 25 footshocks of $1.0 \mathrm{~mA} / 2 \mathrm{~s} / \mathrm{session}$ daily through PD31-40); or a combination of FS and RS]. Naïve animals were left undisturbed in their home cages. At adulthood, animals were tested for anxiety responses (elevated plus-maze, EPM; PD65), cognitive function (novel-object recognition test, NOR; PD66-67), and locomotor response to amphetamine (PD68-69). One week after the behavioral experiments, the activity of VTA DA neurons was evaluated using in vivo electrophysiology (PD77-102). Three parameters of activity were measured: population activity, ie, the number of spontaneously active DA neurons per electrode track; average firing rate; and the percentage of action potentials occurring in bursts. We also evaluated whether the exposure to the combination of FS and RS in adulthood (PD65-74) produced behavioral (PD99-103) and electrophysiological changes ( $>$ PD111) similar to the adolescent stressors. In another experiment, we sought to determine if a lesion within the plPFC would increase the vulnerability to FS exposure during adolescence in the DA system activity in rats as adults. The plPFC lesion was induced by infusing ibotenic acid $(5 \mu \mathrm{g} / 0.5 \mu \mathrm{l})$ bilaterally into the plPFC in rats at PD25. Six days after surgery, adolescent rats ( $n=6-9$ /group) were submitted to FS (daily through PD31-40). At adulthood, they were tested in the EPM (PD65), NOR test (PD66-67), locomotor response to amphetamine (PD68) and activity patterns of VTA DA neurons (PD77-94).

Results: All stressors impaired body weight gain and induced anxiety-like responses in the EPM. FS and FS+RS also 
disrupted cognitive function as assessed by the NOR test. Additionally, only animals exposed to the combination of FS and RS showed a dopaminergic hyper-responsivity in terms of amphetamine hyperlocomotion and increased VTA DA population activity resembling that observed in animal models of schizophrenia. Interestingly, the increased number of spontaneously active DA neurons was confined exclusively to the lateral VTA, which project to associative striatal regions analogous to those found to be hyper-responsive in schizophrenia patients. In contrast, no change was observed when rats were exposed to the combination of FS and RS during adulthood, underscoring that adolescence is a developmental period of particular susceptibility. Unlike intact rats, animals with a plPFC lesion exposed only to the FS during adolescence showed DA hyper-responsivity. However, plPFC lesioned animals exposed to FS displayed a more widespread increase in DA neuron activity, with significant differences in both medial and lateral VTA regions. Given that the medial and central parts of the VTA send projections to the MPFC and amygdala and these projections play a role in emotional states, an increased DA activity in these VTA subregions may reflect a mechanism related to a disruption of the plPFC control of amygdala reactivity to stress.

Conclusions: Our results are in agreement with previous studies showing long-lasting changes induced by stressful live events during adolescence. The impact on DA system activity, however, seems to depend on higher-level multiple stressors. Furthermore, a failure of the plPFC to regulate the impact of stress, which may be present in at-risk individuals, increases the vulnerability to stress consequences. Thus, predisposition to stress hyper-responsivity, or exposure to substantial stressors, during adolescence can initiate a cascade of events that result in a schizophrenia-like profile in adults. This data can provide information with respect to identifying markers for schizophrenia vulnerability early in life and, by mitigating the impact of stressors, prevent the transition to psychosis in susceptible individuals.

Keywords: Stress, Schizophrenia, Adolescence, Prefrontal Cortex, Dopamine.

Disclosure: Nothing to disclose.

T203. An Integrative Approach to Test the Relationship Between Social Impairment and the Development of Psychosis

Eva Velthorst*, Avi Reichenberg, Iliyan Ivanov, Sean Froudist-Walsh, Douglas Ruderfer, Eli Stahl, Gunter Schumann

Icahn School of Medicine at Mount Sinai, New York, New York, United States

Background: Despite the known importance of impaired social functioning in the pathway to psychosis, there is no clear understanding about the etiological underpinnings of this relationship. A number of hypothesis have been proposed, including the idea that (1) social impairment is an independent contributor to psychosis risk, (2) social impairment and psychosis are two independent outings of the same genetic vulnerability, and that (3) social impairment may be the confounding results from brain abnormalities that are directly associated with psychosis risk. Our objective was to combine imaging, clinical and genome-wide association data to dissect how early disruptions in social functioning are related to psychotic experiences.

Methods: We combined data on social functioning, the social brain (fMRI faces task), SCZ and ASD polygenic risk scores (PRS) and psychotic experiences (CAPE scores) from 2,257 participants (mean age 14.4 $(\mathrm{SD}=.41)$ ) of the multi-centered IMAGEN study (Schumann et al, 2010) that were followedup between from age $14-18$. By means of univariate analyses of covariance and regression analyses, we mapped the severity and developmental trajectories of social functioning (assessed at age 14, 16 and 18) and examined the interrelationship between genetic vulnerability, deviations in the social brain areas and psychotic experiences at age 18. Results: We found compelling evidence for the first hypothesis, indicating independent associations between social functioning problems $(t=-10.69, p<.001)$, and between PRS for psychosis $(p=.001)$ and the development of psychotic experiences at age 18. There was no significant interaction between $\mathrm{PRS}^{*}$ social functioning, which may indicate that peer problems and high polygenic risk are two independent pathways to psychotic experiences. Our data are currently being linked to data on deviations in the social brain, which will be presented at ACNP as well.

Conclusions: Our results underscore both the significance and complexity of premorbid social functioning in the pathway to psychosis and may indicate that early social impairment and high polygenic risk are two independent pathways to psychotic experiences. There is a paucity of knowledge about the genetic contribution to the development of social impairment in psychosis and our findings may provide important new directions to this field.

Keywords: Social Functioning, Psychosis, Imaging, Polygenic Risk Score.

Disclosure: Nothing to disclose.

\section{T204. A Gene-Based Study of Acoustic Startle Latency}

Alicia K Smith, Tanja Jovanovic, Varun Kilaru, Adriana Lori, Lauren Gensler, Samuel S Lee, Seth Norrholm, Bruce Cuthbert, Bekh Bradley, Kerry J Ressler, Erica Duncan*

Emory University, Decatur, Georgia, United States

Background: The acoustic startle response and its modulations have been very well studied in schizophrenia. In humans the eyeblink component of the startle response is easily measured by electromyographic recording of the right orbicularis oculi muscle. Latency of acoustic startle is the time required from the presentation of the startling auditory stimulus until the startle response is elicited. It is determined by the time required for the auditory signal to travel through the 3-synapse subcortical circuit that mediates the startle response and thereby provides an index of neural processing speed. Latency is prolonged in subjects with schizophrenia compared to healthy controls and is highly heritable (90\%). We previously reported significant associations of latency with single nucleotide polymorphisms (SNPs) on genes relevant to schizophrenia. We now report a gene-based 
analysis of latency in a larger sample, followed by a SNP based analysis in an independent sample to determine genetic associations with latency as a potential inroad into genetically based vulnerability to psychosis.

Methods: The subjects in the first sample were 380 individuals tested as part of the Grady Trauma Project, a primarily African American inner city cohort with a variety of psychiatric diagnoses and including some subjects without psychiatric diagnosis. Startle testing was conducted by means of a Biopac M150 system according to published methods from our group. DNA was obtained from saliva samples, and genotyping was performed with the Omnil-Quad and OmniExpress BeadChips. We applied gene-based testing using the minSNP method implemented in the FAST package to identify genes that associate with acoustic startle latency, while controlling the false discovery rate at 5\%. A pathway analysis of significant genes was conducted using DAVID. Next, a SNP-based replication study was conducted on a Veterans Affairs (VA) cohort of 185 subjects with schizophrenia and 139 psychiatric controls. Startle testing was conducted using a computerized EMG startle response monitoring system (SR-LAB, San Diego Instruments) according to published methods from our group. Genotyping was performed on DNA samples in a 384-well format using iPlex Gold kits and the Sequenom MassARRAY system. Amplification and extension primers were designed by SpectroDESIGNER software. The MassARRAY ${ }^{\mathrm{TM}}$ typer software was used to assign the genotype calls. Candidate SNPs were selected from genes that were significant in our genebased analysis and were associated with startle and/or schizophrenia in the animal or human literature. Because we had no assumptions about the mechanism of latency heritability, we examined the association between latency and each SNP with separate linear regressions using an additive model (e.g. 0,1 , or 2 copies of a reference allele), including age, race, sex and diagnosis as additional variables. Results: The gene-based analysis of the Grady Trauma Project cohort yielded 2870 genes that associated with startle latency after multiple test correction (FDR). A pathway analysis indicated that these genes were enriched for relevant biological processes including neurotransmission (pcorr $=$ $0.0029)$ among others. For example, latency-associated genes included DISC1 $(p=.0038), \mathrm{ERBB} 4(p=0.00011)$, NOS1AP $(p=0.00053)$, and NRG1 $(p=0.0026)$, all of which genes have been implicated in SCZ. We next focused on the significantly associated genes relevant to SCZ and/or startle in a SNP-based replication study in the VA cohort. rs901561 (NRG1) predicted latency (Beta $=0.20, p=0.004)$, indicating that subjects with the AA and AG genotypes had slower mean latency than subjects with GG genotype. Similarly, rs2082552 in DISC1 significantly predicted slowing of latency (Beta $=0.01, p=0.03$ ) indicating that subjects with the AA genotype had slower latency than GG subjects, with AG subjects' latency being intermediate.

Conclusions: Latency of the acoustic startle response, an index of neural processing speed, is a potential endophenotype for schizophrenia. Latency is prolonged (i.e. slower) in schizophrenia and is highly heritable. A gene-based analysis of GWAS data revealed an association of latency with genes known to be implicated in schizophrenia: NRG1, DISC1, ERBB4, and NOS1AP. Our SNP based replication study confirmed a strong association of latency with genetic variation in NRG1 and DISC1. An association of slowed latency with SNPs on these genes is consonant with the well replicated significance of these genes in schizophrenia GWAS studies. Our findings also fit with prior endophenotype findings for these genes in a large consortium study of schizophrenia probands and their families (confer Consortium on the Genetics of Schizophrenia, COGS). NRG1 has previously shown association with another startle endophenotype (prepulse inhibition) and cognitive phenotypes in humans, and with prepulse inhibition in animals. DISC1 is associated with P50 gating, the antisaccade task, and two cognitive endophenotypes in the COGS study. The gene encodes a protein that modulates cortical growth and development, which could explain why malfunctions in the gene could increase latency and slow neuronal processing speed. These results need replication in larger datasets; however, our latency findings may provide a foundation from which we can characterize a distinct subgroup of schizophrenia patients and discover the neurobiology underlying the association of slowed latency with schizophrenia risk.

Keywords: Schizophrenia, Latency of Acoustic Startle, Genetics.

Disclosure: Nothing to disclose.

\section{T205. Early Parental Loss and Family History of Mental Illness in Relation to Schizophrenia: A Case-Control Study}

Ofer Agid*, Cynthia Siu, Gagan Fervaha, Robert Zipursky, Huma Shireen, Hiroyoshi Takeuchi, George Foussias, Gary Remington

Centre for Addiction and Mental Health, Toronto, Canada

Background: Previous studies suggest that environmental stressors early in a child's development may interact with a genetic predisposition to increase the risk of developing schizophrenia. Environmental factors can influence the expression of neurodevelopmental defects and hence the risk of schizophrenia. The primary objective of this casecontrol study was to investigate the association between early parental loss (EPL) and schizophrenia risk. Secondary objectives were to investigate the associations and interaction between EPL, family history of mental illness and gender on risk of schizophrenia.

Methods: We conducted a case control study in which effects of early parental loss (EPL before the age of 17 years) were evaluated in 1,600 participants: 800 patients with a diagnosis of schizophrenia (based on DSM-V) compared to heathy control subjects. Cases were matched to control subjects on a one-to-one basis according to age ( \pm 5 years) as well as gender, and researchers were blind to EPL status. Matched controls were drawn from individuals in the Greater Toronto Area who completed the General Health Questionnaire $(\mathrm{GHQ}>6)$ and reported no psychiatric medications use. Selection of controls was carried out by a market research company, Ipsos Canada, (http://www.ipsos.ca/en/), via the internet. EPL was defined as the loss of a parent before the subject reached the age of 17 years due to (i) death or (ii) separation resulting from a parent permanently leaving the home and residing elsewhere. The definition of 
$\overline{\mathrm{S} 414}$

separation did not require that there be no contact between the subject and the parent but did clearly stipulate that the parent was no longer resident in the home from the time of the separation.

Logistic regressions were applied to estimate the effects of early parental loss (EPL) on risk of schizophrenia. Propensity analyses were applied to control for bias in comparison groups (EPL vs. no EPL) and prognostic imbalances in age, gender, family history of mental illness, and smoking status. Similar analyses were conducted to evaluate the influences of age, gender and family history of mental illness and their interactions with EPL on schizophrenia risk.

Results: In this case-control study, 654 schizophrenia cases (63.4\%) and 160 controls (18.3\%) had early parental loss. Early parental loss was significantly associated with an increased risk of schizophrenia (adjusted odds ratio $=5.6$, 95\%CI: $4.5,7.1$ ), after exact matching on age (mean 23.8 years), gender (72.6\% male), family history of mental illness (36.6\%), and smoking status (35.6\%). There was significant difference in frequency of family history of mental illness between those with $(403 / 1032)$ and without schizophrenia $(125 / 872)$ (adjusted odds ratio $=2.7,95 \%$ CI: 2.1, 3.5), indicating significant association between family history of mental illness and risk of schizophrenia. In addition, we found significant quantitative interaction between EPL and family history of mental illness for development of schizophrenia in logistic regression $(p=0.036)$. The adjusted odds ratio for association between schizophrenia and EPL was 3.31 (95\%CI 2.1, 5.2) and 6.8 (95\% CI: 5.2, 9.1) for subjects with and without family history of mental illness, respectively. Significant interaction was also found between EPL and gender $(p=0.043)$, with increased risks of developing schizophrenia in male subjects (odds ratio $=6.8,95 \%$ CI 5.0, 9.2) compared to female subjects (odds ratio $=3.2,95 \% \mathrm{CI}$ $2.2,4.8)$.

Conclusions: These findings suggest that EPL alone or in interaction with family history of mental illness is associated with an elevated risk of schizophrenia. EPL does not increase the risk of schizophrenia uniformly for all individuals. There is significant interactive influence of family history of mental illness on the association between EPL and schizophrenia, suggesting some gene-environmental interaction for the development of schizophrenia. Though EPL, gender, and family history increased the risk of schizophrenia, not everyone with these risk factors developed the illness, suggesting that other (protective) factors are also at play (e.g., resilience). Genetic predisposition may influence the degree of susceptibility of the individual to the effects of early environmental stress, and may also determine the psychopathological entity to which the individual is rendered more vulnerable as a consequence of the stress. These issues will need to be disentangled in studies which take both genetic and environmental factors and their interactions into account and seek to define their respective contributions to schizophrenia.

Keywords: Schizophrenia, Environmental Risk Factors, Early Parental Loss.

Disclosure: Sunovion: Research Support, Self.
T206. Inflammatory Processes and Schizophrenia: Two Independent Lines of Evidence From a Study of Twins Discordant and Concordant for Schizophrenic Disorders

Silke Braun, René Bridler, Norbert Müller, Markus Schwarz, Erich Seifritz, Matthias Weisbrod, Alexandra Zgraggen, Hans Stassen*

\section{University Hospital of Psychiatry, Zurich, Switzerland}

Background: The concept of twin concordance involves quantifying the resemblance between co-twins in an "objective" and reproducible way. Yet quantifying resemblance in the case of complex psychiatric traits like schizophrenic disorders leads to methodological problems, as the yes-no dichotomy of diagnostic schemata does not allow one to assess between-subject differences in psychopathology patterns sufficiently accurate. Therefore, we relied on a multidimensional, quantitative concordance measure that provided a high resolution and differentiation when assessing the resemblance between subjects regarding psychopathology patterns.

Methods: In a first normative step, we gauged the concordance measure's performance by (1) comparing the psychopathology patterns of 269 index cases suffering from functional psychoses with the respective patterns of the 350 "affecteds" among their 1,501 first-degree relatives; (2) systematically comparing the psychopathology patterns of 100 unrelated patients with a diagnosis of schizophrenic disorders with each other; and (3) detailing the within-pair concordance of elementary traits among 2,734 healthy twin pairs. The psychopathology assessments were based on the syndrome-oriented instruments SSCL-16 and SSCL-16 Supplement which extend the DSM-V and ICD-10 definitions of psychiatric disorders by replacing the yes-no dichotomy of diagnostic schemata by dimensional quantities, thus enabling the inclusion of details of psychopathology patterns that do not reach diagnostic thresholds and would otherwise be ignored. In the second step, we determined the extent to which (1) the observed variation of between-subject psychopathology concordance among 100 schizophrenic patients, and (2) the observed variation of within-pair psychopathology concordance among 71 twin pairs with schizophrenic disorders, could be explained by Immunoglobulin M (IgM) levels.

Results: For our family data, the multidimensional, quantitative concordance approach revealed striking similarities between the psychopathology profiles of index cases and the corresponding profiles of their affected first-degree relatives. In particular, concordance analyses yielded for all syndrome profiles under investigation approximately normal distributions with robust and virtually identical concordance rates. The mean values lay around $0.536 \pm 0.091$ (concordance $53.6 \%$ ), as long as the key syndromes of schizophrenic disorders were included. As to the role of active immune processes in the context of schizophrenic disorders, we found that there exists a $25-30 \%$ subgroup of patients for whom aberrancies of the inflammatory response system, as quantified through IgM levels, appeared to be linked to the pathogenesis of schizophrenic disorders $(r=0.7515 / 0.8184$, $p<0.0001)$. None of the cytokines under investigation had a significant influence on the observed IgM levels, thus indicating that acute infections played a minor role in this 
study, if at all. The variation of within-pair psychopathology concordance among twins with schizophrenic disorders was found to be "explainable" in part by chronically elevated IgM levels $(24.5 \%$ of observed phenotypic variance; $p=0.0434)$, thus suggesting that monozygotic twins concordant for schizophrenic disorders may possess a less "robust" variant of the inflammatory response system which can more easily be triggered by exogenous factors than the more "robust" variants of discordant pairs.

Conclusions: Based on samples of quite respectable size, our results provided two independent lines of evidence for an involvement of the inflammatory response system in the pathogenesis of schizophrenic disorders: (1) there existed a subgroup of patients for whom aberrancies of the inflammatory response system appeared to be linked to the pathogenesis of schizophrenic disorders; (2) the variation in $\mathrm{mz}$ concordance regarding schizophrenic disorders was in part "explainable" through chronically elevated IgM levels. Although our data did not establish "causality" in a strict sense, they may have cleared the way for an early identification of patients with schizophrenic disorders for whom the inflammatory response system is a target for therapeutic intervention. We expect that our findings will lead to new treatment strategies with focus on a more personalized medicine.

Keywords: Twins, Within-Pair Concordance, Psychopathology Patterns, Inflammatory Response System, Vulnerability. Disclosure: Funded in part through the 6th EU Framework Programme for Research and Technological Development (Marie Curie Action: 035987; EUTwinsS).

T207. Safety, Pharmacokinetics (PK) and Clinical Effects of PF-04958242, an $\alpha$-Amino-3-Hydroxy-5-Methyl-4Isoxazolepropionic Acid (AMPA) Positive Allosteric Modulator (PAM) in Subjects With Stable Schizophrenia

Barbara Evans, Nicholas DeMartinis, Francois Gaudreault, Jessica Mancuso, Laura Zumpano, David Walling, Michael Kelley Erb, Martin Bednar, Brendon Binneman*

\section{Pfizer, Inc., Cambridge, Massachusetts, United States}

Background: PF 04958242 is being developed for the treatment of cognitive impairment associated with schizophrenia (CIAS). PF 04958242 is a potent and highly selective positive allosteric modulator of $\alpha$ amino 3 hydroxy 5 methyl 4 isoxazolepropionic acid (AMPA) receptors (an AMPA potentiator) that represents a mechanistically novel approach to the treatment of CIAS. In response to glutamate, postsynaptic AMPA receptors produce a rapid membrane depolarization that removes the magnesium block from co localized $\mathrm{N}$ methyl $\mathrm{D}$ aspartate (NMDA) receptor-gated ion channels and allows calcium influx into the cell. The resulting intracellular cascade activates kinases and transcription factors, which induce long-term potentiation and gene expression. This process, which produces changes in synaptic morphology and strength, is believed to underlie learning and memory. Pharmacologic antagonism of NMDA receptors in healthy humans and genetic manipulation of NMDA receptors in rodents produce cognitive impairment and behavioral effects suggestive of schizophrenia; postmortem studies of subjects with schizophrenia have found decreased AMPA receptor density in the hippocampus to be among the strongest evidence for dysregulation of glutamatergic transmission in schizophrenia.

PF-04958242 has been evaluated in 4 preclinical models in two species (rat and non-human primate [NHP]), including 3 models with NMDA receptor antagonist-mediated deficits and a rat functional model examining fluorodeoxyglucosepositron emission tomography (FDG-PET). Overall, these preclinical studies, in aggregate, predicted a minimal efficacious plasma exposure of $2.4 \mathrm{ng} / \mathrm{mL}$ in clinical studies. These preclinical models have translated to prior clinical studies, where PF-04958242 has demonstrated pharmacological activity both via functional Magnetic Resonance Imaging (fMRI) detected reduction in activity in the left inferior parietal cortex of healthy subjects undergoing a $2 \mathrm{D}$ spatial working memory (WM) task, $p=0.0246$ and behaviorally via an attenuation of ketamine-induced cognitive impairment on the Hopkins Verbal Learning Test Revised - Immediate Recall (HVLT-R-IR), $p=0.0787$ at exposures predicted to be efficacious.

Methods: 2 studies were conducted to evaluate the safety, tolerability and PK of multiple escalating doses of oral PF-04958242 administered to subjects with stable schizophrenia receiving antipsychotic medication. The studies were randomized, double-blind, sponsor-open, placebo-controlled, parallel-group, multiple ascending dose studies of PF-04958242 $(N=51)$ or placebo $(N=18)$ administered daily for 14 consecutive days. Exploratory objectives included an evaluation of PF-04958242 on cognitive behavioral endpoints.

Results: 69 subjects with schizophrenia were enrolled in these studies. PF-04958242 was safe and well-tolerated. Exploratory efficacy analyses demonstrated a statistically significant dose-related improvement on the Measurement and Treatment Research to Improve Cognition in Schizophrenia (MATRICS) Consensus Cognitive Battery (MCCB) WM Domain at the highest dose evaluated after 2 weeks of dosing. These results are supported by a significant relationship between average plasma PF-04958242 concentration at steady-state (Css,avg, ng/mL) and the MCCB WM Domain (change from baseline to 14 days).

Conclusions: PF-04958242 was safe and well-tolerated after multiple doses. The exploratory efficacy data from the MCCB WM domain provides clinical translation from preclinical models and support further evaluation in longer term efficacy studies in subjects with cognitive impairment associated with stable schizophrenia.

Keywords: Cognition, Working Memory, Schizophrenia, AMPA Receptors, Glutamate.

Disclosure: Pfizer Inc.: Employee, Self; Collaborative Neuroscience Network, Employee, Self.

T208. Transcriptomic Alterations in Psychiatric Disease and Rodent Models: Inflammatory and Redox Pathways

Thomas Lanz ${ }^{*}$, Simon Xi, Patricio O'Donnell

Pfizer, Inc., Cambridge, Massachusetts, United States

Background: Schizophrenia is a neurodevelopmental disorder with risk factors of both genetic and environmental 
$\overline{S 416}$

origins. Several neurodevelopmental animal models have been developed in which a genetic manipulation or an environmental insult is introduced to alter the normal trajectory of the developing brain. Certain features of schizophrenia pathology, such as deficits in parvalbumin interneurons, have been recapitulated in such animal models. The present study aimed to compare transcriptional alterations in neurodevelopmental animal models to post-mortem schizophrenia brain.

Methods: We first analyzed prefrontal cortex, hippocampus and striatum from subjects with schizophrenia by microarray. We than collected tissues from several developmental rodent models, including the MAM rat, neonatal ventral hippocampal lesion rat, the Df1/+ model of $22 \mathrm{q} 11$ microdeletion syndrome, a truncated DISC1 mouse model, and a prenatal poly I:C mouse model. Animal model tissues were analyzed by RNAseq, and select pathways significantly enriched in the schizophrenia data were examined across animal models.

Results: Increased expression of numerous genes involved in inflammatory pathways was observed in schizophrenia, including pro-inflammatory cytokines (e.g. IL-6 and IL-2). Several signaling pathways were enriched based on genes that were reduced in schizophrenia, but the pathway with the largest burden of down-regulated transcripts was oxidative phosphorylation. The oxidative phosphorylation pathway was significantly enriched in the Df1/+ mouse, though changes in select redox related genes could be observed across models. While several models showed enrichment in select inflammatory pathways, the MAM rat showed the greatest number of these. Several inflammatory pathways enriched in schizophrenia PFC were enriched in MAM rat PFC, such as IL-6 signaling, acute phase response signaling, and $\mathrm{nF}-\mathrm{kB}$ signaling.

Conclusions: Transcriptional profiling of post-mortem schizophrenia brain samples suggested an increased inflammatory state and an altered state of redox homeostasis. Several neurodevelopmental animal models showed signs of altered inflammatory pathways and some alterations in redox-related genes at ages when loss of cortical parvalbumin has been reported. The MAM rat in particular showed a significant overlap in pathways found to be enriched in schizophrenia. Taken together, these data suggest that some elements of schizophrenia molecular pathology can be recapitulated in neurodevelopmental animal models.

Keywords: RNAseq, MAM, 22q11, Redox Dysregulation, Inflammation.

Disclosure: Pfizer: Employer, Self.

\section{T209. Ectopic Mossy Fiber Pathfinding in the Hippocampus Occurs in Bipolar Patients and is Recapitulated in a Mouse Model of Psychiatric Disease}

Soichiro Nakahara*, Megumi Adachi, Noah Walton, Carrie Heusner, Hiroshi Yamada, Mickey Matsumoto, Hiroyuki Ito, Katsunori Tajinda

Astellas Pharma, Tsukuba, Japan

Background: A recurring challenge in the discovery of psychiatric drugs is the identification of the pathophysiological signature(s) that underpin symptoms. Because the hippocampal dentate-CA circuit is likely an impacted region underlying cognitive dysfunction and emotional regulation in psychiatric disorders, we investigated the pathfinding characteristics of dentate gyrus mossy fibers in the hippocampus of psychiatry patients (and subsequently a mouse model of psychiatric disease) in an attempt to correlate pathophysiological alterations with information coding deficits appreciated in psychiatric populations.

Methods: Postmortem brain tissues of healthy controls, along with schizophrenia, bipolar disorder, and major depression patients were donated from the Stanley Medical Research Institute. Mossy fiber distribution and maturational state of granule cells was profiled using immunohistochemistry (IHC) in the hippocampus of both human subjects ( $n=7-9$ slices/group) and mice $(n=10$ slices from 5 animals). For stratification analysis, we applied principal component analysis for all human subjects based on the pattern of mossy fiber distribution and the expression levels of neuronal maturation markers. For mechanistic studies, we examined the involvement of microglia engulfment $(n=5-6$ animals) via IHC, western blotting and/or qPCR. Electrophysiological properties of mossy fiber synapses in the hippocampus (including fEPSP and gamma oscillation activity) were measured via field recording $(n=8-9$ slices from 6 animals).

Results: Ectopic mossy fiber distribution was observed in stratum oriens (SO) region in the CA3 of male bipolar disorder patients, although the main bundle of mossy fiber remained primarily within the stratum lucidum (SL) region. These patients displayed increased calretinin (immature neuronal marker) and decreased calbindin (mature marker) expression. PCA indicated that this population of bipolar patients was distinct from control subjects, suggesting that patients possessing ectopic mossy fiber pathfindings also harbor immature granule cells. CaMKII $\alpha$ heterozygous knockout (hKO) mice, a longstanding psychiatric mouse model, display similar ectopic mossy fiber localization, as well as increased calretinin and doublecortin (immature neuronal marker) expression and decreased calbindin expression in granule cells. This suggests these animals may be a suitable model in which to study the mechanisticand functional basis for these aberrancies in translational validity.

Using this model, we attempted to identify the molecular underpinnings of the aberrant axonal pathfinding. CaM$\mathrm{KII} \alpha$-hKO mice displayed deficits in microglial engulfment on mossy fiber terminals coinciding with increased CD47 expression on mossy fibers in the SO and decreased MECP2 expression in immature granule cells, indicating one possible mechanism for ectopic mossy fiber distribution. Examining the relationship between these constituent factors in dissociated hippocampal primary cultures, we found that CaMKII $\alpha$ overexpression increased MECP2, whereas MECP2 overexpression decreased CD47 expression. We hypothesize that MECP2 reduction in CaMKII $\alpha$-hKO mice leads to increased CD47 expression, resulting in the observed ectopic sprouting. Field recordings in the hippocampal slices indicated that the mossy fiber-CA3 connection was weakened in CaMKII $\alpha$-hKO mice, while the directionality of gamma oscillation between SO and SL was inverted, suggesting ectopic gamma oscillations occur in these animals. 
Conclusions: We have identified an ectopic projection of mossy fibers to the SO subfield in male bipolar patients that is accompanied by systemic immaturity of granule cells neurons within the dentate gyrus. This ectopic mossy fiber sprouting may result in inefficient conduction in the axonal tract and generate aberrant gamma oscillations, potentially leading to deficits in information encoding. Mechanistic studies in a mouse model displaying similar pathophysiological aberrancies indicate that pathfinding deficiencies arise at least in part - from microglia engulfment deficits and reduced MECP2 expression. As such, the relationship between these factors in bipolar disorder patients may represent a promising avenue for future study of the relevance of neuron-microglia interaction (neuroinflammation) in psychiatric disorders.

Keywords: Mossy Fiber, Camk2a, Bipolar Disorder, $\gamma$ Oscillation, Microglia.

Disclosure: Astellas Pharma: Employer, Self; Astellas Research Institute of America: Employer, Self.

T210. Cognitive Impairment and Clozapine Response in Treatment-Resistant Schizophrenia - A Cross-Sectional Study

Shinichiro Nakajima*, Yusuke Iwata, Eric Plitman, Jun Ku Chung, Philip Gerretsen, Wanna Mar, Vincenzo De Luca, Gary Remington,

Ariel Graff-Guerrero

Centre for Addiction and Mental Health, Tokyo, Japan

Background: Response to clozapine is proposed to be a marker by which patients with schizophrenia can be stratified into biologically distinct subgroups. While cognitive impairment is a core symptom of schizophrenia, few studies have examined the relationship between cognitive function and response to clozapine in patients with treatment-resistant schizophrenia.

Methods: This present study included 16 patients with schizophrenia/schizoaffective disorder who responded to clozapine (age: $43.2 \pm 11.9$ years; female 37.5\%), 19 patients who did not respond to clozapine (age: $44.6 \pm 11.3$ years; female $26.3 \%$ ), and 19 healthy controls (age $44.1 \pm 12.5$ years; female $31.6 \%)$. Participants were age- and sex-matched. The following cognitive assessments were administered: Executive Interview (EXIT), Finger Tapping (FT), Grooved Pegboard (GP), Letter Fluency (F, A, and S) (LF), LetterNumber Span (LNS), Mini-Mental Status Exam (MMSE), Repeatable Battery for the Assessment of Neuropsychological Status (RBANS), Stroop Test (Color and Color-Word tests), Trail Making Test A and B (TMT), and Wechsler Test of Adult Reading (WTAR). Cognitive functions were compared among responders, non-responders, and healthy controls using analyses of variance. Tests with an associated $p$-value less than $0.0022(0.05 / 23)$ were considered significant. If there was a significant difference among them, post-hoc Tukey's tests were conducted. Post-hoc tests with an associated $p$-value less than $0.0038(0.05 / 13)$ were considered significant since there were 13 cognitive domains in which significant differences were found among the 3 groups.
Results: PANSS total, positive subscale, and global subscale scores were higher in non-responders than in responders $(\mathrm{t}(33)=4.7, p<.001 ; \mathrm{t}(33)=6.1, p<.001 ; \mathrm{t}(33)=4.2$, $p<.001$, respectively), while no difference was found in clozapine dose between the groups $(\mathrm{t}(33)=0.90, p=.38)$. Significant differences were found in scores of the WTAR, EXIT, RBANS Immediate Memory, Visuospatial/Constructional, Language, Attention, and Delayed Memory domains, GP (dominant and non-dominant hands), LF $F$ test, LNS, Stroop Color-Word tests, and TMT B among the 3 groups $(\mathrm{F}(2,46)=9.62, p<.001 ; \mathrm{F}(2,51)=27.09, p=<.001$; $\mathrm{F}(2,51)=10.99, p<.001 ; \mathrm{F}(2,51)=8.62, p=.001 ; \mathrm{F}(2,50)=$ $37.71, p<.001 ; \mathrm{F}(2,50)=15.14, p<.001 ; \mathrm{F}(2,50)=15.71$, $p<.001 ; \mathrm{F}(2,50)=9.00, p<.001 ; \mathrm{F}(2,50)=8.31, p=.001$; $\mathrm{F}(2,44)=12.88, p<.001 ; \mathrm{F}(2,49)=12.45, p<.001 ; \mathrm{F}(2$, $51)=18.06, p<.001$, respectively). No differences were found in MMSE, FT (dominant and non-dominant hands), LF A and $S$ tests, Stroop Color test, and TMT A scores $(\mathrm{F}(2$, $50)=0.11, p=.90 ; \mathrm{F}(2,51)=4.76, p=.04 ; \mathrm{F}(2,51)=4.77$, $p=.01 ; \mathrm{F}(2,50)=4.75, p=.01 ; \mathrm{F}(2,50)=2.84, p=.07 ; \mathrm{F}(2$, $50)=1.44, p=.25, \mathrm{~F}(2,51)=6.52, p=.003$, respectively), and score ratios of Stroop Color and Color-Word tests and TMT $\mathrm{A}$ and $\mathrm{B}(\mathrm{F}(2,43)=4.42, p=.02 ; \mathrm{F}(2,51)=5.38, p=.008$, respectively).

Among these 13 domains, there was no domain where any difference was found between responders and nonresponders. WTAR, EXIT, RBANS Language domain, LF F test, and Stroop Color-Word test scores were lower in nonresponders than healthy controls without any significant differences among other comparisons $(p<.001 ; p<.001$; $p<.001 ; p=.001 ; p<.001$, respectively). Both responders and non-responders performed significantly worse than healthy controls on the RBANS Total scale, Immediate Memory, Visuospatial/Constructional, Attention, and Delayed Memory domains, GP (dominant and non-dominant hands), LNS, and TMT B without any significant differences between responders and non-responders $(p<.001$ and $p<.001$; $p<.001$ and $p<.001 ; p=.001$ and $p<.001 ; p<.001$ and $p<.001 ; p=.002$ and $p<.001 ; p=.002$ and $p<.001 ; p=.003$ and $p=.001 ; p<.001$ and $p<.001 ; p<.001$ and $p<.001$, respectively).

Conclusions: Regardless of response to clozapine, patients with treatment-resistant schizophrenia appear to have impairments in attention, processing speed, working memory, cognitive control/executive function, and visual learning. These results are consistent with the finding that cognitive symptoms are generally unresponsive to antipsychotic treatment. Since cognitive deficits are among the strongest predictors of functioning in patients with schizophrenia, further research is warranted to elucidate the biological mechanisms underlying these symptoms in this patient population.

Keywords: Treatment-Resistant Schizophrenia, Clozapine, Cognition.

Disclosure: Nothing to disclose. 
T211. Olanzapine Abolishes the Ability of Central Insulin to Inhibit Hepatic Glucose Production

Chantel Kowalchuk, Celine Teo, Virginia Wilson, Denise Belsham, Adria Giacca, Gary Remington, Margaret Hahn*

Centre for Addiction and Mental Health, Toronto, Canada

Background: Atypical antipsychotics (AAP) such as olanzapine (OLA) are widely prescribed but associated with high rates of type 2 diabetes (T2D). Historically the risk of T2D was attributed to their weight gain propensity, which varies among AAPs. However, existing work shows that: a) independently of weight gain AAPs have an immediate effect, and b) they act at least in part via the brain, to perturb glucose homeostasis. Intriguingly, links between central insulin pathways and AAP therapeutic efficacy have been proposed. Thus understanding brain-mediated glucose dysregulation may be critical not only to treating these side-effects, but to maximizing AAP efficacy.

Methods: Here, we used our established rodent model of AAP-induced glucose dysregulation to examine if OLA causes insulin resistance via an impairment of hypothalamic insulin sensing. Gold-standard pancreatic clamps were used to measure glucose kinetics (hepatic glucose production (HGP) and peripheral glucose disposal). This procedure allows maintenance of basal peripheral insulin levels and separate manipulations of central insulin levels. Rats were treated with an acute subcutaneous dose of OLA $(2 \mathrm{mg} / \mathrm{kg})$ or vehicle during the clamp procedure according to our established model of OLA-induced insulin resistance. A intracerbroventricular(ICV) infusion of insulin $(30 \mu \mathrm{U}$ over 210 minutes) or vehicle (VEH) was administered during the clamp procedure. The treatment groups were as follows (ICV-peripheral): VEH-VEH $(n=8)$; VEH-OLA $(n=7)$; INS-OLA $(n=6)$; INS-VEH $(n=6)$.

Results: There were no differences between any treatment groups in glucose or insulin levels during the basal or clamp phase. The glucose infusion rate (GIR), a measure of whole body insulin sensitivity, was increased in the INS-VEH and INS-OLA groups relative to VEH-VEH and VEH-OLA, an effect driven by glucose uptake. Glucose uptake during the clamp phase was significantly higher in the INS-OLA $(p<0.05)$ and INS-VEH $(p<0.01)$ relative to both groups receiving ICV-VEH. The INS-VEH group demonstrated significant suppression of HGP relative to basal phase $(83.26 \%+/-22 ; p<0.05)$, an effect which was no longer observed when OLA was co-administered (i.e. INS-OLA). Hepatic glucose suppression (\% clamp relative to basal) was significantly higher in the INS-VEH group than all other treatment groups $(p<0.001)$, which did not differ significantly from each other.

Conclusions: We demonstrate that OLA, a high metabolic liability AAP, abolishes the well-established ability of a central insulin infusion to suppress HGP. These findings suggest that OLA induces central insulin resistance. This work is critical to furthering our understanding of a very serious adverse effect (risk of T2D), which through the brain may overlap with treatment dimensions of schizophrenia.
Keywords: Atypical Antipsychotics, Insulin Resistance, Type-2 Diabetes, Intracerebroventricular, Central Nervous System.

Disclosure: Nothing to disclose.

T212. Synaptic Plasticity: A New Strategy to Reveal Early Phenotypes in DISC1 (L100P) Mutant Mice and to Test Candidate Treatments

Daniela Tropea*, Ines Molinos, Emilie Petit, David Quigley, John Waddington, Aiden Corvin

Trinity College Dublin, Dublin, Ireland

Background: Mice mutant for rare, highly penetrant risk variants can be useful in dissecting the molecular mechanisms involved in neuropsychiatric disorders. The gene Disrupted in Schizophrenia 1 (DISC1) has been associated with increased risk for several conditions, such as depression, autism and schizophrenia. Mice mutant for Discl (L100P) display morphological, functional and behavioral deficits that are consistent with impairments observed across these disorders.

Methods: Here we use protocols normally used to elicit synaptic reorganization, to test the ability of the mutants to reorganize the circuitry, and to measure the effects of potential treatments.

Results: Molecular analysis in vitro reveals that, in response to altered stimulation (chemical Long Term Potentiation cLTP), the L100P mutants have a reduced expression of PSD95 and PCREB compared to WT. However, if during the challenge the cultures were treated with a plasticity modulator: insulin-like growth factor 1 (IGF1), the L100P mutants showed a reduced dysregulation of the candidate cellular marker.

Conclusions: These findings suggest that synaptic plasticity protocols can be used to reveal abnormal phenotypes in L100P mutants, even before onset of the symptoms. The same strategy may be used to uncover unexpected cellular phenotypes for other risk gene mutants, and to test new candidate treatments.

Keywords: Synaptic Plasticity, DISC1, Insulin-like Growth Factor 1.

Disclosure: IGF1: Patent, Self.

T213. Variability of 128 Schizophrenia-Associated Gene Variants Across Distinct Ethnic Populations

Kazutaka Ohi*, Takamitsu Shimada, Toshiki

Yasuyama, Takashi Uehara, Yasuhiro Kawasaki

Kanazawa Medical University, Ishikawa, Japan

Background: The second Psychiatric Genomics Consortium (PGC II), the largest available genome-wide association study (GWAS) investigating the genetic risk factors for schizophrenia, previously identified 128 independent schizophrenia-associated gene variants (GVs). Schizophrenia is a common polygenetic disease affecting approximately $0.5-$ $1 \%$ of individuals across distinct ethnic populations. The current study examined the genetic variability of GVs across different ethnic populations. 
Methods: To assess the genetic variability across different populations, the "variability indices" (VIs) of the 128 schizophrenia-associated GVs were calculated. We used 2,504 genomes from the 1000 Genomes Project taken from 26 worldwide samples comprising five major ethnicities: East Asian (EAS: $n=504$ ), European (EUR: $n=503$ ), African (AFR: $n=661$ ), American (AMR: $n=347$ ) and South Asian (SAS: $n=489$ ).

Results: The GV with the lowest variability was rs36068923 $(\mathrm{VI}=1.07)$. The minor allele frequencies (MAFs) associated with this GV were $.189, .192, .256, .183$ and .194 for the EAS, EUR, AFR, AMR and SAS populations, respectively. The GV with the highest variability was rs7432375 (VI = 9.46). The MAFs associated with this GV were .791, .435, .041, .594 and .508 for the EAS, EUR, AFR, AMR and SAS populations, respectively. The allele frequencies of 86 GVs significantly differed between the EAS and EUR populations $(p<3.91 \times$ 10-4). The GV with the highest variability was $\mathrm{rs} 4330281$ $(p=1.55 \times 10-138)$. The MAFs associated with this GV were .023 and .519 for the EAS and EUR populations, respectively. The GV with the lowest variability was $\operatorname{rs} 2332700 \quad(p=$ $9.80 \times 10-1)$. The MAFs associated with this GV were similar between these populations (i.e., .246 and .247 for the EAS and EUR populations, respectively). Interestingly, the mean allele frequencies of the GVs did not significantly differ between these populations $(p>.05)$.

Conclusions: Although genetic heterogeneities were observed in the schizophrenia-associated GVs across ethnic groups, the combination of these GVs might increase the risk of schizophrenia.

Keywords: Population Genetics, Schizophrenia, GenomeWide Association Studies, Genetic Variability, 1000 Genomes Project.

Disclosure: Nothing to disclose.

\section{T214. The Validity and Sensitivity of PANSS-6 in the Clinical Antipsychotic Trials of Intervention Effectiveness (CATIE) Study}

Soren Dinesen Ostergaard ${ }^{\star}$, Leslie Foldager, Ole Mors, Per Bech, Christoph Correll

\section{Aarhus University Hospital, Risskov, Denmark}

Background: The 30-item Positive and Negative Syndrome Scale (PANSS-30) is frequently used in research, but considered too time consuming for clinical use. We recently demonstrated that a six-item version of PANSS (PANSS-6: $\mathrm{P} 1=$ Delusions, P2 = Conceptual disorganization, P3 = Hallucinations, $\mathrm{N} 1=$ Blunted Affect, $\mathrm{N} 4=$ Social withdrawal, N6 = Lack of spontaneity/flow of conversation) may be a more practical alternative to PANSS-30. The aim of the present study was to test the validity and sensitivity of PANSS-6 further via a reanalysis of data from the Clinical Antipsychotic Trials of Intervention Effectiveness (CATIE) study.

Methods: First, we tested the scalability of PANSS-6 and PANSS-30. Scalability is present when each symptom item in a rating scale provides unique information regarding syndrome severity. Subsequently, we tested the level of correlation between the total scores of PANSS-6 and PANSS-30 in order to determine whether PANSS-6 conveys similar information as PANSS-30. Finally, to test whether PANSS-6 was equally sensitive as PANSS-30 in detecting differences in antipsychotic efficacy, we compared the effect of the five antipsychotics studied in CATIE, using the total scores of PANSS- 6 and PANSS-30 as outcomes.

Results: For the 577 subjects contributing data to the scalability analyses (those with complete PANSS ratings at baseline, month 1, month 3, and month 6), PANSS-6 was scalable, whereas this was not the case for PANSS-30. In the 1,432 subjects in the intention to treat (ITT) sample, the total scores on PANSS-6 and PANSS-30 were highly correlated (Spearman coefficient $=0.86$ based on 5,081 ratings). In the ITT sample, PANSS-6 and PANSS-30 identified the same statistically significant differences in antipsychotic efficacy, namely that olanzapine was superior to risperidone (PANSS-6 $P$-value $=0.0003 \&$ PANSS-30 $P$-value $=0.0003)$ and ziprasidone (PANSS-6 $p$-value $=0.0018 \&$ \& PANSS-30 $P$-value $=0.0046)$.

Conclusions: PANSS- 6 is a brief and scalable, clinicianbased rating scale for schizophrenia that adequately measures symptom severity and antipsychotic efficacy. Research should test acceptability of PANSS- 6 for routine, measurement-based care.

Keywords: Schizophrenia, Rating Scales, Psychiatric Measurement.

Disclosure: Alkermes, Bristol-Myers Squibb, Forum, Gerson Lehrman Group, IntraCellular Therapies, Janssen/J\&J, Lundbeck, Medavante, Medscape, Otsuka, Pfizer, ProPhase, Sunovion, Supernus, Takeda, and Teva, Christoph Correll: Financial Involvement with a pharmaceutical or biotechnology company, a company providing clinical assessment, scientific, or medical products or companies doing business with or proposing to do business with ACNP over past 2 years/Income Sources \& Equity of $\$ 10,000$ per year or greater/Financial Involvement with a pharmaceutical or biotechnology company, a company providing clinical assessment, scientific, or medical products or companies doing business with or proposing to do business with ACNP which constitutes more than $5 \%$ of personal income/Grants from pharmaceutical or biotechnology company, a company providing clinical assessment, scientific, or medical products directly, or indirectly through a foundation, university, or any other organization., Self

\section{T215. Should Antipsychotic Medications for Schizophrenia be Given for a Lifetime? A Naturalistic Follow-Up Study}

Ira Glick*, John Davis

Stanford University School of Medicine, Stanford, California, United States

Background: Antipsychotic medication has been the treatment of choice for schizophrenia for many decades. What has not been clear - since a double blind randomized controlled trial is not feasible- is how long after the initial episode or onset of antipsychotic treatment to continue medication to achieve the best outcome. We designed a small, clinical study to retrospectively examine medication adherence and outcome. 
Methods: This is a naturalistic study of 35 patients with chronic schizophrenia examining antipsychotic medication adherence from 8-50, average 21 years after onset of antipsychotic treatment. The sample was derived from all patients in one physician's academic clinic. Information was gathered on medication adherence, long-term global outcomes (based on both patient ratings as well as a blindclinician's assessment on both a Global Outcome Scale and the Global Assessment of Function scale), and the patientrated Satisfaction with Life Scale. Spearman's rank order correlations were used to relate medication adherence to global outcomes and life satisfaction, as were linear regression models adjusted for demographic and clinical characteristics.

Results: A total of 35 patients, mean age 45, and mean 21 years of possible medication since onset of treatment were assessed. Medication adherence was a statistically significant predictor of better long-term global outcomes and life satisfaction, both in Spearman's rank order correlations and in covariate-adjusted linear regressions (all $p$-values $<0.01$ ). Poor medication adherence was associated with poor outcomes, often disastrous, with low life satisfaction. Other variables did not explain the difference between those who adhered and those who didn't.

Conclusions: In this naturalistic study, patients who adhered to antipsychotic medication had better long-term outcomes. Study limitations include the potential for residual confounding. This sample provides data consistent with the recommendation for long-term, continuous antipsychotic treatment for chronic schizophrenia. Further research on other patient samples, including VA clinics and low-income clinics, is warranted.

Keywords: Schizophrenia, Psychopharmacology, Treatment. Disclosure: Janssen: Consultant, Self; Otsuka: Consultant, Research, Lecture honorariums, Self; Forrest: Consultant, Self; Takada: Consultant, Self; Teva: Consultant, Self; Synovian: Consultant, Self; Envivo: Research, Self; Alkames: Research, Self; Lilly: Research, Self; Neurex: Research, Self; Pfizer: Research, Self; Allergen: Research, Self; Johnson \& Johnson: Equity, Self.

\section{T216. Rebalancing of Activity in Frontal Cortical Populations During Sleep}

Brendon Watson*, Daniel Levenstein, John P Greene, Jennifer N Gelinas, Gyorgy Buzsaki

Weill Cornell Medical College, New York, New York, United States

Background: Sleep has many regulatory effects on the brain and body. A major hypothesis of the action of sleep on the brain is that while waking activity upregulates activity and synaptic strengths, sleep must homeostatically downregulate activity and synaptic strength.

Methods: We implanted silicon probes into the frontal cortex of 11 male Long-Evans rats to record neural activity over Wake-Sleep cycles in these animals. We used spike sorting and clustering do identify 1100 neurons and classified putative excitatory and putative inhibitory neurons. We also identify brain states including WAKE, SLEEP, NREM, REM and Microarousal using automated algorithms. We then identify changes in spiking rates of various classes of neurons over these various brain states.

Results: Here we describe a novel form of rebalancing of frontal cortical circuits during sleep wherein the neurons most active in wake have their spike rates downregulated over sleep whereas the neurons least active in wake have their spike rates upregulated over sleep. This leads to a relative homogenization of spike rates by the end of sleep. Furthermore, we show that REM, NREM and microarousals each play unique roles in the overall spike rate regulation over sleep.

Conclusions: These results indicate a novel target of homeostasis in the brain and may have implications for both sleep and beyond.

Keywords: Sleep, Homeostasis, Neuron, Electrophysiology, Rat.

Disclosure: Nothing to disclose.

\section{T217. Microglial Associated Circadian Regulation of Perineuronal Net Composition}

Harry Pantazopoulos*, Emrah Yildiz, Phoebe Seltzer, Lilla Turiak, Joseph Zaia, Sabina Berretta, Magdalena Ardent

Harvard Medical School, Belmont, Massachusetts, United States

Background: Perineuronal Nets (PNNs), extracellular matrix structures that envelop subpopulations of neurons and restrict synaptic plasticity, were long thought to be stable, cartilage-like structures of the brain. Recent studies however suggest that PNN composition, particularly the biochemical characteristics of one of the main components, i.e. chondroitin sulfate proteoglycans (CSPGs) is modified during learning tasks, possibly allowing for formation of new synapses in response to environmental stimuli. New synapses are believed to undergo strengthening during sleep, part of the 'memory consolidation' process. We tested the hypothesis that PNNs are regulated in a circadian manner, possibly allowing for increased plasticity during active periods and contributing to strengthening of new synapses during sleep. The protease cathepsin-S has been reported to cleave CSPGs, and was shown to be expressed in a circadian manner in the prefrontal cortex, coinciding with rhythmic changes in dendritic spine densities. We tested the hypothesis that expression of cathepsin-S may vary according to circadian rhythms in association with similar rhythmic PNN composition.

Methods: Total numbers and numerical densities of PNNs labeled with wisteria floribunda agglutinin lectin (WFA), or with the antibody cat-301 directed against the aggrecan core protein, were quantified in the amygdala and thalamic reticular nucleus (TRN) in a cohort of postmortem brain samples from normal human subjects (12-15 subjects) and plotted by time of death for each subject. Glycomics analysis was carried out using mass spectrometry on free-floating sections from the human mediodorsal thalamus to quantify specific sulfated chondroitin sulfate (CS) disaccharides. In addition, WFA was used to quantify PNNs, and anticathepsin-S was used to quantify expression of this protease in microglia in a cohort of wild type male $129 \mathrm{sv}$ mice housed 
in a 12:12 light: dark cycle, sacrificed every 4 hours across the 24-hour cycle in the amygdala, thalamus, hippocampus, and habenula. A second cohort of C57Bl6 mice housed in constant darkness was used to confirm circadian rhythms of markers of interest in the absence of light entrainment.

Results: In human, we observed a 'rhythmic-like' relationship of WFA labeled PNNs in the amygdala and TRN. In addition, mass spectrometry analysis of CS disaccharides in the mediodorsal thalamus revealed a rhythmic-like relationship with time of death of non-sulfated CS, as well as the 4 S to $6 \mathrm{~S}$ sulfation ratio. In contrast, aggrecan immunoreactive (IR) PNNs in the human amygdala did not show a 'rhythmic-like' relationship with time of death. In mice, we observed a similar rhythmic distribution of WFA labeled PNNs in the hippocampus, habenula, TRN and in the lateral and basolateral amygdala nuclei. We confirmed rhythmic PNN composition in the hippocampus in a second set of mice housed in constant darkness. Circadian rhythms of cathepsin-S IR microglia in the hippocampus of mice were antiphase to the rhythm of WFA labeled PNNs. Furthermore, incubation of tissue sections with cathepsin-S eliminated WFA labeling of PNNs, providing evidence that this microglial-derived protease alters PNN components.

Conclusions: Our data shows that PNN numbers vary in a circadian manner in several brain regions in human and rodents, and that cathepsin-S expression varies in a complementary pattern. These results suggest that PNN composition varies in a circadian manner and that microglia may contribute to this process through rhythmic expression of the protease cathepsin-S. Rhythmic modification of PNNs may contribute to circadian regulation of neuronal firing properties, increased plasticity during active periods, and memory consolidation during sleep.

Keywords: Circadian Rhythm, Schizophrenia, Microglia, Bipolar Disorder, Parvalbumin Interneurons/Perineuronal Net.

Disclosure: Nothing to disclose.

\section{T218. Selective Inhibition of Orexin-2 Receptors Prevents Stress-Induced ACTH Release in Mice}

Pascal Bonaventure ${ }^{\star}$, Su Jin Yun, Michelle Wennerholm, Jonathan Shelton, Michael Letavic, Brock Shireman, Timothy Lovenberg, Christine Dugovic

Janssen Research \& Development, LLC, San Diego, California, United States

Background: Orexins are peptides produced by lateral hypothalamic neurons that exert a prominent role in arousal-related process, including stress by activating orexin-1 (OX1R) and orexin-2 (OX2R) receptors located widely throughout the brain. Selective pharmacological blockade of OX2R promotes sleep and in contrast, pharmacological or genetic selective inhibition of OX1R minimally affects sleep. Stress or orexin administration stimulates hyperarousal, ACTH and corticosterone release, and selective OX1R blockade can attenuate several stress-induced behavioral and cardiovascular responses but not the hypothalamic-pituitary-adrenal (HPA) axis activation. As opposed to OX1R, OX2R are predominantly expressed in the paraventricular hypothalamic nucleus which is involved in the HPA axis regulation. In the present study, we investigated the effects of a psychological stress elicited by cage exchange on ACTH release in two models (pharmacological and genetic) of selective OX2R inhibition.

Methods: The cage exchange stress procedure was performed in male C57Bl6 mice. The animal was removed from its home cage and placed into a dirty cage previously occupied by another animal for at least one week. As a control procedure, the animal was removed from its home cage and returned to the same cage (brief handling). Mice were dosed with vehicle or the OXR antagonists (JNJ-42847922, a selective OX2R antagonist or SB-649868, a non-selective OXR antagonist $30 \mathrm{~min}$ prior to cage exchange. After $20 \mathrm{~min}$, blood was drawn by a submandibular punch. The same procedure was performed in non-treated OX2R knockout and corresponding wild type mice. An Elisa kit was used to measure serum ACTH. To assess whether the intrinsic and distinct sleep-promoting properties of each antagonist could account for the differential stress response, a separate group of mice chronically implanted with electrodes for EEG sleep recording were orally dosed with JNJ-42847922 or SB-649868 during the light phase.

Results: Cage exchange-induced stress produced a significant increase in ACTH plasma levels. Mice lacking the OX2R exhibited a blunted stress response. Stress-induced ACTH release was absent in mice pre-treated with the selective OX2R antagonist JNJ-42847922 (30 mg/kg po), whereas pretreatment with the dual OX1/OX2R antagonist SB-649868 (30 $\mathrm{mg} / \mathrm{kg}$ po) only partially attenuated the increase of ACTH. The EEG sleep recording study indicate that both compounds reduced the latency to NREM sleep without affecting its duration, however a prominent REM-sleep promoting effect was observed only in mice treated with the dual OX1/OX2R antagonist.

Conclusions: While it is well established that selective pharmacological blockade of OX2R is sufficient to promote sleep and OX1R minimally affects sleep, the differential contribution of OX1R and OX2R signaling in HPA axis is unclear. Here we demonstrate that in a psychological stress model, increased ACTH secretion can be prevented by a selective OX2R antagonist which is consistent with the predominant expression of $\mathrm{OX} 2 \mathrm{R}$ in the paraventricular hypothalamic nucleus. These data complement a previous study where we demonstrated that selective OX1R blockade did not affect the cage exchange stress-induced ACTH release in mice. Based on these new preclinical data demonstrating that a selective $\mathrm{OX} 2 \mathrm{R}$ antagonist prevented stress induced ACTH secretion, we propose that a selective OX2R antagonist might be suitable for the treatment of hyperarousal insomnia characterized by overactivity of the HPA axis.

Keywords: Orexin Receptor Antagonist, HPA Axis, Acute Stress, Hyperarousal, Insomnia.

Disclosure: Janssen Research and Development: Employee, Self. 
T219. Fronto-Cerebellar and Other Resting Connectivity Circuits Demonstrate Inverse Correlations to Delay Discounting in Smokers and Controls

Betty Jo Salmeron*, Miji Um, Sufang Li, Thomas Ross, Elliot Stein, Yihong Yang, Li Ming Hsu

National Institute on Drug Abuse, Baltimore, Maryland, United States

Background: Delay discounting (DD) refers to the reduction in current value of a reward to be received at some point in the future. Individuals with substance use disorders (SUD), including smokers, are known to discount more steeply than healthy individuals. Imaging studies during the act of choosing between an immediate reward and a larger, delayed reward activate frontal, ventral striatal and posterior cingulate regions. Investigators have conceptualized the choice as a product of a single evaluation system or as a competition between separate systems for immediate and delayed rewards. DD, however, can also be viewed as a personality trait, as it is stable over time and highly heritable. As such, it may be possible to identify a neural signature of DD in resting fMRI data, since connectivity of brain regions at rest is thought to underlie how they are engaged when behavior is required. $\mathrm{DD}$ is also thought to relate to impulsivity, which has been shown to have some novel neural underpinnings in several SUDs, a result consistent with the fact that impulsivity both predisposes to SUDs and is worsened by them. In this study, we took a data-driven approach to identifying neural circuits relating to DD in healthy controls and smokers as well as those that differ between them.

Methods: 34 smokers and 34 matched controls were recruited under a protocol approved by Institutional Review Board at National Institute on Drug Abuse Intramural Research Program (NIDA-IRP). Participants completed a computerized DD task, and the log-transformed discount rate calculated from this was used in all correlations. They also underwent a 6-minute whole brain eyes open resting scan (3T Siemens TRIO) with repetition time (TR) $=2000$ $\mathrm{ms}$; echo time $(\mathrm{TE})=27 \mathrm{~ms}$; flip angle $(\mathrm{FA})=80^{\circ}$ along with an MPRAGE anatomical scan. After standard preprocessing steps, functional connectivity strength (FCS) maps were generated for each participant by attributing to each voxel the sum of functional connectivity to all other voxels passing a minimum threshold $(r>0.2)$ of connectivity with that voxel. An LME analysis identified regions where DD was significantly related to FCS resulting in two, rather large clusters which were subjected to a modularity analysis to identify regions within these clusters that were more homogeneous. These clusters were then used as seed regions to identify circuits contributing to significant FCS correlations with DD. This was done by creating individual maps of connectivity with each seed region which were used as the dependent variable in an LME with DD and Group (smoker/ control).

Results: Smokers and controls did not differ on DD. FCS was significantly related to DD in two large frontal clusters. The modularity analysis subdivided the first cluster into right (R) lateral OFC, $\mathrm{R}$ frontal pole and $\mathrm{R}$ dlPFC clusters. It subdivided the second cluster into bilateral subgenual ACC (sgACC) and R mPFC. Using these five clusters as seeds, main effects of DD were found in five circuits: right frontal pole:left (L) lingual gyrus, $\mathrm{R}$ frontal pole:L cerebellum, $\mathrm{R}$ dlPFC:R inferior parietal lobule, sgACC:R frontal pole (BA10) and sgACC:cerebellar tonsil. No circuits were identified for the $\mathrm{R}$ lateral $\mathrm{OFC}$ or $\mathrm{R}$ mPFC clusters. Interactions between $\mathrm{DD}$ and Group were identified in $\mathrm{R}$ frontal pole:R cerebellum, R lateral OFC: L inferior frontal gyrus, R lateral OFC:L cerebellum and sgACC:R lingual gyrus. Visual inspection of the interaction effect in scatterplots between connectivity strength and discounting rate showed that high discounting controls had higher connectivity strength in $\mathrm{R}$ frontal pole: $\mathrm{R}$ cerebellum, $\mathrm{R}$ lateral OFC:L cerebellum and sgACC:R lingual gyrus circuits, while high discounting smokers had lower connectivity strength in these circuits. The $\mathrm{R}$ lateral OFC: $\mathrm{L}$ inferior frontal gyrus circuit, on the other hand, showed opposite relationship: high discounting controls had lower connectivity strength while high discounting smokers had higher connectivity strength in the circuit.

Conclusions: This data driven method identified, at the FCS step, frontal regions previously associated with DD such as $\mathrm{mPFC}$, dlPFC and OFC, as well as novel regions (sgACC and frontal pole). The circuits underlying the FCS results, however, are novel and include fronto-cerebellar circuits about which little is known and circuits involving frontal pole. Frontal pole is a phylogenetically new region that may relate to learning from feedback and was associated with both main effects of DD and interactions with group. Cerebellum, notably regions associated with executive control networks, was involved in circuits showing high connectivity in high discounting controls but low connectivity in high discounting smokers. High discounting smokers, on the other hand, showed high connectivity between $\mathrm{R}$ lateral OFC and L inferior frontal cortex while this was associated with low discounting in controls. Lateral OFC is associated with learning new associations, especially from punishment, while L inferior frontal cortex has been associated with inhibition of learning from undesirable information, making this inverse relationship with DD in smokers and controls particularly interesting. Reversals of these correlations in smokers compared to controls may indicate a reversal of the direction of influence between regions in smokers.

Keywords: Delay Discounting, Resting State Functional Connectivity, Nicotine Dependence.

Disclosure: Nothing to disclose.

\section{T220. Sativex Associated With Behavioral-Relapse Prevention Strategy as Treatment for Cannabis Dependence, a Pilot Study}

Jose Trigo*, Alexandra Soliman, Gregory Staios, Lena Quilty, Benedikt Fischer, Tony George, Jurgen Rehm, Peter Selby, Allan Barnes, Marilyn Huestis, Bernard Le Foll

Centre for Addiction and Mental Health, Toronto, Canada

Background: Cannabis is the most commonly used illicit drug. The current lack of pharmacological treatments for cannabis use disorder (CUD) warrants the use of novel 
approaches and further investigation of promising pharmacotherapy. In a previous laboratory study, we observed that high doses of Sativex ( 1:1, $\Delta 9$-tetrahydrocannabinol (THC)/cannabidiol (CBD) combination) were well tolerated and significantly reduced cannabis withdrawal during abstinence as compared to placebo. In this pilot study, we assessed the use of self-titrated dosages of active $\sim 1: 1 \mathrm{THC} /$ $\mathrm{CBD}$ or placebo in combination with Motivational Enhancement Therapy and Cognitive Behavioral Therapy (MET/ CBT) for the treatment of cannabis dependence among treatment-seeking community-recruited cannabis-dependent subjects.

Methods: Forty treatment-seeker community-recruited cannabis-dependent subjects underwent a double-blind, placebo-controlled randomized clinical trial (RCT) consisting in a 12 weeks treatment phase (up to 113.4 of THC/105 $\mathrm{mg}$ of $\mathrm{CBD}$ ) and MET/CBT, followed by a 12 week followup phase.

Results: $\sim$ 1:1 THC/CBD was well tolerated by all participants (average daily doses ranged 10.9 THC/10.2 mg CBD to $36.2 \mathrm{THC} / 33.5 \mathrm{mg} \mathrm{CBD}$ ). We did not observe any serious adverse events on any of the experimental conditions. Sevenday point prevalence cannabis abstinence was at week 13: $41.2 \%$ for $\sim 1: 1 \mathrm{THC} / \mathrm{CBD}$ group and $30 \%$ on the placebo group; and at week 24: $29.4 \%$ for 1:1 THC/CBD group and $20 \%$ on the placebo group (NS). The results of this pilot RCT revealed a decrease in cannabis use in the group receiving the $\sim 1: 1$ THC/CBD combination, as compared the placebo group. Interestingly, we identified that some participants did not use the sprays much (i.e. 'low medication intake' group, average $8.4 \mathrm{mg}$ THC/ $7.8 \mathrm{mg} \mathrm{CBD}$ ), while other participants clearly used the medication more (i.e. 'high medication intake' group, average $49.2 \mathrm{mg} \mathrm{THC} / 45.5 \mathrm{mg} \mathrm{CBD}$ ). By analyzing the results based on the medication intake levels, we observed that cannabis use greatly differed in the high vs low medication intake subgroups. We found a significant effect of $\sim 1: 1 \mathrm{THC} / \mathrm{CBD}$ on cannabis use as compared to cannabis intake at baseline during treatment (weeks 3 and 5$10)$ and follow-up (weeks 13,16 and 21-23) phases $(P<0.05)$, while the placebo group did not show significant changes as compared to cannabis intake at baseline. Similarly, better outcomes on craving and withdrawal appeared in the high medication intake subgroup when using $\sim 1: 1 \mathrm{THC} / \mathrm{CBD}$.

Conclusions: This pilot study indicates good tolerability of $\sim 1: 1$ THC/CBD combinations among participants with CUD. Our pilot study supports the possible efficacy of $\sim$ 1:1 THC/CBD at high doses combinations with MET/CBT. Due to the promising results study, further systematic exploration of $\sim 1: 1 \mathrm{THC} / \mathrm{CBD}$ combinations as a treatment option for CUD should be performed.

Keywords: Cannabis Dependence, THC, Cannabidiol, Withdrawal, Craving.

Disclosure: Nothing to disclose.
T221. Chronic Ethanol Exposure Alters GABA(A)-R Expression Through Epigenetic Mechanisms That are Prevented by HDAC Inhibition

\section{J Peyton Bohnsack, Todd K O'Buckley, A Leslie Morrow $^{*}$}

University of North Carolina School of Medicine, Chapel Hill, North Carolina, United States

Background: Alcohol Use Disorders are a leading cause of preventable death and account for $\$ 234$ billion dollars in lost economic costs in the United States. Nevertheless, few therapeutic interventions for the treatment of alcohol use disorders are available. Chronic ethanol exposure in rodent models causes $\mathrm{GABA}(\mathrm{A})$ receptor (GABA(A)-R) hypofunction in the cerebral cortex, associated with a decrease in the prominent $\alpha 1$ subunit and increase in $\alpha 4$ subunit expression. These changes in receptor expression contribute to many of the phenotypic symptoms associated with alcohol use disorders, including anxiety, benzodiazepine cross-tolerance, insomnia, and increased seizure susceptibility. Previous work by other groups demonstrated that histone deacetylase (HDAC) inhibitors can prevent GABA(A)-R hypofunction in the VTA and elevations in anxiety-like and drinking behavior after chronic ethanol consumption. Therefore, we examined the role of histone deacetylation in ethanol effects on GABA(A)-Rs in cerebral cortex and cortical cultured neurons.

Methods: We evaluated the effects of HDAC inhibitors on the effects of chronic ethanol exposure ( $5 \mathrm{~g} / \mathrm{kg}$, i.g., 14 days) followed by $24 \mathrm{~h}$ withdrawal. The HDAC inhibitor, Trichostatin A (TSA), was administered on the three days prior to sacrifice $(2 \mathrm{mg} / \mathrm{kg}$, i.p.). We then performed open field locomotor, loss of righting reflex, qPCR, western blot, and chromatin immunoprecipitation assays to determine if TSA would prevent changes induced by chronic ethanol exposure. We then utilized a cultured cortical neuron model that recapitulates the changes found after chronic ethanol exposure in vivo to determine the role of HDACs in ethanol-induced changes in GABA(A)-R expression using overexpression and lentiviral knockdown strategies. Statistical significance was determined using 2-way ANOVAs and Student's unpaired two-tailed t-tests unless otherwise noted. Results: Chronic ethanol exposure reduced GABA(A)-R $\alpha 1$ subunit expression by $32 \pm 2 \%$, that was prevented by TSA $(p<0.001)$. TSA further prevented ethanol-induced decreases in overall locomotor activity $(32 \pm 1 \%, p<0.05)$, and the duration of zolpidem-induced $(60 \mathrm{mg} / \mathrm{kg})$ loss of righting reflex $(48 \pm 6 \%, p<0.05)$. Ethanol exposure reduced histone pan-acetylation by $22 \pm 5 \%(p<0.05)$ and TSA administration with ethanol increased acetylation by $123 \pm 13 \%(p<0.0001)$. Quantitative chromatin immunoprecipitation assays revealed there were specific decreases in acetylation associated with the Gabral promoter and gene following chronic ethanol exposure $(75 \pm 5 \%)$, that were prevented by the administration of TSA $(p<0.05)$. Decreases in histone-3 acetylation were accompanied by a selective increase in HDAC2 $(46 \pm 15 \%, p<0.01)$ and HDAC3 $(35 \pm 9 \%, p<0.05)$ expression in the chromatin fraction and HDAC2/3 enrichment on the Gabral promotor $(250 \pm 15 \%, 283 \pm 10 \%$, respectively, $p<0.0001)$. Mechanistic studies in cultured cortical neurons demonstrate that ethanol 
(50mM) exposure for 4 hrs decreased GABA(A)-R $\alpha 1$ subunit levels in the membrane fraction by $17 \pm 3 \%(p<0.01)$ and this was prevented by co-exposure to TSA (500 nM). Next, HDAC2 was overexpressed in cortical neurons using FuGENE HD reagent (DIV 15) to determine if overexpression caused changes in GABA(A)-R subunit expression. HDAC2 overexpression decreased $\alpha 1$ expression $(40 \pm 5 \%, p<0.01)$. Next, we next specifically inhibited HDAC2 using a lentiviral-shRNA strategy (DIV0). Knockdown of HDAC2 prevented the ethanol-induced decrease in $\alpha 1$ expression in cortical neurons, indicating that ethanol activation of HDAC2 is sufficient to regulate GABA(A)-R $\alpha 1$ subunits in cultured cortical neurons. Pharmacological inhibition of HDAC3 using RGFP966 (80 nM and $8 \mathrm{nM}$ ) also completely prevented the ethanol-induced reduction in $\alpha 1$ expression. Finally, targeted acetylation of the Gabra1 promotor using a novel dCas9-P300 construct prevented the decreased GABA(A)-R $\alpha 1$ caused by ethanol (dCas9-P300 + EtOH, $553 \pm 182 \%, p<0.0001)$. This effect was not observed when the dCas9-P300 construct was targeted to a distal exon. Conclusions: The summation of the present studies demonstrate a novel mechanism for the development of alcohol dependence involving regulation of GABA(A)-Rs by HDAC2 or HDAC3 and a putative therapeutic avenue to inform treatment of alcohol use disorders. Further, these studies demonstrate a novel mechanism for regulation of the most abundant GABA(A)-R, providing a new target for many CNS pathways dependent upon GABA inhibition for proper function.

Keywords: Alcohol Dependence, Epigenetics, GABA-A Receptors, HDAC 2/3.

Disclosure: Nothing to disclose.

T222. $\beta 3$ Integrin, Focal Adhesion Kinase and Cofilin as a Signaling Pathway for MMP-9 Induction of Transient Synaptic Plasticity in Cocaine Relapse

Coti Garcia-Keller*, Michael Scofield, Ana-Clara Bobadilla, Sade Spencer, Cara Monforton, Townsend Reeves, Stewart Bryant, Peter Kalivas

Medical University of South Carolina, Charleston, South Carolina, United States

Background: Cocaine use elicits neuroplasticity within the nucleus accumbens core (NAcore) that leads to increased vulnerability to relapse, even after protracted abstinence. Matrix metalloproteinases (MMPs) are inducible endopeptidases that degrade extracellular matrix (ECM) proteins (such as fibronectin, laminin and thrombospondin) as well as nonECM signaling molecules, and reveal an RGD domain that binds and signals through integrins. Integrins are heterodimeric receptors composed of $\alpha \beta$ subunits, and their primary signaling kinases are the focal adhesion kinase (FAK) and integrin linked kinase (ILK). Previous results show that $\beta 3$ integrin is upregulated after cocaine selfadministration and MMP-9 activity is increased during cuedreinstatement of cocaine and promotes transient synaptic plasticity (t-SP: increases in spine head diameter (dh) and AMPA/NMDA). Here we endeavor to understand if $\beta 3$ integrin signaling through FAK and cofilin (actin depolymerization factor) is necessary to promote synaptic growth and regulate actin polymerization during $\mathrm{t}-\mathrm{SP}$ produced during drug-seeking.

Methods: Following a single 2-hour session of food training, adult Sprague-Dawley rats were trained to self-administer cocaine or yoked-saline on an FR1 schedule where responses on an active lever resulted in a drug infusion paired with discrete cues for 10 days, followed by 10-14 days of extinction where lever pressing no longer had any programmed consequences. Animals were assigned to one of the following experiments for each of the different conditions: saline, extinguished and reinstated. 1) Animals were microinjected with an antisense oligo sequence (morpholino) to downregulate the expression of $\beta 3$ Integrin in the NAcore during 5 days or FAK inhibitor $10 \mathrm{~min}$ before cue-induced reinstatement of cocaine and heroin. 2) Animals received daily morpholino treatment for 5 days and were sacrificed after 15 min cued-reinstatement to measure changes in spine dh and density. 3) Animals were sacrificed after 15 min of extinction or cued-reinstatement and p-FAK (active) and p-Cofilin (inactive) expression was measure on NAcore labeled spines with AAVC2-hSYN-ChR2-YFP virus. 4) Animals were microinjected with FAK inhibitor before 15 min of extinction or cued-reinstatement to measure MMP activity (zymography). The contrateral hemisphere of each animal was used as a control. 5) As controls, sucrose seeking and locomotor activity was studed after FAK inhibitor or $\beta 3$ Integrin morpholino microinjection in the NAcore. All experimental protocols in animal studies were approved by the Institutional Animal Care and Use Committee at MUSC and were conducted in accordance with the National Institutes of Health Guide for the Care and Use of Laboratory Animals.

Results: $\beta 3$ Integrin down-regulation and FAK, but not ILK, inhibition reduced cocaine drug seeking and prevented the increase in spine dh produced during $15 \mathrm{~min}$ of cue-induced reinstatement compared to control animals. Also, dh frequency was leftward shift in $\beta 3$ integrin antisense injected animals compared to control morpholino sequence after cued-reinstatement session, indicating that the increase in spine dh induced by MMP activation during t-SP was blocked. Importantly, neither the FAK inhibitor nor $\beta 3$ Integrin morpholino impacted cued sucrose seeking or locomotor activity following intra-NAcore microinjection. Immunohistochemistry in NAcore labeled spines, revealed increased p-FAK localization in dendritic spines of extinguished and cue-reinstated animals, but p-Cofilin was increased only in the cue-reinstated condition suggesting these proteins comprise part of the pathway regulating the increase in the spine dh.

Conclusions: These data propose that $\beta 3$ Integrin, FAK and Cofilin constitute a signaling pathway downstream of MMPs activation that is involved in promoting transient synaptic growth induced by cocaine-conditioned cues that reinstate drug seeking.

Keywords: Matrix Metalloproteinase-9 (MMP-9), Relapse, Integrin, Dendritic Spines.

Disclosure: Nothing to disclose. 
T223. Sex-Dependent Effects in the Effects of Unpredictable Stress Upon Alcohol Sensitivity

Sema Quadir, Eugenie Guezlian, Mason Palmer, Douglas Martin, Jennifer Kim, Daniel Colin, Praveena Motupalli, Karen Szumlinski*

University of California, Santa Barbara, Santa Barbara, California, United States

Background: A high rate of co-morbidity exists between alcohol use (AUDs) and affective disorders, suggesting common biological underpinnings in the etiology of these diseases. In support of this suggestion, a history of unpredictable, chronic, mild stress (UCMS) increases sensitivity to both the psychomotor and rewarding properties of alcohol - a phenomenon known as stress-alcohol crosssensitization. Stress reactivity is sexually dimorphic in both humans and laboratory animals, with females typically exhibiting greater stressor sensitivity than males. However, no study to date has examined for sex differences in stressalcohol cross-sensitization of relevance to our understanding of the etiology of AUDs or disease co-morbidity.

Methods: As females tend to be more stressor-reactive than males, we hypothesized that female mice would exhibit greater stress-alcohol cross-sensitization than age-matched males. To test this hypothesis, adult male and female mice on a mixed C57BL/6J X 129X1/SvJ background were subjected to an 11-day UCMS procedure, while control animals were injected daily with saline (IP; vol=0.02 ml/g). In the first study, mice were then injected with $2 \mathrm{~g} / \mathrm{kg}$ alcohol (IP), assayed for locomotor cross-sensitization and then tested for alcohol consumption under continuous-access conditions. In the second study, a dose-response approach was employed $(1-4 \mathrm{~g} / \mathrm{kg}$ alcohol) to assay for sex differences in the effects of UCMS upon alcohol-induced locomotion and motor coordination/intoxication. Additional cohorts of mice were allowed to binge-drink alcohol following stressor presentation. Given evidence that binge drinking augments stressorreactivity, subgroups of male and female mice were included in Study 2 that were allowed to binge-drink alcohol for 2 weeks under modified Drinking-in-the-Dark (DID) paradigm (2-h access to 5, 10, 20 and $40 \%$ alcohol) prior to undergoing UCMS/control procedures, testing for alcohol sensitivity and subsequent binge-drinking to examine for additive or synergistic effects upon behavior. As the results of the 2 nd study did not indicate an additive effect of sequential binging and UCMS upon alcohol sensitivity, in a third study, mice underwent binge-drinking and UCMS procedures simultaneously, prior to testing for alcohol sensitivity and intake.

Results: In study 1 , female mice exhibited greater sensitivity to $2 \mathrm{~g} / \mathrm{kg}$ alcohol and consumed more alcohol under continuous-access procedures than males. In this singledose study, UCMS augmented both alcohol-induced locomotion and intake in males, with no UCMS effect apparent in females. In Study 2, we detected significant interactions for locomotor activity between alcohol dose and sex (females $>$ males), dose and binge-drinking history (water $>$ DID), and dose and UCMS history (UCMS > controls at $1 \mathrm{~g} /$ $\mathrm{kg}$ ) for locomotor activity. We did not detect any additive effect of prior binge drinking and stressor history upon alcohol-induced locomotion or motor co-ordination in either sex and, irrespective of binge-drinking history and sex, UCMS reduced the motor-incoordinating effects of $3 \mathrm{~g} / \mathrm{kg}$ alcohol. When tested for their righting reflex, a sexdependent difference in the ability to right was apparent in non-binging mice, with UCMS increasing intoxication in males, but reducing intoxication in females. UCMS history potentiated subsequent binge-drinking in alcohol-naive mice of both sexes and in male bingers. While a prior bingedrinking history did not alter subsequent binging in males, female bingers exhibited greater alcohol intake that was insensitive to prior stress history.

Conclusions: These data indicate that the subject factors of sex, prior stressor history and prior binge-drinking history interact in complex ways in mice to impact sensitivity to alcohol's motor-stimulating, motor-incoordinating and intoxicating effects, as well as influence subsequent heavy drinking. While we have yet to complete our characterization of the effects of simultaneous drinking and stress upon alcohol sensitivity and intake, the available data argue that while females tend to exhibit greater stressor-reactivity alcohol-sensitivity and binge-drinking than males, they are less vulnerable to stressor-induced potentiation of alcohol behavioral sensitivity, of potential relevance to the etiology of affective disorder and alcoholism co-morbidity.

Keywords: Binge Drinking, Unpredictable Stress, Co-Mobidity, Cross-Sensitization.

Disclosure: NIAAA grants AA016650 and AA024044 to KKS, the UCSB Faculty Research Assistance Program to SGQ and MP and the McNair Scholars Program to EG.

\section{T224. Synaptic Plasticity Induced by THC+CBD Self- Administration Resembles Opioids, Not Psychostimulants}

Sade Spencer*, Daniela Neuhofer, Danielle Schwartz, Michael Scofield, Constanza Garcia-Keller, Peter Kalivas

Medical University of South Carolina, Charleston, South Carolina, United States

Background: It is well established that chronic consumption of addictive drugs leads to lasting changes in structure and function in brain reward nuclei including the nucleus accumbens core (NAcore), however the nature of these changes may differ between drug classes. As a prominent example, stimulants like cocaine increase complexity and numbers of dendritic spines on NAcore medium spiny neurons (MSNs) while opiates like heroin have the opposite effect. Likewise, cocaine increases AMPA:NMDA ratios at cortico-accumbens synapses while heroin does not. Nonetheless, both types of drugs produce analogous behavioral profiles including a high susceptibility to relapse after withdrawal suggesting that it may be the magnitude rather than the direction of the neuroadaptation that is most important for influencing future behavior. Cannabis is the most widely used illicit drug worldwide, and the main psychoactive component of cannabis is $\Delta 9$-tetrahydrocannabinol (THC). THC on its own can produce unpleasant side effects including increased anxiety, but cannabis contains over 60 cannabinoids and more than 400 additional chemicals. Cannabidiol (CBD), a non-psychoactive 
$\overline{S 426}$

cannabinoid, can counter some of the aversive properties of THC by producing anxiolytic and anti-psychotic effects. Here we combined THC and CBD in a rat intravenous self-administration model in order to assay enduring neuroadaptations produced by chronic cannabinoid use. Importantly, our model consistently produces cue-induced reinstatement leading to us to hypothesize that we might similarly observe enduring morphological and physiological changes in NAcore MSNs motivating the liability to relapse. Methods: Adult Sprague-Dawley rats were trained to selfadminister $\mathrm{THC}+\mathrm{CBD}$ in a 10:1 ratio on an FR1 schedule (1.5 hrs per day) where responses on an active lever resulted in a drug infusion (1-4 $\mu \mathrm{g} / \mathrm{kg}$ per infusion) paired with discrete light and tone cues for $10+$ days followed by $7+$ days of extinction where lever pressing no longer had any programmed consequences. Following extinction, animals were tested for reinstatement of drug seeking to drug-paired cues or sacrificed to assay various biological endpoints in the NAcore. Whole-cell patch clamp electrophysiology was used to measure AMPA:NMDA ratios and NMDA-LTD. Diolistic labeling and confocal microscopy was employed to quantify changes in dendritic spine morphology. In vivo zymography was adapted to measure matrix metalloproteinase activity. All experimental protocols in animal studies were approved by the Institutional Animal Care and Use Committee at MUSC and were conducted in accordance with the National Institutes of Health Guide for the Care and Use of Laboratory Animals. Results: Rats extinguished after THC+CBD selfadministration showed marked reinstatement of pressing on the active lever compared to extinction in response to THCpaired cues $(\mathrm{F}(3,160)=39.84, p<0.0001)$. THC+CBD selfadministration occluded NMDA-LTD in NAcore $(p<0.05$, unpaired t-test). Delta9-tetrahydrocannabinol self-administration also reduced the density of dendritic spines on NAcore MSNs (t-test by segment $p=0.006$; $t$-test by animal number $p=0.1289$ ) but did not affect spine diameter (t-test by segment $p=0.3699$; t-test by animal number $p=0.5259$ ). However, extinction from $\mathrm{THC}+\mathrm{CBD}$ self-administration did not alter basal properties of synaptic physiology or AMPA: NMDA ratio $(p=0.6493$, unpaired t test $)$ in NAcore. Likewise, we found no basal effect of THC+CBD on matrixmetalloproteinase (MMP) activity ( $p=0.6188$, unpaired $t$ test), which is permissive for synaptic plasticity.

Conclusions: In summary, these data illustrate that THC $+\mathrm{CBD}$ self-administration and extinction results in enduring changes in morphological and physiological plasticity in the NAcore and suggests both distinct and overlapping mechanisms between cannabinoids and other drugs of abuse may underlie the high susceptibility to relapse engendered by chronic drug use. Overall, we find that the basal neuroadaptations produced by $\mathrm{THC}+\mathrm{CBD}$ self-administration are more similar to those produced by opiates than psychostimulants. Yet heroin, cocaine, and now THC have all been shown to reduce NMDA-LTD, and increasing evidence indicates that this loss in the ability to induce synaptic plasticity might be a potential hallmark of addiction. Future studies will investigate if and how relapse to THC seeking further modulates synaptic plasticity, as we have shown that transient synaptic potentiation at NAcore synapses is also a shared feature of cue-induced relapse across abused substances.
Keywords: Delta9-Tetrahydrocannabinol, Nucleus Accumbens, Long-Term Depression, Self-Administration.

Disclosure: Nothing to disclose.

\section{T225. HDAC5 and NPAS4 Regulate Cocaine Reward- Environment Associations and Reinstatement of Drug Seeking}

Makoto Taniguchi*, Maria Carreira, Yonatan Cooper, Daniel Guzman, Evan Balmuth, Jaswinder Kumar, Laura Smith, Nobuya Koike, Joseph Takahashi, Tae-Kyung Kim, David Self, Yingxi Lin, Christopher Cowan

Medical University of South Carolina, Charleston, South Carolina, United States

Background: Drug addiction is defined as a long-lasting disorder characterized by compulsive drug seeking and consumption despite adverse, negative consequences to the individual. The persistence of craving and high incidence of relapse following prolonged periods of abstinence in the addicted patient population is a major hurdle for therapeutic treatment. A central goal for current drug abuse research is to understand the molecular mechanisms that include pathological plasticity in brain reward circuits and underlie the development and persistence of drug addiction. Although complex in nature, one key mechanism involved in relapse involves the formation of enduring associations between the primary rewarding properties of drugs and the environmental cues linked to drug use. Although initially these external cues are motivationally neutral, their association with drug use can encourage repeated drug seeking and taking. Increasing evidence suggests that epigenetic regulation of gene expression by histone modifying enzymes contributes to persistent, maladaptive synaptic and behavioral plasticity associated with the development of addictive-like behaviors. Previously, we demonstrated that cocaine exposure induces the transient nuclear accumulation of the class IIa histone deacetylase, HDAC5, through a signaling pathway involving the D1 dopamine receptor, cAMP signaling and PP2A phosphatasedependent dephosphorylation of three HDAC5 residues. Overexpression of a dephosphorylated form of HDAC5 in the nucleus accumbens (NAc) reduced the development, but not expression, of cocaine conditioned place preference (CPP), suggesting that transient nuclear accumulation of HDAC5 limits cocaine's ability to produce reward-associated behaviors. However, the genomic targets of nuclear HDAC5 that mediate these effects, and the influence of nuclear HDAC5 on volitional cocaine administration, were unknown. Methods: We identified HDAC5 binding peaks, including Npas4's activity-sensitive enhancer region, in striatal neurons using chromatin immunoprecipitation followed by highthroughput sequencing (ChIP-seq). Npas4 gene and protein expression and HDAC5 association on the Npas4 enhancer region were examined in NAc and striatal tissues after exposure to novelty, stress and/or cocaine or in cultured primary striatal neurons. Characterization of the Npas4 genomic enhancer region was performed using a luciferase reporter gene. HDAC5 and NPAS4 manipulations in vivo were accomplished with adult mice or rats receiving intra-NAc infusions of adenoassociated viruses (AAVs) expressing HDAC5 (WT or S259A/ 
S279A/S498A, 3SA), Cre recombinase, or Npas4 shRNA. Mice were either WT (C57BL/6) or homozygous floxed-Npas4 (C57BL/6 backcrossed), as appropriate. We examined cocaine reward related behaviors using several behavioral paradigms, including rat intravenous cocaine self-administration (IVSA), mouse cocaine CPP and mouse locomotor sensitization. Control behavioral testing also included the sucrose preference test, contextual fear conditioning, and the open field test.

Results: Virus-mediated overexpression of nuclear HDAC5 (3SA) in the rat medial NAc significantly reduced cue- and cocaine prime-induced drug seeking in the cocaine IVSA extinction-reinstatement assay. In contrast, no significant effects were observed on stable cocaine intake or extinction training. To identify HDAC5 target genes that mediate its role in cocaine reward-related behavior, we performed ChIPseq and identified numerous candidate genes, including Npas4 - an activity-induced neuronal transcription factor. NPAS4 is rapidly and transiently induced by glutamatergic synaptic activity, and it is reported to regulate inhibitory and excitatory synapse density in a cell-type specific manner. We identified a $\sim 400$ bp enhancer region in the 5' end of the Npas4 gene that is necessary and sufficient to mediate depolarization-induced activation of NPAS4 expression, and we show that HDAC5 3SA blocks the activity-dependent expression of Npas4 via this enhancer region, and endogenous HDAC5 is recruited to the Npas4 enhancer following cocaine administration. Hdac5 $\mathrm{KO}$ mice show elevated levels of Npas 4 mRNA in the NAc. We found that exposure to novel context induced transient NPAS4 mRNA and protein expression in a small subset of FOS+ neurons in the NAc. Conditional genetic deletion or reduction of Npas 4 in the NAc significantly reduces cocaine CPP, without altering psychomotor responses to cocaine, natural reward behavior, anxiety-like behavior, or fear-related contextual memory.

Conclusions: In this study, we found that enhanced nuclear accumulation of HDAC5 in the NAc reduces cue- and drug prime-induced reinstatement of drug seeking behaviors in cocaine IVSA. Using an unbiased, genome-wide analysis of HDAC5 binding sites, we identified the activity-sensitive enhancer of Npas4 as a novel genomic target, and we show that reduction of Npas4 expression, like overexpression of dephosphorylated HDAC5, in the adult NAc reduces cocaine $\mathrm{CPP}$, without altering the sensitivity to cocaine's motor activating effects, natural reward preference or aversive learning and memory. Together our data suggested that HDAC5 regulates the association between external and internal cues associated with drug administration and cocaine reward, at least in part, through repression of Npas4 expression.

Keywords: Epigenetic, Cocaine, HDAC.

Disclosure: Nothing to disclose.

T226. Resting State Functional Connectivity in Tobacco Smokers and Electronic Cigarette Users: Correlations With Gut Bacterial Diversity

Ramiro Salas*, Christopher Stewart, Richard De La Garza, Kaylah Curtis

Baylor College of Medicine, Houston, Texas, United States

Background: Electronic cigarettes (ecigs) are devices that deliver nicotine and are increasingly popular, especially among adolescents and young adults. A major question in the field is the extent to which tobacco cigarettes and ecigs affect brain functioning and if differences occur between these exposures. Of additional interest, preliminary data in both human and rodent models showed that the bacterial microbiome may be differentially altered by ecig use vs. cigarette smoking.

Methods: We used brain functional MRI to study resting state functional connectivity (RSFC) in a sample of current cigarette smokers $(N=6)$, ecig users $(N=8)$, and nonsmoking controls $(N=7)$. We also collected stool and oral samples for microbiome analyses. Bacterial profiles were generated to assess the total and viable bacterial populations by V4 16 S sequencing of extracted DNA and RNA (cDNA), respectively. Alpha- and Beta-diversity metrics were used to compare bacterial diversity and composition between cigarette smokers or ecig users, and matched controls. For RSFC we used a series of regions hypothesized to be important for tobacco use disorder (accumbens, striatum; inferior, medial, superior, orbitofrontal prefrontal cortices; precuneus; anterior, medial, and posterior cingulate cortices) as seeds in an ROI-to-ROI analysis. For microbiome studies, we collected stool samples and used operational taxonomic units (OTUs) as a measure of bacterial diversity in a preliminary analysis. Although inconclusive, increased microbial richness is usually associated with good health measures.

Results: We found differences in RSFC between cigarette smokers and ecig users in several regions, including: accumbens/mid cingular (higher in cigarette smokers), med prefrontal/precuneus, medial prefrontal/cerebellum crus 2, frontal medial orbital/temporal inferior cortex, and precuneus/thalamus (higher in ecig users). In most cases, nonsmoking controls had intermediate values between cigarette smokers and ecig users. Next, we performed correlations between RSFC and OTUs in participants for which we had both kinds of data. We found that in all cases except nucleus accumbens/med cingulum, RSFC negatively correlated with OTUs. We are currently collecting additional data to complete these studies.

Conclusions: In conclusion, our data suggests that ecig use has effects in certain brain regions opposite that seen in cigarette smokers. In addition, RSFC between the regions that showed differences between cigarette smokers and ecig users negatively correlated with OTUs. If these preliminary data are confirmed in a larger cohort, the microbiome may be implicated in the effects that cigarette smoking and ecig use have on brain functioning, providing a novel therapeutic target.

Keywords: Tobacco Smoking, Electronic Cigarette (E-Cigarette), Gut Microbiome, Resting State Brain Imaging, Medial Prefrontal Cortex.

Disclosure: Nothing to disclose. 
T227. When Passion Persists: Cocaine Patients Exhibit "Mesolimbic Persistence" to $33 \mathrm{msec}$ Drug Cues That is Both Medication-Sensitive and Positively Correlated With Years of Prior Cocaine Use

Anna Rose Childress*, Kanchana Jagannathan, Paul Regier, Stefanie Darnley, Jesse Suh, Teresa Franklin, Zachary Monge, Kimberly Young, Reagan Wetherill, Elliott Berkowitz Sturgis, Kyle Kampman, Michael Gawrysiak, Daniel Langleben, Regina Szucs-Reed, Charles O'Brien

University of Pennsylvania, Philadelphia, Pennsylvania, United States

Background: Addicted individuals often experience cuetriggered responses (subjective arousal and drug motivation) that recur long after the last dose of their preferred drug. In the "cue-vulnerable" individual, these behavioral responses can be remarkably resistant to extinction: they persist despite repeated exposure to real-world cues, and in the absence of continued drug use. Using brain imaging tools, we and others have begun to characterize the brain response to drug cues. Most of these imaging studies of cue-triggered brain responses have been based in simple "magnitude" comparisons, e.g., comparing the averaged BOLD fMRI response for repetitions of a target (e.g., cocaine cues) vs. a neutral comparator. In an effort to model the real-world phenomenon of "persistence", we have recently used an analytic approach that measures the change in the brain response to multiple repetitions of an individual cue type (e.g., drug, sex, aversive, neutral), in the first vs. second half of a task. We hypothesized that our cocaine patients would exhibit "persistence" in the mesolimbic response to drug cues (with sustained, or even increased, responding in the second half of the task) - while healthy young controls would show the usual decline (extinction) in response to repeated, nonreinforced appetitive (sexual) stimuli. We also predicted that the "persistence" response might be positively correlated with years of prior cocaine use (offering more "conditioning trials") and that the "persistent" brain response might be modulated by candidate medications with known actions on the mesolimbic dopamine (DA) system (the GABA B agonist baclofen, and the nicotinic partial agonist, varenicline). All of the tests reported here were carried out using our special 33 msec cue probe that captures the 'earliest' brain response to the cues, prevents conscious recognition, and thus precludes secondary responses (e.g., embarrassment, regret) that could confound the primary motivational response.

Methods: In a "fast" event-related BOLD fMRI paradigm, male cocaine inpatients ( $n=85$ total: $n=64$ unmedicated; $n=10$ baclofen $(20 \mathrm{~m} . g$. t.i.d in the week prior to scanning); $n=11$ varenicline ( $1 \mathrm{mg}$ b.i.d. in the week prior to scanning), and healthy young male controls $(n=18$; average age 22.4 years) were exposed to cocaine-related and to comparison (sexual, aversive and neutral) cues of $33 \mathrm{msec}$ duration. Each cue (48 presentations of each cue category) was "backwardmasked" by a 467 msec neutral stimulus to prevent conscious recognition. A standard SPM 8 pipeline was used for image preprocessing (realignment, normalization, smoothing). The focus of the current analyses was "persistence", as measured by pre-planned contrasts comparing (especially) mesolimbic brain responses in the first half vs. second half of the task, within each cue category (drug2-drug1; sex2-sex1, etc.). For the unmedicated cocaine patients, we additionally tested for correlations between years of cocaine use or age with the effects of interest, using each as a separate, single covariate of interest in the a priori contrasts.

Results: As expected, the healthy young controls showed a robust mesolimbic (ventral tegmental area, ventral striatum, pallidum, amygdala, medial orbitofrontal cortex and insula; $2<\mathrm{t}<5$; peak $\mathrm{t}$ in ventral striatum/pallidum, 3.47) in response to the $33 \mathrm{msec}$ sexual cues in the first half of the task, followed by an equally dramatic decline (same brain regions) in the second half of the task (their response to drug cues was much smaller, and also rapidly declined). For cocaine patients, the 'persistence' analysis revealed an increase in responding to drug cues across the task (drug2drug1) in the bilateral ventral striatum and pallidum that was indeed positively correlated with years of cocaine use $(2<\mathrm{t}<5$; peak $\mathrm{t}$ in ventral striatum/pallidum, 3.83). Age was not significantly correlated with the 'persistent' cue response. Cocaine patients receiving the GABA $\mathrm{B}$ agonist baclofen showed an encouraging decline in the mesolimbic response to drug cues across the task; in contrast, patients taking varenicline actually showed recruitment of (mesolimbic) responding to repeated drug, sexual and aversive stimuli.

Conclusions: These findings highlight the potential utility and clinical relevance of capturing the "persistent" limbic brain response to drug cues, even when these cues occur completely outside conscious awareness. Healthy young controls do not exhibit persistence, either to relevant (sexual) or novel (drug) stimuli - but many of our cocaine patients do, and the response is well-correlated with their prior years of cocaine exposure. Encouragingly, a medication such as baclofen - that increases inhibition of VTA cell firing and thus inhibits DA release to cues - may have the potential to prevent the 'persistence', and to reset or restore the brain's ability to extinguish responding to non-reinforced stimuli. The results with varenicline are not positive, but they underscore the sensitivity of the $33 \mathrm{msec}$ paradigm, and the 'persistence' analysis, for screening candidate medications. Worth noting, these results with "persistence" in the appetitive domain of addiction have direct parallels in feardriven disorders (e.g., anxiety, phobias, PTSD), where the core pathology is the "persistent", non-extinguishing response to learned cues for danger. The familiar co-morbidity between the (appetitive and aversive) disorders of "persistence" may reflect an underlying, shared brain vulnerability the (over-)learned response to cues - that could be treated with a single, "extinction-encouraging" medication.

Keywords: Functional MRI (fMRI), Cocaine, Extinction, Cue Reactivity.

Disclosure: Nothing to disclose. 
T228. Alcoholism Drug Repurposing From Computational Analyses With High Drinking in the Dark Mice

Angela Ozburn*, Laura Ferguson, Pamela Metten, Dayne Mayfield, Igor Ponomarev, Robert Adron Harris, John Crabbe

Portland VA Medical Center, Portland, Oregon, United States

Background: The FDA has approved only three drugs for treatment of alcoholism - disulfiram, naltrexone, and acamprosate. One of the impediments to discovery of new therapeutic compounds has been that existing animal models used for screening typically do not voluntarily drink to intoxication. However, recent efforts have led to animal models of drinking to intoxication (i.e. Drinking in the Dark, DID). Further work has been carried out to develop selectively bred mice for High Drinking in the Dark (HDID-1 and HDID-2), by mating individuals reaching the highest blood alcohol levels (BALs) after a second daily DID session. HDID mice achieve BALs between 170 and $150 \mathrm{mg} \%$ after 32 (HDID-1) and 25 (HDID-2) selected generations, respectively; this is approximately twice the $80 \mathrm{mg} \%$ level defined by the NIAAA as binge drinking. Binge drinking is a strong predictor of alcohol use disorder diagnosis and has deleterious health consequences, particularly in US combat veteran populations.

Methods: We used regional brain tissue from HDID-1 male mice and compared gene expression profiles between HDID mice and their non-selected HS/Npt control line with data from the Library of Integrated Cellular Signatures (LINCS), which comprises gene expression response profiles for $>19,000$ compounds in multiple cell lines, including responses to many FDA-approved compounds. We generated a list of genes that have increased or decreased expression in alcohol-naïve HDID mice vs HS. We then used this input to query LINCS and identify compounds with signatures that have a strong connectivity to the disease signature. We prioritized compounds and tested the effects of selected compounds on binge-like drinking in HDID-1 mice. We hypothesized that these novel drugs would normalize HDID-1 expression differences from HS and would successfully predict drug efficacy to reduce binge-like drinking.

Results: Testing has been carried out successfully in separate groups of male and female HDID-1 mice for two targets, a phosphodiesterase type 4 (PDE4) inhibitor and a Bruton's tyrosine kinase (BTK) inhibitor. We first tested the PDE4 inhibitors rolipram and apremlilast, and both drugs significantly reduced binge-like alcohol drinking. Additionally, generalizability of these findings has been established via testing carried out in another mouse strain at an additional site. Notably, BTK inhibition (via administration of terreic acid) also resulted in a significant reduction in alcohol intake and associated blood alcohol levels.

Conclusions: Our analysis and testing approach successfully identified novel compounds that drastically reduce alcohol intake and blood alcohol levels in HDID mice. Therefore, we conclude that other novel compounds suggested by our investigation could have therapeutic potential for the treatment of Alcohol Use Disorders.
Keywords: Alcohol Intake, Pharmacotherapy, Drug Discovery - New Approaches, Gene Network Analysis.

Disclosure: These studies are a part of the Integrative Neuroscience Initiative on Alcoholism-Neuroimmune, an NIAAA-sponsored consortium effort (U01 AA10760). Additional support: NIH (R24 AA020245, F31 AA024332, AA017234), US Department of Veterans Affairs Awards (IK2 BX002488, 101BX000313, and 02488) and BBRF NARSAD Young Investigator Award.

\section{T229. Organic Cation Transporter 3: An Unsuspected Player in the Actions of Psychostimulant Drugs}

Harald Sitte*, Felix Mayer, Diethart Schmid, W. Anthony Owens, Georgianna Gould, Mia Apuschkin, Isabella Salzer, Stefan Böhm, Peter Chiba, Piper Williams, Hsiao-Huei Wu, Ulrik Gether, Wouter Koek, Lynette Daws

\section{Medical University of Vienna, Vienna, Austria}

Background: Medications used to treat psychiatric disorders and psychostimulant abuse often fail to provide therapeutic benefit. For example, selective serotonin reuptake inhibitors (SSRIs), which target the serotonin transporter (SERT) and are the frontline treatment for depression, do not provide symptom relief for the majority of patients. In the case of psychostimulants such as amphetamine, which target the dopamine transporter (DAT), strategies targeting DAT have yielded little to no benefit in treating abuse of these drugs. A rapidly growing literature supports a prominent role for organic cation transporters, particularly the organic cation transporter 3 (OCT3) in regulating biogenic amine neurotransmission. Here we discuss recent findings pointing to these transporters as important players modulating the effects of psychostimulants.

Methods: We obtained functional measures of transporter activity using uptake assays in cell-based systems in vitro by radioactive and fluorescent ligand flux measurements; we determined monoamine clearance in vivo by high-speed chronoamperometry. We examined transporter phosphorylation sites by mass spectrometric analysis. Locomotor activity was assessed using beam break measurements. Radioligand binding and western blotting using specific antibodies were used to examine transporter expression.

Results: Our results demonstrate that these transporters significantly contribute to the action of amphetamines and are a yet underappreciated but nevertheless important, clinically relevant target of psychostimulants.

Conclusions: Our studies hence contribute to the basic understanding of psychostimulant drug action which are widely used and abused. The poster will also discuss OCT3 as a promising target for the development of novel drugs with improved therapeutic outcome for psychostimulant abuse.

Keywords: Psychostimulants, Amphetamine, Organic Cation Transporters.

Disclosure: Nothing to disclose. 
T230. Maladaptive Behavioral Regulation in Alcohol Dependence: Role of Kappa-Opioid Receptors in the Bed Nucleus of the Stria Terminalis

Brendan Walker*

Washington State University, Pullman, Washington, United States

Background: An important role of the endogenous opioid dynorphin (DYN) and its receptor, the kappa-opioid receptor (KOR) has been established for the treatment of addictive disorders such as alcohol dependence. The extended amygdala is critically involved with the regulation of motivational and emotional behavior in a manner that promotes personal survival and propagation of the species and is defined by functional connectivity between the central nucleus of the amygdala (CeA), the nucleus accumbens (Acb), and a third important brain structure: the bed nucleus of the stria terminalis (BNST). Recent data strongly implicates the DYN/ KOR system in the extended amygdala, including the BNST, in the regulation of alcohol self-administration in dependence. Until more information is established regarding the role of the BNST in alcohol dependence, a critical gap in our knowledge base will exist and prevent the development of comprehensive strategies for the treatment of alcohol dependence.

Methods: Using a combination of molecular and neuropsychopharmacological approaches, the current study evaluated KORs in the BNST for their contribution to escalated alcohol self-administration during acute withdrawal in alcohol dependent rats. To this end, male Wistar rats were trained to self-administer alcohol, with a subset of animals undergoing stereotaxic intra-BNST cannula guide implantations. All animals were subjected to an alcohol-dependence induction procedure using intermittent alcohol vapor exposure (14 h alcohol exposure/10 h alcohol withdrawal daily) with control animals air-exposed. Following onemonth of alcohol vapor (or air) exposure, animals were tested for alcohol self-administration at a time-point equal to six-hours into withdrawal until stable responding was achieved. Non-cannulated animals were sacrificed during acute withdrawal to collect the BNST via brain microdissection for later quantitative reverse transcription PCR (RTqPCR) while those animals that were previously cannulated received intra-BNST KOR antagonist infusions to functionally assess the role of BNST KORs in alcohol dependence.

Results: In self-administering alcohol-dependent animals, Oprk1 mRNA expression was shown to be elevated in the BNST compared to non-dependent animals. Furthermore, intra-BNST infusions of a KOR antagonist dose-dependently attenuated escalated self-administration during acute withdrawal without altering non-dependent alcohol selfadministration.

Conclusions: These results functionally confirm an important role of dysregulated KORs in the BNST and together with previous results showing recruitment of DYN / KOR system in the Acb and CeA during dependence, illustrate the importance of the extended amygdala in the maladaptive behavioral regulation observed in alcohol dependence.

Keywords: Kappa Opioid Receptor, Bed Nucleus of the Stria Terminalis, Alcohol Self-Administration, Gene Expression, Alcohol Dependence.

Disclosure: Nothing to disclose.
T231. Neurokinin-1 Receptor Antagonism Attenuates Alcohol Intake in Multiple Models of Escalated Consumption

Jesse Schank ${ }^{\star}$, Courtney King, Kenner Rice, Markus Heilig, Nelson Britta

University of Georgia, Athens, Georgia, United States

Background: We have previously demonstrated that the neurokinin-1 receptor (NK1R) mediates stress-induced reinstatement of alcohol seeking as well as escalated alcohol consumption in alcohol preferring $(\mathrm{P})$ rats. Specifically, we have found that NK1R antagonism, either when administered systemically or infused directly into the nucleus accumbens (NAC) shell, attenuates the expression of footshock-induced reinstatement. Additionally, we have demonstrated that NK1R antagonism, either systemic or via direct infusion into the central nucleus of the amygdala $(\mathrm{CeA})$, attenuates escalated alcohol self-administration in $\mathrm{P}$ rats, a strain of rats that has been selected over many generations for high alcohol preference and shows NK1R upregulation in the CeA. It is unclear if non-genetic models of escalated alcohol self-administration are mediated by NK1R signaling, and if so, what brain regions govern this effect.

Methods: In the experiments presented here, we use two methods of inducing escalated alcohol intake in outbred Wistar rats: yohimbine pretreatment and intermittent access schedules. In addition to triggering reinstatement of alcohol seeking, yohimbine injection can induce an increase in alcohol self-administration rates. Intermittent access (Monday, Wednesday, and Friday availability) of $20 \%$ alcohol in two bottle choice procedures has also been shown to consistently induce elevated alcohol consumption relative to continuous access schedules.

Results: In these experiments, we found that escalated alcohol consumption induced by both yohimbine injection and intermittent access are attenuated by systemic administration of the NK1R antagonist L822429. There was no effect of NK1R antagonism on baseline alcohol self-administration or consumption of $20 \%$ alcohol on a continuous access schedule. To determine if alterations in NK1R expression occur following intermittent access to alcohol, we sacrificed rats following two bottle choice procedures. We found that, when compared to continuous alcohol access or access to water alone, NK1R expression was increased in the NAC and dorsal striatum, but not the amygala.

Conclusions: Taken together, these results suggest that NK1R upregulation contributes to escalated alcohol consumption that is induced by genetic selection, acute yohimbine injection, and intermittent access schedules. However there appears to be a dissociation between the regions involved in these behaviors with amygdalar upregulation contributing to genetic predisposition to escalated consumption and striatal upgregulation driving escalation that is induced by environmental exposures. Future experiments will examine the impact of viral vector driven overexpression of the NK1R in discrete brain regions in attempts to phenocopy the consumption of rats exposed to intermittent access.

Keywords: Alcohol Intake, Neuropeptides, Stress Models.

Disclosure: Nothing to disclose. 
T232. Effects of a Heroin-Tetanus Toxoid Conjugate Vaccine on the Behavioral Pharmacology and Pharmacokinetics of Heroin in Nonhuman Primates

Matthew Banks ${ }^{\star}$, Paul Bremer, Justin Poklis, Kim Janda

Virginia Commonwealth University, Richmond, Virginia, United States

Background: Opioid addiction represents a significant and growing public health issue. Although there are FDAapproved pharmacotherapies for opioid addiction, these drugs have undesirable effects that limit their clinical utility. For example, methadone also possesses abuse liability and naltrexone non-discriminately antagonizes both abused mu-opioid agonists and prescribed mu-opioid agonists for pain management. The high recidivism of heroin addiction and undesirable effects of current pharmacotherapies highlights the need for preclinical research to develop novel pharmacotherapies for heroin addiction. The present study aim was to determine the effects of a novel heroin-tetanus toxoid conjugated vaccine on the behavioral pharmacology and pharmacokinetics of heroin in rhesus monkeys.

Methods: Adult, male rhesus monkeys $(n=4)$ were trained to respond under a fixed-ratio 30 schedule of food presentation during daily experimental sessions that consisted of 5 components. Each component consisted of a 25-min timeout period and a 5-min response period. Behavioral sessions were conducted 5 days per week and test session were usually conducted on Tuesdays and Fridays. Initially, cumulative dose-effect functions were determined for heroin $(0.01-1.8 \mathrm{mg} / \mathrm{kg}, \mathrm{IM})$ and oxycodone $(0.01-3.2$ $\mathrm{mg} / \mathrm{kg}, \mathrm{IM}$ ) alone and following pretreatment with naltrexone $(0.032 \mathrm{mg} / \mathrm{kg}, \mathrm{IM})$. Mu-opioid agonists were tested up to doses that suppressed rates of operant responding greater than $50 \%$ of the control rate. In addition to these behavioral dependent measures, pharmacokinetic studies were also conducted in the same monkeys to determine 6-acetylmorphine and morphine plasma levels after heroin $(0.32 \mathrm{mg} / \mathrm{kg}, \mathrm{IM})$ administration. Subsequently, the herointetanus toxoid conjugate vaccine was administered at weeks $0,2,4$, and 11 . Heroin and oxycodone cumulative dose-effect functions were redetermined at weeks $6,9,10,13,14$, and 15 . Heroin $(0.32 \mathrm{mg} / \mathrm{kg}, \mathrm{IM})$ pharmacokinetic studies were redetermined at week 7 .

Results: Under baseline conditions, both heroin and oxycodone dose-dependently decreased rates of operant responding and the corresponding mean ED50 values (SEM) were $0.1(0.03)$ and $0.2(0.06)$, respectively. Acute naltrexone pretreatment produced an 8-fold and 6.7-fold shift in the potency of heroin and oxycodone to decrease rates of operant responding, respectively. Following, herointetanus toxoid conjugate vaccine administration, there was a significant 4.3-fold shift in the potency of heroin to decrease rates of operant responding. In contrast, the potency of oxycodone to decrease rates of operant responding was nonsignificantly shifted 1.4-fold. This heroin potency shift was time dependent and diminished as a function of time following the last heroin-tetanus toxoid vaccine administration. For the pharmacokinetic studies, heroin-tetanus toxoid vaccine administration significantly increased plasma 6-acetylmorphine levels approximately 14-fold, whereas plasma morphine levels were unaltered. Plasma heroin levels were not detectable either before or after vaccine administration.

Conclusions: Overall, these experiments suggest two main findings. First, these results provide behavioral evidence of efficacy and selectivity for a heroin-tetanus toxoid conjugate vaccine to shift the potency of heroin to decrease rates of operant responding in rhesus monkeys. Second, these results provide pharmacokinetic evidence that the heroin vaccine is sequestering 6-acetylmorphine in the plasma. In summary, these results support further preclinical research evaluating the effects of a heroin-tetanus toxoid conjugate vaccine as a candidate immunopharmacotherapy for heroin addiction.

Keywords: Heroin, Nonhuman Primates, Pharmacokinetics, Behavioral Pharmacology.

Disclosure: Nothing to disclose.

\section{T233. Dopaminergic Dynamics Underlying Sex-Specific Cocaine Reward Processing}

Erin Calipari*, Barbara Juarez, Carole Morel, Deena Walker, Efrain Riberio, Charu Ramakrishnan, Karl Deisseroth, Ming-Hu Han, Eric Nestler

Icahn School of Medicine at Mount Sinai, New York, New York, United States

Background: Drug addiction is a debilitating neuropsychiatric disorder characterized by high levels of drug intake, drug seeking and repeated cycles of abstinence and relapse. While both males and females become addicted to cocaine, females transition to dependence faster, take more cocaine, experience more adverse consequences and have more difficulty remaining abstinent in both humans and animal models. The underlying neural mechanisms controlling these sexualdimorphisms in motivated behaviors and how they mediate the increased abuse liability of cocaine in females remain unclear. There are several reports of menstrual-cycle dependent fluctuations in cocaine craving, suggesting that estrous-cycle effects may be involved. However, most of these investigations used ovariectomized females with hormone replacement to observe these effects, ablating the natural fluctuations in hormones observed with the estrous cycle. Here, we define a sex-specific neural mechanism contributing to this enhanced cocaine reward in females.

Methods: Using a combination of in vivo electrophysiology of ventral tegmental area (VTA) dopamine neurons and fast scan cyclic voltammetry in the nucleus accumbens (NAc), in vivo calcium imaging and conditioned place preference, we were able to record pathway specific VTA and NAc responses to contextual cues that were previously paired with cocaine in male mice and in intact females over different stages of the estrous cycle. Using ELISAs we measured circulating levels of ovarian hormones and determined their relationship to estrous cycle dependent fluctuations in reward processing. Finally using designer receptors exclusively activated by designer drugs (DREADDs) we manipulated mesolimbic dopamine system activity to directly link downstream alterations in dopamine transporter function to activity dependent changes at the level of the VTA.

Results: Here we identified an estrous-cycle dependent enhancement of dopaminergic function in both the VTA and NAc, which acts to increase dopaminergic responses to 
salient stimuli. This enhancement is converged with an increased ability of cocaine to augment synaptic dopamine levels due to a robust increase in cocaine's ability to bind directly to the dopamine transporter and inhibit its uptake function selectively during estrus. These changes were linked to alterations in circulating estradiol levels, which predicted changes in both VTA firing rate as well as cocaine effects at the dopamine transporter. Using DREADD-induced activation of VTA dopamine neurons to enhance firing we were able to mimic the effects of estrous cycle on the dopamine system and subsequent reward processing in both diestrus females and males. Importantly, these changes lead to not only enhanced reward processing during estrus, but potent and long-lasting associations between the rewarding effects of cocaine and associated environmental cues that extend to other stages of the estrous cycle. Females that were exposed to cocaine during estrus had enhanced VTA and NAc responses to cocaine-associated cues, as compared to males and females in diestrus, that were correlated with drug seeking. These enhanced responses to environmental cues, in the absence of drug, occur even at later stages in the estrus cycle, and acts to enhance motivation and drug seeking.

Conclusions: Together we define a basic mechanism by which estrus-cycle dependent changes in the dopamine transporter underlies susceptibility to cocaine addiction by promoting persistent cue-reward associations that can precipitate relapse. Since enhanced drug seeking is characteristic of individuals who transition to addiction, our findings which reveal novel sex-specific factors controlling drug seeking are critical to guiding evidence-based therapeutic interventions for female addicts.

Keywords: Cocaine Seeking, Dopamine, Calcium Imaging, Sex Differences, Reward Processing.

Disclosure: Nothing to disclose.

\section{T234. Inhalation Self-Administration of Addictive Drugs via E-Cigarette Technology in Rats}

Michael Taffe*, Sophia Vandewater, Jacques Nguyen, Maury Cole

The Scripps Research Institute, La Jolla, California, United States

Background: Addiction to psychostimulants such as methamphetamine (MA) or cocaine, as well as to cannabis and other drugs interferes with many aspects of personal health, vocational performance, interpersonal relationships and financial well-being. Operant intravenous selfadministration models in experimental species have proven to be highly effective in many investigations into the effects of, and treatment for, drugs of abuse. Studies have shown that smoked MA leads to a similar subjective high at lower plasma exposure, and a more rapid diminution of that high, relative to i.v. or intranasal administration in humans. Despite higher rates of treatment admissions for smoked versus other routes of cocaine administration, increasing proportions of inhalation MA users and a majority of cannabis users who smoke, there are no convenient models of inhalation self-administration available for rodent research.
Methods: We have developed an e-cigarette based technology that can deliver physiologically and behaviorally relevant doses of psychostimulants and $\Delta 9$-tetrahydrocannabinol (THC) to rats via vapor inhalation within a sealed homecage style chamber. The present study determined if inhaled MA, 3,4-methylenedioxymethamphetamine (MDMA), 3,4methylenedioxypyrovalerone (MDPV) or THC serve to reinforce operant behavior. Groups of rats were trained to nose poke for 2-min epochs of exposure to vaporized drug in a propylene glycol (PG) vehicle in $1 \mathrm{~h}$ sessions. Initial response requirements of FR1 were gradually incremented to FR5.

Results: Stable or gradually increasing numbers of stimulant vapor epochs were obtained. Responses on the drugassociated hole gradually increased relative to the alternate hole; $89 \%$ selectivity was observed in some groups. Pretreatment with the dopamine D1-like receptor antagonist SCH23390 decreased responding for MA vapor and dose related changes in drug deliveries were observed for THC.

Conclusions: This study shows that rats will make nose poke responses to obtain access to vaporized drugs. This establishes a new model of intrapulmonary self-administration of drugs in rats which uses a technology (e-cigarette) that is increasingly being adapted in human users for non-nicotine drug use.

Keywords: Self-Administration, Cannabis Dependence, Methamphetamine, Electronic Cigarette (E-Cigarette).

Disclosure: La Jolla Alcohol Research, Inc.: Company, Self.

\section{T235. New BAC-Derived Transgenic Models for Studies of Alpha-5 Nicotinic Acetylcholine Receptor Subunit Function}

Nailyam Nasirova, Lely Quina, Evelyn Lambe, Eric Turner*

University of Washington School of Medicine, Seattle, Washington, United States

Background: The alpha- 5 nicotinic receptor, encoded by the Chrna5 gene, is a non-channel forming subunit of the pentameric nicotinic receptor that has been shown to alter channel properties (Kuryatov et al, 2008; Bailey et al, 2010). Chrna5 is co-located with two related genes, Chrna3 and Chrnb4, in the mouse and human genome. However, in the rodent CNS, Chrna5 mRNA is generally not co-expressed with that of Chrna3/b4, perhaps because Chrna5 and Chrna3/b4 are transcribed on opposite chromosomal strands, and thus may be independently regulated. The Chrna5/a3/b4 gene cluster is of special interest because markers at this locus and a Chrna5 coding polymorphism have been associated with smoking (Berrettini and Doyle, 2012). Chrna5 is expressed in brain areas that are implicated in drug reinforcement, such as the prefrontal cortex and ventral tegmental area, and in the interpeduncular nucleus, which has been shown to mediate nicotine withdrawal symptoms. However, the mechanism by which Chrna5 and its polymorphisms affect behavioral responses to nicotine remain largely unknown. Generally, reporter genes integrated into BAC constructs disrupt the expression of the native gene. However, this is confounded in the case of the co-localized Chnra5/a3/b4 genes, since the available BACs 
contain all of the genes in the cluster, leading to overexpression of the untargeted genes. In prior work, we have employed a Chrna5-GFP BAC transgenic to mark Chrna5expressing neurons for gene expression studies (Hsu et al, 2013), and related BAC transgenics have been used in other studies (Frahm et al, 2011). In the current work we have reengineered a Chrna5 BAC-cre transgene (Chrna5[delta] a3b4Cre) in order to map the regulatory elements of the locus and create a suitable model for functional studies.

Methods: To generate the Chrna5[delta]a3b4Cre transgene, an existing Chrna5 BAC integrant containing a crerecombinase expression cassette was obtained from the GENSAT project. BAC recombineering was employed to excise the Chrna3 and Chrnb4 genes from the BAC vector. The resulting construct containing 20kb of Chrna5 upstream regulatory sequence was transferred to a plasmid vector and injected into mouse oocytes to generate three transgenic lines. In order to assess the extent of cre-recombinase expression in Chrna5[delta]a3b4Cre transgenic mice, founders were crossed with a reporter strain B6.Cg-Gt(ROSA) 26Sortm6(CAG-ZsGreen1)Hze/J (Ai6, gift of Hongkui Zeng, Allen Institute for Brain Science), which expresses the fluorescent reporter ZsGreen in the presence of cre. To identify the specific efferent projections of cortical neurons that express Chrna5-cre, the prefrontal (prelimbic + infralimbic) cortex of adult mice was injected with a mixture of color-contrasting AAV reporter viruses activated or inactivated by cre.

Results: The transgenic reporter expression pattern induced by Chrna5[delta]a3b4Cre was compared with the expression of Chrna5 mRNA and a previously described Chrna5GFP reporter containing the intact Chrna5/a3/b4 locus (Hsu et al, 2013). Only one of three lines mediated expression in layer 6 of the cortex, in a pattern strongly resembling endogenous mRNA expression. In contrast, all three lines expressed correctly in the interpeduncular nucleus (IP). Immunostaining of the IP for afferent medial habenula (MHb) fibers shows that Chrna5[delta]a3b4Cre marks a population of IP neurons that receive cholinergic input from the ventral $\mathrm{MHb}$, but not peptidergic input from the dorsal MHb. Two Chrna5 [delta]a3b4Cre lines mediated expression in the habenula itself, but the variable pattern of expression did not correlate with the expression of Chrna3/b4 mRNA observed there, nor with the expression of the ventral $\mathrm{MHb}$ cholinergic marker ChAT. In contrast, the Chrna5[delta]a3b4Cre lines showed very sparse expression in the ventral tegmental area (VTA). Immunostaining for tyrosine hydroxylase, a marker of dopaminergic neurons, showed rare co-localization with Chrna5[delta]a3b4Cre-driven reporter expression. Thus Chrna5[delta]a3b4Cre does not reproduce the endogenous pattern of Chrna5 mRNA expression in the VTA. Local injection of adeno-associated virus (AAV) was then used to trace efferent fibers of Chrna5[delta]a3b4Cre in the prefrontal (prelimbic + infralimbic) cortex of adult mice. The availability of AAV reporter strains both activated and silenced by cre-recombinase in contrasting colors allowed specific mapping of efferent fibers of Chrna5[delta]a3b4Cre, transgenic line 3. Efferent fiber terminals of Chrna5 neurons were located in the lateral hypothalamus, anteromedial thalamus, amygdala, VTA, and paramedian raphe. In contrast, labeled fibers were sparse in the dorsal and ventral striatum.
Conclusions: We conclude that regulation of the mouse Chrna5 gene locus is discrete from the adjacent Chrna3/ Chrnb4 loci. Gene regulatory elements that govern Chrna5 expression in layer 6 of the cerebral cortex and the IP, but not the VTA, lie within $(20 \mathrm{~kb})$ of the Chrna5 transcriptional start site. While Chrna5[delta]a3b4Cre gives only a partial replication Chrna5 mRNA expression in the CNS, specific expression in cortical layer 6 and the IP may be very useful for isolating pre- and post-synaptic roles of neurons expressing Chrna5. Chrna5-cre expressing neurons project strongly to areas associated with the regulation of affective states and reinforcement. Chrna5[delta]a3b4Cre mice thus provide a useful model for physiological, behavioral, and optogenetic studies of the function of the alpha-5 nicotinic receptor subunit, and the subset of cortical and tegmental neurons which express this receptor.

Keywords: Interpeduncular Nucleus, Habenula, Nicotine, Prefrontal Cortex, Nicotinic Acetylcholine Receptors.

Disclosure: Nothing to disclose.

\section{T236. A Genome Wide Association Study Reveals Association of Genetic Markers With Length of Abstinence in Alcoholics Treated With Acamprosate}

Victor Karpyak*, Jennifer Geske, Man Choi (Ada) Ho, Julie Cunningham, Antony Batzler, Daniel Hall-Flavin, Terry Schneekloth, Mark Frye, Richard Weinshilboum, Doo-Sup Choi, Joanna Biernacka

\section{Mayo Clinic, Rochester, Minnesota, United States}

Background: Acamprosate supports abstinence in some alcoholics and is believed to counteract the "relief craving" associated with increased brain glutamate levels. Yet not every patient responds to acamprosate treatment and predictors of response are unknown. We previously investigated associations between acamprosate response and genetic variation in pathways responsible for glycine and glutamate neurotransmission, and found that abstinence length in acamprosate-treated alcoholics is associated with the GRIN2B rs2058878 variant. Here we present results of the first genome wide association study (GWAS) searching for genetic markers associated with abstinence length in alcoholics treated with acamprosate.

Methods: Illumina HumanCore genotyping array was used to genotype DNA samples collected from Illumina HumanCore genotyping array was used to genotype DNA samples collected from alcoholics treated with acamprosate in a single-arm study performed by the Mayo Clinic Center for Individualized Treatment of Addiction (CITA). Data from 297 subjects with 3-month treatment outcomes were used to perform a GWAS. Following quality control and imputation, over 5 million single nucleotide polymorphisms (SNPs) with minor allele frequency $\geq 0.05$ were analyzed. Cox proportional hazard models were used to test for association of genotype with the length of abstinence (time to first alcohol use measured by Time Line Follow Back) during the first 3 months of acamprosate treatment. Statistical models were adjusted for covariates previously found to be associated with treatment outcome in the study sample, including: number of sober days between the last drink and initiation of acamprosate, craving score at baseline (measured by Penn 
Alcohol Craving Scale) and recruitment site. Top association signals were queried in databases such as GTEx and Braineac to identify potential expression quantitative loci (eQTLs).

Results: Shorter abstinence was found to be associated with the minor allele of $r s 140982243(p=1.01 \times 10-8)$; this SNP lies in a gene-rich region on chromosome 19, which includes the genes GTF2F1 (encoding subunit 1 of a general transcription initiation factor that binds to RNA polymerase II and helps to recruit it to the initiation complex) and PSPN (encoding a neurotrophic factor, belonging to the GDNF family known to promote survival of ventral midbrain dopaminergic neurons). Other top association findings include rs72819704 $(p=9.5 \times 10-7)$ located in a gene rich region on chromosome 17 that includes PCGF2 (encoding transcriptional repressor polycomb group ring finger 2 , that may play a role in neural cell proliferation and development). eQTL analysis suggests an association between rs72819704 and PCGF2 expression in the frontal cortex $(p=0.0016)$.

Conclusions: Our findings indicate that abstinence length in acamprosate-treated alcoholics may be associated with sequence variation in genes encoding proteins involved in neural cells function, development and survival. Future research should focus on replication of these findings and investigation of the potential functional role of the identified variants.

Keywords: Alcohol Use Disorders, Pharmacogenetic Response, Genome-Wide Association Studies.

Disclosure: Nothing to disclose.

\section{T237. Animal Modeling Polysubstance Addiction} Vulnerability in Mental Illness: Concurrent Alcohol and Nicotine Use in Rats With Neonatal Ventral Hippocampal Lesions

Robert Chambers*, Alena Sentir, Richard Bell, Eric Engleman

Indiana University School of Medicine, Indianapolis, Indiana, United States

Background: Substance use disorders, particularly those involving multiple substances are common in patients with major mental illnesses, such as schizophrenia. Building preclinical animal models of these poly-comorbidities will be important to advancing our neuroscientific understanding of these complex, real-world clinical conditions.

Methods: A widely studied and well-validated neurodevelopmental rat model of schizophrenia (produced by neonatal ventral hippocampal lesions (NVHL)) was studied in comparison to healthy (SHAM-operated) controls in adult self-administration of ethanol and nicotine consumed concurrently. To encourage concurrent oral ethanol with iv. nicotine self-administration in adulthood, all rats were first given 1 hour daily, limited free access to a 19-day sequence of a cross-tapering sucrose and ethanol solution from mid to late adolescence (Post-natal days 35-63). Adult rats aged 64 days were then allowed to acquire lever pressing for oral access to the final ethanol solution ( $2 \%$ sucrose/ $10 \%$ ethanol) concurrently with access to i.v. nicotine $(0.015 \mathrm{mg} /$ $\mathrm{kg} / \mathrm{inj}$ ) over 15 acquisition sessions spanning 3 weeks. Thereafter, rats were tested in 2 sets of 5 extinction sessions, where in the first set, access to nicotine was denied, but alcohol continued. For the second set, access to both drugs was denied. Finally, all rats proceeded through 3 reinstatement sessions where the effects of nicotine re-exposure and alcohol access on bar pressing was examined.

Results: NVHL and SHAM rats did not differ in amounts of free access ethanol solution consumption during adolescence. However, in adulthood, self- administration via instrumental responding for oral ethanol solution and iv. nicotine was greater in NVHL compared to SHAM rats. During acquisition, NVHL-based differences in bar pressing for drug delivery generally increased across sessions and was most strongly expressed for nicotine compared to alcohol, even as the largest fraction of overall bar pressing by both groups of rats were directed at the ethanol lever. Lesionbased differences in bar pressing was also more robust for hits that actually delivered the drug as opposed to time-out responses (when drug was not available) or hits on the blank (non-drug paired lever). In subsequent extinction sessions, rats showed expected extinction patterns in responding specific to each drug-paired lever as first nicotine then both drugs were withheld. However, NVHL rats persisted in showing greater drug-seeking on both the ethanol and nicotine-paired levers across these sessions. In reinstatement, all rats showed increased lever responding upon the reintroduction of ethanol access, while NVHL rats persisted in responding significantly more on the nicotine paired lever. Conclusions: This is the first demonstration of concurrent multi-substance use vulnerability in a previously well validated animal model of mental illness. Neurodevelopmental ventral hippocampal abnormalities of the ventral hippocampus may thus set up the brain not only for mental illness but single and polysubstance addictions. Extending this line of preclinical modeling in future research may inform improved, integrative prevention and treatment strategies for complex but common real world behavioral health comorbidities.

Keywords: Nicotine Addiction, Alcohol Dependence, Schizophrenia.

Disclosure: Nothing to disclose.

T238. Pain Interference and Psychological Wellbeing in Patients Receiving Medication Assisted Therapy for Opioid Treatment (MAT)

Tanya Alim*, Peggy Compton, Kathryn Cavallo, Beverlyn Settles-Reaves

Howard University Hospital, White Plains, Maryland, United States

Background: The prevalence of chronic pain in patients on medication assisted therapy (MAT) for opioid treatment is high; rates have been estimated to range from $55-60 \%$ (Eyler. 2013; Voon et al, 2015), compared with $31 \%$ in the general population. The literature is sparse utilizing the multidimensional McGill Pain Questionnaire (MPQ) to assess pain in patient receiving MAT. We report on a small sample of patients receiving MAT with chronic neuropathic pain $(n=22)$, examining the somatic and affective qualities of their pain, as well as the degree to which their pain interferes with life activities. Cold-pressor pain responses were 
available on a subset of subjects $(n=13)$, and pain-related correlates of responses are described.

Methods: As part of screening for a larger study, 22 participants were included were between the ages of 18-62 (15 male and 7 female), stable in MAT and reported an active chronic pain condition. Data was collected during a single session using both interview and self-report measures. In addition to demographics and drug use history, qualities of the pain were assessed using the McGill Pain Questionnaire (MPQ) and pain interference captured with the Brief Pain Inventory (BPI). A random subsample of 13 subjects (3 female) underwent a single standardized cold-pressor trial with pain threshold and tolerance measured.

Results: The sample was older (mean age $=56 \mathrm{yr}, \mathrm{SD}=$ 4.69). On average, self-reported illicit drug use was low; days of heroin, cocaine and marijuana use were less than one in the past month, although urine toxicology found 3 of the subjects positive for amphetamine, 7 positive for cocaine, 4 positive for THC, 4 positive for methamphetamine and 4 positive for opioids other than MAT opioid. On a scale of 110 , with 10 being worst, subjects rated their average pain at $6.1(\mathrm{SD}=2.19)$, with average worst pain rated at 6.7 and least at 4.9. Sleep $(x=5.8, S D=3.28)$ and walking $(x=5.8$, $\mathrm{SD}=2.98$ ) appeared to be the life domains with which the pain interfered the most, followed by enjoyment with life $(x=5.3, S D=3.16)$, overall activity $(x=5.1, S D=2.76)$, and work $(x=5.0, S D=3.14)$. Pain interfered least with mood $(\mathrm{x}=4.8, \mathrm{SD}=3.03)$ and the ability to relax $(\mathrm{x}=3.2, \mathrm{SD}=$ (3.36). The mean total somatic score on the MPQ was 17 $(\mathrm{SD}=8.07)$ and affective score, 3.65 (SD 3.59). As anticipated, subjects appeared hyperalgesic on cold-pressor pain testing with mean pain threshold of $14.05 \mathrm{sec}(\mathrm{SD}=7.51)$ and pain tolerance of $24.20 \mathrm{sec}(\mathrm{SD}=11.29)$. Cold-pressor pain tolerance was negatively and significantly correlated with pain interference with mood, ability to relax and ability to enjoy life.

Conclusions: In this sample of patients on MAT, severity of chronic pain was reported as relatively high, and with a greater somatic than affective component. Significant pain interference was noted in multiple life domains, and poor tolerance for pain predicted greatest pain interference with psychological well-being, including enjoyment, mood and ability to relax.

Keywords: Opioid, Chronic Pain, Psychiatric Comorbidity. Disclosure: Nothing to disclose.

\section{T239. Effect of Dreadd-Mediated Modulation of Gq-Coupled Signaling in Lateral Habenula Neurons on Cue-Induced Reinstatement of Cocaine Seeking in Rats}

Sunila Nair*, Jonathan Browne, Melissa Estabrook, John Neumaier

University of Washington, Seattle, Washington, United States

Background: The lateral habenula ( $\mathrm{LHb}$ ), an epithalamic nucleus located in the dorsal diencephalon is an important regulator of midbrain dopaminergic systems that are known to be involved in the reinforcing properties of cocaine. We previously determined that DREADD-induced activation of $\mathrm{Gi} /$ o-coupled signaling in LHb neurons increases operant cocaine self-administration, but decreases cocaine-priming induced reinstatement of cocaine seeking. In contrast, we found that activation of Gq-coupled signaling in $\mathrm{LHb}$ neurons decreases both operant cocaine self-administration and cocaine-induced reinstatement of lever responding. Here, we further examined the effect of DREADD (hM3Dq)-induced transient activation of Gq-coupled signaling in $\mathrm{LHb}$ neurons on reinstatement of cocaine seeking induced by contingent tone and light cues.

Methods: Firstly, male Long-Evans rats were injected with adeno-associated virus expressing hM3Dq into the $\mathrm{LHb}$ and were implanted with jugular venous catheters. Approximately two weeks after viral infusions, rats were trained to self-administer cocaine $(0.75 \mathrm{mg} / \mathrm{kg} /$ infusion $)$ on a fixed ratio 1 reinforcement schedule in the presence of contingent tone-light cues and the operant response was subsequently extinguished. Following extinction, rats were re-exposed to contingent tone and light cues in the presence or absence of clozapine-N-oxide (CNO) ( 1 and $3 \mathrm{mg} / \mathrm{kg}$ ), the inert ligand for DREADD's to determine the effect of activation of Gqcoupled signaling in $\mathrm{LHb}$ neurons on cue-induced reinstatement of cocaine seeking. Secondly, a distinct cohort of rats was infused with viral vectors as described above and trained for five days (two trials/day) on a rotarod. Rats were then injected with vehicle or CNO (1 and $3 \mathrm{mg} / \mathrm{kg}$ ) to determine the effect of activation of hM3Dq in the LHb on motor coordination.

Results: Our results indicate that activation of $\mathrm{hM} 3 \mathrm{Dq}$ in LHb neurons CNO (3 mg/kg, i.p) has no effect on cueinduced reinstatement of cocaine seeking. Further, CNO (1 and $3 \mathrm{mg} / \mathrm{kg}$ i.p) - induced activation of hM3Dq in the LHb had no effect on motor co-ordination in the rotarod test.

Conclusions: Taken together, our results suggest that DREADD-mediated transient activation of Gq-coupled signaling in LHb neurons selectively decreases cocaine priming-induced reinstatement of cocaine seeking and that this effect is not due to a deficit in motor co-ordination in rats.

Keywords: Cocaine Seeking, Relapse, Lateral Habenula, DREADDs.

Disclosure: Nothing to disclose.

\section{T240. Modafinil for the Treatment of Cocaine Use Disorder; Follow-Up Results}

\author{
Kyle Kampman*, Kevin Lynch, Charles O'Brien
}

University of Pennsylvania, Philadelphia, Pennsylvania, United States

Background: Modafinil is a medication approved for narcolepsy and shift work sleep disorder. It has both dopaminergic and glutamatergic activity that could be useful for the treatment of cocaine dependence. Modafinil has reduced cocaine subjective effects in human laboratory trials, reduced cocaine self-administration in human laboratory trials and has reduced cocaine use in cocaine dependent patients in some clinical trials. In a previously reported clinical trial of modafinil for the treatment of cocaine dependence, $23 \%$ of modafinil-treated subjects maintained abstinence from cocaine for the last 3 weeks of an 8-week trial compared to $9 \%$ of placebo-treated subjects. The current 
study evaluated the durability of treatment response in modafinil and placebo responders 1 and 4 months after trial completion.

Methods: This was an 8-week, double blind, placebo controlled parallel group clinical trial involving 94 DSM IV cocaine dependent subjects without concurrent alcohol dependence. Subjects received $300 \mathrm{mg}$ of modafinil or identical placebo each day along with weekly individual cognitive behavioral relapse prevention psychotherapy. The primary outcome measure was cocaine use measured by selfreport, and confirmed by twice weekly urine drug screens. At 1 and 4 months after the end of the medication phase of the trial, subjects returned for a follow up visit. At each follow up visit, a urine drug screen was obtained; the Addiction Severity Index (ASI) and the Clinical Global Impression Scale (CGI) were administered.

Results: During the medication phase of the trial, the odds ratio (OR) in favor of abstinence for modafinil vs. placebo was $2.28(p=.038)$ and modafinil-treated subjects were significantly more likely than placebo-treated subjects to be abstinent from cocaine during the last 3 weeks of the trial, $23 \%$ vs. $9 \%, \chi^{2}=3.9, p<.05$. Modafinil-treated subjects were also more likely than placebo-treated subjects to rate themselves as "very much improved" on the CGI $(\mathrm{OR}=$ $2.15, p=.02$ ). There was no significant difference between the modafinil-treated subjects and the placebo-treated subjects in ASI Composite Scores in any of the 7 ASI domains at 1 or 4 month follow up. There was also no difference in CGI scores between the two groups at the 1 or 4 month follow up. Nearly half $(45 \%)$ of the modafiniltreated subjects who achieved three weeks of abstinence at the end of the medication phase of the trial maintained abstinence from cocaine at the 1 and 4 month follow up. None of the placebo-treated subjects abstinent at the end of the medication phase of the sustained abstinence at the 1 and 4 month follow up.

Conclusions: In this trial, modafinil treatment was associated with reduced cocaine use, and greater self-reported and observer-reported overall improvement in cocaine dependent subjects during the medication phase. During the follow up phase, a significant proportion of modafinil-treated subjects who achieved end of medication phase abstinence from cocaine were able to maintain abstinence.

Keywords: Cocaine Addiction, CNS Clinical Trials, Pharmacotherapy, Substance Abuse.

Disclosure: Indivior: Grant Support, Consultant, Self; Opiant: Consultant, Self; Alkermes: Grant Support, Self; Braeburn: Grant Support, Self.

\section{T241. Individual Variation in Model-Free and Model-Based Reinforcement Learning and Methamphetamine-Taking Behavior in Rats}

Stephanie Groman*, Bart Massi, Daeyeol Lee, Jane Taylor

\section{Yale University, New Haven, Connecticut, United States}

Background: The emergence and persistence of drug taking behaviors may be due to disruptions in reward-guided learning. In particular, stimulant-dependent individuals are impaired in their decision-making behaviors. Recent studies, using a multi-stage decision making task, have suggested that the disruptions in goal-directed behavior might be due to dysfunction in prospective, or model-based, learning and not in retrospective, or model-free, learning. However, it is unclear if model-based deficits are a cause or a consequence of chronic drug use.

Methods: To determine how model-free and model-based learning systems are involved in the process of addiction, we examined decision-making processes in rats using a novel multi-stage decision making (MSDM) task before and after rats self-administered methamphetamine. Twenty male, Long Evans rats were trained on the MSDM task and then they self-administered methamphetamine in 6-hour daily sessions for 14 days. Following one week of forced abstinence from methamphetamine, performance on the MSDM was reassessed and subsequently brain tissue was collected for biochemical analyses.

Results: Prior to drug exposure, there were marked individual differences in the degree to which rats used model-free and/or model-based learning strategies on the MSDM that paralleled those observed in humans. Furthermore, individual differences in model-free learning, but not model-based learning, predicted future methamphetaminetaking behaviors $(\mathrm{R}=0.56 ; p=0.02)$ : rats with greater modelfree learning took less methamphetamine than those rats with poorer model-free learning. Methamphetamine selfadministration significantly disrupted model-free learning $(p=0.006)$ with a similar trend for model-based learning $(p=0.12)$.

Conclusions: These data suggest that chronic exposure to drugs of abuse impairs both model-free and model-based learning and that individual differences in model-free processes might be a behavioral marker of vulnerability for drug use. Ongoing biochemical analyses will determine the role of different neurotransmitter systems in model-free and model-based learning strategies to identify novel pharmacological targets for the prevention and treatment of addiction. Keywords: Addiction, Methamphetamine, Reward-Based Decision-Making, Computational Neuroscience, ModelFree And Model-Based Learning.

Disclosure: Nothing to disclose.

T242. Childhood Trauma as a Premorbid Marker of
Orbitofrontal Cortex Structural Integrity in Cocaine
Addiction, Beyond Drug Use and Demographic Effects

Keren Bachi*, Muhammad A. Parvaz, Gabriela Gan, Scott J Moeller, Rita Z Goldstein, Nelly Alia-Klein

Icahn School of Medicine at Mount Sinai, New York, New York, United States

Background: Adverse childhood experiences are major stressors during development that may affect vulnerability to addiction. As a potential premorbid marker, adverse childhood experiences may be associated with aberrant brain development, potentially contributing to the reductions in gray matter integrity in the prefrontal cortex observed in individuals with cocaine use disorders (iCUD). Since iCUD report disproportionately more adverse childhood experiences, we hypothesized that childhood stressors contribute to reduced structural integrity of the prefrontal cortex above 
and beyond contributing drug effects and demographics. The association between adverse childhood experiences and structural morphological abnormalities in iCUD can help identify biomarkers that could predispose individuals to developing addiction and enhance prevention and treatment. Methods: All 76 participants completed the Childhood Trauma Questionnaire (CTQ; a standardized self-report inventory that measures emotional, physical, and sexual abuse, and emotional and physical neglect), and regional gray matter concentration (GMC) was obtained using voxelbased morphometry in SPM on T1-weighted magnetic resonance imaging scan. We compared adverse childhood experiences and brain structural integrity between 29 healthy controls with low childhood trauma (CON; CTQ mean, SD: $34.2 \pm 5.1$ ), 23 iCUD with low childhood trauma (CUD-L: $33.2 \pm 5.4$ ), and 24 iCUD with high childhood trauma (CUD$\mathrm{H}: 57.9 \pm 11.1)$; low versus high childhood trauma was determined based on a median split $(=43)$ of the total CTQ score among all iCUD. The three groups were matched on gender (men; CON: 20; CUD-L: 20; CUD-H: 18), race (black; CON: 22; CUD-L: 19; CUD-H: 16), and age (CON: $41.9 \pm 7.9$; CUD-L: 47.0 \pm 7.9 ; CUD-H: $45.8 \pm 7.8$ ), but differed significantly in education $(\mathrm{CON}>\mathrm{CUD}-\mathrm{L}=\mathrm{CUD}-\mathrm{H}$, $p<.001$ ), used as a covariate in all analyses. We also inspected selected lifetime drug use (in years) comprised of cocaine (CON: NA; CUD-L: 18.5 \pm 8.8 ; CUD-H: 16.8 \pm 9.5 ), alcohol (CON: 9.3 \pm 10.1 ; CUD-L: 14.8 \pm 12.1 ; CUD-H: $17.3 \pm 10.1$ ), and cannabis (CON: $.6 \pm 2.1$; CUD-L: $5.9 \pm 7.5$; CUD-H: $10.3 \pm 12.2$ ). Whole-brain group comparisons controlled for education, age, and total intracranial volume using height threshold puncorr $<.01$ and extent threshold of $\mathrm{k}>100$ voxels. False discovery rate correction for multiple comparisons at the cluster level was applied $(\mathrm{pFDR}<.05)$. Multiple regression in SPSS was then used to evaluate the contribution of childhood trauma to GMC variance, beyond demographics and select drug use variables.

Results: A main group effect was observed in the right lateral orbitofrontal cortex (OFC) (883 voxels; peak-voxel: $\mathrm{x}=29$, $\mathrm{y}=47, \mathrm{z}=-14$, cluster-level pFDR-corr $=.02)$. Follow-up whole-brain comparisons showed significantly reduced GMC in CUD-H as compared to CUD-L (1537 voxels: $\mathrm{x}=30, \mathrm{y}=48, \mathrm{z}=-12$; cluster-level $\mathrm{pFWE}$-corr $=.035)$ and CON (15097 voxels: $\mathrm{x}=39, \mathrm{y}=41, \mathrm{z}=-15$; cluster-level pFWE-corr <.001). The CUD-L and CON groups did not differ. The hierarchical regression (where GMC in the right lateral OFC was the dependent variable and two hierarchical blocks were tested including, first: age, education, total intracranial volume, lifetime drug use, and second: the CTQ scores) revealed that GMC in the right lateral OFC was not significantly affected by the demographic and drug use variables (first block $p=.839$ ), while the CTQ scores (second block) accounted for $48.4 \%$ of variance in this dependent variable in the CUD groups $(\mathrm{F}=31.8, p<.001)$.

Conclusions: Above and beyond effects of lifetime drug use or demographics, our results point to a unique and significant contribution of adverse childhood experiences, predating drug use, to structural integrity of the OFC in cocaine addiction, possibly due to a particular impact of childhood trauma and associated excessive environmental stress. As one of the last brain regions to fully develop in humans, the OFC may be particularly vulnerable to the effects of early stress, where exposure to even brief periods of intense stress is sufficient to cause significant structural remodeling within the rodent prefrontal cortex, and involve impairments in OFC-dependent cognitive functions via excessive stimulation of dopaminergic and noradrenergic receptors. Thus, these findings may have important clinical implications as they underscore a link between premorbid environmental stress and OFC GMC in addicted individuals, with a potential impact for developing individualized interventions and timely prevention efforts.

Keywords: Cocaine Addiction, Orbitofrontal Cortex, Childhood Trauma, Gray matter Concentration, Voxel-Based Morphometry.

Disclosure: Nothing to disclose.

\section{T243. Effects of TAAR 1 Agonists on Nicotine-Taking and -Seeking Behaviors in Male Rats}

\author{
Jianfeng Liu, Yanan Zhang, Jun-Xu Li*
}

State University of New York at Buffalo, Buffalo, New York, United States

Background: Trace amine-associated receptor 1 (TAAR1), a $G$ protein coupled receptor, modulates the dopaminergic system and dopamine related behaviors. Selective TAAR1 agonists could inhibit the rewarding effects of stimulants, e.g. methamphetamine and cocaine, reducing drug intake and relapse in animal drug addiction model. However, nothing is known about the role of TAAR1 in nicotine addiction or its underlying mechanism.

Methods: In the present study, we tested the effects of selective TAAR1 full agonist RO5166017 and partial agonist RO5263397 on nicotine-taking behavior (intravenous selfadministration) and nicotine-seeking behavior (reinstatement model). For nicotine taking behavior, rats were trained to self-administer nicotine $(0.003,0.01,0.03$, and $0.1 \mathrm{mg} / \mathrm{kg} /$ infusion) for $1 \mathrm{hr}$ per day under a fixed ratio 3 schedule of reinforcement followed by a 30 -s time-out period. Infusions were accompanied by a 5-s illumination of the stimulus light above the active lever and the house light was extinguished for the duration of the time-out period. For nicotine-seeking behavior, rats were trained for $0.03 \mathrm{mg} / \mathrm{kg} /$ infusion nicotine self-administration for 15 days in total. The response requirement was gradually increased from FR 1 to FR 3 over a period of 7 days, and then maintained for 8 days under FR 3. Rats were then given six daily extinction sessions, during which lever presses had no consequence (no drug or cues).

Results: Nicotine self-administration followed an inverted U-shaped pattern, with the peak number of injections (about 9-10 injections in $1 \mathrm{hr}$ ) maintained by $0.03 \mathrm{mg} / \mathrm{kg} /$ infusion. Pretreatment with RO5166017 (5.6 and $10 \mathrm{mg} / \mathrm{kg}$, i.p.) and RO5263397 (3.2 and $5.6 \mathrm{mg} / \mathrm{kg}$, i.p.) $10 \mathrm{~min}$ before nicotine self-administration each day dose-dependently decreased the dose-effect curve of nicotine injections and total nicotine intake. For the reinstatement of nicotine-seeking behavior, RO5166017 (10 mg/kg, i.p.) was administrated $10 \mathrm{~min}$ before cue- or drug $(0.3 \mathrm{mg} / \mathrm{kg}$ nicotine, i.p.)-induced reinstatement of nicotine-seeking. Both cue- and drug-induced reinstatement of nicotine-seeking were significantly inhibited by RO5166017. 
Conclusions: These results indicate that TAAR1 plays an essential role in nicotine-related behaviors, suggesting that TAAR 1 may represent a novel target for the treatment of nicotine addiction.

Keywords: Nicotine Addiction, Self-Administration, Reinstatement, Trace Amine-Associated Receptor 1.

Disclosure: Nothing to disclose.

\section{T244. Analysis of Astroglial Complexity at the Single Cell Level}

Michael Scofield*, Hao Li, Siemsen Ben, Kathryn Reissner, Peter Kalivas

Medical University of South Carolina, Charleston, South Carolina, United States

Background: A myriad of labs around the world investigate the morphological properties of neurons in different brain regions, often observing changes brought on by chemical insult, disease states, or exposure to drugs of abuse. To date, the state of the art level of analysis for the morphometric properties of neurons is of course the analysis measurement and classification of spines, individual synapses that protrude from a dendritic shaft. Indeed, using this level of analysis, the field has revealed dynamic changes in the amount, shape and size of these structures that have been functionally linked to biologically relevant events. A large body of work confirms the importance of quantifying alterations in the morphological properties of neurons at this single synapse level in an effort to gain a better understanding of synaptic plasticity. Understandably, this level of analysis of astrocytes, the nonneuronal cells responsible for ion buffering and glutamate uptake among other things, has not advanced quite as rapidly. Yet as studies continue to demonstrate that astroglia play an important in maintaining synaptic communication and in regulating synaptic plasticity, the study of astrocyte structure becomes increasingly important. Here we describe a set of methods used to analyze complexity of the labeled astrocytes at the single cell level. Moreover, we detail methods for analysis of the size and shape of the finest individual astroglial processes.

Methods: Using a viral vector that allows for membrane specific expression of GFP selectively in astrocytes, and confocal microscopy, we are able to isolate and image individual astrocyte cells. Then using software modules in Imaris, we create $3 \mathrm{D}$ models from our confocal datasets to determine both the area and volume of each cell. From this point, we generate filaments that accurately recapitulate the architecture of the astroglial plasma membrane in 3D. From these filaments we can obtain measures of astroglial morphological complexity including levels of branching and sholls intersections. In addition, using this technique we are also able to measure the diameter of the finest astroglial processes that interdigitate the synaptic machinery. Results: We have developed and characterized a simple workflow for evaluating the complexity of astrocytes using confocal microscopy and Bitplane Imaris software. 3D analysis of the astrocyte plasma membrane reveals that it far more complex than the GFAP filament skeletons, a signal that has been previously used as a metric for astroglial morphology and complexity. Moreover, using a previously published data set where cocaine exposure caused reduced volume and surface area of accumbens astrocytes, we show that the markers of astrocyte complexity discussed above were also reduced. Finally, we present a method for the measurement of the very fine astrocyte processes that insulate the pre and postsynapse at a level akin to what is the current state of the art for spines along a dendritic shaft. Conclusions: Analysis of glial morphological complexity as it relates to synaptic transmission is revealing alterations caused by exposure to drugs of abuse that could be easily missed if an analysis of branching or complexity of the more central skeletal GFAP filament is performed. Moreover, our data demonstrate that much like neurons, astrocytes are dynamic with a less static morphology than previously hypothesized. This supports the hypothesis that astrocytes actively alter levels of synaptic contacts in order to influence plasticity. Measurements made with these techniques may allow us to link changes in the size of astroglial processes to alterations in level of synaptic coverage, potentially providing insight to changes in glutamate uptake, ion buffering, trophic support and synaptic communication that accompany neuropsychiatric diseases.

Keywords: Astrocyte, Nucleus Accumbens, Computational Neuroscience.

Disclosure: Nothing to disclose.

\section{T245. Increased Striatal Dopamine Release Under Expectation of Alcohol as a Risk Biomarker for Alcohol Use Disorders: A Positron Emission Tomography Imaging Study With [11C]raclopride}

Lawrence Kegeles*, Guillermo Horga, Rassil Ghazzaoui, Rachel Rosengard, Xiaoyan Xu, Mark Slifstein, Ismene Petrakis, Stephanie O'Malley, John Krystal, Anissa Abi-Dargham

Columbia University, New York, New York, United States

Background: The dopaminergic response to alcohol challenge has been studied in humans but has not been compared to alcohol-cue related response in alcoholics and at-risk subjects. We used Positron Emission Tomography (PET) imaging with the $\mathrm{D} 2 / 3$ receptor radioligand [11C] raclopride to examine the effects of administering an alcohol or placebo beverage to subjects with alcoholism compared to healthy controls with and without family history of alcoholism.

Methods: We enrolled 65 participants in three groups: subjects with alcoholism (ALC, $n=15$ ) as well as control subjects who were family history negative (FHN, $n=34)$ or family history positive (FHP, $n=16$ ) for alcoholism. Subjects consumed a placebo $(n=65)$ or alcohol $(n=63)$ beverage prior to [11C]raclopride PET scanning $(n=128$ scans). Groups were matched for age and sex and were given the drinks in counterbalanced order. A linear mixed-effects model was used to compare binding potential BPND in the two drink conditions and the percent change $\triangle B P N D$ between conditions across striatal subregions. The effects of alcohol on BPND were assessed. Effects of group, drink order, and sex on BPND and $\triangle B P N D$ were evaluated. Subjective effects of beverage consumption were rated with the Subjective Effects of Alcohol Scale. 
Results: Alcohol resulted in greater dopamine release than placebo across all groups, most pronounced in ventral striatum (VST) $(p<.001)$. There were no main effects of group, drink order, or sex on VST BPND or $\triangle \mathrm{BPND}$, or sex by group interaction. However, there was a drink order by group interaction $(p=.02)$ whereby FHP subjects' $\Delta$ BPND response to the drink conditions was markedly different from the FHN and ALC groups. FHP subjects showed a much larger BPND reduction following placebo - nearly as great as following alcohol - when placebo was consumed first. This contrasted with FHN and ALC subjects, whose responses to placebo were substantially less than to alcohol in both drink orders. Subjective effects of alcohol showed a similar pattern.

Conclusions: FHP participants showed a distinct dopamine release (and subjective) response pattern: when they consumed the placebo drink first, VST DA release was essentially as great as alcohol-induced release. This hyperresponsivity of the dopaminergic system in FHP participants to placebo consumed first may represent an excessive response to alcohol-related cues and may constitute a risk factor for alcoholism. Further investigation is needed to determine whether the hyperresponsivity of the dopaminergic system to alcohol-related cues associated with family history is an independent risk factor for alcoholism.

Keywords: PET Imaging, Alcohol Dependence, At-Risk, Biomarker.

Disclosure: Nothing to disclose.

T246. Pharmacogenetic Effects of DAT1 Variation on Aripiprazole Response Among Non-Treatment-Seeking Individuals With Alcohol Use Disorder

Joseph Schacht*, Konstanin Voronin, Patrick Randall, Raymond Anton

Medical University of South Carolina, Charleston, South Carolina, United States

Background: Dopamine signaling regulates many aspects of Alcohol Use Disorder (AUD). Alcohol cues and alcohol selfadministration both increase dopamine release in the ventral striatum in animal and human models of AUD. The dopamine transporter (DAT) is the primary mechanism for dopamine clearance in this region, and a 40-base-pair variable number tandem repeat (VNTR) polymorphism in the 3' untranslated region of the dopamine transporter gene (DAT1), whose most common allelic variants are 9 and 10 repeats, affects expression of the DAT protein, such that the 9-repeat (9R) allele is associated with lower DAT expression. This allele, which has also been associated with increased striatal response to reward, may thus engender greater synaptic dopamine concentration and prolonged dopamine effects after phasic release produced by alcohol cues or alcohol self-administration. The atypical antipsychotic aripiprazole has been investigated as a treatment for AUD, with the hope that its unique partial agonist property at dopamine receptors might allow it to stabilize dopamine tone among individuals with abnormally high or low dopamine tone as a function of their alcohol exposure and/ or genetic background. A large multisite clinical trial of aripiprazole found no effects on the primary drinking outcome (percent days abstinent), but reported positive drug effects on other outcomes, including drinks per drinking day and an alcohol biomarker, that were highly variable, suggesting the possibility of a pharmacogenetic interaction. The current study examined whether DAT1 VNTR genotype may moderate aripiprazole effects on laboratory-based AUD phenotypes.

Methods: Eighty-three non-treatment-seeking individuals with AUD (mean age $=27,76 \%$ male, 9.2 drinks per drinking day, with $57 \%$ heavy drinking days in the past 90 days) were randomized to receive aripiprazole (titrated to $15 \mathrm{mg}$ ) or placebo for eight days. Subjects were genotyped for the DAT1 VNTR (genotype frequencies: 9/9, 0.17; 9/10, 0.39; $10 / 10,0.44$ ) and completed an fMRI alcohol cue reactivity task on day 7 and an alcohol self-administration paradigm (bar lab) on day 8. In the bar lab, subjects were administered a priming dose of alcohol and were subsequently offered the choice to consume up to eight additional drinks. Primary outcomes analyzed were alcohol cue-elicited activation of the ventral striatum (anatomically defined) and the number of drinks individuals chose to consume in the bar lab.

Results: Subjects who carried the 9R allele were compared to 10R homozygotes. DAT1 genotype significantly moderated the effect of aripiprazole on both primary outcomes, such that aripiprazole, relative to placebo, reduced cue-elicited ventral striatal activation $(p=.02)$ and bar-lab drinking $(p=$ $.04)$ only among individuals who carried at least one copy of the $9 \mathrm{R}$ allele. Interestingly, there was some evidence that aripiprazole had the opposite effects on these outcomes among 10R homozygotes.

Conclusions: Collectively, these data suggest a novel pharmacogenetic interaction between variation at the DAT1 VNTR and aripiprazole response in AUD, such that individuals who carry the allele of this variant associated with lower DAT expression (and potentially greater synaptic dopamine accumulation or prolonged effects in the striatum) displayed reduced cue-elicited ventral striatal activation and reduced drinking with aripiprazole, presumably due to dopamine receptor blockade or stabilization. This further suggests that aripiprazole's partial agonist property may be beneficial among individuals predisposed to enhanced dopamine effects but ineffective or potentially deleterious among others; this mixed profile could account for previous negative clinical trial results. Further exploration of whether DAT1 genetic variation may predict aripiprazole effects among individuals with AUD is warranted.

Keywords: Atypical Antipsychotics, Dopamine Transporter, fMRI/Imaging Genetics, Pharmacogenetics, Aripiprazole.

Disclosure: Nothing to disclose.

T247. Chronic Extended Access (18-H) Intravenous Cocaine Self-Administration Procedure: Pattern of Cocaine Intake and Role of the Kappa Opioid Receptor

Marta Valenza*, Eduardo R Butelman, Mary Jeanne Kreek

Rockefeller University, New York, New York, United States

Background: Cocaine addiction is a chronic brain disease characterized by compulsive drug intake and dysregulation 
of brain reward and stress systems. Addicted patients typically undergo cycles of active cocaine use, abstinence and relapse. Few preclinical studies have modeled the natural longitudinal course of cocaine addiction and its molecular consequences. Furthermore, the precise role of exacerbated cocaine intake over time ("escalation") remains unclear. Identifications of changes in the pattern of cocaine intake during the development of addictive-like states may allow better treatments for vulnerable subjects. Extended access self-administration protocols are powerful methodologies to model the advanced stages of addiction, which may include changes in the daily pattern and amount of total intake. Several protocols have been published in literature; however, few have a duration of drug access greater than 12 hours per day, potentially resulting in limited construct validity. We therefore characterized the behavioral pattern of intake during our recently developed 18-hour intravenous cocaine self-administration protocol, analyzing behavioral difference between high- and low-intake rats, across chronic exposure (14 consecutive daily sessions). Moreover, since a promising pharmacological target to relieve distress during abstinence/ withdrawal, thus potentially preventing relapse, is the kappa opioid receptor (KOPr), we tested the effect of its blockade in relieving neuroendocrine signs of stress and depression, such as serum corticosterone and brain-derived neurotrophic factor in rats in early (30-hour) withdrawal from chronic cocaine self-administration and compared it to yokedsaline rats.

Methods: Adult male Sprague Dawley rats were implanted with a catheter in the jugular vein under isoflurane anesthesia. Upon recovery, rats were trained to press a lever to receive $0.5 \mathrm{mg} / \mathrm{kg}$ cocaine unit doses, associated with a light cue (FR1). Training sessions lasted 2h; rats then underwent a 14-day self-administration paradigm, in which they had free access to cocaine for 18 hours/day. Analysis of daily intake and hourly active lever presses during the 18-h self-administration procedure was performed. Yoked-saline rats were run concurrently and paired with cocaine rats, and received an equal number of infusions of physiological saline. Cocaine and yoked-saline rats were sacrificed 30 hours in withdrawal, their brains were dissected and brain regions were analyzed through custom microarray analysis as well as qPCR gene expression. An additional cohort was devoted to the serum CORT and BDNF quantification comparing cocaine-exposed and yoked-saline rats that received acute systemic administration of a selective short-acting kappa opioid receptor antagonist (LY2444296; 0, $3 \mathrm{mg} / \mathrm{kg}$ ). Data were analyzed with factorial or repeated measures two-way ANOVA, followed by the Student Neumann Keul's test for multiple comparisons. Significance was set at $p<0.05$.

Results: Over 14 days of 18 -h self-administration sessions, the hourly pattern of intake changed between the early sessions and the later ones. Thus during the first week of access rats showed greater intake during the first hours of each operant session, whereas during the second week of self-administration rats showed the greater intake during the last hours of the sessions. This change in behavior across days was particularly pronounced in high-intake rats, compared to low-intake rats. Additionally, rats in early withdrawal from chronic extended access cocaine selfadministration showed depressive and anxiety-like behavior compared to yoked-saline rats, as well as an overall reduction in circulating serum CORT. Administration of a selective short-acting kappa opioid receptor antagonist affected serum CORT level. Rats re-exposed to the self-administration sessions after 2 weeks of abstinence showed cocaine intake comparable to the last session before withdrawal. Of note, there was a significant positive correlation between rat cocaine intake during the last day of withdrawal and the first session of re-exposure.

Conclusions: Taken together, these results demonstrate that the pattern of self-infusions of cocaine changes over time during chronic extended access $18 \mathrm{~h}$ self-administration sessions, as well as a characteristic pattern of within-day circadian changes of self-exposure. A single dose of $\mathrm{KOPr}$ antagonist with "medication-like" duration of action prevented cocaine withdrawal-induced neuroendocrine changes. Further studies are needed to determine the effectiveness in preventing relapse-like and depressant-like or anxiety like behaviors in specific addictive diseases.

Keywords: Kappa Opioid Receptor, Cocaine Addiction, SelfAdministration, Withdrawal.

Disclosure: Nothing to disclose.

\section{T248. Investigating Endocannabinoid Metabolism in Stress-Related Psychiatric Illnesses: Neuroimaging Studies With the Novel FAAH Probe, [11C]-CURB}

Isabelle Boileau*, Stephen Kish, Bernard Le Foll, Belinda Williams, Esmaeil Mansouri, Rachel Tyndale, Don Richardson, Romina Mizrahi, Pablo M. Rusjan, Markus Heilig, Sylvain Houle, Junchao Tong

Centre for Addiction and Mental Health, Toronto, Canada

Background: Preclinical and brain imaging studies (of CB1 receptors levels) have implicated endocannabinoid signaling and status of Fatty Acid Amide Hydrolase (FAAH), the enzyme metabolizing the major endocannabinoid anandamide in stress-related neuropsychiatric conditions including substance use disorders (SUD) and post-traumatic-stress disorder (PTSD).

The major specific aim of our work was to provide the first in vivo measurement of FAAH levels in brain of individuals with SUD (cannabis and alcohol) and PTSD.

Methods: Subjects with PTSD $(n=7)$ and with cannabis (CUD) $(n=10)$ and alcohol use disorder (AUD) $(n=14)$ and matched controls completed [11C]CURB/PET scans with arterial sampling. Cannabis users were scanned after overnight abstinence and alcohol users during early ( $\sim 5$ days) and late abstinence (+3 weeks). An irreversible 2-tissue compartment model was used to assess FAAH levels $(\lambda \mathrm{k} 3)$ in MRI delineated regions of interest.

Results: Relative to healthy matched controls, early abstinence from cannabis and alcohol in CUD and AUD was associated with significantly lower FAAH ([11C]CURB $\lambda \mathrm{k} 3$ ) across all brain regions examined (CUD: $-14-17 \%$, AUD: $-14-19 \% ; p<0.05)$. Lower FAAH levels in AUD tended to recover after 3 weeks abstinence $(n=11)$. In contrast PTSD was associated marginally elevated FAAH ([11C]CURB $\lambda \mathrm{k} 3$ ). Cerebral blood flow (arterial spin labeling MRI) did not affect the quantification of $\lambda \mathrm{k} 3$. 
Conclusions: We report the first preliminary evidence that FAAH activity and presumably endocannabinoid tone are affected in stress-related neuropsychiatric conditions. These findings have implication for the translation of the FAAH modulation strategy to the clinic.

Keywords: Positron Emission Tomography Imaging, Endocannabinoids, Substance-Related Disorders, Post-Traumatic Stress Disorder.

Disclosure: Nothing to disclose.

\section{T249. Identification of Genetic Loci Influencing Vulnerability to Cocaine Addiction in Selectively Bred High Responder and Low Responder Rat Lines}

Zhifeng Zhou, Peter Blandino, Qiaoping Yuan, Pei-Hong Shen, Huda Akil, David Goldman*

National Institute on Alcohol Abuse and Alcoholism, Rockville, Maryland, United States

Background: High responder (bHR) and low responder (bLR) Sprague-Dawley rat lines were selectively bred for exploratory locomotion, a behavioral trait closely correlated with novelty-seeking, impulsive response to reward. These rat lines are widely used as a genetic model to investigate the neurobiology of addiction. The purpose of this study was to identify the genes and loci that are responsible for intrinsic differences in exploratory locomotion between the bHR and bLR strains.

Methods: Exome-based genomic sequencing of bHR and bLR rats was conducted to uncover the genetic variants in gene-coding regions that segregate between the two lines. Quantitative trait loci (QTL) analysis was then performed in the F2 rats derived from intercross between bHR and bLR rats, with densest coverage of the segregating genomic regions identified by exome-sequencing. Targeted genetic association analysis was also carried out in two unrelated human population samples, Finnish and African American, with drug and alcohol addictions and related personality traits by genotyping selected SNPs in human genes orthologous to the genes located at the most significant QTL peak in the bHR $x$ bLR F2 rats.

Results: A total of 8,123 partially and fully segregating SNPs (Fisher Exact Test, $P<10-4)$ were identified by exomesequencing. Among those variants, 1,582 SNPs showed full allelic segregation between the $12 \mathrm{bHR}$ and $12 \mathrm{bLR}$ rats sequenced. 862 partially and fully segregating missense variants located in 620 genes were also identified. For QTL analysis, a panel of 416 SNPs was genotyped in 314 F2 rats for genome wide QTL analysis with enhanced coverage of the segregating regions. A genome-wide significance level of LOD score $3.99(\alpha=0.05)$ was determined by permutation $(n=1,000)$ test. The strongest QTL peak $(\mathrm{LOD}=7.766)$ located at SNP S126771200 on chromosome $1(126,771,200$ bp) accounted for over $10 \%$ of the total variance in motor activation, and we identified seven genome wide significant QTL regions. The combined effects of the QTLs with genome-wide significance explained approximately half of the genetic variance in novelty induced locomotion. Human genetic association analysis also linked APBA2, a human ortholog of the rat gene located at the center of the strongest QTL, with substance addictions and related behavioral phenotypes, convergent with previous molecular and animal behavioral studies.

Conclusions: Via combined exome sequencing and F2 linkage analysis, we identified seven genetic loci influencing novelty-induced hyperlocomotion, a behavior trait related to vulnerability to drug addiction that had been selected in bHR and bLR rats. These results demonstrate that our approach of combining exome-based genomic sequencing in the selectively bred parental lines with QTL analysis in the F2 animals with dense coverage of the segregating genomic regions is an effective strategy for locus identification in model organisms. Keywords: Quantitative Trait Locus, Exome Sequencing, Locomotion, Selectively Bred bHR and bLR Rats.

Disclosure: Nothing to disclose.

\section{T250. Effects of Acute Exposure to Cannabis Smoke on Working Memory}

Barry Setlow*, Shelby Blaes, Caitlin Orsini, Shandera Ferguson, Sara Heshmati, Shannon Wall, Marcelo Febo, Adriaan Bruijnzeel, Jennifer Bizon

University of Florida College of Medicine, Gainesville, Florida, United States

Background: Cannabis is the most widely used illicit drug in the United States and worldwide, and cannabis use is reported to cause cognitive impairments. Studies in animal models show that acute administration of delta-9tetrahydrocannabinol (THC, the primary psychoactive component of cannabis) or synthetic cannabinoid receptor type 1 (CB1) agonists can impair performance on cognitive tasks that depend upon the hippocampus and prefrontal cortex. Given that the primary route of cannabis use in humans is through smoking, however, there is comparatively little research in animal models that has investigated this route of administration. The primary goal of these experiments was to determine how acute exposure to cannabis smoke affects performance in a delayed response working memory task in rats that depends upon the integrity of the prefrontal cortex. A secondary goal was to determine whether any such effects differ in males and females, as there are reported sex differences in sensitivity to cannabinoids.

Methods: Adult male $(n=7)$ and female $(n=8)$ Long-Evans rats were trained in a food motivated delayed response working memory task in operant test chambers. In each of the 40 minute sessions in this task, rats had to remember the location of a response lever over a variable delay period that ranged from 0-24 s. Smoke exposure began once rats reached stable performance in the task. One hour prior to sessions in the working memory task, rats were placed in a ventilated chamber where they were exposed to smoke generated by burning either cannabis (5.3\% THC, $\sim 0 \% \mathrm{CBD})$ or placebo ( $0 \%$ THC, $0 \%$ CBD) cigarettes in an automated cigarette smoking machine. A semi-randomized within-subjects experimental design was used such that each rat was exposed to smoke from $0,1,3$, and 5 cigarettes of each type, with at least a 48-hour washout period between successive exposures. Three weeks after the last smoke exposure, the effects of the CB1 receptor antagonist rimonabant (SR141716A) on task performance were evaluated. Using a randomized

(1)


$\overline{\mathrm{S} 442}$

within-subjects design, rats received i.p. injections of rimonabant $(0.2,0.6,2.0 \mathrm{mg} / \mathrm{kg})$ or vehicle $(5 \%$ Tween 80 and $20 \%$ DMSO in $0.9 \%$ saline) 10 minutes before testing in the working memory task, with at least a 48 -hour washout period between successive injections.

Results: Prior to smoke exposure sessions, male rats performed more accurately than females on the working memory task, particularly at longer delays. Analysis of performance in females revealed no effects of estrous cycle. Exposure to smoke from cannabis cigarettes had no effects on working memory accuracy in males, but enhanced performance in females. This enhancing effect was not evident following exposure to placebo smoke, in which there were trends toward impairing effects of exposure in males. Acute administration of the $\mathrm{CB} 1$ antagonist rimonabant impaired working memory accuracy; however, this effect was carried largely by the highest dose, which also reduced the total number of trials completed.

Conclusions: The results of these experiments suggest that passive exposure to cannabis smoke can enhance performance on a prefrontal cortex-dependent working memory task. The fact that this effect was evident only in female rats is consistent with evidence that females are more sensitive than males to the behavioral effects of cannabinoids under some conditions. In addition, the absence of these enhancing effects following placebo smoke exposure suggests that smoke itself (or stress/arousal resulting from passive smoke exposure) was not the cause of enhanced performance. Ongoing experiments will determine whether enhancing effects of cannabis smoke on working memory performance are mediated by $\mathrm{CB} 1$ receptors and mimicked by $\mathrm{THC}$ administration at doses that produce blood THC levels comparable to those obtained with smoke exposure.

Keywords: Marijuana, Cannabinoid, Working Memory, Rats.

Disclosure: Nothing to disclose.

\section{T251. Nicotine Withdrawal and Deficits in Cognitive Flexibility: Contribution of Frontostriatal Neurochemical Mechanisms}

Vinay Parikh*, Robert Cole, Matty Zimmerman, Munir Kutlu, Thomas Gould

Temple University, Philadelphia, Pennsylvania, United States

Background: Nicotine addiction continues to be a leading cause of preventable death worldwide. Despite the plethora of available treatments for smoking cessation, smoking relapse after attempts to quit remains high. The behavioral and cellular mechanisms underlying nicotine dependence and withdrawal are not fully understood. Cognitive flexibility is the ability to switch strategic responses adaptively between changing environmental stimuli. Loss of cognitive control during nicotine withdrawal decreases an individual's ability to maintain cognitive flexibility. It is possible that impairments in cognitive flexibility and underlying neurochemical circuits in nicotine addicts may foster maladaptive behaviors that affect individuals' ability to refrain from taking drugs. The present study was designed to examine the effects of spontaneous nicotine withdrawal on cognitive flexibility in mice using an operant strategy set-shifting task. Because frontostriatal circuits are critical for cognitive flexibility and brain-derived neurotrophic factor (BDNF) modulates glutamate plasticity in these circuits, we also explored the effects of nicotine withdrawal on these neurochemical substrates.

Methods: Adult male C57BL/6J mice were trained in an operant task that required them to switch from using a spatial response-driven strategy to a visual cue-based strategy to achieve rewards. Animals were exposed to either chronic nicotine $(18 \mathrm{mg} / \mathrm{kg} / \mathrm{d})$ or saline using subcutaneous miniosmotic pumps for 14 days. During the treatment duration, the animals remained on the spatial lever discrimination phase of the task. Spontaneous nicotine withdrawal was induced by removing the pumps and the animals were tested on the strategy switching phase until criterion $(\geq 80 \%$ correct responses for 3 consecutive days) was attained. Following behavioral testing, brains were extracted for q-PCR and immunoblot analysis of BDNF mRNA and protein expression. In another cohort of animals, the effects of nicotine withdrawal and BDNF responsiveness were assessed on striatal glutamatergic transmission using in vivo amperometric recordings.

Results: Mice undergoing nicotine withdrawal required more trials to attain strategy switching criterion as compared to the controls $(\mathrm{F}(1,15)=89.0, p<0.001)$. Error analysis show that animals withdrawn from nicotine committed higher perseverative errors as well as strategy maintenance errors ( $p=0.03 \& p=0.02$; respectively). Anxiety symptoms observed during nicotine withdrawal in mice were associated with higher perseverative responding to the previously reinforced stimulus. BDNF mRNA expression increased in the prefrontal cortex (PFC) following nicotine withdrawal. Surprisingly, the level of the mature form of BDNF protein of these animals declined in the PFC $(p=0.02)$ but was elevated in the dorsal striatum (DS; $p=0.02$ ). Higher DS:PFC BDNF ratios were inversely related to the task acquisition. BDNF protein levels remained unaltered in the nucleus accumbens (NAc) during withdrawal. Glutamate release following the local application of BDNF in the DS was suppressed in nicotine withdrawal mice as compared to the saline group $(p<0.01)$. On the contrary, the amplitude of BDNF-induced glutamate spikes was significantly higher in the NAc during nicotine withdrawal (nic $=16.8 \pm 1.9 \mu \mathrm{M}$ vs $\mathrm{sal}=8.69 \pm$ $1.1 \mu \mathrm{M})$.

Conclusions: Our data illustrate that robust impairments in strategy switching observed in nicotine withdrawal mice resulted from a generalized behavioral disinhibition. Moreover, withdrawal-related affective dysfunction may in part account for some of the observed deficits in cognitive flexibility. Nicotine withdrawal-related disruption in the trafficking of mature BDNF between the PFC and DS, and altered glutamate responsiveness to BDNF in the DS and NAc is indicative of aberrations in BDNF signaling in discrete frontostriatal glutamate circuits. As BDNF is critical for cognitive and affective processes, therapeutic strategies aimed at normalizing BDNF signaling and restoring frontostriatal plasticity may improve relapse rate in smokers. Keywords: Nicotine Addiction, BDNF, Glutamate, PFC, Cognition.

Disclosure: Nothing to disclose. 
T252. Cocaine Dependence and Thalamic Functional Connectivity: A Multivariate Pattern Analysis

Chiang-Shan Li, Sheng Zhang, Sien Hu, Rajita Sinha, Robert Malison, Marc Potenza

Yale University School of Medicine, New Haven, Connecticut, United States

Background: Cocaine dependence is associated with deficits in cognitive control. Previous studies demonstrated that chronic cocaine use affects the activity and functional connectivity of the thalamus, a subcortical structure critical for cognitive functioning. However, the thalamus contains nuclei heterogeneous in functions, and it is not known how thalamic subregions contribute to cognitive dysfunctions in cocaine dependence. For instance, chronic cocaine users showed hypo-activation of the thalamus during a visual spatial attention task (Tomasi et al, 2007b). In a longitudinal study, decreased error-related activation of the thalamus predicts relapse and time to relapse to drug use in cocaine dependent individuals (Luo et al, 2013). On the other hand, increased thalamic activations were observed in individuals with cocaine dependence and other addictive disorders when they were exposed to drug cues or situational factors related to drug use (Gozzi et al, 2011; Jia et al, 2011; Tomasi et al, 2007a). This seeming inconsistency may relate to functional heterogeneity of the thalamus, with some subregions mediating craving and others subserving control of craving. Understanding how thalamic cortical circuits respond to different psychological constructs and behavioral contexts will help elucidate the multifaceted etiologies of cocaine addiction.

Methods: To address this issue, we used multivariate pattern analysis (MVPA) to examine how functional connectivity of the thalamus distinguishes 100 cocaine-dependent participants (CD) from 100 demographically matched healthy control individuals (HC). We characterized six task-related networks with independent component analysis (ICA) of fMRI data of a stop signal task and employed MVPA to distinguish $\mathrm{CD}$ from $\mathrm{HC}$ on the basis of voxel-wise thalamic connectivity to the six independent components. First, the fMRI data of $100 \mathrm{CD}$ and $100 \mathrm{HC}$ were analyzed by ICA to generate 30 networks, from which six task-related networks were selected for further analysis. A thalamus mask was applied to generate feature set including only voxels within the thalamus for MVPA. MVPA was applied using leaveone-out cross-validation to obtain true accuracy rates in group classification. Classification features were selected and support vector machine (SVM) classifier was trained based on the training data set. During testing, the same features from the testing data set were applied to the trained SVM classifier to obtain classification results (CD or HC). This procedure was repeated until each of the $100 \mathrm{CD}$ and $100 \mathrm{HC}$ was selected once as the validation data. The mean accuracy rate was computed to index overall accuracy. The selected thalamic voxels from each ICA network were analyzed across 200 leave-one-out cross validation runs. Statistical significance of the classification was examined using a permutation scheme. Finally, we explored the correlation of these thalamic connectivities with clinical and performance variables. In addition to the thalamus, we examined other masks with a similar volume, including the caudate and middle orbital frontal gyrus, for comparison.
Results: In an unbiased model of distinct training and testing data, the analysis correctly classified $72 \%$ of subjects with leave-one-out cross-validation $(p<0.001)$, superior to comparison brain regions with similar voxel counts $(p<0.004$, two-sample $t$ test). Thalamic voxels that form the basis of classification aggregate in distinct subclusters, suggesting that connectivities of thalamic subnuclei distinguish CD from HC. Specifically, a total of $513 \pm 10$ features were selected at $p<0.05$, uncorrected. The results of MVPA showed that, with connectivity to the motor cortical network, bilateral ventrolateral nuclei (VL) possibly including mediodorsal nuclei (MD) distinguished $\mathrm{CD}$ from HC. Compared to $\mathrm{HC}, \mathrm{CD}$ showed significantly lower functional connectivity between this cluster and the motor cortical network ( $p=0.0002$, twosample t test). With connectivity to the right frontoparietal network, three clusters, each in bilateral ventroposterior lateral nuclei (VPL) and pulvinar (PUL), and left VL distinguished $\mathrm{CD}$ from HC. Compared to $\mathrm{HC}, \mathrm{CD}$ showed significantly higher functional connectivity in the right VPL/ PUL ( $p=0.0001)$ and lower connectivity in the left VPL/PUL $(p=0.0002)$ and left VL $(p=0.0002)$. With connectivity to the left frontoparietal network (IC027), clusters in the area of bilateral VL and right MD distinguished $C D$ from HC. Compared to HC, CD showed significant lower connectivity in bilateral VL (all p's $<0.00001)$ and higher connectivity in right $\mathrm{MD}(p=0.0009)$. With connectivity to the midline cortical-subcortical network, clusters in the area of bilateral $\mathrm{MD}$ distinguished $\mathrm{CD}$ from $\mathrm{HC}$. Compared to $\mathrm{HC}, \mathrm{CD}$ showed higher connectivity in bilateral MD (all p's < 0.0001). With connectivity to the cuneus-precuneus network, clusters in the area of bilateral VPL distinguished CD from HC. Compared to HC, CD showed higher connectivity to VPL (all p's $<0.000001$ ). Further, linear regressions provided suggestive evidence for a correlation of the thalamic connectivities with clinical variables and performance measures on the stop signal task. In particular, Pearson regression across subjects showed positive correlations between the stop signal reaction time and right MD connectivity to the left frontoparietal network in $\mathrm{CD}(p=0.007, \mathrm{r}=0.27)$ but not $\mathrm{HC}(p=0.39, \mathrm{r}=0.09)$.

Conclusions: In summary, MVPA demonstrated thalamic functional connectivities as a potential diagnostic circuit marker of cocaine addiction. The intricate patterns of thalamic subregional connectivities that distinguish cocaine-addicted from non-addicted individuals confirm the utility of this computational approach in psychiatric neuroscience research.

Keywords: Thalamus, Cocaine Addiction, ConnectivityBased Parcellation.

Disclosure: Nothing to disclose.

\section{T253. Effects of Guanfacine on a Laboratory Model of Cannabis Withdrawal and Relapse}

Margaret Haney*, Ziva Cooper, Gillinder Bedi, Evan Herrmann, Sandra Comer, Stephanie Collins Reed, Richard Foltin, Frances Levin

Columbia University, New York, New York, United States

Background: Medications to improve outcome for the treatment of cannabis use disorder (CUD) are a needed option to 
reduce the high rates of relapse observed in placebocontrolled clinical trials. In the human laboratory, we have shown that the $\alpha 2 \mathrm{a}$ adrenergic receptor agonist, lofexidine, improved sleep during cannabis withdrawal and reduced a laboratory measure of cannabis relapse (Haney et al, 2008). Yet lofexidine has a short half-life, so was administered frequently throughout the day $(0.6 \mathrm{mg}$, QID) in that inpatient study. Morever, in a recent clinical trial lofexidine was poorly tolerated even at a lower target dose $(0.6 \mathrm{mg}$ TID), with $40 \%$ of patients experiencing dizziness, fatigue and pressor effects (Levin et al, 2016). A possible alternative is guanfacine hydrochloride $\left(\right.$ Tenex $\left.^{\circledR}\right)$, an $\alpha 2$-adrenergic agonist that is FDA-approved for the treatment of hypertension which has been shown in some studies to reduce opioid, alcohol and nicotine withdrawal symptoms. The objective of this study was to test whether a single daily administration of guanfacine at bedtime would reduce cannabis withdrawal symptoms, particularly sleep disruption, while producing little daytime fatigue or pressor effects relative to placebo.

Methods: The study comprised two 19-day outpatient/ inpatient phases, with one phase testing placebo and the other testing guanfacine maintenance in counter-balanced order. The 10-day outpatient phase preceding each 9-day inpatient phase was used for medication clearance or dose induction (target dose: $2.0 \mathrm{mg}$ ). Nontreatment-seeking, daily cannabis smokers came to the laboratory every 48 hours while outpatient for measures of blood pressure, medication compliance (riboflavin), ongoing cannabis use (timeline followback, urine toxicology) and side effects. During the inpatient phases, participants repeatedly smoked active cannabis (5.6\% THC) under controlled conditions on the first 2 inpatient days in order to standardize recent cannabis exposure and to allow assessment of guanfacine's effects on measures of cannabis intoxication. For the next 4 days, no cannabis was available (withdrawal), followed by 3 days when active cannabis was available for self-administration (relapse). Participants had to pay for self-administered cannabis using their study earnings. Ratings of mood, drug-related effects, sleep, cardiovascular effects, food intake and cognitive task performance were assessed repeatedly throughout each inpatient day.

Results: Male $(n=13)$ and female $(n=2)$ daily cannabis smokers $(4.7+3.3$ grams/day) completed the study. Cannabis Withdrawal: Under placebo medication conditions, withdrawal from cannabis produced: (1) significant sleep disruption (e.g., decreased sleep efficiency and sleep satisfaction, increased latency to fall asleep), (2) significantly worsened mood (increased cluster ratings of 'miserable' and 'irritable'), and (3) small but significant increases in systolic and diastolic pressure and decreases in heart rate relative to active cannabis administration. Guanfacine significantly improved objective measures of sleep (efficiency and latency) during withdrawal, and attenuated withdrawal-related ratings of 'miserable' and 'irritable.' Guanfacine had no effect on ratings of fatigue or cluster ratings of 'bad effects' (including dizziness) relative to placebo. Guanfacine produced small but significant decreases in systolic and diastolic blood pressure and heart rate, independent of cannabis condition; no dose was held due to symptoms of hypotension (BP < 90/50, lightheadness, dizziness). Cannabis relapse: Under placebo medication conditions, half of the participants (53.3\%) 'relapsed,' i.e., paid to self-administer cannabis after 4 days of abstinence, purchasing $5.9 \pm 1.0$ cannabis puffs ( $5 \mathrm{sec} / \mathrm{each})$. Guanfacine did not alter the amount of cannabis self-administered relative to placebo. Data analysis is still underway for other outcome measures (cognitive task performance, food intake, and outpatient measures).

Conclusions: Once/daily guanfacine administration at bedtime produced several positive signals in this model of medications development for CUD. The medication was well tolerated and significantly improved sleep and reduced the negative mood symptoms of cannabis withdrawal relative to placebo. In fact, guanfacine is the only non-cannabinoid receptor agonist tested in our laboratory model (out of 13 medications) to significantly improve withdrawal-related mood symptoms. The failure of guanfacine to alter cannabis self-administration is noteworthy, as our working hypothesis is that medications that reduce both self-administration and cannabis withdrawal in the laboratory provide the strongest justification for clinical trial testing. Nonetheless, the findings support further studies to optimize guanfacine's effects on cannabis withdrawal and relapse or combine guanfacine with other medications for the potential treatment of CUD.

Keywords: Marijuana, Noradrenergic, Treatment. Disclosure: Nothing to disclose.

\section{T254. OPRM1 Asp40 Interacts With DAT1 VNTRs in Predicting Naltrexone Response in an Alcohol Use Disorder Randomized Clinical Trial}

\section{Raymond Anton*, Sarah Book, Konstanin Voronin, Patricia Latham, Patrick Randall}

Medical University of South Carolina, Charleston, South Carolina, United States

Background: There is growing interest in "personalized" treatment of AUD with the opiate antagonist, naltrexone. Whether the putative mu opioid receptor polymorphism OPRM1 asp40 status influences naltrexone response is controversial, with retrospective studies being positive (Oslin. 2003; Anton. 2008) and one prospective study reportedly negative (Oslin 2015). We had previously reported (Anton 2012), in those not seeking treatment for AUD, that naltrexone effects on drinking were influenced by an interaction of the OPRM1 asp40 allele and VNTR variation in the dopamine transporter gene (DAT1-SLC6A3). We wanted to examine this effect in treatment seeking AUD individuals participating in a clinical trial for naltrexone (ntx.) designed to examine genetic predictors of response.

Methods: 146 Caucasian alcohol dependent individuals (DSM IV) with a mean $80 \%$ heavy drinking days (HDD) were randomized to naltrexone $(50 \mathrm{mg}$ ) or placebo, based on their OPRM1 allele status (asp40 vs. asn40 homozygotes taqman PCR) on a one to one basis in a blinded fashion. In an exploratory fashion we evaluated DAT1 VNTR (using primers from ABI, PCR, and chromatographic separation) status in these participants. Smoking (40\%), antidepressant use $(33 \%)$, and sex (30\% Female) were equally distributed across groups. Nine sessions of Medical Management (wks. $1,2,3,4,6,8,10,12,16)$ were provided and drinking assessed by TLFB during the 16-week treatment. Percent heavy drinking days (\%HDD) over the 4 study months were analyzed in 
mixed model of medication (ntx vs. plac.) x OPRM1 allele (at least one asp40 allele vs. asn/asn homozygotes) x DAT1 (at least one 9 VNTR vs. 10/10 homozygotes) x time (study month).

Results: Of 146 evaluable individuals $n=73$ were OPRM1 asp40 carriers and 73 asn 40 homozygotes. DAT1 9 carriers and 10/10 homozygotes were not significantly different across OPRM1 and medication groups (group size range 1224). Study completers (73\%) and complete drinking data (86\%) did not differ across groups. There was a significant four-way interaction $(p=0.015)$ such that those Asp40 carriers who also had DAT1 10/10 genotypes and those Asn40 genotypes that who were DAT1 9 carriers all had less \%HDD over the course of the study when on naltrexone compared to placebo. This was not modified by sex, age, antidepressant use or smoking status. The same pharmacogenetic relationships held when percent days abstinent $(p=0.022)$, drinks per day $(p=0.051)$ and drinks per drinking day $(p=0.024)$ were similarly analyzed.

Conclusions: While great emphasis has been placed on the OPRM1 asp40 predicting naltrexone response, results have been inconsistent. One reason is that OPRM1 asp40 might be interacting with other genetic variants (epistasis). One important possibility is the functional VNTR variant in the dopamine transporter coding (DAT1) gene. Many reports have indicated DAT1-VNTR 9 carriers to have lower DAT function leading to more synaptic dopamine availability underlying more reward and cue sensitivity. We previously reported in a sub-acute drinking experiment in nontreatment seeking AUD individuals, that OPRM1 asn40/ DAT1 9 carriers had less drinking on naltrexone that was also observed in OPRM1 asp40/DAT1 10/10 carriers. Replication in treatment seeking individuals in this study lends credence to that observation. Overall, this data suggests that genetically based dopamine system variation/tone may influence how opiate systems might respond and interact with medication. This could have both important scientific meaning and clinical implication in regards to pharmacogenetic treatment response and personalized medicine initiatives.

Supported by NIAAA R01AA017633 and K05AA017435.

Keywords: Naltrexone, Pharmacogenetic Response, AUD, OPRM1 Gene, DAT1 Gene

Disclosure: Alkermes: Past Consultant - Supporter of ACTIVE, Self; Lilly: Past Consultant - Supporter of ACTIVE, grant, Self; Lundbeck: Past Consultant - Supporter of ACTIVE, Self; Xenoport: Past Consultant - Supporter of ACTIVE, Self; Indivior: Past Consultant - Supporter of ACTIVE, Self; Ethypharm: Supporter of ACTIVE, Self; Otsuka: Supporter of ACTIVE, Self; Pfizer: Supporter of ACTIVE, Self.

\section{T255. Protein Kinase C Epsilon Inhibitors Reduce Alcohol Consumption in Mice}

Robert Messing*, Dan Wang, Jingyi Wang, Angelo Blasio

The University of Texas at Austin, Austin, Texas, United States

Background: Although alcohol use disorder is a major public health problem worldwide, only three drugs have been approved by the FDA for treatment: Disulfuram, naltrexone and acamprosate. Our previous studies have provided strong evidence that the enzyme protein kinase $\mathrm{C}$ epsilon (PKCepsilon) promotes ethanol consumption and reward. We recently identified a lead compound derived from the commercially available Rho-associated coiled-coil forming protein kinase (ROCK) inhibitor Y-27632, which inhibits PKCepsilon. We tested whether this lead, compound 1.0 and its analogs are efficacious in reducing ethanol drinking in mice.

Methods: Novel compounds were tested for inhibitory activity in vitro using PKC assays, and in vivo in mice for their ability to inhibit ethanol consumption in an MWF intermittent access, 24- hour, 2-bottle choice procedure, to alter ethanol clearance following i.p. administration of $4 \mathrm{~g} / \mathrm{kg}$ ethanol, and to prolong the duration of the ethanol-induced loss of the righting reflex.

Results: Compound 1.0 and 3 analogs inhibited PKCepsilon with $\mathrm{Ki}<20 \mathrm{nM}$. All four compounds weakly inhibited conventional PKCgamma at $10 \mu \mathrm{M}$, while compounds $1.3,1.4$ and 1.7 showed little activity against atypical PKCzeta at that high concentration. All inhibited the highly related novel PKCs, PKCdelta and PKCtheta, but showed some selectivity within the novel PKC subfamily being less potent in inhibiting PKCdelta and PKCtheta than PKCepsilon. Compound 1.0 was screened against a panel of 395 non-mutant kinases using a binding competition assay at a concentration of $200 \mathrm{nM}$ and was found to be selective, inhibiting only 10 other kinases to $<35 \%$ of control activity. A pharmacokinetic study with $1.0(20 \mathrm{mg} / \mathrm{kg})$ and $1.3(40 \mathrm{mg} / \mathrm{kg})$ showed that these compounds enter the brain and achieve a brain/ plasma ratio at 4 hours of 0.89 and 0.16 , respectively. These two compounds dose-dependently reduced voluntary ethanol consumption in an intermittent ( 3 days per week) access procedure in which mice drank on average $5.4 \pm 0.2 \mathrm{mg} / \mathrm{kg}$ to achieve a mean BEC of $104 \pm 10 \mathrm{mg} / \mathrm{dL}$ in the first 4 hours of drinking. In addition, these compounds prolonged the ethanol-induced LORR in wild type but not PKCepsilon knockout mice, and they did not alter ethanol clearance.

Conclusions: These data indicate that compounds 1.0 and 1.3 are relatively selective PKCepsilon inhibitors that reduce voluntary ethanol consumption in mice. In conclusion, PKCepsilon inhibitors may prove useful as new therapeutics for alcohol use disorder.

Keywords: Protein Kinase C, Alcohol Self-Administration, Kinase Inhibitor.

Disclosure: Patent US8785648B1, Self.

\section{T256. The Role of Glutamate in Mediating the Reinstatement of Cocaine-Seeking is Altered by Both a History of Stress and Combined Alcohol and Cocaine Self-Administration}

Lori Knackstedt*, Bethany Stennett, Marek Schwendt

University of Florida, Gainesville, Florida, United States

Background: Drug addiction is often co-morbid with other psychiatric disorders. Furthermore, the majority of addicted individuals are polysubstance users. Particularly common is the combination of alcohol and psychostimulants: $50-90 \%$ of cocaine addicts also drink alcohol. Poorer health outcomes 
$\overline{S 446}$

and greater resistance to treatment are reported for patients with comorbidities. Few pre-clinical studies have directly examined the behavioral or neurobiological underpinnings or consequences of comorbidity. Here we present data utilizing rat models of both comorbid PTSD + cocaine addiction and comorbid cocaine and alcohol use that indicate that these comorbidities alter the neurobiology of cocaine relapse relative to cocaine alone.

Methods: We used a predator stress model of PTSD in which rats undergo a single 10-minute exposure to 2,4,5trimethylthiazoline (TMT). Rats are then tested 7 days later in both the elevated plus maze (EPM) and acoustic startle response (ASR) to classify rats as PTSD-like, Resilient or intermediate. Resilient and PTSD rats subsequently underwent cocaine self-administration, extinction and were tested for cue-primed reinstatement. To model alcohol-cocaine coabuse, rats were trained to drink unsweetened ethanol $(20 \%$ $\mathrm{v} / \mathrm{v}$ ) in the home cage for two weeks prior to beginning cocaine self-administration. Immediately following daily operant cocaine self-administration sessions, rats were given access to either $20 \%$ ethanol or water in the home cage. Following extinction training, rats were tested for cocaine and cue-primed reinstatement during a microdialysis session with probes inserted into the nucleus accumbens core (NAc). Glutamate content in microdialysis samples was quantified with HPLC.

Results: Approximately 20\% of rats were classified as "PTSD-like" based on meeting the dual criteria of scoring above the median ASR and below the median open arm entries in the EPM. An equal number of rats were classified as "Resilient" upon scoring below the median ASR and above the median open arm entries in the EPM. Rodents exposed to TMT that developed a PTSD-like phenotype displayed both blunted extinction learning and enhanced cue-primed reinstatement of cocaine-seeking relative to Controls and Resilient rats. The antibiotic ceftriaxone $(200 \mathrm{mg} / \mathrm{kg})$, which has been repeatedly demonstrated to prevent cue-primed reinstatement of cocaine-seeking in non-trauma exposed animals, did not prevent cue-primed reinstatement in PTSD animals but did in Control and Resilient rats. However, administration of the mGlu5 positive allosteric modulator CDPPB prior to only the first 5 sessions of operant and fear extinction followed by 5 days of ceftriaxone $(200 \mathrm{mg} / \mathrm{kg})$ completely prevented cue-primed reinstatement in PTSD rats when animals were tested without either ceftriaxone or CDPPB onboard. The number of lever presses during reinstatement tests did not differ between groups that consumed water or alcohol following operant cocaine sessions. However, rats that self-administered alcohol and cocaine did not display increased glutamate release in the NAc during either cocaine- or cue+cocaine-primed reinstatement tests as did rats that self-administered only cocaine. Ceftriaxone $(200 \mathrm{mg} / \mathrm{kg})$ did not alter relapse in rats that self-administered both cocaine and alcohol as it does in rats that self-administer only cocaine.

Conclusions: These findings indicate that rats with an anxious phenotype (PTSD-like) following predator stress exposure display enhanced cue-induced relapse of cocaineseeking that is resistant to treatment with ceftriaxone. Medications that reduce cocaine-seeking in non-trauma exposed patients may not be effective in PTSD patients and these patients may require polytherapy to reduce symptoms of both disorders. Positive allosteric modulation of mGlu5 receptors in combination with ceftriaxone represents an attractive target to reduce cocaine-seeking in the PTSD population. Furthermore, glutamate transmission in the NAc does not mediate relapse to cocaine-seeking in animals that consume alcohol with cocaine and medications targeting glutamate, such as ceftriaxone, may not be effective therapies for preventing relapse in cocaine addicts that also consume alcohol. The results of these studies indicate that the neurobiology underlying cocaine-seeking is altered by comorbid disease.

Keywords: GLT-1, Addiction, Cocaine, Alcohol, PTSD. Disclosure: Nothing to disclose.

\section{T257. Stressful Events During Medically Assisted Opioid Treatment: Comparison With Daily Stress}

Kenzie Preston*, Karran Phillips, William Kowalczyk, David Epstein

National Institute on Drug Abuse, Baltimore, Maryland, United States

Background: Studies using ecological momentary assessment (EMA) have shown a strong relationship between stress and craving when both are assessed at random times in participants' natural environments. We recently completed the first EMA study in which participants were asked to report every stressful event they underwent, as soon as it happened. The participants were receiving outpatient treatment for drug dependence; their data enabled us to examine craving for drugs in the context of discrete episodes of stress as well as fluctuations in randomly assessed background stress.

Methods: Outpatients $(N=190)$ maintained on daily buprenorphine or methadone self-reported stress, craving and mood and behavior on electronic diaries for up to 16 weeks. Participants initiated an entry each time they felt more stressed than usual (event-contingent entries; ECs) and made randomly prompted entries (RPs) three times per day. In both types of entries, participants rated the severity of stress and craving and the context of the report (location, activity, companions). Stressful events were also identified in terms of consequences ("a hassle", "something that could spoil the day", or "something that could do more than spoil the day"), causes, and nature of the emotional response (stressed, overwhelmed, or anxious). We compared context and severity of stress, and its relationship to craving, in ECs versus RPs.

Results: Participants made nearly 45,000 RP entries, and 166 participants (87\%) reported at least one stress event in an EC entry, for a total of $1818 \mathrm{EC}$ entries. In the EC entries, participants specified their emotional responses as feeling stressed (60\%), overwhelmed (32\%), and/or anxious $(25 \%)$. The most common precipitants were interpersonal conflict (19\%), just thinking about stress (18\%), having too much to do (16\%), money problems (17\%), and "other" (10\%) with family and other miscellaneous problems the most frequently cited other. Mean $( \pm$ SEM) ratings of stress severity (0-10 scale) increased linearly $(\mathrm{F}(1,200)=100.27, p<.0001)$ across the categories of hassle $(4.0 \pm 0.1)$, day spoiler (5.1 $\pm 0.1)$, or more than a day spoiler $(6.1 \pm 0.1)$. Craving for 
heroin and cocaine also increased linearly across those stressseverity categories [heroin: $\mathrm{F}(1,114)=69.91, p<.0001$; cocaine: $\mathrm{F}(1,114)=13.06, p=.0005]$ and with numerically rated severity of background $(\mathrm{RP})$ stress [heroin $(\mathrm{F}(1,571)=$ 1282.29, $p<.0001)$ and cocaine $(\mathrm{F}(1,571)=411.65$, $p<.0001)]$. Compared to RPs, stress ECs were significantly $(p<.05)$ less likely to be reported when participants were with their spouse or a child and more likely when participants were with acquaintances, coworkers, strangers, friends, or someone they frequently used with (gate partner). Compared to RPs, stress ECs were significantly $(p<0.05)$ more likely to be reported when participants were arguing, walking or riding, on the phone, talking, waiting, or working, and less likely when they were resting, watching TV, eating, or on the internet.

Conclusions: We found that it is feasible to ask participants to report all stressful events in real time. Our qualitative and quantitative measures of severity each showed that a broad spectrum of stressor severities was reported. The contexts of the stressful event reports differed somewhat from those of the highest ratings of ongoing background stress in randomprompt entries. Nevertheless, background stress and patient-initiated stress-event reports had similarly positive relationships with craving for both heroin and cocaine. Further analyses of EMA and urine drug screen data should provide additional information on the relationship between drug use and daily-life stress.

Keywords: Craving, Acute Stress, Substance Abuse, Opioids, Cocaine.

Disclosure: This work was supported by the Intramural Research Program, National Institute on Drug Abuse, NIH.

\section{T258. Amygdala Volume, Resting State Functional Connectivity, and NEO Impulsiveness (N5) in Cocaine Use Disorders}

Bryon Adinoff*, Ingrid Kepinski, Hong Gu, Sabrina Blackledge, Yihong Yang, Elliot Stein

University of Texas Southwestern Medical Center, Dallas, Texas, United States

Background: The neural correlates of substance use disorder (SUD) often overlap with those of personality, specifically domains associated with negative affect and impulsivity. Alterations in amygdalar size, activity and connectivity, in particular, has been associated with disorders of negative affect. Healthy controls, for example, show a negative correlation between neuroticism and right amygdalar gray matter volume (Omara et al. 2005) and a positive correlation between neuroticism and right amygdala-dorsomedial prefrontal cortex (dmPFC) functional connectivity (Cremer et al. 2010). Healthy controls also demonstrate a positive correlation between neuroticism and white matter integrity in tracts interconnecting the amygdala and PFC (Xu et al. 2012), as well as with amygdalar activation during high emotional conflict (Haas et al. 2007). In SUD individuals, the amygdala is activated during craving and is strongly implicated in drug-seeking behaviors and drug reinforcement. Although the PFC is critical for top-down behavioral control (inhibition, constraint), impulsivity is negatively correlated with resting state functional connectivity (rsFC) between the right amygdala and the anterior cingulate in healthy controls (Gu et al, 2010; Kerr et al, 2014). Bi-directional connectivity between the prefrontal cortex (PFC) and the amygdala is considered to be a key neural mechanism for the over-expression of negative affect (Belcher et al, 2014). "Impulsiveness" (N5), a subdomain of the Five Factor Model trait of Neuroticism, captures aspects of both negative affect and impulsivity ("Sometimes I do things on impulse that I later regret"). N5 is significantly different between CUD and control participants (Lobue et al, 2014) and we have observed that of all NEO-PR-I and TCI subdomains, N5 best differentiates between the two groups. Based upon these findings, we used a pre-existing database of healthy controls and individuals with CUD to explore the Impulsiveness by Group interaction effects on 1) right amygdalar volume and 2) right amygdalar rsFC (whole brain exploration).

Methods: 21 healthy controls and 42 2-4 week abstinent, treatment-seeking CUD participants, all completed the NEOPI-R and a 6-min rsFC and structural MRI scan. Individual right amygdala volume was determined using FreeSurfer and adjusted by participant's total subcortical gray matter volume. The corresponding amygdala mask was extracted individually and then used as the seed region to generate rsFC connectivity maps. The relationships (controlled for age, gender and years education) between the behavior and imaging indices were tested by the Group x Impulsiveness interaction effects on (1) right amygdalar volume and (2) voxel-wise right amygdalar rsFC strength using a General Linear Model. For whole-brain voxel-wise rsFC analysis, effects were considered significant at pcorrected $<0.05$ (uncorrected $p<0.02$ combined with a cluster threshold of 112 voxels).

Results: Main Effects: There was not a significant difference in right amygdalar volume between the control and CUD group. Group interaction of right amygdalar volume and Impulsiveness (N5): There was a significant Group interaction in the right amygdalar volume and Impulsiveness relationship $(p<0.003)$. This interaction was driven by a positive correlation between right amygdalar volume and Impulsiveness in the CUDs $(\mathrm{r}=0.50, p=0.001)$ and a nonsignificant negative correlation in controls $(\mathrm{r}=-0.37, p=$ $0.10)$ Group interaction of right amygdalar $\mathrm{rsFC}$ and Impulsiveness (N5): There was a significant Group interaction in the right amygdalar rsFC strength and Impulsiveness relationship $(p<0.05)$. This interaction was driven by a positive correlation between Impulsiveness and right amygdalar-PFC rsFC strength in healthy controls ( $\mathrm{r}=$ $0.67, p=0.001)$ and no correlation in the CUD group $(\mathrm{r}=$ $0.16, p=0.31$ ).

Conclusions: The presence of CUD altered the relationship between 1) Impulsiveness (N5) and right amygdalar volume and 2) Impulsiveness and right amygdalar functional connectivity with regions involved in affect regulation and response inhibition. These findings suggest a neural diathesis in individuals at risk for CUDs and/or are a result of chronic cocaine use. Increased right amygdalar volume has been associated with impulsivity in psychiatric populations (Gopal, et al, 2011). However, Impulsiveness, as a subdomain of neuroticism, represents not only impulsivity but also a propensity towards negative affect. In general, high neuroticism individuals are more likely to experience stress and/or trauma, and to react more strongly to it, as well as to develop SUDs. In trauma exposed populations, smaller 
amygdala volume has been observed (Morey et al, 2012), along with associated increases in amygdalar reactivity towards negative stimuli (Lidell et al, 2005). While the right amygdala of our control group showed decreases in volume that would be expected of high-impulsiveness individuals that may be more vulnerable towards increased experience of stress and/or trauma, our CUD group showed the reverse. This suggests that prolonged cocaine use may result in a differential reactivity towards negative affective experiences than would be expected in non-CUD individuals. Greater connectivity between the amygdala and prefrontal cortices would indicate greater top-down control over emotional responses. In our controls, connectivity appeared to increase with Impulsiveness (N5), suggesting that healthy control individuals may increasingly call on engagement from topdown inhibitory processes with higher trait Impulsiveness. High N5 CUD individuals appeared not to have similarlyenhanced top-down control; however, our study cannot determine whether this is due to the chronic effects of cocaine use or was pre-existent to the onset of drug initiation.

Keywords: Impulsiveness, Amygdala-Based Networks, Cocaine Addiction.

Disclosure: Supported by DA023203, NIDA Intramural Research Program, and UTSW Center for Translational Medicine UL1TR000451.

\section{T259. Enhanced Transition From Impulsive to Compulsive Ethanol Seeking Behavior is Associated With Increased ERK- $\Delta$ FosB Activity During Ethanol Extinction}

Phillip Starski, Alfredo Oliveros, Allen Cui, Sun Choi, Doo-Sup Choi*

Mayo Clinic College of Medicine, Rochester, Minnesota, United States

Background: Individuals with Alcohol use disorder (AUD) often display an increase in risk-prone behavior, resulting in a propensity for compulsive (acting without forethought to prevent a consequence) conduct. Compulsivity is a major barrier for individuals who are trying to maintain prolonged abstinence (extinction) for their addiction, where the development of habitual behavior (drinking) gradually stops due to the removal of the stimulus (alcohol). However, the molecular mechanisms underlying compulsive ethanol seeking behaviors and abstinence have remained largely unexplored.

Methods: We investigated whether male C57BL/6J prefer a sucrose-ethanol (SE) reward over a sucrose (S) reward in the 5-choice serial reaction time task (5-CSRTT) through ultrasonic vocalization analysis. We then tested the animals for various impulsive and compulsive behaviors using the 5CSRTT between mice given an S reward or a SE reward. After continuous testing, we removed the reward to induce extinction. Finally, we examined ERK1/2 and $\Delta$ FosB protein expression in the amygdala and nucleus accumbens during extinction.

Results: We found that SE mice emitted positive ultrasonic vocalizations during 5-CSRTT pre-training and 5-CSRTT testing, suggesting increased positive affective state during ethanol seeking. Interestingly, SE-mice displayed exacerbated compulsivity during early 5-CSRTT testing with a reward and during extinction. The SE-mice also exhibited increased behavior during spontaneous relapse. Using Western blot analysis, we revealed that $\Delta$ FosB protein expression is increased in the amygdala in both S-mice and SE-mice compared to unconditioned control mice along with FosB and ERK1/2. Additionally, an active form of ERK1/2 (phosphorylation of ERK1/2, Thr202/Tyr204) was also increased in S-mice compared to both unconditioned and SE-mice in both the amygdala and nucleus accumbens. Delta FosB was also increased in the nucleus accumbens of S-mice compared to the unconditioned control. Whereas, SE-mice had lower amounts of total ERK1/2 compared to unconditioned and S-mice.

Conclusions: Our present study demonstrated that amygdala $\Delta$ FosB and pERK expression play an essential role during ethanol extinction. Since $\Delta$ FosB is implicated in other psychiatric disorders, our findings suggest that dampening $\Delta$ FosB expression in the amygdala may be correlated with reward extinction or withdrawal associated psychiatric symptoms.

Keywords: Impulsivity, Compulsive Models of Drug Use, Alcohol Use Disorder.

Disclosure: Nothing to disclose.

\section{T260. The Role of Microglia in the Neuroimmune Effects of Acute Binge Alcohol}

Thomas Jordan Walter*, Leon Coleman, Liya Qin, Fulton Crews

University of North Carolina, Carrboro, North Carolina, United States

Background: Microglia are the resident macrophages of the central nervous system. Dysregulation of microglia has been implicated in the pathogenesis of a variety of neuropsychiatric diseases, including alcohol use disorders. However, the effects of alcohol on microglia in vivo have not been thoroughly defined. Furthermore, studies causally examining the role of microglia in the brain neuroimmune response to alcohol have not been undertaken.

Methods: First, in order to define the effects of acute binge alcohol on microglia in vivo, C57BL/6J mice were gavaged with a single dose of alcohol $(3,4.5$ or $6 \mathrm{~g} / \mathrm{kg}, 25 \% \mathrm{v} / \mathrm{v})$ and sacrificed at $0,6,12,18,24$ or 48 hours post-gavage. The brains were isolated and the expression of various microglial and neuroimmune genes was assessed by RT-PCR. Next, in order to define the role of microglia in the neuroimmune effects of acute binge alcohol, microglia were depleted from the brains of mice using a colony stimulating factor 1 receptor (CSF1R) inhibitor, PLX5622, from Plexxikon Inc. The mice were gavaged with a single dose of alcohol $(6 \mathrm{~g} / \mathrm{kg}$, $25 \% \mathrm{v} / \mathrm{v}$ ) and sacrificed at 18 hours. The brains were isolated and the expression of various neuroimmune genes was assessed by RT-PCR.

Results: Acute binge alcohol decreased expression of the microglial genes Iba1 and CD68 during the initial intoxication period (6 hrs), but increased expression of the same genes later during the withdrawal period (12-24 hrs). Acute binge alcohol caused similar changes in multiple pro- 
inflammatory genes. For example, TNF $\alpha$ and Ccl2 expression increased, but not until the withdrawal period (12-24 hrs). Dose-response curves showed that high doses of alcohol altered the expression of microglial and neuroimmune genes, while low doses did not. Lastly, microglia were depleted from the brains of mice using the CSF1R inhibitor PLX5622. Microglial depletion blunted the alcohol-induced increase in TNF $\alpha$ and $\mathrm{Ccl} 2$, but did not change the response of IL-1 $\beta$ or IL- 6 to alcohol. Interestingly, microglial depletion enhanced the alcohol-induced response of anti-inflammatory genes such as IL-10, IL-4 and IL-1ra.

Conclusions: These results show that acute binge alcohol causes dynamic changes in the expression of microglial and neuroimmune genes. Microglial depletion decreased expression of some pro-inflammatory genes and enhanced expression of multiple anti-inflammatory genes to alcohol. Targeting microglia may be an effective means of treating alcohol-induced pathology related to neuroimmune system dysregulation.

Keywords: Microglial Activation, Ethanol, Neuroimmune. Disclosure: Nothing to disclose.

\section{T261. Hnrnph1 Deletion Shifts Methamphetamine Preference and Reinforcement via a Mechanism Involving Increased Dopaminergic Innervation in the Mesocorticolimbic System}

Neema Yazdani*, Eric R Reed, Qiu T Ruan, John Shahin, Farzad Mortazavi, Douglas Rosene, W Evan Johnson, Karen Szumlinski, Camron Bryant

Boston University School of Medicine, Boston, Massachusetts, United States

Background: In humans, sensitivity to the subjective and physiological responses to amphetamine can be explained, in part, by heritable genetic factors that may underlie risk/ resilience toward substance abuse. We recently identified Hnrnph1 (heterogeneous nuclear ribonucleoprotein $\mathrm{H} 1$ ) as a quantitative trait gene for the locomotor stimulant properties of methamphetamine (MA). Here, we wished to test the hypothesis that Hnrnph1+/- mice also exhibit reduced MA reward and reinforcement. Furthermore, based on our previous transcriptome analysis of the QTL suggesting a deficit in midbrain dopaminergic neuron development, we examined dopaminergic neuronal innervation of the dorsal and ventral striatum as a neuroanatomical mechanism underlying reduced MA sensitivity.

Methods: Extending Hnrnph1 to MA addiction-relevant behaviors, we conducted conditioned place preference (CPP) and oral drug self-administration (SA) assessments in Hnrnph1+/- mice. CPP consisted of baseline preference assessment (Day 1), alternating saline and MA training (Days 2-5), consolidation (Days 6-7), and final MA preference assessment (Day 8). Oral SA consisted of MA training at low (5-80 mg/L) and high (160-400 mg/L) doses of drug for 4-5 days per dose. To test the hypothesis that altered sensitivity to MA reward/reinforcement was caused by changes in dopaminergic innervation of the striatum, we conducted immunohistochemical analysis of tyrosine hydroxylase (TH) or hnRNP $\mathrm{H}$ in the mesocorticolimbic circuit.
Results: Hnrnph1+/- mice showed a rightward shift in the inverted U-shaped MA dose-place preference curve, whereby they were less sensitive to low dose reward $(0.5 \mathrm{mg} / \mathrm{kg}$, i.p.) and more sensitive to high dose reward (2 $\mathrm{mg} / \mathrm{kg}$, i.p.), relative to wild-type mice. Interestingly, MA intake $(\mathrm{mg} / \mathrm{kg} /$ day) in Hnrnph1+/- mice was significantly higher at low doses $(80 \mathrm{mg} / \mathrm{L})$ and significantly lower at high doses (160$400 \mathrm{mg} / \mathrm{L}$ ). In parallel with changes in intake, Hnrnph1+/mice also presented significantly fewer active nose-pokes for MA delivery at high MA doses. IHC analysis of TH in Hnrnph1+/- mice indicated a pronounced increase in dopaminergic innervation in the ventral striatum. Additionally, hnRNP $\mathrm{H}$ was expressed pan-neuronally throughout the adult prefrontal cortex and striatum, and excluded in glia.

Conclusions: These studies provide novel evidence that Hnrnph1 deletion perturbs catecholaminergic innervation and/or catecholaminergic expression in the striatum that is associated with profound changes in MA reward and intake. Keywords: Methamphetamine, Addiction, Genetics.

Disclosure: Nothing to disclose.

\section{T262. miR-132 in the Nucleus Accumbens Regulates Cocaine Intake}

Alexander Smith*, Purva Bali, Paul Kenny

Icahn School of Medicine at Mount Sinai, New York, New York, United States

Background: Chronic cocaine use results in maladaptive neuroplasticity that underlies development of compulsive drug seeking and vulnerability to relapse, even after protracted abstinence. Targeting this plasticity is seen as a potential point of pharmacotherapeutic intervention. Extensive experimental efforts have shown that dopaminergic and glutamatergic plasticity within the striatum following extended access to cocaine self-administration contributes to escalation of cocaine intake and development of compulsivelike drug seeking behavior. MicroRNAs are small ( 21-23 nucleotide) noncoding RNAs that bind to complementary regions of 3' UTRs in target mRNAs to regulate their stability and translation. Many microRNA are enriched in neurons and exhibit unique regional expression patterns. MicroRNA-132 and -212 are transcribed from the same gene, and are both enriched in the striatum. It was recently established that miR-212 plays a key role in regulating the motivational properties of cocaine. Specifically our laboratory has shown that miR-212 expression is increased in dorsal striatum of rats with extended access to cocaine. Moreover, virus-mediated overexpression of miR-212 in striatum dramatically decreased cocaine intake in rats with extended access to the drug. Conversely, antisense oligonucleotide-mediated knockdown of miR-212 in striatum increased cocaine intake in rats under extended access conditions. miR-132 and miR-212 have identical seed regions (i.e. nucleotides 2-8 that predict target binding), and thus have identical predicted targets. However, our laboratory has generated data showing that they have differential effects on mRNA and protein expression, indicating that non-seed region differences may confer diverse biological roles for these two miRNA. A particularly interesting target that is differentially affected by miR-132/212 is cylindromatosis 
(CYLD), a K63 deubiquitinase that regulates signaling of the IkK kinase and thus NF-kB transcription factor activity.

Methods: For in vitro experiments, HEK293 cells were used to determine the effects of miR-212 and miR-132 on CYLD mRNA and protein expression, using qPCR and western blots, respectively. For in vivo experiments, male Wistar rats were implanted with chronic indwelling catheters and intracranial guide cannulae, and then trained to selfadminister cocaine in the presence of a light conditioning cue. Following acquisition of stable cocaine selfadministration $50 \mathrm{pmol}$ of a locked nucleic acid (LNA) antisense against miR-132 was injected into the nucleus accumbens one hour prior to beginning self-administration for three consecutive days. CYLD expression following LNAanti-miR-132 was examined using qPCR.

Results: We show that miR-132 significantly decreases CYLD mRNA and protein expression in cell culture, while miR-212 does not. We have also validated that in vivo LNAanti-miR-132 increases CYLD mRNA in the rat striatum. Furthermore, while we previously showed that LNA-antimiR-212 increased cocaine self-administration, here we show that LNA-anti-miR-132 in the nucleus accumbens decreases cocaine intake during restricted access cocaine selfadministration.

Conclusions: These data bring to light differential effects of two miRNA that are in the same cluster, and have identical seed regions. This indicates that non-seed region regulation of miRNA-mRNA interaction confers biologically relevant differential effects of these two miRNA. Further experimentation is ongoing to examine the cellular effects of CYLD, focusing on its regulation of NF-kB signaling, as well as GluA1 insertion.

Keywords: Cocaine Addiction, MicroRNA, Epigenetics. Disclosure: Nothing to disclose.

\section{T263. Stress and Chronic Ethanol Interactions on Drinking and Cognitive Contro}

Ellen Rodberg, Carol den Hartog, David Moorman, Elena Vazey*

University of Massachusetts Amherst, Amherst, Massachusetts, United States

Background: Stress is a risk factor implicated in the transition from initial alcohol drinking to dependence, as well as relapse following abstinence. Ethanol consumption is influenced by the intersection of genetic and environmental factors. In particular, a history of repeated cycles of intoxication and withdrawal reliably escalates drinking in rodents. Additionally, chronic stress increases ethanol consumption in rodents with a history of ethanol dependence. Chronic exposure to stress or alcohol can drive neuroadaptations in areas such as prefrontal cortex (PFC) and locus coeruleus (LC), disrupting cognition and contributing to alcohol use disorders. Here we examined interactive effects of chronic ethanol exposure and stress on volitional ethanol drinking and prefrontal dependent cognition.

Methods: Adult male C57BL/6J mice were trained to drink ethanol $(15 \%, \mathrm{v} / \mathrm{v})$ on a $1 \mathrm{hr} /$ day 1 -bottle choice schedule until a baseline was established. Mice were exposed to weekly cycles of chronic intermittent ethanol (CIE) or air-control vapor exposure (Air), followed by test cycles of $1 \mathrm{hr} /$ day ethanol drinking. Mice were split into non-stress control (NS) or stress groups. Stress groups received 10 minutes of forced swim stress (FSS) 4 hours before each drinking test. This schedule produced four experimental groups: control, Air/NS; ethanol-dependent no stress, CIE/NS; nondependent stress, Air/FSS; or ethanol-dependent stress, CIE/FSS. Prefrontal dependent cognition was assessed using object/context recognition and attentional set shifting. After testing brains were collected for histological analysis of neuronal activity using immunohistochemistry for c-Fos and DeltaFosB.

Results: CIE/FSS mice escalated ethanol intake faster than CIE/NS during CIE cycles one and two. CIE/FSS mice consumed more ethanol than Air/NS across all test cycles. In the object/context recognition task, CIE/FSS mice performed at chance, indicating that stress and ethanol interactions disrupted context specific recognition memory. In the attentional set shifting task, CIE/FSS mice required more trials to reach criterion during the extradimensional shift than all other groups, indicating that combined chronic ethanol and stress impaired behavioral flexibility. We are currently analyzing neuronal signaling in PFC and LC to characterize circuit changes underlying this transition.

Conclusions: Together, these findings show that previous ethanol exposure and stress escalates drinking and disrupts normal cognitive functions. This "double-hit" may facilitate transitions to alcohol dependence.

Keywords: Behavioral Flexibility, Prefrontal Cortex, Alcohol Dependence.

Disclosure: Nothing to disclose.

T264. How Does Baclofen Affect the Subjective and Physiological Responses to Alcohol Cues and Drinking? A Human Laboratory Study in Anxious Alcohol-Dependent Individuals

Mehdi Farokhnia*, Melanie Schwandt, Mary Lee, Lisa Farinelli, Jared Bollinger, Jonathan Amodio, David Spero, Thomas Lionetti, Lorenzo Leggio

National Institutes of Health, Bethesda, Maryland, United States

Background: The GABAergic system plays a key role in the neurobiology of alcohol consumption, and has received considerable attention as a potential target for medication development for alcohol use disorder. Baclofen, the prototypic GABA-B receptor agonist, has been investigated as a promising pharmacotherapy; however, not all patients with alcohol dependence respond to this medication. Different studies suggest that baclofen might be particularly efficacious in those alcoholic patients suffering from high levels of anxiety. Furthermore, the biobehavioral effects of baclofen are not well understood. In the present study, our goal was to investigate the subjective and physiological effects of baclofen in relation to alcohol drinking and cue-induced craving in a population of anxious alcohol-dependent individuals.

Methods: This was a between-subject, double-blind, placebocontrolled, randomized human laboratory study. Non- 
treatment seeking alcohol-dependent individuals with high anxiety (Spielberger State Trait Anxiety Inventory - trait version score $\geq 40$ ) were randomized to receive baclofen (30 $\mathrm{mg} /$ day) or placebo. Thirty-nine individuals were enrolled and thirty-four participants completed the study. After taking the study medication for at least a week, an experimental session was conducted in a private bar-like laboratory room. This session was a combination of two procedures: alcohol cue-reactivity followed by alcohol selfadministration. During the alcohol cue-reactivity session, participants were exposed to visual, tactile, olfactory and proprioceptive stimuli associated with the beverage in a water trial followed by two consecutive alcohol trials. Subjective craving for alcohol, as well as cue-induced physiological changes were assessed. For the alcohol selfadministration session, forty minutes after having a fixed priming drink which was designed to raise the breath alcohol concentration (BrAC) to $0.03 \mathrm{~g} / \mathrm{dl}$ (alcohol challenge phase), more alcohol was presented in the form of two trays with four mini-drinks each. During this two-hour period, participants could choose to drink any or all glasses (free choice phase) and three dollars was provided as an alternative reinforcer for not drinking each mini-drink. Total amount of alcohol consumed during the free choice phase was recorded as the primary outcome; alcohol craving, subjective response to alcohol drinking, BrAC, mood and anxiety symptoms were also evaluated.

Results: No significant medication effect was found on the outcomes assessed during the alcohol cue-reactivity session (p's $>0.05$ ). Similarly, there was no significant effect for the medication group on the total amount of alcohol consumed during the free choice phase $(p>0.05)$. However, there was a significant medication $\times \max$ alcohol challenge $\mathrm{BrAC}$ interaction effect $[\mathrm{F}(1,25)=5.22, p=0.03]$; while the placebotreated patients showed a trend-level positive correlation between their max BrAC during the alcohol challenge phase and the total amount of alcohol consumed during the free choice phase $(\mathrm{R} 2=0.22, p=0.06)$, this association was blunted in the baclofen group ( $\mathrm{R} 2=0.08, p=0.25)$. Notably, there was no significant medication effect on the BrAC, indicating no change in the pharmacokinetics of alcohol $(p>0.05)$. There were significant main effects for baclofen in increasing subjective effects of alcohol related to 'feeling intoxicated' during both the alcohol challenge $[\mathrm{F}(1$, $26.3)=6.02, p=0.02]$ and free choice $[\mathrm{F}(1,26.3)=4.92$, $p=0.03$ ] phases, as well as 'feeling high' during the free choice phase $[\mathrm{F}(1,28.6)=4.36, p=0.04]$. Also, baclofentreated participants reported significantly lower scores on the Fatigue/Inertia factor of the Profile of Mood States compared to the placebo group $[\mathrm{F}(1,35.2)=8.70, p=0.005]$. As for the physiological data, baclofen-treated individuals had significantly lower heart rates during both the alcohol challenge $[\mathrm{F}(1,30.5)=9.45, p=0.004]$ and free choice $[\mathrm{F}$ $(1,32.5)=17, p<0.001]$ phases, as well as a trend-level higher mean arterial pressure during the alcohol challenge phase $[\mathrm{F}(1,26.8)=4.12, p=0.05]$.

Conclusions: The results of this human laboratory study suggest that baclofen modulates some of the behavioral and physiological effects of alcohol without affecting alcohol pharmacokinetics. These findings suggest that baclofen, combined with alcohol, may result in an amplification of alcohol effects, which may in turn lead to reduced alcohol drinking. By contrast, baclofen does not seem to reduce cueinduced craving for alcohol. Future larger studies are needed to better understand baclofen's mechanisms of action in relation to alcohol seeking behaviors.

Keywords: Baclofen, Alcohol, Human Laboratory Study, Biobehavioral Effects, Subjective Response.

Disclosure: Nothing to disclose.

\section{T265. Academic Achievement Based on Neuroimaging, Measures of Initiation of Cannabis and Alcohol use, and Prior Performance}

\author{
Alejandro Meruelo*, Norma Castro, Susan Tapert \\ University of California, San Diego, San Diego, California, \\ United States
}

Background: Academic performance has important bearings on scholastic advancement, including the opportunity to attend college, graduate school, and other career-determining educational programming. The role of substance use and brain maturation in predicting academic performance have not be extensively explored. Memory, attention, and planning have been thought to influence success in high school (Jung and Haier. 2007; Shaw. 2007). However, the specific brain regions involved in predicting academic performance have not been as carefully explored. Cannabis use has been associated with poorer academic performance, higher rates of high school dropout, and reduced socioeconomic status and employability (Henry et al, 2007; Schulenberg et al, 1994; Paulson et al,1990; Mensch and Kandel. 1988; Bryant et al, 2003; Hawkins et al, 1992). Though smaller studies have been conducted, it is not yet known whether initiation of cannabis use is associated with poor academic performance, or if those doing poorly are more likely to engage in cannabis use. Similarly, alcohol use has been reported to be associated with decreased years of schooling and dropout rates (Chatterji. 2006; Chatterji and DeSimone. 2005; Cook and Moore. 1993; Gil-Lacruz. 2007). Conflicting studies have suggested such effects are minimal and not significant (Evans. 2003; Koch and Ribar. 2001). Similarly, it is not clear whether initiation of alcohol use is associated with poor academic performance, or if those doing poorly are more likely to engage in alcohol use. Here we address these gaps.

Methods: We analyzed brain MRI morphometry metrics (surface areas, cortical thicknesses, and subcortical volumes) in early adolescence (age 12-14 years) as predictors of academic performance (GPA) over high school using a naïve Bayesian classifier approach with $n=170$ subjects. We also examined metrics of middle school academic performance (GPA at 7th, 8th, and 9th grade) and initiation of cannabis and alcohol use (age of first use, number of drug use days, length of use, use during junior year, any use) in predicting GPA. Subjects were divided into high (GPA $\geq 3.54 ; n=87$ ) and low (GPA $<3.54 ; n=83$ ). Covariance analysis was performed to look at subject demographics. We examined predictive features from the 343 neuroimaging parameters available (surface areas, cortical thickness, and subcortical volumes), and 36 metrics of middle school academic performance/initiation of alcohol and cannabis use. We applied several algorithms for selection and reduction of 
attributes, in addition to performing principal component analysis for the latter 36 metrics.

Results: Cortical thickness measures performed better than surface areas or subcortical volumes as predictors. We identified 15 cortical thickness regions most predictive of academic performance, with a sensitivity of 0.57 and specificity of 0.73 using 10 -fold cross validation. Three new and previously unidentified predictive regions were found: the left hemisphere fusiform, bilateral insula, and left hemisphere paracentral regions. Independent t-test analyses on these 15 features identified 10 that showed significance at the $P<0.05$ level. We applied the same 4 algorithms for selection and reduction of 36 attributes. We also used principal components analysis using metrics of middle school academic performance and of initiation of cannabis and alcohol use (age of first use, number of drug use days, length of use, use during junior year, any use) to account for the greatest variance in GPA at the junior level of high school. Prior academic performance predicted high school academic performance less well than cortical thickness features but better than measures of drug use, with a sensitivity of 0.57 and specificity of 0.56 . Beyond prior GPA, age of alcohol use onset predicted academic performance better than any other measures of drug use, with a sensitivity of 0.54 and specificity of 0.52 .

Conclusions: Early cortical thickness metrics predicted academic performance at the junior and senior level of high school better than 7th, 8th, and 9th grade GPA, which predicted better than age of alcohol use onset, which predicted better than any other substance use metrics for cannabis, alcohol, or other drug use. Cortical thickness metrics reflect a more accurate snapshot of adolescent brain development trajectory that predicts performance better than middle school GPA based on easier coursework than encountered in high school. Prior performance has often been found to be a reasonable predictor of future performance; e.g., SAT scores and college performance. Nonetheless, it was notable that middle school GPA was more predictive of high school academic performance than any metrics of substance use (cannabis, alcohol, or other drug use). Of all substance variables, age of onset of alcohol use was found to be most predictive of high school academic performance. That alcohol over cannabis use predicted performance better is consistent with findings that alcohol use has longer lasting effects on school performance and impact of cannabis on school performance is less clear. Compared to alcohol, much higher levels of cannabis and more frequent use are required to affect similar deterioration in school performance. Our findings suggest a basis for improving current screening practices for early alcohol and cannabis use, and those struggling in school at a young age. Keywords: Magnetic Resonance Imaging, Artificial Learning, Adoelscent Academic Performance, Cannabis Use, Alcohol Use.

Disclosure: Nothing to disclose.
T266. Initial Response to Intranasal Nicotine Young Adults With and Without Attention Deficit Hyperactivity Disorder

Scott Kollins ${ }^{\star}$, Francis McClernon, Joseph English, Denny Hood, Kenneth Perkins

\section{Duke University School of Medicine, Durham, North Carolina, United States}

Background: Individuals with Attention deficit hyperactivity disorder (ADHD) are at increased risk for a range of adverse smoking outcomes, including earlier initiation, faster progression to regular use, heavier smoking/nicotine dependence, more severe withdrawal, and worse cessation outcomes. It has been speculated that nicotine and cigarette use serves a self-medicating function for those with ADHD given the well-documented effects of nicotine on attention and related processes. However, little is known about the behavioral or pharmacological mechanisms that confer risk for smoking outcomes among patients with ADHD, especially early in the process of experimentation. The purpose of the present investigation was to model an initial smoking experience in non-smoking young adults with and without ADHD using a novel intranasal delivery approach. We hypothesized that the subjective and reinforcing effects of nicotine would differ between the groups, thus offering some insight into the processes that might potentially lead to worse smoking outcomes.

Methods: Ninety-four young adults (mean age $=21.8$ years) with $(n=31)$ and without $(n=63)$ a verified clinical diagnosis of ADHD were enrolled in the experimental protocol. Participants were excluded if they reported having ever smoked more than 2 puffs of a cigarette in their lives and smoking status was verified biologically (via urinary cotinine and expired breath $\mathrm{CO}$ ) and through social contacts. Other exclusionary criteria included presence of any other DSM-V Axis I condition, major medical conditions, and positive urine drug screen for any illicit substances. Following Screening, participants completed 5 experimental sessions. During the first 3, they were exposed to 3 different fixed doses of intranasally administered nicotine $(0.0,0.5,1.0$ $\mathrm{mg}$ ), which were given under blinded conditions and in counterbalanced order. Vital signs, self-reported subjective effects, and neurocognitive measures were collected after each administration. During the last 2 sessions, participants chose to self-administer nicotine or placebo under 2 different conditions - one in which self-administration immediately preceded a cognitively demanding task; and one in which self-administration occurred prior to a low demand condition. Across experimental days, general linear models were used to evaluate the effects of dose and group on a range of outcomes.

Results: The ADHD and Control groups did not differ in terms of estimated IQ, sex, or race/ethnicity. The ADHD group was somewhat younger (20.9 years vs. 22.2 years; $\mathrm{t}=$ $-2.9, p<0.01)$ and age was thus used as a covariate in all analyses. There were dose-dependent increases in heart rate $(\mathrm{F}=3.3, \quad p<0.05)$ across groups. Nicotine also dosedependently increased systolic and diastolic blood pressure across groups, though this effect was not statistically significant. In general, ADHD participants reported a pattern of self-reported subjective effects consistent with greater 
positive effects and lower negative effects of nicotine, compared to non-ADHD participants. There were dosedependent increases in self-reported ratings of "Feel drug" on a Visual Analog Scale, although there were no group differences. ADHD participants reported significantly higher ratings of "Good Effects," "Like Drug" and "Would Like to Take Drug Again", (F-values $>5.8$, $p$-values $<0.02)$. There was also a trend for ADHD participants to report lower ratings of "Bad Effects" $(\mathrm{F}=2.9, p=0.09)$. On a validated measure of Initial Reactions to nicotine, ADHD participants reported significantly higher ratings of Pleasant Sensations $(\mathrm{F}=16.0, p<0.001)$ and a trend toward higher ratings of Dizziness $(\mathrm{F}=3.2, p=0.08)$. During self-administration sessions, there was a trend for a main effect of group and a condition $\mathrm{x}$ group interaction. This pattern was characterized by the ADHD generally choosing to self-administer nicotine more than the Control group across both conditions. There was a significant difference between the groups during the low demand conditions with the ADHD group choosing to take more nicotine $(\mathrm{t}=2.5, p<0.02)$.

Conclusions: These findings demonstrate for the first time that a clinical group at risk for adverse smoking outcomes exhibits differential response to initial exposure to nicotine. Across measures, individuals with ADHD self-reported greater positive responses to nicotine. Moreover, individuals with ADHD self-administered higher levels of nicotine, especially under conditions of low cognitive demand. Given that initial reactions to smoking experiences have been shown to be predictive of later smoking behavior, these findings have substantial implications for prevention efforts. Young people with ADHD experience a qualitatively different experience to initial exposure to nicotine and under some conditions, choose to self-administer nicotine significantly more than their non-ADHD peers. These results highlight the importance of preventing even isolated experimentation with nicotine/smoking among youth with ADHD.

Keywords: ADHD, Nicotine Addiction, Behavioral Pharmacology.

Disclosure: Akili Interactive: Consulting and Research Support, Self; Alcobra: Consulting and Research Support, Self; Atentiv: Consulting, Self; Ironshore: Consulting and Research Support, Self; Medgenics: Research Support, Self; Arbor: Research Support, Self; Neos: Research Support, Self; Neurovance: Consulting and Research Support, Self; Purdue Pharma: Consulting and Research Support, Self; Rhodes: Consulting and Research Support, Self; Shire: Consulting and Research Support, Self; SK Life Science: Consulting, Self; Sunovion: Consulting and Research Support, Self; Tris: Consulting and Research Support, Self.

\section{T267. Differential Cerebrovascular Perfusion and Metabolism After Prolonged Exposure to Cannabis in Male and Female Users}

Francesca Filbey*, Sina Aslan, Hanzhang Lu

The University of Texas at Dallas, Dallas, Texas, United States

Background: The primary psychoactive ingredient in cannabis, $\delta$-9-tetrahydrocannabinol (THC), relaxes arterial walls resulting in lower blood pressure and increased blood flow to tissues. In the brain, THC binds to ubiquitous cannabinoid receptors (CB1), which are present in arterial tissue 1 and regulate the microvascular environment via dose-dependent dilation of cerebral arterioles 2. Despite the known cardiovascular effects of $\delta$-9-tetrahydrocannabinol (THC), its long-term effects on brain perfusion and metabolism remain largely unexplored. The aim of this study was to evaluate differences in cerebrovascular function in chronic cannabis users relative to non-using controls using global and regional resting cerebral blood flow (CBF), oxygen extraction via oxygenation of blood in superior sagittal sinus, and cerebral metabolic rate of oxygen (CMRO2) as a marker of aggregated neural activity.

Methods: 73 cannabis users and 101 non-users were recruited for this study. Cannabis users were recruited based on self-reported history of regular cannabis use with a minimum of 5000 lifetime occasions, as well as daily use over the preceding 60 days. Verification of cannabis use was conducted via presence of THC metabolites during gas chromatography/mass spectrometry. The non-using controls were recruited based on the absence of daily marijuana use at any period in their lifetime, in addition to no current illicit drug use in the past 60 days. MRI scans were performed on a 3 Tesla MR system. A body coil was used for radiofrequency transmission and an 8-channel head coil with parallel imaging capability was used for signal reception. We used several MRI techniques to investigate brain metabolism at rest: a time-of-flight angiogram to visualize the internal carotid arteries and vertebral arteries, a phase contrast MRI to measure whole brain blood flow by positioning the imaging slab perpendicular to internal and vertebral arteries3, T2-Relaxation-Under-Spin-Tagging (TRUST) MRI to assess blood oxygenation of the brain via superior sagittal sinus, and pCASL MRI sequence to measure regional blood flow of the brain. Last, a high resolution T1 weighted image was acquired as an anatomical reference. Duration of cannabis use, total number of lifetime occurrences, and $\mathrm{THC} / \mathrm{Cr}$ were collected as measures of cannabis use. Voxel wise correlation analyses were conducted to determine correlations between cerebrovascular function with measures of cannabis use and cognition while controlling for age and gender. For voxel wise correlation, we applied a clusterdefining magnitude threshold of $p<0.02$ with minimum volume of 341 voxels $(2,728 \mathrm{~mm} 3)$ and for main effect analysis we applied a $p<0.005$ with minimum volume of 156 voxels $(1,248 \mathrm{~mm} 3)$ to achieve FWE cluster correction of $p<0.05$.

Results: There were no significant differences between the groups on whole brain blood flow $(p=0.18)$. However, there was higher regional $\mathrm{CBF}$ in the users compared to non-users in the right pallidum/putamen and lower regional CBF in left angular cortex (BA 39/40) (FWE cluster corrected $<0.05$, $\mathrm{k} \geq 1,248 \mathrm{~mm} 3$ ). The oxygenation extraction fraction (via TRUST) was trending higher in users compared to non-users $(p=0.067)$. This was inversely correlated with total $\mathrm{CBF}$ $(r=-0.60$ and $p<0.001)$ for all participants. No significant difference between the groups was found with $\mathrm{CMRO} 2$ although there was a positive correlation between $\mathrm{CMRO} 2$ and levels of THC metabolites in the users $(p=.05, \mathrm{r}=.33)$. Secondary analyses revealed significant 'group $x$ sex' interactions such that male users had higher oxygenation 
extraction fraction compared to male non-users $(p=0.03)$, whereas no significant difference was found between the female users and non-users $(p=0.58)$. CMRO2 was trending lower in the male users compared to male controls $(p=0.08)$. Male users had higher $\mathrm{CBF}$ in the right pallidum/putamen region whereas controls had higher CBF in left middle/ superior frontal cortex (BA9) (FWE cluster corrected $<0.05$, $\mathrm{k} \geq 1,248 \mathrm{~mm} 3$ ). Female controls showed higher $\mathrm{CBF}$ in the right angular cortex compared to users (FWE cluster corrected $<0.05, \mathrm{k} \geq 1,248 \mathrm{~mm} 3$ ).

Conclusions: Because CBF and brain function are closely coupled, it is likely that widely observed alterations in brain functional activation may be partly explained by latent cerebrovascular changes. These findings demonstrate significant cerebrovascular effects as a result of prolonged exposure to cannabis that is both region- and sex-specific that should be considered in both research and clinical applications related to THC.

Keywords: Cannabis, THC, MRI, Cerebral Blood Flow. Disclosure: Nothing to disclose.

T268. Dorsal Anterior Cingulate Glutamate is Associated With Engagement of the Default Mode Network During Exposure to Smoking Cues

Amy Janes*, Jennifer Betts, J Eric Jensen, Scott Lukas

Harvard Medical School, Belmont, Massachusetts, United States

Background: When exposed to smoking cues, nicotine dependent individuals activate brain regions overlapping with the default mode network (DMN), a network of regions involved in internally-focused cognition. However, the DMN has not been directly evaluated in the context of smoking cue reactivity. The salience network $(\mathrm{SN})$, which includes the dorsal anterior cingulate cortex (dACC), is thought to interact with the DMN and aids in directing attention toward salient internal or external stimuli. One possibility is that neurochemical variation in $\mathrm{SN}$ regions such as the dACC impact DMN reactivity to personally relevant stimuli such as smoking cues. This is consistent with emerging evidence suggesting an association between midline cortical glutamate (Glu) and activity in brain regions overlapping with the DMN.

Methods: Data analysis included 18 nicotine-dependent individuals in our primary cohort and $N=14$ in our replication sample. Using functional magnetic resonance imaging (fMRI) whole brain activation to the smoking vs. neutral contrast was compared to the DMN region of interest (ROI) defined by Smith et al. (2009) by calculating the cross correlation between these maps. To more directly evaluate how the DMN responds to smoking $>$ neutral cues, betaweights from the DMN ROI were extracted using FSL's featquery from each individual. The Pearson's correlation coefficient was then calculated between DMN beta-weights and dACC Glu/Creatine as measured by magnetic resonance spectroscopy for each cohort to assess the association between these measures.

Results: There was substantial overlap between the brain map generated by the whole brain smoking vs. neutral contrast and the DMN ROI as defined by Smith et al. $(r=0.46)$. Further, activation within the DMN ROI was significantly less suppressed during exposure to smoking relative to neutral cues $(t=5.2 p<0.001)$, and also there was a positive association between $\mathrm{DMN}$ reactivity to smoking relative to neutral cues and dACC Glu $(r=0.56, p<0.02)$. This finding was confirmed in the independent replication cohort $(r=0.64, p<0.02)$.

Conclusions: The current findings confirm that the DMN is less suppressed when smokers view smoking relative to neutral cues, suggesting that smoking cues engage selfrelevant processing. Furthermore, these results indicate that dACC Glu is associated with enhanced DMN engagement when nicotine-dependent individuals are exposed to selfrelevant smoking cues and suggests a neurochemical link between the DMN and SN.

Keywords: Nicotine, Default Mode Network, Glutamate, fMRI, Anterior Cingulate Cortex.

Disclosure: Nothing to disclose. 